$$
\begin{aligned}
& \text { Piny } \\
& 2 \cos \operatorname{sen}
\end{aligned}
$$

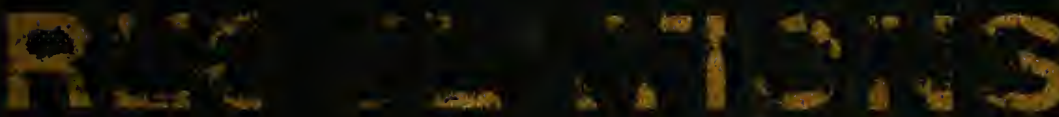

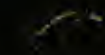

$$
\begin{aligned}
& \text { P.t? } \\
& \text { ADOLPH }
\end{aligned}
$$





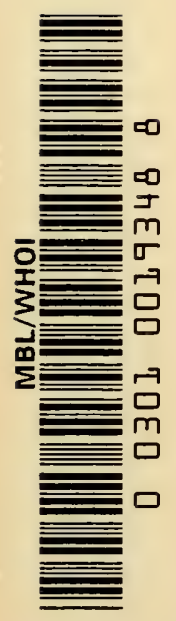





\section{PHYSIOLOGICAL REGULATIONS}

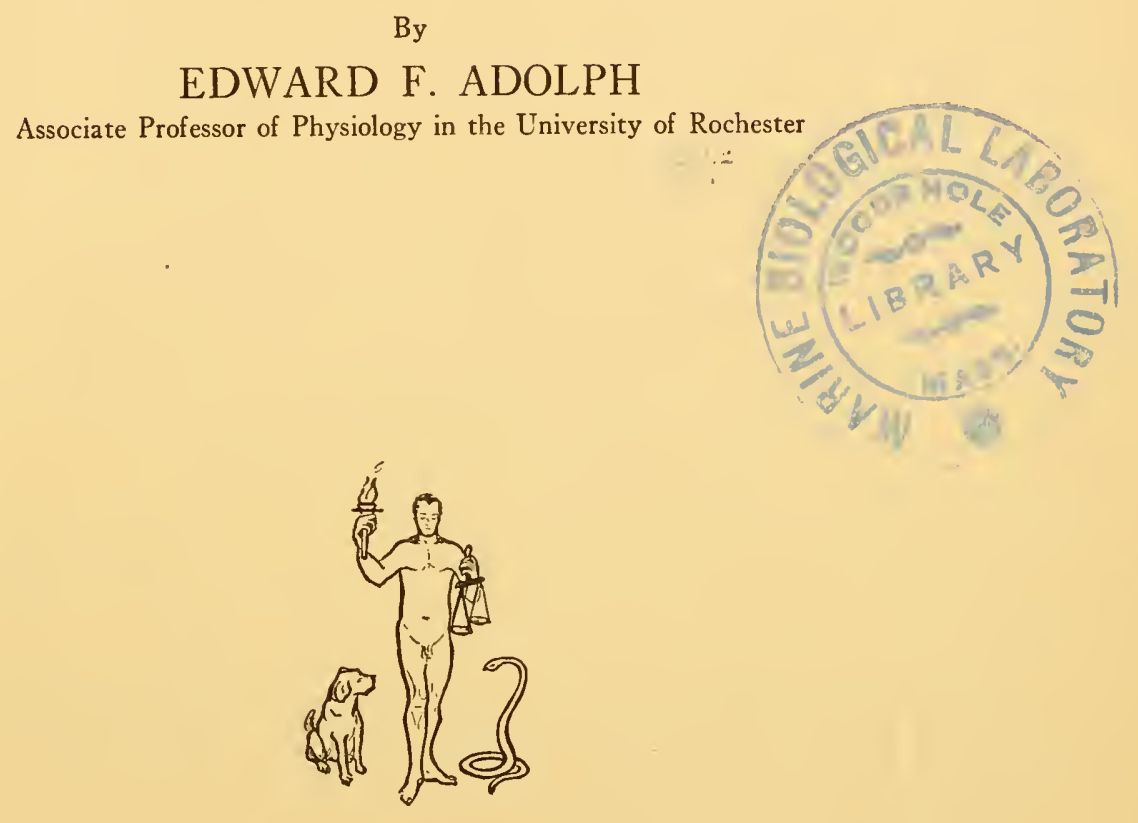

THE JAQUES CATTELL PRESS

LANCASTER, PENNSYLVANIA

1943 
Copyright, 1943, by

THE JAQUES CATTELL PRESS

PRINTED IN U. S. A. THE SCIENCE PRESS PRINTING COMPANY LANCASTER, PENNSYLVANIA 
To the memories of JOHN SCOTT HALDANE 1865-1936 and LAWRENCE JOSEPH HENDERSON 1878-1942

Bold explorers and teachers of a larger physiology Investigators of physiological regulations 
"Life is an example of the way in which an energy-system in its give and take with the energy-system around it can continue to maintain itself for a period as a self-centered, so to say, self-balanced unity. Perhaps the most striking feature of it is that it acts as though it 'desired' to maintain itself."

-C. Sherrington: Man on His Nature, p. 84; Cambridge, 1941. 


\section{PREFACE}

"His body temperature is always the same; if it were not he would be sick." That empirical rule is rediscovered daily. To physiologists the rule is a challenge to seek further relations among bodily functions. How are the specific properties of an organism maintained? What sorts of processes are provided so that the temperature is not upset in a body subjected to twenty-fold increases in heat production and to winter's icy blasts?

Physiological regulations are patterns of processes; the outcome of all those operating characteristics that assure the constancy of a property such as body temperature. The creature's endowments are not merely material; activity is provided continuously. Of all the possible combinations of chemical velocities, physical responses, and organismal behaviors that are possible, those very ones prevail that fit into a scheme of self-perpetuation. This scheme is the object of investigation. The invisible pattern according to which in a given physiological state, whether of action or of rest, these particular velocities are operating and others not, constitutes regulation.

Physiology seems to me more than a science of individual working parts. I found it fun to compare the manners in which a kidney excretes various substances. But then I wondered whether there was some rhyme in the heights of the several thresholds and the hypertonicities of various urines. It next became obvious that measured ingestion saved excretion many a day's work. Evidently excretion is subservient to organism, a kidney to a body. The scale of renal clearances seems utterly arbitrary until one thinks of the millions of body cells that have to tolerate and even transform what the kidneys do not excrete.

At one time I supposed that the pattern of physiological research was fixed once for all. The newcomer merely went into more detail with modified apparatus. Now, I believe that fingers and levers are to be supplemented by concepts. There is no limit to the patterns of physiological investigation, for every concept adds a pattern of search. Physiology is more than a technology, more than information. It develops new aspects at every turn; as long as it lives it will include the unorthodox. The unorthodox of today becomes the standard of tomorrow; let no one make a 
religion of an emphasis. Let not wisdom scoff at strange notions or isolated facts. Let them be explored. For the strange notion is a new vision, and the isolated fact a new clay, possible foundations of tomorrow's science.

Lest anyone shy at the title before he has sampled the book, I may state that it describes interrelations among a limited number of rather familiar facts. It is not a derivation of the imagination, nor an exposition of a hypothesis, nor a development of a nebulous idea. Whatever ideas went into the work were, I think, either substantiated or killed. The presentation that results therefrom takes on an almost mechanical plan consisting of numerical data and of generalization from them. Often the facts seem exciting enough so that no imagery or creed is required. This book is not a survey nor a review; it is a report of an investigation that became too extensive for presentation in a journal. It would be gratuitous in such a work to note what raw materials were omitted, or even what other lessons might be drawn from the same sludge. Let it be supposed, as for Basil Montag'u, that "He puts the facts before us in the full confidence that they will produce on our minds the effect which they have produced on his own" (T. B. Macaulay: Essay on Lord Bacon).

The best evidence that the study of regulations actually helps one to understand plysiology, lies in predictions about organisms. Here is some substance $J$ always present in muscle. That fact per se implies that there is a source and a sink for J. But further, the fact suggests that acquirement of $J$ is probably faster whenever its content tends to diminish. So metabolism is viewed as an intricate pattern of interrelated processes which persists by virtue of the very multiplicity of its quantitative steps. Once the steps have been enumerated, their correlations become self-evident. Truly each relation is new fruit, which would be discovered to be "necessary" to some future brilliant hypothesis, if it were not firmly indicated by present facts.

Those who prefer to take their facts on faith may avoid wading through the presentation of details by reading the concluding section of each chapter. In this way the progress of the inductive development may be partially followed, leading at the close to certain generalizations concerning the functional constitution of organisms. 
I wish to acknowledge the generous encouragement of the late Professor L. J. Henderson, who furnished the environment for the inception of this specific investigation. He took the trouble to read the manuscript shortly before his untimely death. Possibly years ago he and the late Professor J. S. Haldane unwittingly implanted a curiosity about the regulative aspects of physiology. I am grateful for the comradeship of my students who have shared in the laboratory studies concerning water and heat metabolism.

Material aid was accorded the prosecution of this work through various administrative officers of the University of Rochester. The publication is made possible by the School of Medicine and Dentistry of the University.

October, 1942

E. F. Adolph 



\section{ABSTRACT}

This is a monograph recording an investigation in quantitative physiology. Regulations in organisms are maintenances of relative constancies. They are described from suitable data by selected relations of the following sorts:

(1) Variations, especially in the successive values found for one sort of measurement in an individual. These represent the results of all those events that antagonize the occurrence of wider variations.

(2) Changes and exchanges as correlated with excesses over, or deficits under, the control values (contents). These are rates of processes, and express what is done to recover the usual physiological state.

(3) Behaviors that exhibit preferences for environments which either promote or prevent exchanges of diverse components.

Water is chosen as the prototype of component that tends to be in quantitatively regular amounts in living units. Data for the dog show in how far water contents (body weights), intakes, and outputs vary from hour to hour and from day to day; and how the rates of intakes and outputs are modified with each experimentally provided content. The latter relation is investigated both in stationary states of unusual content, and in recoveries from them. Preservation of content may be regarded as a pattern of particular relations among the rates provided in diverse paths of exchange.

Man, frog, and many other species of animals, including several of invertebrates, manifest similar relations in water exchange. Organs, tissues, and cells are also recognized as having the pattern. From these materials the general features common to many living units are formulated. Quantitative differences among species are also ascertained. A variety of other measurements, particularly upon blood and other parts, is correlated with water content, to characterize the physiological states of water excess and deficit. Thus an intensive and comprehensive account of water relations of animals and their tissues results.

Analogous data are set forth concerning other quantities than water. Some of these are heat, glucose, oxygen, carbon dioxide, lactate, frequency of heart beat, and blood pressure. For each a similar pattern of equilibration is found. 
The uniformities and the contrasts among them are pointed out. The general forms of variation in content, of time relations in recovery, and of rates of exchange in relation to excesses and deficits (equilibration) are induced.

Simultaneous equilibrations of two or more such quantities are studied. The interplay of quantities indicates the organism's choices in handling at one time the various items that call for recovery. It is inferred that the organism is a compound of relations among components in mutual adjustment. Descriptive procedures are illustrated that exhibit the multiple relations in comprehensible manners.

These selected relations among data are manifestations of the processes commonly meant by the term physiological regulations. They provide a quantitative means of visualizing what organisms do to maintain constancy not only of composition, but of energies, forces, structures, and functioning. 
Preface

Abstract

Chapter I. Introduction

$\oint$ 1. The project

2. How regulations may be studied ...................................................................................... 4

3. Extensions of the study …………........................................................................................... 7

4. Mode of treatment ................................................................................................................. 8

5. Outline of the investigation ................................................................................................. 11

PART A. WATER RELATIONS OF ANIMALS

Chapter II. Water Exchanges of Dog …………....................................................................... 17

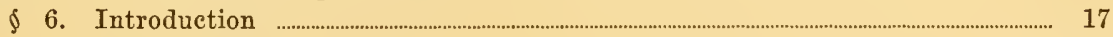

7. Sudden excesses of water ……............................................................................................... $\quad 18$

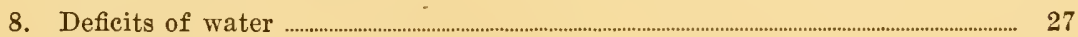

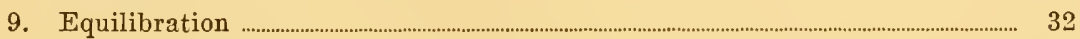

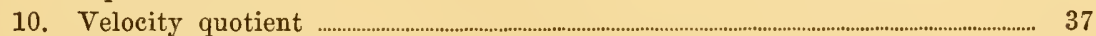

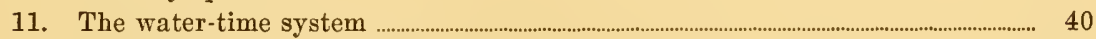

12. Stationary states of excess .................................................................................................. 41

13. Stationary states of defieit .................................................................................................................... $\quad 47$

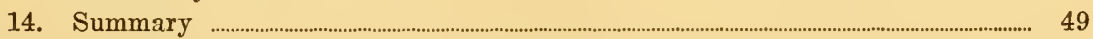

Chapter III. Other Types of Water Inerement (Dog) ................................................................. 50

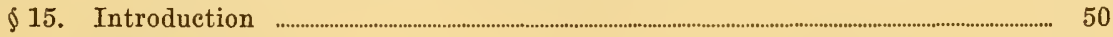

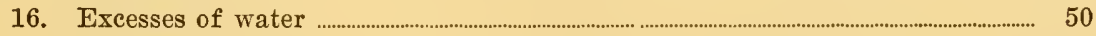

17. Deficits of water ....................................................................................................... 59

18. Distinctions among water increments ......................................................................................... 62

19. Modifications of water content at balance ........................................................................... 68

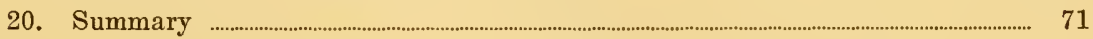

Chapter IV. Variabilities of Water Relations (Dog) ……..................................................................... 73

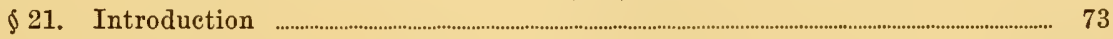

22. Variations of water content ............................................................................................... $\quad 73$

23. Variations of turnovers ....................................................................................................................... $\quad 78$

24. Variations in rates at diverse loads .................................................................................................. 84

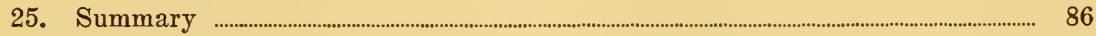

Chapter V. Water Relations of Man .......................................................................................................... 88

\$26. Introduction ............................................................................................................................ 88

27. Single ingestions by mouth ................................................................................................... 88

28. Repeated ingestions by mouth .................................................................................................. 93

29. Water privation ………................................................................................................................................. $\quad 95$

30. Equilibration and recovery ................................................................................................................... 100

31. Comparison with dog ................................................................................................................................. 102

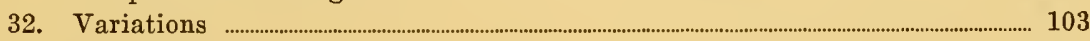

33. Characterizations and tests ...................................................................................................................... 105

34. Diverse types of water load .............................................................................................................. 107

35. Summary ........................................................................................................................................................... 108

Chapter VI. Water Relations of Frog …........................................................................................................ 110

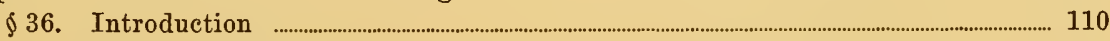

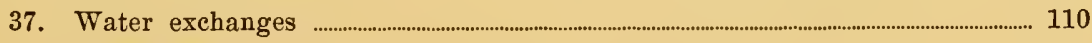

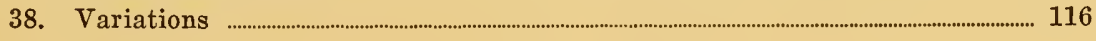


39. Some other types of water load

40. Summary ............................................................................................................................................................ 120

Chapter VII. Water Relations of Other Species ……............................................................................. 122

$\$ 41$. Introduction ........................................................................................................................................ 122

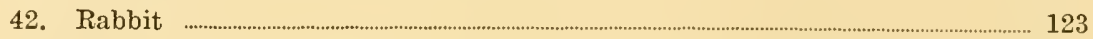

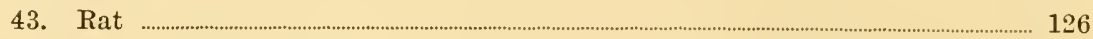

44. Garter snake. Reptiles ...................................................................................................... 132

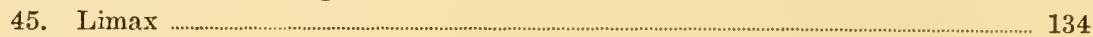

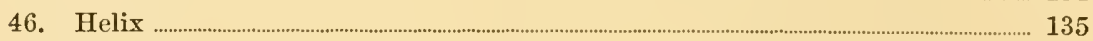

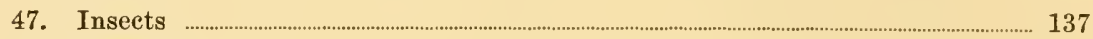

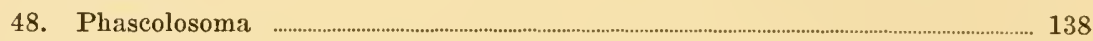

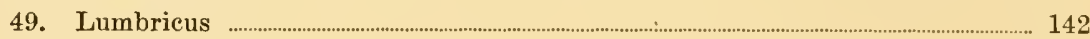

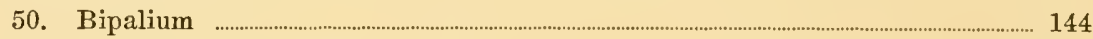

51. Arbacia egg. Echinoderm eggs ......................................................................................... 144

52. Freshwater Zoothamnium ........................................................................................................ 146

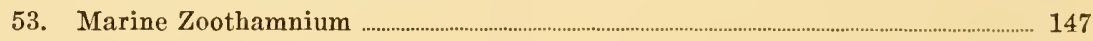

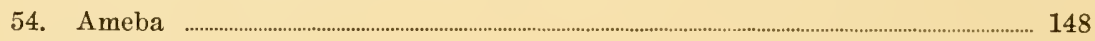

55. Note on plants ................................................................................................................................... 148

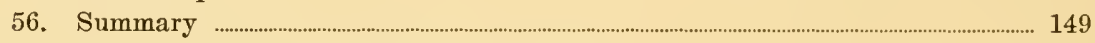

Chapter VIII. Equilibrations in Parts of Organisms ....................................................................... 151

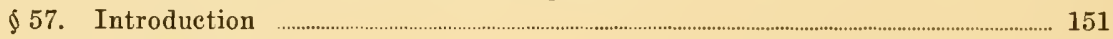

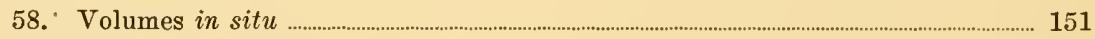

59. Blood and plasma …............................................................................................................................... 153

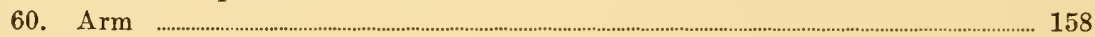

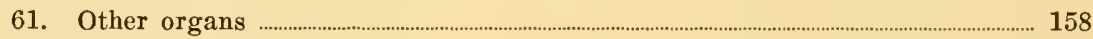

62. Cells and nuclei .......................................................................................................................................... 160

63. Summary of parts in situ ............................................................................................... 160

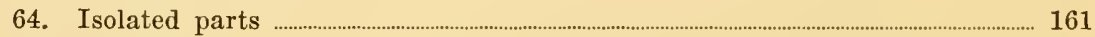

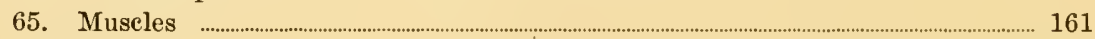

66. Cells ............................................................................................................................................................... 164

67. Summary ................................................................................................................................................. 166

Chapter IX. General Features of Water Exchanges ….................................................................... 168

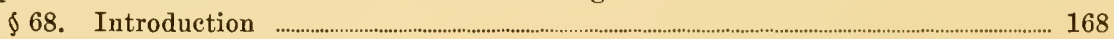

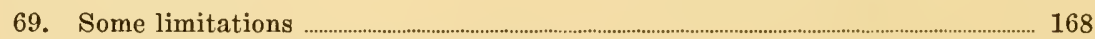

70. Some parameters comparing exchanges …................................................................................. 170

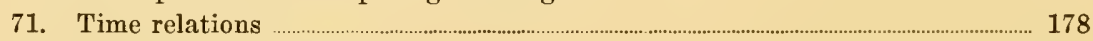

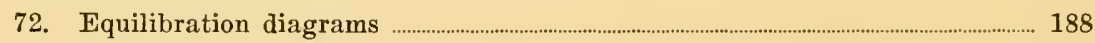

73. Behavior ........................................................................................................................................................... 195

74. Water contents and turnovers ...................................................................................................... 195

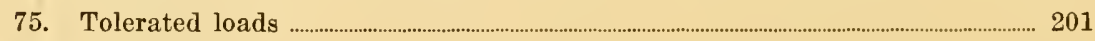

76. Summary .................................................................................................................................................................... 202

Chapter X. Some Other Correlatives of Water Content (Dog) ................................................... 206

\$ 77. Orientation .......................................................................................................................................... 206

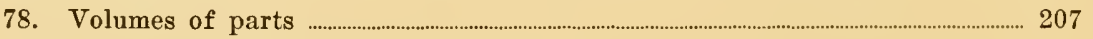

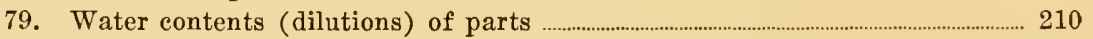

80. Blood and plasma ................................................................................................................................... 213

81. Concentrations of urine ……................................................................................................ 224

82. Concentrations of other body fluids …................................................................................... 225

83. Others compositions .................................................................................................................... 225

84. Correlated metabolisms and behaviors ..................................................................................... 227 
85. Quantitative characterizations of water loads …........................................................ 233

86. Summary ........................................................................................................................................ 236

Chapter XI. Some Other Correlatives of Water Content (in Other Species) .................... 238

§ 87. Introduction ...................................................................................................................................................... 238

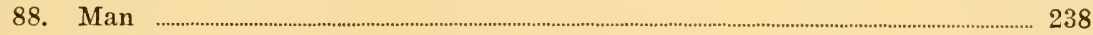

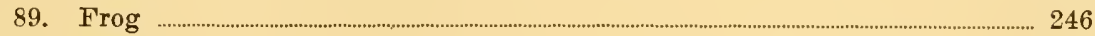

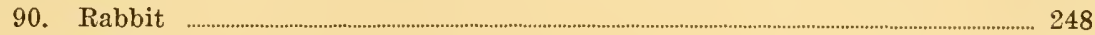

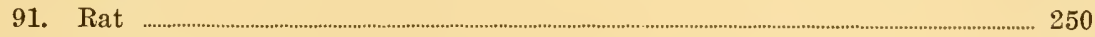

92. Comparisons ............................................................................................................................................ 251

Chapter XII. Further Correlatives of Water Content and Exchanges ................................... 255

§93. Introduction ............................................................................................................................................... 255

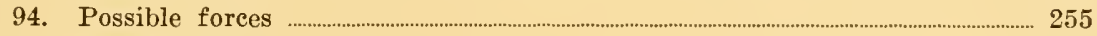

95. Permeability ........................................................................................................................................... 258

96. Body size, age ........................................................................................................................................ 263

97. Temperature ............................................................................................................................................ 275

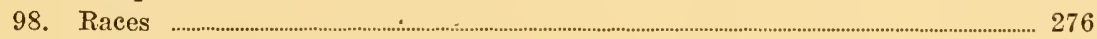

99. Summary …................................................................................................................................................................ 277

Chapter XIII. Water Balances and Exchanges. Recapitulations ........................................... 279

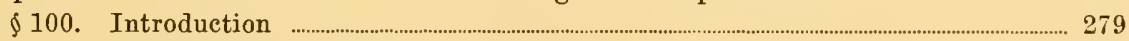

101. Classification of variables …..................................................................................................... 279

102. Scopes of the classes of variables .......................................................................................... 280

103. Interrelations of the variables .................................................................................................... 284

104. Procedures or steps used ............................................................................................................... 285

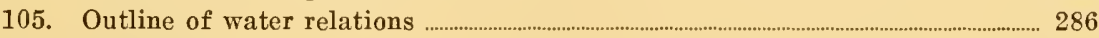

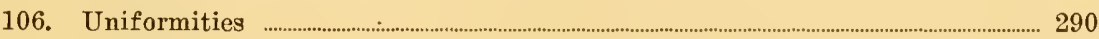

107. Diversities ................................................................................................................................................................... 292

108. Agents and types of load ................................................................................................................... 294

109. General theories of water constancy ................................................................................... 296

110. Summary ......................................................................................................................................... 297

\section{PART B. REGULATIONS OF SEVERAL COMPONENTS} AND IN GENERAL

Chapter XIV. Heat

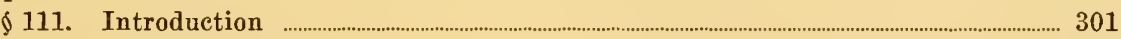

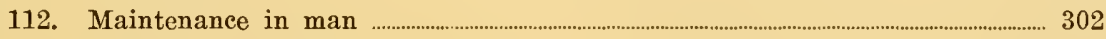

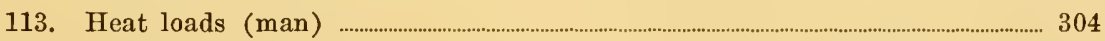

114. Recoveries (man) …................................................................................................................ 307

115. Heat exchanges of rabbit ........................................................................................ 312

116. Comparisons among species ................................................................................................................. 318

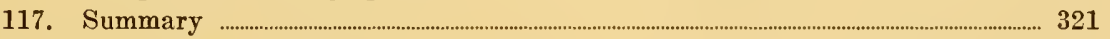

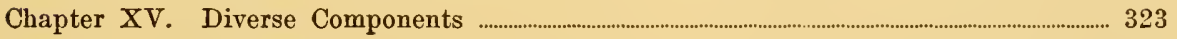

118. Total substance ................................................................................................................................................ 323

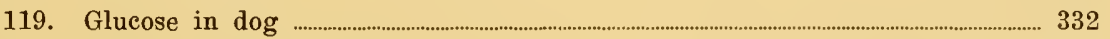

120. Carbon dioxide in man ……..................................................................................................... 340

121. Oxygen in man ........................................................................................................................................... 343

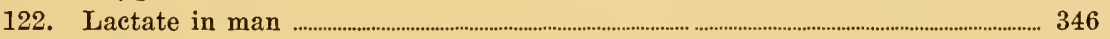

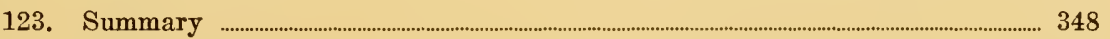

124. Heart frequency ..................................................................................................................................... 350

125. Arterial blood pressure ………………......................................................................................................... 352

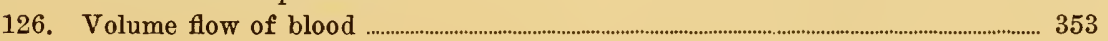


PAGE

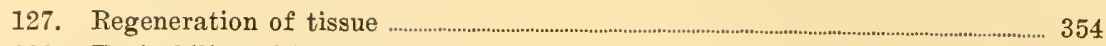

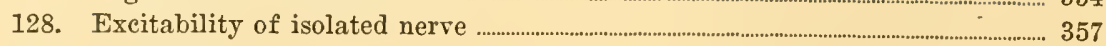

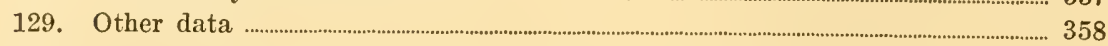

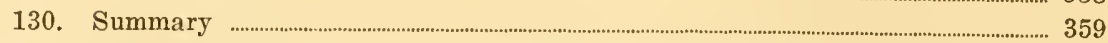

Chapter XVI. Uniformities and Comparisons among Components ............................................ 361

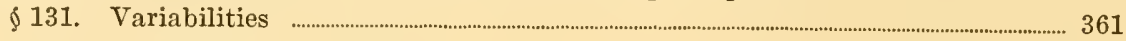

132. Behavior and maintenance ........................................................................................................ 364

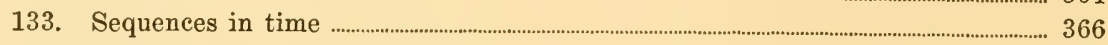

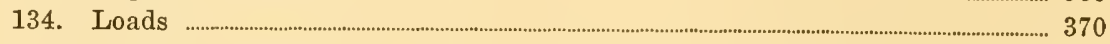

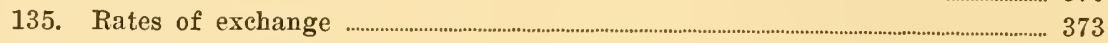

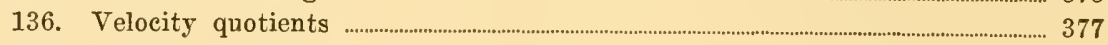

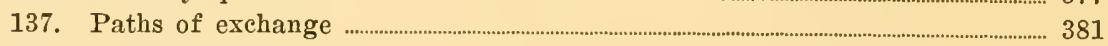

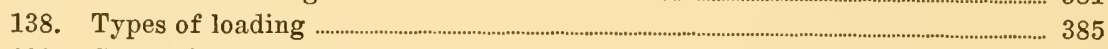

139. Comparison of kinetic parameters ............................................................................................... 386

140. Changes in tissues, other metabolisms, etc. ........................................................................ 389

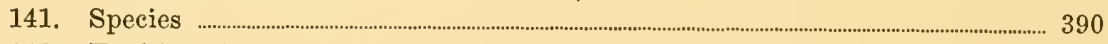

142. Equilibration diagrams ................................................................................................................... 394

143. Summary of variables studied ….......................................................................................... 396

144. Types of relations ................................................................................................................................... 398

Chapter XVII. Interrelations among Components …............................................................................4.400

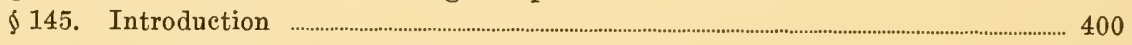

146. Heat load and water load in man ...................................................................................................... 400

147. Several components during physical exercise (man) ....................................................... 405

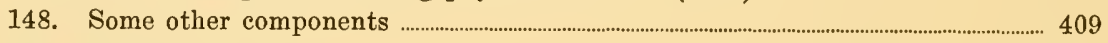

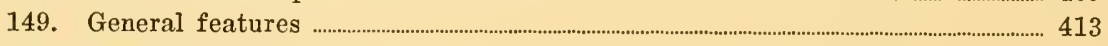

150. Meanings of interrelations .................................................................................................................. 419

Chapter XVIII. Choosing Physiological Variables ......................................................................................... 422

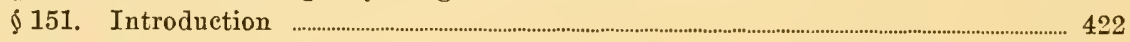

152. Present procedures ................................................................................................................................................ 422

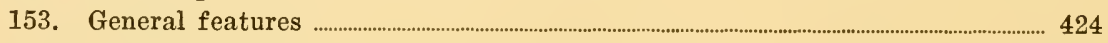

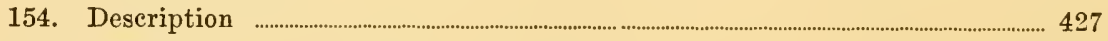

155. Other modes of procedure …........................................................................................................... 431

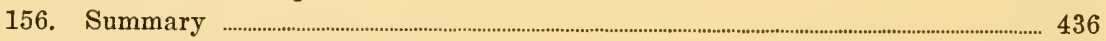

Chapter XIX. Physiological Regulations ........................................................................................................... 437

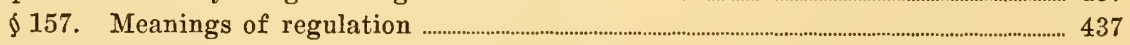

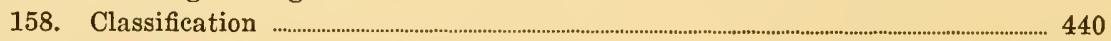

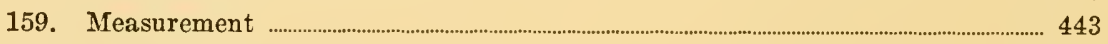

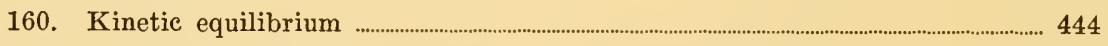

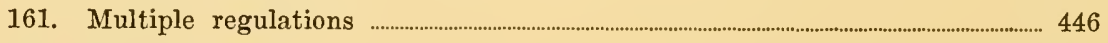

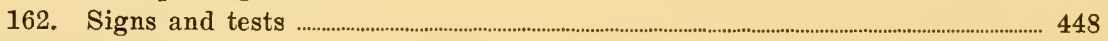

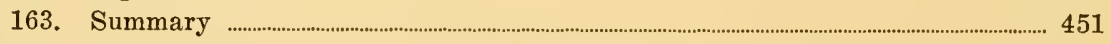

Chapter XX. Some Speculations Concerning Regulations .................................................................. 453

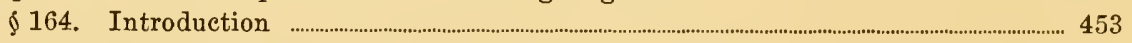

165. Distinctions between generalizations and theories .......................................................... 454 .

166. Maxima and minima ............................................................................................................................... 455

167. Origins of equilibrations ..................................................................................................................... 458

168. Forees in biological equilibria ...................................................................................... 461

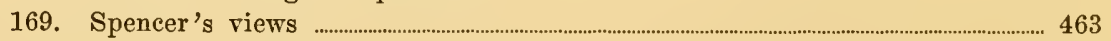

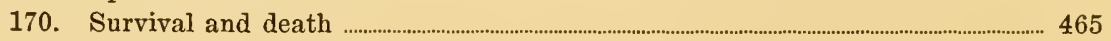

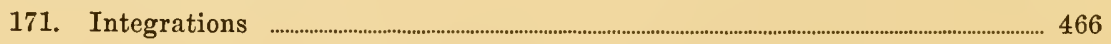


172. List of theories

173. Summary

175. Abstract of the investigation ……......................................................................................... 471

176. Equilibrations

177. Alternative studies of the same material

475

178. Contributions made 476

179. Conclusions concerning physiological constancies 477

180. Retrospect 


\section{LOCATIONS OF TABLES}

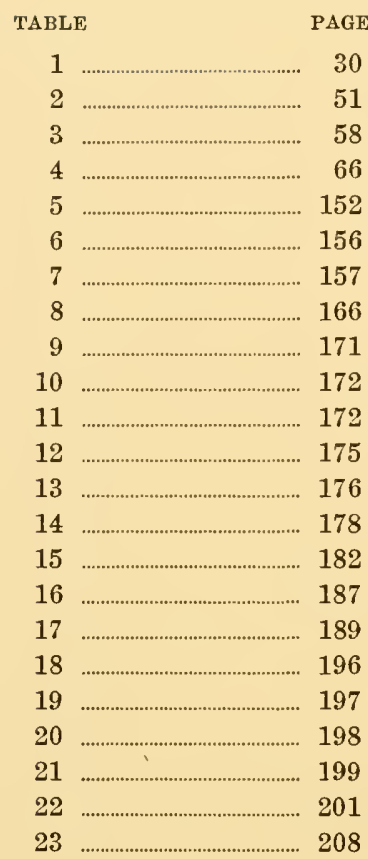

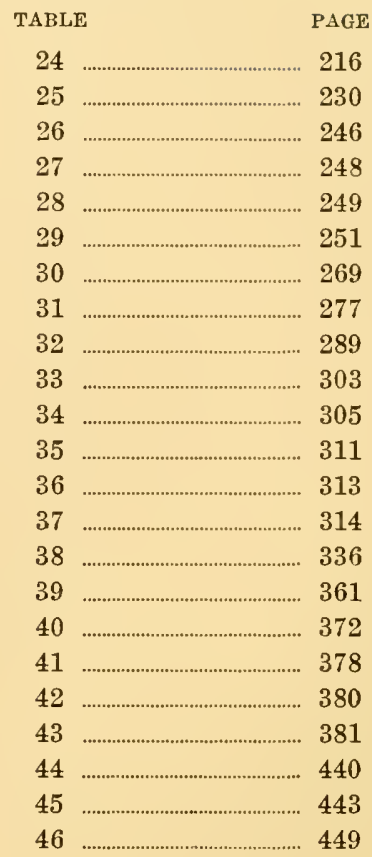




\section{Chapter I}

\section{INTRODUCTION}

$\$ 1$. A living organism is much the same day after day, not only in form but in function. If I count my heart beats for a minute each morning before getting out of bed, I find the frequency of beats only less constant than the length of my foot. The foot looks like a permanent mold in which matter is cast. Actually it swells and shrinks with each pulse of blood coming to it, each change of posture, and each meal. Its substance changes from month to month. The beats of the heart appear to be accurately meted out without a visible pendulum. During a day many an acceleration of frequency occurs; but when rest or other uniform state of body prevails, the frequency quickly returns to its characteristic value. That constancy represents a physiological regulation.

Every biologist, indeed every owner of a pet, expects his animal to be nearly the same every day; to show the same movements, the same responses to signals, the same posture, and the same food consumption. If not, he decides there is "something wrong." The certainty that he feels about the animal's health is similar to that which he feels about the performance of his automobile, his kitchen, or his piano. He has come, by experience, to expect uniformities of particular sorts. Failure of the car's motor to start is for him as uncommon as failure of the car's fender to keep its shape. Accumulated experiences seem to make a man more and more confident of always finding particular features that he can count on in his machine or instrument.

In much the same way, the biologist becomes intimate with the uniform characteristics of each animal dealt with, and the physician notices small departures from regularity in his old patient. All this is a tribute to constancy of physiological function, a recognition that each living individual tends in many ways to be like itself at each observation.

Regulations mean, then, in physiological science, those selfmanagements of organisms which result in constancy of function. What do animals do to maintain their physiological constitutions and activities? Functional arrangements evidently work hand-inhand with structural, chemical, and other kinds of arrangements; 
it would be quite arbitrary to consider traditionally functional aspects of organisms by themselves, and the term physiological merely indicates the aspect to be stressed here above others.

And now the questions are: how may this recognition of constancy be removed from the limbo of vagueness, and subjected to quantitative study? What shall be measured to assure me of the concrete existence of regulations? How may a physiologist record what an organism is doing to maintain its functions so constant?

Why does anyone investigate constancies anyway? Various excuses have been set forth by others for this perennial interest. Any property seems worth knowing about, just in proportion to its prevalence. Constancy of function is a feature of whatever constituents and manifestations have been observed in all organisms. By the degree of constancy I can characterize diverse manifestations in one species, can compare similar manifestations in diverse species, can ascertain what each animal does to insure continuance of function, and can observe how the animal manages many properties simultaneously. If I had not guessed at ways to investigate how constant various components are, and what happens when a component is disturbed, I might have been content with lip service to the existence of constancies. But now that some of my guesses have found substantiation in data and correlations, I an not satisfied with anything less than a quantitative treatment of the elements concerned. There is nothing in physiology or any other science to indicate that understanding is promoted by not intimately prying into the relations involved in a class of phenomena.

I propose first to outline briefly the subject of maintenance of physiological constancies, giving the methods, results, and conclusions that I have found appropriate. Thereafter, a more detailed and ordered treatment of the material will be given, leading to each of the generalizations to be mentioned. Since my generalizations have arisen in the study of water relations of animals, it is most direct to use water content as the property whose constancy shall be visualized (Part A). Whatever further features can be found with respect to content or manifestations of other components of organisms can be noted later (Part B).

In an early stage of physiological science the statement was often made that an organism's water content is kept constant by virtue of kidneys, or integuments, or some other structures. Later 
it was asked how those structures "knew" when the content was the same as usual, and "knew", what to do when it differed. Further it was observed that the regularity of water content is related to systematic modifications in the exchanges through those or other structures, in accordance with the actual contents prevailing. To certain contents the body is indifferent or acquiescent; in the presence of unusual amounts of the same components there is violent action that usually succeeds in changing them. Moreover, however satisfying it may be to know that kidneys or alimentary tracts are pathways that care for the exchanges of water, it is soon realized that many organisms exist without those organs, and yet probably every organism has means of correcting unusual water contents. Further still, an organ to remove excesses and another organ to make up deficits serve the body only so long as each grades its activities to whatever disturbance of content prevails, and only so long as the two organs are related to one another, in agreement as to what water content will be accepted by both.

Is an organism actually exposed to unlimited loss, or to unlimited gain? This topic concerns structural, mechanical, chemical and other features that somehow protect the body from suffering rapid changes, in the mode of existence peculiar to its species.

But is water content entirely a matter of faster and slower exchanges? Does its constancy not depend on availability of water in the environment? Water content is usually part of a stationary state that requires a source and a sink; these are environmental features toward which the behavior of the organism is frequently found to be appropriate.

Biologists in all ages have visualized one or another of these questions. Taken together, their views ( $\$ 157$ ) form a valuable background to an intensive treatment of self-maintenances. As long as 2400 years ago, Alcmaeon stated that "The preservation of health consists in an accurate adjustment of forces." This is the oldest known generalization of physiology. It represents the vague realization that the normal state of the organism is maintained by specific processes which resist change. And today it persists as a fruitful generalization that can be sharply delineated. By methods that were scarcely tried by my predecessors I can hope to fit together observations that help in comprehending the manner in which constancies of many properties are maintained. I seek to describe the operating characteristics of these maintenances. 
The peculiar method of this description is to use detailed and quantitative materials to lead up to a picture of certain types of regulation. Then the maintenance of a specific constancy in an organism may appear no longer as a conjecture or a pronouncement, but as a conclusion from many observations. Regulation takes on a patterned, and at times a geometrical, concreteness. Heretofore the abstraction of regulation has been an accepted part of physiology, though often regarded more as a frill than as an inevitable aspect of everyday phenomena. Usually it is conceived in the form in which Claude Bernard (1878, p. 144) presented it. He postulated that "in animals having free existence there necessarily exists a group of arrangements controlling the losses and the gains in a manner that maintains the quantity of necessary water in the internal medium" of the body. A quantitative description of any considerable group of such arrangements has been given only for blood (Henderson, '28) ; and blood has commonly been referred to as though it were a special sort of system, as though to it alone did that scheme for representing interrelations apply.

By sifting and putting together selected data I ascertain what occurs when some quantity (content) of the organism gets too large or too small for continuance. In this way a circumscribed functioning of the organism is quantitatively described, which later (chapter XIX) proves to be a part of a larger functioning.

\section{§2. How REgulations MaY BE STUdied}

The approximate definitions of regulations, indicated above, at once suggest means of investigating them. What procedures are available for measuring constancies in living bodies, and what features are present by virtue of which the constancies are perpetuated? I think that there are broad categories of measurements which characterize the maintenances of all sorts of properties, and that in the end it will be quite unnecessary to restrict specifications to water content or to heat content or to any other one. The following phenomena appear to be implicated.

(1) Exchanges. In a stationary state which is the organism, constancy of content means a rate of gain equal to a rate of loss. But, of numerous components, as water, animals do not characteristically have equal inflow and outflow in every minute of life. Both undergo endless fluctuations. They are compatible with con- 
stancy in so far as the content is juggled by compensations. First gain exceeds loss, then loss exceeds gain, and in diverse degrees. The mean rate of gain is found by measurement to exceed the rate of loss when the content of substance is lower than usual. In other words, the modifications of exchange that are seen when content is disturbed, whereby gain is faster when content is low and loss is faster when content is high, indicate the way in which exchanges are modified so that in the average instance the usual content tends to be restored.

This relation of exchanges to contents describes how corrections of content occur. It specifies the precise connection between processes of gain and processes of loss, and the actual gradation of each to the content. Corrections of unusual contents may be thought of roughly, though not strictly, as internal regulations.

Though faster or slower exchanges stand ready to compensate when the organism has gotten into trouble, provisions for keeping out of trouble, for preventing departures from constancy, might save many a need for cure. Those processes of the body and its parts that discourage excesses and deficit of some property $J$ in the routine of life are therefore observable means of preserving constancies. One method of preservation is complete isolation; a dead animal in a museum might remain constant in that way. Since complete isolation is incompatible with much of living, partial isolation appears more often. To judge when it is present is feasible in two ways. It may be shown that a dog, or some portion of its surface, exchanges $J$ more slowly than does a sponge soaked with saline solution and of equal size, shape, temperature, and so forth. Or, it may be shown that modifying or removing the surface hastens the exchanges. In general, the constitution of the organism is such that the environment exchanges with the body more slowly than it would with another constitution believed to be less differentiated.

(2) Behavior in selecting among environments. Given the physicochemical constitution of the organism, its peculiar gearing to rates of exchange, and its sensitivity to changes of content, still a factor in its preservation is the keeping of itself in environments that are not too hard on it. By selection of surroundings, the organism may obtain the cheapest maintenance if it find a place in which to be temporarily isolated, or in which water or other substance is optimally available. In general the organism seeks some conditions, and avoids others. 
To accomplish this, the organism uses sensory and locomotory (sensorimotor) equipment. There is thus a distinction between the behaviors of alimentation and absorption, which are exchanges; and the behaviors of making water available (hydrotropism) or absent. The latter is a frequenting of certain environments, a group of external regulations.

The study of selection among environments is carried out by well-recognized methods. A rat $(\$ 43)$ is compelled to pass certain obstructions to obtain water. Or, a rat is put in an apartment having both moist atmospheres and dry ones; when poor in body water it shows preference for the moist ones, thereby minimizing its further water loss, while when rich in body water it is more indifferent about moisture. In other words, there is a correlation between the animal's water content and the water content of the environment that it frequents.

(3) Variability of content. This answers the question, how constant is the content of component J? And, how far does the content change before something is done about it? In a first approximation an investigator might choose a hundred individuals under specified conditions and ascertain the content of $J$ in each. But sooner or later it might be found that the individuals chosen did not belong to a homogeneous population. It seems to me preferable to make, if possible, a hundred determinations of content upon one individual at successive equal intervals of time. All the while the individual studied will be maintaining itself under conditions that the observer believes are adequate, or under those particular conditions that the animal itself frequents.

The outcome is a succession of values of content. There are various ways of summarizing this succession. If it be shown that the points are random in time, then a distribution of frequencies of content, or its standard deviation, might characterize the variability. Without that demonstration I believe the physiological variations are best accounted by ascertaining the differences between successive points. These differences are what the organism allows itself in the way of fluctuations of body substance. Contents outside the range observed simply do not occur spontaneously; this organism in this environment sees to it that they do not oceur. Such a variability can be measured in respect to most kinds of content, and represents the net outcome of all regulatory processes. 
By the three aspects: modifications in exchanges, behavior toward environments, and variability of contents, regulations are to be described. Information that falls in these categories will be the materials examined.

\section{§ 3. Extensions of the STUdY}

Each of the three ways of studying self-maintenances of constancies is applicable, so far as I can now ascertain, to all organisms and portions of them (chapters XIV to XVI). All three can be further studied (to limited extents) by interfering with what the organism is doing, i.e., by surgical, pathological, or pharmacological means, especially in breaking connections among its functional units.

One means of gaining insight into regulations is to observe to what extents various animals are provided with them. Provisions for correcting the contents of some components are absent or incomplete to diverse degrees in immature animals. Or, comparisons of species show regulations of a few components (as, heat) in some but not in others. In some, regulations are faster than in others; in some they are lacking over certain ranges of bodily content. Instances are found in which compensations become more rapid with practice, and even in which non-existent ones may be called into being. All these special instances may help in grasping how self-maintenance is carried on.

Any compensation appears as a correlation between content of $J$ and some feature of exchange. Of all the possible exchanges, organisms are patterned to modify the exchange of $J$ itself, which alone restores the content of this particular component. To limited extents, however, other contents and exchanges are modified (chapters X to XII), usually to smaller extents. A study of the numerous correlations among measurable properties yields a notion of the number of strains suffered by the organism whenever it has departed from balance of $J$.

Further, each of the properties that is disturbed is itself undergoing adjustment toward restoration. That gives a picture of the interrelations existing among components whereby simultaneous processes act to bring the organism to its most persistent state (chapter XVII). Only with violence to the organism does the investigator study its components one at a time. Nevertheless, after examination of what organisms do when single components 
are varied in content, uniformities of pattern are evident. These general features form quantitative pictures of the manner of regulations. Instead of having a different basic scheme for handling each component, the organism apparently assigns to some portion of itself the fulfilling of the specific operations, consistent with the general pattern that it has for other components. While the specific physical machines for handling excesses of water, heat, oxygen, and glucose might be infinite in variety, the kinetics of restoring content is practically singular. That general fact calls for intensive study.

In brief, diverse sorts of living units and of a variety of properties, may be examined in the light of relations of compensation, preference, and variability. Studies of stages in development of the individual, comparisons among species and tissues, and examinations of simultaneous disturbances of many properties, are indicated. The task is to discover those features that are common to numerous kinds of regulated properties.

\section{\$4. Mode of treatment}

Every investigation appears to start from vague notions about the relations among observable phenomena. Having supposed that certain quantities might inform me about regulations, and tested the suppositions, I find scarcely worth mentioning what the working hypotheses were and what vicissitudes they have undergone. Then how shall I proceed to record the results, contributing order to the concrete observations?

(1) Description. There are often said to be two kinds of scientific ordering, causal and mathematical. In physiology the causal has frequently been essayed, and has not, I believe, revealed all that can be discovered with respect to regulations. I propose to try the other procedure, indicating descriptive relations among processes that seem concerned in maintenances of organisms. I will ask how, and not the unanswerable why. It is often supposed that mathematical descriptions do not satisfy the desires for understanding in the way that causal orderings do. That seems to depend on the individual scientific appetite. For I find that mathematical relations in physiology, even as in modern physics, may prove just as explanatory as any others that have been proposed. Of that no one can judge who has not attempted to use them.

Readers may feel the omission of the usual excuses, arguments, 
and sophistries that ordinarily accompany statements of fact derived by inspection, much as they might miss their own clothing. The practice of combining facts with non-descriptive inferences is so prevalent that facts seem not to sit comfortably in some minds without that kind of reasoning. The urge toward rationalization is as though natural phenomena did not subsist in their own strength but needed bolstering before they become acceptable. Statements are said to be tedious and dry; they are supposed to need flavoring with imagination and hypothesis before they are presentable. I propose to depend heavily on correlations for flavors and significances. If a reader were to overlook this explicit procedure ( $\$ 154)$ he might make the mistake of supposing that some data presented have no connotations, as though they had been set down in useless and thoughtless disorder. Recognition of this purpose rather than of some other purpose is a means of grasping quickly the plan and ends of the investigation.

(2) Quantitative data are obtained and utilized at every point possible. They make precise whatever phenomena are studied. They allow conclusions to be set down in concrete numbers and dimensions. They avoid certain of the intrigues of words and statements whose generality would require further investigation.

The data selected in what might seem at first to be a prolix profusion lead by induction to conclusions concerning maintenances and regulations. "Illustrations" have been suggested heretofore in the pursuit of these same general phenomena. To me the factual material would not be sufficient for the present inductions so long as it was qualitative. Even in what is currently termed quantitative biology, only qualitative conclusions are regularly drawn. But numerical relations seem to me to be precise parts of conclusions; phenomena are not just large or small, more or less, yes or no, proved or disproved. Equations and graphs may express more forms of relations than words do $(\$ 152)$.

(3) Generalizations result from the quest for uniformities among the quantitative descriptions of particular kinds of physiological phenomena. Instead of inferring that a relation observed will turn out to be a constant one, it seems preferable to count how frequently, with what variability, and under what varieties of conditions each uniformity appears. The reiteration of certain quantities, of combinations, of correlations, becomes the basis of under- 
standing the apparent vagaries and diversities of living units $(\S 153)$.

(4) Interrelations among measured phenomena become extended and multiple. A considerable part of scientific activity (sometimes believed to be the whole of science) is the placing of facts in relation to one another. It might be said that any datum may be viewed in an almost infinite number of ways. Ordinarily the first observer of fact A places it in relation with facts B, C, etc., arbitrarily according to his own knowledge, interests, and interpretations. This prejudices the future relations in which the fact $\mathrm{A}$ will be thought of, considered, and used; especially this limits the relations of which the discoverer will think. Another person is, so far as I can discern, freely entitled to reisolate the fact A and put it in relation with facts $\mathrm{D}$, E, etc. Often the second scientist will put the fact $A$ to as fruitful use as the first did.

Nevertheless, the ignoring of those relations among facts that have previously been promulgated, and the emphasizing of other relations, often arouses resentment. Divorcing facts from connotations is perhaps as important as, and is often more difficult than, finding connotations for them in the first place. The plan of recording an investigation is a responsibility; but to enable another to separate the observed fact from the scheme of representation is also a responsibility.

I know of no means of predicting beforehand which, of the semiinfinite number of correlations that are possible, will prove to be more interesting than the average, in the eyes of many generations of scholars. After correlations are established, the positive and negative ones look happier than the zero ones; and those with small perturbations have more statistical significance than others. But it seems as though any correlation that is made, takes on significance to the investigator as he observes it and thinks upon it ( $\S 144$ and $\$ 150)$. Hypotheses are gradually formulated about it, and an interrelation that to one physiologist looks arid will to another be pregnant with meaning.

Hence, the procedures are such as to emphasize: description of relations, quantitative data and comparisons, generalization from similarity of relations and multiple interrelations. No doubt the conclusions to be found depend upon the particular data utilized; insofar as they have been tested, the conclusions that are reached appear to be of considerable generality. 


\section{§5. Outline of the investigation}

The plan of this investigation is the equivalent of an interweaving of at least five studies. Each of them could be undertaken separately, but their interplay multiplies the inductions that can be formulated from the data presented.

(1) Water exchanges in animals.

(2) Rates (kinetics) of certain classes of physiological processes; time sequences.

(3) Quantitative comparison of like functions in diverse species and individuals.

(4) Organ and tissue exchanges; the study of specializations, localizations, paths, and routes.

(5) Components (constituents, endowments, and properties); similarities and contrasts in their metabolisms and economies.

Emphasis throughout is upon interrelations among simultaneously occurring activities.

Some features into which this study delves, incidentally to its chief purpose of describing physiological regulations, are:

Water metabolism of man (chapter V)

Tolerance curves $(\S 71$ and $\S 133$ )

Stationary states in organisms $(\$ 138)$

Variabilities of organisms (chapter IV, and \$131)

Recovery processes ( $\$ 71$ and $\$ 133$ )

Signs of disturbance and disease $(\$ 162)$

Interacting maintenances (chapter XVII)

Temperature regulations (chapter XIV)

Selection of environments ( $\$ 43$ and $\S 132)$

Storage and depots $(\$ 79)$

Ontogeny of regulatory processes $(\$ 96$ and $\$ 115)$

Comparative physiology ( $\$ 107$ and $\S 141$ )

Cells and tissues as regulated units (chapter VIII)

Volumes of distribution ( $\$ 58$ and $\$ 80$ )

Blood volumes ( $\$ 59$ and $\S 80$ )

In designing the investigation, it is useful to distinguish certain categories of measurement with which the data will deal. The variables initially selected for study fall into six classes, none of which excludes from membership in other classes: 
(1) Rate of exchange (R), e.g., Total gain of energy, of water

(2) Species or Living Unit (U), e.g., Dog, erythrocyte

(3) Path of exchange ( $p)$, e.g., Urinary, radiative

(4) Tissue (portion) studied (s), e.g., Blood, whole body, cell

(5) Component (J), e.g., Water, heat, pressure

(5a) Type of displacement (f), e.g., Privation, injection

(5b) Quantity of one component (C), e.g., Excess, deficit

(6) Temporal parameter (t), e.g., Time, duration

The number of kinds of correlation among 6 variables is 57, or taken two at a time is $(6 \times 5) /(1 \times 2)$ or 15 . The number of specific data available in each class to be correlated is semi-infinite.

The procedure chosen is, at first, to keep the component $(J)$ constant, then the species or individual or living unit (U) constant. Within the study of one component, the type or means of disturbance (f) influencing the component is recognized; later the tissue or other portion measured $(\mathrm{s})$ is differentiated. This reduces any one set of data to three variables ( $J, p$ and $t$ ) to be dealt with; $p$ often can also be selected and held constant through one series of data. The other three are entered in separate correlations, until summaries calling for regrouping are required. A more complete view of the course pursued may be gained after part of the treatment has been covered ( $\$ 101$ and $\$ 152)$.

Not all the possible combinations of variables are presented. To do so would be a completion but also a tedium. Those omitted are not less important to the organism, so far as anyone can judge; but are of three classes: those for which suitable data were not obtained, those unfamiliar to me, and those in which the correlation did not seem to me to yield illuminating relations. Limitations are imposed also by the number of variables that can be conveniently and profitably handled at one time. Variables, in fact, may be numerous and unknown, but the ones recognized are chosen for their reproducibility, statistical significance, and apparent interrelations. Only by continual classification and limitation can the investigation be kept, at each point in its progress, within comprehensible limits. Some of the studies here presented may appear to be exhaustive. But I am the last one to consider them so, for hosts of lacunae and possible extensions have come into view. It is conceivable that physiology of the future will be still more quantitative, more detailed, and more interested in interrelations and variabilities. 
Of course, most of the objectives were not apparent, or even implicit, in the initial conscious formulations of this investigation. As in all studies, possibilities became evident as the methods and results accumulated. Initially the research appeared only as a means of relating certain quantities from studies of water in organisms. Thereafter the generality of the relations and their role in regulations gradually unfolded. It is also fair to say that I have long hoped to find general methods of understanding regulations in organisms, and it is almost usual that methods turn up to the conditioned (prepared) mind.

The inquiry is divided into two chief parts: Part A deals with selected data and aspects of water exchanges and water content. Generalizations among measured quantities concerned with maintenance of water metabolism lead, in PART B, to comparison and extension of the same types of description to other components and exchanges. In this way certain general methods of study and some general properties of physiological processes are examined. 



\section{Part A WATER RELATIONS OF ANIMALS}





\section{Chapter II}

\section{WATER EXCHANGES OF DOG}

$\$ 6$. Of the several approaches to the study of regulations, the compensations manifested when an animal has unusual amounts of water in the body call for intensive consideration. The object is to find how the water content is restored after it is disturbed from complacency, after water is forcibly added to or subtracted from what is usually there.

Among the relations of water content, it seems to me desirable to study features available in all organisms and their parts and aggregates. The amount of water present in the living unit, and the rate at which it is gained or is lost are, in that respect, suitable quantities. Each has distinct dimensions. The amount of water present is measured either in physical units, or else in physiological units that are obtained by comparing the state of the organism having much or little water with its control state as it exists before or after or omitting the treatment or condition. The physical unit is the liter or the gram; the physiological unit is a relative measure, such as a relative increment of weight.

The initial investigation of water exchanges by living units is limited, while dealing with one individual animal $(\mathrm{g})$ in one set of observations ( $f$ ) and by one path of exchange $(p)$, to variables of four dimensional sorts: Water increment $(\Delta W)$, water exchange $(\mathrm{R}$, or $\delta \mathrm{W} / \Delta \mathrm{t})$, time elapsed $(\mathrm{t})$ after establishment of a new water content, and velocity quotient ( $\mathrm{k}$ or $1 / \Delta \mathrm{t}$ ). Rate of exchange, $R$, is thus a ratio of a quantity of component to an interval of time; and velocity quotient, obtained by dividing a rate of exchange by a content, is a reciprocal of an interval of time. Intensive treatment of these four quantities (in chapters II to IX) concerns the one bodily component water.

With regard to the component water, excesses are first imposed in the form of water by stomach and deficits in the form of privation of water. Two types of water excess or deficit are successively studied: the temporary state following a single administration or deprivation of water $(\$ 7$ to $\$ 10)$ and the stationary state in which excess or deficit is maintained approximately constant for some period of time $(\$ 12$ and $\$ 13)$. Total exchanges are mea- 
sured, followed by separation into paths: Urinary water, evaporative water, fecal water, water formed in metabolism (oxidative), and water ingested as such (ingestive). One species (dog) is initially studied, and the whole body is a unit, it being of no concern for the present where within the body the water is.

Confusion may be avoided by further defining a few quantities to be used, particularly in connection with the graphical representation of data. Any departure of water content (W) from the control content $\left(\mathrm{W}_{0}\right)$ is designated as water load, $\pm \Delta \mathrm{W}$. Quantitatively the unit of load is defined as an amount of water, weight, or other equivalent equal to one-hundredth of the control live weight of the body $\left(B_{0}\right)$ or $\Delta W=100\left(W-W_{0}\right) / B_{0}$. Each type of modification of the water content has a slightly different definition within the general class; each may be measured by a partially different procedure, or under different conditions; and some will be in milliliters per gram, others in grams per gram.

Similarly, interval of time $\left(t_{2}-t_{1}\right)$ is designated as $\Delta t$. Usually the unit of time is the hour, but the hour selected may be various: the initial hour, the hour of maximal load, an hour's duration of an instantaneous rate, hour computed from half-hours, quarter-hours, minutes, or days, and many others. It seems to me that clarification is obtained by multiplying distinctions just so far as actual or probable differences exist in the phenomena described.

While the dogs are endowed with the diverse increments of water content, their rates of total water exchanges $(\delta \mathrm{W} / \Delta \mathrm{t})$ are measured, during the passage of time $(\mathrm{t})$ after the increment is established. Rates are expressed in per cent of body weight per hour, or $\delta \mathrm{W} / \Delta \mathrm{t}=100\left(\mathrm{~W}_{2}-\mathrm{W}_{1}\right) / \mathrm{B}_{0} \Delta \mathrm{t}$.

Thus I have elected an experimental situation and a set of variables to be measured. Their definition constitutes the design of the initial investigation.

\section{\$7. Sudden Excesses of water}

Water contents are conveniently modified by administering known quantities of water to dogs that are initially in control state. How long do excesses remain? Knowledge that water was the only liquid or solid given the dog saves the great trouble of evaluating the water contents of dogs sacrificed and analyzed at diverse intervals in each test. The quantities of water present and the exchanges are both measured by body weight, since the amounts of 
other substances lost and gained are small in comparison to water and are known to be almost independent of water content.

In each actual test a dog unfed for 16 hours but allowed water ad libitum is given water by stomach tube (at zero time). Urine is collected, and body weight is measured, every 0.25 hour. In plan the test requires little apparatus, for all measurements are made with a graduated cylinder, a sensitive balance, and a clock. In

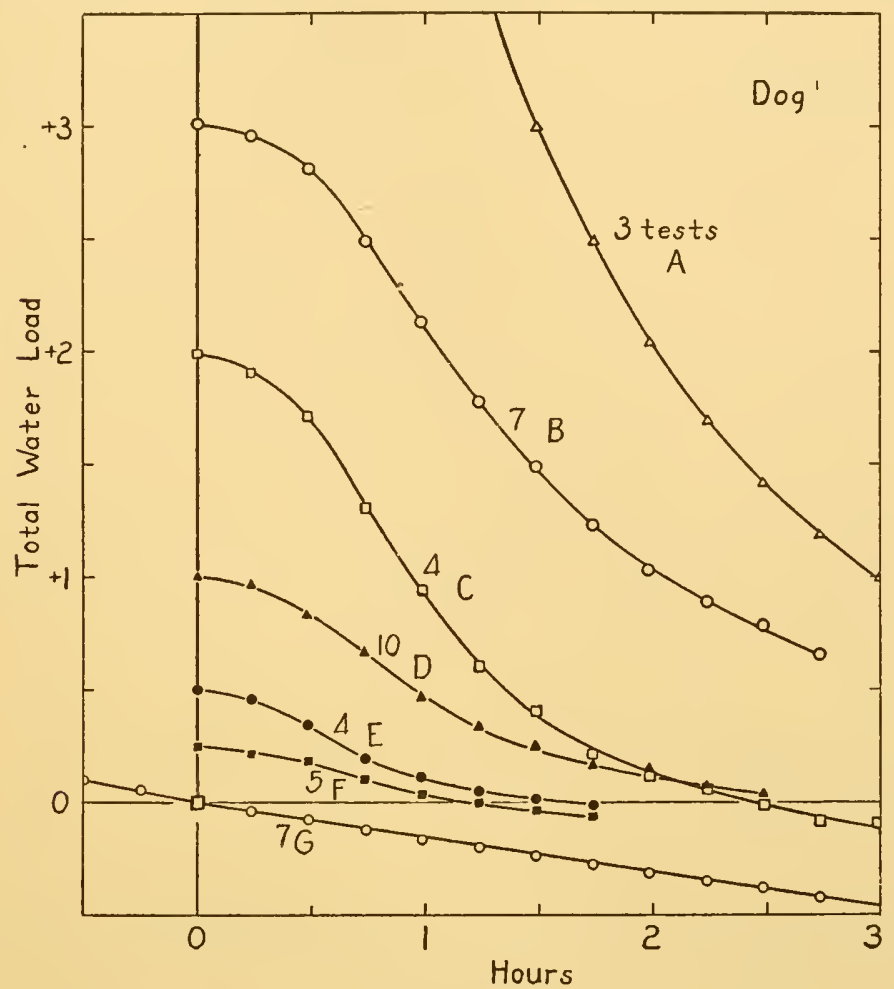

FIG. 1. Course of total water load (per cent of the body weight, $B_{0}$, at zero time) after diverse single quantities of water are given by stomach tube. Dog, four individuals. The number of tests indicated is also the number of measurements averaged for each point. In series $A$ the initial water load was 6.14 per cent of $B_{0}$. New data of Kingsley and Adolph.

practice each dog needs to be trained to stand still during several hours, to take a stomach tube without struggling, and to submit to continuous observation. Previously a bladder fistula or an exteriorization of the urinary bladder is surgically produced. Ultimately many computations are required to place the results in coordinated and comparable form. 
In the course of time (fig. 1), water is lost until the initial weight is approximately reattained. The rate at which water is lost increases as more is administered, and the whole process of recovery lasts but little longer. For each curve, all tests in which similar amounts of water were initially given contribute values of relative body weight; all those values occurring in the same serial interval of time are averaged arithmetically.

Rates of elimination are also averaged arithmetically, though with equal suitability harmonic means might have been used. The rates of elimination (fig. 2) vary with time as well as with the

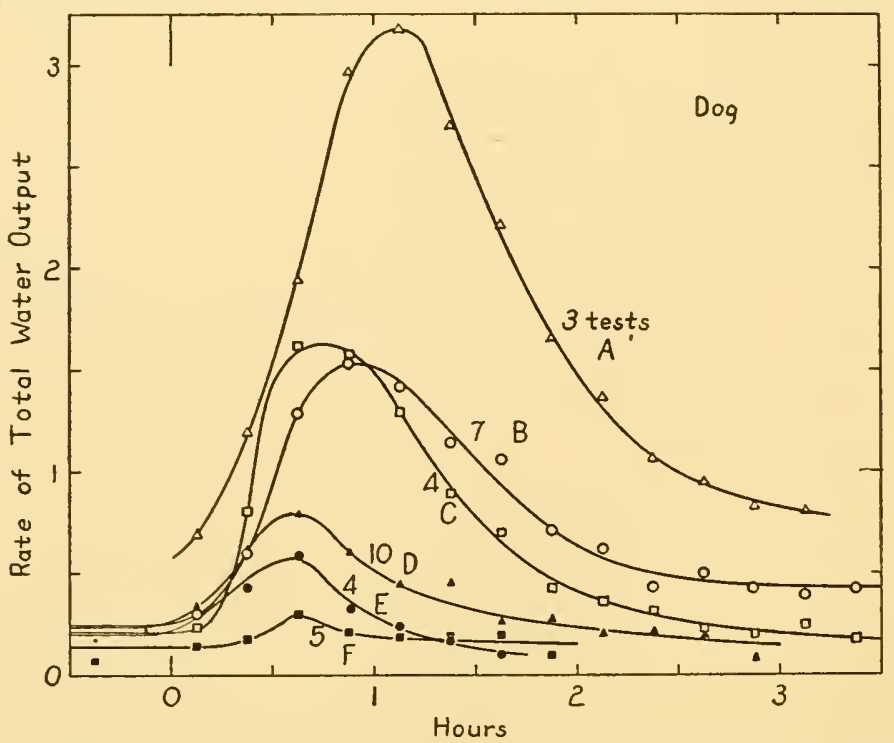

FIG. 2. Rate of total water output ( $\%$ of $\mathrm{B}_{0} /$ hour $)$ in relation to time after diverse quantities of water (shown in figure 1) are given by stomach tube. Each rate is obtained from the change of body weight during 0.25 hour, and the average is plotted at the middle of the period in which it prevailed.

quantity of water put into the stomach. Initially there is a lag period in which loss is unmodified; thereafter rates increase quickly to maximal, followed by more gradual fall. With regain of control water content, the rate of water output approaches the initial rate.

Very similar relations are shown (fig's. 3 and 4) by measuring the exchanges through urinary channels alone. Exact comparisons and differences between total losses and urinary losses are not computed, inasmuch as figures 3 and 4 contain added tests that are not represented in figures 1 and 2 .

In this general account of mean results, no account is taken of 


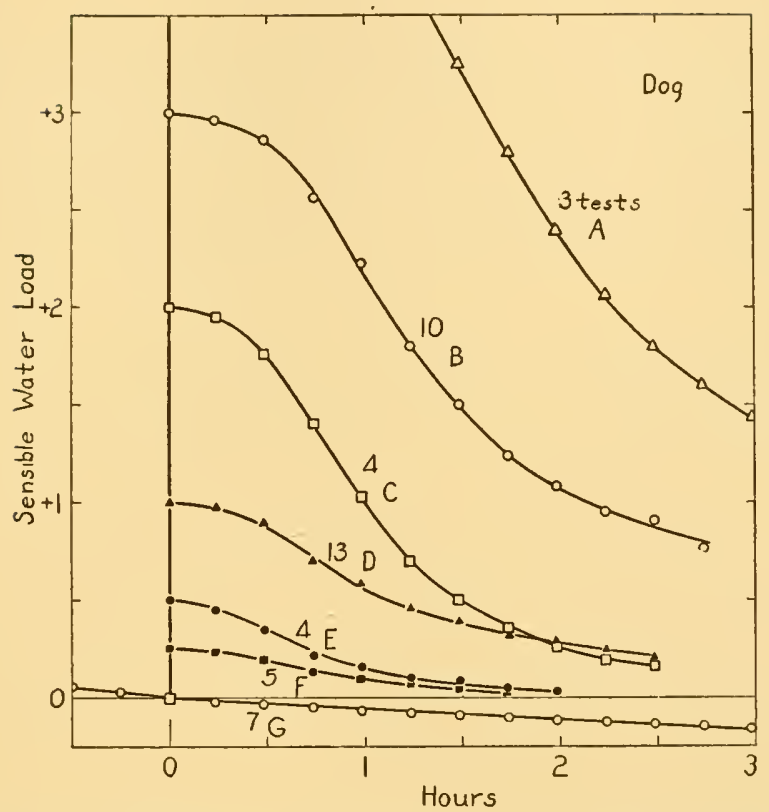

FIg. 3. Course of sensible water load ( $\%$ of $B_{0}$ ) after water is given by stomach in a single dose. Sensible load is total ingesta minus urine collected. The same tests are represented as in figure 1 , plus a few additional one on the same four individuals.

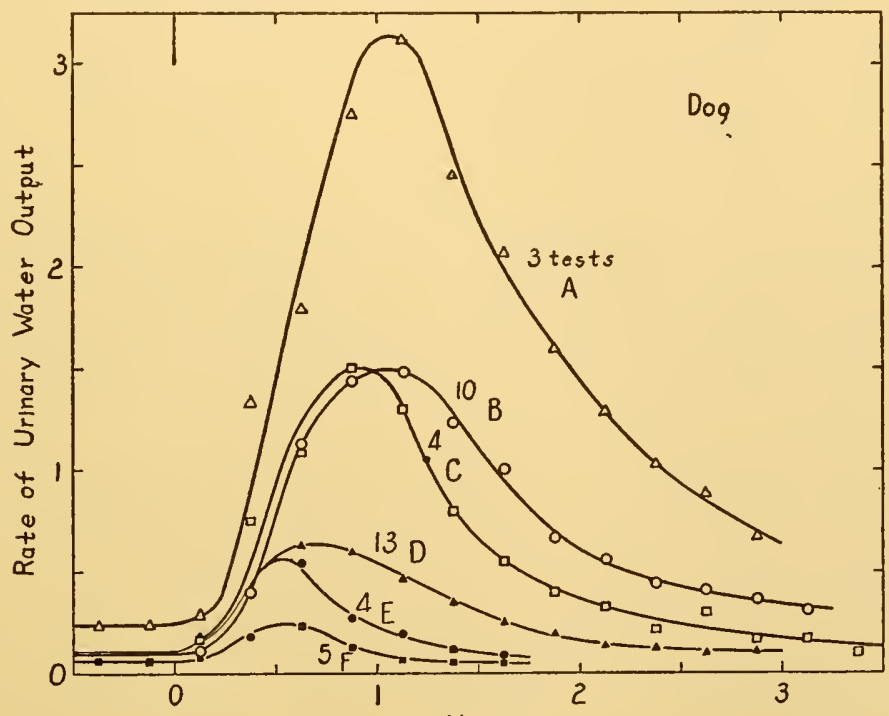

Hours

FIG. 4. Rate of urinary water output ( $\%$ of $\mathrm{B}_{0} /$ hour) in relation to time after a single dose of water is given by stomach tube. Same tests as in figure 3 , plotted in manner of figure 2. 
the variation among tests upon one individual. Also, recognizable differences exist among individuals, as though the capacities for metabolizing water were of diverse orders; these too are disregarded. Further, there is significant acclimatization in an individual that has been repeatedly given water in large doses, whereby the speed of disposal is increased. Such matters may await later treatment.

The course of water exchanges is partially different if, instead of the dog as weighed, one considers water content of the dog minus the alimentary tract. Then the water in stomach and intestine is

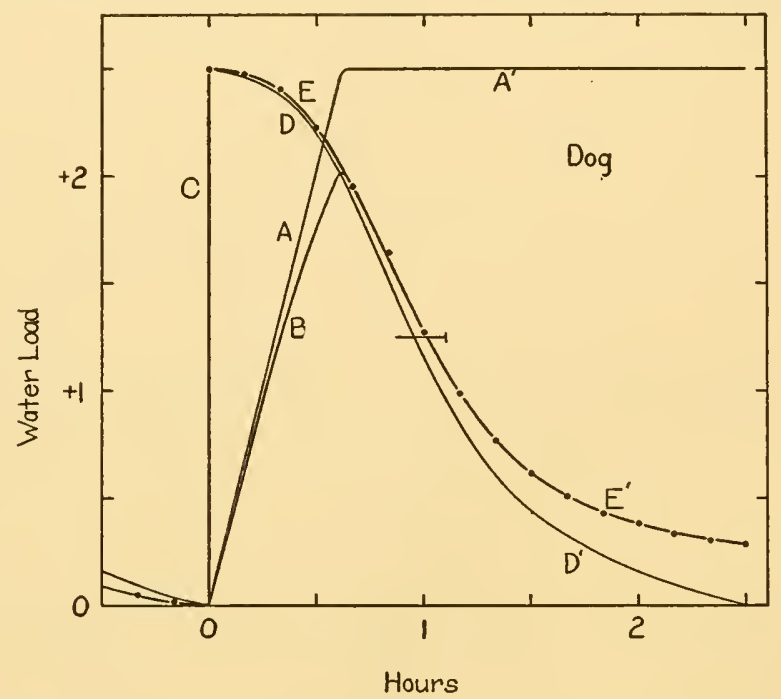

FIG. 5. Course of water load (\% of $\mathbf{B}_{0}$ ) after water is given by stomach tube. Loads represent the means in 9 individuals upon each of which several tests of urinary water output were completed. Absorbed volumes are the means in those 9 individuals when killed at various intervals within 0.6 hour after water administration, in order to find how much water remained in the alimentary tract. Data of Klisiecki et al. ('33a). Redrawn by permission of the Royal Society of London.

counted as being outside the body, and the rate of absorption modifies the increment or load of water present at any instant. Absorption has been directly measured at only one load administered; with considerable variation among individuals, the amount absorbed was found to be proportional to time elapsed up to complete absorption in 0.6 hour. The load characterizing the dog minus alimentary tract is indicated in figure 5. This diagram serves partially to locate the water at each instant during the test. Actually most of the water given is still unexcreted when absorption is apparently complete. 
Water is gained during these periods of water excess in only small amounts. When water is offered, the dog consistently refuses to drink it. Small quantities are being continually formed in the body by oxidation of organic compounds containing hydrogen, and possibly by other processes, which do not vary significantly with water content or load (Bidder and Schmidt, 1852; Rubner, '02, p. 62; Heilner, '07; Lusk, '12).

In general, losses of water are enormously increased, while gains of water are somewhat decreased, for some hours after water is given by stomach. The greatest modification is in rate of urinary output.

The full amount of water ingested is only rarely realized or returned in the urine before the rate of urine formation comes back to approximately that of the control state. After administration of more than 2 per cent of the body weight, about 76 per cent is returned as urine in 3 hours (fig. 3 ). In total output the corresponding return is 82 per cent of the volume ingested (fig. 1). If corrections are made for the basal (control) rates of water loss, either urinary or total, the return is still less. Apparently the body does not treat quite all the water administered as excess. Somewhat greater returns than those exhibited occur only after special preparation for the experiment, consisting in a previous administration of an excess of water on the same day (Klisiecki et al., '33a; Kingsley and Adolph).

Not only can the returns be ascertained after diverse periods of time have elapsed, but from figures 1 to 5 may be read the times required for initiation of diuresis, for maximal rates of excretion, for half return or half-life (or any other fraction) of the administered load, and for cessation of diuresis. All of these intervals of time except that for initiation are longer as the load is greater.

Figures 1,3 and 5 represent loads in relation to time. This relation in certain components of organisms is commonly termed a tolerance curve; the designation may be applied therefore to all curves relating load to time. I suppose the "logic" of the word tolerance is that the curve indicates how much added component the organism tolerates by removing it. High tolerance for water means fast disposal of an excess or a deficit of it, the opposite of indifference toward the increment. Rates of exchange in relation to time (figs. 2 and 4 ) may be designated as exchange curves.

Water content in control conditions is approximately propor- 
tional to body weight, being about 62 per cent of it, in the intact dog (see table 18). A 1.0 per cent load or increment $( \pm \Delta W)$ as based on body weight, is therefore a 1.6 per cent increment in body water.

Loads of water may be classified (fig. 5) into:

(1) Total Load = Excess body weight, CDD'

(2) Sensible Load=Excess administered, minus urine excreted, CEE'

(3) Administered Load = Excess administered, C

(4) Absorbed Load = Excess administered and not in the alimentary tract, $\mathrm{AA}^{\prime}$

(5) Absorbed Total Load= Excess absorbed, minus amount lost judged by body weight, $\mathrm{BD}^{\prime}$

(6) Absorbed Sensible Load = Excess absorbed, minus urine excreted, $\mathrm{BE}^{\prime}$

It might be considered poor technique to employ a large number of types of water load. In reality the number studied is the smallest number suitable for the purposes in hand. In the course of study it is poignantly observed that several confused variables are easily and usually thrown into a single category by virtue of having the same dimensions or names. The distinction of varieties of water contents may be a step toward clarity and precision in the study of the physiology of water.

The tolerance curves shown above are approximately confirmed in unanesthetized dog's by less complete data of other investigators (Falck, 1872 ; Molitor, '26a ; Rioch, '30 ; Abe, '31a ; Hatafuku, '33a ; Theobald, '34; Pickford, '36; Rydin and Verney, '38).

Now I have described what happens to loads and rates of exchange in the course of time. By correlating the ordinates of figure 2 with the simultaneous ordinates of figure 1, I compare rates of water elimination at diverse contents of water in the body. The course of each test or set of tests may be followed on the new coordinates (figs. 6 and 7). If corrected for absorption (fig. 8), only the early portion is modified. At any one time after the initial 0.5 hour (fig. 2) the rates of water loss increase with the excesses present. During a chosen interval of total elapsed time this is also true (fig. 9). After a while a maximal rate of excretion is attained, and thereafter at any one load (fig. 6) the rate of excretion is roughly independent of time. I believe it is signifi- 
cant, however, that at a given load slightly faster rates follow more recent maximal rates.

The measurements of water exchanges after sudden establishment of water excesses lead to a relation of output to content that is rather uniform, for one is roughly proportional to the other. More exactly, if $R_{W}$ is rate of water output at load $+\Delta W$, a is rate of output at no load, and $\mathrm{k}^{\prime}$ is a coefficient of proportion, then $\mathrm{R}_{\mathrm{w}}-\mathrm{a}=\mathrm{k}^{\prime}(\Delta \mathrm{W})$. In figure $6, \mathrm{a}=0.15 \%$ of $\mathrm{B}_{0}$ hour, and $\mathrm{k}^{\prime}=$ $0.7 /$ hour.

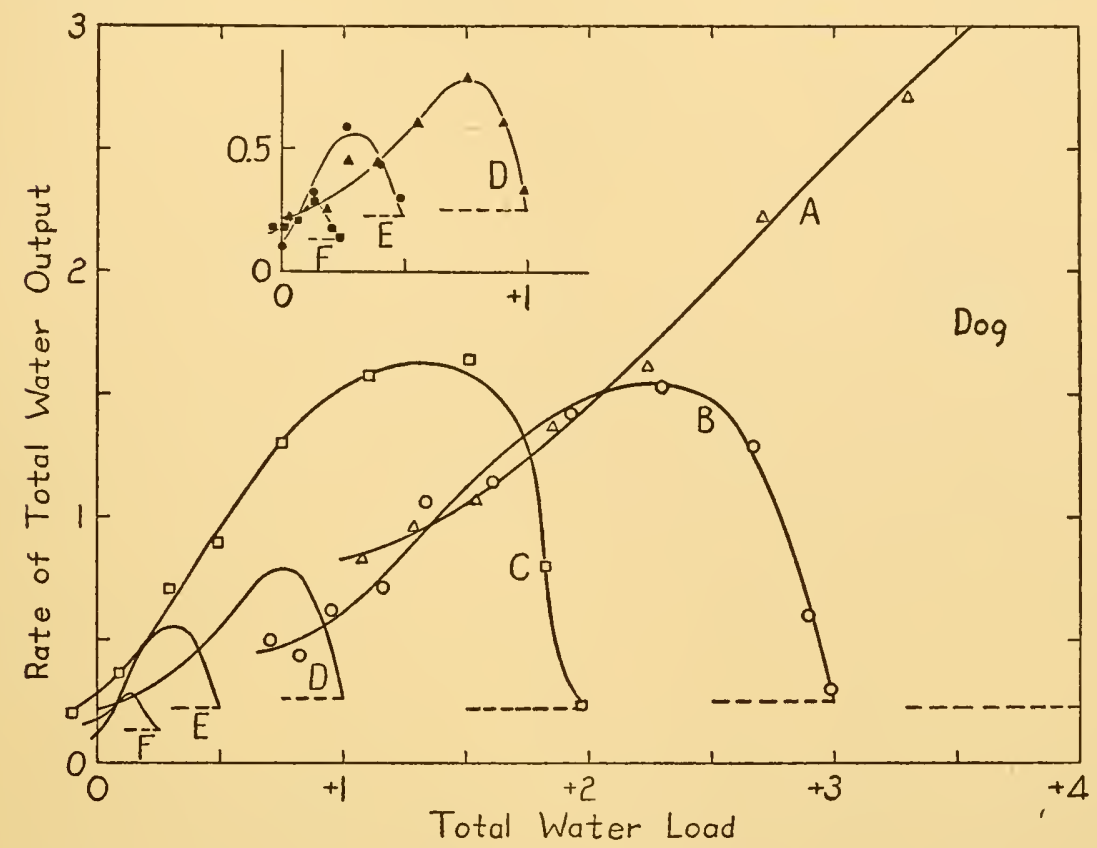

Fig. 6. Rate of total water output ( $\%$ of $B_{0} / \mathrm{hr}$.) in relation to total water load ( $\%$ of $\mathbf{B}_{0}$ ). Each point in figure 2 is plotted against the mean load in figure 1 , in the corresponding 0.25 -hour interval.

Under the conditions chosen, only urinary losses of water, and losses by no other paths, increase; evaporative losses from skin and from lungs do not, losses do not. Gains by ingestion of water are nil and metabolic production of it is independent of water increment. The net exchange of water (loss - gain) is on the average infinitely increased over that in control conditions, for in them loss equals gain over sufficient periods of time.

In summary, excess of body water suddenly created in dogs by gastric administration of water leads to rates of water loss typi- 


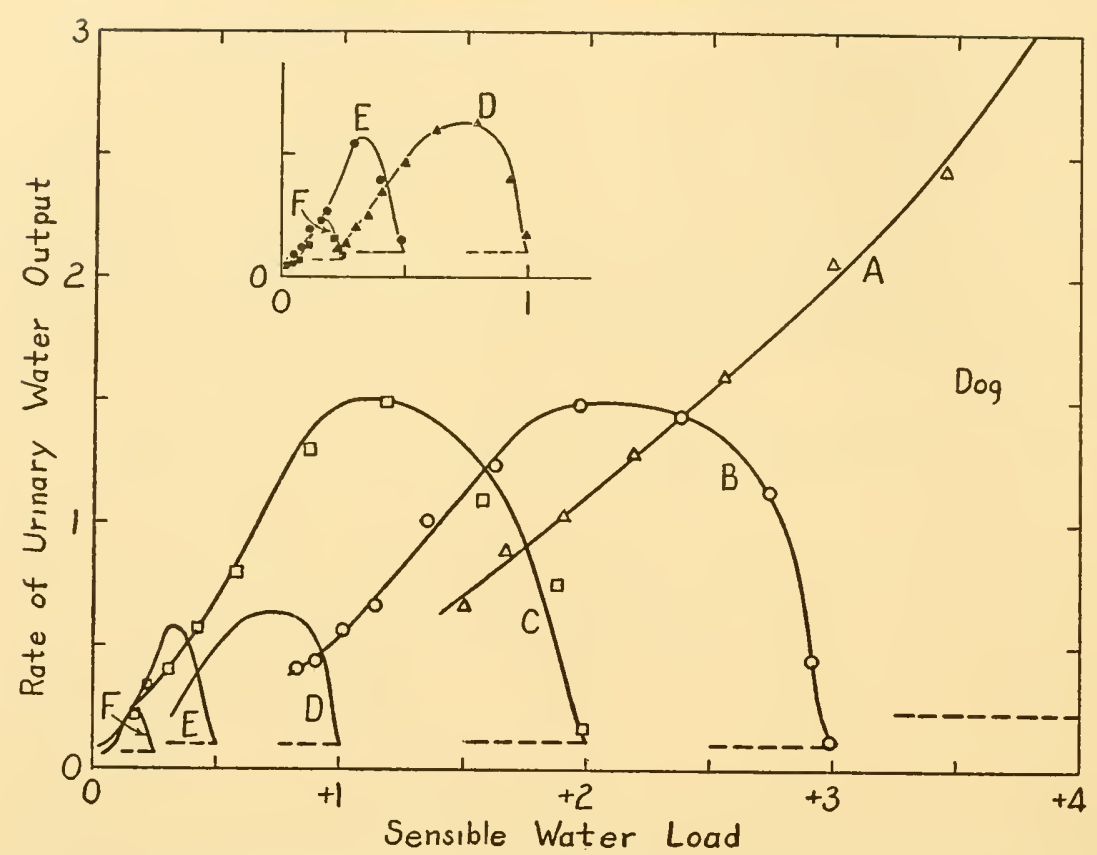

FIG. 7. Rate of urinary water output ( $\%$ of $\mathrm{B}_{0} / \mathrm{hr}$.) in relation to sensible water load $\left(\%\right.$ of $\mathrm{B}_{0}$ ). Each point in figure 4 is plotted against the mean load (in figure 3 ) of the corresponding 0.25 -hour interval.

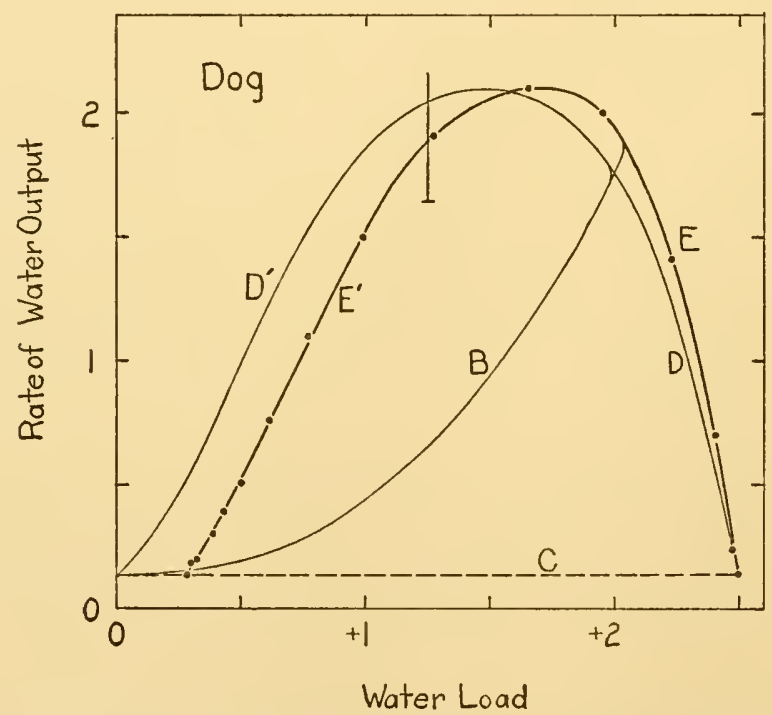

Fig. 8. Rate of water output ( $\%$ of $B_{0} / h r$.) in relation to water load ( $\%$ of $B_{0}$ ). Same data as in figure 5 , each point representing a period of 0.25 hour. Data of Klisiecki

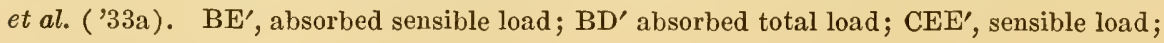
$\mathrm{CDD}^{\prime}$, total load. 
cally greater than usual. At each time, after a latency which is related to absorption of the water from the alimentary tract, the rate is proportional to the load. A family of curves of water tolerance is thus described. Recovery of water content ensues within a few hours; it takes but little longer after large excesses than after small. The urinary route is the only path of output

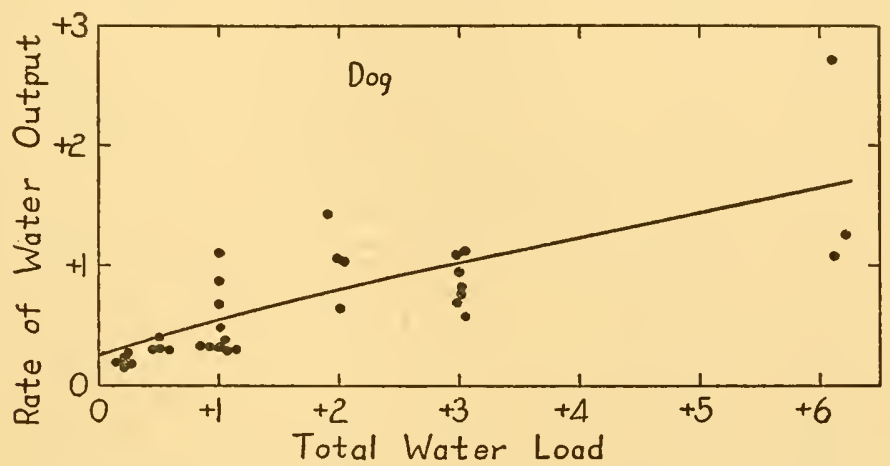

Fig. 9. Rate of total water output ( $\%$ of $\mathrm{B}_{0} / \mathrm{hr}$.) within the first 1.0 hour after a single administration of water by stomach, in relation to administered water load. Each point represents one of the tests of figures 1 and 2. Data of Kingsley and Adolph.

in which rates of loss are significantly modified during this recovery, and through it nearly all of the excess is eliminated. All this is what the dog does to compensate for having more water than usual.

\section{\$8. Deficits of water}

It seems natural to expect the dog to deal with water deficits just as effectively as it deals with excesses. Initial states of water shortage are established in the body by administering food which is constant both in composition and in amount, but allowing with it insufficient water for maintenance. After one to five days of partial privation, with loss of body weight and of water content, water is offered to the dog (Adolph, '39a).

Promptly the dog drinks (fig. 10) amounts of water which on the average slightly exceed the amounts of water shortage in the body as judged from deficits of body weight. The weight is more than restored for the time being, within 1 to 4 minutes from the time water was first offered. Thus the dog closely matches its deficit by its intake, without waiting for absorption of the water drunk to ascertain whether it has enough. The mean rate of ingestion $(\delta \mathrm{W} / \Delta \mathrm{t})$ is initially enormous, and just as promptly falls to 
almost nothing. The speed of drinking and the sudden cessation are equally dramatic.

When the loads or deficits of water $(\Delta W)$ are correlated with the amounts ingested in the first 5 minutes $(\delta \mathrm{W} / \Delta \mathrm{t})$, a linear relation or regression is obtained (fig. 11).

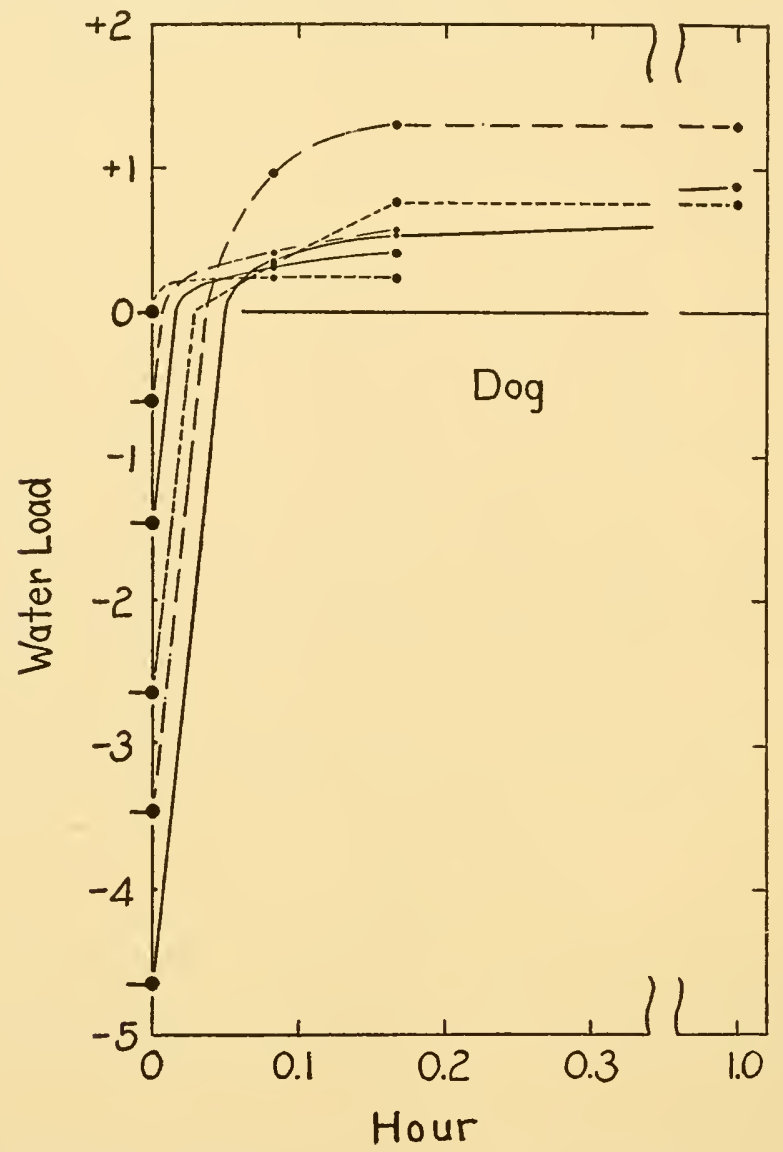

FIG. 10. Amounts of water ingested $\left(\%\right.$ of $\left.B_{0}\right)$ following the establishment of diverse negative water loads. Forty-one tests on 3 dogs are averaged in groups of 8 taken in order of load established. Drinking is at top speed until the control body weight is regained. Additional data of Adolph ('39a).

Gains by the oxidative path are independent of water content, so far as is known (data of Straub, 1899). Even in the extreme states of deficit no augmentation of rate of oxidation appears to occur. This is a matter for measurement, for no argument that hydrogen is or is not too expensive to turn into mere water, is logic so convincing as the dog's metabolic actions. 
Drinking and absorbing are the counterpart of excreting. Gain and loss are equivalent and symmetrical aspects of the recovery and the maintenance of water content. To regard water intake and water output as wholly separate functions of the dog is to

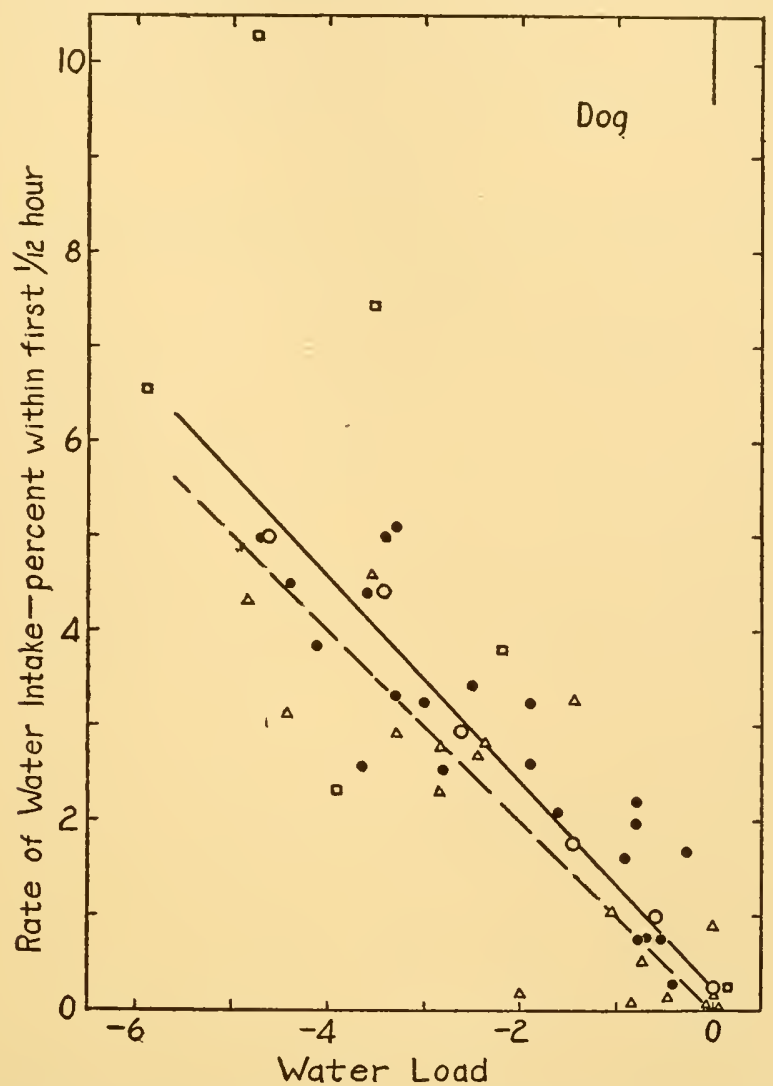

FIG. 11. Amounts of water ingested ( $\%$ of $\mathrm{B}_{0} / 0.08$ hour) in relation to negative total water load $\left(\%\right.$ of $\mathrm{B}_{0}$ ). The same 41 tests on 3 individuals as in figure 10 are indieated, together with the regression of ingestion rate on load (solid line and open circles). If the dogs had taken the exact amounts of the deficits, the dash line would have resulted. The equation of the solid line is $R=0.28-1.073 \Delta \mathrm{W}$; of the dash line, $R=-\Delta W$. The coefficient of variation (with respect to ordinates) of the points from the solid line is \pm 32 per cent. Redrawn from Adolph ('39a).

ignore their high (negative) correlation. The reproducibility, too, of rate of initial drinking is just as high as of rate of urinary output $(\$ 24)$.

Diverse features concerned with water ingestion are quantitatively known (table 1). Capacities of stomach and small intestine, rates of emptying of stomach, rates of absorption, maximal 
rates of drinking, maximal drafts, frequency of gulps; these and many more are characteristic, and may be observed and correlated in the same detail as the diverse excretory activities. The multiplicity of the tabulated features is a reminder of the interrelation of processes in water intake.

Throughout the period of water privation the losses of water are (fig. 12) reduced below those prevailing in water balance.

TABLE 1

Features of water intake of dogs. The measurements are means, observed unless otherwise noted among 4 individuals that received with their food about half of the water gained; while being studied in other respects (Adolph, '39, p. 77)

\begin{tabular}{|c|c|c|c|}
\hline Quantity measured & Volume, ete. & $\%$ of $\mathrm{B}_{0}$ & $\%$ of $\mathrm{B}_{0} / \mathrm{hr}$ \\
\hline Body weight $\left(=\mathrm{B}_{0}\right)$ & $18,600 \quad \mathrm{gm}^{2}$ & 100.0 & $\ldots \ldots \ldots \ldots \ldots \ldots . . . \cdot$ \\
\hline $\begin{array}{l}\text { Body surface (computed) } \\
\text { Mean water gain/day }\end{array}$ & $\begin{array}{l}7,800 \mathrm{~cm}^{2} \\
1,100 \mathrm{ml}\end{array}$ & 6.0 & 0.25 \\
\hline 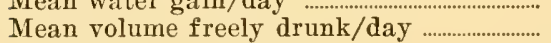 & $540 \mathrm{ml}$ & 2.9 & 0.121 \\
\hline 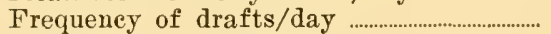 & 9 & & \\
\hline Ordinary volume/draft ........................................... & $60 \mathrm{ml}$. & 0.32 & 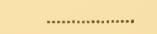 \\
\hline Maximal volume/draft ... & $1,390 \mathrm{ml}$. & 7.5 & ............. \\
\hline Maximal frequency of gulps/minute ......... & 175 & 0.018 & ........ \\
\hline Maximal volume swallowed/minute & $580 \mathrm{ml}$. & $\begin{array}{l}0.010 \\
3.1\end{array}$ & 187.0 \\
\hline $\begin{array}{l}\text { Capacity by gastroclysis } \\
(2 \text { indiv. of Falek, 1872) }\end{array}$ & $1,400 \mathrm{ml}$. & 10.5 & \\
\hline $\begin{array}{l}\text { Gastric emptying, volume/minute } \\
\quad(2 \text { indiv. of Moritz, '01) } \\
\quad(1 \text { indiv. of Best and Cohnheim, '10) ... }\end{array}$ & $\begin{array}{ll}17 & \mathrm{ml} \\
11 & \mathrm{ml}\end{array}$ & 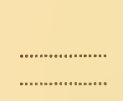 & 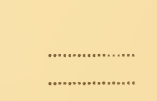 \\
\hline $\begin{array}{l}\text { Absorption, volume/minute } \\
\quad(7 \text { indiv. of Klisiecki et al., '33a) ............ }\end{array}$ & $7.2 \mathrm{ml}$. & 0.072 & 4.3 \\
\hline $\begin{array}{l}\text { Capacity by proctoclysis } \\
(5 \text { indiv. of Falck, 1873) }\end{array}$ & $540 \mathrm{ml}$. & 4.5 & 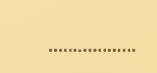 \\
\hline
\end{tabular}

Both urinary water and evaporated water are economized, the latter but slightly. The dog's "water minimum" is the rate of total water output at a water load of $\Delta \mathrm{W}-4$ or less; it is about $0.17 \%$ of $\mathrm{B}_{0}$ /hour in the dogs studied.

What conditions prevailed in the above tests of compensatory processes? Specifications for measurements of water exchanges in dogs are: (1) materials are made available and means are made available for the recovery or attempt at recovery of the animal toward its control state; (2) It is usually desirable that during the actual recovery other substances (food) than water are not available; (3) Total exchanges of water are measured by differences of body weight, and not merely those exchanges by paths that can be identified separately; (4) No anesthesia nor immediate operative 
procedure is imposed, no muscular exercise is permitted or other unusual influence of which the experimenter is aware; (5) Random days but similar hours are chosen for recoveries from diverse water loads; and (6) Various factors are arranged, according to experience in physiological experimentation and to acquaintance with the species, so that measurements are as comparable as feasible.

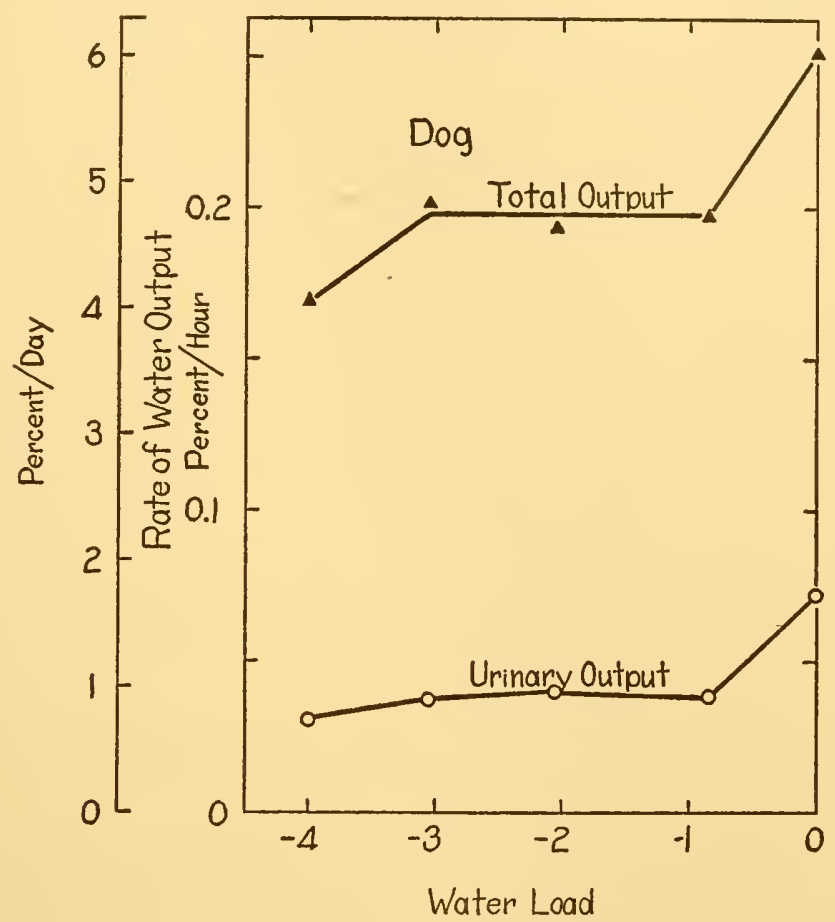

FIG. 12. Mean rates of water output ( $\%$ of $B_{0} / \Delta t$ ) at diverse negative water loads $\left(\%\right.$ of $\mathrm{B}_{0}$ ) in 2 dogs. Each measurement represents a 24 -hour period, 5 being average for each point of total loss, and 10 for each point of urinary loss. Additional data of Adolph ('39a). The results are approximately confirmed by fewer data of Straub (1899) and Spiegler ('01).

In a few words, the water exchanges are such during recovery from privation that gain greatly exceeds loss, and such that gain is augmented and loss is diminished as compared with the control exchanges. Both factors are in directions (of signs) that tend to restore water content to its control value. Of gains, drinking alone is proportional to the negative water load. Detailed features of the drinking process emphasize its physiological nature. Of losses, those by the urinary path are reduced. Each load is linked to an 
intake and an output. Their difference (net rate) is the rate at which the water load is being removed.

\section{\$9. Equilibration}

What quantitative account can now be given of the compensations for water loads? Since coordinates have the same dimensions both in excesses and in deficits of water content, the data so far considered may be combined on a common scale of water loads (fig. 13). The first one hour since recovery started is chosen as the interval of time in which rates of water exchange are measured. To figures 9 and 11 are added values for total gain of water in

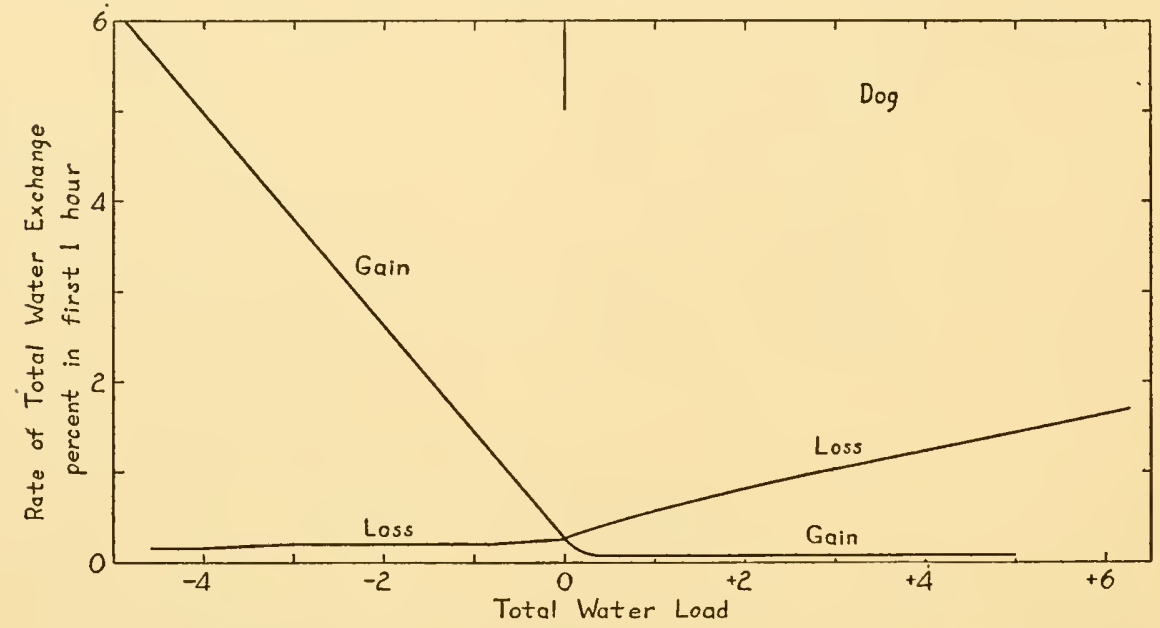

FIG. 13. Rates of total water exchanges $\left(\%\right.$ of $B_{0}$ within the first 1.0 hour of recovery) in relation to total water load $\left(\%\right.$ of $\left.\mathrm{B}_{0}\right)$ in dog. Equilibration diagram. In negative loads the data are taken from figures 11 and 12 ; in positive loads from figure 9 and from computed gain by oxidation.

excesses (by oxidation, data of Heilner, '07) and total loss of water in deficits (fig. 12) ; also figure 11 is corrected by means of figure 10 to yield data for another duration of time (1.0 hour) than that originally considered. At the same time, the two diverse modes of producing initially new water contents (privation, gastric administration), one for negative loads, the other for positive, are carefully labelled.

In figure 13 the rates of water exchange $(\delta \mathrm{W} / \Delta \mathrm{t})$ are compared with the coincidental water loads of the body $(\Delta W)$. This type of diagram, always with coordinates of like dimensions, will often recur in this investigation, and $I$ therefore give it the distinctive name (suggested by Dr. L. J. Henderson) of "equilibration dia- 
gram." It explicitly embodies a description of what takes place to correct the organism's water content. All the processes simultaneously occurring may be collectively termed equilibration.

Some features of the dog's equilibration diagram for water are: (1) The curves have but one crossing, at which gains equal losses. (2) After any departure from the water content at which this crossing occurs, water contents tend to be restored to it. The slope of each curve represents an equilibrating increment, or velocity quotient, of recovery either by gain or by loss of water. (4) High net rates of exchange mean rapid recovery. (5) Conparisons of maximal and minimal rates for gain and for loss can be

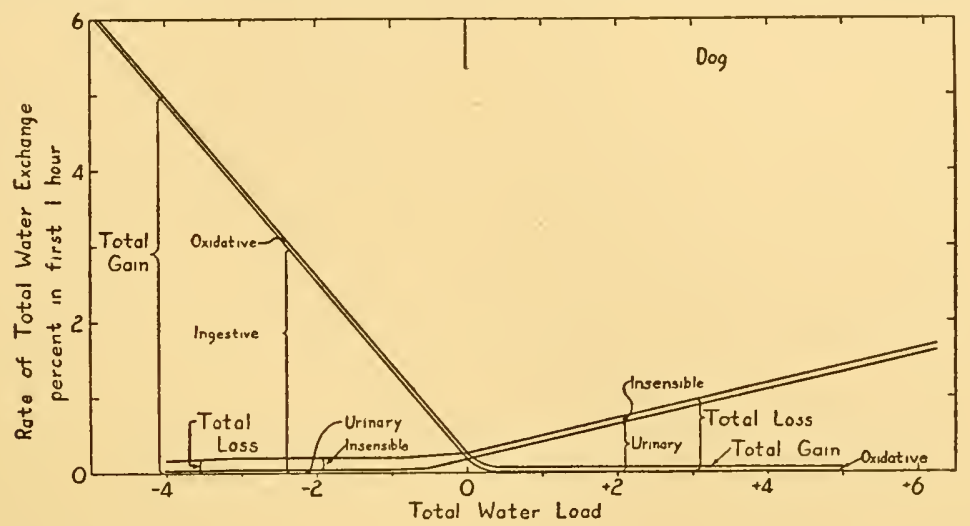

FIG. 14. Partitions or rates of water exchanges ( $\%$ of $B_{0} /$ hour) at various water loads $\left(\%\right.$ of $\mathrm{B}_{0}$ ). Losses are taken from figures 7 and 12 ; gains are from figure 11 and from the data of Straub (1899), Heilner ('07) and Lusk ('12), which indicate that rate and R.Q. of oxidation, and hence the production of water by oxidation, all are approximately independent of water content both in negative and in positive loads.

made. (6) The relative role of diverse paths at each water load may be investigated (fig. 14).

The partition of losses and gains among paths (fig. 14) is, of course, provisional. At present, outputs by three paths have been measured: urinary (sensible), evaporative (insensible), and fecal. The output by the last is too small to be shown graphically. It is noteworthy that in the dog evaporative exchange exceeds urinary at negative loads. While evaporative loss diminishes slightly where water conservation is greater, according to figure 12, this fact fails to be properly represented in figure 14 because the values recorded in figures 6 and 7 were transferred, instead of scaling the evaporative losses to their proper controls at zero load. It may be said with excellent approximation that the urinary fraction only 
is concerned in adjustments (recoveries) by water loss. Only two paths of gain are recognized: ingestive and oxidative (metabolic). Evidently oxidative formation of water is not a means of restoring. water content after a deficit; drinking alone is responsible for adjustments by water gain.

The ratio of maximal rate to minimal rate of total exchange (or of some distinguishable path of exchange) is a convenient measure of adaptability with respect to this function. It may be termed the ratio of modification. This ratio amounts in the dog, reading values from figure 13 , to $3.3 / 0.17=19$ for total loss, 100

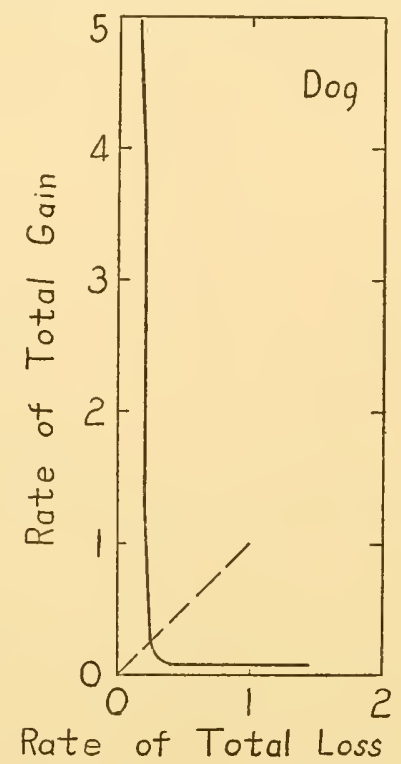

FIG. 15. Rate of total water gain ( $\%$ of $B_{0} /$ hour) in relation to total water loss ( $\%$ of $\mathrm{B}_{0} /$ hour). The curve is transformed from figure 13 , representing the first one hour of recovery of water content. Similar data of figure 29 below, representing rates in steady loads, fall on approximately the same curve. The dash diagonal line indicates equality of gain and loss.

for total gain, 103 for urinary loss, etc. This indicates the enormous range of rates over which the exchanges operate, each rate appearing under circumstances appropriate to the equilibration as a whole.

The quantitative contrasts in rates of exchange are further expressed in figure 15. High rates of gain are found only with low rates of loss, and vice versa. Water balance occurs only at relatively low rates of both. The mutual exclusion of high rates of activity has often been recognized qualitatively in the concept 
of "reciprocal action of organs." It is now possible to say that a path of intake (ingestive) is precisely correlated, rate for rate, with a path of output (urinary). This continuous juggling of swallowing against excreting, represents the dog's mode of restoring and governing its water content. Were the rate of one to be somehow disturbed in its relation to the rate of the other, different recoveries and different balances would be found.

It seems to me that the equilibration diagram yields a concrete picture of the physiological activities by which water balance is restored after disturbance of it. Among only total exchanges, there are four ways in which the dog might restore its water content. In deficits its gain might increase, its loss might decrease; actually recovery depends almost entirely on the former. In excesses its loss might increase, its gain might decrease ; actually both occur, but the former is more highly modified. Conceivable alternatives, such as no modification of any exchange, would not accomplish recovery. An increase in loss or a decrease in gain when deficit prevails, would oppose recovery. Of the four possible modifications, the dog has three, and could maintain its water content with only two; that situation actually occurs in other species.

Subtracting rates of loss from rates of gain, I obtain the rates of net exchanges (fig. 16). For convenience all net rates are considered to be positive. The relations shown compare the rates of recovery at various displacements from balance. For example, at $\Delta \mathrm{W}$ of $\pm 2 \%$ of $\mathrm{B}_{0}$ the restoration is more rapid (in the first hour) in negative increments than in positive ones.

So long as processes of exchange are quantitatively fixed in relation to water load, the water content inevitably slides back toward its control value. This control value is the only one at which ordinarily gain approximately equals loss. Hence it represents the only state in which constancy of water content persists; in fact, equality of gain and of loss characterizes this state of balance with respect to water. All other rates indicated in the equilibration diagram prevail only temporarily at loads that the organism quickly abolishes.

What can be said about this unique physiological state of balance with respect to water? Not only is the dog usually found in this state, but in it gain and loss continue indefinitely, representing the steady turnover of water. The magnitude of the turnover may be thought of in relation to many factors; it includes the "require- 
ments" of water in heat loss, in solute excretion, in swallowing of food, in water content of food, and in others. It is possible to inquire whether any one factor is likely to limit the smallness of turnover, to prevent the animal from getting along without water. So far as I can see, no one factor is limiting, and several of them insure that some water exchange will always go on.

In another direction, it is possible to inquire what properties of the dog distinguish its water losses from those of a sack of salt solution. The sack might be of rubber, allowing no exudation, or of any one of many materials that allow water to pass at diverse rates. Eimer ('26) found that the body surface of the dog lost by evaporation about $0.017 \%$ of $\mathrm{B}_{0}$ /hour to air of $23^{\circ}$ to $27^{\circ} \mathrm{C}$. and

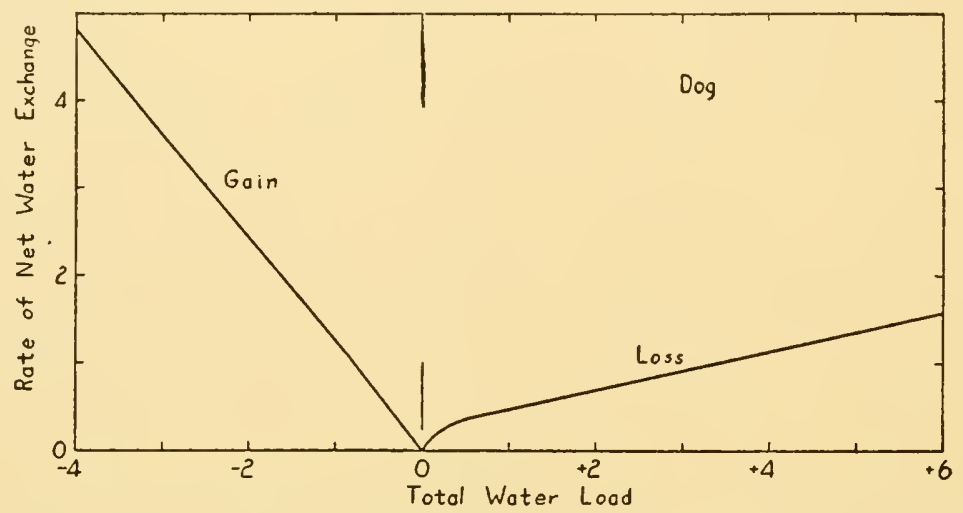

FIG. 16. Rate of net water exchange ( $\%$ of $B_{0} /$ hour) in relation to total water load $\left(\%\right.$ of $\left.B_{0}\right)$. Net exchanges are either total gains minus total losses or total losses minus total gains. These curves are derived from figure 13, and represent the rates of recovery within the first 1.0 hour of recovery.

33 to 62 per cent relative humidity (43 determinations on 4 individuals). This is about $5 \mathrm{gm}$. per square meter of skin surface an hour (Trolle, '37). Local evaporation from man amounts to about $6 \mathrm{gm} . / \mathrm{m}^{2}$ hour (Pinson), while from a water bath kept at $32^{\circ} \mathrm{C}$. it varied from $48 \mathrm{gm} . / \mathrm{m}^{2}$ hour when still, to $240 \mathrm{gm} . / \mathrm{m}^{2}$ hour when stirred steadily. Air motion and other conditions promote evaporation still further. All of this indicates that the dog's body surface does not allow free evaporation, but allows an amount small enough to be consistent with its heat turnover. Modification of evaporative rate, of course, depends chiefly on the respiratory surfaces; but such modification is found to be unrelated to water load except at extreme excesses.

If the dog, except the head, be immersed in water, exchanges 
dissimilar from those of the sack might also be revealed. The only direct tests available are not on dog but on man. No detectable water is then taken through the skin; but output through it is wholly inhibited, except when solutes are added to the bath (Whitehouse et al., '32).

The dog's respiratory surfaces appear to lose water enough to saturate very nearly all the air that is pumped in and out of them (Hemingway, '38). No system appears to have been devised in terrestrial animals whereby oxygen may be taken out of air without giving up to the air at least an equal number of molecules of water.

Altogether the paths of water exchange have a group of properties that remain fixed in states of balance. They represent anatomical, chemical, and functional arrangements suitable for the continuance of water turnover and content. Two of the paths, the kidneys and the alimentary tract, modify their activities in accordance with water load. This fact is expressed quantitatively in the equilibration diagram, which indicates in what degree each of the exchanges enters into the recovery from each possible load.

\section{\$10. Velocity QUOTIENT}

The equilibration diagram has the coordinates $\Delta \mathrm{W}$ and $\delta \mathrm{W} / \Delta \mathrm{t}$. The quantities $\Delta \mathrm{W}$ and $\delta \mathrm{W}$ have the same dimensions in both variables, and represent the same substance (water). Care is exercised to correlate only simultaneously occurring quantities. Dividing rate by load, $\delta \mathrm{W} / \Delta \mathrm{t} \div \Delta \mathrm{W}$ equals $1 / \Delta \mathrm{t}$, the velocity quotient. Its significance is clearer if written $\delta \mathrm{W} /(\Delta \mathrm{W} \times \Delta \mathrm{t})$; it is the volume of water restored or removed, relative to the increment to be restored or removed, per interval of time. If, for instance, an excess of $2 \%$ of $\mathrm{B}_{0}$ is present in the dog's body (fig. 6) and three-fourths of it $\left(1.5 \%\right.$ of $\left.\mathrm{B}_{0}\right)$ is removed per hour of recovery, the $1 / \Delta \mathrm{t}$ equals 0.75 /hour. The larger this quotient is, the faster is the recovery.

From the data of figure 13 a train of values of $1 / \Delta t$ is obtained (fig. 17), all within the first 1.0 hour of recovery. Similarly figure 16 furnishes a series of other values (fig. 18). Two kinds of quotients are thus available, depending on whether the rates of total gain and loss are used (total, fig. 17) or the rates of net water exchange (net, fig. 18). Whereas net exchanges give rather constant values of $1 / \Delta t$ at diverse loads $(\Delta W)$, total exchanges do not, for near zero load the latter ratios approach infinity. 


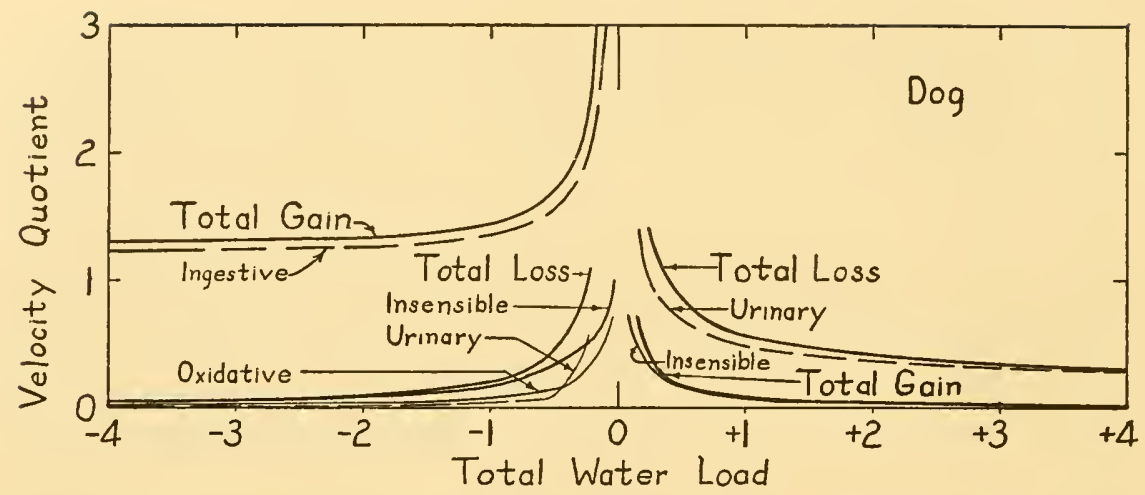

FIG. 17. Velocity quotient (1/hour) in relation to total water load ( $\%$ of $B_{0}$ ). These velocity quotients are obtained by dividing each rate of exchange by load. Total exchanges (derived from figure 13), and partitioned exchanges (derived from figure 14) are represented.

Single paths of exchange may also be represented (fig. 17). In extreme loads the difference between values for total loss and for urinary loss becomes negligible; in negative loads the difference between values for total gain and ingestive gain is very small.

The rule emerges that only that fraction of the exchange which is modified greatly with load has values of velocity quotient that approach constancy. Only the compensatory exchanges are readily

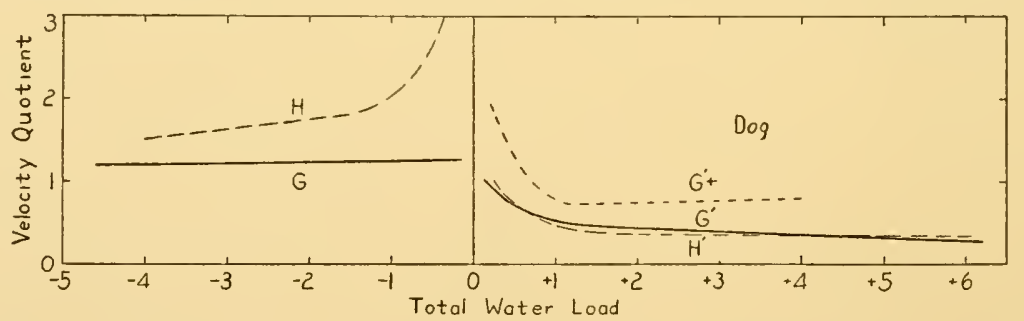

Fig. 18. Net velocity quotient ( $1 /$ hour) in relation to total water load (\% of $B_{0}$ ). These velocity quotients represent rate of net water exchange divided by total water load. Line $\mathrm{GG}^{\prime}$, first 1.0 hour of recovery after single privations or ingestions of water (derived from figure 16); line $G^{\prime}+$, later times after single ingestion (derived from figure 6 ); line $\mathrm{HH}^{\prime}$, in steady water loads (derived from figure 29, below).

described by the quotient; whenever it is constant, the rate of exchange is approximately proportional to the excess or deficit that prevails. This is a property that will be found to characterize very many processes of recovery.

In the dog velocity quotients are greater in negative increments of water than in positive increments, the mean values of $1 / \Delta t$ (net) in the first hour of water equilibration being $1.33 /$ hour and 
$0.41 /$ hour. These values show in numbers how much more is done in that interval of time to compensate for deficits.

Velocity quotients vary not only with loads but also with time (fig. 19). The largest variations are in initial periods, for in these periods the ingestion in deficit is sudden while the excretion in excess is gradually augmented. After the first hour, velocity quotients in positive loads often become nearly constant (fig. 19, $\mathrm{A}$ and $\mathrm{B})$. This indicates that the quotients found during falling

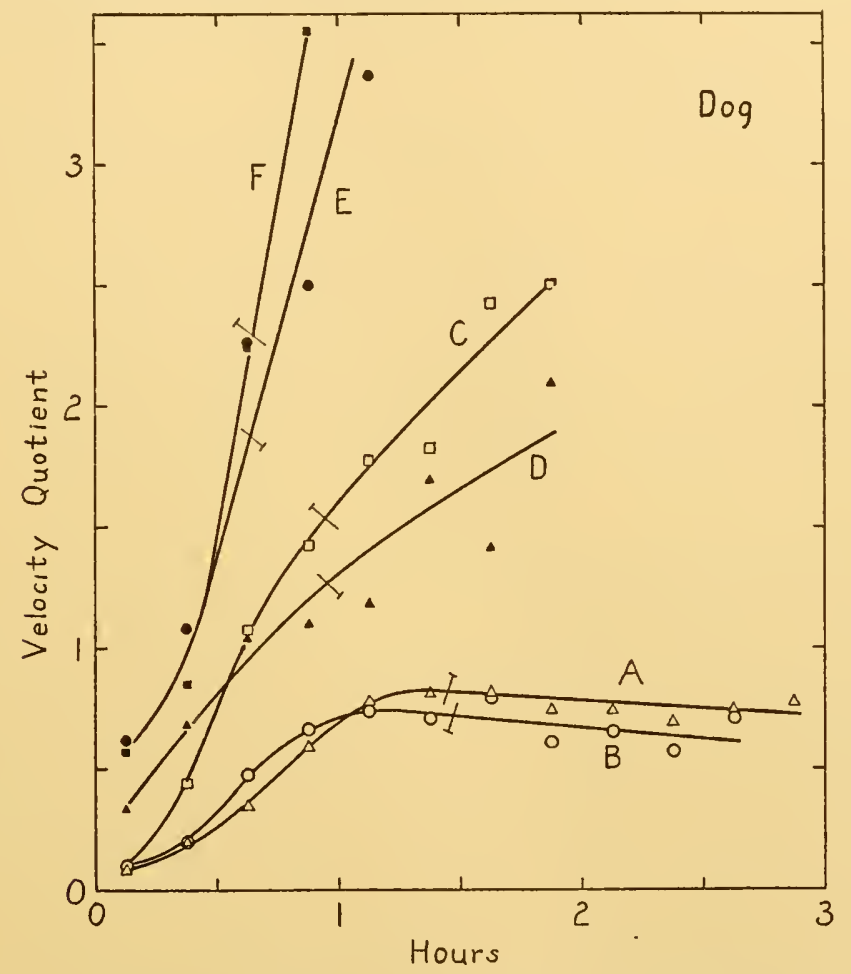

FIG. 19. Total velocity quotient (1/hour) in relation to time after single administration of water by stomach. These velocity quotients are obtained by dividing rate of total water output by total water load, using the data of figure 6 . Times at which half the administered load had been disposed of are indicated $(\perp)$ from figure 1.

rates of excretion and at loads above $+1 \%$ of $B_{0}$ serve to characterize more broadly the exchanges that prevail.

Under limited conditions, then, the velocity quotient (rate/load) compares numerically the exchanges that occur at various loads, by various paths, at various times. Very often it is constant throughout considerable ranges of load or of time. It will be seen later $(\$ 71)$ that it is a parameter in an equation that describes the 
kinetics of recovery from water load. It allows numerical representation of the very exchanges that constitute adjustment of water content.

\section{\$11. The Water-time System}

The data presented have been restricted to variables of four dimensions: $\Delta \mathrm{W}$ (water load), $\delta \mathrm{W} / \Delta \mathrm{t}$ (rate of water exchange), $\mathrm{t}$ (time), and $1 / \Delta \mathrm{t}$ (velocity quotient). Each variable appears in diverse conditions, and continued care is necessary to limit the data in any one comparison to those values obtained simultaneously or under stated conditions.

Correlations among these four variables taken two at a time are of six types:

$\Delta \mathrm{W}$ vs. $\delta \mathrm{W} / \Delta \mathrm{t}$ equilibration diagram (figs. $6,11,13$ )

$\Delta \mathrm{W}$ vs. $\mathrm{t}$

tolerance diagram (figs. 1, 5)

$\Delta W$ vs. $1 / \Delta \mathrm{t}$

$\delta \mathrm{W} / \Delta \mathrm{t}$ vs. $\mathrm{t}$

load-velocity diagram (fig. 17)

$\delta \mathrm{TV} / \Delta \mathrm{t}$ vs. $1 / \Delta \mathrm{t}$

exchange diagram (fig. 2)

exchange-velocity diagram (not exemplified)

$1 / \Delta$ t vs. t velocity diagram (fig. 19)

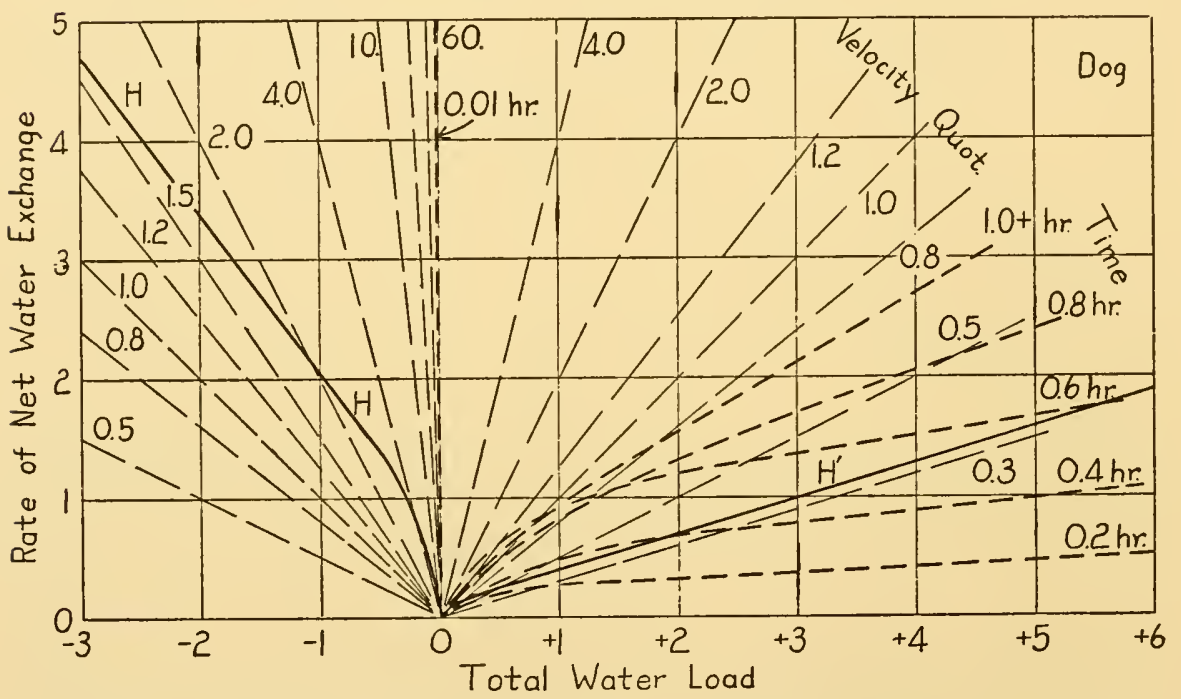

FIG. 20. Contour diagram interrelating rate of net water exchange ( $\%$ of $\mathrm{B}_{0} /$ hour), total water load ( $\%$ of $B_{0}$ ), time (hours), and velocity quotient (1/hour). Dog. Heavy lines represent sequelae of single ingestions of water by stomach, derived from figures 2 and 6 in positive loads, and from figure 10 in negative loads. Solid lines $H H^{\prime} H^{\prime}$ represent the dogs maintained at various steady water loads, derived from figure 29 of section 13. 
Three variables at a time can be represented in one diagram by one set of accessory contours. With a three-dimensional figure, four variables could be represented. For the present system of variables that is unnecessary, since one of the above four variables is a ratio between two others, and a two-dimensional figure is adequate (fig. 20). On this one diagram may be represented the data contained in all the foregoing figures.

These four variables may be said to constitute the water-time system for this particular species under the prescribed types of load and the named conditions of environment. Such a system is delimited by the describer. Correlations among these variables characterize the responses to disturbances of water content with respect to time and to accuracy of recoveries.

\section{\$12. Stationary states of excess}

Thus far the responses have been described that follow a sudden addition of water or end a privation of water. Other physiological states have also been studied, in which the increment of water in dogs in maintained steadily. Do they also help in understanding equilibrations?

Positive increments $(+\Delta W)$ are created by administering repeated doses of water by stomach at equal intervals of time (figs. 21 and 22). The process of loading the body with water is most effective at first, for then nearly all the water put into the stomach stays in the body. With the passage of time and the increase of load, output becomes faster (figs. 23 and 24). Still, only at low rates of water administration ( $\mathrm{M}$ and $\mathrm{N}$ ) does the rate of output come to equal (at 1.5 hour) the rate of forced intake. Output also becomes stationary over a period of time, and might remain so for an indefinite period, if intake continued. When water administration ceases (at 2.5 hours) excretion is well under way, and no lag occurs in the recovery; in other words, the maintenance of a steady output and the beginning of a recovery output are one process. Throughout recovery the rate of water loss is closely proportional to water load, or, the falling curves (as well as the rising curves) of figures 21 and 22 are exponential with time.

All this is shown in still another way by comparing simultaneous rates of intake and of output (fig. 25). The sequence of exchanges is emphasized by this correlation.

When the rates of water output are correlated with the loads 
of water prevailing (figs. 26 and 27), it becomes explicit that the rates during loading are not equivalent to those during unloading. Hence even the procedure of repeated administration has its time factors, but not of a sign that would be concerned with lag in absorption of administered water. During unloading, rates are nearly linearly proportional to the loads.

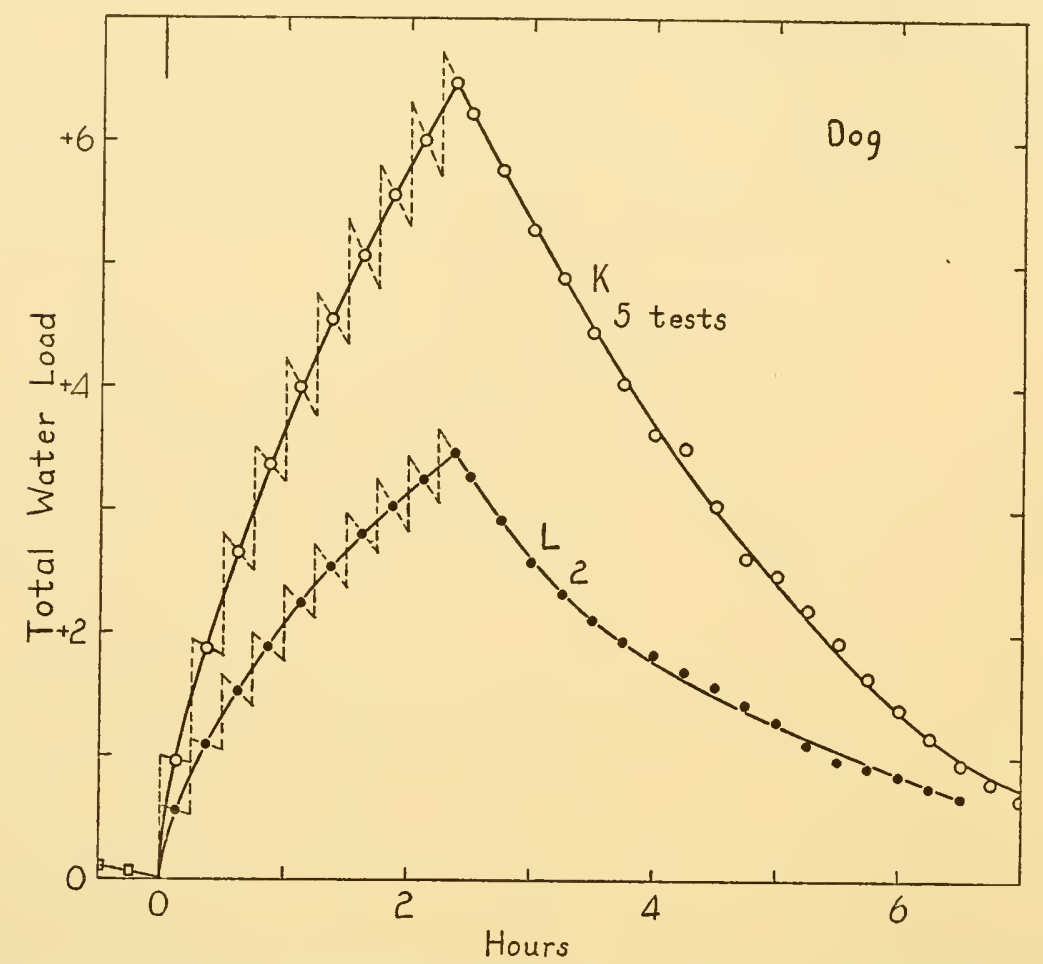

Fig. 21. Course of total water load ( $\%$ of $B_{0}$ ) when water is administered by stomach tube in ten equal portions ( 1.00 or $0.60 \%$ of $\mathrm{B}_{0}$ each) during the first 2.5 hours. Dog, 2 individuals. The actual loads found by weighing are connected by dotted lines, while the mean loads of each 0.25 hour are marked by points. After 2.5 hours the points are actual body weights. New data of Kingsley and Adolph.

Comparing figure 26 with figure 6 , and figure 27 with figure 7 , I find that the rates of output during recovery from repeated water administration are less than during recovery from single doses. I think the difference is significant in spite of the fact that diverse individuals, and the same individuals in dissimilar stages of acclimatization to water excesses, were used in some of the tests that are averaged. Hence it may be concluded that the rate of recovery depends not only upon the magnitude of the load but also upon the 
duration and course of the previous loading. Slow and prolonged administration yields slower excretion at any one load. Further evidence of difference is observed in other ways. The highest output rates in series $K$ to $N$ are found when intake rate exceeds output rate; series $\mathrm{A}$ to $\mathrm{F}$ further exaggerates this factor. During: intake the rate is higher than after it, as though incoming water is more promptly excreted than water already incorporated.

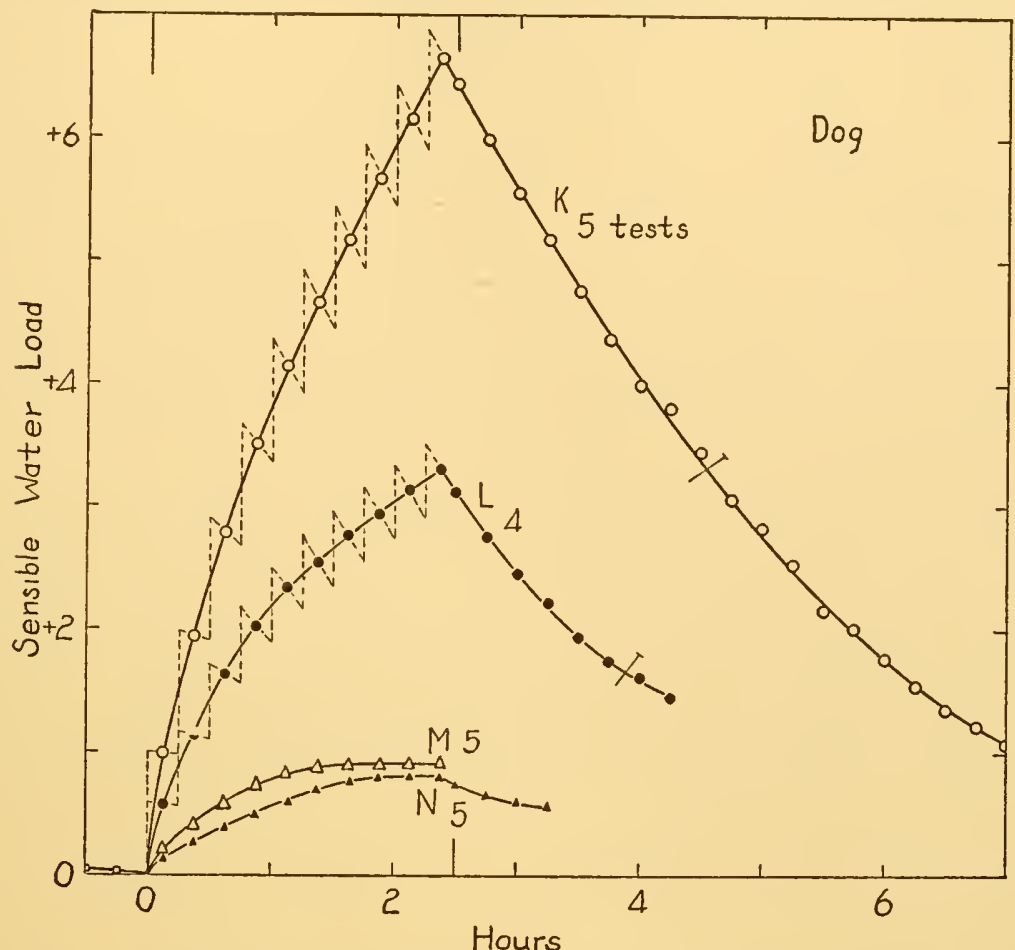

FIG. 22. Course of sensible water load $\left(\%\right.$ of $\left.\mathrm{B}_{0}\right)$, i.e., ingested water minus urine excreted, with repeated water administration. In the first 2.5 hours, water is given by stomach tube in ten equal portions, and the loads are designated as in figure 21 . Half of the maximal load is returned at $\perp$. The total amounts of water given are: $\mathrm{K} 10.0 \%$ of $\mathrm{B}_{0}, \mathrm{~L} 6.0 \%$, M $2.14 \%$, N $1.40 \%$. Data of Kingsley and Adolph.

Which of the data shown may be considered characteristic of the stationary state of load? A stationary state strictly exists only in those limited periods where neither contents nor rates of exchange manifest a marked trend. A marked trend is (for the present) one in which the component may be shown to have changed, either consistently or statistically significantly, within three of the successive periods of time in which its exchange is measured. 


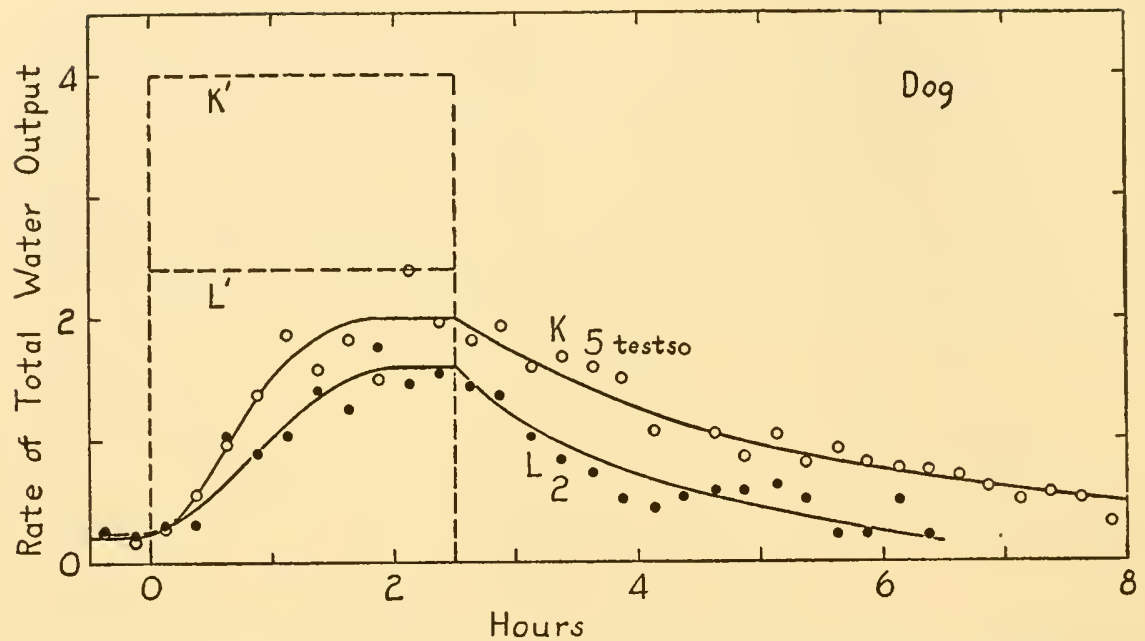

FIG. 23. Rate of total water exchange ( $\%$ of $\mathrm{B}_{0} / \mathrm{hr}$.) in relation to time. In the first 2.5 hours water is given by stomach in the equal portions at the rates of intake $K^{\prime}$ and $L^{\prime}$. Rates of output are ascertained every 0.25 hour. The same tests are represented as in figure 21 .

Actually, the data show that both in the stationary state and in the recovery state following it (i.e., during diminishing loads), the rate bears the same proportionality to load. In other words, after the load ceases to increase, the rates of output in series $K$ and $L$ are regularly related to water load, and not to time. This conclusion could only be guessed until the consistent data were obtained based

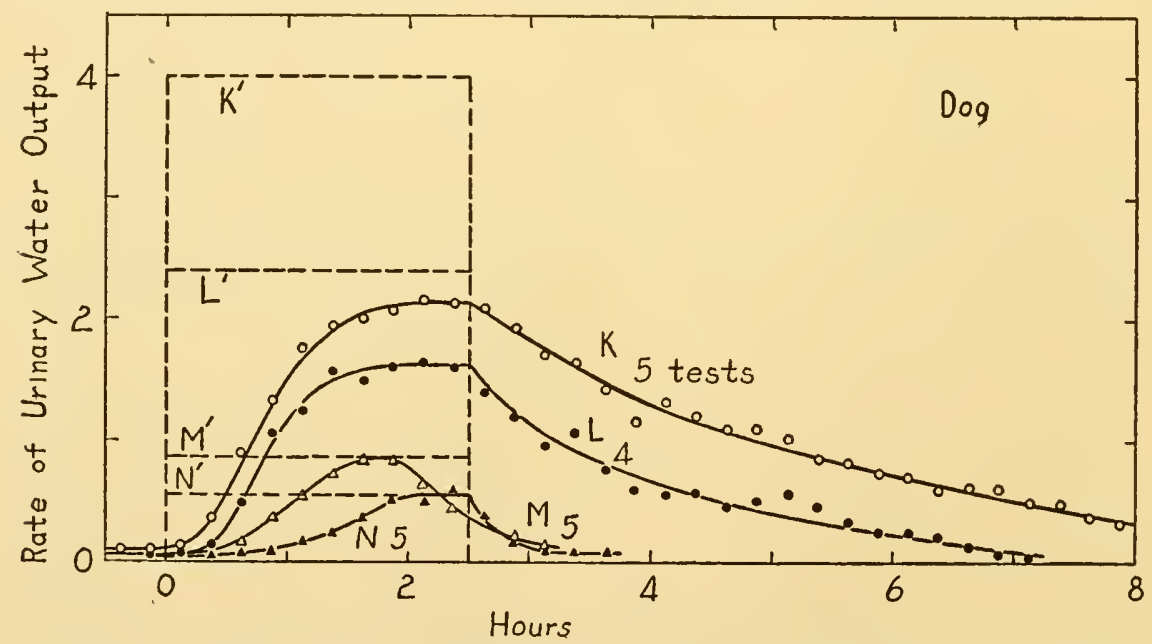

Fig. 24. Rate of urinary water output ( $\%$ of $\mathrm{B}_{0} / \mathrm{hr}$.) in relation to time. The same tests, with repeated water intake by stomach, are represented in figure 22. 


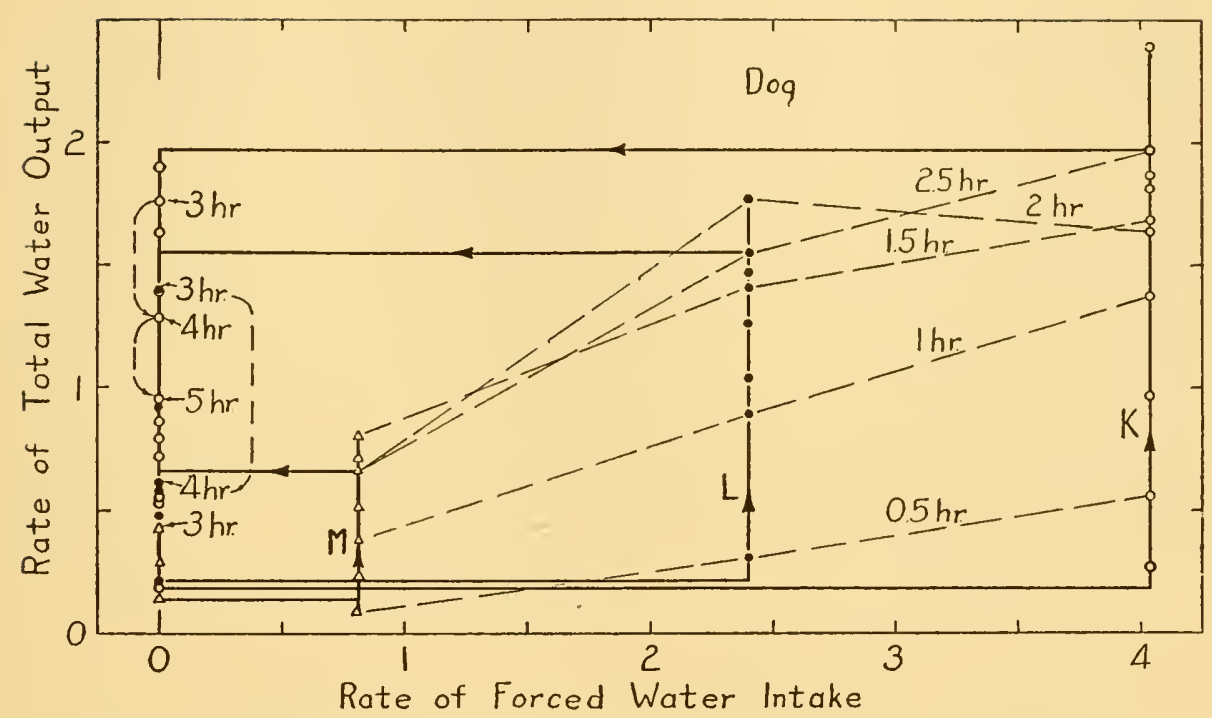

FIG. 25. Rate of total water output ( $\%$ of $\mathrm{B}_{0} / \mathrm{hr}$.) correlated with simultaneous rate of forced water intake ( $\%$ of $\mathrm{B}_{0} / \mathrm{hr}$.). Water is given by stomach tube every 0.25 hour for 10 periods. Times are indicated by light dash lines; heavy lines follow the mean progress of any one rate of administration. The data are the same as in figures 23 , $M$ representing one test additional.

upon many loads. Hence the rate of loss seems to be linear with load within the range of increments studied (fig. 26).

More extreme positive loads of water were observed in dogs by Greene and Rowntree ('27) and by Harding and Harris ('30). Most of their data are incomplete for the present purpose, since either loads (body weights) were not reported or rates of loss were

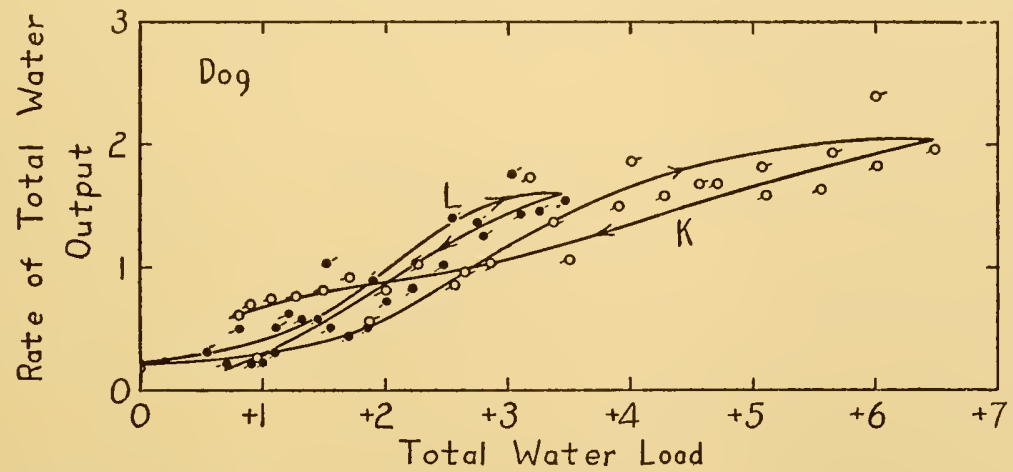

FIG. 26. Rate of total water output ( $\%$ of $\mathrm{B}_{0} / \mathrm{hr}$.) in relation to total water load $\left(\%\right.$ of $\mathrm{B}_{0}$ ). Repeated water intake during 2.5 hours, followed by recovery. The data are those represented in figures 21 and 23 . In rising loads points are knobbed to the right, in falling loads to the left. 
ascertained only over periods lasting several hours. Many of their rates are higher than in figure 26, the maximum being $3.4 \%$ of $\mathrm{B}_{0}$ /hour. But whether they are still linearly proportional to water load cannot be ascertained; I infer they are not. Certain of the data given by Harding and Harris indicate that in some individuals low rates of water output accompany large loads, as though under stress of continued administrations of large amounts of water ( $10 \%$ of $\mathrm{B}_{0}$ /hour), water automatically accumulates (up to $+20 \%$ of $B_{0}$ ) in individuals that excrete slowly.

Descriptive physiology may proceed in the correlation of quantities without inquiring whether the hypophysis, or any other organ, regularly influences the excretion of water. Very often

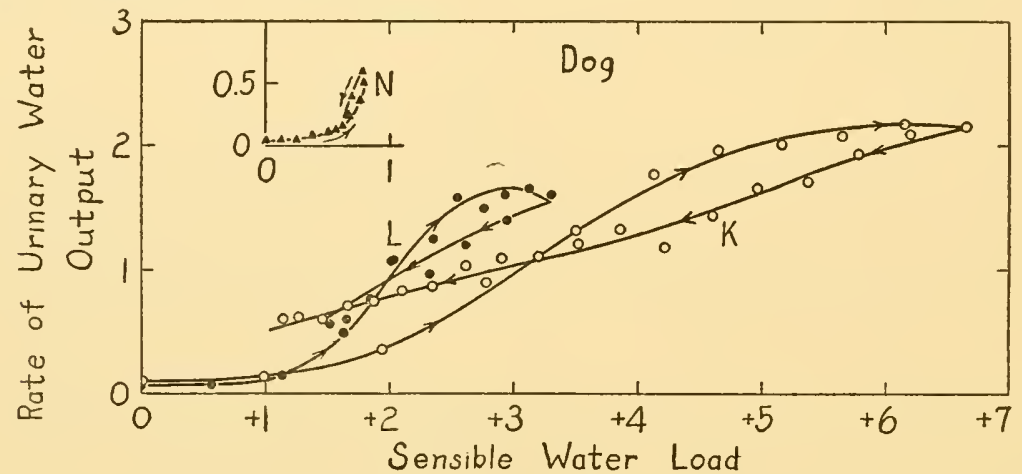

Fig. 27. Rate of urinary water output ( $\%$ of $\mathrm{B}_{0} / \mathrm{hr}$.) in relation to sensible water load $\left(\%\right.$ of $\left.\mathrm{B}_{0}\right)$. The data are from figures 24 and 22 , water being given by stomach every 0.25 hours for ten periods up to maximal load.

such an organ is regarded as a dictator of water exchanges. From the relations shown above it is evident that the dictator, if such there be, precisely grades the exchange to the load. Whether that fact robs the dictator of his title, is of little moment. To know in what anatomical part, if any, the grading of exchange to load is managed, is a project for research, but is not required for the present description of water maintenance. For, the quantitative relations shown in equilibration diagrams are explanations of water regulation to the same degree as relations of any other kind that have been discovered.

What has been shown is that repeated gastric administrations of water at brief intervals ultimately produce a stationary rate of water loss. At diverse rates of administration, the resulting losses are proportional to water loads. The same proportionality con- 
tinues during recovery after administration ceases, but each rate of loss is somewhat less than when sudden excesses are given the dog to obtain an equal load.

\section{\$13. Stationary states of Deficit}

Negative increments of water are readily maintained in dogs with fistula of the esophagus. Water taken into the mouth escapes into a measuring vessel, thus indicating the amount drunk by the dogs, but does not enter the remainder of the body to modify its water content. On constant diet these dog's may receive inadequate amounts of water by stomach, creating water (weight) deficits of

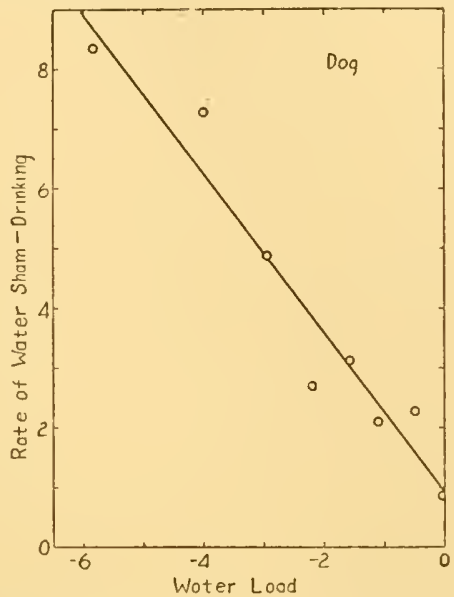

FIG. 28. Rate of sham-drinking of water $\left(\%\right.$ of $B_{0} / h r$.) in relation to negative water load $\left(\%\right.$ of $\left.\mathrm{B}_{0}\right)$ in two dogs with esophageal fistulas. Each measurement is the mean for a period of 24 hours. Twelve such measurements are averaged, in order of water or weight deficit, for each of the points plotted, giving the regression of rate on load. Redrawn from Adolph ('39a).

diverse magnitudes. Hour after hour the sham-drinking intermittently continues, at mean rates (fig. 28) proportional to the deficit prevailing.

The intermittent drinking of the dog with esophageal fistula approaches in velocity only momentarily the sudden drinking of the dog that ingests (Adolph, '39a). But spread over an arbitrary interval of one hour or more the steady rate is found to surpass the initial rate. The intermittent character of drinking ordinarily allows time for absorption to follow ingestion. Evidently the alimentary tract meters the water taken, before any postabsorptive influence upon bodily composition occurs. The subsequent failure of the postabsorptive factors to confirm the earlier alimentary fac- 
tors as to whether water was obtained, releases more alimentary activity (Bellows, '39).

Data are now available for a second equilibration diagram that concerns stationary states of water loads (fig. 29). Comparing in it the positive increments with equal negative increments, I note that all rates of net gain surpass rates of net loss. It is as though intake were on a larger scale (oversize) than output. The same statements are represented in another form by the velocity quotients (fig. 18, $\mathrm{HH}^{\prime}$ ).

At any one load, simultaneous rates of gross gain and of gross loss of water may be compared numerically by taking their ratio.

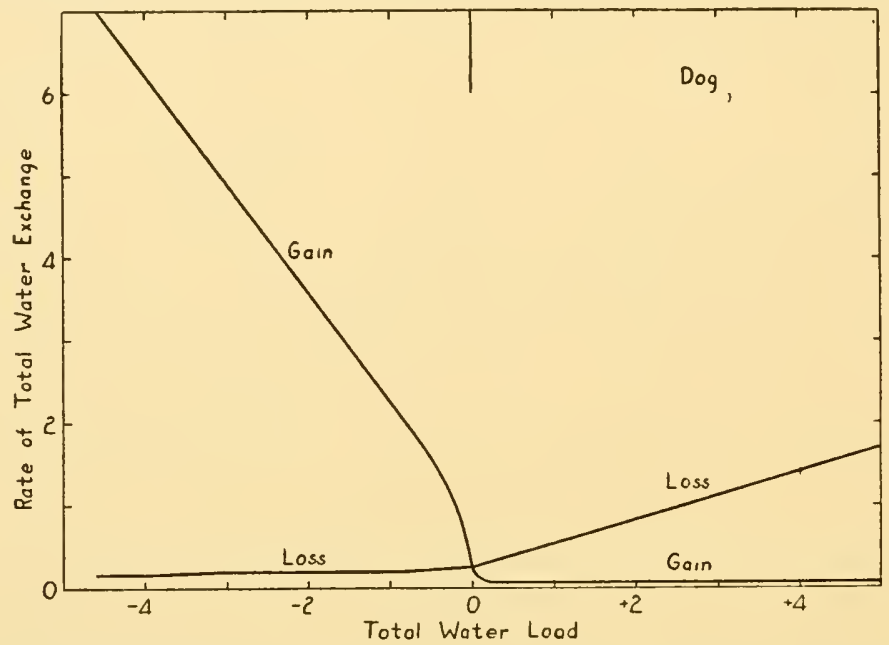

FIG. 29. Rates of water exchanges ( $\%$ of $\mathrm{B}_{0} /$ hour) in relation to water load (\% of $\mathbf{B}_{0}$ ) in steady states of load. Equilibration diagram. In negative loads the data are from figures 28 and 12 ; in positive loads the losses are from figure 26, the gains from figure 13.

This ratio (total gain/total loss) has been termed by Huber ('24) the water economy, or economy quotient. At balance the economy quotient is 1 . The economy quotient thus measures the relative role of gain and of loss during attempts to recover.

Thus, in selected conditions of nearly steady water load, uniform rates of water exchange are observed. In them the equilibration diagram measured is of wide generality, for it is independent of time. Whenever the water load is known, total and partitioned exchanges are also known. Whenever one rate of total exchange is known, the others and the water content may be predicted. 


\section{\$14. Summary}

To find how the dog adjusts the content of water in its body, unusual amounts of water are experimentally provided, and subsequent movements of water into and out of the body are observed. It is found that excesses are removed chiefly through the kidneys, at rates that are nearly proportional to the excesses; while deficits are made up with amazing promptness and exactitude by drinking. Both recoveries are by modifications in rates of exchanges that already are operating in turnover.

Alternatively, excesses may be experimentally maintained by continual addition of more water, and deficits by not allowing the water that is drunk to be absorbed (esophageal fistula). Then the time elapsing since the water load was imposed ceases to be an important factor; the rate of exchange is stationary.

In all circumstances the relations between exchange and load serve to describe the processes concerned in recovery from water load. Such relations, represented in equilibration diagrams, indicate the events by which the usual water content is restored, and ordinarily is maintained. Partial representations are afforded by various numerical means: velocity quotient, ratio of modification, and economy quotient. These are several ways of comparing the modifications of water exchanges that occur in the presence of increments of water content. They concern only four sorts of variables which for convenience are said to constitute the watertime system of the dog.

The relative effectivenesses of the separate paths of exchange (urinary, evaporative) are rated according to the speeds with which water flows through them at various increments. The net effects in compensating for unusual water contents are the algebraic sums of these speeds. The fact that each rate of net water exchange is proportional to water load appears to be a condensed account of what the dog does to compensate for any disturbance of its water content. 


\section{Chapter III}

\section{OTHER TYPES OF WATER INCREMENT (DOG)}

$\$ 15$. It seems useful to examine further compensatory exchanges of water, before the dog's other manifestation of water regulation are examined. In the investigation of water equilibration already presented, only one general method of producing excess and only one of producing deficit are considered; positive loads are imposed through administration by stomach and negative loads through privation. These two types of positive and negative increment, and various sorts of stipulated conditions, were arbitrarily chosen; scores of alternatives are possible.

Data exist for water exchanges following some of the alternative modifications of water content, and I now inquire what features of the physiological recoveries are similar, and what ones differ, among them. There is no way other than actual comparison of finding whether or not, for instance, what Keith ('24) called "dehydration" is the physiological equivalent of what Gamble ('29) called "dehydration." How does the dog indicate equivalence among possible deficits or excesses of water? Does "excess" of water content always call forth polyuria, and one rate of urinary output?

\section{\$16. ExCESSES of WATER}

Types of water load may be provisionally grouped according to manners of their production.

a. Does an anesthetized dog with water load differ from the same individual unanesthetized? It is widely recognized that it usually does; the present object is to treat the differences as quantitative ones. In positive loads of water, the rates of elimination are diminished under the influence of ten out of eighteen narcotics tested by Bonsmann in a variety of concentrations. A few results are shown in table 2. The other narcotics, such as papaverine in the dose tested, do not diminish water diuresis. None of them augments the returns by significant amounts, indicating that, as usual, processes are not hastened by imposed agents.

In negative loads the rates of water intake under anesthesia are zero. When stated thus, any alternative seems preposterous; but that does not keep physiologists from doing experiments which 
TABLE 2

Urinary water losses of dogs, in the first 2.0 hours after giving water by stomach plus administering narcotic or anesthetic

\begin{tabular}{|c|c|c|c|c|c|}
\hline Physiological state & $\begin{array}{l}\text { Num- } \\
\text { ber of } \\
\text { tests }\end{array}$ & $\begin{array}{l}\text { Water } \\
\text { given, } \\
\text { \% of } \mathrm{B}_{0}\end{array}$ & $\begin{array}{c}\text { Urinary } \\
\text { water loss } \\
\text { in } 2 \mathrm{hrs} ., \\
\% \text { of } \mathrm{B}_{0}\end{array}$ & $\begin{array}{l}\text { Urinary } \\
\text { water loss } \\
\text { in } 2 \text { hrs., } \\
\text { \% of the } \\
\text { water } \\
\text { given }\end{array}$ & Source of data \\
\hline 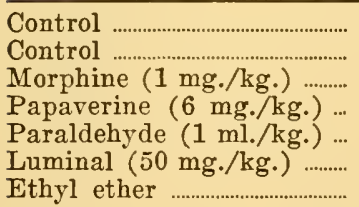 & $\begin{array}{r}4 \\
20 \\
16 \\
3 \\
8 \\
11 \\
4\end{array}$ & $\begin{array}{l}1.99 \\
2.20 \\
1.99 \\
2.15 \\
1.77 \\
2.18 \\
2.78\end{array}$ & $\begin{array}{l}1.74 \\
1.56 \\
0.37 \\
1.62 \\
0.90 \\
0.38 \\
0.55\end{array}$ & $\begin{array}{l}87 \\
71 \\
19 \\
75 \\
51 \\
18 \\
20\end{array}$ & 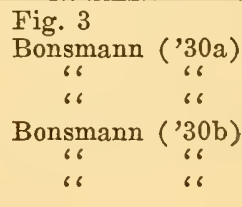 \\
\hline
\end{tabular}

presuppose that water balance is restored or maintained during anesthesia. Actually, no method has been devised of demonstrating whether an animal that is in water balance before anesthesia continues to be so under anesthesia. Therefore all such tests rest on the supposition that anesthesia has not shifted the relation between water content and water balance.

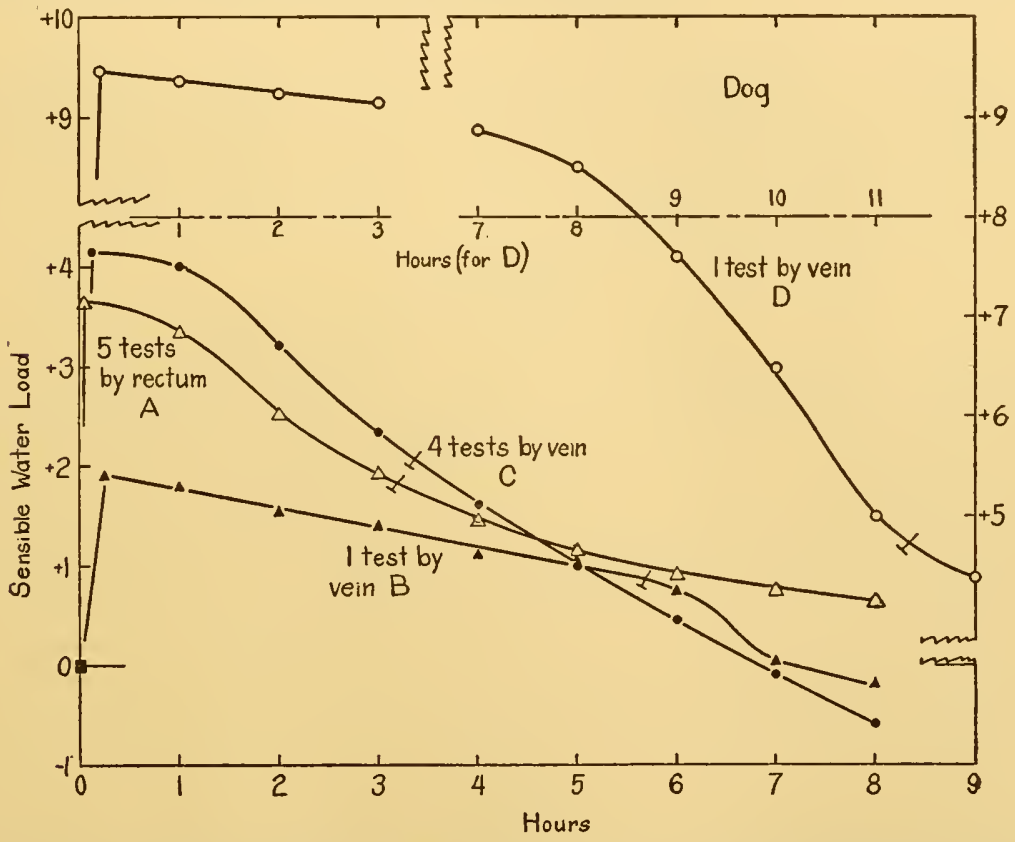

FIG. 30. Course of sensible water load $\left(\%\right.$ of $\left.B_{0}\right)$, i.e., forced intake minus urinary output. Dog. Water given by rectum (A), mean of 5 tests on one individual; data of Falck (1873). Water given by vein (B, C, and D) during 0.2 hour; data of Falck (1872). At $\perp$, half the load is returned in urine. 


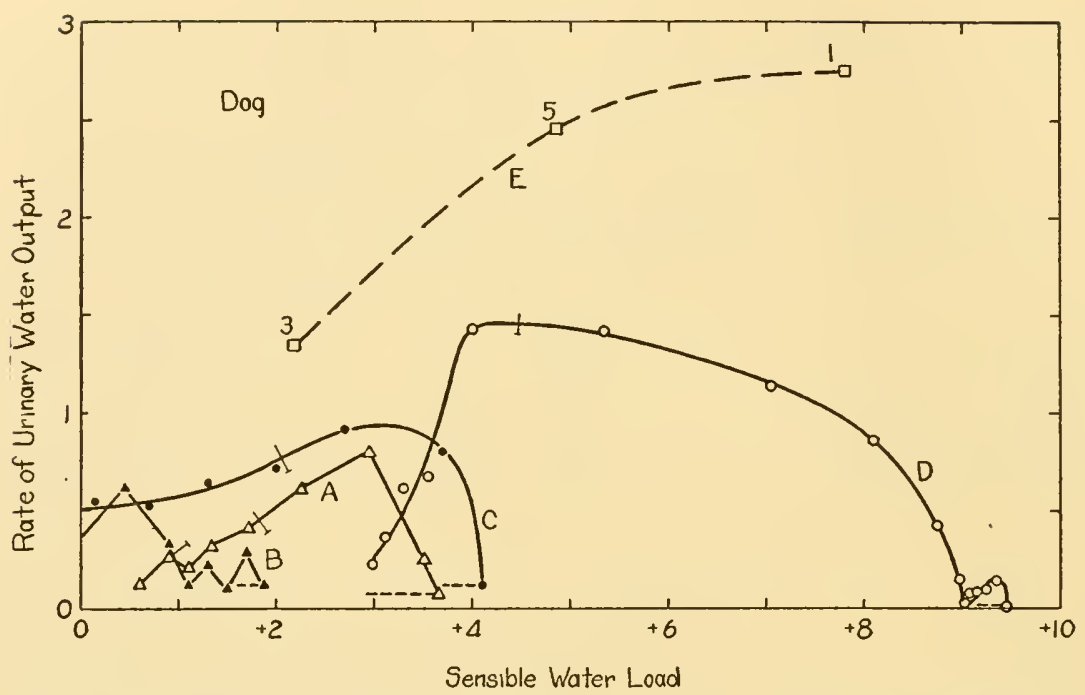

Fig. 31. Rate of urinary water loss ( $\%$ of $\mathrm{B}_{0} /$ hour) in relation to sensible water load $\left(\%\right.$ of $\left.\mathrm{B}_{0}\right)$. A, water administered by rectum; $B, C$, and $D$, water administered by vein; $\mathrm{E}$, maximal rate in the third hour after giving water by stomach to the same 3 individuals. Each load is the mean during 1.0 hour. Same data as in figure 30 , of Falck (1872, 1873).

b. Various route's of administering water have been studied. Falck (1872) gave $9 \%$ of $\mathrm{B}_{0}$ of water by vein (figs. 30 and 31 ) with results far different from those when the same individuals received

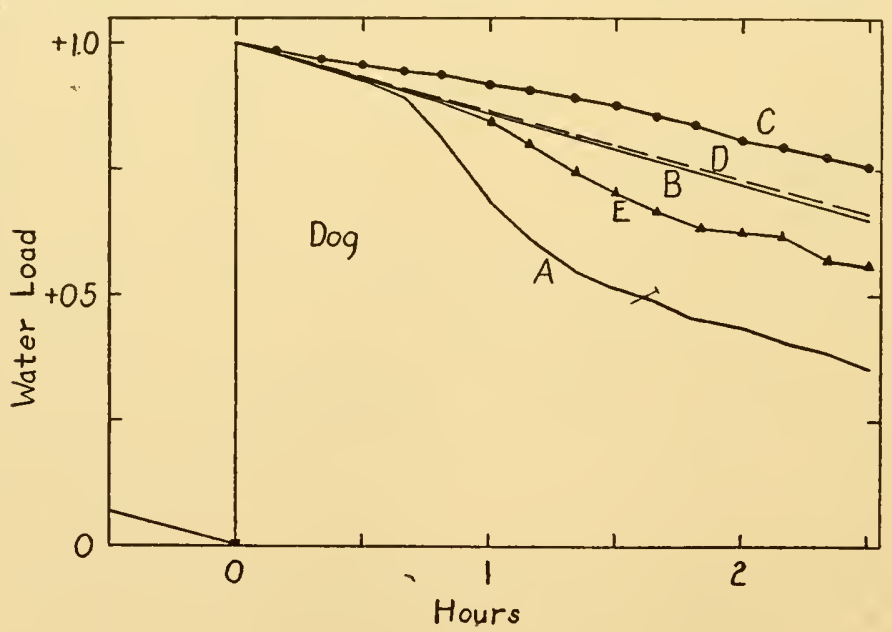

FIG. 32. Course of sensible water load after single equal volumes of water are introduced by five types of procedure. Dog. Each curve is the mean of 4 tests and individuals with bladder fistulae. A, tapwater introduced quickly by stomach; B, tapwater subcutaneously in 0.2 hour after zero time; C, tapwater by vein in 0.2 hour; $D$, tapwater subeutaneously in 1.0 hour; E, tapwater by vein in 1.0 hour. Data of Hashimoto ('14). 
water by stomach. The diuresis that follows infusion is delayed, less intense, and greatly prolonged; thus diuresis is not apparent for six hours, during which oliguria prevails (D). Even with smaller administrations (1.9 and $4.1 \%$ of $\mathrm{B}_{0}, \mathrm{~B}$ and $\mathrm{C}$ ) seven hours are required to eliminate in urine the entire volumes injected. At any one load, the rates are all lower than when the water is given by stomach. But $15 \%$ (Chiray et al., '38) to $21 \%$ (Falck) may be tolerated when given by vein.

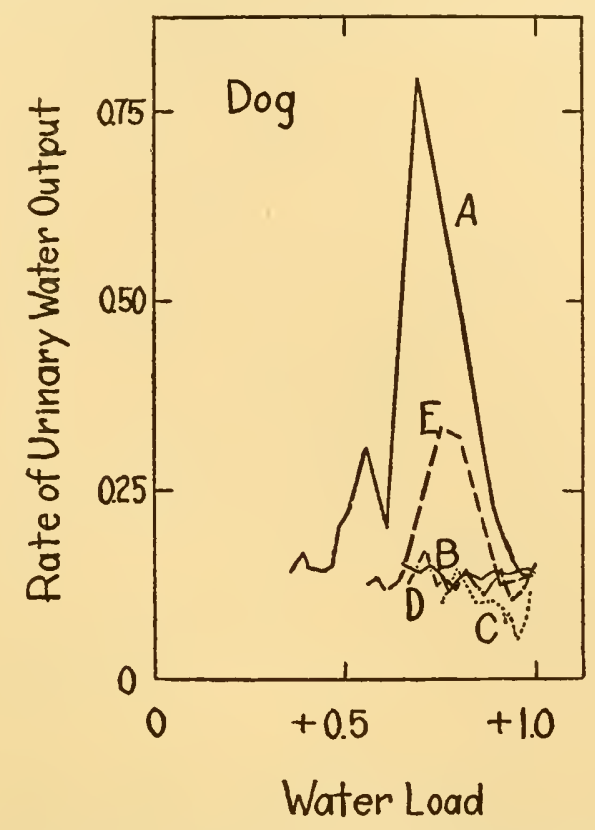

Fig. 33. Rate of urinary water output ( $\%$ of $\mathrm{B}_{0} /$ hour) in relation to sensible water load $\left(\%\right.$ of $\left.\mathrm{B}_{0}\right)$. Single equal volumes of water are administered by five types of procedure. Same data of Hashimoto as in figure 32.

Hashimoto ('14) injected water by vein in less amount (1\% of $\mathrm{B}_{0}$ ). When given during a period of time equivalent to Falck's (0.1 to 0.2 hour) only oliguria results (C, figs. 32 and 33). But given during 1.0 to 1.2 hour (E) some diuresis occurs, yet only sufficient to return as urine during it one-fifth of the fluid administered.

Water given subcutaneously in the amount of $1 \%$ of $B_{0}$ yields no diuresis whether injected rapidly (B) or slowly (D, fig. 32).

When water is given by rectum $\left(3.6 \%\right.$ of $\mathrm{B}_{0}$, tests $\mathrm{A}$ in figs. 30 and 31 ) diuresis is prompt, but less in rate and less prolonged than after the same load is given by stomach. 
The upshot is that water is not the same everywhere. The site of the water and the rate of administration of it are distinguishable factors. It may be inferred that the water content of the body is, after one of these administrations, eventually adjusted to its initial value; meanwhile an excess is present, often for many hours. It was found above $(\$ 13)$ that sudden additions of water lead to faster elimination than gradual additions of equal amounts. Hence any decrease in rate of passage or absorption is likely to decrease the rate of recovery. By no known rule is the rate of output regularly limited by a capacity of the kidneys ; time relations throughout the body are factors.

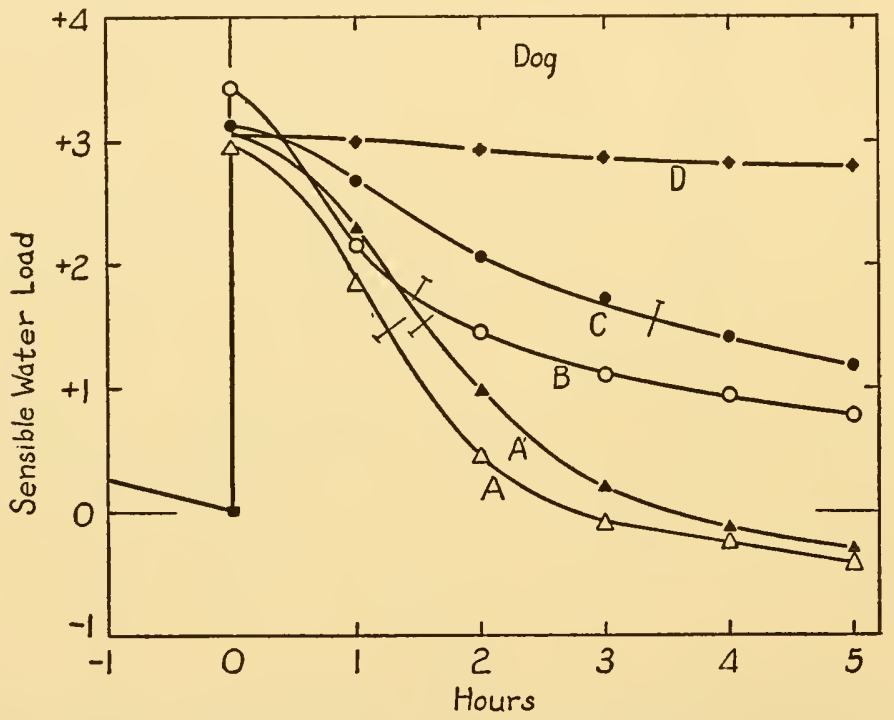

FIG. 34. Course of sensible water load in dogs subjected to four diverse regimes. Single doses of water are given by stomach. Each point represents the mean of 6 or 7 tests on as many individuals, the same individuals being catheterized hourly in each set. $A$ and $A^{\prime}$, standard state; $B$, fed thyroid substances; C, deprived of food for 8 days but allowed water ad libitum; $\mathrm{D}$, deprived of food and water for 8 days. In $\mathrm{D}$ the sensible water load does not represent the total water load, since water balance did not prevail at zero time as it presumably did in the other tests. Data of Hatafuku ('33a, '33b).

c. Dogs subjected to various regimes are given water by stomach. If deprived of food and water for 8 previous days (D, fig. 34), they show no diuresis when $3 \%$ of $\mathrm{B}_{0}$ of water is given. If previously given no food but allowed water ad libitum (C) they have diuresis, yet only half of the water given is returned in urine within 4 hours; while all of it is returned by the same individuals upon control days. It is quite arbitrary to define water balance under such regimes. 
If fed with thyroid substance, diuresis follows the introduction of water to the stomach just as promptly, but sometimes yields less complete returns (B, fig. 34). The same procedure (so far as stated) in another laboratory showed continued greater rates of water output and equally complete returns during thyroid feeding (B, fig. 35). Here is a regime, the only one known at present, that augments the exchange above the usual. Privation of food superimposed on the procedures mentioned diminishes the response to that without thyroid administration (Hatafuku, '33b).

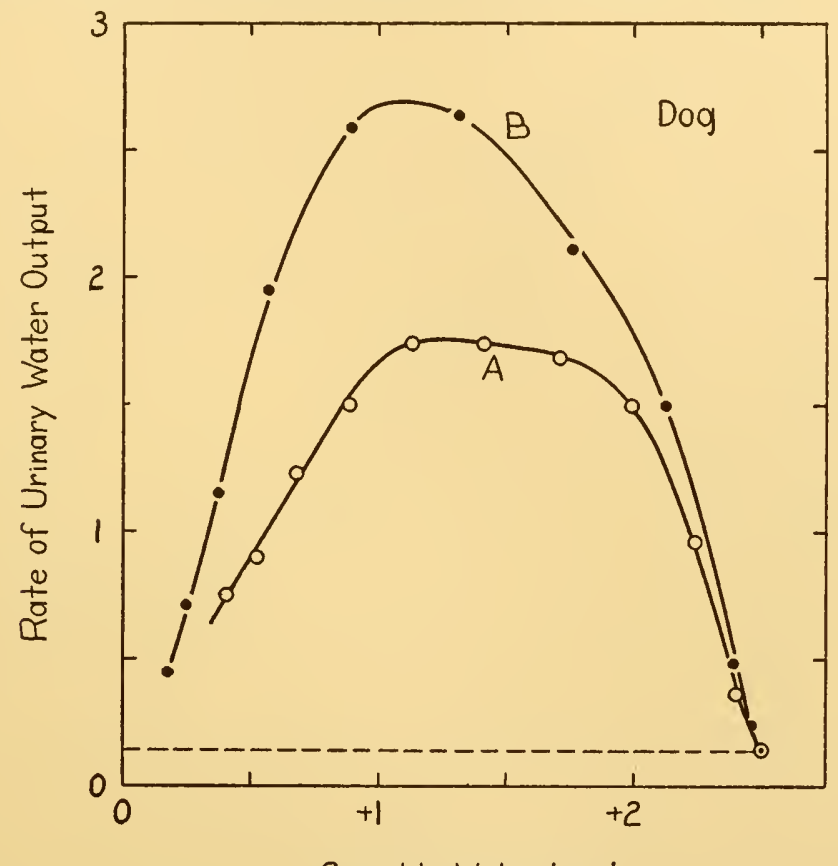

Sensible Water Load

FIG. 35. Rate of urinary water output in relation to sensible water load during the elimination of single doses of water given by stomach. A, standard state; B, thyroxinfed. Each point represents the mean for four dogs, in 18 and 20 tests altogether, the same four individuals with "extended" ureters being used for both sets A and B. Data of Klisiecki et al. ('33b, p. 534).

If the dog is injected with pituitrin, the introduction of water to the stomach induces no diuresis for some hours (Molitor, '26a; Klisiecki et al., '33), after which the effects of pituitrin disappear. Altogether less than two-thirds of the water administered is returned in urine.

Poisoning with phosphorus (C, fig. 36) appears to reduce the 
rate of output of water in recovery from water excess. But administering the drug novasurol (D) seems to remove the effect of phosphorus treatment.

Physical exercise of running prevents or reduces the usual response to water introduction by stomach (Rydin and Verney, 38). This inhibition of diuresis may intervene at any time after diuresis has begun, and may outlast the exercise by various lengths of time.

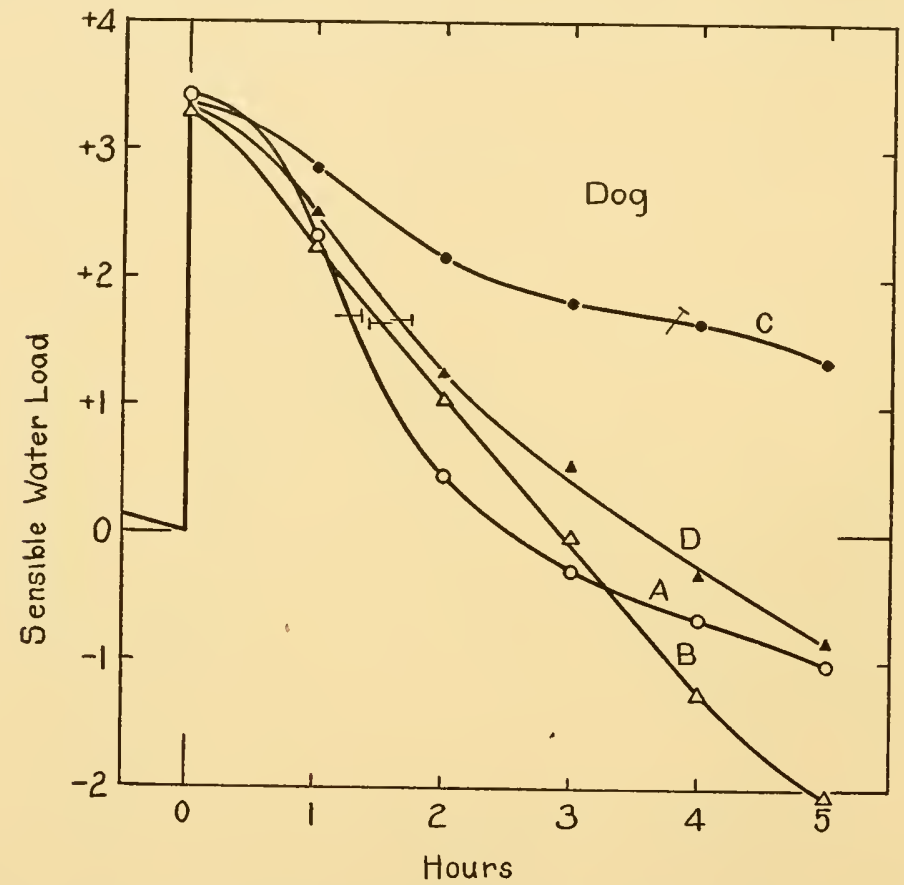

FIG. 36. Course of sensible water load after single ingestions of water by stomach. Each of four individuals is tested once in each series. Data of Abe ('31c). A, in control state; B, given novasurol intramuseularly; C, treated with phosphorus; D, treated with novasurol and phosphorus.

The general conclusion is that many regimes give rise to physiological states that modify the recovery of water balance.

d. Various solutions may be substituted for water to constitute positive loads. Given by stomach, such solutions induce almost any degree of polyuria and oliguria, according to the solute, its concentration and amount (Chanutin et al., '24; Rioch, '30; Melville, '36; Kaunitz, '37). Given by vein the return is no more complete (D, fig. 37) than when water is substituted (fig. 30). Superimposed upon diver'se negative loads of water (fig. 37) almost no 
diuresis might be observed. Given by peritoneum still other relationships are expected (Darrow and Yannet, '35) ; for oliguria and aposia (not drinking) now prevail together.

Enough instances have been cited to illustrate the variety of responses (table 3 ) that may be observed after administration of excesses of fluid to dog's. To consider the dog's under these various administrations, conditions, regimes, and solutions in a single category leads to confusion. Some of their contrasts are indicated in the right half of figure 38. In diverse sets of measurements after water is given by stomach under supposedly identical conditions,

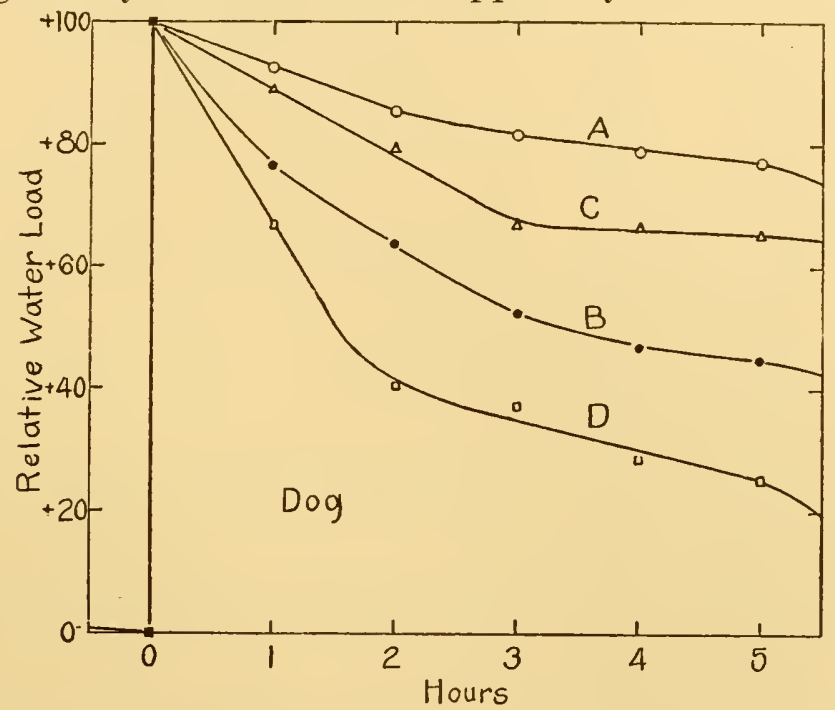

Fia. 37. Course of total water load (relative to volume infused) in dogs in four states. About $8 \%$ of $\mathrm{B}_{0}$ of $0.15 \mathrm{M}$ sodium chloride solution is infused by vein at zero time. Data of Davis and Dragstedt ('35). A, 2 tests after deprivation of drinking water for 12 days; B, 11 tests after continued total loss of pancreatic juice; C, 3 tests after total loss of gastric juice for 8 to 11 days; D, 3 tests in control state.

the velocity quotient varies between 2.4 and $0.3 / \mathrm{hr}$. The highest rates occur with thyroid administration (velocity quotient $3.5 / \mathrm{hr}$.) ; the lowest with pituitrin and with intravenous water (velocity quotient $0.02 / \mathrm{hr}$.). Yet all these varieties of water excess (and others too) are commonly referred to as states of "hydration," "positive water balance," "hydremia," and the like. In reviews (e.g., Adolph, '33, p. 348) observations from one or several of them are quoted in the same sentence as being coordinate facts, as occurring or predicted for all forms of water excess.

Once it is recognized that the sequelae, of any one means or of 


\begin{tabular}{|c|c|c|c|}
\hline 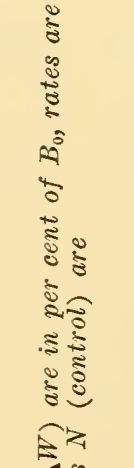 & 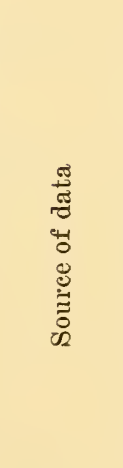 & 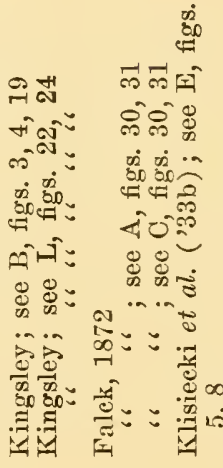 & 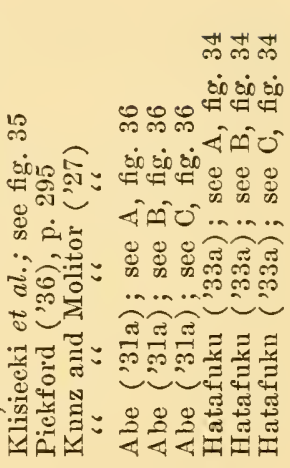 \\
\hline 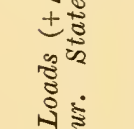 & 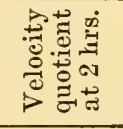 & 跑 & 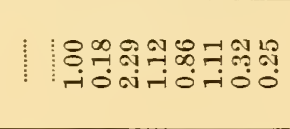 \\
\hline 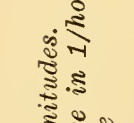 & 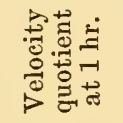 & 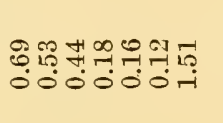 & 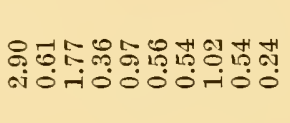 \\
\hline 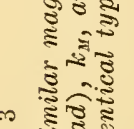 & 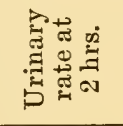 & 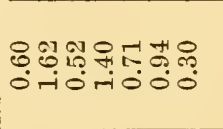 & : \\
\hline 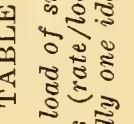 & 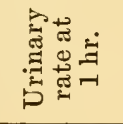 & 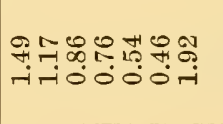 & 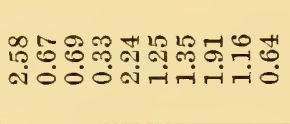 \\
\hline 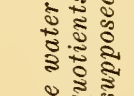 & 苛 & 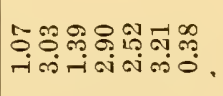 & 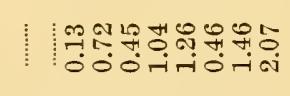 \\
\hline 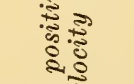 & 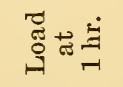 & 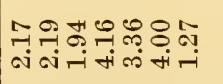 & 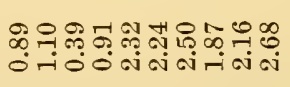 \\
\hline 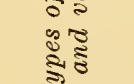 & 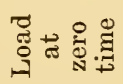 & 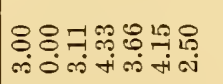 & 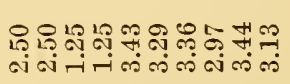 \\
\hline 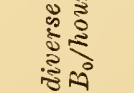 & 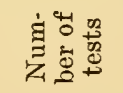 & 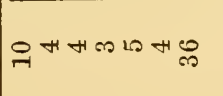 & 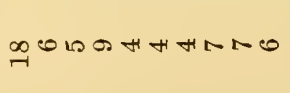 \\
\hline 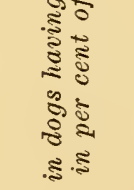 & 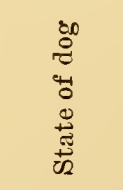 & 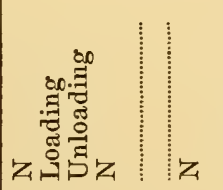 & 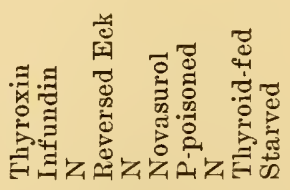 \\
\hline 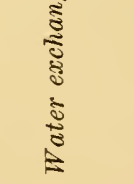 & 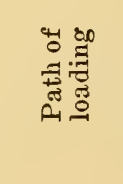 & 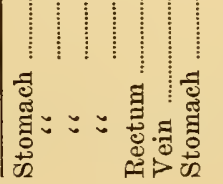 & 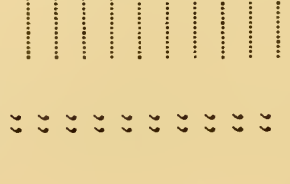 \\
\hline
\end{tabular}


any one agent that produces water excess, are not uniform, quantitative relationships can supersede categorical statements in describing the enhancement and inhibition of water diuresis. At one load and time the rates of recovery of water content are diverse but are numerically comparable.

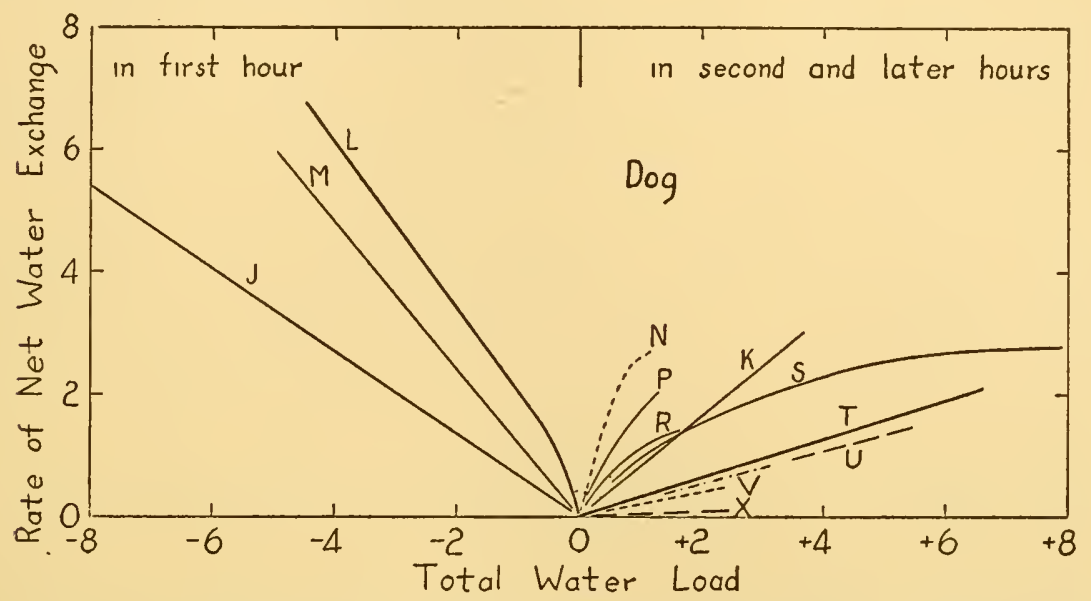

FIG. 38. Comparison of net exchanges of water in dogs subjected to several types of water load. Ordinates, $\%$ of $\mathrm{B}_{0}$ /hour; abscissae, $\%$ of $\mathrm{B}_{0}$. The curves selected are each derived from 4 or more tests, as indicated in figures $39(\mathrm{~J}), 16(\mathrm{M}), 29(\mathrm{~L}, \mathrm{~T}), 35(\mathrm{~N}, \mathrm{P})$, $34(\mathrm{R}, \mathrm{V}), 6(\mathrm{~K}), 31(\mathrm{~S}, \mathrm{U})$; and treated with pituitary extracts studied by Molitor, '26a (X). Gains in negative loads are compared in the first 1.0 hour of recovery, except for the steady ingestion (L) by the dog with esophageal fistula; losses in positive loads are compared at times after 1.0 hour, being the maximal rates of net exchange found.

\section{$\$ 17$. Deficits of water}

Water-drinking ordinarily follows water deficit. No dog unassisted gains water by route other than the mouth; dogs without food drink less (Kleitman, '27) ; dogs after physical exercise drink more (Gerhartz, '10); dogs ingest other amounts of any solution offered them in place of water (Wettendorff, '01). Both excretion and ingestion are quantitatively graded activities; so also are mobilization and absorption.

An example of recovery from another type of water deficit is the sequel of sucrose infusion (Keith and Whelan, '26). After two hours of intravenous administration of $1.46 \mathrm{M}$ solution, four hours are allowed for further loss of water through diuresis accompanying excretion of the sugar. Then water is offered during one to five hours, with the recoveries shown in figure 39. The quantities ingested are less at every deficit than those of figure 11, as figure 40 
shows. Some of the differences might be due to reliance upon body weight alone as a measure of water deficit in both situations. The recovery from "dehydration" by sucrose infusion may differ from the recovery from the first type of water deficit cited (privation of dietary water, fig. 11) less than many other types do; there are no suitable measurements of water ingestion after other types. In addition to the types listed in table 3, some that have been termed "dehydrations"' in dogs are: privation of food as well as of water (Mayer, '01), hemorrhage (Wettendorff, '01), catharsis by magnesium sulfate (Tobler, '10), pyloric obstruction (Gamble and Ross, '25), intestinal obstruction (Haden and Orr, '23), removal of pancreatic juice (Gamble and McIver, '28), superficial burns (Butler et al., '31), gas poisoning (Underhill, '19), histamine dosage (Underhill and Kapsinow, '22), insulin administration (Drabkin and Shilkret, '27), adrenal insufficiency (Loeb et al., '33), and parathyroid treatment (Shelling et al., '38). While certain features such as decreased body weight or increased concentration of hemoglobin in whole blood may be common to all these states, the danger of having a single term for all the states lies in the prevalent assumption that other characteristics such as water load and water exchange will be uniform. On the contrary, not only qualitative, but particularly quantitative, diversities may be peculiar to each type of modification.

The statement is repeatedly made that "dehydration is accompanied by reduction in the urine flow ... and by sensation of thirst'" Adolph, '33, p. 349; Gregersen, '38, p. 917). A search makes it evident that investigators who produced states of "dehydration" in dogs did not heretofore report the rates of urine flow, and no one has yet directly measured the sensation of thirst in dogs. Perhaps the statement is correct sometimes; but no attempt has been made to limit the term "dehydration" to instances where the reduction and the sensation have been demonstrated. The investigators had, of course, other criteria, either in the blood or in the previous loss of fluid, of the fact that the body had less water than before. The term "dehydration" has many meanings, therefore, often being not equivalent to "negative water load." No term in previous usage appears to be sufficiently exact to characterize an experimental state of water content and exchange.

A second measure of recovery in negative loads of various types is the retention of injected solutions (fig. 37). Dogs in water 


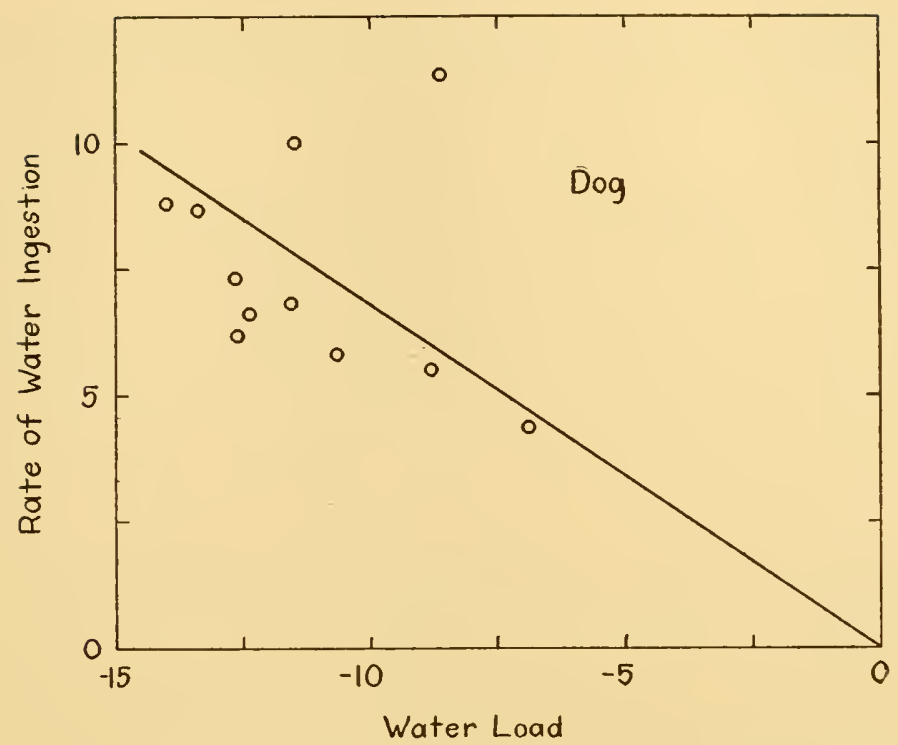

FIG. 39. Water intake in relation to water load after sucrose infusion by vein. A 50 per cent $(1.46 \mathrm{M})$ sucrose solution is injected by vein during 2 hours until $16 \mathrm{gm}$. sucrose/kg. of $\mathrm{B}_{0}$ have been given. Then 5 hours more elapse before water is offered in limited amounts (for about 1 hour). Data of Keith ('24) and Keith and Whelan ('26).

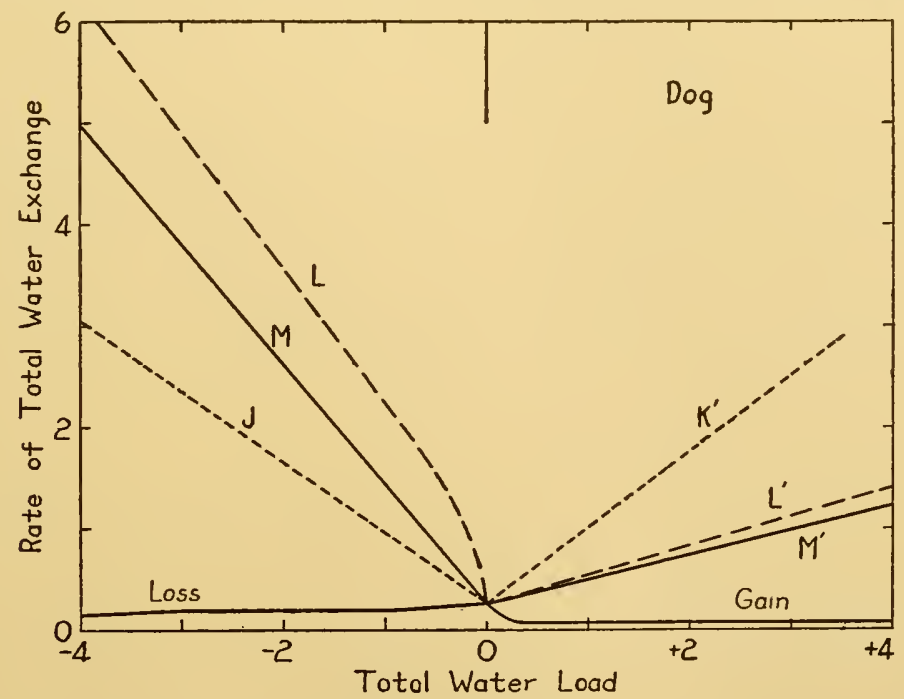

FIG. 40. Rate of total water exchange in relation to total water load. Quantitative comparison of equilibration diagrams in dog. $\mathrm{J}$, gain in the first 1.0 hour of recovery after sucrose injections (from fig. 39); $\mathrm{K}^{\prime}$, loss in periods after the first 1.0 hour of recovery after single administration of water by stomach (from fig. 6) ; L, L', gain and loss in stationary states of defiicit and excess (from fig. 29); $M, M^{\prime}$, gain and loss in the first 1.0 hour of recovery from deficit and excess of water (from fig. 13). 
balance rapidly eliminate most of the sodium chloride solution infused; those previously depleted do not. Comparable results follow the infusion of $0.28 \mathrm{M}$ glucose solution in each of the types of depletion.

The present study is being strictly limited to what occurs after the positive or negative increment of water content has been established. The additional paths, such as loss by rectal diarrhea, drainage of saliva or nasal secretions or lymph, or loss of blood or sweat, that might be involved in securing the water losses (loading), are not concerned in recovery except as the types of load brought about are distinctive of the particular manner of initial loss.

With the recognition of many qualitative types of water deficit, the expedient indicated is to designate each type by the procedure used in loading. Before the types are some day quantitatively characterized, the rates of exchange that prevail during recoveries from diverse increments may be measured.

\section{§18. Distinctions among water increments}

Meanwhile how shall I judge when a water deficit exists? In one type of water privation, constant food is being added to the body. In "dehydration" by sucrose a fluid is added to the blood stream such that more fluid than given is eventually lost from the body. In vomiting, dissolved substances, especially electrolytes, are lost in diverse proportions. In hemorrhage all substances are lost in the proportions present in the blood, but this is not the proportion present in the body as a whole. Only arbitrary judgments of what constitutes a deficit are available; but once a criterion is chosen its consequences are provisionally accepted for purposes of classification and comparison.

When $0.15 \mathrm{M}$ solution of sodium chloride is infused, the disturbance of water content is termed an excess (positive load). Is $0.30 \mathrm{M}$ also an excess? The answer seems to depend in part on whether the urine subsequently formed is more concentrated or less concentrated than the fluid administered. A systematic study of continuous intravenous infusions (Wolf and Adolph) compares the gains with the outputs of water, after 7 hours during which steady rates of output are gradually attained. With a particular rate of inflow $\left(0.9 \%\right.$ of $\mathrm{B}_{0}$ /hour) the outputs become equal to the intakes if the salt concentration infused is either $0.11 \mathrm{M}$ or $0.29 \mathrm{M}$. 
Between these limits water is slowly added to the body; below $0.11 \mathrm{M}$ the water is mostly excreted and draws some salt from bodily reserves with it; at concentrations higher than $0.29 \mathrm{M}$ the water leaves the body faster than it enters, but some sodium chloride stays behind. It is clear that the mere infusion of fluid is no guarantee that a positive load of water is established. The precise relations to solute and to previous depletion determine what regulatory activities toward water come into play; and the relations are already sufficiently complex that no one is likely to predict the responses to these types of water load from a knowledge of some other type.

If $3.4 \mathrm{M}$ solution of sodium chloride is infused, the dog promptly drinks water (Gilman, '37; Bellows, '39). The drinking itself might be regarded as a criterion of water deficit, even though the body weight meanwhile is increased. If $6.7 \mathrm{M}$ solution of urea is infused in equal volume, less drinking results; is the deficit less?

One distinction that is useful is between an absolute deficit and a relative deficit, the latter representing a change in the proportion of water to at least one, several, or all other constituents of the body. In addition, an increment that is initially a relative deficit may become during the processes of adjustment and metabolism a relative excess, and vice versa. In both, however, recovery with respect to water consists in net loss of water in excess and net gain of water in deficit, for no other events constitute a restoration of water content. The rule of procedure which emerges is that every state of water content requires quantitative characterization by rates of water exchanges at least. Other characteristics may be studied to great advantage; such will be considered later (chapter X).

Whereas in the water exchanges considered in chapter II only five chief paths are distinguished: urinary (sensible), evaporative (insensible), fecal, ingestive, metabolic (oxidative); in the water increments of other magnitudes and other types additional paths may be involved. Water in loads above $+10 \%$ of $\mathrm{B}_{0}$ arouses intense salivation and actual large losses of water thereby (Weir, Larson and Rowntree, '22), especially when pituitrin is also administered (Theobald, '34). Vomiting is a response to rapid water administrations, but only when the water is put into the alimentary tract (Rowntree, '22). The partition of water exchanges among paths thus shows large contrasts among the several types that have been investigated. 
It is apparent that an increment of water content $( \pm \Delta W)$ may be obtained by means that may not ordinarily be thought of as disturbing the component water, nor as displacing it in a recognized direction. There is no certain means of foretelling which conditions affect water load and which not. Indeed, it becomes probable that there are relatively few states in which the organism can be found that do not involve water loads and water exchanges. It is only arbitrarily and for present purposes that I concentrate attention on increments of water and omit other modifications that accompany them.

A limited method by which water exchange can be known surely to involve the same type of water load over a period of a few hours is to use a single kind of analysis or measurement for both rate $(\delta \mathrm{W} / \Delta t)$ and load $(\Delta \mathrm{W})$, provided water and oxygen alone are available from without. Thus, the weight changes of the dog give both data (exchange and increment) from one difference of weights taken at two times.

In summary, a few factors may be specified that affect water exchanges under diverse types of water loads. An increment of water $( \pm \Delta W)$ is not often just a change in water content, even though no other chemical constituent of the organism be known to have changed.

(1) Time (since ingestion or privation of water) makes a difference in the rates of water exchange.

(2) Means of addition of water matter. Thus, intravenous injection of water was found to produce highly variable results; the administration being sometimes termed "unphysiological."

(3) Means of subtraction of water matter. Thus, the sequelae of catharsis by rectum may not à priori be confused with those of water privation.

(4) Any accompaniment of addition or subtraction may be of consequence. There probably is no "pure" change of water balance.

(5) Ration or regime upon which the water load is superimposed may matter. Thus, whether the alimentary tract is empty or full while recovery is proceeding may be important.

In general, each type of water load concerns water and a variety of circumstantial factors such as time, locality, responses incidental to introducing or subtracting the water. The bodily system studied is never a homogeneous one, as though a solution were diluted or 
concentrated with prompt mixing; but a highly diversified one, such that only one type of carefully specified procedure may give a set of reproducible results. What at first appears to be an increment of a single chemical entity is in reality an arbitrarily chosen complex; indeed, no increment without a "complex" seems physiologically possible.

The term "hydration," like "dehydration" and many other terms that might be cited, may have been first used (in connection with animals) to designate a particular change in the organism. Later supposedly similar changes were assigned the same name. The early attempt to group like states has now probably passed its usefulness, and a need prevails to separate these states according to their dissimilarities. A dozen types of water excess are here compared (table 3), and at least thirty more have been experimentally observed in part. Additional procedures and responses may at any time be distinguished and the present types be subdivided accordingly.

Finally, I recognize that many investigators are more interested in what goes on within the dog than in the overall responses to water load. How does the body recognize the presence of load? What tissues are excited, what ones transmit messages in accordance with the load present? Implicit in the fact that exchanges are correlated with load and with one another, is the existence of coordination and its machinery. To small degrees their locations may be made out by methods of isolation and interference, making use of physical, chemical, pathological, and surgical procedures of various sorts. All those tools are also, however, specifications of the diverse types of water load; they are conditions of recovery. Here the emphasis is not upon the parts played by each anatomical or chemical bit of the organism; yet the same facts are included in the account exhibited above. Those facts seem to me to furnish help in the study of regulations in this one respect, namely, how differently do dogs get along when their compensations are abolished? If all are abolished for long, dogs do not survive. But over limited periods of time each path of exchange, each means of communication and distribution, and each excitable tissue may be out of commission.

Specific physiological factors for recovery of water balance in the dog are far from intimately known. For polyuria to follow, water may be administered by many routes; the most prompt 
PHYSIOLOGICAL REGULATIONS

\begin{tabular}{|c|c|c|c|c|c|c|c|c|c|c|}
\hline $\begin{array}{l}8 \\
0 \\
\dot{5} \\
\vdots \\
\vdots\end{array}$ & 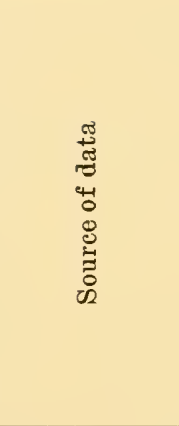 & 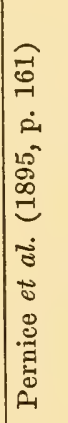 & 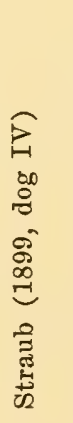 & 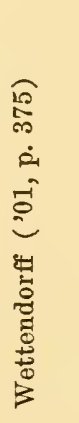 & 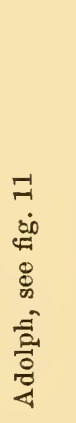 & 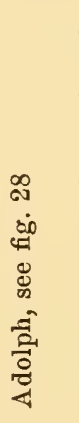 & 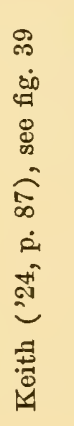 & 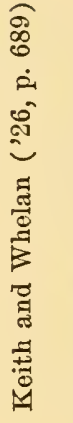 & 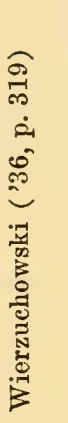 & 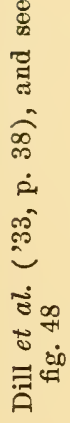 \\
\hline 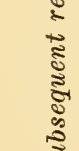 & 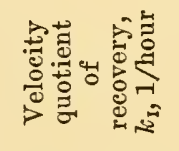 & 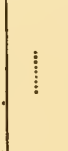 & 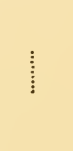 & 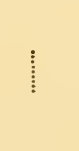 & 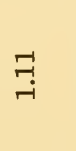 & $\underset{+}{\stackrel{\text { I }}{4}}$ & $\begin{array}{l}\infty \\
0 \\
0\end{array}$ & $\stackrel{\overbrace{}}{\sim}$ & 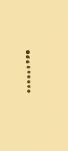 & \\
\hline $\begin{array}{l}\infty \\
0 \\
0 \\
\text { క్ } \\
0\end{array}$ & 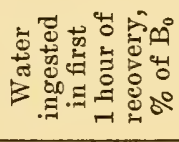 & $\vdots$ & $\vdots$ & $\vdots$ & is & $\stackrel{\check{\infty}}{\infty}$ & 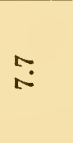 & $\overline{0}$ & $\vdots$ & $\vdots$ \\
\hline 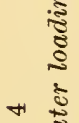 & 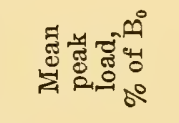 & $\begin{array}{l}0 \\
\text { के } \\
1 \\
1\end{array}$ & $\stackrel{\infty}{\infty}$ & $\begin{array}{c}\text { ฯy } \\
\text { तi } \\
1\end{array}$ & $\begin{array}{l}0 \\
\stackrel{+}{+} \\
1\end{array}$ & $\begin{array}{c}\infty \\
10 \\
10 \\
1\end{array}$ & 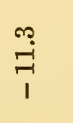 & $\stackrel{\infty}{\leftarrow}$ & $\underset{1}{\stackrel{0}{-}}$ & $\begin{array}{l}0 \\
10 \\
10 \\
1\end{array}$ \\
\hline E- & 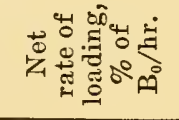 & $\stackrel{\circ}{\circ}$ & $\stackrel{0}{\stackrel{0}{0}}$ & $\stackrel{m}{\overparen{0}}$ & $\stackrel{\infty}{0}$ & $\stackrel{\infty}{\circ}$ & 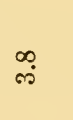 & n் & $\begin{array}{l}\infty \\
\text { i }\end{array}$ & $\vec{a}$ \\
\hline $\begin{array}{l}\Delta \\
0 \\
\& \\
\&\end{array}$ & 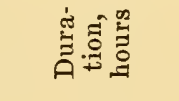 & ठั & $\mathscr{8}$ & $\stackrel{\infty}{\varrho}$ & $\stackrel{\infty}{\not+1}$ & మే & N & $\infty$ & 0 & 0 \\
\hline 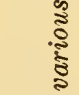 & 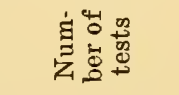 & -1 & -1 & $H$ & $\infty$ & $\stackrel{\sim}{\sim}$ & $N$ & $\infty$ & 10 & $r$ \\
\hline 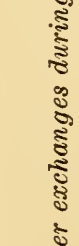 & $\stackrel{\overrightarrow{0}}{\stackrel{0}{A}}$ & 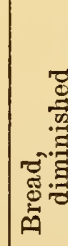 & 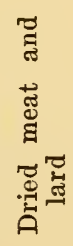 & 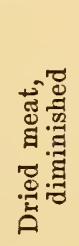 & 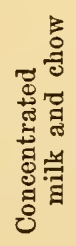 & 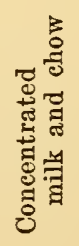 & & & & \\
\hline$\triangleq$ & 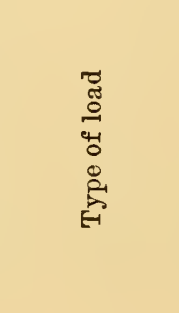 & 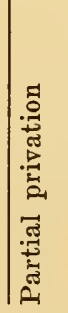 & 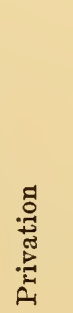 & 苗 & 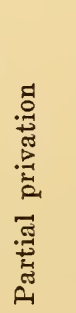 & 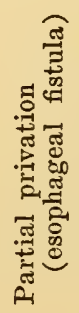 & 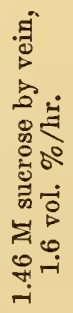 & 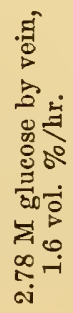 & 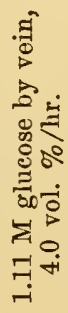 & 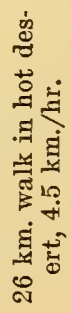 \\
\hline
\end{tabular}


elimination of the excess follows introduction by stomach. A whole circulatory system and at least one intact kidney are required. The kidney need not be innervated, nor need any adrenal be present. For polyposia to follow, deficit is created in the presence of intact swallowing machinery, but no one group of cranial nerves need be intact. The esophagus may be disconnected from the remainder of the alimentary tract. Some day all that information may possibly be arranged so as to explain water regulations to students of physiology. At the present time it seems to form little but a special paragraph in the anatomical story of the dog.

The diversities of water exchanges shown among the types of load-production that have been cited (tables 3 and 4) are: (1) Rates of both intake and output sometimes are increased during recoveries (sucrose, sodium chloride). (2) Rates of both may be decreased, thus preventing recovery (luminal, ethyl ether). Water excesses may be temporarily retained, with less rates of output than are shown after introduction of water by stomach to control individuals. (4) Polyuria may appear only after a delay of some hours, or (5) polyuria may disappear before the positive load of water has been completely returned.

The exact evaluation of the statistical significances of the diversities awaits further data. Physiological classification of the types of load might be based on the rates of exchange or the velocity quotients that result, thus minimizing the emphasis upon agents and conditions that prevail.

What are the uniformities found among all the types of water load that have been mentioned? (1) Some change occurs either of absolute water content ( $\mathrm{ml}$. per $100 \mathrm{gm}$. wet weight) or of relative water content (ml. per gm. dry weight, or weight of some component). But the relative content may differ in sign as well as in magnitude from the absolute content. (2) Rates of water exchange are modified with load. (3) Other uniformities are concerned with quantities outside of water exchanges and water contents of the whole body. (4) The fact that some features such as increased rates of urinary loss are common to several types of water increment is no guarantee that other features such as rates of salivation will also appear common. (5) Many possible relations of water in the body do not occur in water loads of any sort. Some of these are: increased rates by paths other than ingestive, urinary or salivary; increased rates of ingestion in relative water excess; augmentation in rate of loss without any lag. 


\section{\$19. Modifications of water content at balance}

While positive and negative increments of water have been examined, little has been said of states in which water balance prevails. These states are both kinetic and stationary. When balance is defined as equality of intake and output, further qualifications are still needed, for in the dog intake is usually intermittent, and output is ordinarily continuous. Moreover, diverse unusual balances can be maintained by repeated ingestions (i.e., in stationary states of load as fig. $24, \mathrm{~N}$ ), for then within limits the average rate of output equals the rate of intake.

Hence it is desirable to specify that the term usual water balance applies to those states in which neither intake nor output is forced, where neither privation nor manipulative procedures interfere, and where sufficiently long periods (usually 24 hours) elapse, so that rhythms of feeding and sleeping shall be minimized. In particular cases control dogs may be put under more rigidly or less rigidly uniform restrictions of diet, movement, temperature, and the like; it seems quite impossible to define water balance with great generality and yet with rigor.

Often a steady balanced state is approached, when the environment or the body is changed, that differs from the state that would be recovered in the original environment or organic state. The modifications of water content and the rates of these shifts (total, net; gain, loss) may sometimes be measured also during the transition.

I know of no data that compare accurately an equilibration of $\mathrm{W}_{0}$ that has been shifted by a known content with an unshifted equilibration diagram in the dog. What is here discussed are, therefore, consequences of partial data as generalized in the light of relationships so far outlined.

Some instances of modified turnovers are as follows. If dogs are deprived of anatomical connections between hypophysis and brain, the intake and the output of water (turnover) increase enormously and in two cycles (Bellows and Van Wagenen, '38). Administration of desoxycorticosterone induces persistently high turnover (Ragan et al., '40). Surgical imposition of Eck fistula increases the exchanges of water (fig. 41), with gradual recovery toward usual rates. Chloroform-poisoning gives a smaller increase of turnover; but ligation of bile-duct and certain other surgical procedures do not. Where on the scale of water contents 


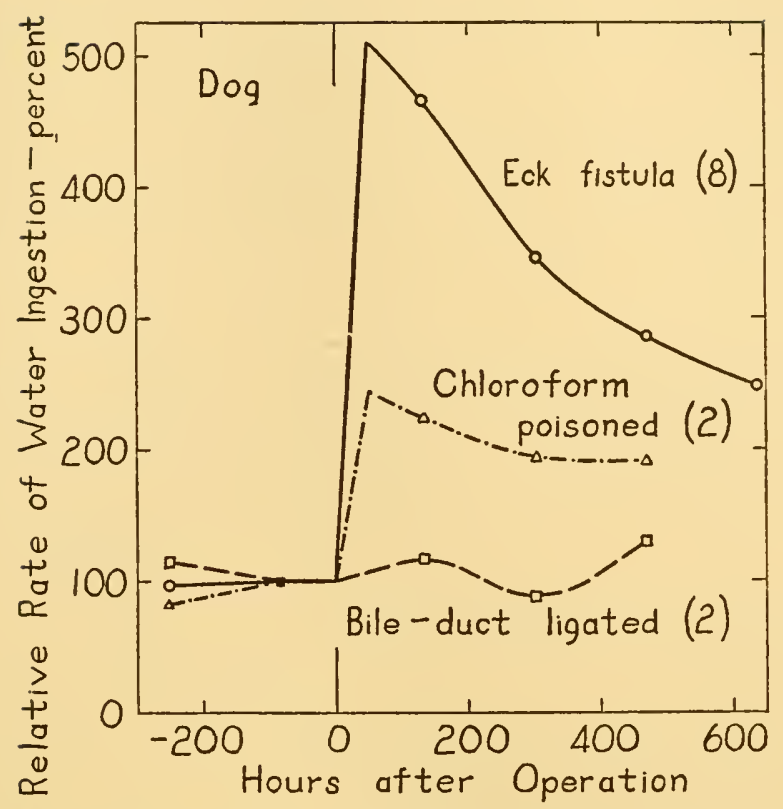

FIG. 41. Rate of water ingestion (relative to last control period), in relation to time before and after surgical operation. Each period of time lasts 1 week; the number of individuals averaged is indicated for each series. Data of Crandall and Roberts ('36).

the operated individuals fall, compared with themselves before operation, is unknown. Rates of turnover are the only items that are known to be augmented in all those instances.

Any content of water at which gain of water equals loss of water evidently represents a state of water balance. A positive content at balance is upon this definition one in which the new $\mathrm{W}_{0}$ exceeds the control $W_{0}$, whether or not rates of exchanges are modified (fig. 42). Criteria for measuring a positive or a negative content at balance may be of diverse kinds, and a shift in $W_{0}$ perhaps as judged from body weight may be positive, while in $\mathrm{W}_{0}$ perhaps as estimated from analyses of tissues is negative. A growing dog is

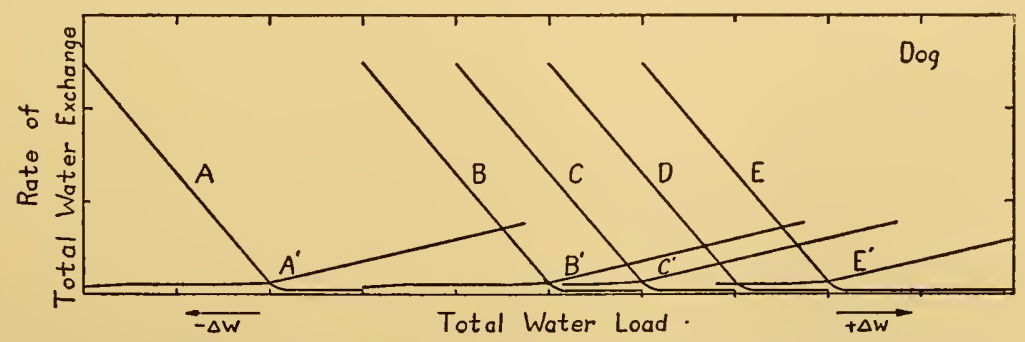

FIG. 42. Equilibration diagrams representing possible relations of the dog's rates of total water exchange to control water content $\left(W_{0}\right)$ and to one another. 
in just this situation; the total water in the body increases with age but the proportion of water to dry matter decreases (Thomas, '11). Privation of food may accomplish the opposite (Böthlingk, 1897; Witsch, '26), decreasing the total water and increasing the proportion of it in the body. A drink of $0.08 \mathrm{M}$ solution of sodium chloride increases the water content both "absolutely" and relatively, but one of $3.4 \mathrm{M}$ only absolutely.

No sharp criterion (other than recovery itself) is alone sufficient to distinguish $(a)$ shifts of content at balance $\left(W_{0}\right)$ from $(b)$ changes of water content $(\Delta W)$ without shifts of the $W_{0}$ to which it will return when allowed. Possible criteria would be the demon-

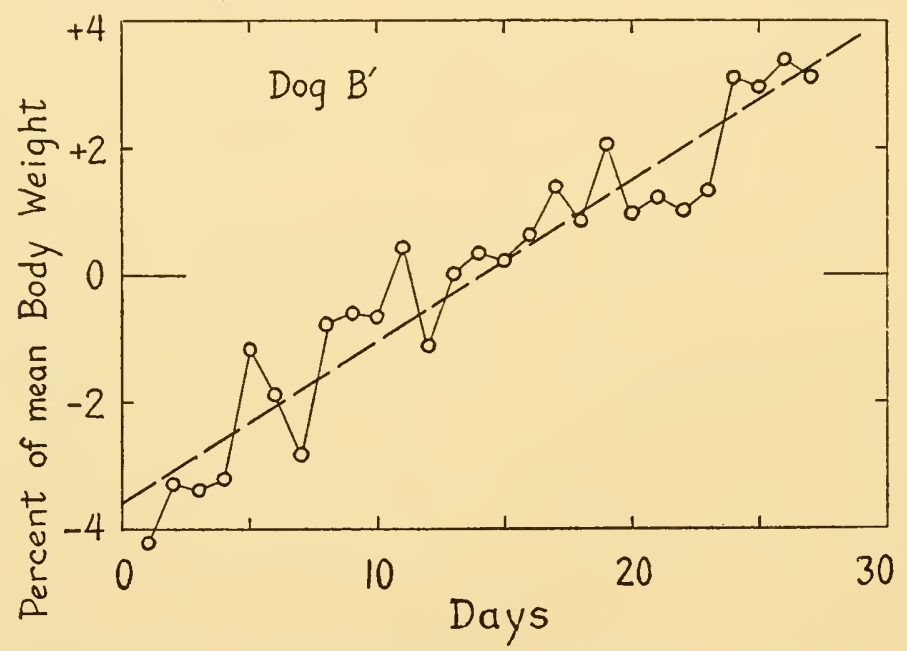

FIG. 43. Sequence of body weights upon 27 successive days. The dog received constant food once daily; water was continuously available. New data.

stration that rates of exchange at some three or four diverse water contents have changed (fig. 42). Biochemical analyses that could determine the water content are at present much less accurate than the measurements of physiological recoveries that ensue. Over periods of time exceeding a few days, or in inconstant conditions of food intake, it is customary to credit changes of body weight (i.e., of water content) to shifts of the zero water load (e.g., see fig. 43). In general, wherever prompt adjustment of water content to zero load does not occur when conditions are judged proper for recovery, it is tentatively supposed that $W_{0}$ has suffered change.

One further step is suggested. When it becomes possible to select some criterion by which the water content of the dog is measured independently of the water exchanges, then diverse modifi- 
cations of exchanges may be represented in a series of equilibration diagrams (fig. 42). In them the shapes of the curves for intake and for output have not changed, it is supposed, but only their water contents at balance have shifted in diverse degrees. In practice it is hardly possible to distinguish case $\mathrm{BB}$ from case $\mathrm{CC}$, since both show the same pattern of equilibration, and the displacement of $W_{0}$ in absolute or relative value might be quite small.

Inhibition of intake and output together (e.g., by anesthesia or pituitrin injections) may not change the analyzable content but does temporarily modify exchanges. In such cases $(\$ 16, \mathrm{a}, \mathrm{c})$ there are two control or "normal" rates of exchange, one for the individual treated, the other for modal individuals or the same individual untreated.

Case DB', which exhibits both polyuria and polyposia, might be judged to be in some state of diabetes insipidus, or of Eck fistula, or other unusual character. Cases like DE', which have small turnovers of water, exhibit oliguria plus oligoposia.

If either the curve for water intake alone $\left(\mathrm{BB}^{\prime}\right.$ to $\left.\mathrm{AB}^{\prime}\right)$ or the curve for water output alone $\left(\mathrm{BB}^{\prime}\right.$ to $\left.\mathrm{BE}^{\prime}\right)$ shifts, then the value of $\mathrm{W}_{0}$ also changes. This contrasts with shifts of $\mathrm{W}_{0}$ which may occur without any modification of the relative curves ( $\mathrm{BB}^{\prime}$ to $\left.\mathrm{EE}^{\prime}\right)$ for water exchanges.

Changes in water content at balance $\left(\mathrm{W}_{0}\right)$ undoubtedly are as worthy of study as are those that excite equilibrations. The difficulties in their accurate examination are greater, for $W_{0}$ shifts only in conjunction with contents of other components. Hence the criteria of body weight and sensible water content are usually unsuitable, and two values of $W_{0}$ can be compared only by (1) full accounting for all water exchanges between the two, or (2) chemical analysis of paired individuals. Comparison of this kind reveals, however, the definite nature of the physiological state of the dog with respect to water.

\section{\$20. Summary}

Excesses and deficits of water may be produced in the dog in various ways and under diverse conditions. Each type of load requires distinct denomination, and accurate measurement of the responses to it. Several states of narcosis, routes of water administration, modifications of regime, and kinds of solutes added to the water administered are here compared. Most rapid negative loadings are obtained by intravenous infusion of solutions of 
sugars (table 4, column 5). By various means water exchanges in the diverse types of positive and negative load may be abolished altogether, or may be tripled in rate (tables 3 and 4 , columns 7 ), as compared with those prevailing in the two types of water loads of $\$ 7$ and $\$ 8$.

A few criteria are suggested for classifying difficult types into positive loads and negative loads. The relative rates of various water exchanges during recovery themselves usually serve as criteria of the direction and amount of disturbance in water content. Sometimes unusual paths of water exchange aid in adjusting the contents.

The content of water at balance is also modified by many agents. The rates of exchanges (turnover) that prevail at balance may or may not suffer modification at the same time. Each state of the dog with respect to water therefore calls for characterization both in regard to water content and in regard to modifications in rates of water exchange, as greater and smaller loads of both signs are present.

In the story of regulations of water content, there could be a chapter describing the behavior of the dog toward water in the environment. What acumen does the dog show in finding water and avoiding water? Does it hasten its water gain and minimize its water loss by choosing appropriate surrounding's when it is in water deficit? Unfortunately for the present enterprise, no quantitative information is available in answer to those questions. This particular method of regulation has been studied especially in the rat $(\$ 43)$ and in insects $(\$ 47)$.

Qualitatively there is much evidence that dogs, like most other animals, seek out and stay in environments that favor their maintenance of water content. They avoid desert areas, they systematically search for sources of water supply. In other words, they use sensorimotor abilities to evade serious difficulties in supplying themselves with water, and in surrounding themselves with a paradise of water. By such choices they forestall the frequent use of compensations on any large scale; this is a prevention that precludes the need for cure. Behavior can be thought of as a separate line of defense against water loads, modifications of water exchange as an insurance when behavior fails. More strictly both are coordinate and specific means of maintaining and recovering water content. 


\section{Chapter IV}

\section{VARIABILITIES OF WATER RELATIONS (DOG)}

$\$ 21$. Physiological regulation concerns the preservation of regularity in some property that might otherwise show larger changes. It seems to me that a way to measure how much preservation occurs is to ascertain how much regularity prevails. That task is accomplished by finding the natural distribution of physiological states, or, more specifically, of diverse water contents. Any maintenance of content or exchange may be regarded as the systematic prevention of unusual states or contents.

It may be realized that such a measure of regulation fails to distinguish between what the organism does to preserve itself and what the environment contributes. This realization is a part of the discovery that the organism and its environment are inseparable. The presence of water instead of liquid ammonia in the animal body is a tacit recognition of the fact that the environment lavishly aids in supplying water and does not abound in ammonia. It seems to me quite inadequate to consider the anatomical boundaries of the organism as the boundaries of a physiological system; for the initiation of isolation is the end of the stationary state. Even an excretory organ works in continuous reference to atmosphere and hydrosphere, whether or not the correlation between them be one that is explicit in the reports of experiment.

Suitable methods of characterizing whatever irregularities occur in the dog's water content are first required. Preferably the distribution of contents is observed in a single individual at successive equal intervals of time. Is the distribution random? What parameters lend themselves to expressing it?

\section{$\$ 22$. Variatrons of Water content}

The fluctuations of water content in the individual dog may be analyzed like any other characteristic. Most methods of study ask the blunt question, are the fluctuations random? And in what respects are the fluctuations non-random?

According to one definition, regulation is that portion of the change in content of water that is non-random. It will shortly be evident, however, that in many series of data on water content no 
such kind of regulation prevails. Instead, the physiological states of water content turn out to be random according to most criteria of randomness, but confined within the restricted range permitted to them. Two problems therefore arise, to see in how many respects the time-series of water contents is random, and to derive parameters by means of which the variability among water contents may be compared with the variabilities of other components.

Data consist in the mere sequence of body weights (fig. 43) in a dog consuming the same kind and amount of food each day, living under conditions arbitrarily fixed. The important aspect is that measurements are made at equal intervals of time in an individual upon uniform regime.

(1) There is a marked trend in the series. Hence to analyze the fluctuations as deviations from a mean (C.V. \pm 2.09 ) is of no significance for the study of regulation of water content as such.

(2) The trend may be found $(B=17.335 \mathrm{~kg} .+0.0460 \mathrm{t})$, and the fluctuations may be regarded as deviations from it. The root mean square of this deviation amounts to only $\pm 0.62 \%$ of $\mathrm{B}_{\mathrm{M}}$.

(3) More simply, first differences between successive values may be taken, their root mean square obtained, and divided by $\sqrt{2}$ to correct for the fact that each value enters twice in the series. This parameter I designate standard difference, and relative to the mean ordinate, coefficient of difference $(\mathrm{C} \Delta)$. Here $\mathrm{C} \Delta$ is $\pm 0.67 \%$ of $B_{M}$. Only on one-third of days is the shift of weight greater than this.

The latter two parameters, which are in any random series (as here) identical, serve to characterize fluctuations. They do not, however, utilize all the information about temporal sequence. In that lies more grist for the algebraic mill.

(4) For instance, the frequency of inversions of body weight may be counted. In a random series they occur in 50 per cent of the first differences. Here they occur $73 \pm 10.2$ per cent of the times, which is not very significantly different. After the \pm sign the standard error is shown.

(5) Frequency of movements toward the line of trend may be counted. Randomly they occur in 75 per cent of the first differences; here they occur in $64 \pm 10.0$ per cent.

(6) Numbers of points succeeding in one direction may be observed. Such a succession is termed a run, and when first and last point are both included in each run, it randomly consists of 2.5 
points (Kermack and McKendrick, '37). Here the mean size of run is $2.30 \pm 0.18$.

(7) Numbers of points succeeding from one maximum to the next may be observed. This succession is termed a gap, and usually consists of 4 points (Kermack and McKendrick). Here the mean gap is $3.67 \pm 0.37$.

Hence, of many possible tests, the five ( 3 to 7 ) that have been selected for their wide applicability all indicate randomness in the dog's daily body weights. Regulation, therefore, consists not in steering the body weight from one day to the next, but in preventing wider fluctuations. From a somewhat different aspect this means that one day is too long an interval for the steering to be visible.

Accordingly, fluctuations at shorter intervals of time may be examined. At one-hour intervals, in which either no food is furnished but water is allowed, or both food and water are allowed ad libitum, the fluctuations are no longer random. If no attention were paid to their succession, the non-randomness would not be evident; only the parameter $\mathrm{C} \Delta=0.098 \%$ would be known. Instead it may be noted (figs. 45 and 46 ) that within 4 hours there are no inversions, and the runs are the full length of the series. No longer is the body weight fluctuating; it is diminishing throughout. That fact leaves two possibilities; either regulation is absent for several hours on end, or body weight is no longer a measure of water content. Evidence to be presented below indicates that both are factors.

When observations are extended over a sufficient number of hours, runs succeed one another. The fact that they are greater than 4 hours in length (actually 4.5) implies that inversions of body weight (when the dog drinks) are at such intervals. When the dog is weighed every 0.25 hour, the same result is exaggerated; the mean run has 18 points. Such an event as drinking is, hence, anything but random in occurrence; instead it comes at rather regular intervals, and by it the dog obtains amounts of water that carry the body through several hours. The fact of periodicity is the clear expression of non-randomness, and indicates a characteristic of regulation additional to "restricted fluctuations." Thus, over daily intervals only fluctuations are evident, but over hourly intervals periodic factors are apparent. The latter reflect the fact that the dog does not sip water every quarter-hour or even 
every hour, but waits longer intervals to raise the body's water content.

A special study was accordingly made (fig. 46) to find how much and how frequently dogs drink, under four sets of conditions at one particular time of day. (1) With water continuously available, but without food, dogs drink within 2 hours only in one of ten tests (E). The single amount drunk is somewhat less than the intervening deficit of body weight.

(2) With food, water is taken by dogs once to four times after each meal (A, B, C,) even though a meal of dry food be eaten every hour (b). (3) If water is not available for 1 or 2 hours, it is not drunk if offered at the close of either period. At the close of 3 hours (not shown), it is drunk in half the tests, and then in amounts equal to about half the deficit. (4) If the dog be warmed for 1 hour, thus increasing the deficit of water, water is drunk as soon as offered in every test but one, whether offered during the heating (D), immediately afterward (G), or 0.5 hour afterward.

From this I conclude that a dog does not sip water at short intervals. Most water is taken, as is well known, shortly after food is eaten. But in the absence of feeding or heating, water is likely to be drunk about every 4 hours, in amounts less than sufficient to restore the body weight. However, the lapse of time is probably less closely correlated with drinking than the lapse of body weight is, for when deficit is hastened by heating, drinking occurs as soon as $-0.5 \%$ of $\mathrm{B}_{0}$ has been reached. That is the greatest change of water content that a dog usually allows without doing something to remove it.

Whether the fluctuation of water content represents a load or a shift of content at balance, cannot be entirely decided. When a dog stands hour after hour in a stall, losing water by evaporative and urinary paths, leaving water untouched, I may conclude that either $(a)$ the dog is running into negative loads, and the sensitivity of the processes leading to water ingestion is too low to act, or $(b)$ the dog is staying in water balance, water not being required to replace that lost until food has also been taken or until the clock gets around to some other hour. On the latter criterion the usual losses of water are eliminations of excesses that arise as metabolism proceeds, and the sensitivity of the responses by intake is possibly as great as of those by output. On the former criterion of water content the organism sacrifices water to the benefit of excre- 
tion, and intake of water lag's behind other means of compensation. In this instance, as in very many others, constancy of all quantities is evidently impossible. I can decide to what quantity the organism is apparently insensitive only in terms of some specified measurement. Actually the weight of evidence is that both $a$ and $b$ are partial factors, basal body weight changing in a trend while $\pm \Delta W$ oscillates about it.

In all this there is no implication as to whether the dog is better or worse served by having a small range of water contents, i.e., a stricter constancy of it. There is no evidence that great constancy is more fit, or that infrequent inversions of net exchange are cheaper, or whether some mean between them is optimal. The present concern is to find just what constancy prevails under arbitrarily chosen conditions, when the state of the dog together with the conditions impinging preserve the content of body water within the fluctuations observed. Insofar as the fluctuations are limited both in amount and in time sequence, their evaluation describes the organism as a preserved unit. For often "constancy is in itself evidence that agencies are acting, or ready to act, to maintain this constancy', (Cannon, '32, p. 281).

The physiological significance of variations in content is, I believe, that the limitation of the variations measures the maintenance of that content. Whenever content tends to change, activities on the part of the organism intervene to oppose the change. If this could be said in mathematical language alone, many possible misunderstandings would be avoided. For, such a form of expression appears to hold fewest connotations and implications. On the other hand, numbers and symbols would convey little idea of an organism's constancy and maintenance, were physiological terms not placed in parallel.

Other measures of water content might be used in place of (1) body weight, and each would fluctuate when observed at 24-hour intervals. (2) Chemical analysis of a group of dogs, killed one or more every day, would measure content. (3) Metabolic retention, measured as total gain of water minus total loss of water, would be another. (4) Sensible retention (intake of water as such minus output of water as such) could be measured in 24-hour periods. (5) The volume of distribution (see $\$ 58$ ) of a substance such as urea or sulfanilamide could be repeatedly ascertained on one individual. 
In order that the variations found shall be clearly of physiological significance, the deviations among control measurements of each quantity recorded are appreciably less than the deviations to be evaluated. This stipulation probably rules out all analytical methods (2, above). The method of measurement is preferably such as not to modify the general physiological state of the animal without cognizance being taken of that fact; this reduces the utility, for instance, of method 5 .

Successive variations of water content and their statistical parameters indicate in part what sort of "governor" controls the diverse processes by which certain departures in content do not persist. Each serves to characterize the maintenance of the dog's water content as a whole, treating it as a specific project in hydraulic engineering. Some of the particular characterizations suggested are: the coefficient of difference between successive body weights at a series of time intervals; and frequency of inversion in the direction of change of water content, or reciprocally the mean time that intervenes before each inversion or compensation appears. Additional numerical factors may easily be devised, each adding to the completeness with which performance is evaluated.

\section{\$23. VARIATIONS OF TURNOVERS}

Rates of exchange that prevail in those states and conditions selected as control ones likewise vary. The data to be mentioned

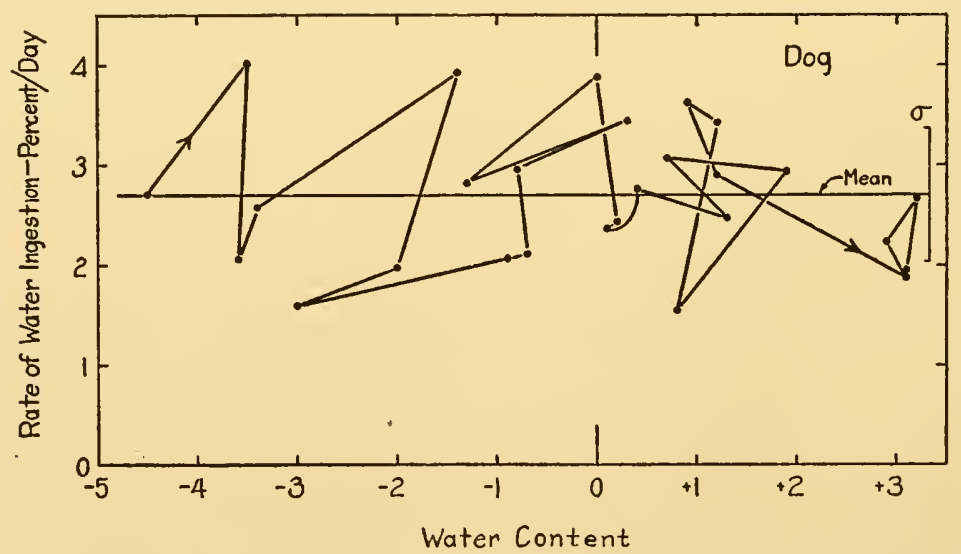

FIG. 44. Simultaneous variations of rates of water drinking and of body weight (or water content). Water content is in $\%$ of $B_{0}$, zero of its scale being the mean of the 27 values of body weight. Measurements were made in 24-hour periods on 27 consecutive days during which uniform food, containing additional $3.05 \%$ of $\mathrm{B}_{0}$ of actual plus potential water, was eonsumed daily. Dog $B^{\prime}, 18.0 \mathrm{~kg}$. New data. 
are listed in table 12. Measured in 24-hour periods the ingestive water intake of dog $\mathrm{B}^{\prime}$ (fig. 44) has a coefficient of difference (CA) of \pm 12.2 of the total water gain. Meanwhile the urinary water output has $\mathrm{C} \Delta \pm 22.7$. Here too, is evidence of activities that prevent unusually low and high rates of water exchange. Part of the smaller variation of intake may be connected with the fact that about half of the water (actual plus potential) came with the food, which was provided in constant daily amount. The free water alone varied by $\mathrm{C} \Delta \pm 22.2$.

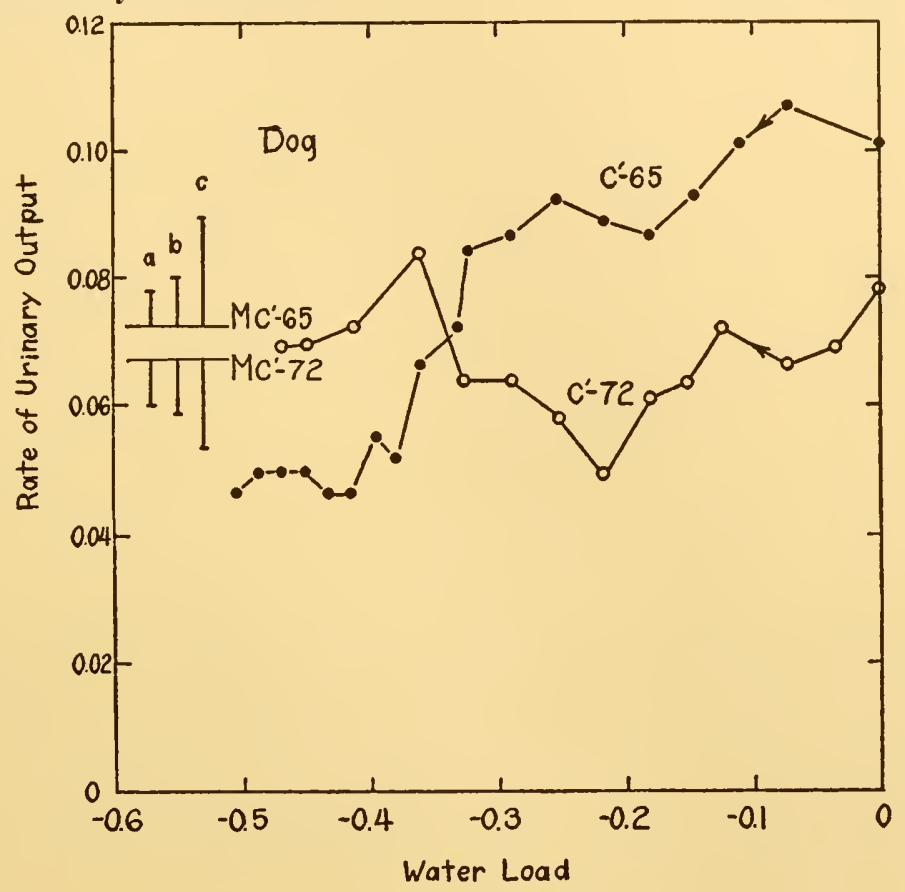

FIG. 45. Rate of urinary water output in relation to total water load (body weight) upon two days in water balance. Dog $\mathrm{C}^{\prime}, \mathrm{B}_{0}=13,885 \mathrm{gm}$. Urine was collected in consecutive 0.25 -hour periods from a bladder fistula. Water was continuously available, but was refused throughout the measurements. $M=$ mean rate. $I=$ root mean square of differences (standard difference) in (a) 0.25 -hour periods, (b) 0.5-hour periods, (c) 1.0-hour periods. New data of Kingsley and Adolph.

In shorter periods (fig. 45) variations in rate of urinary output in individuals with fistulous bladders are nearly the same as in 24 hours. At 1.0-hour intervals $\mathrm{C} \Delta$ is \pm 16 , at 0.25 -hour intervals \pm 19 . These values and an examination of figure 20 suggest that rates of urinary output are smoothed out over periods of time greater than 0.25 hour. Instead of jumping about at random, rates 
tend to be similar to those just preceding and just following them. Inversions in direction of change (accelerations and decelerations) occur 11 times out of 29 possible ones (38 per cent). All changes of rate are gradual; they too look as though a mechanical "governor" exerted an inertia that prevented sudden fluctuations. Or, whatever is concerned in exchanges exerts influences that tend to be uniform and continuous. Trends endure for hours rather than either for quarter-hours or for days.

Comparing paths of water exchange, I find that ingestive exchange is less variable than urinary over periods of 24 hours, but

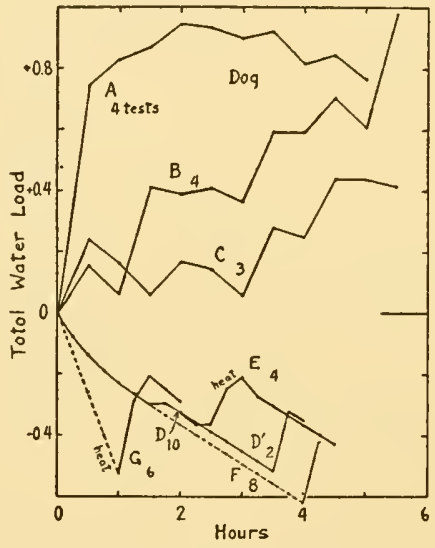

FIG. 46. Total water load $\left(\%\right.$ of $\left.\mathrm{B}_{0}\right)$ in relation to time in dogs nearly in water balance. The number of tests is indicated in each series. Water was available to the two dogs at all times except on dotted lines. A, one-fourth of daily food was eaten at zero time. B, one-twenty-fourth of daily food was eaten every hour. C, one-eighth of daily food was eaten at 0 and at 3 hours. $D$ and $D$, control regime $=$ no food or heat. $\mathrm{E}$, heated by radiator during third 1.0-hour. F, no food or water for four hours, then presented with water. G, heated during first 1.0-hour (no water), then presented with water. New data of Robinson and Adolph.

in periods of 1 hour and 0.25 hour is much more variable ( $\mathrm{C} \Delta \pm 75$, \pm 180 ) as ascertained in tests of the sort shown in figure 46. Evaporative exchange varies about as much as urinary, within each of the time intervals mentioned.

Each of the quantities concerned in water intake (table 1), and in water output, is equally capable of evaluation by its variations. In the end I would learn precisely which are the more uniform processes and which less uniform in the handling of water by the dog, and what periodicities are characteristic of each.

Adjustment of water content is by means of the exchanges represented in the fluctuations that are being studied. Content is a 
subtrahend of gains and losses $(\$ 4)$, which in turn are sums of exchanges by several paths. The interrelations among the rates of exchange are indices to regulations. Does each rate vary at random? Or does path $\mathrm{p}_{1}$ compensate for the vagaries of $\mathrm{p}_{2}$ ? Or is $p_{1}$ positively correlated with $\mathrm{p}_{2}$ at any one time? Thus, Rowntree ('22, p. 131) says: "The total output of water is determined by the total intake." Is there evidence that $p_{1}$ is an independent variable, while $\mathrm{p}_{2}$ behaves in accord with it?

In the data of figure 44 there is found no correlation between ingestive gain and urinary loss. In short periods of time also

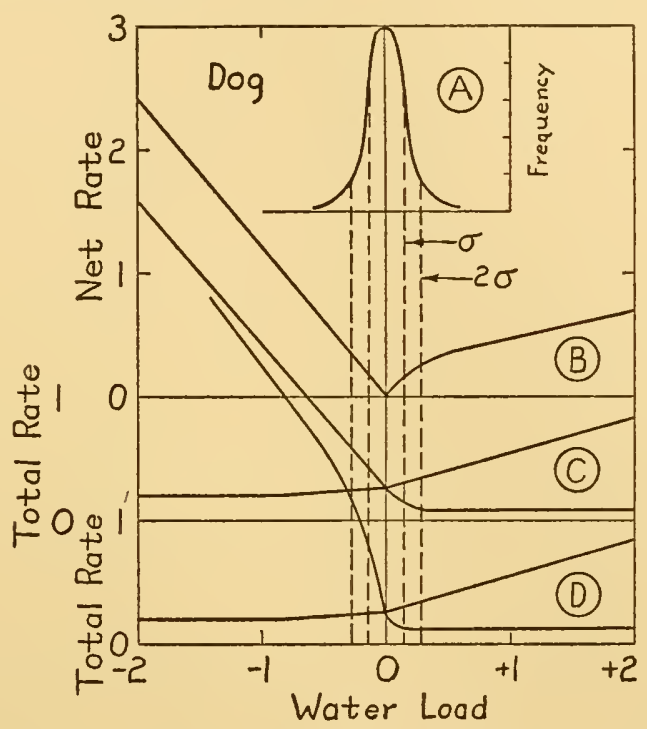

FIG. 47. Frequency of occurrence of diverse water loads ( $\%$ of $B_{0}$ ) when measured at hourly intervals (A) compared with rates of water exchange $\left(\%\right.$ of $\left.B_{0}\right)$ at diverse water loads $(B, C, D)$. A represents data from table 12, row 2. B, net rate in first 1.0hour of recovery, from figure 16. C, total rate in first 1.0-hour of recovery, from figure 13. $\mathrm{D}$, total rate in stationary state, from figure 29.

(fig. 45) there is no correlation between the two, since ingestion did not occur. Even if there were a correlation, there is no way of ascertaining, I believe, whether $\mathrm{p}_{1}$ influences $\mathrm{p}_{2}$, or vice versa, or whether both are coordinated by some other factors.

Very often dogs exhibit rates of urinary loss greater than the average at 0.5 to 1.0 hours after each spontaneous ingestion of water. It is possible to say that increased intake results, after a lag, in increased output. The events form a sequence. Another correlation found is that ingestion follows low rates of loss. In the 
first correlation, output is modified after intake has changed; in the second, intake is modified after output has changed. Separation in time is also no guarantee that one determines the other.

The variation found in any one individual that is maintaining water balance is to be related to the form of the equilibration diagram (fig. 29). Any departure of water content from the usual involves a load, and a load inevitably means a rate of intake unequal to rate of output. The variability found is an expression of the occurrence of each load; some minimal departure from balance appears before measurable steps for its correction go into effect (fig. 47). Thereafter the rate of the correction (recovery) depends upon the difference between rate of intake and rate of output. If a large difference of rates (large net rate) occurs at a small load, it provides a rapid means of recovery, a deterrent to variations of content. Quantitatively, the standard difference at hourly intervals of time (fig. 45 ) is $0.098 \%$ of $\mathrm{B}_{0}$. At loads of $\pm 0.098 \%$ of $\mathrm{B}_{0}$ the economy quotients (rate of intake/rate of output) are 1.4 and 0.7 (compared with 1.0 at balance) as inferred by interpolation upon the diagram (fig. 47, C) for the physiological maintenance of water relations of a dog under the conditions specified. Variation of content and equilibration of content are two aspects of maintenance, showing what does not happen and what does happen, respectively, when the water content is near balance.

The avoidance of extreme rates of exchange has been studied with respect to certain other components and species by Gasnier and Mayer ('39). They defined several useful measures of constancy in turnover. (1) Precision is the ratio of gain to loss in a chosen period of time. Here this ratio is termed economy quotient. (2) Fidelity is the difference (latitude) between largest and smallest precision, or presumably any other measure of distribution of precisions. (3) Sensibility is the percentage of successive observations of rate between which the sign of the difference has changed (inversions have occurred). (4) Rapidity is the shortest interval of time at which the gain most usually equals the loss, or over which the precision is near to 1 . These are ready methods other than first differences of working with data upon variability of exchanges in dog's that are maintaining water balance. Each of the four might be illustrated from the data of figures 44 and 45 . Each term has here a specific definition that may not be confused with some other definition or connotation of the same word. 
Departing from conditions that I chose to regard as standard ones, I may measure variations in water content when the total exchange. (turnover) of water is enhanced, as in physical exercise (fig. 48). It appears that water is not only more liberally ingested, but more exactly restores the body weight, in a hot climate than in a cool one. In other words, a high turnover now maintains more uniform contents than a lower turnover. In a like manner a variety

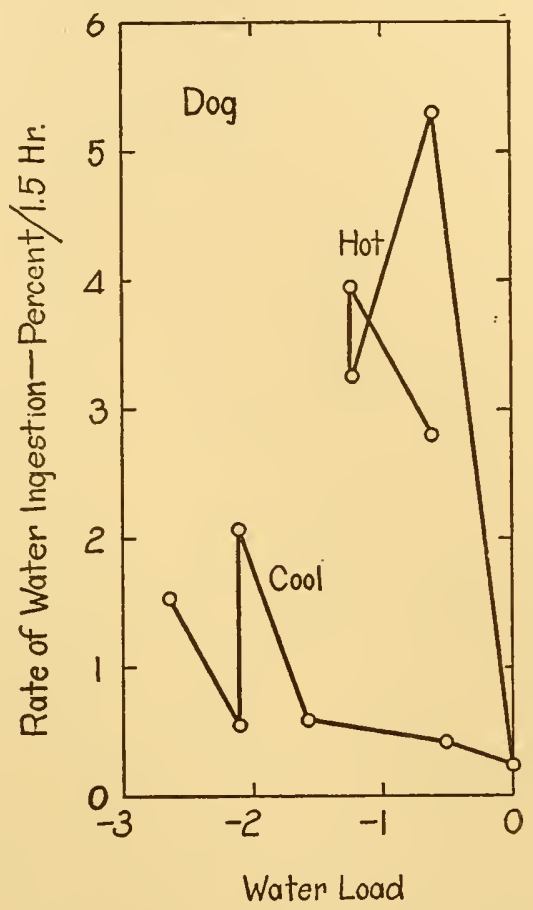

Fig. 48. Simultaneous fluctuations in rates of water drinking and in water load (or body weight, in \% of $B_{0}$ ) in a dog (16.0 to $18.9 \mathrm{~kg}$.) walking on leash in two tests in different climates and drinking ad libitum after each 1.5 hours. In the hot desert the dog made up 96 per cent of the total weight losses by concurrent drinking; in the cool elimate 63 per cent. Data of Dill et al. ('33).

of conditions might be studied; in each one the constancy with which water content is maintained would be ascertained quantitatively. It is still unknown whether the variability of water exchanges increases with the variability of the environment. Similarities and differences among individuals in any one set of conditions also remain to be found.

In rating the accuracies (fidelities) of maintenance no assumption is implied as to whether the dog functions "better" by having 
more constant rates of water exchange than less constant ones; that is a question for investigation only after some criterion of "better" to which even the dog will agree has been discovered.

In brief, the rates of exchange that occur under standard conditions for water balance vary by measured coefficients, rates through two paths fluctuating simultaneously by diverse amounts. Available data do not indicate that adjustments are more regular or accurate by gain than by loss, or vice versa, nor that control is exerted upon intakes more or less vigilantly than upon outputs. Both are coordinate in the maintenance of water content. Only in the average of successive periods of time does total gain equal total loss.

\section{\$24. Variations in Rates at Diverse LOADS}

Recognition that contents and rates each vary while the dog is in a state of water balance allows the introduction of variabilities into the equilibration diagram. First of all, the point $\mathrm{W}_{0}$ on the abscissae (fig. 47, C) is actually not a point but a zone. Since periods of 1.0 hour were used in the measurements of rate that go into it, this zone has a width of $\sigma$ or \pm 0.10 per cent of the body weight. It may be supposed as a crude guess that the variability of any body weight that enters into the estimates of water load is at least this large. Second, the ordinate rate of turnover $\left(R_{0}\right)$ is a zone about \pm 16 per cent of $0.25 \% / \mathrm{hr}$. or $\pm 0.04 \% / \mathrm{hr}$. in height. Rate and load together give a rectangle of random variations $0.08 \% / \mathrm{hr} . \times 0.20 \%$, that usually includes $2 / 3 \times 2 / 3=4 / 9$ of the observations. Third, each series of rates for which averages have been plotted, such as figure 2 , has a variation peculiar to itself. Every point in every graph might therefore be rendered as a rectangle or ellipse. These rectangles or ellipses might also be employed, with modifiers for numbers of observations, as measures of error and of significance.

One question that may be decided is whether the variation of rates at several water loads is proportional to the mean rate or whether it is independent of the mean. The former is approximately the case, and for this reason coefficients of variation are much more useful than standard deviations in characterizing it. Next, its magnitude may be found. Among 7 tests on two individuals (B, fig. 2), in the first 1.0 hour after water was put into the stomach, the rate of total loss varied by C.V. \pm 24 , of urinary loss varied by C.V. \pm 27 , or identical values. Further, selecting 24 tests 
on three individuals (all those having water deficits exceeding $2 \%$ of $B_{0}$, fig. 11 ), the rate of gain varied by C.V. \pm 32 . It may be concluded that ingestive gain and urinary output are managed with equal uniformity. There is here no evidence that drinking is less accurately controlled by the dog than other exchanges.

Another question is whether the variation is different if instead of averaging those measurements of rate taken at one time I ascertain the distribution of rates at one load. The variability turns out to be similar. Further it would be possible to find the distribution of times or of loads at which uniform rates prevail.

Measurements of variability in rates of exchange make possible the detection of unusual responses of the individual. How small a rate of water excretion, following the ingestion of $3 \%$ of $\mathrm{B}_{0}$ of water, shall be considered pathological? There are data of Falck (E, fig. 31) which, so far as the methods and conditions of measurement are described, duplicate those of Kingsley (fig. 2). But the rates of output are much smaller and the lags much greater. It is difficult to believe that three individual dogs used by Falck, consistent among themselves, were genetically different from three used by Kingsley, fairly consistent among themselves. Diversities of method, race, diet, conditioning and acclimatization that will produce such wide divergencies of result remain to be precisely investigated in some one laboratory.

Again, "distilled" water by stomach in quantities of 1 and $2 \%$ of $\mathrm{B}_{0}$ did not invoke diuresis, while tap water did (Hashimoto, '14). All distilled water is not similar. But few investigators would have considered it worthwhile to test the differences among samples of water, yet to disregard a difference in water administered is physiologically confusing. Unfortunately Hashimoto did not demonstrate that the fact of being distilled water was what mattered in those tests, nor did he show that the difference was a significant one.

In general at diverse rates of water exchange, the variations are relative to the mean rates. In recoveries from water deficits the initial water intakes vary by C.V. \pm 32 . In recoveries from water excesses the initial outputs vary by C.V. \pm 24 . Hence the responses to negative and to positive water loads are of equal accuracies. Unusual responses may be adjudged by reference to these manifest variabilities. 


\section{\$25. Summary}

Variations of water content and of rate of water exchange in the dog are indications of the latitude permitted in the regulations of these quantities. Fluctuations outside the usual are opposed by activities or processes that tend to restore water balance or flow. Often the fluctuations found are not random in their sequences.

Differences among successive values are influenced by durations of period of measurement, numbers of individuals, conditions of observation, and other factors. From any one set of measurements, however, accurate characterizations of regulatory activities, with respect to water content or to rates of water exchange, are obtainable in terms of statistics of distribution.

Variabilities of water content or exchange may be correlated with variations in the environing conditions, and in states of the dog both present and previous. Evidence exists especially in water ingestion, as part of turnover, of long-time oscillations. The fluctuations permitted characterize, equally with the mean position of each content and rate of exchange, the "normal" or balanced state.

There is no evidence that during turnover water intake sets the pace for water output or vice versa, or that one path of output compensates for irregularities in another path of output. During recoveries, rates of ingestive gain in water deficits are about equal in variability to rates of total loss in water excesses. The variabilities in usual individuals being known, unusual features of compensations of water content in other individuals may be detected.

This marks the end of the formal account of the water-time relations of the dog. Even within the limited selections of variables and of types of water loads the account is far from exhaustive.

The dog's recoveries from sudden excesses of water and recoveries from deficits of water were studied in relation to amounts of water load $( \pm \Delta W)$. Total exchanges, net exchanges, and partitioned exchanges were each covariates of load, their regressions forming equilibration diagrams. The responses were evaluated in terms of modification ratio, velocity quotient, tolerance, promptness of modification, maximal rates, and completion of recovery. Stationary states of water load were similarly investigated. It was found that excesses of water in the body were eliminated, almost exclusively through the kidneys, at rates proportional to the load. Deficits of water were dispelled by ingestion of amounts that were 
slightly in excess of the believed load and proportional to it (chapter II).

Recoveries from numerous types of water excess and of water deficit were compared, each type representing diverse states of the $\operatorname{dog}$ and diverse conditions for recovery. Several criteria were found whereby a water load might be distinguished from a new water content in water balance. It was suggested that all modifications of water exchange and of water content may be classified qualitatively, while within each class quantitative diversities prevail. These types represent the physiological states of the dog, the diverse accompaniments of the water administration, and the paths by which water is administered and returned. Selection of those environments in which water losses are minimized and access to water is easy, may be of significance in maintaining the characteristic water content (chapter III).

Physiological variation of water content in dogs serves as a measure of its regulation. With respect to short periods of time, successive variation often differs from random variation, denoting a temporal characteristic of possible governors. Several methods of evaluating and comparing the variabilities and their sequences are suggested, both for contents and for rates of exchange. Rate of water gain is not more variable than rate of water loss in periods of 24 hours, but in periods of 1 hour gain is much the more variable of the two. No further evidence was found that content is regulated more accurately either at gain or at output, and compensations for departures from balance occur equally at both. In exchanges during recoveries the variabilities are relative to the mean rates rather than absolute (chapter IV). 


\section{Chapter V}

\section{WATER RELATIONS OF MAN}

\$26. As the physiological pattern of the dog with respect to water exchanges has been described, so it is possible to characterize quantitatively the water exchanges of man. In early crude studies it was implicitly supposed that all mammals are alike and that approximate facts about one species would hold for all. Now, it is easily demonstrated that the similarities, extensive though they be, do not extend to all characteristics of water relations. In fact, it becomes apparent that precision of description differentiates species and individuals physiologically with respect to these very characteristics. One description, even when thorough, cannot suffice for two descriptions. Descriptions of two or more species allow the identification of uniformities as distinct from diversities.

Does a man compensate for disturbances of water content just like a large-sized dog? Does identity of organs of exchange mean that functions are quantitatively alike? If not, are the rates of water exchange correlated with other differences between the species?

Proceeding with the investigation in somewhat the same manner for man as for dog, I analyze analogous data on water exchanges. At first the data are restricted to the same four kinds of variables (water content $\Delta \mathrm{W}$, water exchange $\delta \mathrm{W} / \Delta t$, time $t$, and velocity quotient $1 / \Delta \mathrm{t}$ ). The responses are described following single ingestions by mouth, repeated ingestions by mouth, and desiccations by privation. From these, an equilibration diagram is obtained.

\section{\$27. Single ingestions by mouth}

Tolerance curves (fig. 49) for excesses cover longer times than in the dog. Or, in the larger species the half-life of the water load is g'reater. The curves are little modified when alimentation and absorption (see fig. 62) are taken into account.

The coefficient of difference in rates of total water loss between successive control periods of 1.0 hour each is \pm 13 per cent of the mean rate or $\pm 0.020 \%$ of $B_{0} /$ hour, and in rates of urinary excre- 


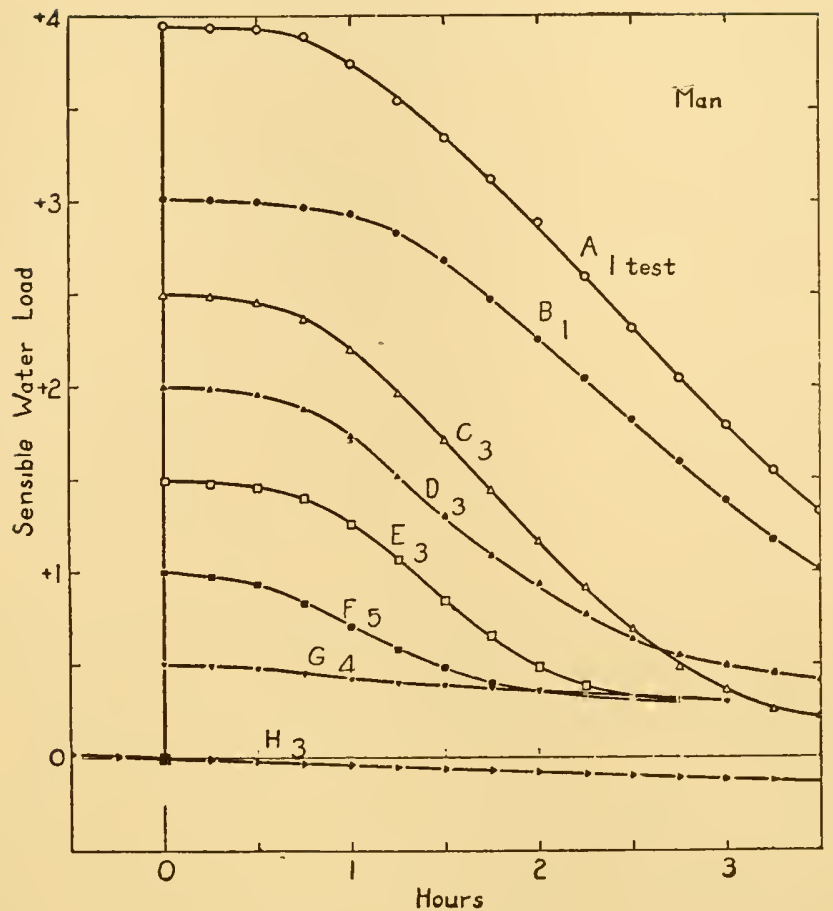

FIG. 49. Course of sensible water load $\left(\%\right.$ of $\left.B_{0}\right)$ after diverse amounts of tap water were ingested. Man. All tests (as indicated) in which the same quantity of water (relative to body weight) was ingested are averaged. $\perp=$ half of administered load was returned. New data of Kingsley and Adolph.

tion is \pm 21 per cent of the mean rate of $\pm 0.012 \%$ of $B_{0} /$ hour. With these as criteria of significant increases in rate, the latent period of diuretic response may be said to end when the urinary

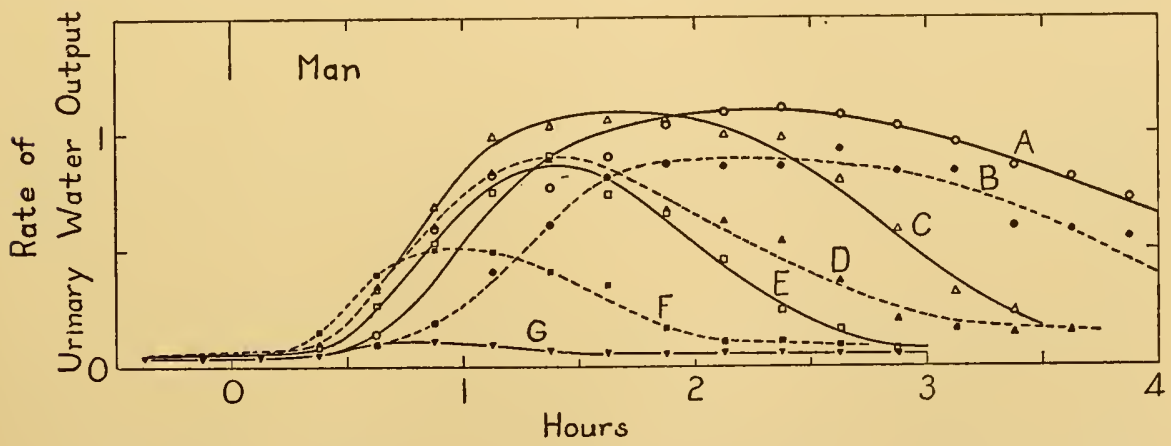

FIG. 50. Rate of urinary water output in relation to time. At zero time diverse amounts of tap water were ingested; all tests in which the same quantity of water (relative to body weight) was ingested are averaged. Same tests as in figure 49 . 
rate exceeds the control by double this deviation. The latency varies from 0.3 to 0.7 hour (fig. 50 ).

The maximal rate of water loss is regularly attained later with large administered loads than with small (fig. 50).

Completion of diuresis may be said to occur either (a) when the rate of urinary water loss once more comes within \pm 42 per cent of the rates characteristic of control periods at the same hour, or (b) when the rate decreases by less than it fluctuates in successive periods of measurement. The times in hours required to complete the diuretic response are related to the volumes administered $\left(\Delta \mathrm{W}_{\mathrm{c}}\right)$ by the equation $\mathrm{t}_{\mathrm{c}}=1.3+1.0 \Delta \mathrm{W}_{\mathrm{e}}$. Similar rules hold for diverse fractions of unloading, such as, for the half-life of the sensible water load.

The volumes of water finally eliminated (returned) differ from the volumes ingested by a constant positive amount, and not by a uniform fraction of the initial load. Part of this volume of retention vanishes when a preparatory water administration just precedes the test. Three manners of computing the volumes eliminated yield nearly similar returns: (a) total water loss, (b) urinary water loss, (c) total or urinary water loss in excess of that eliminated in equal control periods, either at some arbitrary time or at completion of diuresis. If one must know how large a drink of water can be disposed of in 3 hours, the first comparison is used. If, however, the body is allowed unlimited time in which to adjust its water content, the second or third might be preferred. Losses by the several paths are found not to increase significantly with water load.

It has been previously concluded that all the water ingested by a man in water balance is returned as excess urine by the time the diuresis ends (Adolph, '21). The evidence now available is more extensive and precise; from it (fig. 49) the conclusion appears to be that in man as well as dog the urine that issues at rates above control ones is significantly less than the water administered. This may mean that some of the water drunk takes the place of that which is being "catabolized" or made available for excretion from other sources. It may be supposed that the man or dog is never "saturated" but at best maintains himself in slight deficit, and so retains small absolute amounts of the water ingested. Other data (A, fig. 51) indicate returns in urine alone that equal 
the loads administered. How to obtain one or the other result at will is not known; a priming ingestion 3 hours previous to the test somewhat augments the return, however.

Initial rates at which excess water is eliminated increase with water load up to a maximal rate (fig. 52). The later rates are

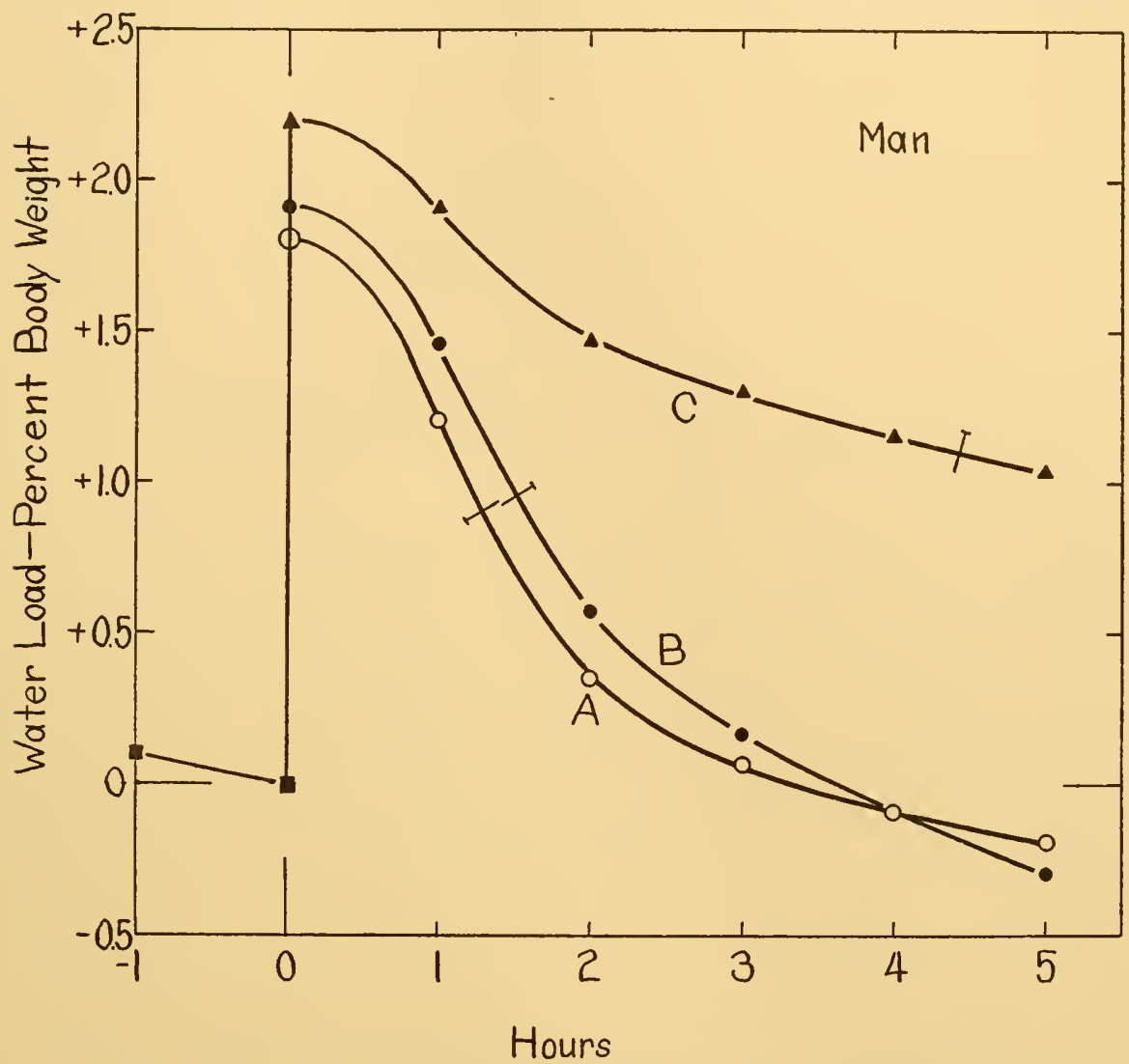

FIG. 51. Course of sensible water load $\left(\%\right.$ of $\mathrm{B}_{0}$ ) after a single ingestion. Individuals with two types of hepatic disease (jaundices) were compared with control individuals. Only in one, obstructive jaundice, was the excretion of excess water in urine significantly retarded. A, 12 control individuals; B, 7 patients with diagnosis of catarrhal jaundice; C, 4 patients with diagnosis of obstructive jaundice with cancer of the bile duet. Data of Abe ('3la).

more rapid, and still later rates decline. Once the rates of elimination have begun to decrease (fig. 53), the rates are significantly independent of time and are related to load.

Net velocity quotients $(1 / \Delta t)$ are highest at moderate loads (about $+1 \%$ of $\mathrm{B}_{0}$ ). The maximal values attained indicate what 


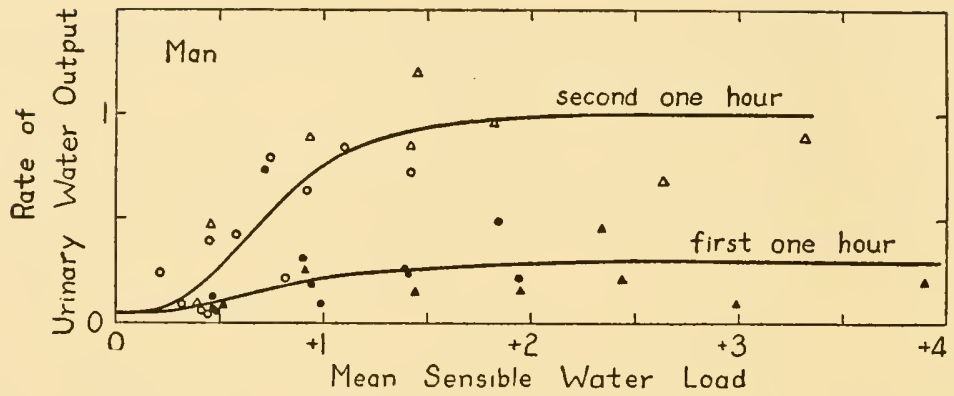

FIG. 52. Rate of urinary water output ( $\%$ of $\mathrm{B}_{0} /$ hour) in relation to mean sensible water load $\left(\%\right.$ of $\mathrm{B}_{0}$ ). Each point represents the total urine put out in one hour in a single test of figure 49 . Two subjects are distinguished by triangles (K) and circles $(\mathrm{N})$. For each test a single ingestion of water occurred at zero time.

the organism can do in recovering from a single ingestion of water if given time to get under way and if given an optimal load. Values of $1 / \Delta t$ may, of course, be of largest magnitude when values of $\delta \mathrm{W} / \Delta \mathrm{t}$ are not maximal.

The sort of data discussed above is confirmed in a less systematic way by early studies of Falck (1852), Ferber (1860), and many others. Those studies differed mainly in furnishing measurements only at 1-hourly intervals, in not relating exchanges to body weight, in employing only one subject, and in measuring urinary output but not total output of water. Falck demonstrated that the time relations of the compensatory urinary output differed but little when water was administered by rectum instead of by mouth.

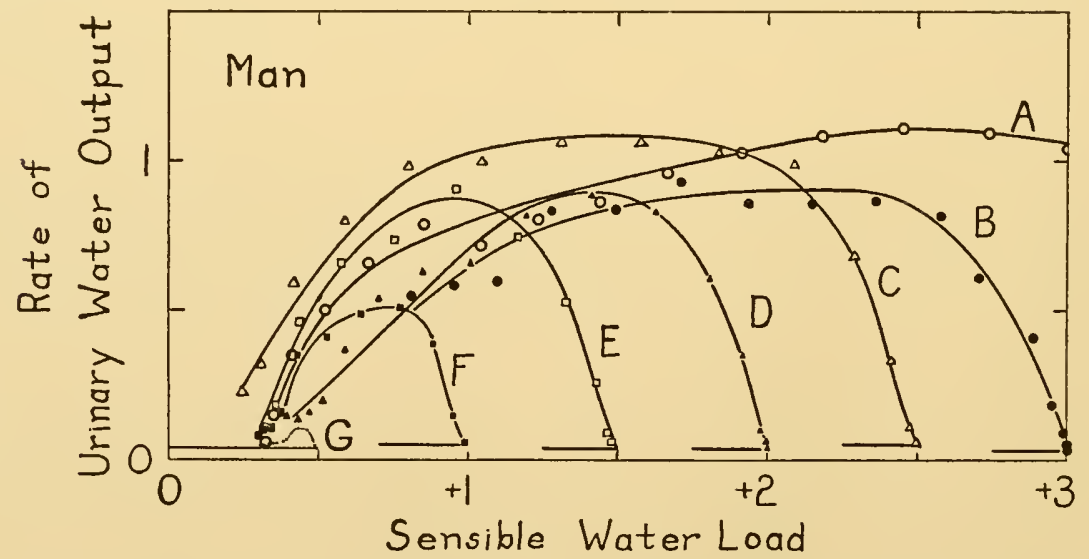

FIG. 53. Rate of urinary water output in relation to sensible water load after single ingestion of water. The same tests are averaged as in figures 49 and 50 . 
While augmented excretion is proceeding in the presence of water excess, the opportunity to drink water is consistently refused. The only known accession of water is the slow formation by oxidations; its rate is uninfluenced by water excess (Carpenter and Fox, '30).

In general, the rates of elimination of excess water by man are smaller than by dog (relative to body weight), and are slower in accelerating. Rates are less nearly proportional to loads. The amounts of water returned in diuresis are in both species less by an absolute volume than the amounts administered. It may be stated that after water excess the total water losses do not differ significantly from the urinary water losses.

\section{§ 28. Repeated ingestions by MoUth}

What happens when the accession of water to the body continues? Water is ingested in 8 to 16 equal portions at intervals of 0.25 hour (Kingsley and Adolph, new data). Latent periods for appearance of diuresis vary widely, being very long at small rates of ingestion. Times required to reach maximal rates of diuretic response also are diver'se in much the same proportion. Ultimately stationary rates of elimination of water are attained; and at some rates of ingestion a steady state of content also is maintained for an hour or more. In these tests the three factors of intake, output, and load are physiologically fixed with relation to one another. Even in moderate loads the rate at which the body excretes water appears to reach a limit (figs. 54 and 55). But temporarily at least this rate of excretion may exceed the rate of intake (Wendt, 1876).

Velocity quotients $(1 / \Delta t)$ in steady states of ingestion and load resemble those that prevail during decreasing rates of water excretion after single ingestion, if compared at equal loads. The quotients decrease with load, whether the loads be total or sensible, and whether the rates of exchange be total or urinary.

After ingestion of water ceases, rates of elimination gradually decrease, with diverse periods of persistence and diverse decelerations. After those states have prevailed in which elimination rates equal ingestion rates, cessation of diuresis requires less time than attainment of its maximal rate does. But where ingestion rates are very high, some hours may be consumed in completing the 


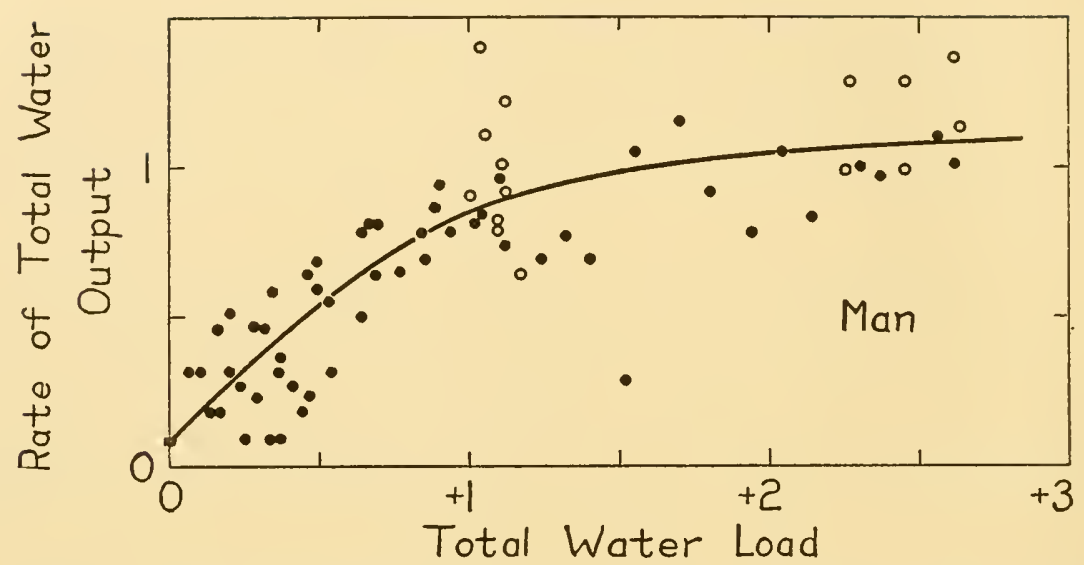

FIG. 54. Rate of total water output ( $\%$ of $\mathrm{B}_{0} /$ hour) in relation to total water load $\left(\%\right.$ of $\mathrm{B}_{0}$ ). Stationary state. One individual in 5 tests. Each point represents the rate that prevailed during one period of 0.25 -hour, beginning 1.75 -hour after the first of ten equal ingestions at 0.25 -hour intervals, and continuing thereafter. Open circles, during water ingestion; solid points, after ingestion ceased. Data of Kingsley and Adolph.

elimination of excesses, indicating definitely that water loss has previously failed to maintain proportionality to water load.

The termination of diuresis appears to be a smooth and orderly event. The diuresis is at these times a correlative of the water load; as the water load disappears, excretion diminishes without measurable lag. Suppose, however, that the excretion were de-

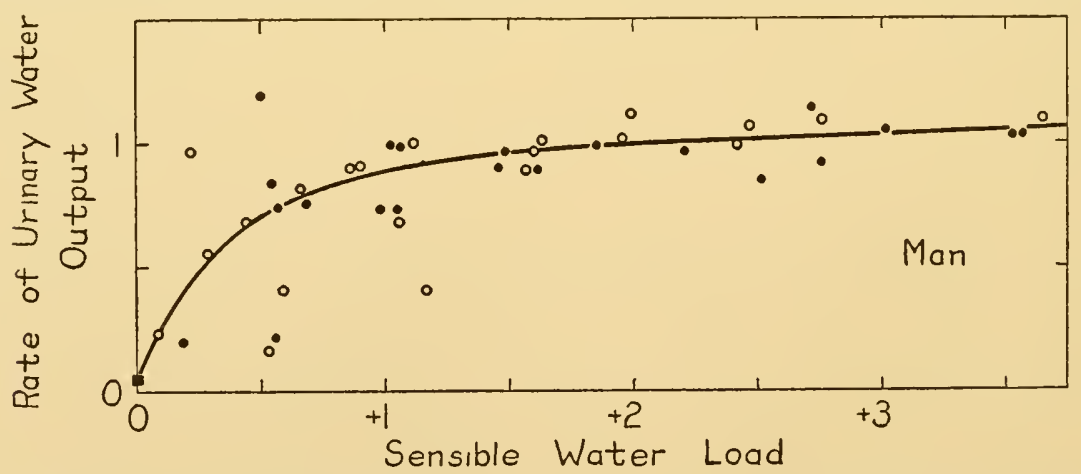

FIG. 55. Rate of urinary water output ( $\%$ of $\mathrm{B}_{0}$ /hour) in relation to sensible water load $\left(\%\right.$ of $\left.\mathrm{B}_{0}\right)$. Three individuals in stationary state, 21 tests. Water was ingested at 0.25 -hour intervals, usually in 16 portions but varying from 8 to 16 . Solid points, maximal urinary rate in any 0.25 -hour period before ingestions ceased; open points, maximal urinary rate (and coincident load) in any subsequent 0.25 -hour period. Data of Kingsley and Adolph. 
pendent upon the load a half-hour previously (that the response occurred with a lag such as occurs at the initiation of diuresis). Then the return in diuresis might easily overshoot the volume ingested; an initial water excess would give rise to an ultimate water deficit. Hence the rate of diminution (deceleration) of water output might conceivably be an optimal process. Were the deceleration faster, overshooting might frequently occur. Were the deceleration slower, the restitution of water balance would appear to be less sensitive to changes, and the tail-end of any diuresis would be much prolonged. Unfortunately no quantitative expression can be given to this virtual optimum, the absence of other factors (as always in problems of optima) being only a supposition. But I wish to emphasize that this deceleration is of consequence to the organism.

Without presenting further data for man, I conclude that the rates of water loss are very similar during stationary rates of water intake and during recovery (beyond the first 1.0 hour) from single water administrations. The only significant diversities are in loads less than $+1 \%$ of $\mathrm{B}_{0}$; in them unidentified factors exert their influences. But in both repeated and single administrations, the constancy of rate of output with all loads above $+1 \%$ of $\mathrm{B}_{0}$ in man is in marked contrast to the dog; it implies a maximal capacity for elimination and an inconstant velocity quotient. It may be noted, however, that some human individuals have exhibited higher rates of elimination than any shown in figures 52 or 55 (e.g., Haldane and Priestley, '16); these individuals appear to augment their losses as the loads increase above +1 or even $+2 \%$ of $B_{0}$. In any case the rates of water excretion are proportional to water load over a narrower range in man than in dog.

\section{$\S 29$. WATER DEFICITS}

Water deficits are studied by the following procedures. The subjects deprive themselves of all liquids and of foods containing large proportions of water, but maintain adequate food intakes. The diet is not strictly constant in some cases; in others it consists of uniform amounts of butter, sucrose, and casein. Usual activities of the subject are continued. After measured losses of weight, water is offered either in ad libitum amounts or in uniform amounts at ad libitum times. The quantities drunk are ascertained, and they represent the gains of water (fig. 56). 


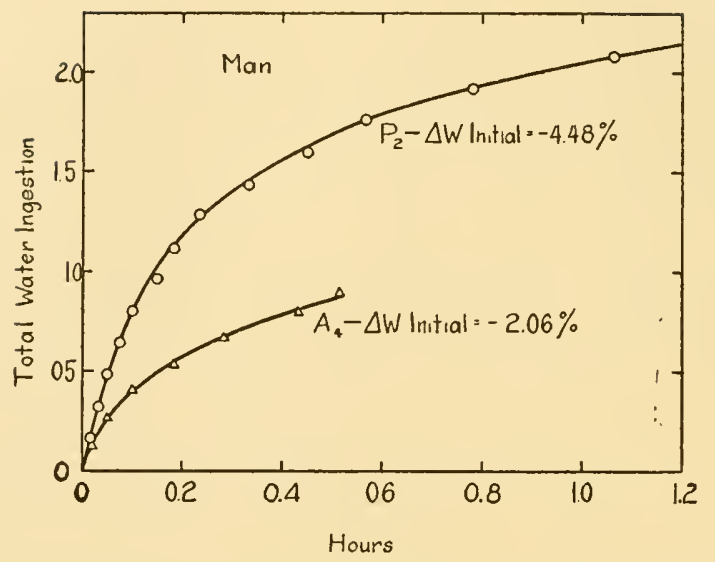

FIG. 56. Amounts of water voluntarily ingested $\left(\%\right.$ of $\left.\mathrm{B}_{0}\right)$ in relation to time after drinking was allowed. In $P_{2}$ the deficit $(-\Delta W)$ was $4.48 \%$ of $B_{0}$ at zero time; in $\mathrm{A}_{4}$ it was $2.06 \%$. In $\mathrm{P}_{2}, 1.45$ hours were required for half recovery, in $\mathrm{A}_{4} 0.75$-hour. New data. The curves very roughly correspond to equations: $\Delta W=-0.63 \log t$ and $\Delta \mathrm{W}=-1.22 \log \mathrm{t}$.

The ingestions are always found to be leisurely, after the first minute or two, and their rates decrease with time (fig. 57). At 1.0 hour the total amount drunk is usually only half or less of the original deficit of water as measured by body weight (fig. 58). Nothing being known about the efficient causes of this characteristically slow drinking, other sorts of "reasons" for it may be imagined.

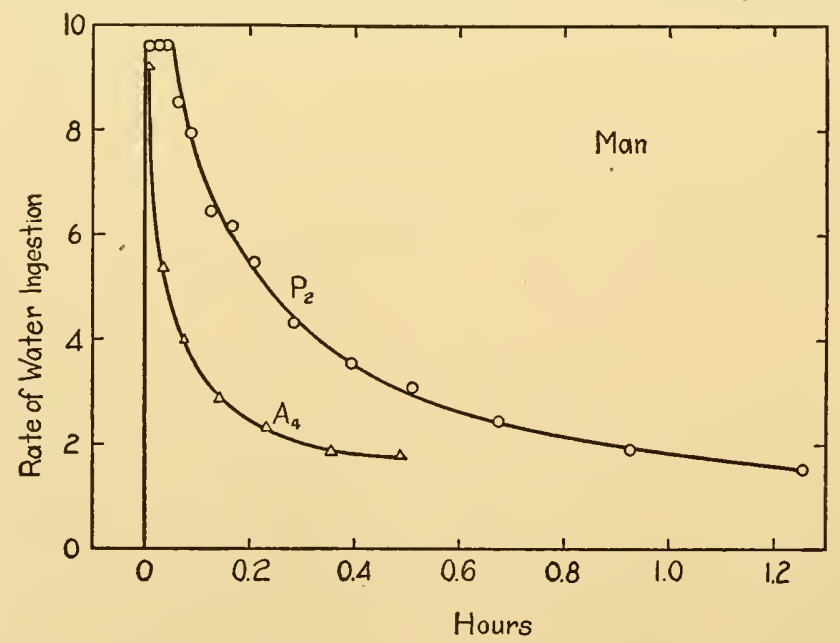

FIG. 57. Rate of water ingestion ( $\%$ of $B_{0}$ /hour) in relation to time after drinking was allowed. Each rate is plotted at the middle of the period observed. Same data as in figure 56. 
One such is that rapid drinking would allow the loss of the new water by diuresis, while slow drinking avoids such an effect of sudden accession. But when tested, the small diuresis that follows slow voluntary drinking is not significantly augmented when water equivalent to two-thirds of the deficit is forced down at once.

At diverse water loads (fig. 59) the rates of gain differed in absolute magnitude. A high correlation between them is evident, and the mean rates of initial drinking are proportional to the deficits.

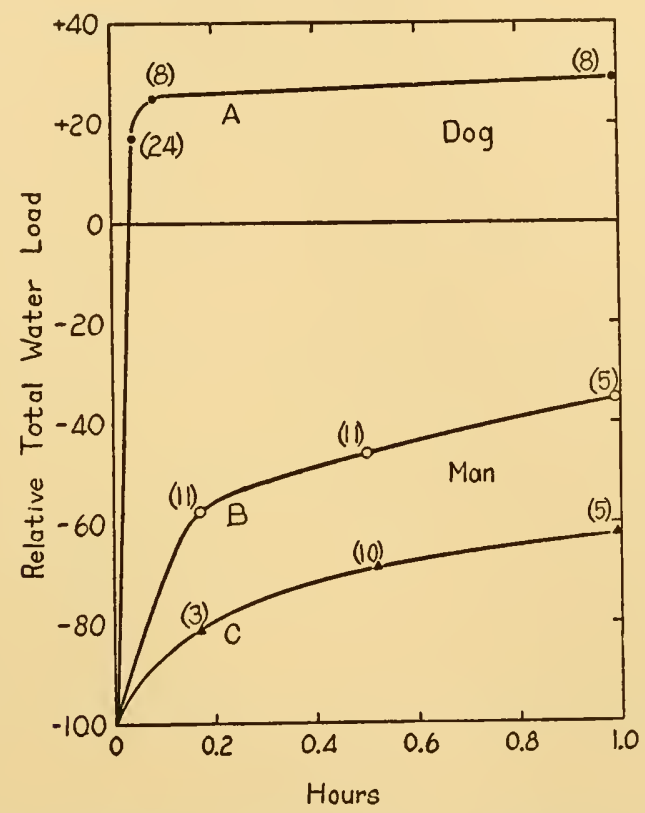

Fig. 58. Relative water load in relation to time. The amounts drunk are computed as percentages of the deficits (loads) that initially prevailed for all tests in which the deficits are $2 \%$ or more of $\mathrm{B}_{0}$. The numbers in parentheses indicate how many tests are averaged. A, dog in laboratory; $\mathrm{B}$, man in desert; $\mathrm{C}$, man in laboratory. At 0.5hour, the difference between desert and laboratory is shown by the $t$ test of Fisher to be significant with a $\mathrm{P}<0.01$. Data of Adolph and Dill ('38), and Adolph ('39a).

To say that the rate of water intake is a psychological measurement, neither adds to nor subtracts anything from the result. No matter how many conditionings and experiences may influence the drinking, evidently the choice of rates is not unlimited. Moreover, most features of ingestion (table 15) are reproducible not only in one individual but among individuals.

The behavior concerned in water drinking belongs to the category of "operant"' behavior (Skinner, '38), the responses of inges- 
tion being measured without reference to any recognized stimulus. Instead they are correlated with water load; alternatively, load can be inferred to accompany some unknown stimulus, as is frequently imagined.

Gain of water by oxidative production is not modified by deficits of water in the body as indicated by the rates of basal oxygen consumption (on 7 days of deficits ranging from -1.5 to $-4.9 \Delta \mathrm{W}$, in 3 tests of Pinson and Wills).

In water privation the rates of water loss diminished significantly below those in states of water balance, when ascertained on

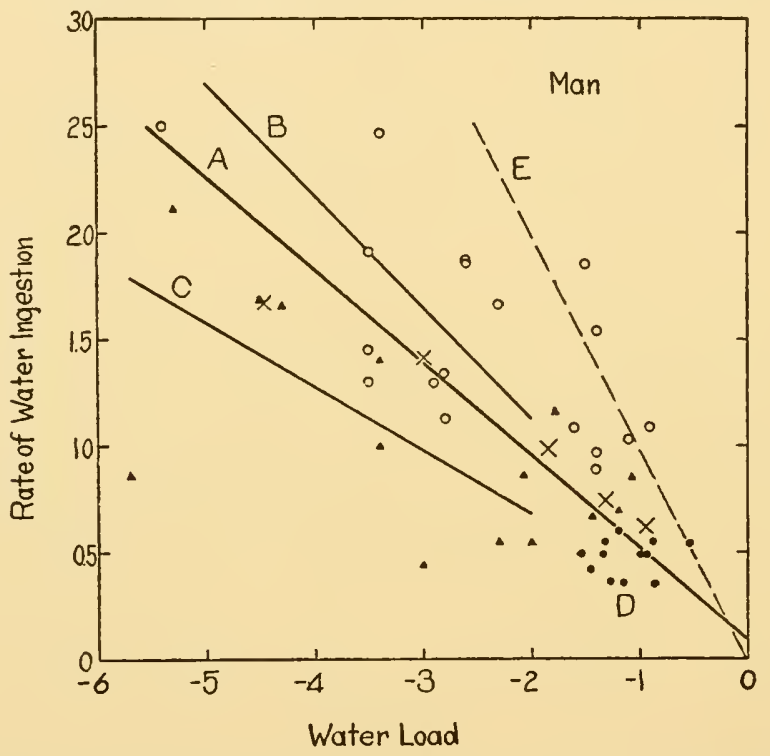

FIG. 59. Rate of water drinking ( $\%$ of $\mathrm{B}_{0} / 0.5$ hour) in relation to water load or deficit ( $\%$ of $\mathrm{B}_{0}$ ). Intakes were each measured in the first 0.5 hour of drinking $a d$ libitum at the end of a period of weight loss. A, regression line representing the group means $(X)$ of 44 tests (10 individuals). B, line representing the 11 tests $(\odot$ ) at $-\Delta W>2$, in the hot desert ( 7 individuals). C, line representing the 10 tests ( $\mathbf{A}$ ) at $-\Delta W>2$, in winter laboratory conditions (4 individuals). D, group of 12 tests ( following physical exercise ( 1 individual). $\mathrm{E}$, hypothesis that $\delta \mathrm{W} / \Delta t=-\Delta \mathrm{W}$. New data.

constant diet in 24-hour periods (Dennig, 1898, 1899; Newburgh and Johnston, '34). The diminution prevails also for urinary loss alone. It holds further for evaporative loss alone, in periods of measurement lasting 0.2 to 3.0 hours (fig. 60). During the first few hours of recovery by drinking, only variable and insignificant modifications of those urinary rates and evaporative rates are found. 
It may be said that water ingestion in man is most rapid when drinking begins. Over a smooth course of intermittent drinking the rate diminishes for an hour or more. In contrast with the dog, drinking is very slow and prolonged (Adolph and Dill, '38), as

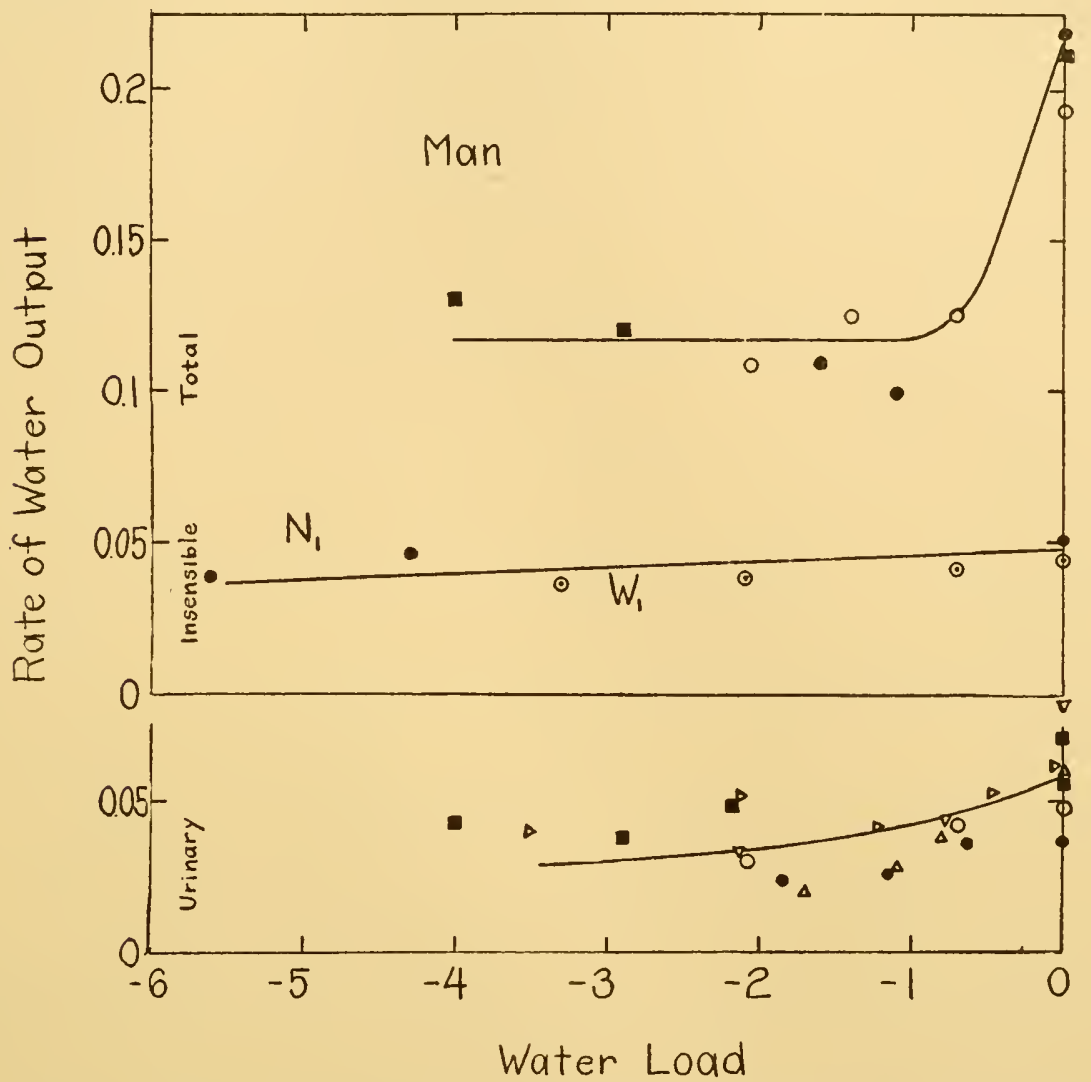

Fig. 60. Rates of water output ( $\%$ of $\mathrm{B}_{0} /$ hour) in relation to water load. Loads ( $\%$ of $\mathrm{B}_{0}$ ) are equivalent to decreases of body weight over periods of 1 to 4 days of usual food intake but inadequate water intake. All rates of output were measured over periods of 0.2 to 3.0 hours; total by loss of body weight and urine; insensible by basal loss of weight lying on a Sauter balance; urinary by collected output. Each letter designates one individual. New data on 4 subjects; and $\mathrm{N} 1$ reported by $\mathrm{Hall}$ and McClure ('36). Urinary plus insensible do not here equal total because separate tests and conditions were concerned in each.

though overshooting of content at balance were fearingly avoided. Water loss by the paths of urinary and of evaporative outputs diminishes significantly. Both the augmented drinking and the diminished loss contribute to the prolonged recoveries from states of water deficit. 


\section{§ 30 . Equilibration and recovery}

Combining the data from single ingestions by mouth and from water privations, I obtain an equilibration diagram (fig. 61). The initial rates of recovery are here represented in the first 1.0 hour; they might be represented over diverse periods of time. Recovery by drinking is most rapid within the first 0.1 hour, while recovery by forming urine is most rapid after 1.0 hour.

The diagram indicates that water balance prevails at only one content. Small departures from the content at balance mean large modifications of exchanges, and ordinarily the body is either in small positive or negative increments at nearly every instant.

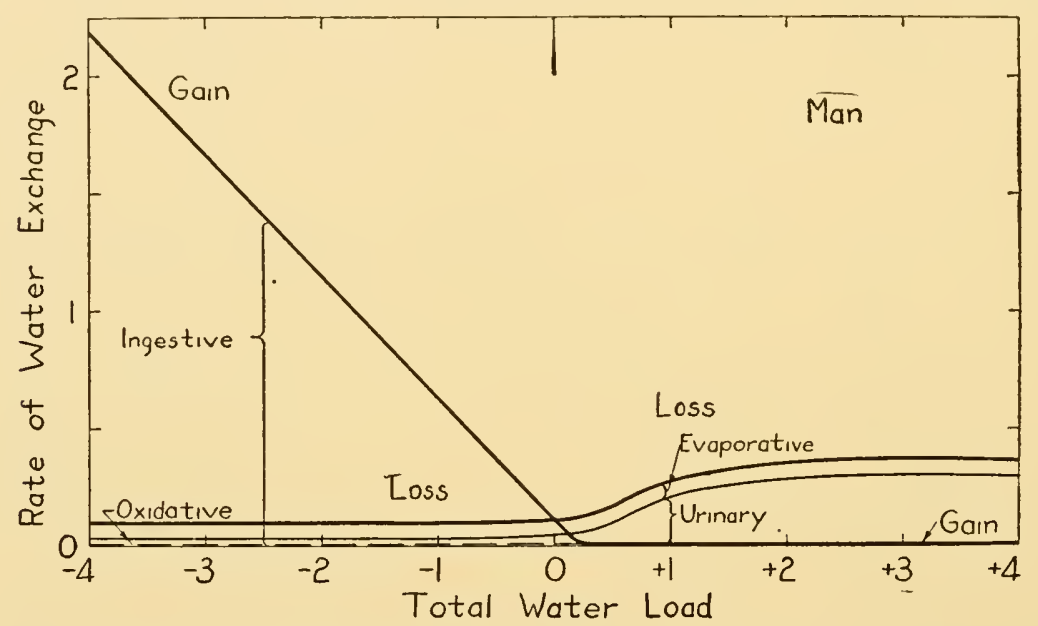

FIG. 61. Rate of water exchange ( $\%$ of $\mathrm{B}_{0} /$ hour) in relation to water load ( $\%$ of $\mathrm{B}_{0}$ ) in man (equilibration diagram in first 1.0 -hour of recovery). Data concerning ingestive gain are obtained from figure 59 in negative load, multiplying by 1.2 (fig. 58) to convert from $0.5 \mathrm{hr}$. to $1.0 \mathrm{hr}$.; concerning urinary loss from figures 60 and 52 . Total loss equals urinary loss, plus $0.07 \%$ of $B_{0}$ /hour as the mean evaporative + fecal losses at all positive loads, they being independent of water load (Hall and McClure, '36). Gain of water by oxidation ( $0.02 \%$ of $B_{0}$ /hour) appears to be nearly independent of water load whether positive (Carpenter and Fox,'30) or negative (Pinson and Wills, new data).

When no factors shift the apparent balance itself, water content is maintained constant within $\pm 0.22 \%$ of $\mathrm{B}_{0}$ in 24 -hour periods and $\pm 0.08 \%$ of $\mathrm{B}_{0}$ in 1 -hour periods ( $\mathrm{C} \Delta$, new data).

The partition of the rates of exchange according to paths (fig. 61) again shows that restoration is predominantly by one path of gain (ingestive) and by one path of loss (urinary).

Net velocity quotients are equal in positive and in negative loads (in the arbitrary initial period of 1.0 hour) only up to $\pm 0.2 \%$ 
of $B_{0}$. At all larger loads recovery by gain is much faster than by loss. Although ingestive gains seem slow, they are at loads above $\pm 2 \%$ of $\mathrm{B}_{0}$ faster than even the maximal rates of urinary losses (fig. 55).

Corrections for alimentary absorption (fig. 62) probably influence the absorbed water loads within 0.6 hour of any ingestion, but not later. Hence they are of no consequence in the equilibration diagram at 1.0 hour, or in its corollaries or derivatives.

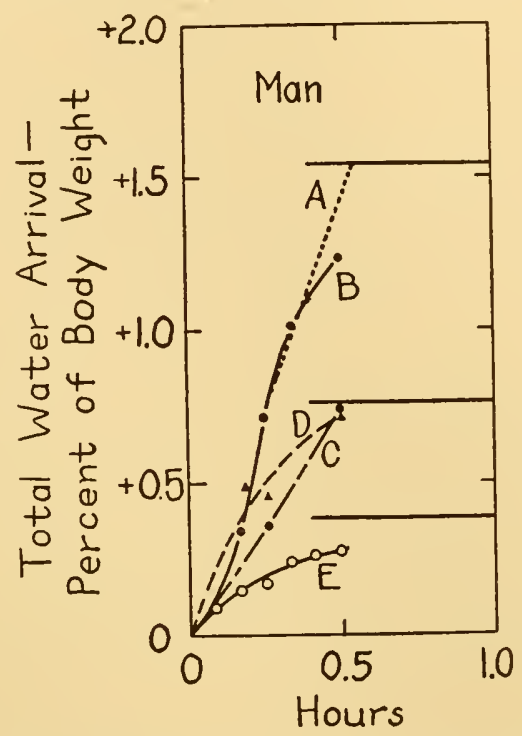

Fig. 62. Water passed through the alimentary tract in relation to time after water was drunk by men in water balance. A, mean of estimations, by 5 methods, of water absorbed from alimentary canal (Baldes and Smirk, '34). B and C, estimations of water disappeared from stomach, by methylene blue volume remaining (Christ, '26). $D$, estimations of water disappeared from stomach, by glucose volume remaining (Moritz, '01), points being the means of 12,18 , and 3 determinations on two subjects. $E$, fluid aspirated from duodenum, means of 5 measurements on 5 subjects (Baird et al., '24). Horizontal lines indicate the loads ingested. Mean body weights are assumed to have been $65 \mathrm{~kg}$.

The equilibration diagram indicates conditions for maintenance of water content in man and for recovery after disturbance of it. It provides standards by which the response of water exchanges by an individual, as a whole and by particular paths, may be evaluated $(\S 33)$.

Behaviors by which men choose environments that favor maintenance of water content are well known, but no systematic studies of them are available. In a general way people do things that are 
appropriate; sometimes by impulse and sometimes by experience. In desert journeys, they travel at night and in shade, minimizing the creation of water deficits through evaporative loss. They are said to become restless when in water shortage, just as infants become restless. Concerning water excesses where choices of environment were permitted, on the other hand, not even such crude facts are known.

\section{§31. Comparison with DoG}

The data interrelating the compensatory processes in man (fig. 61) are obtained under conditions that at present appear to be the same as those prevailing in dog (fig. 13). What similarities and differences occur among the results? These are first and provisional comparisons; ones made below (chapter IX) will concern several species more exhaustively. Heretofore results might have been generalized from one species; now distinctions may also be found.

Initial states are studied under which increments of water content are being dispelled after excess of water has been introduced by mouth or deficit created by privation. The differences are: (a) at any given excess of water (computed as $\%$ of $\mathrm{B}_{0}$ ) the $\operatorname{dog}$ excretes water (per unit of $B_{0}$ ) about twice as fast as the man. Maximal rates of elimination by the dog far surpass those by man. If some other factor than body weight were used, the same conclusion would hold, for the factor cancels out by considering $\Delta \mathrm{W}$ along with its correlative $\delta \mathrm{W} / \Delta \mathrm{t}$; in other words, the net velocity quotients $(1 / \Delta \mathrm{t})$ are greater in dog than in man. (b) In any deficit of water the dog ingested water much faster than man. The dog more than made up the whole deficit in 0.1 hour; the man required some hours to do so. (c) In deficits the sparing of urinary loss is greater in dog than in man. This is related to the fact that dog's urine may carry much more solute, be more highly concentrated. Hence all modifications of water exchange are smaller in man than in dog. (d) All responses are slower, in initiation, in acceleration, in half-life, and in completion, in man than in dog.

These differences are far greater than the differences among individuals (standard deviations) for one species or the other.

Among uniformities between man and dog are found: (n) In the equilibration diagram the rates of loss are represented by a curve having three limbs. The middle steep limb shows rates of 
loss that increase with water excess; at its right a limb shows maximal rates changing little with load. (o) the rates of ingestion are approximately proportional to water deficits, and zero in water excesses. (p) The rates of loss and of gain are equal at one point of balance. (q) In excesses, loss exceeds gain; in deficits, gain exceeds loss; so that in both balance is regained. (r) Losses are slightly decreased in water shortage, both by urinary and by evaporative paths. (s) Oxidative gain is approximately uninfluenced by water load.

Both uniformities and diversities between two species of mammals having been found, it is worth while to inquire which uniformities hold for others as well. The pattern of the equilibration diagram for dog and man may be found in many other species, but its quantitative features show wide diversities of magnitudes.

\section{\$32. VARIATIONS}

A more intimate notion of the exactitude of water balance of man is obtained by the study of fluctuations in content and in exchanges. Near water balance intake is much more variable than output over periods of 0.25 hour (table 12), although one path of it (oxidative gain) is very constant under standard conditions. Often water content (body weight) slowly decreases from quarterhour to quarter-hour until water is taken to restore it in part. No great differences from the dog in this respect $(\$ 22)$ have turned up.

Stability of a physiological function is sometimes said to be a partial measure of, as well as a usual requisite for, the organism's success (in surviving). The criterion of success, however, may equally be otherwise and diversely chosen (leading to confusion of terms unless specially defined), since the small fluctuations of water content now discussed are not known to affect survival. Some kinds of "success" are dependent upon greater rather than less variability. In the social organism, too, great stability is not always beneficial to survival (Pareto, '35, \$2195).

In addition to random variability, it is necessary to distinguish oscillations or rhythms of function, either with respect to time or to some other variable. In the case of water exchanges these periodic fluctuations have been measured in man under continued water drinking. In the average individual, urinary outputs (during uniform hourly intakes) are said to vary in four cycles per day 
(B, fig. 63). Some single individuals do and some do not show these cycles. One way of testing the significance of the data is to compare the variabilities in rates of output in consecutive hours with those in random hours, in second hours, or in third hours. By any test, the extremes of the cycles appear still to overlap to a probability $(\mathrm{P})$ of 0.05 .

Since the apparent cycles coincided with habitual meal-times, although the subjects of the $B$ tests did not eat, food relations were

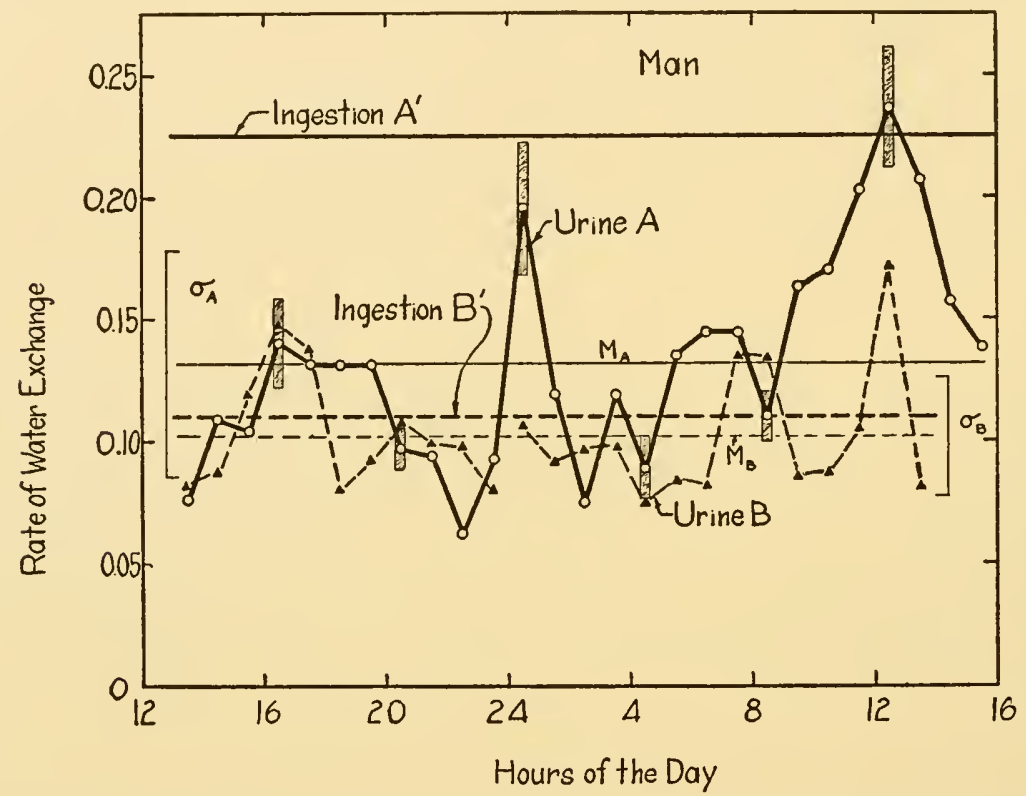

Fig. 63. Rates of urinary water output ( $\%$ of $B_{0} /$ hour) during steady rates of water intake in relation to clock hours. A, mean outputs in 35 tests on 35 men ingesting every hour $0.11 \%$ of $\mathrm{B}_{0}$ of water and $0.13 \%$ of $\mathrm{B}_{0}$ of food (actual plus potential water $=0.225 \%$ /hour $)$. $\mathrm{B}$, mean outputs in 51 tests on 41 men ingesting every hour $0.11 \%$ of $\mathrm{B}_{0}$ of water only. Mean body weights are assumed to have been $68 \mathrm{~kg}$. Means (M) for the 24 or 27 hours and their standard deviations $(\sigma)$ are indicated. At six points on curve $\mathrm{A}$ the standard errors are indicated by rectangles. All are data of Gerritzen ('36).

studied. The day's food was divided into 24 portions and a portion was taken with each hour's drinking water (A tests). The cycles were again possibly apparent, in nearly the same positions on the clock. Hence without further change of regime, which in turn would modify additional balances of the body, uniform rates of water exchanges at various hours cannot be expected. This conclusion means that control tests must be made at the same clock times as other tests in any study of water exchanges in man. 
Another situation in which water exchanges were measured is found when a man walks in hot and in cool environments (Dill et $a l .$, '33). In hot weather the exchanges of water (by evaporation and by drinking) are much larger. The progressive decrease of water content during them, though drinking is allowed, is also large as compared with the dog's in fig. 48. In man drinking is never sufficient to make up for the loss of weight that occurs in the same period of time. Hence no stationary state of water load is reached while the man walked in the desert, yet it is quickly reached in the dog.

Variations, then, in the water loads and water exchanges of man are of two sorts, in rhythms and without apparent rhythms. Use of long periods of time in measuring exchanges, and of average data concerning them, tend to hide both sorts. As with many other activities, what is usual at one time may be incongruous at another. The kinetic equilibration of water content is itself a mean in a set of relations, about which distinguishable modifications oscillate.

\section{\$33. Characterizations and tests}

Given the usual situation that a whole equilibration diagram for all times elapsed cannot be worked out on each man to be studied, what particular tests will yield crucial information? And even when a diagram has been worked out, what points in it are likely to characterize the individual or the species? In many physiological phenomena, empirical tests of this sort have been selected by convenience, usage, and experience, to differentiate experimental and pathological states.

(1) Often, for instance, an individual is either too young or too paralyzed to drink water from a cup, but can swallow water when it is put into the mouth or can receive it by stomach tube. How shall I ascertain whether he is deficient in water content? One test is to administer 1 or $2 \%$ of $B_{0}$ of water and see whether the rate of urinary output more than doubles within 1.5 hours; or, how much of the water appears as urine within 4 hours. For most individuals the position of their water contents at 0 hour, relative to the content at presumptive balance 4 hours later, may thus be ascertained.

A test of a similar sort has been in clinical use for many years, Volhard's "Wasserversuch." In its usual form a subject is given 
one liter of water to drink and the urine excreted is measured in hourly periods for 4 or 5 hours (fig. 51). Quite often no precautions are observed as to whether the subject had opportunity to be in water balance at the start. Hence if little of the water drunk is returned, the individual may be either (a) in initial water deficit, or (b) deficient in elimination. A deficiency of diuretic response to the water drunk is usually presumed to indicate (b1) deficiency of renal function. But it may indicate (b2) deficiencies in any other parts of the metabolizing and mobilizing tissues. Hence further tests are desirable to differentiate these states, such as the duration of the same diuresis; but here I am not concerned with allocating the shortcomings between (b1) and (b2). State (b) can be distinguished from (a) by repeating the test 3 hours after the diuresis that results from the first administration of water.

(2) The maximal rate of urine production after large ingestions of water appears to be a useful characterization of individuals. This establishes either a single point or an equilibration diagram, or a tolerance curve that can be compared with the ranges occupied by other tolerance curves established after the same initial water loads. For instance, in individuals having an administered load of $4.4 \%$ of $\mathrm{B}_{0}$, the fastest urinary output is found to be $0.53 \%$ of $\mathrm{B}_{0}$ /hour, while other individuals excrete $1.10 \%$ of $\mathrm{B}_{0} /$ hour (A, fig. 50). To eliminate that load, diuresis lasts about 12 hours instead of the usual 4 or 5 hours.

(3) Further features of water recoveries may be compared by coefficients that have already been defined, such as economy quotient, ratio of modification, velocity quotient, and increment returned.

Future investigation will undoubtedly lend meaning to several other quantitative characterizations within the four variables studied. Tests are lacking especially in water deficits. This state occurs unintentionally where there is some interference with voluntary water intake; in which case the most sensitive test, ingestion itself, cannot be used. Diminished urinary and evaporative losses are hardly significant without controls in the same individual. Additional indicators from outside the four variables then need to be employed.

For use, each quantity to be compared requires measurement of variabilities. If the variability of one rate of exchange is as 
large as C.V. \pm 80 , that is still a standard. The number of states in which water contents and exchanges are altered also is large. In practice it may be easier to "force fluids" at random than to attempt the differentiation of those states. In theory the intimate connection of water with such a large number of states is of outstanding consequence.

\section{§ 34. Diverse types of water load}

A few studies that furnish portions of equilibration diagrams under conditions other than those used above may be indicated.

(1) Water in excess is ingested with particular quantities of four different foods (data of Petrilli). The subsequent elimination of the water is less than without food, and lasts longer. Anyone can imagine where some of the water goes and where some of the delay may occur.

(2) Water deficits are created by physical exercise instead of by dietary privation. The deficit is produced in 1 or 2 hours instead of in 1 or 2 days, therefore. Subsequent initial ingestions (points D, fig. 59) happen to be in the range of those established for privation alone.

(3) Water deficits follow moderate exertion in very hot atmospheres during 1 to 3 hours. Subsequent drinking is at mean rates somewhat higher (B, fig. 58) than in another state (C), and the rates differ significantly. The difference is chiefly at small deficits (fig. 59). It is possible that the excess of heat content added itself to the deficit of water content in arousing ingestion.

(4) In warm environments the total water losses in balance are modified by the rapid evaporation of sweat. Even in negative water loads the rate of evaporative loss is high; also it is said that sweating is faster at high water contents of the body (Gregory and Lee, '36). But suitable quantitative data are lacking for the construction of an equilibration diagram. Concordantly, the paths of water exchange are very differently partitioned, so that urinary losses are relatively small except where diuresis has been aroused. Evaporative rates sometimes exceed even the most profuse urinary rates; individuals that have been observed under two conditions that regularly result in maximal outputs are able to put out water in sweat faster than in urine.

The water deficit that usually prevails during daily life in the desert (Adolph and Dill, '38) is too small to measure. But it can 
be estimated by indirect means, as follows. The mean rate of total water ingestion is about $0.26 \%$ of $\mathrm{B}_{0} /$ hour, as compared with the rate in other climates of 0.13 . On the equilibration diagram (fig. 61 ) this rate is found at a load of $-0.2 \%$ of $B_{0}$, hence it is the approximate deficit. It is not outside the usual variation of contents found in 24-hour periods in other climates (table 15) nor outside the standard of variation found in that climate.

In the various states considered above, the content at balance $\left(W_{0}\right)$ is believed to be approximately constant. Shifts in the position of $W_{0}$ have been partially investigated in man, particularly in pathological conditions. Examples of such shifts (water retention) are found in lobar pneumonia (Sunderman and Austin, '30), and in treatment with the drug phenylethyl hydantoin (Rockwell, '35). After apparently remaining at the new $W_{0}$ for several days, the body reverts (at crisis) to the original $\mathrm{W}_{0}$.

\section{\$35. Summary}

Investigations of water balance in man are slightly more limited than in the dog, for in man responses in stationary state of water deficit are unknown. Quantitative correlations in the other states of water content are drawn. The same velocity quotients prevail, in the hours after the first, following a single ingestion of water, and after 8 to 16 rapidly repeated ingestions. Several means of testing and characterizing water relations are emphasized: time relations, completeness of return, and maximal rates of exchange.

Man uses all four of the possible means of recovering the usual water content, as does the dog. In deficits, gains are increased while losses are decreased slightly, thus sparing water; in excesses, losses are increased and gains are decreased to the minimum of oxidative formation of water. These four modifications of activities appear to constitute the armamentarium for any adjustment of water content.

Rates of loss are slightly smaller per unit of body weight in man than in dog. In man the rates are less nearly proportional to water load, tending to prolong the life of larger loads.

The most remarkable feature of recovery in man is the slow rate of ingestion after any deficit of water. Often it is asked, why is the drinking leisurely? No other peculiarity of metabolism or structure appears to be known that is especially related to this one. 
Variations of exchanges in believed water balance may occur rhythmically as well as at apparent random.

Diverse parameters are suggested for the characterization of unusual individuals or states.

Differences between man and dog, in equilibration diagrams and their derivatives, may be said to be quantitative only. Qualitatively all features are common to both species, giving rise to the expectation that other animals will exhibit similar patterns of water relations. 


\section{Chapter VI}

\section{WATER RELATIONS OF FROG}

$\S 36$. If the study of water in organisms is to be general, the same variables that are measured in mammals require to be correlated in numerous other kinds of living beings. A frog has many functional differences from a dog, and I now inquire whether and in how far the pattern of water exchanges is different. Is the frog, an animal usually immersed in water, less concerned with constancy of water content? The species Rana pipiens is the one studied except where otherwise stated.

A frog does not drink water by mouth, but takes water into the body through the skin. In older investigations (e.g., of Durig, '01) this fact was ascertained by blocking the gullet in various ways. Equally decisive is the fact that frogs in water gain weight continuously and at nearly steady rates in successive short periods of time. When watched, no frog is seen to open the mouth or to swallow water during the gain of weight. The absence of muscular movements in water intake seems to some persons to confer greater automaticity upon the process in frog than in dog.

A frog puts water out from body through the cloaca, all of it presumably having passed through the kidneys. Only in types of water load other than those studied here does water leave at significant rates through the skin or any other path than the kidneys, so far as is known.

\section{\$37. WATER EXCHANGES}

Recoveries of water content are measured in frogs immersed in water at $20^{\circ} \mathrm{C}$. with nostrils in air. Excesses of water result from sudden injection of distilled water into the peritoneal cavity. Deficits are previously produced by 1 to 24 hours of evaporative desiccation, the frog being temporarily out of water in air (Adolph, '39b). Increments of body weight represent the loads of water.

Figures 64 and 65 indicate the conduct of tests. When the frog's cloaca is closed by ligation the weight increases, corresponding to the rate of entrance of water through the skin. Whenever the cloaca is later opened, urine escapes and the amount of it that has formed since ligation is thus ascertained. When the cloaca is not ligated, changes of body weight indicate net exchanges of water. 


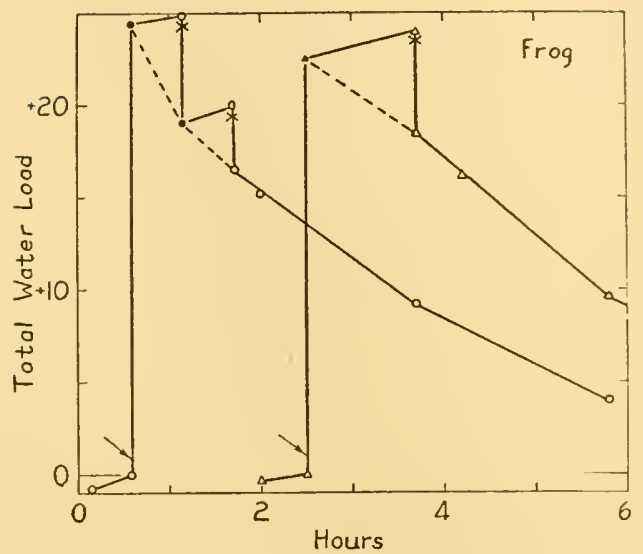

FIG. 64. Course of water load $\left(\%\right.$ of $\left.B_{0}\right)$ in each of two frogs that were injected intraperitoneally (at the arrows) with distilled water. The cloacas were ligated at the time of injections and later opened (at the crosses); the first being ligated twice. The differences of body weight before and after the crosses measure the urine excreted since ligating. Redrawn from Adolph ('39b).

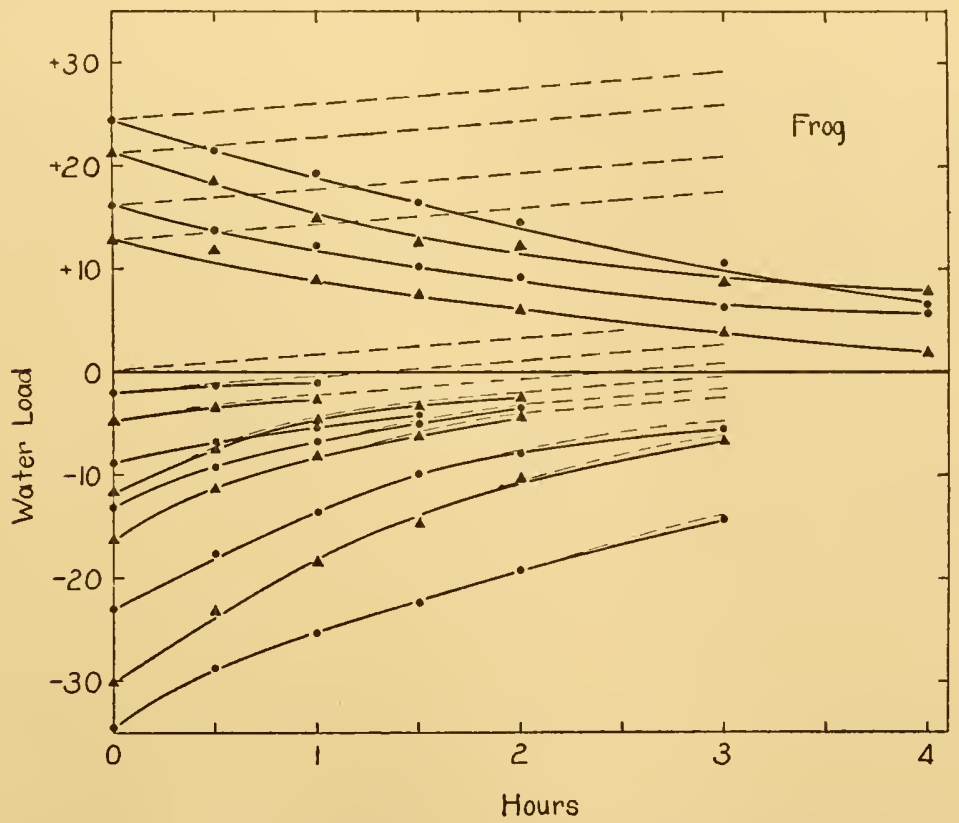

FIG. 65. Course of water load $\left(\%\right.$ of $\left.\mathrm{B}_{0}\right)$ after recovery commenced. Rana pipiens. Points and solid lines, total water load; light dash lines, gross load, which includes urine kept in the body by ligature on the cloaca. The difference between solid and dash line represents total output since zero time. Each line is the mean of 10 individuals grouped in order of the initial loads imposed. Further data of Adolph ('39b). 


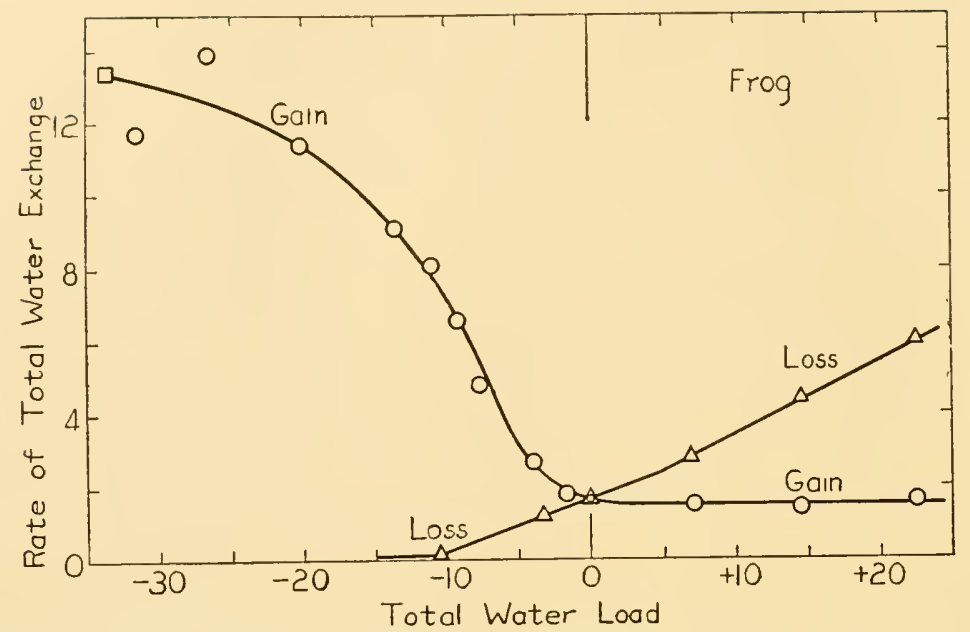

FIg. 66. Rates of water exchange ( $\%$ of $\mathrm{B}_{0} /$ hour) in relation to initial water load ( $\%$ of $B_{0}$ ) (Equilibration diagram). Frog. Rates are all computed from the first 0.5 -hour of recovery, each point representing the mean of 10 measurements on as many individuals. The square represents 10 individuals that did not survive the recovery. Redrawn from Adolph (39b).

In order to yield average data, tests are grouped in tens in the order of the increments of water initially contained in the bodies. The subsequent exchange is then (fig. 65) such that in every case the net weight of the frog tends toward the weight that prevailed before water was subtracted or added. But the gross weight (urine

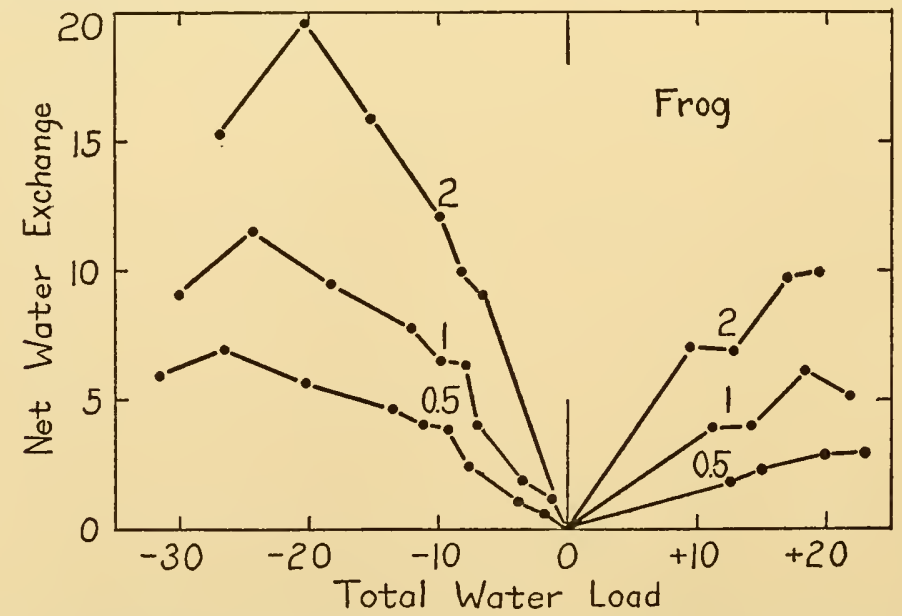

FIG. 67. Net exchange of water $\left(\%\right.$ of $\left.B_{0}\right)$ in relation to mean water load. The exchange is that which occurred within the time indicated in hours of recovery. Each point represents $10 \mathrm{frogs}$, the same as in figure 65. Further data of Adolph ('39b). 
being retained in the cloaca) always increases, at diverse rates that climb with the deficit. When these initial rates are studied individually (Adolph, '39b), the standard deviations of rates among' members of each group of ten are quite uniformly about \pm 27 per cent of their arithmetical means.

Urine formation is measurably present only in positive increments of water content, and in negative increments down to $-10 \%$

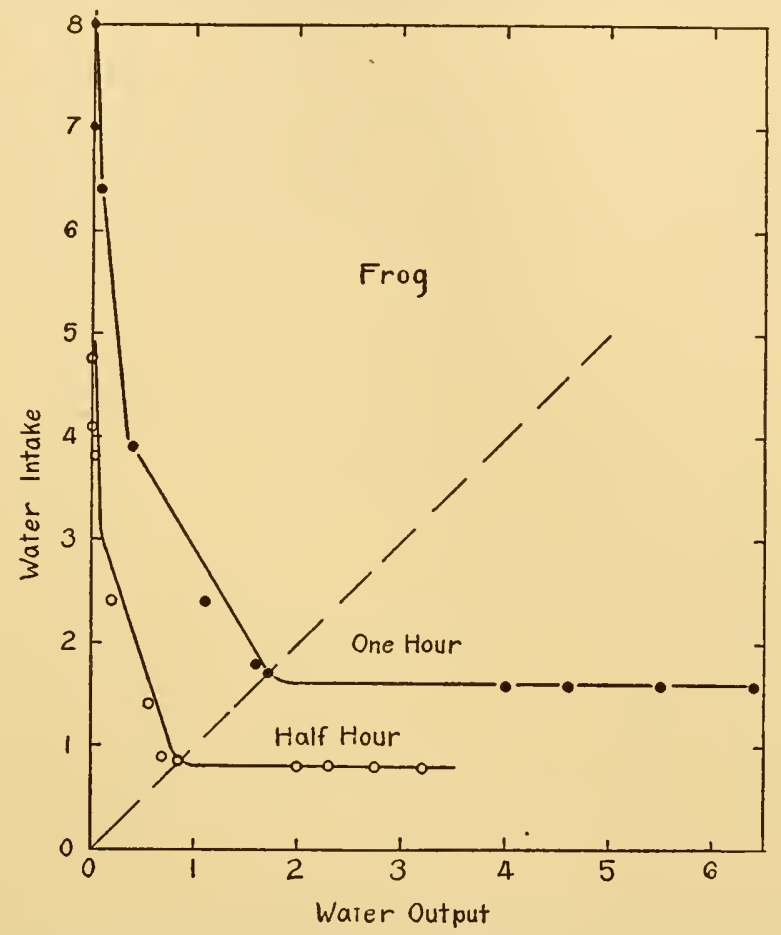

Fig. 68. Water intake ( $\%$ of $B_{0} /$ hour) in relation to water output ( $\%$ of $B_{0} /$ hour) under varying water loads, within the initial 0.5 hour and the initial 1.0 hour of recovery. Each point represents the mean for 10 individuals, as in the same data of figure 66 . The dash line represents the state in which intake and output are equal; this occurs only at water balance.

of $\mathrm{B}_{0}$ (fig. 66). The initial rates are approximately proportional to the water contents of the body above $-10 \%$. Measurements of urine production made over half-hour periods are less accurate than those made over longer periods; nevertheless at each load the average rate of urine production during the first 0.5 hour of recovery is found to equal the rate during 1.0 and 2.0 hours. Hence the intact frog shows no measurable lag in onset of diuresis following intraperitoneal injection of water. 
Initial rates of water intake and of water output together (fig. 66) constitute an equilibration diagram. The intake never falls to nothing; even in the largest water excesses studied, its minimal rate persists. Urine production falls to zero in moderate deficits of water. Its rate is augmented less with excesses of water than intake is augmented with deficits. Since gain and load are little affected by the length of time elapsed as such, the diagram is not greatly modified at longer times than 0.5 hour (fig. 67).

The net exchanges of water (fig. 67) represent the combined accomplishment of the total exchanges as modified at diverse loads. Since body weights are actually being measured it is directly assured that observed gains minus observed losses equal net gains. In 2 hours any deficit is paid off, but only half of any excess is covered.

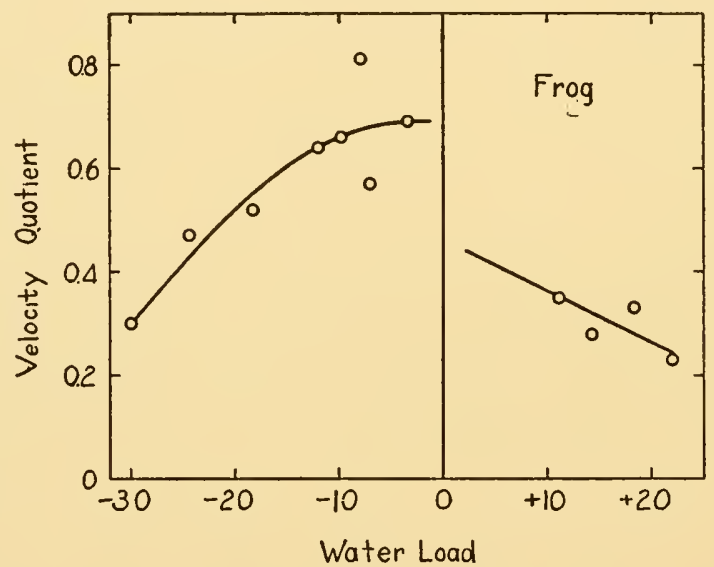

FIG. 69. Net velocity quotient in relation to mean water load. Each point is derived from one group of 10 individuals in the first 1.0 hour of recovery. Load is \% of $\mathrm{B}_{0}$; velocity quotient is obtained from figure 67 by dividing the net rate of water exchange by the mean water load, $=1 /$ hour.

For every rate of water output there is one rate of water intake under the conditions prevailing (fig. 68). The roughly hyperbolic form of the curve relating the two, emphasizes the reciprocal relation of the two processes, as though a high rate in one excluded rapid activity in the other.

Velocity quotients are larger in water deficits than in water excesses (fig. 69). Recovery requires only one-third to one-half the time after moderate desiccation that it requires after water injection. It is most prompt at small loads. Among diverse excesses the net quotients are not much affected by magnitude of water 
load, whereas the quotient for total intake (not shown) is proportional to reciprocal of load.

Initial velocity quotients at small net rates of water exchange exceed those at large net rates (fig.69). As, later in the same tests, the rates become smaller, the quotients remain significantly different (fig. 70). Hence the individual courses of recovery do not exactly recapitulate the array of initial recoveries at identical diverse loads.

A few of the data shown for frogs are confirmed by independent measurements; after desiccation by data of Durig ('01) and Adolph ('32), and after water injection by sparse observations of Adolph ('27).

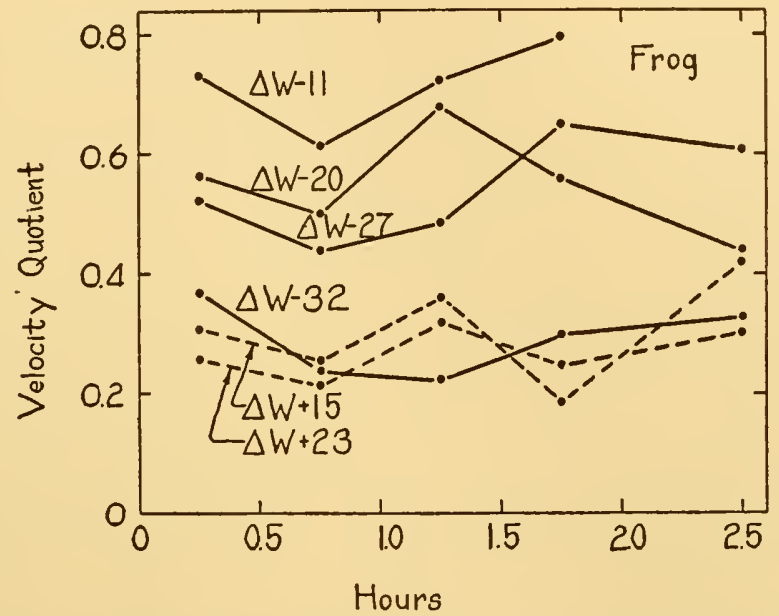

FIG. 70. Velocity quotient (1/hour) in relation to time. Each point is referred to the middle of the period during which it prevailed. Each curve represents a group of 10 individuals starting with the water load indicated. From figure 65.

A biologist unfamiliar with amphibia might expect a frog to gulp water by mouth after being highly desiccated, especially if water is made available to the tongue but not to the skin. Water is not ingested or swallowed. Dogs and men use the quick oral means of obtaining water, but frogs are constituted to sit patiently in water long enough for water to penetrate the skin. No cry of inaptitude in the frog's behavior would help investigators to ascertain whether or not the frog has maximal fitness in its water exchanges; swallowing of water on the part of an animal that has no way of metering it might be more damaging than appropriate.

The accuracy of recovery of water content is indicated by the 
net body weights reattained. Thirty-three frogs that had been desiccated to diverse extents have $\mathrm{C} \Delta \pm 2.87 \%$ of $\mathrm{B}_{0}$ after 6 hours of recovery, and $\pm 2.11 \%$ of $B_{0}$ after 24 hours of recovery. Almost the same difference ( $C \Delta \pm 2.22$ ) is found among frogs kept in water balance for 24 hours, and again among frogs recovering from excesses of water; indicating that the over-all fluctuations of weight are not accentuated by intervening desiccation or hydration. Moreover, the differences are in each of the three groups (deficits, excesses, controls) positive and negative in equal numbers.

The exchanges labelled "intake through the skin" may conceivably be net intakes and not total intakes, since it is not known whether water goes out through the skin concurrently as more comes in through it. This possibility makes no difference in the present study, any more than output through the kidneys depends on whether there is "reabsorption" as well as "filtration," and whether there are any other processes intermediary to excretion.

No significant partition of water exchanges among several paths appears in frogs. Loss by evaporation is exceedingly small, for the frog is nearly completely immersed and breathes air saturated with moisture. Gain by metabolic formation of water amounts to less than $0.01 \%$ of $\mathrm{B}_{0}$ /hour at $20^{\circ} \mathrm{C}$. These quantities cannot be represented at all on the coarse ordinates of figure 66 .

In brief, loads of water imposed on frogs by intraperitoneal injection or by evaporative desiccation, lead to net exchanges appropriate to restoration of control contents. Many recoveries are complete in 6 hours, all are complete in 24 hours. At numerically equal loads, recovery by net intake is faster. In excesses, intake continues at about the usual rate characteristic of balance while output is appropriately modified; in deficits, output helps intake to compensate, output significantly diminishing while intake is increasing. Thus the frog uses three of the four possible modifications by which its exchanges may lead to rapid recoveries of water content.

\section{\$38. Variations}

Above I mentioned the variations that occur in the control state. Single individuals are weighed at hourly (or other) intervals to measure how much the contents and exchanges of water vary, and body weights are found to fluctuate by $\mathrm{C} \Delta \pm 0.77 \%$ of its mean/hour (103 tests, 19 individuals). Errors of measurement are insignifi- 
cant, for weighings repeated at 2-minute intervals differ by only $\mathrm{C} \Delta \pm 0.07$. Water intake in successive periods of 1 hour varies by $\mathrm{C} \Delta \pm 32.3$ per cent of the mean intake (45 observations). Water output in 1-hour intervals is believed to vary by about the same coefficient; but exact data have not yet been obtained in successive periods.

While successive hourly periods show differences of intake averaging $32.3(\mathrm{C} \Delta)$, these same rates deviate from the mean rates of each individual by 31.1 (C.V.). In a random series $\mathrm{C} \Delta$ equals C.V., as here; hence a high rate does not tend to follow a low one, nor vice versa. If there were trends lasting more than one hour, C.V. would tend to exceed $\mathrm{C} \Delta$.

In the initial stages of recovery, the water intakes being augmented as much as eight fold, the variation among the rates (C.V. \pm 27 ) is almost equal to that in turnover $( \pm 31.1)$. This suggests that relative variability overrides whatever absolute variability may be present. Or, the reproducibility of the rate is proportional to the amount of water being handled as intake.

Variability of content in water balance may be considered in relation to mean rate of turnover. In one hour $1.66 \%$ of $\mathrm{B}_{0}$ is on the average taken in and put out again. The standard difference of successive hourly weights $( \pm 0.77 \%)$ is 46 per cent of this; and the mean difference is 37 per cent, mean difference being $0.798 \times$ standard difference. In 24 hours the turnover is $40 \%$ of $\mathrm{B}_{0}$, while the coefficient of difference $( \pm 2.22 \%)$ in 24 -hour intervals is only 6 per cent of the turnover. "Precision" as computed by the method of Gasnier and Mayer ('39) is therefore 95 per cent in 24 hours but only 63 per cent in 1 hour.

The variation of intake $(32.3 \mathrm{C} \Delta)$ might correspond to an inaccuracy of $\pm 0.54 \%$ of $B_{0}$ each hour. This alone almost covers the variation in body weight $\left( \pm 0.77 \%\right.$ of $\mathrm{B}_{0}$ ). Similarly the variation among individuals of output alone $( \pm 23.5 \mathrm{C} . \mathrm{V}$.) is a factor in the content. The variation of content may be viewed as an interplay of both these variations of exchange ; added together, the most usual fluctuation of intake would be $1.66 \times \sqrt{32.3^{2}+23.5^{2}} / 100$ or $\pm 0.65 \%$ of $\mathrm{B}_{0}$. The value 0.65 being less than the value 0.77 there is no significant evidence of mutual compensation, in one-hour periods, between intake and output. Most compensations occur over longer periods of time, but within less than 24 hours. 
Hence, maintenance of water content may be regarded as the resultant of fluctuations in two paths of exchange, imbibitory gain and urinary loss. Within periods of one hour no adjustment of rate of gain to rate of loss is evident, but in successive hours rates of gain compensate one another. In 24 hours, variation of body weight is only thrice as great as in one hour, indicating that adjustments of rates of one exchange to those of the other have intervened to limit the variation found.

\section{§ 39. SOME OTHER TYPES OF WATER LOAD}

Frogs have been subjected to water loads in several diverse ways. What is the course of recovery in those other physiological states and environmental conditions?

(1) Frogs that are pronounced dead, if the criterion of survival be a subsequent muscular activity, gain water at the same rates as living ones. This is the case both near water balance (Brugsch et al., '28) and at extreme water deficits (fig. 66). Yet the rates at those two water contents differ by a factor of 8 , as though the machinery of intake is modified in spite of the concurrent failure of some other activities. Sudden stoppage of the circulation without other immediate injury (Adolph, '31b) likewise indicates that movement of the blood is not necessary in ordinary water exchanges.

(2) Frogs that are kept out of water while provided with various water contents may also be compared (Adolph, '39b). From deficit no recovery occurs even in air saturated with moisture; instead a very slow loss by evaporation continues (which is independent of water load), the frog being slightly warmer than the air (Adolph, '32). Urinary loss is almost zero after the frog has been out of water for two hours. Since gain by oxidation is smaller than the slight continued loss of water, even in deficits water balance is never precisely maintained.

From water excess recovery is by diuresis only (fig. 71). The excess, administered by sudden intraperitoneal injection of distilled water, is to the inside of the frog seemingly the same as in the standard (immersed) conditions; yet the diuresis is much smaller. Urine forms at less than half the rates that prevail in frogs immersed in water that have similar loads (fig. 66); but due to the fact that water also enters the immersed frogs, the net recovery by the frogs in air is slightly more than half as fast. Hence with the stoppage 
of intake, excretion of urine is prevented by more than the intake missed; or paradoxically, water entrance appears to promote net water elimination.

Intermediate conditions between immersion in water and freedom from surrounding water were tested. Very shallow water or wet towels allow nearly as much water intake as deep water, but wet filter paper allows little or no water intake. Gradation of intake by this means is difficult to secure; the phenomenon is almost "all-or-none."

It might seem justified to state that the "wetness of the skin" makes the difference between the recovery of the frog in water and the frog out of water. But this would credit to some one factor a situation that has many possible factors. The physical dif-

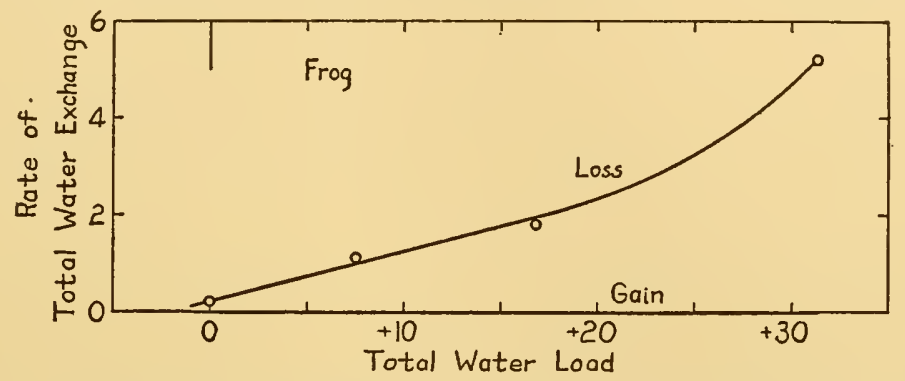

FIG. 71. Rate of water exchange ( $\%$ of $B_{0} /$ hour) in relation to initial water load ( $\%$ of $\mathrm{B}_{0}$ ), in frogs kept out of water in moist air. Intake is zero; output as urine is indicated by points each of which is the mean of 10 tests. Redrawn from Adolph ('39b).

ferences of hydrostatic pressure, temperature, texture of surface, and many others, are all present. The physiological differences may be myriad; the differences are more than skin-deep, since urine is not formed in the skin. So the two types of load are described with fewest implications as belonging (b) to a frog sitting on a dry wire screen in air saturated with moisture instead of (a) to a frog sitting in a glass beaker with water $2 \mathrm{~cm}$. deep.

That a frog depends for constancy of water content upon being in a wet environment is almost obvious. How much time does a frog ordinarily spend out of water? Does it locate water more quickly when already desiccated? Is the movement toward water random or oriented?

I have tested many frogs in water deficit to see how in the laboratory they find their way to water. In the conditions tested, they 
found water only by random movements; once they touched it, the depleted frogs stayed in it. Frogs that did not stumble into it died of water-lack within a few centimeters of a life-saving pool of water. I do not doubt that in outdoor life, frogs have cues that guide them to water; those cues differ from the many that were tested indoors. Though answers to none of the questions are recorded for the frog, certain observations have been made upon another amphibian, Triton (Czeloth, '30). From a distance of $70 \mathrm{~cm}$. the salamander moves directly toward a body of water. It stays in contact with wet soil when possible, and frequents air of high humidity without any liquid water being present. Neither ocular nor nasal senses are required to detect either liquid or humidity. All these actions are ones that result in preservation of the animal's water content.

(3) Water might be expected to move outward through the skin when an excess of water has been injected into the body. It does not; there is not even a decrease in the rate of movement inward in the standard state (a). Of all the methods of influencing water exchanges of frog that have been tested, outward passage of water occurs only under the influence of solutes: (c) when a frog is put into "hypertonic" solutions (Adolph, '31b), and (d) when a frog recovers (by being put into water) after a sojourn in a "hypotonic" solution of sodium chloride (new data). In both these cases some exchanges of solutes are occurring.

In a few words it may be said that dying frogs augment their water intakes as much as living ones of the same water content; frogs out of water lose excesses less rapidly than those immersed in water; and water does not move outward through the skin, except as it either evaporates or else enters a modified medium that may allow exchanges of solutes. Undoubtedly many other physiological states would likewise show water exchanges that differ from those found in the arbitrarily chosen standard type of water load.

\section{\$40. Summary}

The equilibration diagram for water in the frog (fig. 66) is characterized by a curve of losses that is proportional to water content between -10 and $+30 \%$ of $B_{0}$, and a curve of gains that has three limbs, being nearly horizontal at great deficits and in all excesses, and steep at loads between 0 and $-20 \%$ of $B_{0}$. Net gains 
are more rapid than net losses. No data are available in steaảy states of water excess or deficit.

Random variations preserve water content with a $\mathrm{C} \Delta$ of $\pm 2.22 \%$ of $\mathrm{B}_{0}$ in 24-hour periods. This indicates the accuracy with which intake equals output, for $40 \%$ of $B_{0}$ of water is turned over in each of these periods.

Desiccated dead frogs exchange water initially at rates equal to living ones. Frogs kept out of water recover from excesses more slowly than those in water. Water is not lost (excreted) through the skin, when evaporation is prevented, in response to water excesses.

With an entirely different path of water intake (the skin) from that present in mammals, a qualitatively similar diagram is present except that intake through the skin persists when water contents are already excessive. Quantitatively, recoveries occur at rates roughly similar to those in man, and much slower than in dog. Recoveries from deficits exceed in rate the recoveries from excesses, even as they did in man and dog. Quantitative comparisons with other animals will be shown later (chapter IX). Living in an aqueous medium does not mean that fewer or slower compensations are provided for the recovery of usual water contents, nor that the organism is careless of what the content is. 


\section{Chapter VII}

\section{WATER RELATIONS OF OTHER SPECIES}

$\$ 41$. The present account of regulations of water content might be limited to man, or to mammals, or to vertebrates. Such limitation would leave out myriads of other classes of animals; I judge that more insight is to be gained by extending considerations to many kinds. Are regulatory processes present wherever they are sought? Do animals forestall the need for compensations of disturbed water content, by frequenting appropriate environments? Do some species depend on adjustments of output to do the same thing that other species accomplish by adjustments of intake? Are special structures concerned in compensatory exchanges wherever such adjustments are found?

Two kinds of interest (at least) might attach to data concerning water regulations in varied parts of the animal kingdom. General physiology might have its dream come true of knowing exactly how general each of the processes and correlations concerned in water exchanges actually is. Comparative physiology might fairly rate various phyla and species according to their means of disposing of water loads and the kinetics of their exchanges. The perfect dream might require data upon a thousand species. I believe that a fair outline of the features of all water regulations is obtainable from the fifteen or twenty species for which appropriate though partial data exist.

My plan is to present briefly the pertinent information concerning water exchanges in each of those several species. Special methods are required for the study of each, and particular features are to be noted. Thereafter (chapter IX), quantitative similarities and diversities will be ascertained. Comparisons are not limited to animals possessing any one common structure except "protoplasm"; wherever exchanges of water occur they either do or do not serve regulations, and in diverse degrees and patterns.

From knowledge of water relations in dog, man, and frog, the following physiological arrangements might, as a first extrapolation, be expected in other animals : (a) a water content varying less than $\pm 3 \%$ of $\mathrm{B}_{0}$ at intervals of 24 hours, (b) a turnover, (c) a compensatory recovery by augmented rates of loss when excesses 
of water are present, (d) a similar recovery by faster gain when deficits of water are present. The following pages show that these possibilities are realized in a variety of animals, with the exception that in some no turnover has been demonstrated. Also, a greater variety of rates of exchange is uncovered than would thus far be predicted.

\section{§42. RABBIT}

Water excesses introduced by stomach (fig. 72) give rise to diuresis in the rabbit (Lepus). If the data only of series $\mathrm{C}$ existed, it might be concluded that recovery from excess water is both slow and incomplete. In that series the diverse individuals and tests

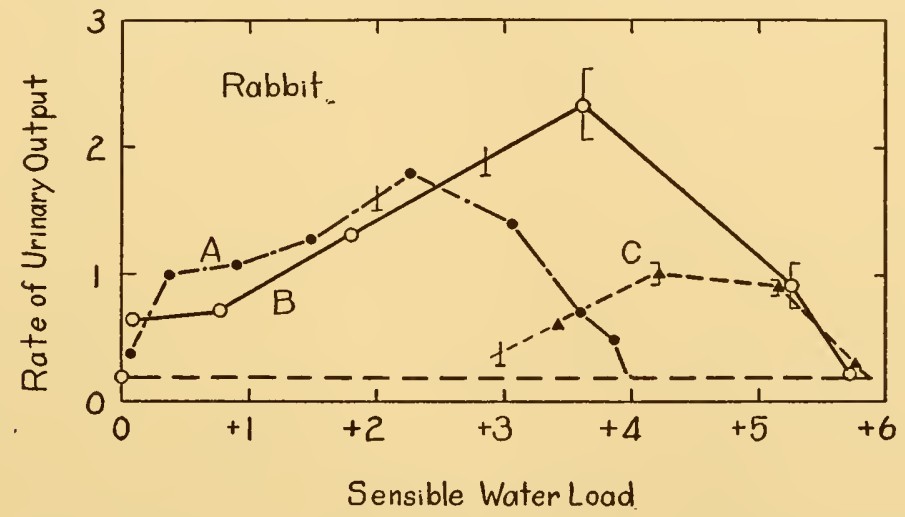

FIG. 72. Rates of urinary output ( $\%$ of $B_{0} /$ hour) in relation to sensible water load (ingesta minus urine) $\left(\%\right.$ of $\mathrm{B}_{0}$ ) in rabbit. $\mathrm{A}$, mean in intervals of 0.5 hour, data of Heller and Smirk ('32a, p. 18); B, mean in hourly intervals, 7 tests and individuals of Abe ('31b, p. 414) ; C, mean in hourly intervals, 32 tests on 11 individuals of Godlowski ('30, pp. 87 to 92 ). Evidently conditions for prompt and unimpeded diuresis do not prevail in series C. Standard errors are shown by brackets at four points; $\perp=$ half of load returned as urine.

are more consistent among themselves than in any other series, yet in 4 hours less than half of the excess water is returned as urine. Comparison with the rabbits of series $A$ and $B$ suggests that those of series $\mathrm{C}$ were in water deficit before the new water was administered.

In rabbit the acceleration of diuresis is about equal to that in dog and man, for maximal rates of urinary output prevail in the second hour after water is placed in the stomach.

When the excesses are very large $(\Delta W+30)$, rates of excretion up to $5.4 \%$ of $\mathrm{B}_{0}$ /hour are found (data of Misawa, '27), but 
they are little greater than at $\Delta W+5$ (data of Frey, '07). Extreme water excesses are established more readily in this species than in dog because vomiting does not occur.

In deficits, attained after privation of water but not of food, the rabbit recovers its body weight in a few minutes of drinking (fig. 73), taking, on the average, amounts slightly greater than the initial loads (fig. 74). That would not have been surmised from earlier studies of Pack ('23); for in his tests the rabbits, deprived for several days of food as well as of water, ingested in 0.5 hour even less water, relative to body weight and to water load, than did men $(\S 29)$.

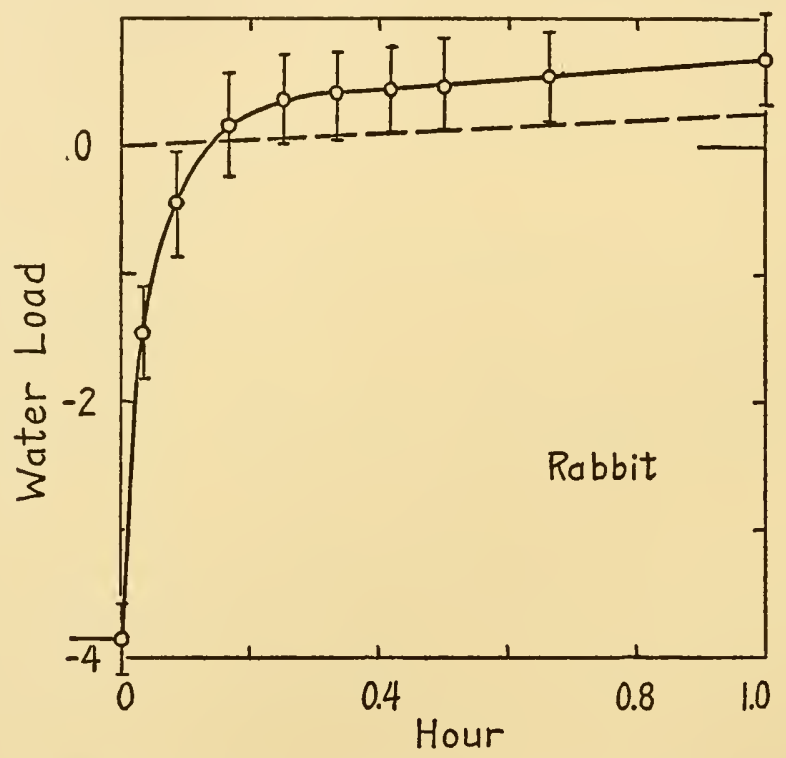

FIG. 73. Course of water load measured by water consumed ( $\%$ of $B_{0}$ ) during recovery from water deficit in rabbit. In each of 10 tests one of two individuals has been deprived of water but allowed food for 24 hours before zero time, when water is freely offered. Bars indicate standard errors of total amounts of water ingested. Dash line indicates mean water consumed in 12 control tests at same time of day in which water but not food is allowed ad libitum without previous privation. New data.

Turnover of water has been ascertained as $13 \%$ of $\mathrm{B}_{0}$ in 24 -hour periods (Gompel et al., '36; Gasnier and Mayer, '37). Most of the water is contained in the (green vegetable) food ordinarily eaten. On dry food (oats) plus water ad libitum, turnover is considerably less $\left(7.0 \%\right.$ of $\mathrm{B}_{0} / 24$ hours; new data).

Variability of water intake in 24-hour periods is in a selected instance ( $\mathrm{H} 1$ of Gompel et al., '36) smaller than has been reported 
for any other species ( $\pm 5.2 \mathrm{C} \Delta$ ). The consistency among rates of intake is partially related to the regime of green food, but differs greatly among ten individuals on the same diet (Gompel et al.). The concurrent urinary output is somewhat more variable ( $\pm 11.8 \mathrm{C} \Delta$ daily in $\mathrm{H} 1$ ), as though drinking is of greater concern. In water content the daily variability of $\mathrm{H1}$ (15 days) appears to be $\mathrm{C} \Delta \pm 1.53$; of individual $\mathrm{Z} 4$ ( 80 days), $\mathrm{C} \Delta \pm 1.49$. The variations are greater than in dog and man by about the same ratio as the turnover rates.

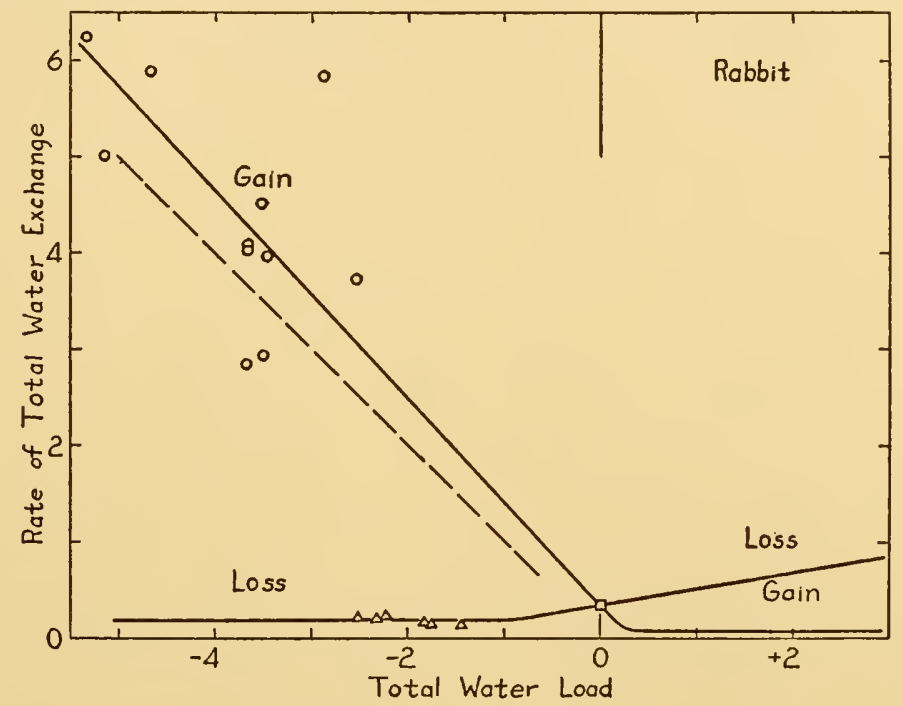

Fic. 74. Rate of total water exchange ( $\%$ of $B_{0} /$ hour) in relation to water load ( $\%$ of $B_{0}$ ). Equilibration diagram for rabbit. In deficits, gain by voluntary ingestion in first 1.0-hour, and loss by all paths, together with turnovers, are measured in two in. dividuals supplied with food; new data. Dash line=theory that intake equals deficit. In excesses, loss is from the animals of series $B$ in figure 72 , but is limited to the first 1.0 hour after loading and has $0.3 \%$ of $\mathrm{B}_{0}$ /hour as evaporative loss added to the urinary loss; gain is oxidative alone, from data of Heilner ('07).

The rabbit is characterized, therefore, by rapid rates of water intake, like the dog's, in recoveries from deficits; and by elimination of small excesses at rates proportional to loads, but of extremely large excesses at a uniform maximal rate. In 24-hour periods the variability of body weight is somewhat greater than that of the larger species dog and man. Rates of intake are generally less variable than in other species observed. Equilibration of water content involves all the processes present in the other two species of mammals, at rates that relative to body weight are as high as in the dog. 


\section{$\S 43 . \mathrm{R}_{\mathrm{AT}}$}

Excesses of water administered by stomach to rats (Epemys) are not fully returned in urine. Figure 75 , confirmed by new tests, shows that about one-third of the water ingested, though absorbed, disappears in evaporation before the original body weight is restored. If this one-third is included in the water excess, which thus represents the total load instead of the sensible load, then the relation (D) between rate and load is like that found in the dog (fig. 8 in $\$ 7$ ). Here is evidence that total water loss is more regularly concerned in recoveries from excesses of content than is urinary loss alone. The clearness of this evidence depends upon

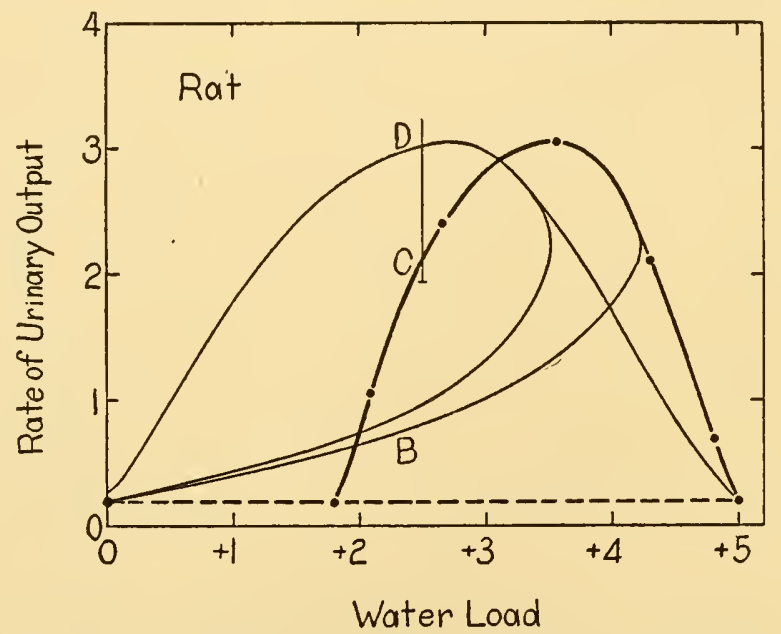

FIG. 75. Rate of urinary water output ( $\%$ of $\mathrm{B}_{0} /$ hour) in relation to mean water load $\left(\%\right.$ of $\mathrm{B}_{0}$ ) established by a single administration of water by stomach in rats. Each point represents a suecessive period of 0.33 hour. D, total load in 18 individuals of Heller and Smirk ('32a, p. 15), and as modified (BD) when absorption is measured in 47 sacrificed individuals. $C$, sensible load in the same 18 individuals, and as modified by absorption (BC). $\perp=$ half of load returned.

study of this species in which evaporative losses are faster (relative to body weight) than in dog or man. Since absorption (B) is complete before diuresis reaches its maximal rate, no account need be taken of the rates of absorption as measured, in describing recoveries over periods later than 1 hour after ingestion.

Recovery from a deficit of water is a gradual process in rats (fig. 76). No matter how the water is presented, only the first minute or two of drinking is rapid and continuous. Here is a species that characteristically drinks at rates intermediate between dog's and man's; still, no special relation of the rate of drinking to 
other diversities of the species is discernible. Drinking is, further, a specific process; for a concentrated solution (M/1) of sodium chloride is consistently refused. But a dilute solution (M/4) is ingested, though in lesser amounts than water; and when its concentration is compared with the "optimal" concentrations of urinary output and of solute intake found by Gamble et al. ('29, '34), it may be inferred that considerable net water is gained or made available after the latter solution has been metabolized.

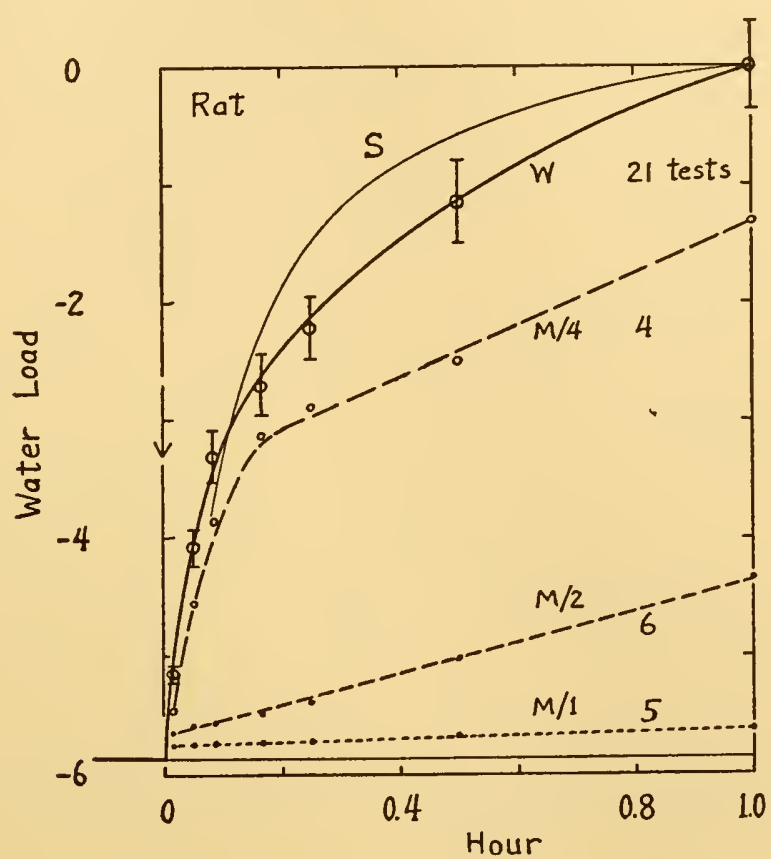

FIG. 76. Course of sensible water load ( $\%$ of $B_{0}$ ) during recovery from water deficits in rat. In each test a rat has been deprived of water but allowed food for 24 hours before zero time. Then water or $\mathrm{NaCl}$ solution $(\mathrm{M} / 4, \mathrm{M} / 2$, or $\mathrm{M} / \mathrm{l})$ is made accessible in a burette. Bars indicate standard errors of amounts of water ingested at diverse times. New data. $\mathrm{S}$ shows the mean ingestion of water in 6 tests of Skinner ('36), the initial and final points being made identical with those of curve W.

The shape of the tolerance curve in recovery from water deficit is independently confirmed by tests of Skinner ('36, '38) which involved a behavior more highly conditioned than drawing water from the open bottom of a burette ( $\mathrm{S}$, fig. 76), namely, pressing a lever to obtain each aliquot of water drunk. Apparently the machinery utilized did not delay the rat in getting its water. In another less detailed study with the "obstruction" method by Warden 
('31), rats attempted to reach an accustomed source of water supply much more frequently after one day's privation of water than ordinarily. With further privation fewer attempts were made; possibly this is related to extinction of the unrewarded response.

A radically different criterion of water deficit is required in the rat from that of initial body weight used in larger mammals and frog. Whereas in $d o g$ and man the food intake is suitably maintained even when water is denied, in rat the food intake is diminished. This diminution itself reduces the body weight of the rat, and represents an appreciable error only in mammals so small that they metabolize rapidly per unit of weight. Water balance, then, is at less than the initial weight. Of the various methods of evalu-

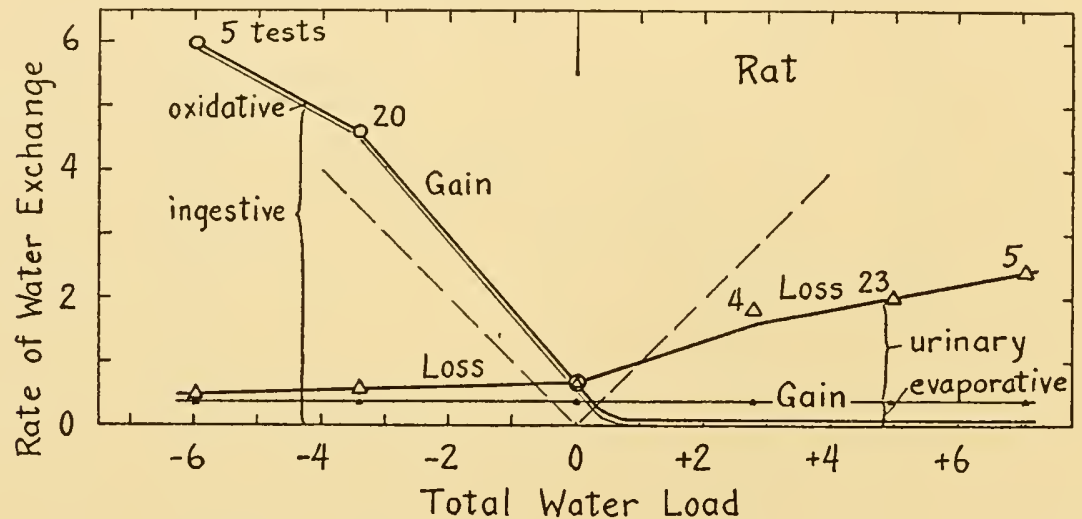

FIG. 77. Initial rate of water exchange ( $\%$ of $\mathrm{B}_{0} /$ hour) in relation to administered water load ( $\%$ of $B_{0}$ ) (Equilibration diagram for water). Rat in first 1.0-hour of recovery. Numerals indicate the number of tests. At the dash lines the hour's exchanges would equal the water loads. New data.

ating the new balance, the one chosen is to find the weights of control rats supplied with water ad libitum but limited to those amounts of food consumed by the individuals to which water was denied. The actual loads of water being thus established, exchanges are compared in the initial hour of recovery (fig. 77). Total gain is the water drunk plus water formed by oxidation; water lost is the total gain minus the measured gain of body weight in the one hour of recovery; part of the loss is represented by urine collected. Clearly the rat takes slightly more than enough water to restore water balance, just as the dog and rabbit do. The chief differences are that the rat requires nearly an hour to ingest this water instead of the few minutes used by those species, and that initial body weight is an insufficient measure of zero load of water. 
Negative increments of water content were tested in one further respect by Verzar ('36). After being deprived of water for 24 hours, in which time I suppose 5 to $7 \%$ of $\mathrm{B}_{0}$ might have been lost, and the actual deficit of water might be $3.5 \%$ of $\mathrm{B}_{0}$, the rats drink 7.3 to $7.8 \mathrm{ml}$. or perhaps $4 \%$ of $\mathrm{B}_{0}$. Of this amount 48 to 66 per cent is found to be absorbed from the alimentary tract in 0.33 hour, indicating that in deficits the rates of absorption are about equal to those in excesses (fig. 75).

When rats are depleted of water by exposure to a hot environment, subsequent water by stomach induces almost as large diuresis as usually, according to Heller and Smirk ('32) and Boyd and Garand ('40). From that it might appear as though water by stomach is not in the rat a "physiological equivalent" of water that has been previously lost by evaporation. But rats so depleted also drink very little (Adolph, '42). However, when rats are depleted by water privation as above, subsequent water by stomach induces only the small diuresis that voluntary drinking does (new data). Whenever the rats end at a weight different from that which was formerly $W_{0}$, the position of $W_{0}$ has changed relative to body weight. This illustrates the fact that choice of controls influences the relationships just as readily as does employment of types of water load.

The turnover of water in rats is higher than in larger mammals per unit of body weight ( 0.60 to $0.75 \%$ of $\mathrm{B}_{0} /$ hour $)$. Most of the loss is evaporative except during water diuresis. The high turnover does not change the form of the equilibration diagram as compared with that found in other species, however.

Variabilities of water content and of water ingestion (new data) indicate accuracies of maintenance and exchange about equal to those of other mammals ( see table 12). Variation of content is not in proportion to the high turnover and small size.

The behavior toward environmental humidity is such as to stabilize the rat's water content. Single individuals are put into a box 3 meters long, wet air flowing into one end of it, dry air into the other. Where does the rat spend most time? At intervals of about 2 hours the flow of air is reversed, so that during one day the animal has four opportunities to shift its location while still frequenting one humidity (A, fig. 78). From 0.4 to 0.8 hour is needed to reverse the gradient of humidity in the box; the tests are therefore summarized by taking the positions of the animal at 1.5 hours 
after each period begins. In 215 such readings each rat tested spent most time in moister air. Since the animal is nearly always found at one end of the box or the other, the data are most readily analyzed in terms of frequencies in two locations. Only rarely does an individual stay at intermediate portions of the long box; but occasionally (fig. 78) a high humidity even in the center of the box is preferred to the otherwise more attractive ends.

When rats are previously in water deficits of diverse extents, established through privation of water but not of food, they frequent moist air more often than when they are in water balance (fig. 79). In extreme deficits of body weight thus induced ( $\Delta W-25$
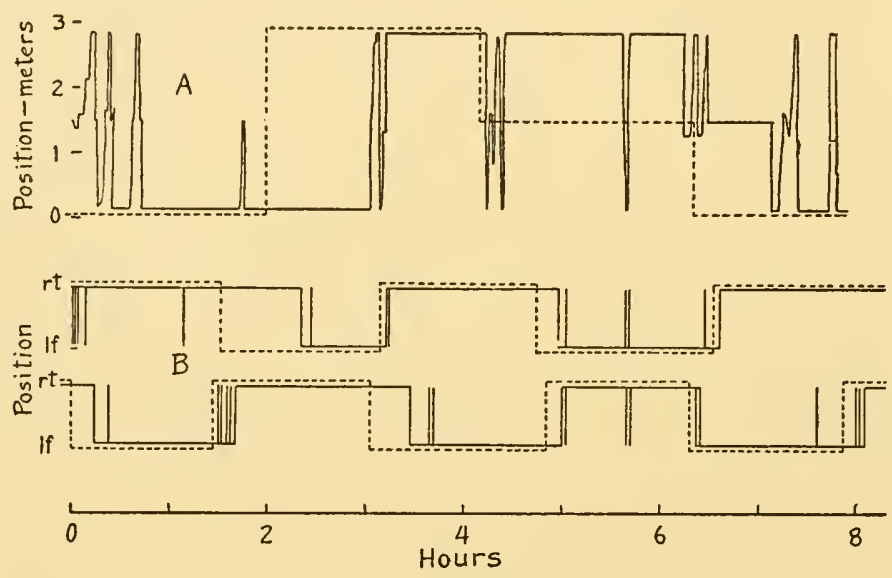

FIG. 78. Position of rat in boxes containing moist and dry air, in relation to time. Location of air of highest humidity is shown by dotted lines; location of rat is shown by continuous line. In $A$, rat $R(\Delta W-26)$ was in the long box. In $B$, rat $L(\Delta W-35)$ was in the two-chamber apparatus during two successive days, remaining in the right chamber overnight. New data.

to -32$)$ the preferences are almost invariable, though in certain few tests the same animals appear to be indifferent to humidity. The correlation of position of rat with water load is statistically significant. The correlation clearly belongs to the category of regulations, for in deficits of water the rat avoids atmospheres in which evaporation is faster.

Having found that rats spend most time in one end or other of the long box, with infrequent tours between them, I used a second apparatus consisting of two chambers connected by a tunnel. The chambers being suspended, positions of the rat could be automatically recorded. In them a stream of moist air displaces dry air within 0.2 hour and correspondingly the rat shifts its position more 
promptly after each reversal of air flow (B, fig. 78). The evidence here too is clear that the rat frequents high humidities, and especially when it is in water deficit.

How the difference of humidities is detected is not known, except that the rat explores before settling in one position. Nor is it known whether humidity is sensed as such or as an effective temperature, as a rate of drying of nasal mucosa, as pain, or as touch. I imagine that many environmental combinations attract and repel a rat; sometimes humidity or some correlative of it overrules other factors in the preferences. Since water deficit reinforces the discrimination, an "operant" behavior is at stake.

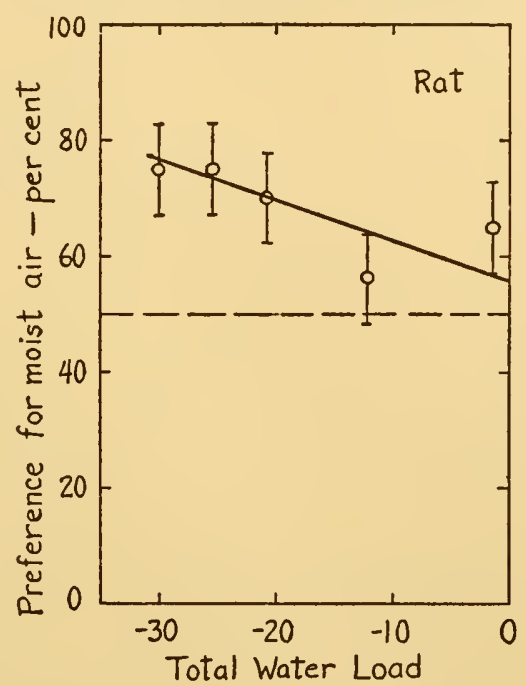

FIG. 79. Preference for moister air (\% of times tested) in relation to water deficit. Rat, 10 individuals. Two hundred tests are divided into 5 equal groups in order of water load. Standard errors are indicated, and show that the 3 groups on the left each differ significantly $(\mathrm{P}<.01)$ from 50 per cent preference. New data.

It is clear, then, that rats frequent air of high humidity and shun air of low humidity; a behavior that in itself tends to preserve water content. Deficit of water in the body augments the frequenting of moist air, thereby economizing water in those physiological states of water shortage where additional means of preservation are appropriate.

In brief, the rat's water exchanges are characterized by its partition of losses, as compared with larger mammals. In turnover about two-thirds of the loss is evaporative; even higher proportions are evaporative in the losses at diverse deficits. But just as 
in other species, urinary loss is the path that adjusts itself so as to compensate diverse positive loads. In the rat diuresis begins and becomes maximal slightly sooner than in dog, man, or rabbit. Ingestion, on the contrary, is slower than in most. When allowed alternative environments, rats choose moist atmospheres, and choose them more frequently in extreme deficits of water. All these kinds of regulations are restorative and preservative of water content.

\section{§ 44. Garter snake. Reptiles}

Perhaps the general processes of water equilibration have now been explored in mammals. What do reptiles do to preserve and

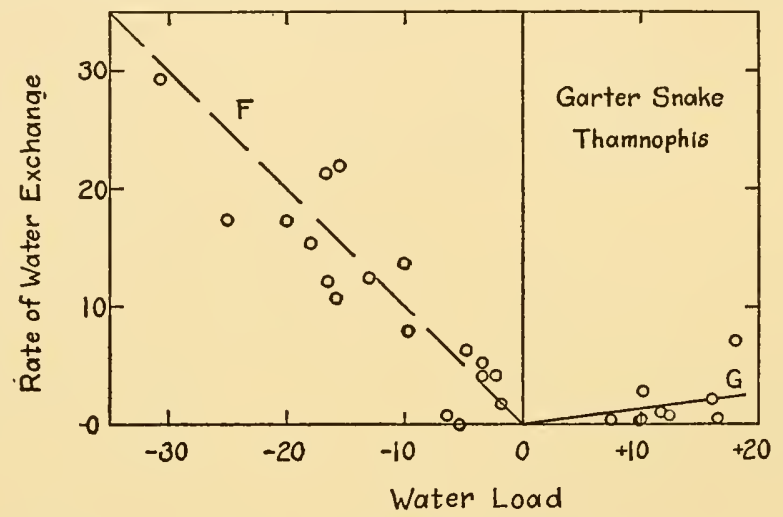

FIG. 80. Initial rate of net water exchange ( $\%$ of $B_{0} /$ hour) in relation to total water load ( $\%$ of $\mathrm{B}_{0}$ ). Equilibration diagram, garter snake, Thamnophis sirtalis. First 1.0 hour. Seven individuals of 6 to 103 grams body weight (without food) have been desiccated for 2 to 12 days at 23 to $28^{\circ} \mathrm{C}$, , or injected intraperitoneally with distilled water. $F$, line representing the data, and the theory that $R_{w}=-\Delta W$. $G$, line fitted by inspection, $R_{w}=+0.14 \Delta W$. New data.

reestablish water balances? The snake, Thamnophis sirtalis, was subjected to diverse water contents, deficits being established by confinement for several days without water, and excesses by sudden intraperitoneal injection of glass-distilled water.

Total water loss from a snake in a closed jar at $25^{\circ}$ to $30^{\circ} \mathrm{C}$. is of the order of $0.02 \%$ of $\mathrm{B}_{0}$ /hour, and does not increase appreciably at molting of the skin. In a wire cage it is greater by 5 fold, indicating the prominent role of evaporation. It is not known where the evaporation is most rapid (lungs, mouth, skin, mucosae).

When allowed water the desiccated snake puts the mouth into the liquid and moves the walls of the mouth rapidly. With considerable accuracy it drinks, usually within 0.1 hour, nearly the amount of 
water by which it is deficient (fig. 80 ). The behavior is like the dog's or rabbit's, and as precise; before the water has time to be absorbed from the alimentary tract the drink has been accurately completed.

More variation is found among individuals with respect to total water output in excesses than with respect to water intake in deficits. After injection of excess water, all individuals lose weight more rapidly, but large individuals show only small augmentations.

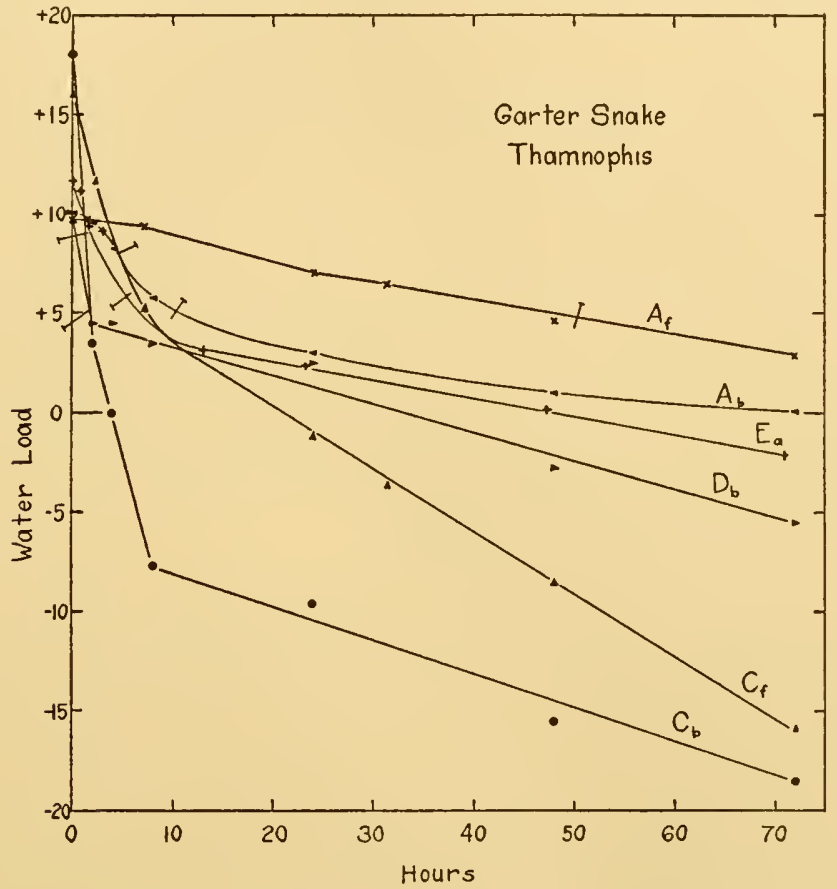

FIG. 81. Course of water load $\left(\%\right.$ of $B_{0}$ ) after the intraperitoneal injection of distilled water. Garter snake. Six tests on 4 individuals, weighing 101, 73, 8 and 11 grams. $\perp=$ half of administered load returned. New data.

As a result, the large individuals take several days to recover their original body weights (fig. 81). The expulsion of liquid from the cloaca in place of the usual semi-solid excreta, indicates one path of augmented output, perhaps the only one.

For certain other genera of reptiles (Chrysemys, Anolis, Phrynosoma, Alligator, Uta, Cnemidophorus) a few new data concern water deficits. In recovery all show sudden gains of water by alimentary drinking. I recall how surprising the sudden and intermittent gains seemed, after I had first measured the smooth and gradual gains of frogs and toads. 
The garter snake appears to maintain its water content (body weight) as accurately as the other small vertebrates that have been studied ( $\mathrm{C} \Delta \pm 1.1$ at 24 -hour intervals). The maintenance is partly by holding on to most of the water it has, day after day; but in addition water is drunk nearly every day. Contrasted with mammals, the turnover is small and is more largely influenced by the humidity and temperature of the environment. The small turnover is said to correspond to the excretion of most non-aqueous materials in solid form and to the extreme resistance to passage of water through the skin. Thamnophis and some other reptiles might be able to supply ordinary water requirements (turnover) by oxidative gain alone, even when only dry food is available; but this has not been demonstrated.

Ingestion into the alimentary tract accurately adjusts the snake's deficits; slow excretion of water through the urinary (cloacal) tract adjusts excesses. Recovery of water content after disturbance is as accurate as in mammals, and there are no recognized points in which adjustment is incomplete. Without knowing how a reptile is inconvenienced by a water load, the fact is plain that no load is permitted to remain.

\section{$\S 45$. Limax}

Though cogent "reasons" are often advanced why mammals, or vertebrates, might value a constant water content, it is often implied that to invertebrate animals such uniformity does not matter. Is it true that these organisms are more indifferent about water, or more slovenly in their operations toward water? Not having those kinds of organs by which vertebrates exchange water, do they still have special equipment for handling water? Are they slower about adjusting it?

Among molluses, terrestrial snails were studied in relation to water by Künkel ('16). In four species of the "naked" pulmonate slug Limax, desiccation is allowed to proceed for a few days, then water is presented (fig. 82). Net gains are recorded only in 24-hour periods, but other of Künkel's observations indicate that most of the intake, which is probably by drinking through the mouth, is immediate. In general the amounts taken nearly compensate the deficits existing. The accuracy of recovery is somewhat less than that in dog or in snake. 


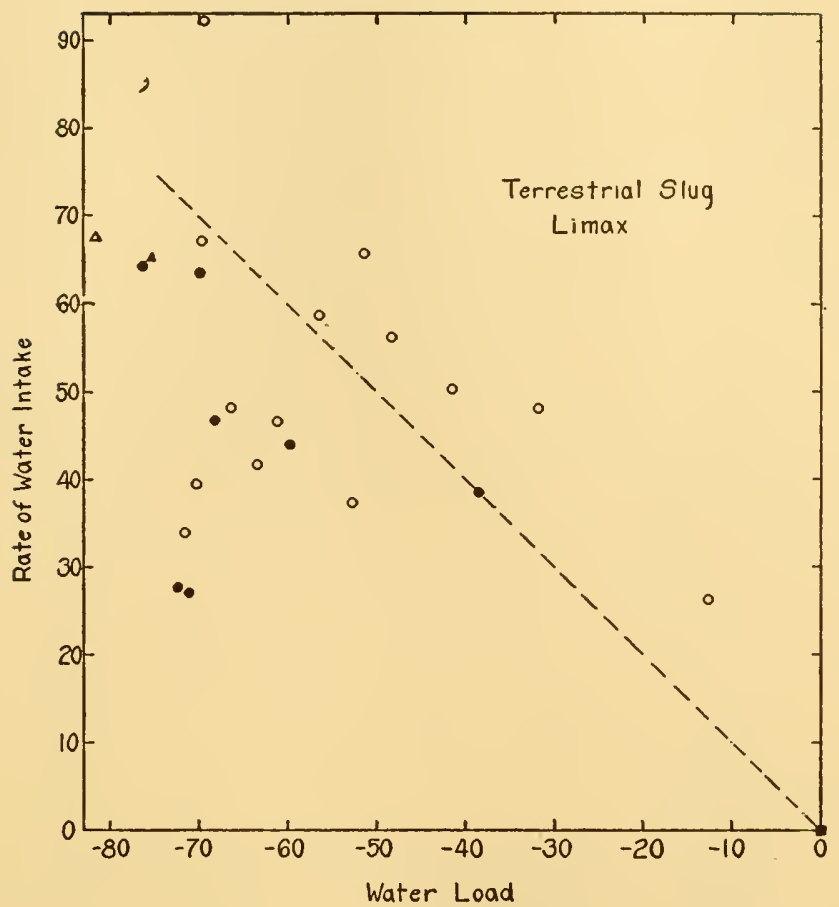

FIG. 82. Rate of initial gain of water ( $\%$ of $\mathrm{B}_{0} / 24$ hours) in relation to water load $\left(\%\right.$ of $\left.\mathrm{B}_{0}\right)$. Terrestrial snail, Limax, four species. For each point an individual weighing 1 to $15 \mathrm{gm}$. is desiccated for 3 to 16 days; then placed for 24 hours where water is available. The line represents the theory that intake in 24 hours equals deficit. Data of Künkel ('16, p. 88 ff.).

\section{§ 46. HeLIX}

Many species of the shelled pulmonate snail Helix were subjected to desiccation. These desiccations with privation of water often lasted for months, involving therefore deficits of other substances as well as of water. "If water and food are available to the snails after desiccation, they begin to eat only after they have drunk', (Künkel, '16, p. 86).

In three species (fig. 83) the returns approximately equal the deficits. "The quantities of water that can be drunk by the snails depend on the water content that the animals have before drinking", (p. 51).

Additional observations show that water is taken through the body (mantle) wall as well as through the mouth. Künkel believes that both paths are ordinarily used. By both paths water apparently ceases to enter when the control volume of the body has been approximately reattained. 
The only known difference between Limax and Helix with respect to water exchange is related to the fact that the latter can prevent loss of water by evaporation, deficits arising but slowly during hibernation or non-locomotion. During hibernation the body fluid gradually increases in concentration of dry residue, recovering its initial concentration suddenly upon emergence and shedding of the epiphragm (Holtz and Brand, '40).

Though Helix loses water very slowly during inactivity, in ordinary existence its weight fluctuates by $(\mathrm{C} \Delta) \pm 8 \%$ of $\mathrm{B}_{0}$ in daily periods (Howes and Wells, '34). Water loss is therefore not

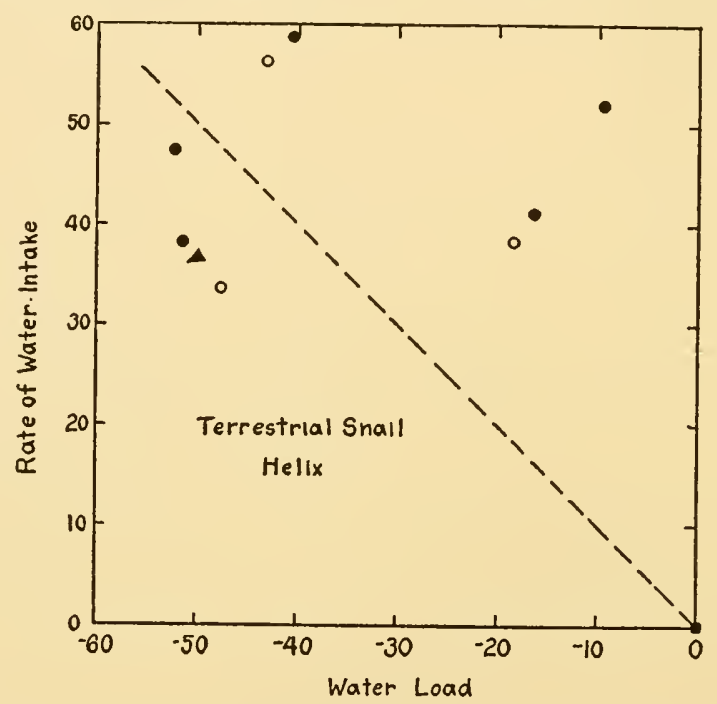

Fig. 83. Rate of initial gain of water ( $\%$ of $\mathrm{B}_{0} / 24$ hours) in relation to water load $\left(\%\right.$ of $\left.\mathrm{B}_{0}\right)$. Terrestrial shelled snail, Helix, three species, 9 individuals. From each body weight ( 0.4 to 19 grams) the weight of the shell has been subtracted. Privation of water lasted 6 to 12 months; then water was available for 24 hours. Data of Künkel ('16, p. 120 ff.).

slow during usual activity. Probably the fluctuations depend upon water intake, for when food but not water is supplied, little irregularity of weight is found. Helix, then, usually does not drink water so frequently as once a day, as though it is insensitive to deficits of $\Delta \mathrm{W}-8$. That picture of inconsistency in Helix is not borne out by the extensive study of analyzable water contents made by Brand ('31), for it would have been expected from the above facts that individuals analyzed at one time would vary by (C.V.) \pm $8 \%$. If Helix be an animal that regularly has a wide range of water contents; it is not representative of invertebrates generally. 


\section{$\S 47$. InsECTS}

The quantitative exchanges of water can scarcely be represented for a single species of insects, for appropriate data are lacking. The qualitative nature of the gains and losses of water, and the tolerance of water deficits, have been outlined for various insect orders by Buxton ('32b). In turnover most gains are said to occur by specific drinking of water, though some kinds do not habitually touch free water. Most losses are believed to be evaporative, except those in water excesses.

The impression might be obtained that the water contents are much less uniform in insect species having non-aqueous environments than in frogs or earthworms. So far it has no quantitative

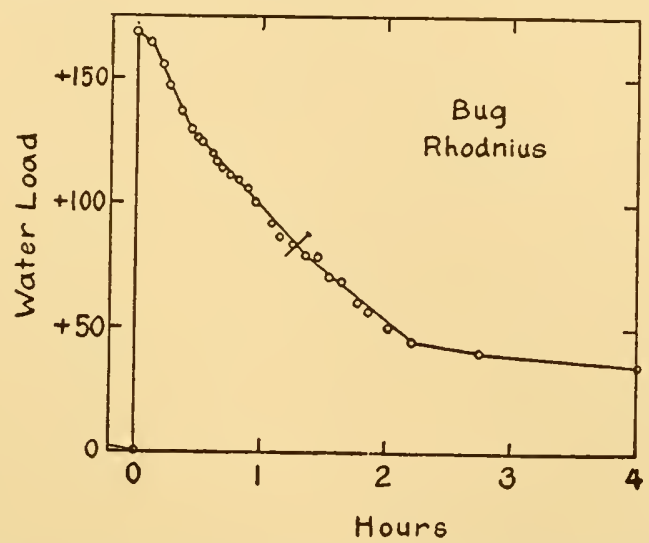

FIG. 84. Course of water load $\left(\%\right.$ of $\mathrm{B}_{0}$ ) after ingestion of rabbit blood. Rhodnius prolixus, 1 individual of $78 \mathrm{mg}$. at $18^{\circ} \mathrm{C}$. The insect took $225 \%$ of $\mathrm{B}_{0}$ of blood, or $169 \%$ of $\mathrm{B}_{0}$ of water. The load plotted is water ingested minus urine expelled (sensible load); an additional $10 \%$ was lost insensibly in 4 hours. Data of Wigglesworth ('31a, p. 414).

support. Variations among analyzed individuals are not greater than in some vertebrate species and many invertebrate ones (see table 19).

Much information indicates that insects do something about a water increment, whether it be a deficit or an excess. For the former, there is no controlled evidence, only surmise, that drinking by mouth is increased by water deficits (Buxton, '32b, p. 277 ; Mellanby, '38, p. 399). For the latter, data concerning excretory recovery of water content are shown in Rhodnius, a blood-sucking bug (fig. 84) after one sudden excess of water (along with other substances) has been taken in the form of rabbit blood. The water is presumably eliminated through Malpighian tubules. 
While many kinds of insects are bighly protected at their surfaces from rapid evaporative losses, others are known to be more vulnerable to such losses. This has given rise to the question, are insects able to choose enviromments that tend to minimize evaporation? There is no doubt that some species frequent atmospheres of a particular humidity; indeed, structures that detect differences of humidity have been described in spiders (Blumenthal, '35) and in mealworm beetles (Pielou, '40). The clearest case of preference for high humidity is in the isopod crustacean Porcellio (Gum, '37) ; it is indifferent to atmospheres of 65 or more per cent relative humidity, but in the region of half saturation distinguishes differences as little as 6 per cent. In general the animal keeps moving rapidly in dry air, becoming quiescent only when it arrives in a moist region; in addition it orients away from dry air if it suddenly comes into a boundary region. Some other species shun wet air (locust, Kennedy, '37; mealworm beetle, Pielou and Gunn, '40).

In the cockroach (Blatta) the preference for moist air is manifest only after previous desiccation (Gunn and Cosway, '38). The correlation between behavior and water deficit then constitutes regulation in the sense of all the studies here considered. For, in the humid air the rate of water loss is believed to be diminished; hence the modification of behavior tends to preserve the body's water content.

To some extent the ability of insects to move toward bodies of water has been investigated, and positive attractions have been demonstrated in several species (Turner, '24; Krijgsman, '30; Hertz, '35). This behavior which in some is dependent upon sight, is an integral part of success in gaining water to compensate for deficits.

Amid the diverse studies of insects in relation to water, data are insufficient to furnish an equilibration diagram, but enough to suggest that each of the constituent compensations exists. Neither turnover, variability of water content in an individual, nor modification ratio can be stated. Certainly behavior toward humidity of environment contributes to maintenance of content in some species; the complete story requires the correlation within one species, of this mode of adjustment with the others.

\section{$\S 48$. Phascolosoma}

Does an animal that lives in the ocean have an easier time maintaining its water content than others? The marine gephyrean 
worm Phascolosoma becomes desiccated by sojourn in sea water more concentrated than that in which it lives, or hydrated by sojourn in diluted sea water. Upon return to normal sea water the body weight in each case reapproaches the control weight (fig. 85). The rates of water exchange gradually diminish (fig. 86), but it may be noted that in positive loads the rates after the first hour are more nearly uniform with time.

At equal loads the exchanges of water, all of which occur through the body wall, are much more rapid as intake than as out-

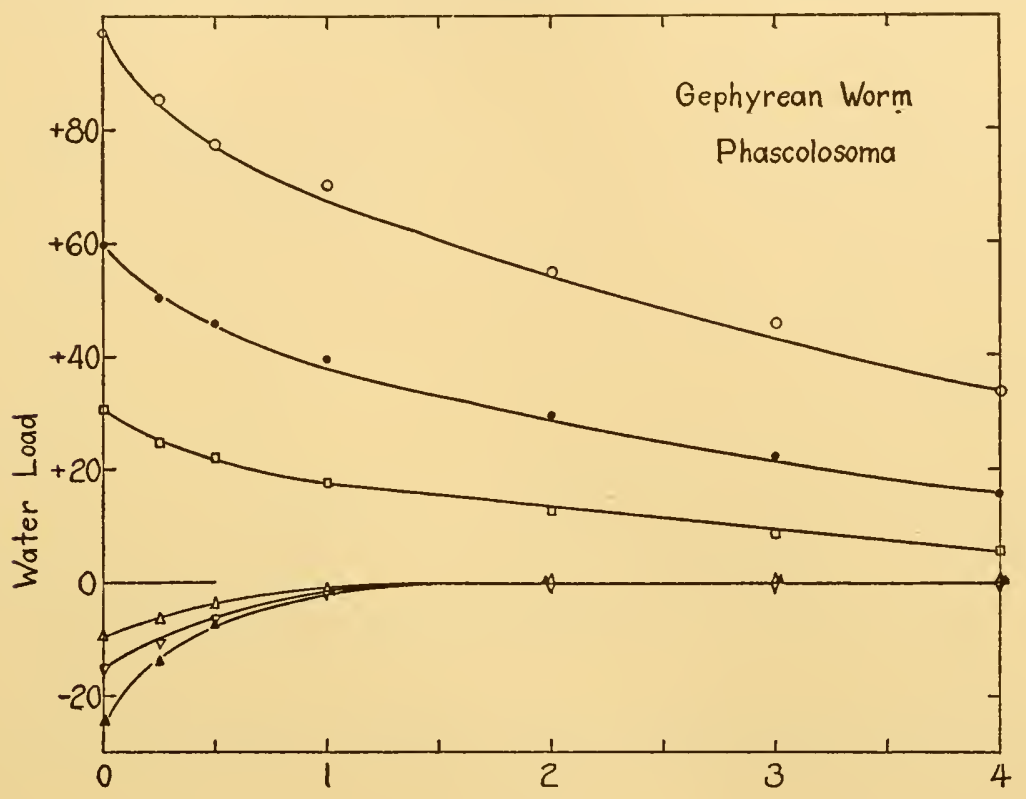

Hours

FIG. 85. Course of net water load $\left(\%\right.$ of $\left.B_{0}\right)$ after return of worms to normal sea water from sea waters of other concentrations. Phascolosoma at $23^{\circ} \mathrm{C}$. Each curve represents the average of 2 to 4 tests ( 5 individuals per test) the points being means of interpolated weights. Additional data of Adolph ('36b).

put. In relation to water load, each gain appears to be continuously proportional to the gradient of osmotic pressure between outside $\left(\mathrm{P}_{\mathrm{e}}\right)$ and inside $\left(\mathrm{P}_{\mathrm{i}}\right)$ the body (Adolph, '36b). The gain follows the equation: $\delta \mathrm{W} / \Delta t=h \sqrt{\pi \mathrm{lB}}\left(\mathrm{P}_{1}-\mathrm{P}_{\mathrm{e}}\right)$, in which the cylindrical worm has weight $B$ and length 1 . The exchanges are at rates roughly proportional to $\mathrm{B}^{2 / 3}$ or $\sqrt{\mathrm{lB}}$ (body surface area), in the range of computed areas from 4 to $20 \mathrm{~cm}$. $^{2}$ The coefficient $h$ has the dimensions of permeability. 
Evidence of various sorts indicates that dissolved constituents (ions) of body fluids and of sea water are in these tests not exchanging between body and environment. Hence desiccation in concentrated sea water is equivalent to desiccation by evaporation; the recovery in sea water following actual evaporation was tested and occurred at the same rates as above. Excesses that were obtained by previously injecting distilled water also showed the same recovery rates as those obtained by previous swelling in diluted sea water.

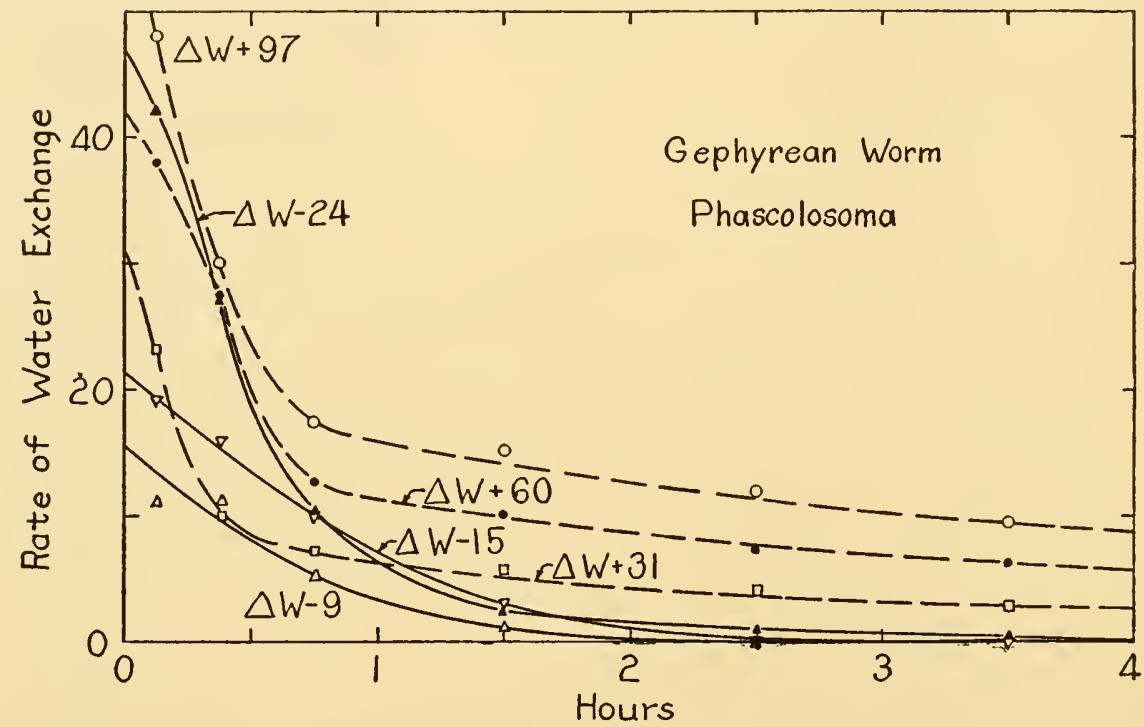

FIG. 86. Rate of net water exchange ( $\%$ of $B_{0} /$ hour) in relation to time after return of worms to normal sea water. Phascolosoma at $23^{\circ} \mathrm{C}$. Same data as in figure 85.

While recovery by intake is faster than recovery by output, yet it is commonly believed that the same forces of water transfer through the same integument are concerned. Actually the kinetics of the two exchanges also differ, as well as the values of $h$ in the above equation; for during water gain $h$ is constant, during loss $h$ is not constant.

Since I am describing the rates of water exchang'e, I preserve $\pm \Delta W$ as abscissae (fig. 87). For other purposes, in view of the supposed relationship of $\delta \mathrm{W} / \Delta \mathrm{t}$ to osmotic pressure in Phascolosoma, $1 /\left(\mathrm{P}_{\mathrm{i}}-\mathrm{P}_{\mathrm{e}}\right)$ might be used as abscissae of figure 108 instead of $\pm \Delta \mathrm{W}$; the internal osmotic pressure $\left(\mathrm{P}_{1}\right)$, being in turn, computed from $1 /(\mathrm{B}-b)$ in which $\mathrm{B}$ is the body volume and $b$ an 
empirically ascertained parameter termed "non-solvent volume." By this device the lines $\mathrm{A}, \mathrm{A}^{\prime}, \mathrm{B}$, and $\mathrm{B}^{\prime}$ actually become straight; strong suppositions may thereupon be formulated for identifying the rates of water exchanges with forces ordinarily termed osmotic pressures. Often, the "permeability coefficient" $h$ is regarded as having some inseparable and indispensable significance in such study of water exchanges, but I find additional comparisons among diverse conditions and species to be also useful.

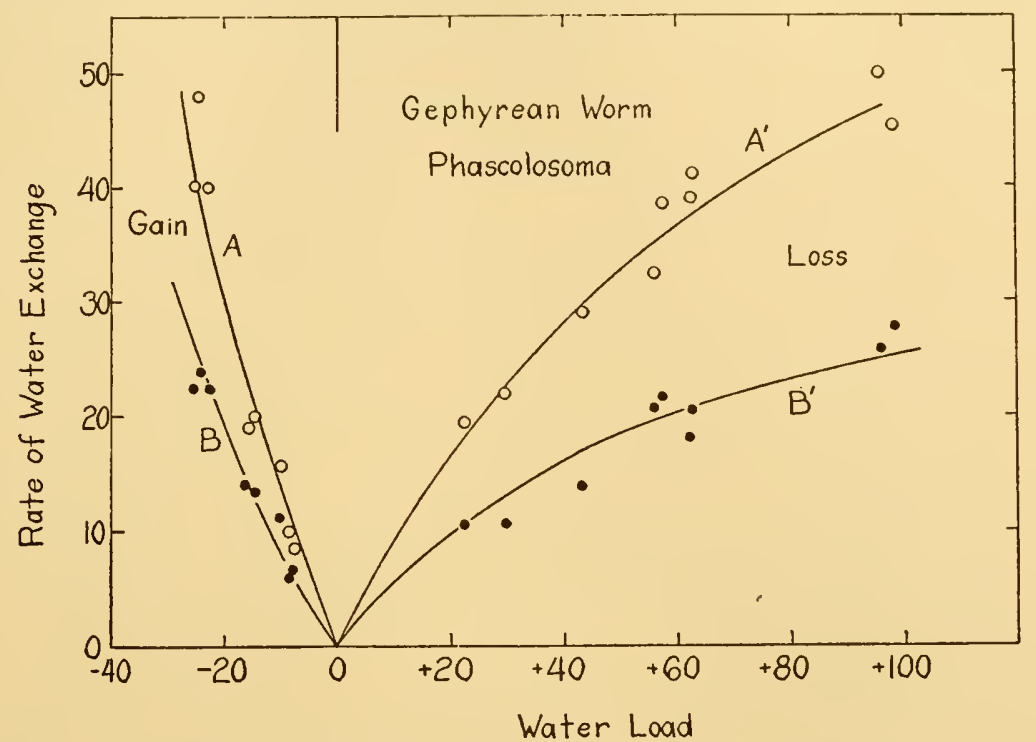

FIG. 87. Initial rate of net water exchange (\% of $B_{0} /$ hour) in relation to initial water load (Phascolosoma). For each point, 5 individuals ( 0.8 to 2.8 grams each) are transferred at zero time from varying concentrations of sea water to normal sea water. $\mathrm{AA}^{\prime}$, exchange in first 0.25 hour $\times 4 ; \mathrm{BB}^{\prime}$, exchange in first 1.0 hour. The curves are drawn so that the ordinates (within each of the two segments) are proportional to the initial difference of total concentrations between worm and new medium. Additional data of Adolph ('36b).

Phascolosoma adjusts its water content without, so far as ascertained, the use of processes that are known to require internal energy. The equilibration diagram is nevertheless similar to others that have been studied; it has only two lines instead of the four usually recorded, since it represents only net exchanges. No turnover has been recognized, unless it be by kinetic interchange of water molecules. Corresponding to the absence of turnover is the small variability of water content (see table 12). The conclusion may not be drawn that all marine animals adjust their water con- 
tents so as to equalize osmotic pressures between body and environment, for sooner or later animals like the teleosts and decapods are studied that pump water, instead of (or as well as) pumping solute, out of or into their bodies.

\section{\$49. LUMBRICUS}

Earthworms live in an environment of earth mixed with fresh water; in the laboratory they are kept in water without earth. The turnover of water is ascertained by the following steps: the worms are handled until constant (basal) weight is attained, then left untouched for 1 to 5 hours; reweighed, rehandled, and finally weighed.

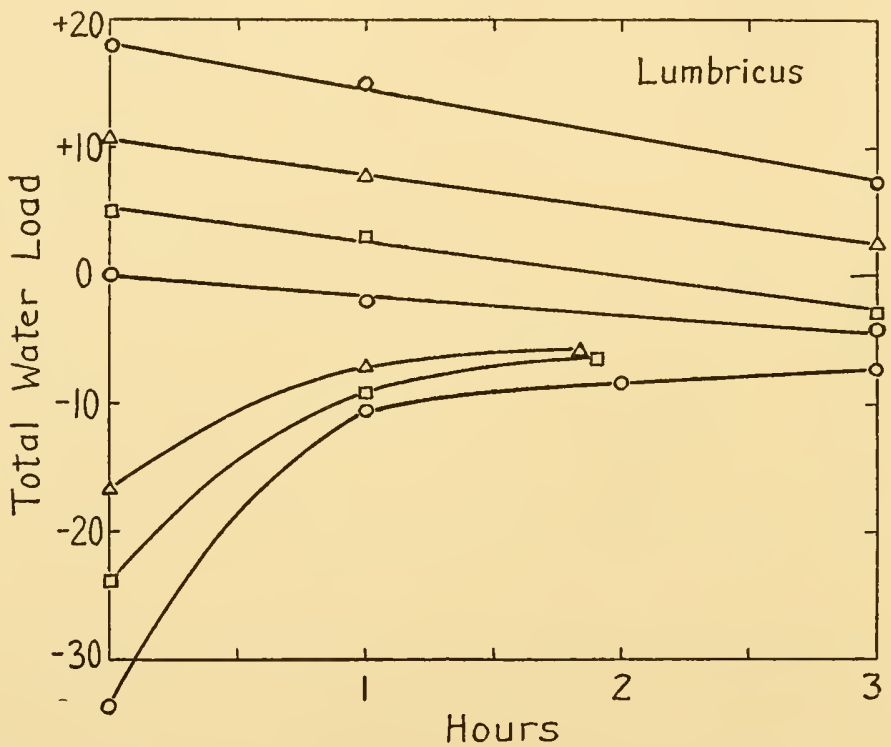

FIG. 88. Course of water load $\left(\%\right.$ of $\left.B_{0}\right)$ in earthworms immersed in fresh water at $18^{\circ} \mathrm{C}$. Each series consisted of 5 individuals just injected with water, or rendered deficient by evaporation. Before each weighing, nephridial anl alimentary reservoirs were emptied by rolling the worms repeatedly. New data of Wolf and Adolph, being similar (in water deficits) to previous data of Wolf ('40a, his fig. 7).

The loss of weight when rehandled represents a minimal value for the water excreted in the period of time elapsed, the evidence being that all of it is held in nephridial reservoirs and alimentary tract while the worm is undisturbed. Nine-tenths of the fluid lost is believed to issue through nephridia (Wolf, '40a); the total turnover is $2.7 \%$ of $\mathrm{B}_{0}$ /hour.

Variability of water content is about $\pm 2 \%$ of $\mathrm{B}_{0}$, as ascertained by weighing individuals at 24 -hour intervals with reservoirs empty. 
This variability is no greater than the frog's (see table 12), though the turnover is almost twice as great.

Negative increments of water are set up by evaporation from worms placed in air; positive increments by intraperitoneal injection of water. After each load has been established, the worms are allowed to recover in fresh water (fig. 88). The control worms of this series happened gradually to lose net weight; in positive loads the net loss is linear with time but slightly faster than in zero load. In negative loads the net gain is at first very rapid, and far from linear with time.

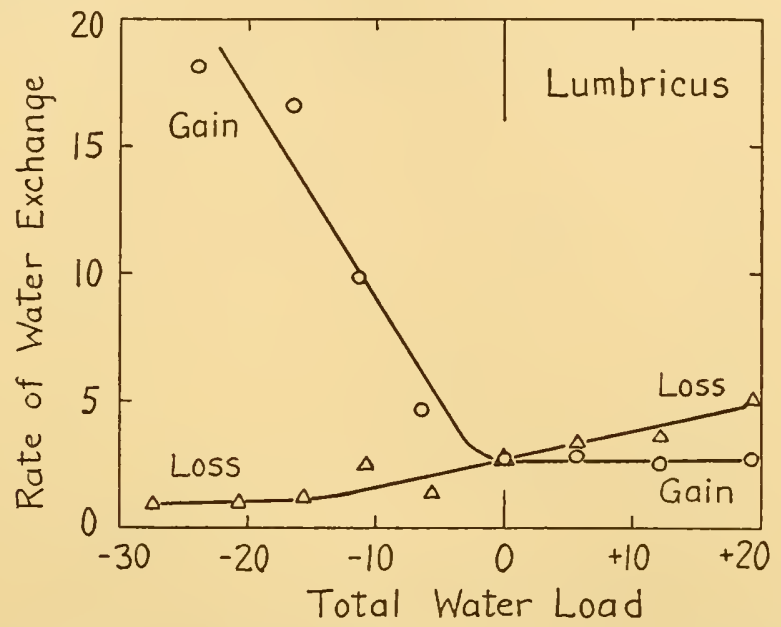

FIG. 89. Rates of total water exchange ( $\%$ of $\mathrm{B}_{0} /$ hour) in relation to mean total water load $\left(\%\right.$ of $\mathrm{B}_{0}$ ). Equilibration diagram of earthworm in fresh water at $18^{\circ} \mathrm{C}$. Each point represents 5 to 22 tests. Data were either in initial or later periods of one hour. First it was ascertained that the total turnover is $2.7 \%$ of $\mathrm{B}_{0} /$ hour (Wolf, '40a). Since in the turnovers later determined the output is $2.0 \%$ /hour greater than the intake (fig. 88), 1.0\%/hour is subtracted from all outputs and added to all intakes on the right of zero load; and the abscissae are moved $1.0 \%$ to the left. New data of Wolf and Adolph.

Since total gain and total loss at diverse loads were measured in the same individuals, a complete equilibration diagram is available (fig. 89). In the initial hour, compensation of each deficit is eight times as rapid as correction of equal excess. Most of the modification is in the rate of intake through the skin. Like the frog's, this gain does not diminish in excesses; in excesses the small modification of nephridial output alone allows slow recovery.

The earthworm, as was said, recovers from water deficits chiefly by faster intake through the skin. The rate of intake increases 
many fold at deficits that do not even double the believed osmotic pressure of the body fluids (Adolph, '27b, p. 56). Diminution of water output through the usual channels contributes but a small portion of the recovery from deficits; in excesses it alone is available. In this there is no feature by which the annelid worm differs from the frog or snake in its ability to compensate for unusual water contents.

\section{$\oint 50$. Bipalium}

Bipalium, a triclad turbellarian worm, is common in greenhouses where sources of water abound. Individuals weighing 0.3 to $2.4 \mathrm{gm}$. were desiccated by Kawaguti ('32) and then placed in tap

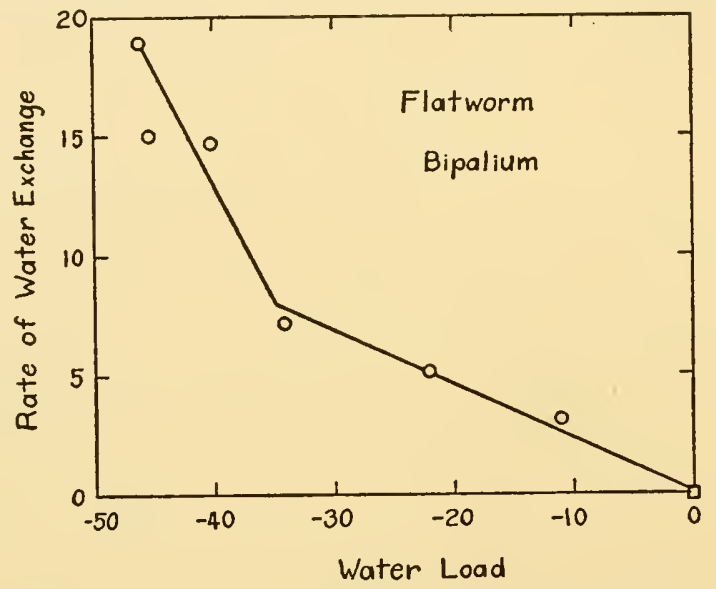

FIG. 90. Initial rate of water intake ( $\%$ of $B_{0} /$ hour) in relation to water load (\% of $\mathrm{B}_{0}$ ). Gain was in first 1.0 hour. Worm Bipalium (triclad turbellarian), 6 individuals weighing about $1 \mathrm{gm}$. each, previously desiceated. On wet cotton at $30^{\circ} \mathrm{C}$. Data of Kawaguti ('32).

water (fig. 90). No effect of body size is evident among the few rates of exchange that he reported. An unusual feature is that the velocity quotient $(1 / \Delta t)$ is greater at very low water contents (large deficits) than in moderate ones. The course of the intake of water is gradual, resembling the earthworm's, and apparently occurs through the entire body surface.

\section{\$51. Arbacia egg. Echinoderm eggs}

Unfertilized eggs of echinoderms furnish considerable information about water exchanges. In the measurements of Lucke and McCutcheon ('27) on sea-urchin Arbacia punctulata, eggs first 
swell in diluted sea water or shrink in concentrated sea water. On subsequent return to normal sea water (fig. 91), mean rates of intake and of output are computed from successive measurements of diameter (d). The rates of exchange in this series turn out to be proportional to the differences of osmotic pressure between egg: and medium. However that may be, for equal differences of it, intake is slightly slower than output, at all temperatures from $12^{\circ}$ to $24^{\circ} \mathrm{C}$.

In this spherical organism the exchanges are probably proportional to surface area $\left(\pi \mathrm{d}^{2}\right)$ and to the gradient of osmotic pressure, according to the equation: $\delta \mathrm{W} / \Delta \mathrm{t}=h \sqrt{\pi} \mathrm{d}^{2}\left(\mathrm{P}_{\mathrm{i}}-\mathrm{P}_{\mathrm{e}}\right)$. The coeffi-

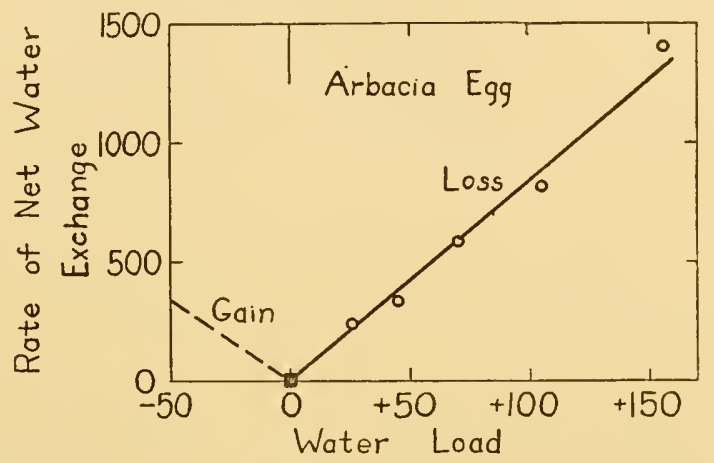

FIG. 91. Rate of net water exchange ( $\%$ of $\mathrm{B}_{0} /$ hour) in relation to total water load $\left(\%\right.$ of $\left.\mathrm{B}_{0}\right)$. Unfertilized egg of the sea urchin Arbacia punctulata in normal sea water at $19^{\circ} \mathrm{C}$. Rate is computed as though it prevailed during the first $1 / 60$ hour; in 6 eggs transferred at zero time from each of 5 dilutions of sea water ( 80 to 40 per cent). Rates of gain in recovery from negative loads were not reported but are believed to be about 20 per cent less than rates of loss at the same positive water loads. Data of Lucké and MeCutcheon ('27).

cient $h$ represents permeability (Lucke et al., '31). When $h$ is constant and turnover is nil, the net equilibration diagram looks much like that for Phascolosoma.

The eggs of half a dozen other species of echinoderms have been studied in an analogous manner (Leitch, '31, '36; Fukuda, '35) in limited respects. For the most part, water exchanges have been measured when eggs are transferred from sea water into various dilutions and concentrations. By analogy with Arbacia it might be supposed that exchanges in recovery depend only on the gradients of osmotic pressure that prevail. The data at hand do not encourage this assumption, since significant differences in $h$ appear within the same species at transfer into 40,50 and 60 per cent sea 
waters. In the early efforts to analyze the factors of water transfer and to rationalize the quantitative relationships, it was important to minimize the irregularities found. In a later analysis it is useful to realize that one permeability coefficient $h$ does not characterize all exchanges of water in any one species.

\section{\$52. Freshwater Zoothamnium}

Zoothamnium, a genus of ciliate protozoa, allows independent measurements of output through contractile vacuoles and of coincident body volume (Kitching, '38). Moreover, both initial states and steady states may be investigated. Two species are examined, one of which lives in fresh water, the other in sea water.

When individuals of the first species are put into one of two chosen media, either a negative or a positive water load is estab-

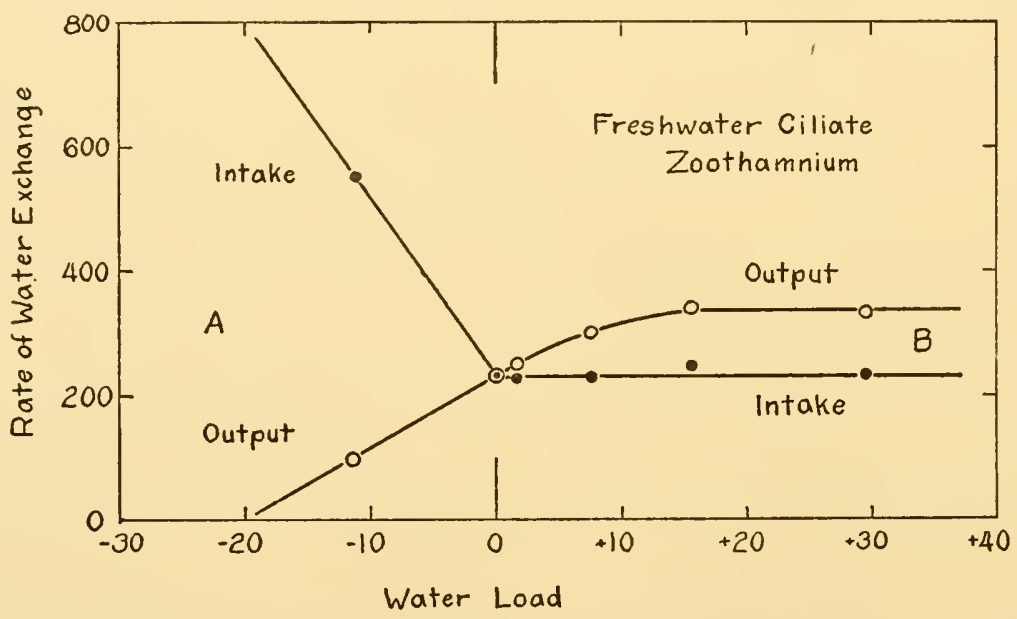

FIG. 92. Initial rate of water exchange ( $\%$ of $\mathrm{B}_{0} /$ hour $)$ in relation to water load $\left(\%\right.$ of $\left.\mathrm{B}_{0}\right)$. Zoothamnium, freshwater peritrich protozoan, at $15^{\circ} \mathrm{C}$. A, an individual returned from $0.05 \mathrm{M}$ sucrose solution to tap water. $\mathrm{B}$, an individual returned from $0.005 \mathrm{M}$ sodium eyanide, which had inhibited contractile vacuolar activity, to tap water. Each point represents recovery during a period of 0.07 to 0.17 hour. Data of Kitching ('38).

lished. On transfer from those media to the control medium (fresh water), water is exchanged at the rates shown in fig. 92. The total water loss is the volume measured as leaving the body through vacuoles, while the gain is the change of volume plus the water loss. Measured in change of volume alone, the rate of net gain in deficits is much greater than the rate of net loss in excesses. It is uncertain whether this contrast in positive and in negative loads is gen- 
eral, since the means (agents) used to attain these loads could be considered as special and peculiar ones.

This and the earthworm are the only invertebrate species for which both turnover's and complete equilibration diagrams, including total as well as net exchanges and loads of both signs, are available at present, so far as I know. Relative to body volume, turnover in Zoothamnium is a hundred times as rapid as in earthworm.

\section{\$53. Marine Zoothamnium}

In a marine species of the same genus, water output is measured in diverse stationary states of water content (fig. 93). Loads are

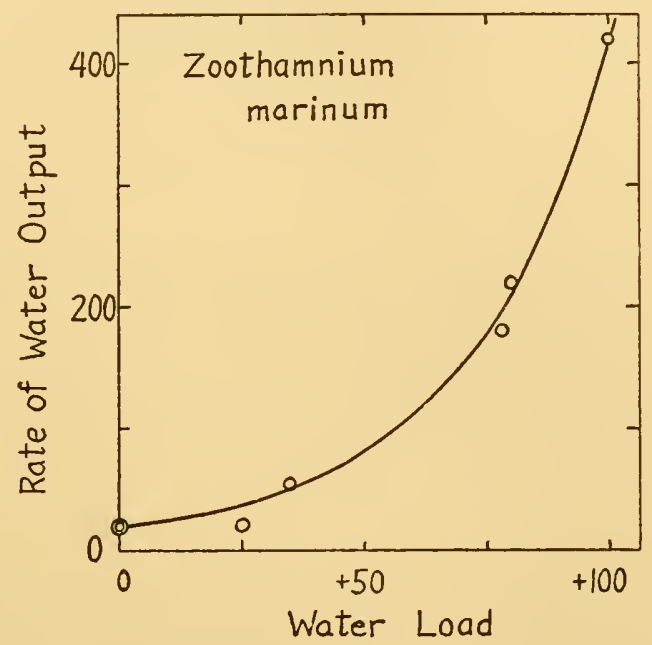

FIG. 93. Steady rate of water output through contractile vacuoles ( $\%$ of $B_{0} /$ hour) in relation to water load $\left(\%\right.$ of $\left.\mathrm{B}_{0}\right)$. Zoothamnium, peritrich ciliate, at $15^{\circ} \mathrm{C}$. Each point represents one test, of rate in diluted sea water, between two measurements of rate in normal sea water. Data of Kitching ('34).

secured by keeping individuals in any dilution of sea water for an hour or more; then body volumes and steady vacuolar outputs are ascertained. Since the body volume remains constant, rate of water intake equals rate of water output.

Very often it is assumed that water continually leaks into such an organism, whereupon the vacuoles pump it out, relieving the body of fluid. The augmentation of rate, amounting to 20 times the usual, and the shape of the curve in figure 93, suggest that the rate of output is proportional to the reciprocal of the concentration of the medium, a fact which allows the assumption of leakage. But the effective concentration of the body substance in each state of 
load being unknown, nothing can be done to test quantitatively the notion that the rate of exchange is proportional to the gradient of believed "osmotic" pressure; it is indeed improbable that the concentration inside the body is the same in all loads.

\section{\$54. Ameba}

The belief is wide-spread that if Ameba regulates its water content, then any animal can. In this freshwater rhizopod, deficits of water $\left(-25 \%\right.$ of $\left.\mathrm{B}_{0}\right)$ are produced by immersion of individuals in $0.2 \mathrm{M}$ to $0.4 \mathrm{M}$ lactose-saline solution (Mast and Fowler, '35, p. 160; '38, p. 303). Recovery of volume, after return to the weak saline solution in which the Ameba proteus has been reared, proceeds during the initial 0.5 hour with velocity quotients of about $0.8 /$ hour. In solutions of similar weak concentrations but of other ions, recovery is slower. Whether any of the recovery occurs by reduction in rate of output through the path of contractile vacuoles was not ascertained.

The maximal rate of turnover measured in contractile vacuoles of $A$. proteus is about $24 \%$ of $\mathrm{B}_{0}$ /hour, with a mean at $23^{\circ} \mathrm{C}$. of $10 \%$ of $B_{0}$ /hour (Adolph, '26, p. 377). The rate of output through vacuoles varies with the salt concentrations of the medium (Müller, '36, p. 360); it is not certain that the body volume is unchanged in these concentrations.

Excesses of water may be introduced into Ameba dubia by micro-injection. According to Chambers and Reznikoff ('26) the water, even in an amount of one-third the body volume, mixes with the body substance. Measurements of subsequent vacuolar output (Howland and Pollack, '27) indicate faster excretion, lasting 0.1 to 0.3 hour, at augmentations 1.16 to 2.9 times the control rate. The response was observed three times in one individual after as many successive injections. Following an injection of one-half the body volume, an augmentation ratio of 3.9 was recorded.

Apparently the rates of compensation of water content in Ameba are, like Zoothamnium, proportioned to its high rate of turnover. That freshwater protozoa are fully equipped for dealing with both deficits and excesses of water is worthy of explicit statement.

\section{\$5. Note on plants}

The water relations studied are equally prevalent in plants. Measurements of transpiration and of potometry now call for cor- 
relation with water load, ascertained either by weight or analyzed water content. It is known, for example, that maize (Maximow, '29, p. 210), wheat (Vassiliev, '36), and many other species (Knight, '17; Pisek and Berger, '38) readily suffer reductions of water content, and that evaporative losses are then diminished. Gain by intake through roots as soon as water again becomes available is presumably faster than in controls. Rates of turnover are often enormous, some plants exchanging their own weight of water every hour (Knight, '17). In aquatic plants with large fronds, half of recovery from desiccation occurs in a few seconds (Kaltwasser, '38).

It is, however, not proposed to analyze further any of the data concerning organisms that are ordinarily considered to belong to the plant kingdom.

\section{§56. Summary}

Of the many kinds of animals whose maintenances of water content might be studied, a small sample is available for future comparisons. Only two species of invertebrates (Lumbricus and Zoothamnium) having turnovers, were examined for rates of exchange during recovery from both excesses and deficits. Both are as well equipped to correct water contents as any mammal.

No turnover of water and no special structures for water exchanges are found in some animals that live in sea water. In water balance any possible turnover is then by the imperceptible replacement of molecule for molecule. In water increments, adjustments still result in an equilibration.

No species has been found without augmentations in rates of the appropriate net exchanges, whenever water loads occur. This fact strengthens the impression that the maintenance of water content in any species may be universally studied by correlating rates of net water exchanges with water loads.

Very often "water diaresis" is considered a wide-spread phenomenon. Extending the term to include all increases in rate of water output by any visible path when positive water increments prevail, actually I find data demonstrating it in 8 species of mammals, one species of birds (Burgess et al., '33; Korr, '39) four of reptiles (fig. 80; Burgess et al.; Boyd and Dingwall, '39; Friedlich et al., '40), one genus of amphibia, one species of fishes (see fig. 102); two species of insects (fig. 84; Lester and Lloyd, '28), one 
genus of crab (Nagel, '34), one kind of annelid worm, and 4 genera of protozoa (fig. 93; Müller, '36). Perhaps a few more species could with further search of published reports be added to that list. I, too, have a sort of faith that all species, and especially those with turnovers, put out water faster when the body has a water excess. That inference is useful, but is not demonstration.

Sometimes "water drinking" is considered to be limited to mammals, birds and reptiles. Though paths may be anatomically different, greatly augmented rates of water gain are found in all species of other classes and phyla in which intakes have been observed during recoveries from water deficits. There is nothing to compel physiologists to restrict the study of compensations by water intake to those animals that have a particular anatomical equipment. Rather, the evidence is that animals of all sorts accelerate their intakes in every deficit, and none trust solely to suppression of output. 


\section{Chapter VIII}

\section{EQUILIBRATIONS IN PARTS OF ORGANISMS}

$\$ 57$. The regulation of water content has now been examined in whole organisms. The individual is a convenient unit for study in that it usually maintains itself in a semi-isolated condition. The question arises: whenever any portion of an individual can be tested, are the same sorts of relations between its water exchanges and water contents found as in the whole body? Such portions (living units) are organs, limbs, tissue masses, cells. Do blood volumes, and fibroblast sizes, also tend to be constant, and are they corrected after disturbances of them?

Equally well, aggregates of individuals might serve as units. They may be the populations of nests, households, farms, herds, towns, forests, continents. Nor may it be assumed that the group is the mere sum of its individual units; for when associated, new conditions impinge upon the individuals. The water exchanges of a whole city could be measured after droughts and after rains, after interruptions of supply and after forced utilizations; such data will not be presented here.

In the study of parts, two sorts of physiological situation may be kept distinct. In one, the portion of an individual remains in place (in situ) and, although loaded directly, recovers while sharing its usual relations with other parts $(\S 58$ to $\S 62)$. In the other, to be considered thereafter, $(\$ 63$ to $\$ 66)$ the portion is isolated.

\section{\$58. VoLUMES IN SITU}

In the organism as a whole, the content of water was very often not identified chemically, but was measured as weight, or (in protozoa and in echinoderm eggs) as volume. In parts of individuals in situ the measurement of volume becomes paramount. This may be made more explicit by designating the increments by $\pm \Delta \mathrm{V}$ instead of by $\pm \Delta \mathrm{W}$.

Of the volumes that are measurable in organisms, many are volumes of distribution. A particular substance in known amount is added to or subtracted from the body by a particular route, and its increment in some tissue is subsequently ascertained. In a restricted sense a volume of distribution is defined (Dominguez, '34) 
as "the volume of body fluid dissolving the substance at the same concentration as the plasma." More generally, a component added may supplement some already present, or if subtracted may decrement that already present; this (positive or negative) increment spreads through a virtual volume of distribution. Going beyond the limits of body fluids as distributees, of substances (heat or pres-

TABLE 5

Mean volumes of distribution, as measured in plasma or whole blood of three species of mammals, after introduction of excess substance into the plasma or into the stomach. The concentrations of the substances marked * were measured in whole blood; the others were measured in plasma

\begin{tabular}{|c|c|c|c|c|}
\hline Distribuend & Dog & Man & Rabbit & Source of data \\
\hline Vital red ........................ & 4.8 & 4.3 & 3.1 & $\begin{array}{l}\text { Smith et al. ('21) } \\
\text { Sunderman et al. ('36) } \\
\text { Utheim ('20, p. 387) }\end{array}$ \\
\hline T 1824 & 4.9 & 4.3 & $\ldots \ldots \ldots .$. & $\begin{array}{l}\text { Gibson et al. ('38) } \\
\text { Gibson and Evans ('37b) }\end{array}$ \\
\hline Trypan red ............................. & $\ldots \ldots \ldots$ & 3.7 & 3.4 & Oka ('38), Takahashi ('35a) \\
\hline 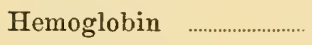 & 4.5 & $\ldots . . . . .$. & $\ldots \ldots . . .$. & Lee et al. ('22, p. 156) \\
\hline Carbon monoxide ${ }^{*}$ & 8.7 & 7.3 & 5.6 & $\begin{array}{l}\text { Smith et al. ('21, p. 351) } \\
\text { Douglas ('10) } \\
\text { Boycott et al. ('09) }\end{array}$ \\
\hline Sulfate & $\ldots \ldots . .$. & 21.0 & $\ldots \ldots . .$. & Lavietes et al. ('36) \\
\hline Sucrose & $\ldots \ldots .$. & 22.0 & $\ldots \ldots \ldots$ & Lavietes et al. ('36) \\
\hline Xylose & 26.0 & $\ldots \ldots \ldots$ & $\ldots \ldots . .$. & Dominguez et al. ('37) \\
\hline 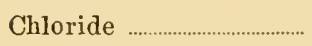 & 27.0 & $\ldots \ldots \ldots$ & 28.0 & Harrison et al. ('36) \\
\hline Thiocyanate ......... & 32.0 & 24.0 & 35.0 & $\begin{array}{l}\text { Crandall et al. }(' 34) \\
\text { Lavietes et al. }(' 36)\end{array}$ \\
\hline Bromide & 32.0 & 27.0 & $\ldots \ldots \ldots$ & Brodie et al. ('39) \\
\hline Iodide & 37.0 & $\ldots$ & $\ldots \ldots \ldots$ & Wallace and Brodie ('37) \\
\hline Glucose $^{*}$ & 50.0 & $\ldots \ldots \ldots$ & 30.0 & $\begin{array}{l}\text { Wierzuchowski ('36) } \\
\text { Sveinsson ('40) }\end{array}$ \\
\hline Creatinine* & 63.0 & $\ldots \ldots . . .$. & $\ldots \ldots \ldots$ & Dominguez et al. ('37) \\
\hline Urea* ${ }^{*} \ldots \ldots \ldots \ldots$ & 63.0 & $\ldots \ldots \ldots$ & $\ldots \ldots \ldots .$. & Painter ('40) \\
\hline Sulfanilamide* & 65.0 & $\ldots . . . . . .$. & $\ldots \ldots . .$. & Painter ('40) \\
\hline
\end{tabular}

sure might be distributed) as distribuends, and of plasmas as distributors, I define a volume of distribution $\left(V_{D}\right)$ as a virtual volume computed to contain an added or subtracted quantity $\left(\Delta \mathrm{G}_{0}\right)$ in that increment of concentration $\left(\Delta \mathrm{G}_{\mathrm{s}} / \mathrm{V}_{\mathrm{s}}\right)$ found by analysis of a chosen sample. Or, $V_{D}$ equals $\Delta \mathrm{G}_{0}$ introduced into or removed from the living unit (of volume $V_{0}$ or weight $B_{0}$ ), divided by $\Delta G_{\mathrm{s}}$ measured in the specified tissue per unit of its volume $V_{s}$. Usually $V_{D}$ is 
expressed as a fraction of the living unit; or $V_{D} / B_{0}=\left(\Delta G_{0} \times V_{s}\right) /$ $\left(\Delta \mathrm{G}_{\mathrm{s}} \times \mathrm{B}_{0}\right)$.

Volumes of distribution have a wide range when diverse components are administered (table 5). In general each distribuend has a smaller volume of distribution in man than in dog, and smaller in rabbit than in either.

Most volumes of distribution have been identified with anatomical "compartments" by hypothesis only. A few of the substances commonly administered as indicators of volume are supposed to attain after certain intervals of time virtually equal concentrations in all the water of the body (urea, sulfanilamide). Others are thought to become distributed in equal concentrations in the water of extracellular localities ( $\mathrm{SCN}^{\prime}, \mathrm{Cl}^{\prime}, \mathrm{Br}^{\prime}$, sucrose, $\mathrm{SO}_{4}{ }^{\prime \prime}$ ), with the exception that erythrocytes at least also contain them. Thus by inference it is said that a certain procedure measures something that approximates "plasma" volume; another, circulating whole "blood" volume; another "extracellular", volume. In general it is a necessary precaution to specify: $V_{D}$ of brilliant vital red volume at 0.1 hour after injection by vein; $V_{D}$ of sodium thiocyanate volume at 3 hours after injection by peritoneum; $V_{D}$ of urea at 2 hours after ingestion by stomach. Perhaps no two methods of measuring volume are likely to agree : either the actual volumes of distribution differ, or some factors in the two procedures are systematic ones. A notion that may be derived is that one procedure is not "superior" to another (though the volume it measures be identical with volume found by other methods), but that each procedure measures reproducibly a unique volume of distribution.

Evidences of regulation now to be mentioned concern either parts of organisms that are volumes of distribution or parts the volumes of which are measured by mechanical or optical methods.

\section{§59. Blood and Plasma}

The volumes of "blood" and of "plasma" are peculiarly suited to the establishment of quantitative excesses and deficits; perhaps this merely means that addition or subtraction is little trouble. Many hypotheses have been erected concerning the lability of those volumes. Choosing increments of volume that directly concern the blood, I here study hemorrhage and transfusion.

In rabbits, "blood" volumes are ascertained as volumes of distribution of trypan red, divided by hematocrit ratio (fig. 94). The 
accompanying "plasma" volumes (fig. 95) are the same volumes of distribution of trypan red, injected anew at each measurement. Transfusion of citrated rabbit blood suddenly enhances both volumes; hemorrhage suddenly diminishes them. Thereafter, changes are most rapid immediately following the experimental procedure; indeed comparisons of the volumes actually injected or withdrawn with the loads measured by the trypan red procedure, show that the fastest adjustments of "plasma" volume occur in the minute dur-

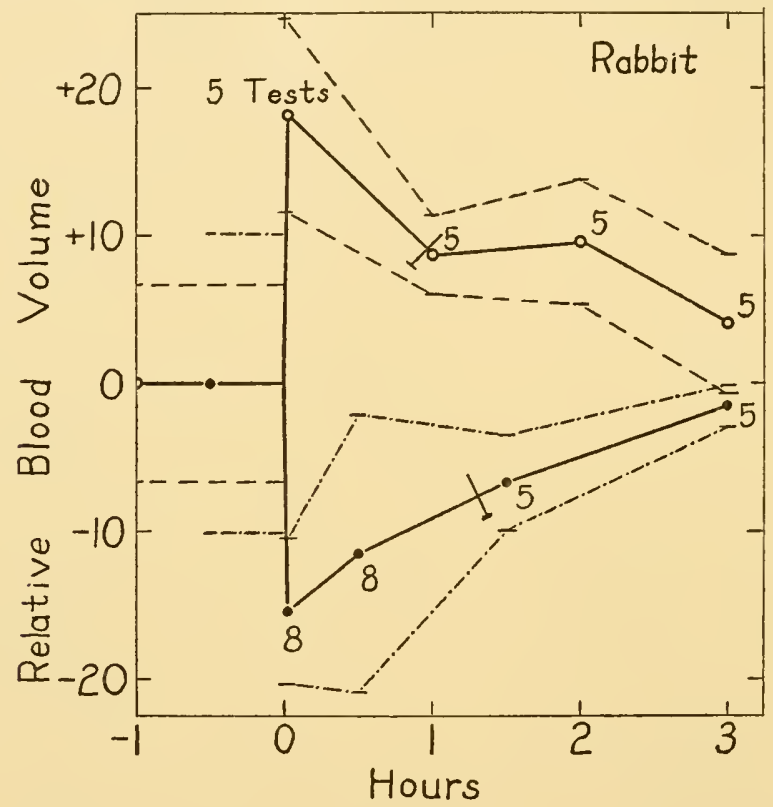

FIG. 94. Course of volume load $\left(\%\right.$ of $\left.\mathrm{V}_{0}\right)$ during recoveries from the transfusion of citrated rabbit blood (open circles) and from hemorrhage (solid circles). Rabbit, mean body weight $2000 \mathrm{gm}$., 5 to 8 individuals and tests. The volumes concerned are the volumes of distribution of trypan red (given by vein) divided by the hematocrit ratios. The enveloping broken lines indicate standard deviations; these are 2 to 3 times the standard errors. Data of Takahashi ('35a, '35b).

ing which the transfusion, and especially the hemorrhage, is administered. "Plasma" fully recovers its initial volume within 0.5 or 1.0 hour, while whole "blood" does not.

During an arbitrary interval of time between the first and second measurements of trypan red volume, the rates at which each volume changes are compared (fig. 96). Following an excess of volume produced by transfusion, the "blood" volume decreases; following a deficit of volume produced by bleeding, it increases. From the total "blood" volumes and the total "plasma" volumes, 


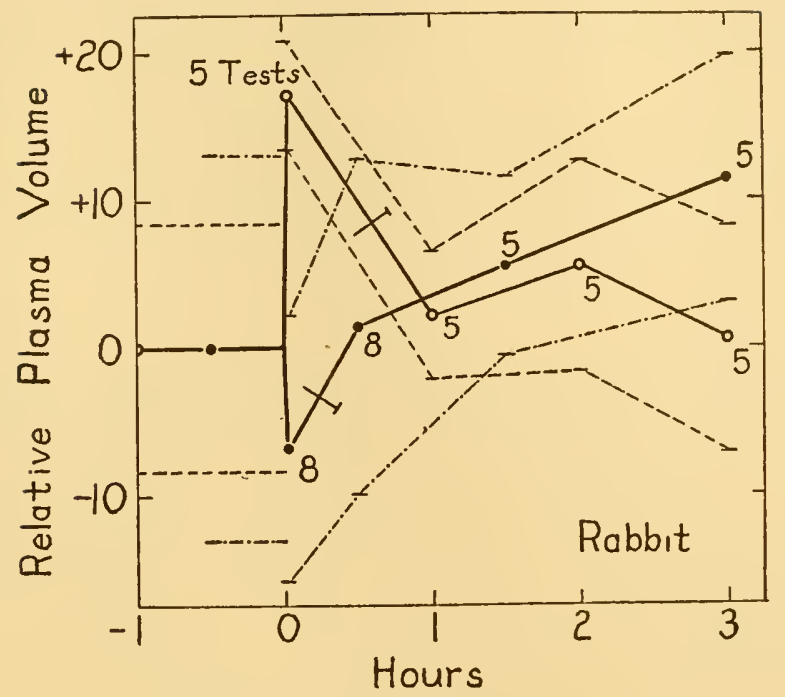

FIG 95. Course of increment in volume of distribution of trypan red given by vein ("plasma volume") ( $\%$ of $\mathrm{V}_{0}$ ), during recoveries from tranfusion of citrated whole blood (open circles) and from hemorrhage (solid circles). Rabbit, same tests of Takahashi (' $35 \mathrm{a}$, ' $35 \mathrm{~b}$ ) as are shown in figure 94 . The enveloping broken lines indicate standard deviations, which are here 2 to 3 times the standard errors.

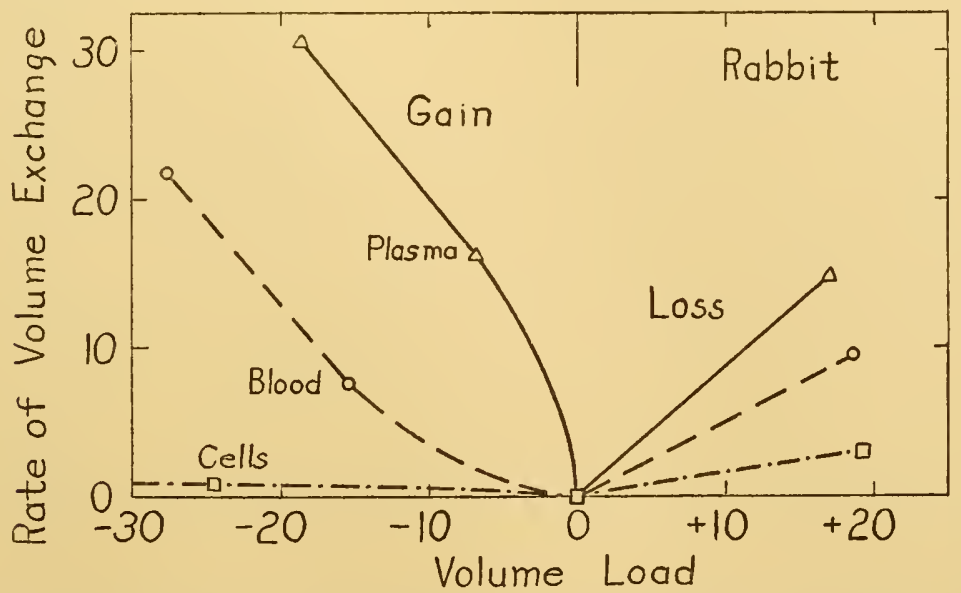

FIG. 96. Initial rate of net volume exchange (\% of $\mathrm{V}_{0} /$ hour) in relation to initial "blood" volume load (\% of $\mathrm{V}_{0}$ ). For each point 2 to 8 individuals were subjected to hemorrhage (negative loads) or transfusion (positive loads); and the changes of volume were measured over the period from 0.03 to 0.50 hour thereafter, and the period from 0.03 to 1.00 hour thereafter, respectively, as indicated in figures 94 and 95 . The volumes concerned are: the volume of distribution of trypan red (termed "plasma"); this volume divided by the hematocrit ratio (termed "blood"); and the difference of blood minus plasma (termed "cells"). Data of Takahashi ('35a, '35b). 
are obtained by difference the supposed total circulating "cells" volumes. Exchanges of "cells" are very much slower than of "plasma." Rates of recovery of volumes are derived from the differences between pairs of successive measurements; but each of them varies in absence of load by a coefficient of difference of about \pm 3 per cent (table 6 ), and hence some of the data are statistically

TABLE 6

Coefficients of difference $\left(C_{\Delta}\right)$ in volumes of distribution in three species of mammals. $C \Delta$ represents $1 / \sqrt{ } 2$ times the root mean square of percentage differences between successive tests on 3 to 6 individuals

\begin{tabular}{|c|c|c|c|c|}
\hline \multirow{4}{*}{$\begin{array}{l}\text { Distribuend ................... } \\
\text { Number of paired tests ... } \\
\text { Interval between tests, } \\
\text { hours }\end{array}$} & \multirow{3}{*}{$\frac{\text { Dog }}{\underset{4}{\text { Vital red }}}$} & \multirow{3}{*}{$\begin{array}{l}\text { Man } \\
\underset{5}{\text { Vital red }}\end{array}$} & \multicolumn{2}{|c|}{ Rabbit } \\
\hline & & & Trypan red & Trypan red \\
\hline & & & 12 & \\
\hline & 0.32 & 170 & 0.33 & 1.0 \\
\hline $\begin{array}{l}\text { C } \Delta \text { of " plasma" volume, } \\
\% \text { of mean }\end{array}$ & 1.11 & 3.47 (C.V.) & 3.08 & 2.91 \\
\hline $\begin{array}{l}\mathrm{C} \Delta \text { of "blood" volume, } \\
\% \text { of mean }\end{array}$ & 1.77 & & 2.84 & 5.26 \\
\hline Source of data & Smith ('20) & $\begin{array}{l}\text { Sunderman } \\
\text { and Austin } \\
(' 36)\end{array}$ & Takahas & $(' 35 a)$ \\
\hline
\end{tabular}

insignificant. Nevertheless all average exchanges of "blood" and of "plasma" are of such a sign that recovery is occurring. Further, reciprocals of increments in concentrations of various other constituents whose circulating amounts are assumed to be fixed, such as hemoglobin, solute refractive index, and colloid osmotic pressure (Onozaki, '35; Nagaoka, '36), indicate proportional changes of water content of the same signs.

The responses to loads are both qualitatively and quantitatively comparable to the net equilibration of water content or body volume in the dog (fig. 16) or other organism as a whole. Net equilibration thus presents a like pattern, regardless of the fact that entirely diverse processes and tissues are concerned in the exchanges of fluid.

No means has been found of ascertaining what over-all volume is both gained and lost during equilibration, or during turnover. Hence total rates are not recorded, as they are for the whole dog or rabbit, but only net rates of exchange. The rate of turnover might be enormous, for it is believed that plasma in every capillary both gains and losses liquid continuously (Schade, '27). Correspondingly the fluctuating variability of "plasma" volume, which 
might however represent mere error of estimate, is considerably larger than for the whole animal.

Attempting to identify some of the tissues concerned in restoring blood volume, Oka ('38a, '38b) induced various surgical and toxicological injuries in rabbits, then repeated the transfusions (table 7). These injuries are known to affect the liver or the kid-

\section{TABLE 7}

Rates of loss of "blood" volume as measured by trypan red injection and hematoorit ratio, in rabbits transfused with citrated rabbit blood. In each series, 5 individuals received by vein 2 per cent of the body weight $\left(B_{0}\right)$ of blood; mean body weight $1750 \mathrm{gm}$. The volume of distribution $\left(\nabla_{D}\right)$ of trypan red was measured at 2 minutes (=initial load) and $28 \mathrm{~min}$ utes thereafter. Standard error is here equal to 0.45 of the standard deviation $(\sigma)$. Data of Oka ('38a,'38b)

\begin{tabular}{|c|c|c|c|c|c|}
\hline State of rabbits & $\begin{array}{c}\text { Mean } \mathrm{V}_{\mathrm{D}} \\
\text { before } \\
\text { operation, } \\
\% \text { of } \mathrm{B}_{0}\end{array}$ & $\begin{array}{c}\text { Mean } \mathrm{V}_{\mathrm{D}} \\
46 \text { hours } \\
\text { later }= \\
\text { after oper- } \\
\text { ation, \% of } \\
\text { new } \mathrm{B}_{0}\end{array}$ & $\begin{array}{l}\text { Initial } \\
\text { "blood" } \\
\text { load, \% of } \\
\text { new } V_{\mathrm{D}}\end{array}$ & $\begin{array}{l}\text { Rate of } \\
\text { loss from } \\
\text { "'blood,", } \\
\% \text { of new } \\
\mathrm{V}_{\mathrm{D}} / \text { hour }\end{array}$ & $\sigma$ of rate \\
\hline 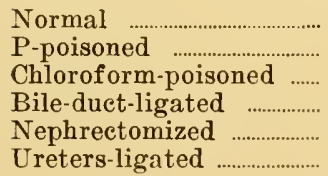 & $\begin{array}{l}6.90 \\
6.69 \\
6.46 \\
6.54 \\
6.67 \\
6.47\end{array}$ & $\begin{array}{l}6.76 \\
6.95 \\
7.52 \\
6.97 \\
6.67 \\
7.71\end{array}$ & $\begin{array}{l}+29.5 \\
+28.8 \\
+26.6 \\
+28.8 \\
+30.0 \\
+25.9\end{array}$ & $\begin{array}{r}13.3 \\
15.6 \\
34.0 \\
-5.6 \\
6.6 \\
12.4\end{array}$ & $\begin{array}{l} \pm 11.0 \\
\pm 17.1 \\
\pm 11.0 \\
\pm 14.7 \\
\pm 16.3 \\
\pm 16.1\end{array}$ \\
\hline
\end{tabular}

neys, whatever else they do. The augmentations in mean rates of volume exchange appear to be significantly greater in chloroform poisoning and significantly smaller in bile-duct ligation; hence effects are in opposite directions. It is hardly to be inferred, however, that the liver alone is specifically inhibited by those agents, nor is the liver demonstrated to have a particular role in restoring "blood" volumes that have been increased by transfusion.

The events that follow when whole blood is added to the volume already circulating differ little, I gather, from those after some other form of water is added to blood, though the initial rates with which recovery occurs may differ for diverse fluids. Several added fluids were compared by Oka, with the result that the differences of exchange rates among them in the initial half-hour of recovery are probably insignificant.

For the study of the equilibration of "plasma" volumes, which were measured (fig. 94) in response to whole blood excesses and deficits, the increments administered might equally be transfusions 
of plasma rather than of whole blood, and plasmapheresis (without replacement of anything but corpuscles) rather than hemorrhage. Suitable data of those sorts scarcely exist, unless in dogs subsequent changes of volumes be inferred from changes of concentration (Weech et al., '33; Calvin et al., '33; Freeman et al., '38).

In rabbit many independent data confirm the recoveries of "plasma" and of "blood" volume above described (e.g., Boycott and Douglas, '09; Nagaoka, '36; Oka, '38). In dog, man, and other species, partial data are not lacking. Those who are familiar with measurements of "blood" volume each have favorite researches that might be considered in this connection. What I would prefer is a thoroughly systematic study, with estimations of volume at initial intervals of only 0.02 hour, that does not yet exist.

In a few words, the maintenances of "blood" and "plasma", volumes depend upon net exchanges of those fluids in appropriate directions. After an excess has been established, net loss is rapid and in proportion to the amount of excess. After a deficit has been established, net gain appears. "Plasma" is exchanged faster than blood "cells." Investigatious designed to discover machinery by which these compensations operate have succeeded only in confirming these bare facts for a number of diverse types of volume load, and in several species.

\section{$\S 60 . \quad A R M$}

The human arm is a suitable unit for measuring volume changes, if by a pressure plethysmograph the volume of tissues minus blood is observed at desired intervals of time. The rates of escape of tissue fluid that had previously accumulated under partial venous stasis are thus ascertained (fig. 97). The rate of decrease of volume in the hydrated arm turns out to be a function of the amount of excess fluid present in it. No methods or data exist to tell whether the gain of volume to an arm previously depleted of some of the usual fluid would likewise be proportional to the deficit. So far as the evidence goes, the "fixed" tissue mass of the arm adjusts its volume after a disturbance of it, in the same manner as the blood of rabbit, or the whole man.

\section{§61. OTHER ORGANS}

Some organs of the animal body are geographically available for enclosure in oncometers and plethysmographs. Of the large 
numbers of studies that have been reported upon the volumes of such organs, none indicates the rates of recovery of volume after diverse amounts of fluid have accumulated in them or been lost from them. Most of the changes measured are probably in amounts of intravascular blood contained in these organs; suitable pressure plethysmographs remain to be applied to livers, kidneys, and muscles.

Changes of volume as inferred from measured changes of concentration in analyzed samples of these organs, would be significant

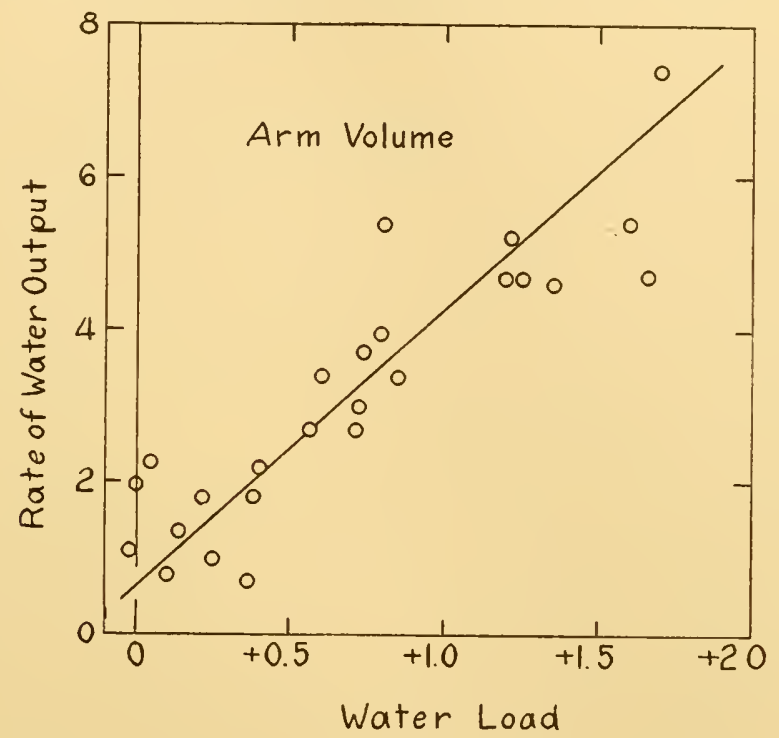

FIG. 97. Rate of volume output (\% of control arm volume/hour) in relation to initial volume load $\left(\%\right.$ of $\left.V_{0}\right)$ in the human arm. The load has been imposed by previous renous congestion, and the subsequent changes in "reduced" arm volume are ascertained in initial periods of 0.17 hour in a plethysmograph at $34^{\circ} \mathrm{C}$. Data of Landis and Gibbon ('33).

only if the changes are larger than the standard errors among samples. Those are usually very large. But occasionally the concentration may be measured optically upon one portion of tissue in situ. By that device control data are ascertained on the same sample, avoiding errors of absolute factors and of variabilities among samples. Further advantages are found in studying recoveries from those increments of volume that are predominantly in the tissue in question rather than in the remainder of the body; such was actually the case for blood, plasma, and arm. 


\section{\$62. Cells ANd NUCLEI}

It would be a pleasure to include in the present study changes in the volumes of cells within animals. Possibly recoveries of volume are ascertainable in circulating erythrocytes (in situ), but they have not been ascertained. Some sort of opacitometer plus enumerator is required to play upon the blood within the blood vessels, calibrated specifically for volume changes. Increments of volume found in drawn blood have probably occurred much more quickly than subsequent measurements can be made.

In some tissues, areas of single cells (Hirsch, '37) and nuclei (Wermel and Portugalow, '35) may be directly measured. The water exchanges might then be ascertained immediately after the microinjection of fluid into or the emptying of material from those units. Eventually other subdivisions of single cells and of unicellular organisms may be similarly observed. The rates of shrinking or swelling in nucleoli, granules, and other units recovering from various disturbances of volume (preferably those that affect the unit more than the remainder), may then be measured, and the equilibration of volume described.

At present this measurement has proven feasible upon the germinal vesicles in ova of invertebrates. In a species each of echinoderm and of annelid eggs, volume changes during swelling of the germinal vesicles (nuclei) are measured in parallel to those of the whole individual (Asterias, Beck and Shapiro, '36; Ceratocephale, Kamada, '36; Arbacia, Churney, '41). The interval of time between swelling of the egg and swelling of the nucleus is much shorter for the echinoderm eggs in sea water than for the worm eggs immersed in a modified Ringer's solution, although the time required to complete the swelling is about the same in all species. Possibly similar contrasts would occur during recoveries from water loads; at least the measurements have been demonstrated to be feasible.

\section{\$63. Summary of Parts in situ}

The studies of recoveries of volume in situ mostly concern volumes of distribution as measured by adding or subtracting components in the body. In one study, "plasma" and "blood" volumes of rabbits are reduced by hemorrhage and augmented by transfusion. Thereupon exchanges appear that effect restorations of volume; "plasma" volumes recover much more quickly than 
"blood" volumes. By transformation of coordinates, net equilibration diagrams are obtained for these volumes. Certain experimental "injuries" to the rabbits modify significantly the rates of plasma exchange, possibly indicating what sites or intermediaries take part in the exchanges. In studies by direct mechanical or optical measurements, volume changes are ascertained in arm, in certain distinct organs, in cells, and in their subdivisions. Only for blood and for arm are concrete portions of the equilibration diagram definitely established.

\section{\$64. Isolated Parts}

Another large field in the investigation of equilibrations of volumes lies in isolated parts of organisms. These parts may be living or dead, or both, according to diverse criteria that are arbitrarily chosen; for, survival after isolation is inevitably a matter of definition.

In general the increments of volume whose recovery is to be studied may be established (d) before isolation, or (e) after isolation. Thus (d), a dog or a frog may be loaded with water, and then its tissues may be isolated and allowed to equilibrate by exchanging fluid with a selected medium. Or (e) the tissues or cells may be removed from the body while in water balance, thereafter loaded and then transferred into the standard medium for equilibration.

Blood or plasma, being liquid, might be studied under isolation by putting samples of either behind uniform or identical partitions of some natural or artificial membrane. While the samples are exchanging water with large volumes of a selected liquid, the rates of transfer would be measured. Other body fluids may be studied in like fashion. In any isolated units, the number of arbitrarily chosen factors will be much larger than within the intact body where these factors are already fixed by the organism. One or another of those factors will seem to someone to be "unphysiological.",

\section{\$65. IsOLATED MUSCLES}

Muscles isolated from frogs are studied by both method (d) and method (e). Positive loads are produced by injecting water intraperitoneally and allowing either 0.5 or 1.7 hours for the water to distribute itself; then the muscles are isolated (method $d$ ) and 
placed for recovery in a standard Ringer's solution. Duplicate muscles are analyzed with respect to dry residue, to find what their mean water content might be. Negative loads are imposed by first isolating the muscles (method e), and allowing them to desiccate by evaporation. At zero time they also are placed in samples of the chosen Ringer's solution.

The tolerance curves (fig. 98) show adjustments toward a common final weight. But the final weight is higher than the initial one,

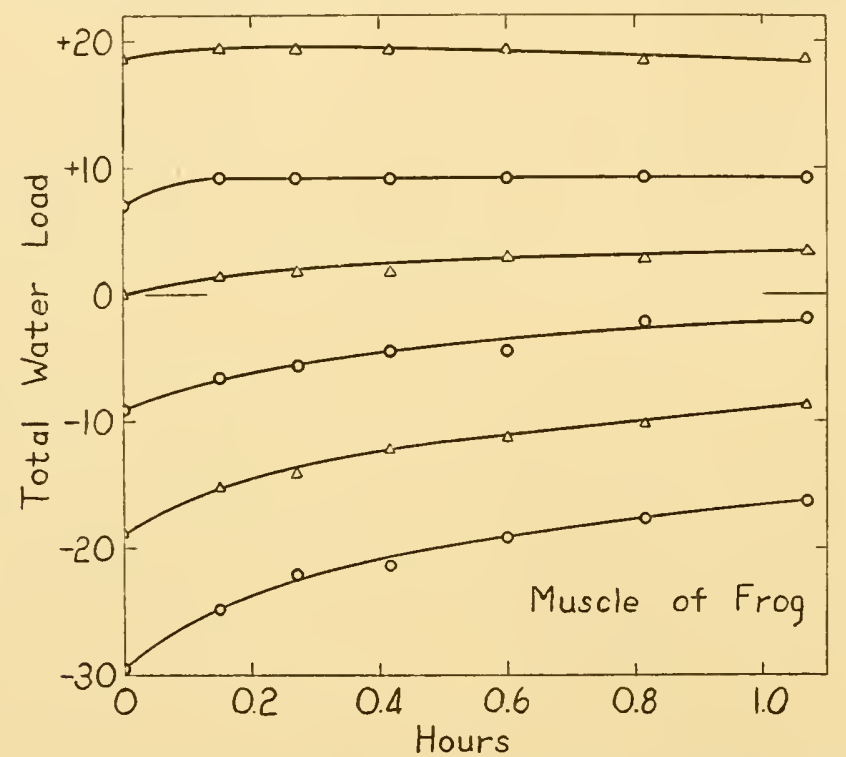

Fig. 98. Course of total water load ( $\%$ of $\mathrm{B}_{0}$ ). Isolated thigh of Rana pipiens, at about $21^{\circ} \mathrm{C}$. The initial weights $\left(B_{0}\right)$ are those found after dipping once into Ringer's solution; later weights are after sojourn in Ringer's solution since zero time. The lower three series ( 8 or 9 thighs each) have been previously desiccated in air, subsequent to isolation; the upper two series (15 to 19 muscles each) previously loaded by injecting distilled water into the peritoneal cavity of the living frog. In the latter case the initial load is computed from analyses of 30 duplicate muscles by the relation $\Delta \mathrm{E}=$ $100\left(\mathrm{D}_{0} / \mathrm{D}_{1}\right)-100$. Data of Wolf (' $\left.40 \mathrm{~b}\right)$.

being in the neighborhood of $+9 \%$ of $V_{0}$. Only muscles having excesses of water greater than $+9 \%$ of $V_{0}$ lose water to Ringer's solution after isolation. Whatever the load, changes occur in the initial 0.2 hour that are independent of the later trends.

In the form of an equilibration diagram (fig. 99), the same data indicate that the volume toward which recovery aims, as well as the rate of exchange, is modified with time. A shift in final volume of the muscle as compared with its original $V_{0}$ (upon which the numerical scale is based) follows the isolation as such (Parry, '36). 
No way is known of rendering the separated muscle like the intact muscle in respect to volume adjustments.

Ingrained in investigators is the desire to say: the changes of volume in blood and in muscle may all be predicted, for the force of osmotic pressure, which is concerned here, produces movements of water proportional to the gradient of concentration of solutes. Such a hypothesis appears valuable, for in part the data agree with it. But the data do not allow the conclusion that only osmotic pressure is concerned. Even after the initial 0.2 hour, many other pressures or forces may be changing along with the volume of the

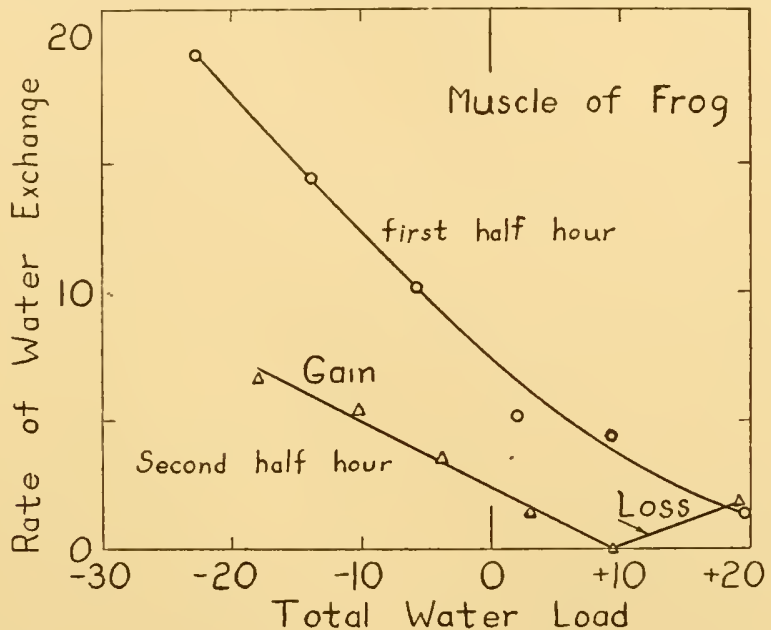

FIG. 99. Rate of water exchange ( $\%$ of $B_{0} /$ hour) in relation to total water load ( $\%$ of $\mathrm{B}_{0}$ ). Isolated thighs of Rana pipiens, immersed in Ringer's solution at $21^{\circ} \mathrm{C}$. Zero load represents the thigh's weight when in the frog that is in water balance. Data (of Wolf, '40b) taken from figure 98.

tissue, and any or all of these might give a like result. In general, one scientist may get a thrill at finding the data in agreement with some prediction, another at finding the unpredicted. Therefore the actual obtaining of any data, however futile and unnecessary to the one, will be a source of satisfaction to the other. Possibly a third might be distinguished, who is interested only in prediction, and who does not find it necessary to retard the production of hypotheses by ascertaining what the facts are.

I believe it is rather distinctive of isolated tissues that following isolation, progressive shifts occur in the water content at balance $\left(V_{0}\right)$. Recoveries from increments are then aiming toward a new content, even as balances in control samples do. This may not be universal, however, and the ideal tissue for study of equilibration 
of water content is one that gets over the stage of "feeling" its isolation, so that for a time equilibration is complete. At present no such tissue is recognizable.

\section{\$66. IsOLATED CELLS}

Isolated cells are susceptible of study by the same methods. With due attention to "survival," freshly isolated blood cells and previously cultured other cells have been subjected to volume changes. But cells rendered visible by direct fragmentation of massive tissues are equally available (Shear and Fogg, '34). Cells already isolated are not considered here; it is more convenient to classify unicellulars of all sorts, as organisms ( $\$ 51$ to $\$ 54)$ rather than as cells.

a. Erythrocytes. For the most part, the rates at which these cells change their volumes have been studied by opacitometers. No instances seem to have been reported in which the changes measured represent recoveries toward the volumes characteristic of cells in vivo. One deterrent to obtaining such recovery data is the belief that they can all be predicted from measurements of rates of swelling and shrinking in initiatory states; however, recovery requires specific measurements. Mammalian erythrocytes are believed to exchange other substances than water, whether they be kept in plasma or in other prepared media (Rous and Turner, '16; Davson and Danielli, '38). Diversities among species may be expected.

b. Leucocytes under certain conditions maintain regular (spherical) shapes, and so their changes of volume may be followed with an opacitometer. The data are limited to swelling in hypotonic solutions, followed by shrinking as recovery supervenes (fig. 100). While it is possible that the absolute rates reported are limited by other factors than the cells themselves, for the present the exchanges may have been at the rates recorded. Loss of water (recovery) occurs somewhat more quickly than the initiatory change of swelling.

c. Fibroblasts in plasma cultures have been measured by micrometry of single cells (Brues and Masters, '36), but not during recovery toward original volume. It appears that fibroblasts from four sources (chick embryo, rat embryo, mouse sarcoma 180, and rat tumor 256) exhibit nearly equal apparent permeabilities to water upon transfer from $0.15 \mathrm{M}$ to $0.05 \mathrm{M}$ sodium chloride solu- 
tions. What would happen if put into solutions containing more of the constituents of plasma or of interstitial fluid, is not known.

Some fibroblasts are observed to engulf fluid from their surroundings (Lewis, '31). This process of "pinocytosis" may be an integral part of the exchanges concerned in turnover of water.

d. Cells in fragmented tissues of mice were studied by Shear ('35) with respect to a particular kind of swelling by bulges. These bulges occur commonly in either usual sodium chloride or Locke's solutions. When later transferred to solutions containing gelatin

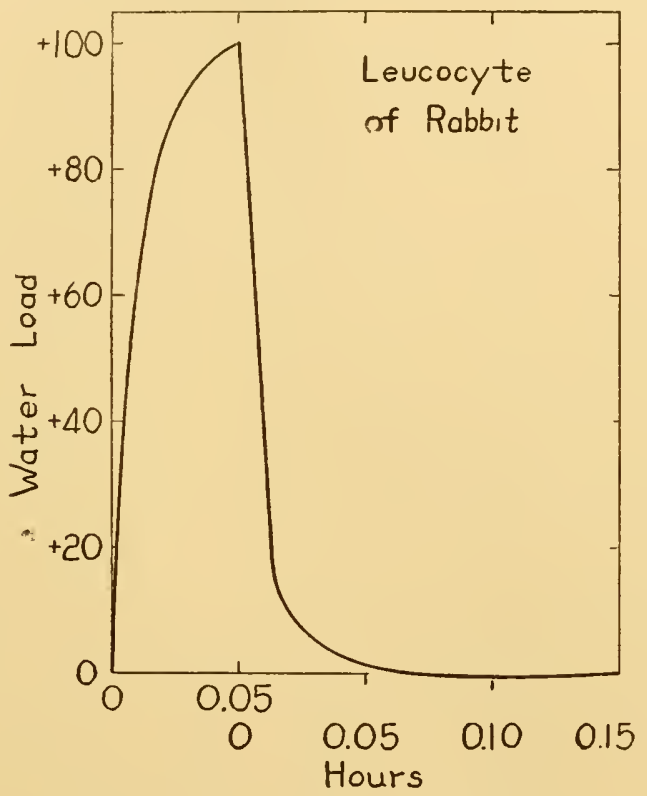

Fig. 100. Course of water load $\left(\%\right.$ of $\left.\mathrm{V}_{0}\right)$. A suspension of rabbit leucocytes in equilibrium with Ringer's solution is put at zero time into a similar solution but 0.4 as concentrated. After 0.05 hour, salt is added to bring the solution back to 1.0 concentration. Water load is estimated from the photoelectric potential ereated by a beam of light passing through the suspension, after calibration. From Shapiro and Parpart ('37). Redrawn by permission of the Journal Cell. Comp. Physiology.

or other proteins the bulges diminish and disappear, at rates that, unhappily, were not ascertained by micrometry. The vacuole-like bulges might be counted as part of the cellular materials or not, as one chooses.

It is apparent that recoveries of water content may be studied in single cells. Permeability itself is a regulated property; even when fixed with respect to water, it allows the usual pattern of equilibration by modifications of water exchange. Among cellu- 
lar units in general, either the whole cell may be concerned in giving up excessive water, or special structures like vacuoles may segregate that water, and either the whole cell or its surface may in deficits take up water. Both exchanges may represent special arrangements for regulatory water contents.

\section{§67. Summary}

How water is handled by living units, is ascertained in parts of organisms in which the volumes can be measured. Volume changes are treated as net changes of water content. Both volume and water content suffer increments of many measurable types, each representing one method of loading the living unit.

In all the tissue masses or cells that have been studied under conditions appropriate for recovery toward balance of volume (as defined previously, $\S 19$ ), initial exchanges are faster as the increments are larger. The directions of the exchange of fluid are such as to restore the masses to the volumes characteristic of control masses. I conclude that portions of individuals show much the same pattern of net compensations that whole organisms do.

Comparisons of exchanges by the diverse tissues and cells studied (table 8 ) are limited by the less accuracy of the data as com-

TABLE 8

Comparisons among net recoveries of water content or volume in tissues and cells

\begin{tabular}{|c|c|c|c|c|c|c|c|}
\hline $\begin{array}{c}\text { Living } \\
\text { unit }\end{array}$ & Type of load & $\begin{array}{c}\text { Initial } \\
\text { period } \\
\text { of re- } \\
\text { covery, } \\
\text { hours }\end{array}$ & $\begin{array}{c}\text { Maximal } \\
\text { rate of } \\
\text { exchange, } \\
\% \text { of } \\
\mathrm{V}_{0} / \text { hour }\end{array}$ & $\begin{array}{c}\text { Mean } \\
\text { peak } \\
\text { load } \\
(\Delta V) \text {, } \\
\% \text { of } V_{0}\end{array}$ & $\begin{array}{c}\text { Velocity } \\
\text { quotient, } \\
k_{1} \\
1 / \text { hour }\end{array}$ & $\begin{array}{l}\text { Half- } \\
\text { life } \\
\text { of } \\
\text { load, } \\
\text { hours }\end{array}$ & $\begin{array}{c}\text { Source of } \\
\text { data }\end{array}$ \\
\hline $\begin{array}{l}\text { Plasma of } \\
\text { rabbit }\end{array}$ & $\begin{array}{l}\text { Hemorrhage } \\
\text { Transfusion }\end{array}$ & $\begin{array}{l}0.5 \\
1.0\end{array}$ & $\begin{array}{l}30.6 \\
15.0\end{array}$ & $\begin{array}{l}-11.0 \\
+\quad 9.6\end{array}$ & $\begin{array}{l}2.8 \\
1.6\end{array}$ & $\begin{array}{l}0.2 \\
0.6\end{array}$ & $\begin{array}{l}\text { figs. } 95,96 \\
\text { figs. } 94,96\end{array}$ \\
\hline $\begin{array}{l}\text { Blood of } \\
\text { rabbit }\end{array}$ & $\begin{array}{l}\text { Hemorrhage } \\
\text { Transfusion }\end{array}$ & $\begin{array}{l}0.5 \\
1.0\end{array}$ & $\begin{array}{r}21.8 \\
9.7\end{array}$ & $\begin{array}{l}-22.2 \\
+13.7\end{array}$ & $\begin{array}{l}0.98 \\
0.71\end{array}$ & $\begin{array}{l}1.3 \\
1.0\end{array}$ & $\begin{array}{l}\text { fig. } 97 \\
\text { figs. } 99,98\end{array}$ \\
\hline$\underset{\text { man }}{\operatorname{Arm} \text { of }}$ & $\begin{array}{l}\text { Stasis of } \\
\text { veins }\end{array}$ & 0.17 & 7.4 & +1.24 & 6.0 & 0.2 & fig. 100 \\
\hline $\begin{array}{l}\text { Muscle of } \\
\text { frog }\end{array}$ & $\begin{array}{r}\text { Isolated and } \\
\text { desiccated }\end{array}$ & 0.5 & 19.2 & -22.8 & 0.84 & 1.4 & \\
\hline $\begin{array}{l}\text { Leucocyte } \\
\text { of rabbit }\end{array}$ & $\begin{array}{l}\text { Isolated in } \\
\text { diluted } \\
\text { Ringer }\end{array}$ & 0.01 & 6000.0 & +68.0 & 88.0 & 0.008 & \\
\hline
\end{tabular}

pared with those in whole organisms. It is clear that the most rapid recovery is in the smallest living unit, the leucocyte. Velocity quotients indicate that exchanges between plasma and the re- 
mainder of the body, and between arm and remainder through transport in plasma, are more rapid than exchanges in isolated muscle that has no vascular circulation. All recoveries are without measurable lag.

Whatever divisions of labor there may be between individuals and their component parts, both kinds of units (whole and part) undergo, each in its own way, adjustments of water content. The vicissitudes of existence may often be diverse for each kind, but also sometimes not. Hence it is possible to select like tasks of regulation for rabbit, for rabbit blood, and for rabbit leucocyte; for example, each might recover from a deficit of water of like magnitude. Roughly, to retur'n half its deficit, the whole body requires 0.02 hour (fig. 74), the blood requires perhaps 1.3 hours (fig. 94), and the leucocyte requires 0.008 hour (fig. 100). Data are not available for comparing recoveries from deficits of one identical type and size that in fact proceeded simultaneously.

A protection to the part is afforded by the fact that it keeps its place in the whole body, just as the whole organism's water content is regulated to some degree by keeping itself in certain environments. For, the body limits the loads to which the part is exposed. And, the body facilitates the exchanges of water by which the part recovers. The relations between the organism and its constituent cells and tissues, therefore, may be equivalent, in part, to those between the environment and the whole individual. 


\section{Chapter IX}

\section{GENERAL FEATURES OF WATER EXCHANGES}

$\$ 68$. The investigation is now provided with an array of materials. These materials offer information, and disclose relations, that seem to explain in part what each kind of organism does with water. Each handles water in a special way, as though there is nothing like it, and as though machinery to handle it is indispensable to life. How particular species, and indeed, other living units, adjust and recover customary water contents, seems to have been ascertained.

The next task is to seek among those materials the constant and the inconstant relations. Instead of letting each species furnish its own story, I now try to find what all species together can tell about how to handle water. Two inclusive questions can be raised. What are the general features of all regulations of water content? And what quantitative factors differentiate the maintenances and compensations in one living unit from those in another? Each question will be answered by asking a series of subsidiary questions.

For the present, the relations to be explored are among the four sorts of variables constituting the water-time system. Three of these (load, rate, time) are measured as such, though often rate of exchange equals change of load in a unit of time. The fourth (velocity quotient) is a particular ratio between two others (rate and load). Additional variables that creep in, fall in the qualitative categories of paths, species, and types of loading.

Those uniformities to be found among species and parts are similarities of correlations among the four variables; they are to be listed here. Diversities among them are of two sorts; one is quantitative differences in correlations among the four variables, which will also be sought. Such diversities are supplemented by a second sort, namely, other correlatives of the four variables, some of which will be ascertained later (chapters X to XII), additional data being considered to that end.

\section{$\$ 69$. Some limitations}

For comparing the water relations of two organisms of great anatomical diversity, such as a mammal and a worm, several dimen- 
sions of reference are available. Occasionally the absolute volumes exchanged are described. Or, the exchanges are expressed as fractions of the volume and the water content of the body, whereby other objectives are gained. Again, time is measured relative to some physiological process (physiological times) instead of by the same clock in all species.

Diversity of conditions and states in each species constitutes a severe limitation to comparisons ; I therefore compare while insisting upon the tentative nature of the results. Particularly, comparisons of water exchanges by isolated tissues with those of tissues in situ may seem fantastic. Probably this opinion is the safer one; perhaps the same view is preferable throughout comparative biology and in all analogies to non-living systems. It is always a propos to say: in this seemingly neat comparison the diversities of surface area, or the presence or absence of a circulation, or the amenities of vitality, are disregarded. So they are, and I think something is disregarded of the hundreds of possible factors, in any conceivable comparison. Hence the investigator chooses between making provisional comparisons and making none; if the former, it is invidious to say that one mode of comparison is better than another; it may rather be said that it is different. Quantitative comparisons have the probable advantages of being implicitly provisional, and of being guided by dimensions and other criteria away from some varieties of confusions. I may confidently expect that some of the features coming to light are independent of what the observer imposes. The utmost judgment will not insure like conditions for two individuals or species, or for two days. Diversity of states in one individual may or may not represent "inherent" variability, so long as there is no possibility of robbing the organism of all environment.

Why not subject all species to the same conditions, it may be asked? Only in the abstract, might it be ideal to use identical means and criteria of water load in all the organisms that are to be studied comparatively. (1) Not all organisms can survive like treatment or like environment. Desiccations of Ameba and of Thamnophis by evaporation, though both technically possible, do not attain the same water deficits in a uniform time. Recoveries of dog immersed in water, Ameba in air, rabbit blood in distilled water, are equally unsuitable. (2) Not all organisms are fur- 
nished with homologous structures and paths. What can be counted as alimentary water in Arbacia egg, as urinary output in leucocytes? (3) Even when organs are supposedly or actually homologous, their perfolmances may be diverse. Thus, frog's urine is not known to be hypertonic to blood plasma under any circumstances; dog's urine is ; both urines are produced by kidneys. In earthworms the output from nephridia may or may not be counted as urine, and as homologous with the renal output in dogs.

Three complete equilibration diagrams were constructed for the dog (fig. 38), all of which are equally valid in representing this species in comparisons of water relations. In fact, at least six types of modification of water content were recognized; some in excess and some in deficit; and there was no unique connection between the particular excess and the particular deficit associated in any one equilibration diagram. Among such diagrams, three or more time relations were utilized: initial (of diverse durations), steady, and maximal rates of water exchange.

In comparisons among living units, whether species or parts of individuals, these several distinctions at least may be maintained. Each of them avoids one kind of confusion.

\section{§ 70. Some parameters comparing exchanges}

Measurable gain was contrasted with measurable loss of water at each load. What methods of evaluating the contrasts between the two, and of exhibiting the modifications of each exchange separately, are useful?

(1) Economy quotients, the ratios of gain to loss, characterize the responses to water increments in the several species (table 9). The fastest recovery from water excess means suppression of gain while loss is maximal; and the fastest recovery from water deficit presupposes the reverse. The economy quotient evaluates how closely this possibly optimal relation is approached.

The dog's exchanges show higher quotients at every negative load than the rat's, which in deficits surpass those of man, frog, and earthworm. In positive loads, values departing most from 1 might be sought. The quotients of dog and man are not most extreme in short periods of time, for in water excesses the frog's diuresis has less lag than the mammal's. By choosing diverse modes of comparison, a case can be made for "superiority" of the quotient in almost any species. Economy quotients are available 


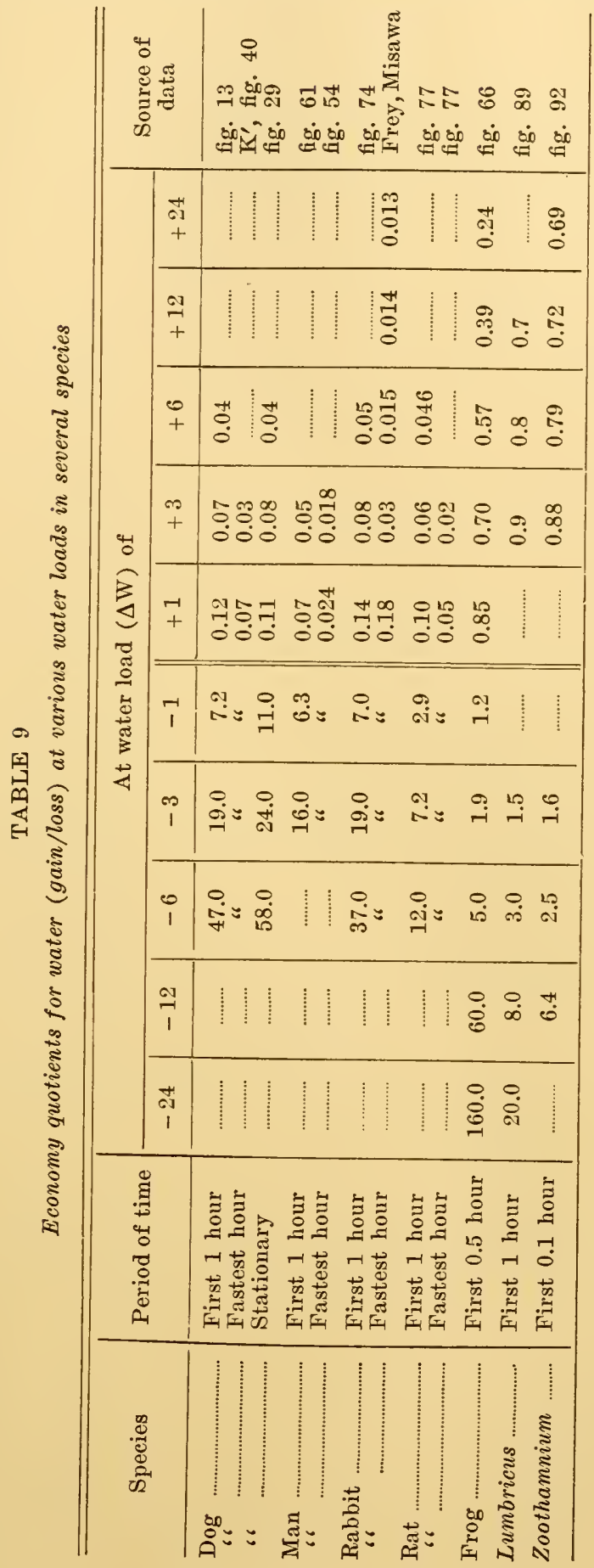


only in those living units whose total gains and total losses are known.

(2) Modification ratios. The responses to change in load, in terms either of total exchanges or of various paths, may be measured by comparing the maximal and minimal rates of exchange found (table 10). Proficient adjustment calls for very rapid movement of water at one time, little at another. At a glance it is apparent that oxidative gain is no factor in recovery. Nor is either fecal or evaporative loss greatly concerned in restoring water balance in any species studied, or under any conditions

TABLE 10

Rates of water exchange (per cent of $B_{0}$, in first 1 hour of recovery) when water load and at

\begin{tabular}{|c|c|c|c|c|c|c|c|c|c|}
\hline \multirow{2}{*}{ Species } & \multicolumn{3}{|c|}{ Total gain } & \multicolumn{3}{|c|}{$\begin{array}{l}\text { Ingestive and } \\
\text { imbibitory }\end{array}$} & \multicolumn{3}{|c|}{ Oxidative } \\
\hline & T.O. & Min. & Max. & T.O. & Min. & Max. & T.O. & Min. & Max. \\
\hline Dog & 0.25 & 0.06 & 6.2 & 0.19 & 0.0 & 6.1 & 0.06 & 0.06 & 0.06 \\
\hline $\begin{array}{l}\text { Dog (stationary } \\
\text { states) }\end{array}$ & 0.25 & 0.06 & 8.5 & 0.19 & 0.0 & 8.4 & 0.06 & 0.06 & 0.06 \\
\hline Man ……………........... & 0.15 & 0.02 & 2.5 & 0.13 & 0.0 & 2.5 & 0.02 & 0.02 & 0.02 \\
\hline Rabbit & 0.54 & 0.07 & 6.4 & 0.47 & 0.0 & 6.3 & 0.07 & 0.07 & 0.07 \\
\hline Rat …………......... & 0.68 & 0.10 & 10.5 & 0.58 & 0.0 & 10.4 & 0.10 & 0.10 & 0.10 \\
\hline Frog & 1.7 & 1.5 & 13.9 & 1.7 & 1.5 & 13.9 & 0.01 & 0.01 & 0.01 \\
\hline Lumbricus ................... & 2.7 & 2.6 & 19.0 & 2.7 & 2.6 & 19.0 & 0.01 & $\cdots \cdots \cdots$ & $\ldots \ldots \ldots .$. \\
\hline Zoothamnium & 230.0 & 230.0 & 255.0 & 230.0 & 230.0 & 255.0 & ........... & $\ldots \ldots \ldots . .$. & $\ldots \ldots . . .$. \\
\hline
\end{tabular}

TABLE 11

Modification ratios, augmentation ratios, and partitions of

\begin{tabular}{|c|c|c|c|c|c|c|c|c|c|c|c|}
\hline \multirow{2}{*}{ Species } & \multicolumn{6}{|c|}{ Modification ratio (Max./min.) } & \multicolumn{5}{|c|}{ Augmentation ratio } \\
\hline & $\begin{array}{l}\text { Total } \\
\text { gain }\end{array}$ & $\mid \begin{array}{c}\text { Inges. } \\
+ \text { imbib. }\end{array}$ & Oxid. & $\begin{array}{l}\text { Total } \\
\text { loss }\end{array}$ & Urin. & Evap. & \multicolumn{2}{|c|}{ Total gain } & \multicolumn{2}{|c|}{$\begin{array}{l}\text { Inges. } \\
\text { + imbib. }\end{array}$} & Oxid. \\
\hline 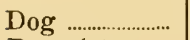 & 100.0 & $\propto$ & 1 & 19.0 & 100.0 & 1.4 & 25.0 & 0.24 & 32.0 & 0.0 & 1 \\
\hline $\begin{array}{c}\text { Dog (sta- } \\
\text { tionary } \\
\text { states) }\end{array}$ & 140.0 & $\propto$ & 1 & 14.0 & 70.0 & 1.4 & & & & & 1 \\
\hline Man ………........... & 125.0 & $\propto$ & 1 & 23.0 & 50.0 & 1.5 & 17.0 & 0.13 & 19.0 & 0.0 & 1 \\
\hline Rabbit & 90.0 & $\propto$ & 1 & 14.0 & & & 12.0 & 0.13 & 13.0 & 0.0 & 1 \\
\hline Rat ………............. & 105.0 & $\propto$ & 1 & 8.0 & 42.0 & 1.7 & 15.0 & 0.15 & 18.0 & 0.0 & 1 \\
\hline Frog & 9.0 & 9.0 & 1. & 60.0 & 60.0 & ....... & 8.0 & 0.9 & 8.0 & 0.9 & 1 \\
\hline Lumbricus ... & 7.3 & 7.3 & $\ldots$ & 5.7 & 5.5 & $\ldots \ldots$ & 7.0 & 1.0 & 7.0 & 1.0 & ... \\
\hline Zoothamnium & 1.11 & 1.11 & ... & 1.23 & 1.23 & ........ & 1.11 & 1.0 & 2.4 & 1.0 & ... \\
\hline
\end{tabular}


listed; for each proceeds almost unchanged at diverse loads. In urinary losses (table 11) the several mammalian species have higher modification ratios and more extreme augmentation ratios than the frog and others. In rates of gain, mammals are outstanding in fitting intake to the state of the body. Those are two of many comparisons among the quantitative compensations in water loads. It will be recalled that total rates and turnovers are unknown for any parts (organs or cells) of organisms.

(3) Variabilities of rates of water exchange in turnover (table 12) may be compared by coefficients either of difference or of

TABLE 10

varies, in several species. Total and partitioned gains and losses are listed at no load (T.O.) extreme loads

\begin{tabular}{|c|c|c|c|c|c|c|c|c|c|c|}
\hline \multicolumn{3}{|c|}{ Total loss } & \multicolumn{3}{|c|}{ Urinary } & \multicolumn{3}{|c|}{ Evaporative } & \multirow{2}{*}{ Fecal } & \multirow{2}{*}{ Source of data } \\
\hline T.O. & Min. & Max. & T.O. & Min. & Max. & T.O. & Min. & Max. & & \\
\hline 0.25 & 0.17 & 3.3 & 0.08 & 0.03 & 3.1 & 0.15 & 0.11 & 0.15 & 0.02 & figs. $2,4,12,44$ \\
\hline 0.25 & 0.17 & 2.3 & 0.08 & 0.03 & 2.1 & 0.15 & 0.11 & 0.15 & 0.02 & figs. $12,23,28,24$ \\
\hline 0.15 & 0.09 & 2.1 & 0.08 & 0.04 & 2.0 & 0.06 & 0.04 & 0.06 & 0.01 & $\begin{array}{l}\text { figs. } 50,54,59, \\
60,61\end{array}$ \\
\hline 0.54 & 0.16 & 2.3 & 0.31 & & 2.2 & 0.17 & & & 0.06 & fig. 74 \\
\hline 0.68 & 0. & 3. & 0.24 & 0.06 & 2. & 0.40 & 0.30 & 0.40 & 0.04 & figs. 77,108 \\
\hline 1.7 & 0.1 & 6.0 & 1.7 & 0.1 & 6.0 & 0.0 & $\ldots \ldots \ldots . .$. & ............... & 0.0 & fig. 66 \\
\hline 2.7 & 0.9 & 5.1 & 2.5 & 0.9 & 4.9 & 0.0 & $\ldots \ldots \ldots . .$. & $\ldots \ldots \ldots .$. & 0.25 & $\begin{array}{l}\text { fig. } 89 \text {, Wolf } \\
\text { ('40a); Davis } \\
\text { and Slater } \\
\text { ('28) }\end{array}$ \\
\hline 230.0 & 220.0 & 270.0 & 230.0 & 220.0 & 270.0 & 0.0 & $\ldots \ldots \ldots . .$. & $\ldots \ldots \ldots . .$. & 0.0 & fig. 92 \\
\hline
\end{tabular}

TABLE 11

water exchanges, in several species, computed from table 10

\begin{tabular}{|c|c|c|c|c|c|c|c|c|c|c|c|}
\hline \multicolumn{6}{|c|}{ (Max./T.O.; Min./T.O.) } & \multicolumn{3}{|c|}{$\begin{array}{l}\text { Ingestive or imbibitory } \\
\text { as per cent of total } \\
\text { gain }\end{array}$} & \multicolumn{3}{|c|}{$\begin{array}{l}\text { Urinary or vacuolar } \\
\text { as per cent of } \\
\text { total loss }\end{array}$} \\
\hline \multicolumn{2}{|c|}{ Total loss } & \multicolumn{2}{|c|}{ Urinary } & \multicolumn{2}{|c|}{ Evap. } & T.O. & Min. & Max. & T.O. & Min. & Max. \\
\hline 13.0 & 0.7 & 40.0 & 0.4 & 1 & 0.7 & 76 & 0 & 98 & 32 & 18 & 94 \\
\hline 9.0 & 0.7 & 26.0 & 0.4 & 1 & 0.7 & & 0 & 98 & 32 & 18 & 92 \\
\hline 15.0 & 0.6 & 25.0 & 0.5 & 1 & 0.7 & 87 & 0 & 99 & 53 & 45 & 97 \\
\hline 4.0 & 0.3 & 7.0 & & & & 87 & 0 & 99 & 58 & & 98 \\
\hline 4.0 & 0.6 & 10.0 & 0.25 & 1 & 0.7 & 85 & 0 & 99 & 35 & 15 & 83 \\
\hline 3.5 & 0.6 & 3.5 & 0.6 & $\ldots$ & & 99 & 99 & 99.9 & 100 & 100 & 100 \\
\hline 1.9 & 0.3 & 2.0 & 0.4 & $\ldots$ & ....... & 99 & 99 & $\ldots \ldots \ldots$ & 93 & 100 & 100 \\
\hline 1.17 & 0.96 & 1.17 & 0.96 & $\cdots$ & $\cdots \cdots .$. & 99 & 99 & $\ldots \ldots . . .$. & 100 & 100 & 100 \\
\hline
\end{tabular}


variation, with respect to (a) total intake or total output, and (b) diverse paths of intake or of output. Rate of total intake is more variable, in successive 1 -hour periods, than rate of output in dog and man, and probably in mammals generally. Variation of intake is about equal to variation of output in frog and perhaps in aquatic organisms generally. In the environments and conditions chosen for study there is also an inverse relation, it seems, between the variability of rate and the steepness of the correlation line in net equilibration diagrams (fig. 47). This steepness is also expressed in net velocity quotients of diverse species.

Among species and environments there is a direct correlation between rate of turnover and variability of content (table 12). Or, the more water passes through the body, the more chance there is for net retention or expulsion of some of it $(\$ 38)$.

Among mammals it is evident that large body size goes with less variation of content (in $\%$ of $\mathrm{B}_{0}$ ), and less turnover (in $\%$ of $\mathrm{B}_{0}$ ).

(4) Maximal rates of exchanges, found at extreme water loads in each of the organisms studies, may not be regarded as absolute values for the species. Provisionally maximal rates of urinary output, evaporative loss, and ingestive gain are recorded in table 10. Table 13 compares the maximal rates observed in whatever species the states of water excess or water deficit were tested. Many possible dimensions of the body might be correlated with these rates; the hope of finding a limiting factor in the structure or use of the alimentary tract or the urinary apparatus or any other one provision cannot be seriously entertained, for single "bottle necks" do not often characterize physiological processes.

(5) Ratio of rates occurring simultaneously in any two paths may represent the partition of flow (table 10) during turnover in balance as well as in recoveries. For example, wishing to characterize adrenalectomized dogs, Uyldert ('38) showed that the ratio: urinary loss/ingestive gain, which was in normal dogs 0.46 , was unchanged by unilateral adrenalectomy, but was increased to 0.64 by either bilateral excision of adrenals or by adding sodium chloride to the diet.

Thus, each specific purpose suggests a feasible method of comparing rates of exchange. If one of them does not emphasize the unusual features in an experimental test or in a species, another will. Altogether it is unlikely that all methods will yield significantly identical results for two species or metabolic states. 


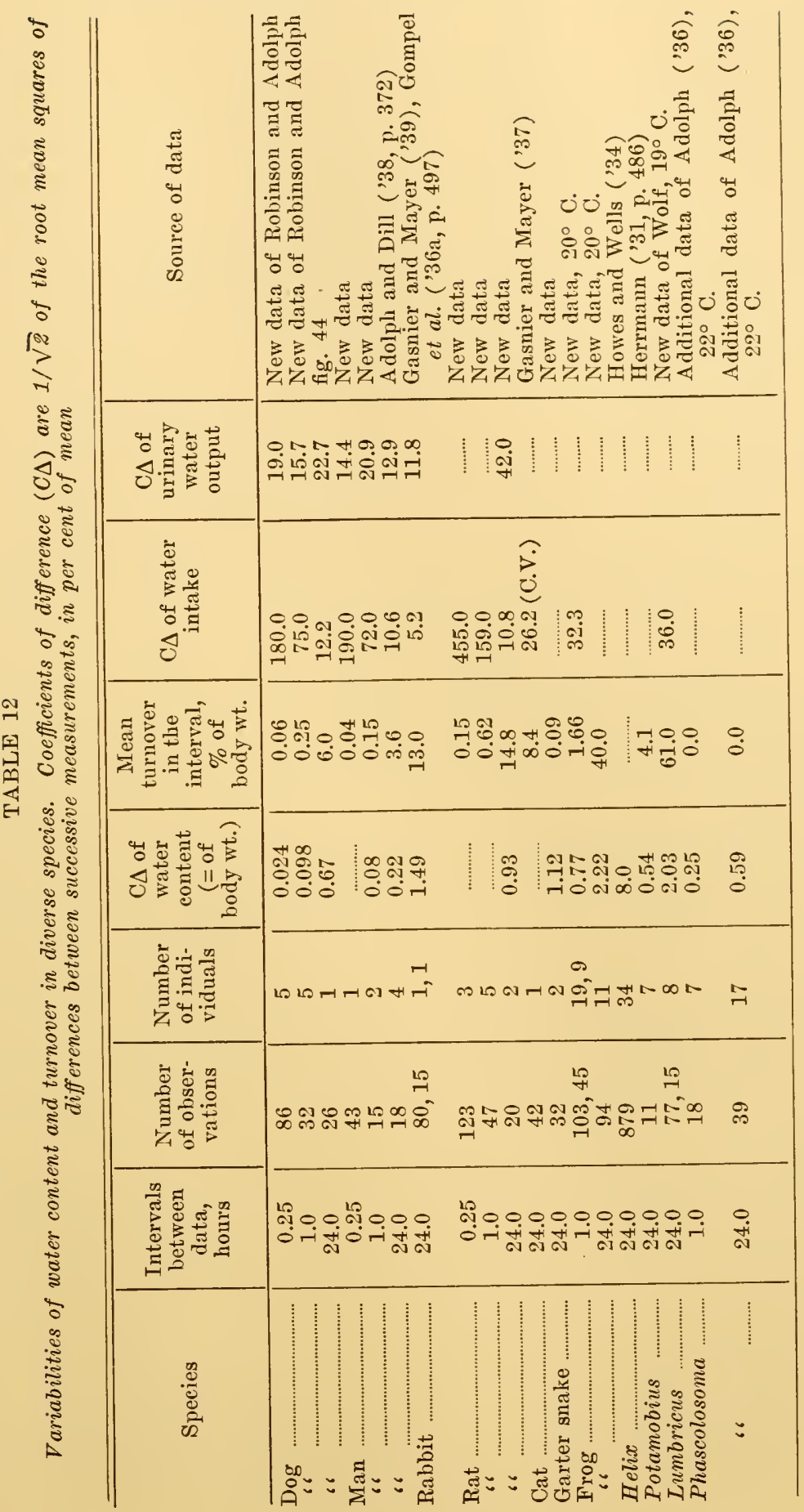




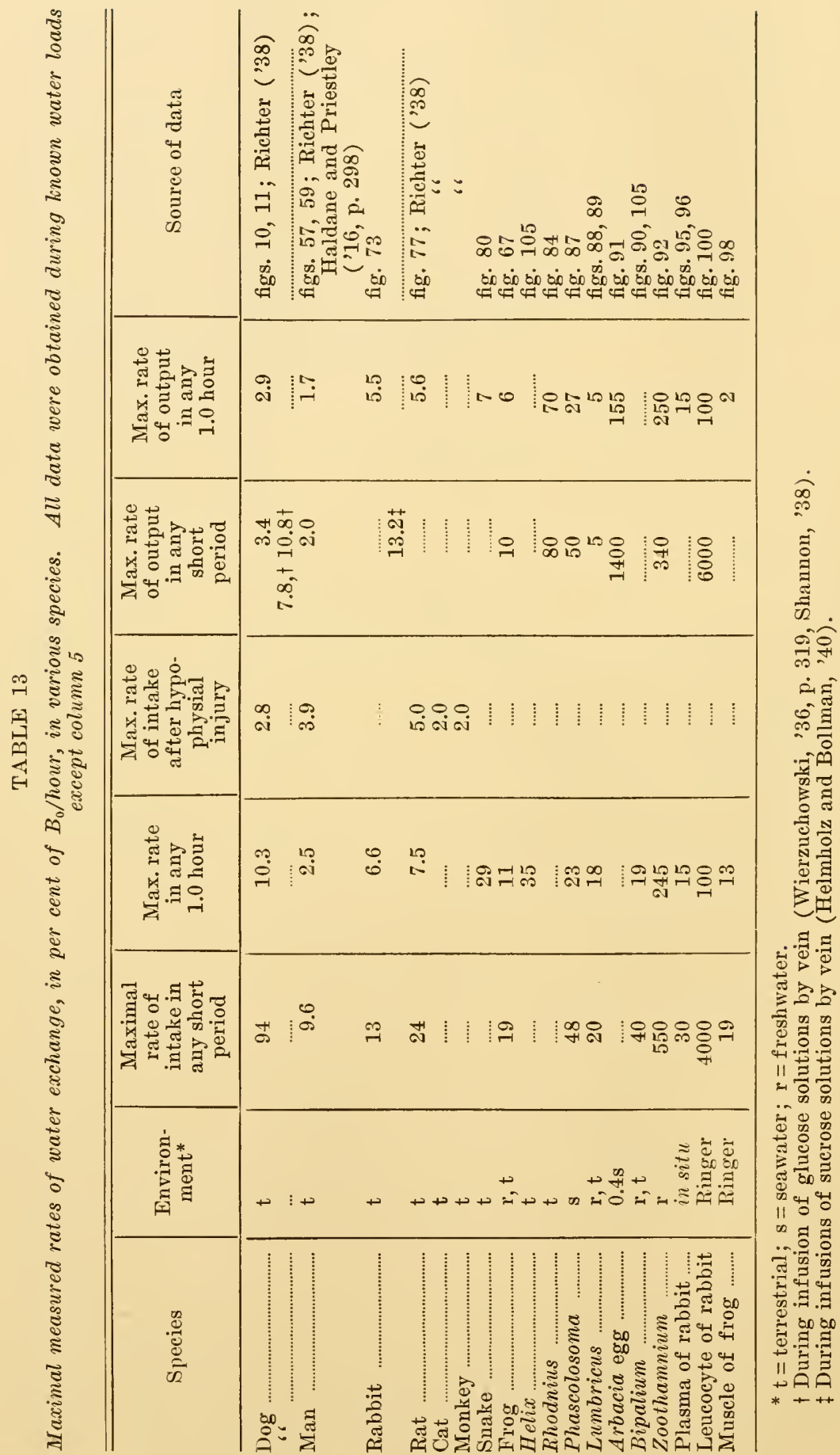




\section{\$71. Thme Relations}

What can be learned by analyzing the time relations in responses to sudden water increments? Initiation of response to load, signalized by acceleration of exchange, is (table 14) without measurable lag in water deficits of all species. In water excesses the responses are more diverse, they appear to begin most promptly where osmosis gives direct passage of water to and from the aqueous environment. Where fluids are excreted by recognized special organs, a few species as frog and Rhodnius appear to accelerate elimination without lag; actually there is a lag, of probable significance (0.04 hour), even in the frog's diuresis (Adolph, '36a). It is possible to guess the extended lag in mammals is a period in which fluid becomes preferentially distributed to tissues before much escapes from the body.

The maximal response (rate) is achieved almost instantly in several species, especially in acceleration of intake. Where this is the case, time is probably not a distinct factor in the exchanges, except as the loads or other quantities change with time.

Completion of recovery, just like the initiation and the maximal stage of recovery, is related to the load of water administered. One criterion of completion (table 14) is the state existing when the rate of exchanges has returned to within \pm 30 per cent (see table 12) of the initial or the control rate; this defines the end of deceleration. The total time elapsed indicated how long the organism is occupied in reattaining balance.

The times required for measurable restitution of basal rates of exchange vary from 0.04 hour (Zoothamnium) to 70 hours (snake). Nearly the whole range is present in one species (snake) when gain is compared with loss. If the brevity of the time occupied in recovery is any indication of a crucial state for survival, then water deficit is the more "dangerous" situation in all species tested. But I have no evidence that death is actually avoided by the great speed of the exchanges observed.

Partial relations of the time for completion or half-completion of recovery (fig. 101) to the body size and to peculiar body structures may be discerned. Thus, Arbacia egg finishes its adjustments rapidly, Phascolosoma, a larger animal, slowly. The circulation of body fluids is a correlative in transport of water, supposedly enabling the 15,000 -gram dog to eliminate through local 


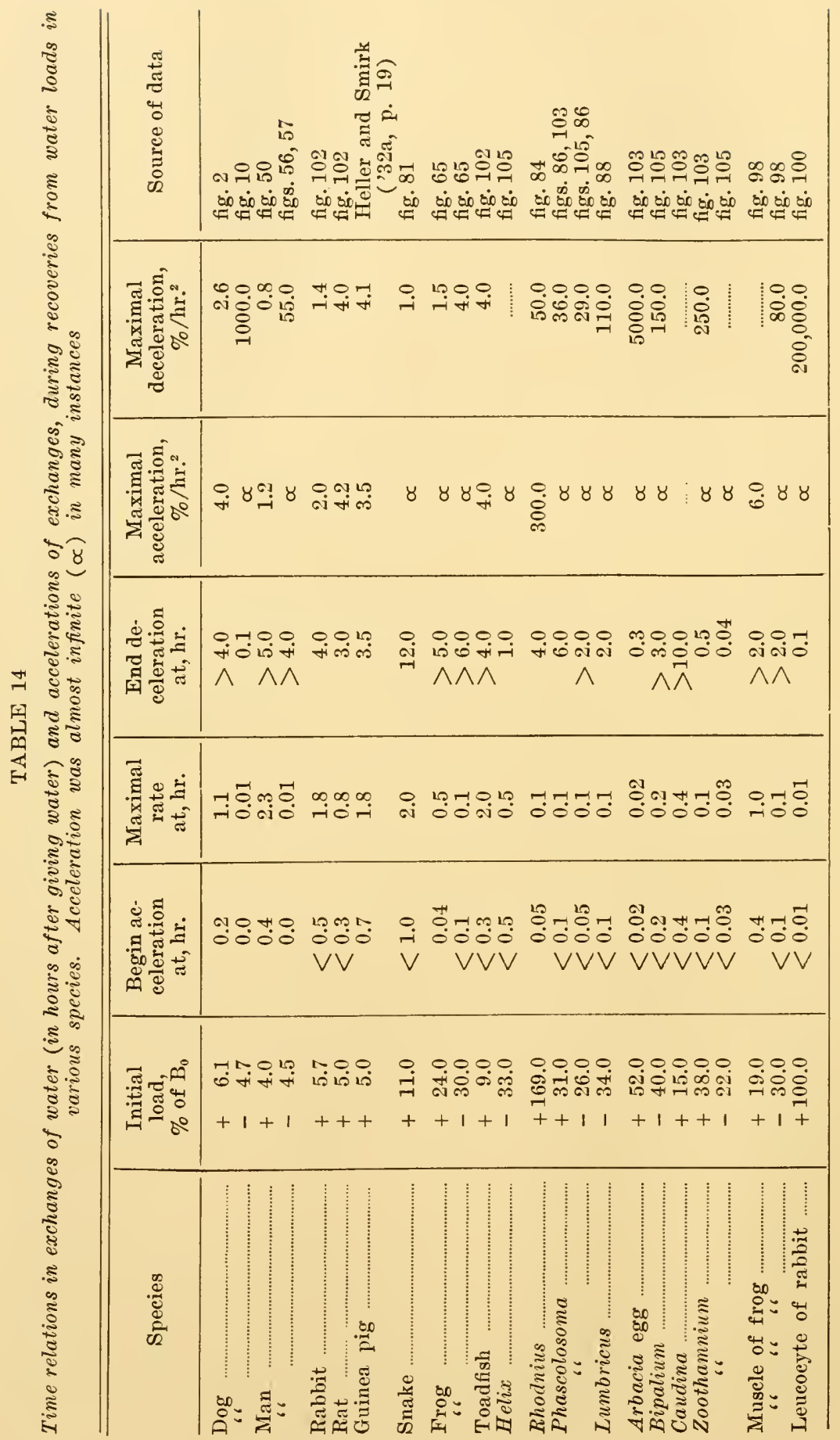


organs, the kidneys, an excess even more rapidly than does the 30 -gram frog.

Accelerations and decelerations of exchanges of water may be compared quantitatively (table 14 ), in $\%$ of $\mathrm{B}_{0} /$ hour ${ }^{2}$. As mentioned, acceleration is apparently instantaneous in some species, hence almost infinite. Deceleration appears to be slower than acceleration in all living units studied; it is greatest in unicellular units.

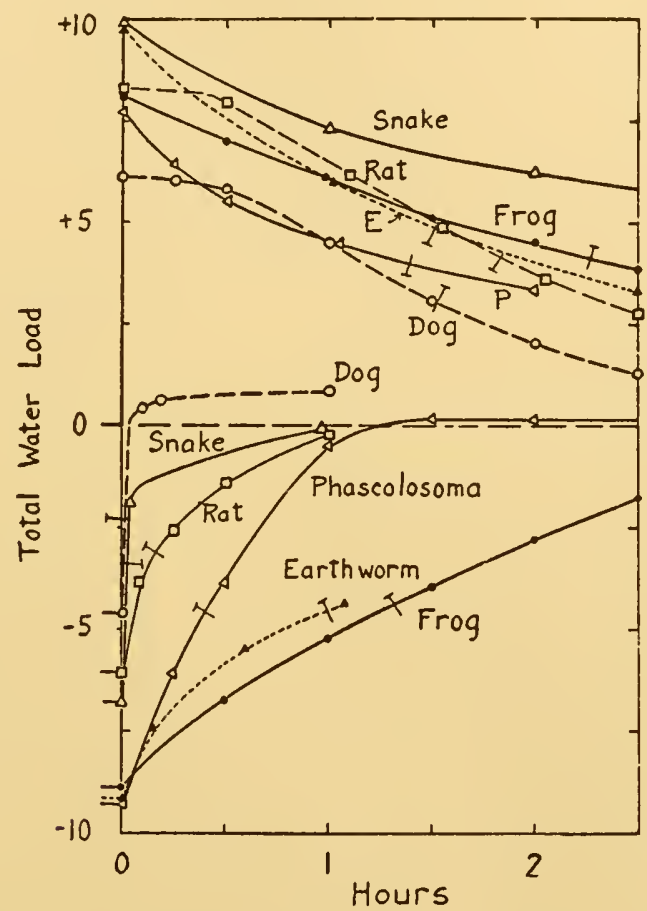

FIG. 101. Courses of total water loads ( $\%$ of $\mathrm{B}_{0}$ ) during recoveries. Comparison of water tolerance curves in 6 species, 3 to 21 tests being averaged for each curve. At each time, the water load is taken as the mean difference of weight increments between the loaded individuals and the control individuals that were similarly denied food but allowed water ad libitum. Dog, from figures 1 (A) and 10; rat, from figure 76 ; garter snake, mean from figure 81 ; frog, from figure 65 ; earthworm, from figure 88 and further data; Phascolosoma, from figure 85.

For comparisons of tolerance curves, initial loads may be equated. Then the relative loads in relation to time (figs. 102 and 103) epitomize those comparisons of eliminations already mentioned. From excesses of water, rats recover most rapidly of any vertebrates. Invertebrates without special circulatory and excre- 
tory organs are in some cases slower than rats, but unicellular ones are faster. In recoveries, by intake, from water deficits (figs. 104 and 105) the contrasts are enormous. If in mammals absorptive rates be substituted for ingestive rates, the diversities are scarcely reduced. In aquatic invertebrates the body size is not the only correlative of rate, since Bipalium is slower than some larger species.

Parenthetically, adjustments by water intake are further analyzed (table 15), the data happening to concern mammals. All species are capable of extreme feats of drinking; how much they drink appears to be graded to the deficits of water imposed. Just

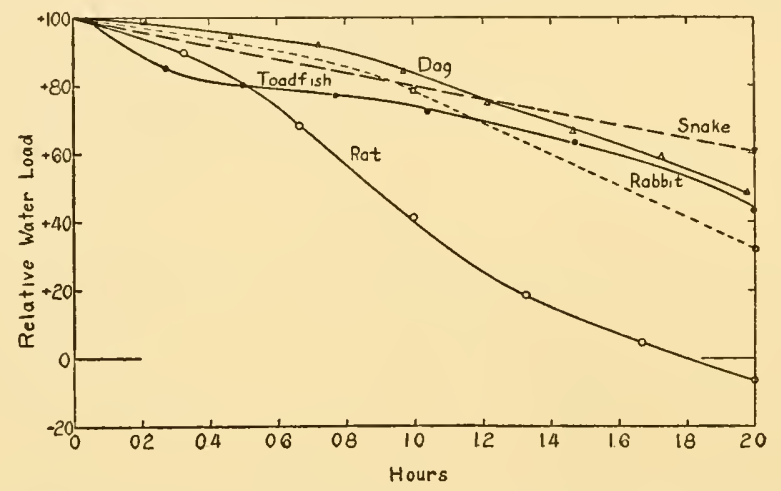

FIG. 102. Course of relative water load (\% of initial load) after recovery began in diverse species of vertebrates that received 5 to $11 \%$ of $\mathrm{B}_{0}$ of excess water. Toadfish, young Opsanus tau, of $6.3 \mathrm{gm}$. kept in sea water at $23^{\circ} \mathrm{C}$., at zero time injected intraperitoneally with $9.5 \%$ of $\mathrm{B}_{0}$ of distilled water; new data. Rabbit, 7 individuals of $1800 \mathrm{gm}$. each given $5.7 \%$ of $\mathrm{B}_{0}$ of water by stomach; data of Abe ('31a); see also figure 72. Rat, 18 individuals of perhaps $250 \mathrm{gm}$. each given $5.0 \%$ of $\mathrm{B}_{0}$ of water by stomach; data of Heller and Smirk ('32a, p. 15); see also figure 75. Snake, eight tests on Thamnophis of 8 to 101 gm., given $10.9 \%$ of $\mathrm{B}_{0}$ of water by intraperitoneal injection; data of figure 80 . Dog, three tests on one individual of $13,870 \mathrm{gm}$., given $6.1 \%$ of $\mathrm{B}_{0}$ of water by stomach; data of figure 1.

as the initial rates of ingestion are directly correlated with $-\Delta \mathrm{W}$, so several of the other measurements listed (duration of draft, rate of swallowing) are found to be proportional to deficit. Among species, many relations to body size are apparent. The frequency of the gulps of which the drafts are composed is quite uniform, and the size of each gulp is closely proportioned to body weight. At one deficit the duration of initial drafts is roughly the same in all species, and hence the rate of swallowing is proportional to body weight. It may be said that the features of intake are geared to 


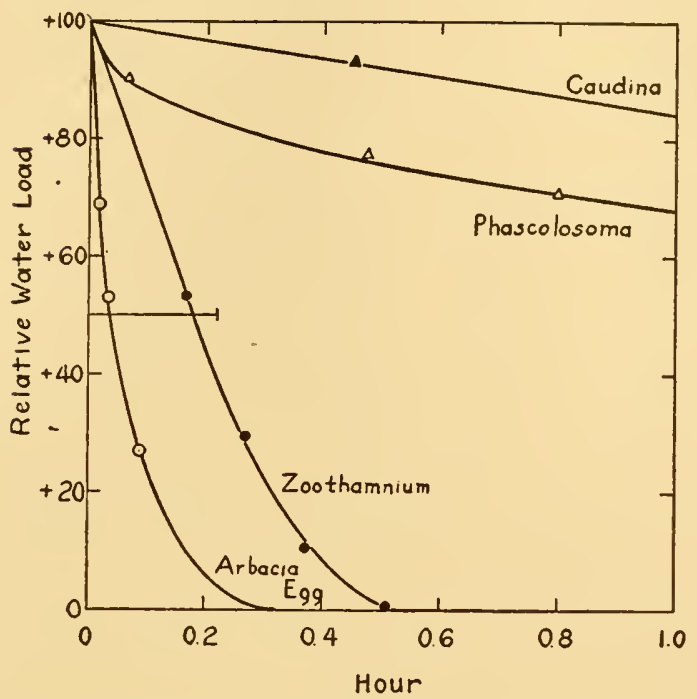

FIG. 103. Course of relative water load (\% of initial load) after recovery from excess began, in diverse species of invertebrate animals. Zoothamnium put from $0.005 \mathrm{M}$ $\mathrm{NaCN}$ into fresh water; initial $\Delta \mathrm{W}+38$; basal body volume $1.8 \times 10^{-8} \mathrm{ml}$; data of Kitching ('38, p. 145). Caudina put from 60 per cent sea water into normal sea water; initial $\Delta W+15$; body weight 27 gm.; $22^{\circ}$ C.; data of Koizumi ('32, p. 277). Arbacia egg put from 60 per cent sea water into normal sea water, initial $\Delta W+52$; average for 6 individuals, body volume $20.3 \times 10^{-8} \mathrm{ml}$; $21^{\circ} \mathrm{C}$.; data of Lucke et al. ('31, p. 416). Phascolosoma put from 60 per cent sea water into normal sea water, initial $\Delta W+44$; average for 5 individuals, $A F$, of body weight $1.9 \mathrm{gm}$. each; $24^{\circ} \mathrm{C}$; additional data of Adolph ('36b).

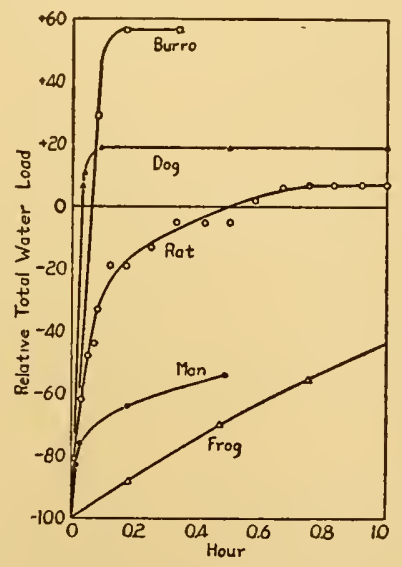

FIG. 104. Course of relative water load (in \% of initial load) after drinking was allowed to begin following the periods of privation. Initial deficits of weight were 4 to $8 \%$ of $\mathrm{B}_{0}$. One test in each of 5 species; Burro, Adolph and Dill ('38); Dog, Adolph ('39a); Rat, new data; Man, new data; Frog, Adolph ('39b). 


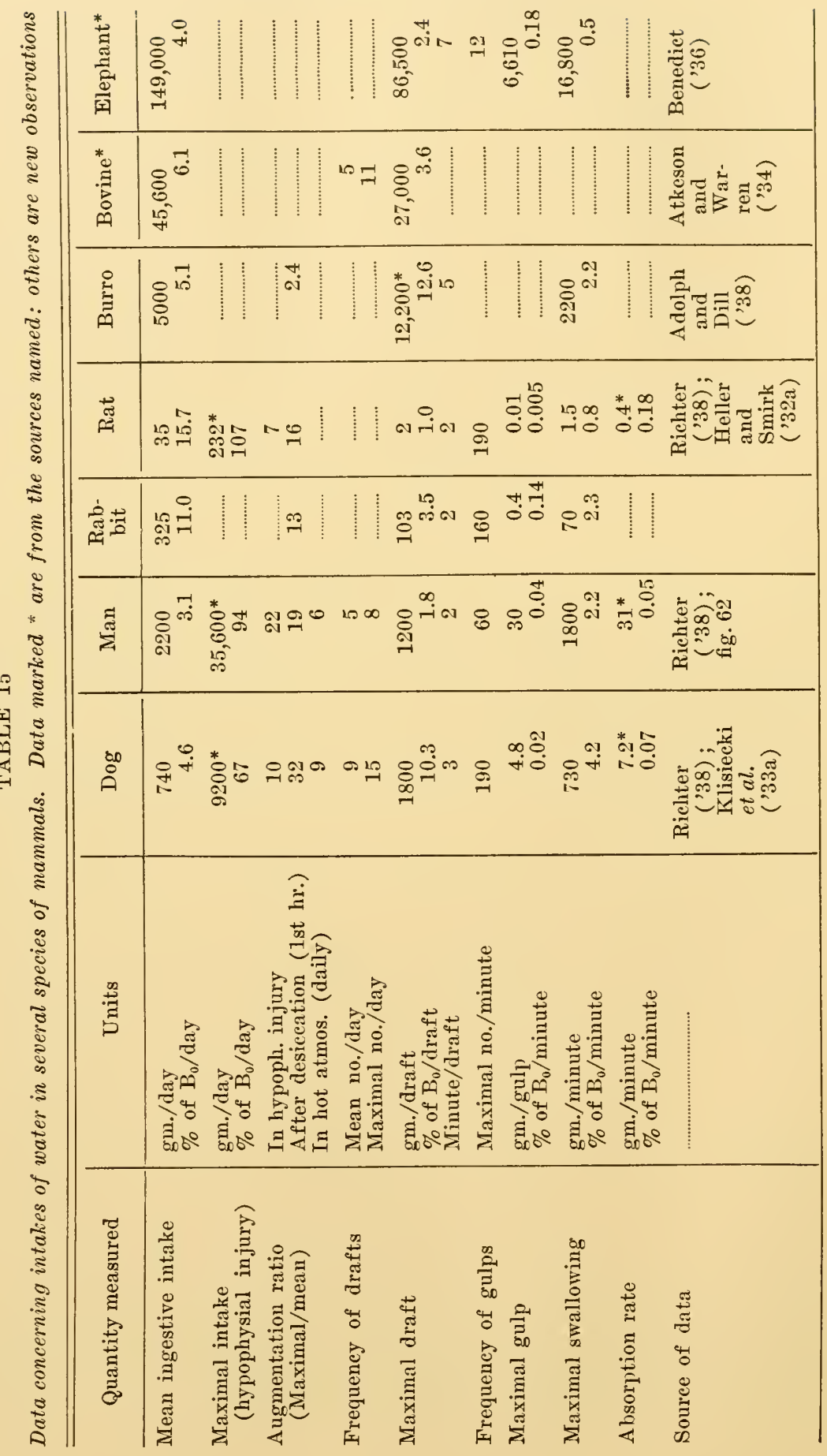


body size so closely that individuals of all species drinking side by side, after depletion of water, would on the average gulp simultaneously and take drafts of equal duration.

The quantities concerning water intake might profitably be observed in other classes of animals for which information now scarcely exists. Frequency of drafts is one such; the snail Helix is known to ingest only once or twice a week (Howes and Wells, '34) ; certain hummingbirds drink every few minutes while on the wing (Ditmars, '37, p. 131), butterflies and other insects take water at intervals quite unknown (Buxton, '32b). Still others like the frog and the termite Termopsis die, presumably of water deficit, without attempting to ingest by mouth water that is available or

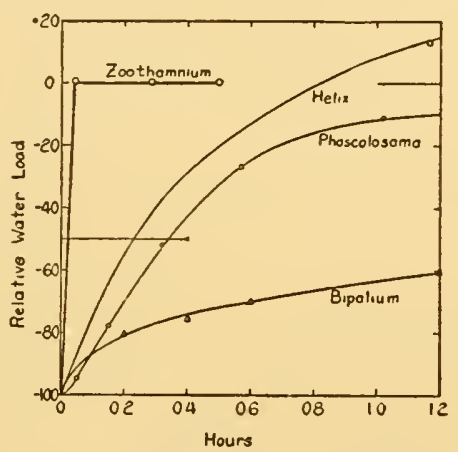

FIG. 105. Course of relative water load (\% of initial load) after recovery from deficit began in diverse species of invertebrate animals. Zoothamnium put from $0.05 \mathrm{M}$ sucrose into fresh water; initial $\Delta W-22$; body volume $1.3 \times 10^{-8} \mathrm{ml}$; data of Kitching ('38, p. 147). Helix pomatia after 4 months' privation of water; initial $\Delta W-33$; body weight $14 \mathrm{gm}$; water placed on mantle only and not on mouth; data of Künkel ('16, p. 45). Phascolosoma put from 150 per cent sea water into normal sea water; initial $\Delta W-26$; average for 5 individuals, $B$; body weight $1.52 \mathrm{gm}$. each; $22^{\circ} \mathrm{C}$.; further data of Adolph (' $36 \mathrm{~b}$ ). Bipalium after 10 hours' desiceation; initial $\Delta W-40$; body weight 1.0 gm.; about $30^{\circ}$ C.; data of Kawaguti ('32, p. 52).

at least present (Cook and Scott, '32). Very numerous are the possible functions with which frequency of drinking is correlated, when water is continuously available. Numerous are the tales extant about habits of drinking in various species; they need not be considered further in this investigation.

Time relations during recoveries in several sorts of living units may be expressed in whole series of tolerance curves upon common scales of load and time (fig. 106). The range of loads studied is very diverse, making for moderate diversity in the magnitudes of exchanges. 
The similarities of time relations among species and tissues, seem remarkable when it is realized that no anatomical arrangement is common to all the living units, and certainly no single variety of physical process is concerned in the water exchanges. The fact to be recognized is that the several living units do the same things in nearly the same sequence while recovering from
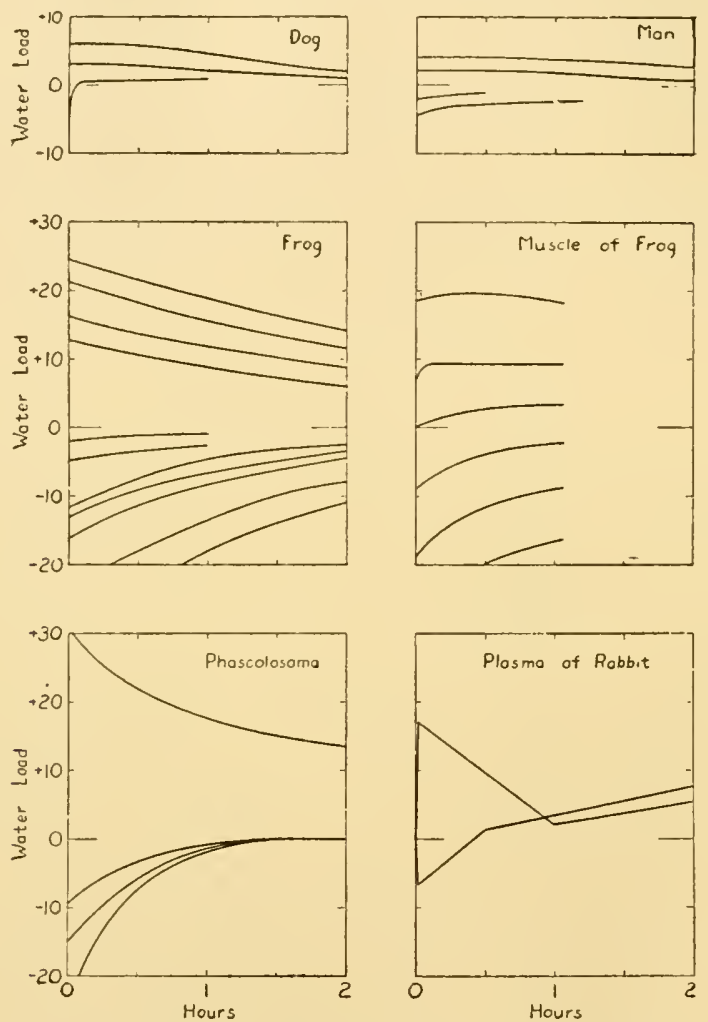

Fig. 106. Tolerance curves for water in six species or tissues; total water load ( $\%$ of $\mathrm{B}_{0}$ or $\mathrm{V}_{0}$ ) in relation to time. All are upon similar seales of coordinates. Dog, from figures 1 and 10; Man, from figures 49 and 56; Frogs in water, from figure 65; Isolated muscle of frog in Ringer's solution from figure 98; Phascolosoma in sea water from figure 85 ; Plasma of rabbit in situ, following transfusion or hemorrhage, from figure 95 .

water loads, without sharing any other unique property that is recognized to be correlated with maintenance of water content.

Diversities among the living units studied are materials in the comparative physiology of water exchanges. Being drawn independently of what organs each species possesses, comparisons are expressed numerically. For instance, I note that a garter snake 
recovers from water deficit more rapidly than a rat, which in turn has a higher velocity quotient than Phascolosoma, man, and frog (fig. 101). The above description characterizes each recovery according to its velocity, its course over a period of time, and its proportionality to load. To fit a rat with a man's slow recovery of water content would apparently be as incongruous as to fit the rat with a man's arm. For, physiological processes have their congruities, however non-material their fixity and invisible their relations to other functions may be.

The fact that all the tolerance curves represented have similar shapes, allowance being made for the fact that recovery from excess starts after a lag in dog, man, and isolated muscle, suggests that they be expressed by a single equation. An equation that satisfactorily fits very many of the data relating load to time $(t)$ is the exponential form $\Delta \mathrm{W}=a e^{-k t}, a$ being a coefficient and $e$ the base of natural logarithms. It may be written in the form $\ln \Delta W=$ $\ln a-k$ t. Values of $k$ therefore express relative slopes of the curves at diverse times.

The rates of exchange may likewise be compared among species during recoveries of water content (fig. 107). Deriving from the same equation

$$
\delta \mathrm{W} / \Delta \mathrm{t}=-a k e^{-k \mathrm{t}}
$$

Dividing rate by load, the quotient is $-k$. This $k$ is identical with the velocity quotient, which is computed either by dividing the rate of exchange by the coexisting load, or by the relation $1 / \Delta \mathrm{t}=k=$ $(\ln \Delta W-\ln a) / t$ obtained by transforming the second equation above.

The quotients (table 16,3 and 4) vary in some species with the increment of water content. But for each species and each type of increment there is a mean value and a range of values. Uniformities become apparent; (1) in many species, values of $-\mathrm{k}$ are nearly constant over considerable ranges of negative load or of positive load. (2) Quotients are higher in deficits than in excesses. (3) Quotients tend to be similar, in one species, for both negative loads and positive loads. (4) Quotients are often greatest near zero load; this region of large quotients includes the most usual contents, the most frequent rates of exchange. (5) At great loads the quotients diminish, except in Phascolosoma's and Bipalium's deficits, whether the chief paths concerned be alimentary tracts or integuments or kidneys. 
The values of quotients depend in part upon the intervals of time allowed for initial recovery, and upon the conditions within and without the organisms. Each species and each part of an individual in a sense competes in speed of water exchanges with each other, be it under supposedly similar or different conditions. When the numerical values of $1 / \Delta t$ are examined, the dog's recovery from deficits and the rat's recovery from excesses are found to be the most rapid; the snake's recovery from excesses is the slowest. There is no apparent relation of velocity quotient to the anatomical elaboration of organs of water exchange, though their spreads of surface are probably factors.
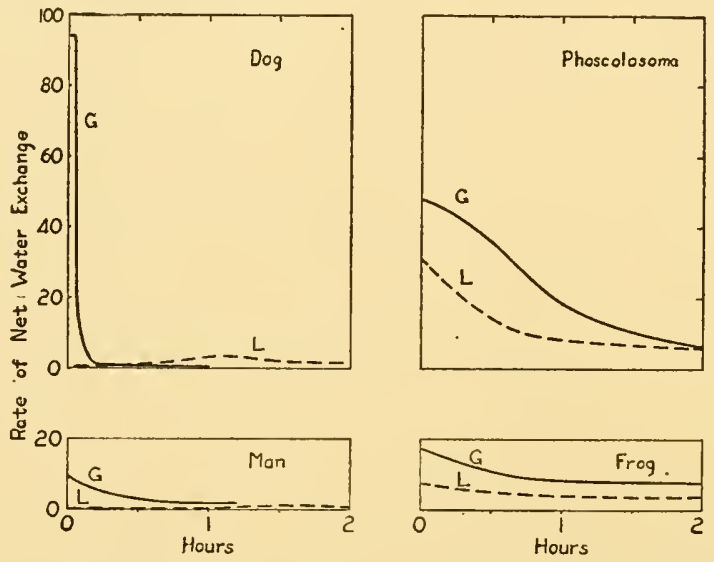

FIG. 107. Exchange curves for water in 4 species; rate of net water exchange (\% of $\mathrm{B}_{0}$ /hour) in relation to time. Gains in deficits, solid lines; losses in excesses, broken lines. Two selected average curves are represented having numerically equal initial loads. Dog, from figures 2 (A) and $10(\Delta \mathrm{W}-4.6)$; Phascolosoma, from figure $86(\Delta \mathrm{W}-24$ and +31$)$; Man, from figures $50(\mathrm{~A})$ and $57\left(\mathrm{P}_{2}\right)$; Frog, from data of figure 67.

Hence it is often possible to represent the whole of a recovery by one parameter, relating water load, rate of net water exchange, and time. In appropriate species the one parameter in turn characterizes the response to load of any magnitude at all times during the recovery. Wherever concentrations either are effective in moving water (as in Phascolosoma) or serve as stimuli to moving water (possibly in $\mathrm{dog}$ ), rate of exchange might have been expected to be proportional to load anyway. But numerous rates are proportional to load also where entirely different processes are inferred (as in intake of man). 


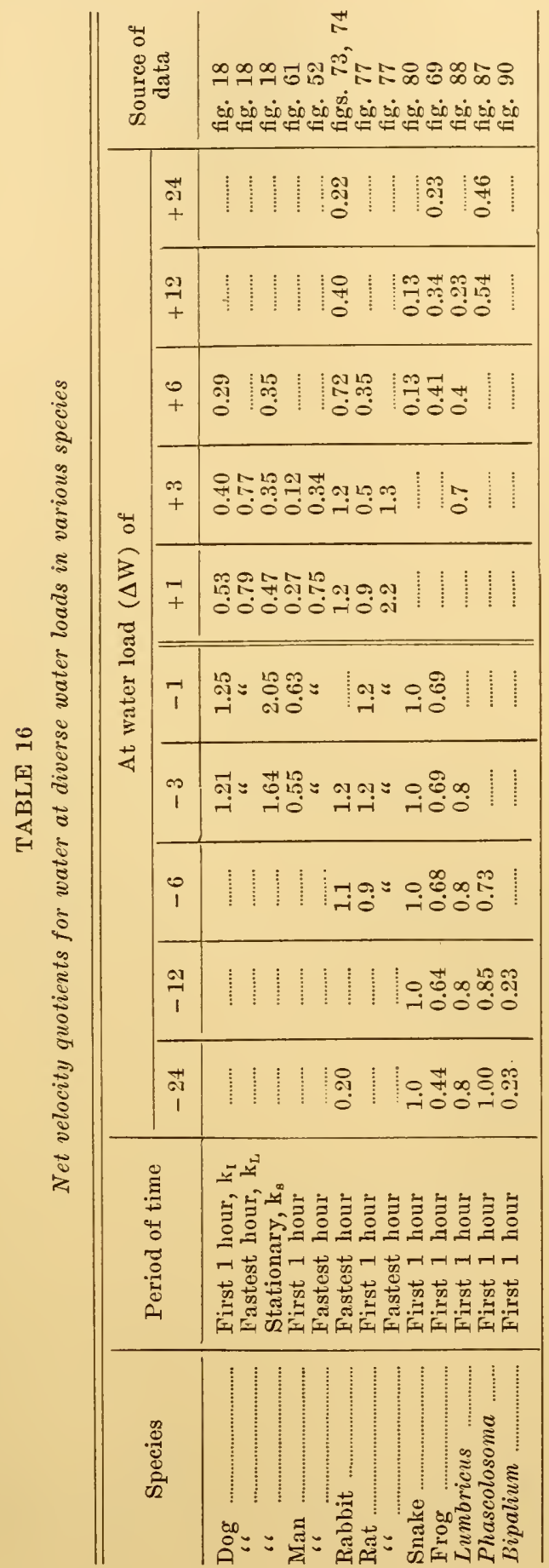




\section{\$72. Equilibration diagrams}

What modifications of water exchanges occur as responses to water increments everywhere, and what ones are limited to some kinds of organisms only? Data may be brought together upon a common scale for 5 species of vertebrates and 2 of invertebrates (figs. 108 and 109).

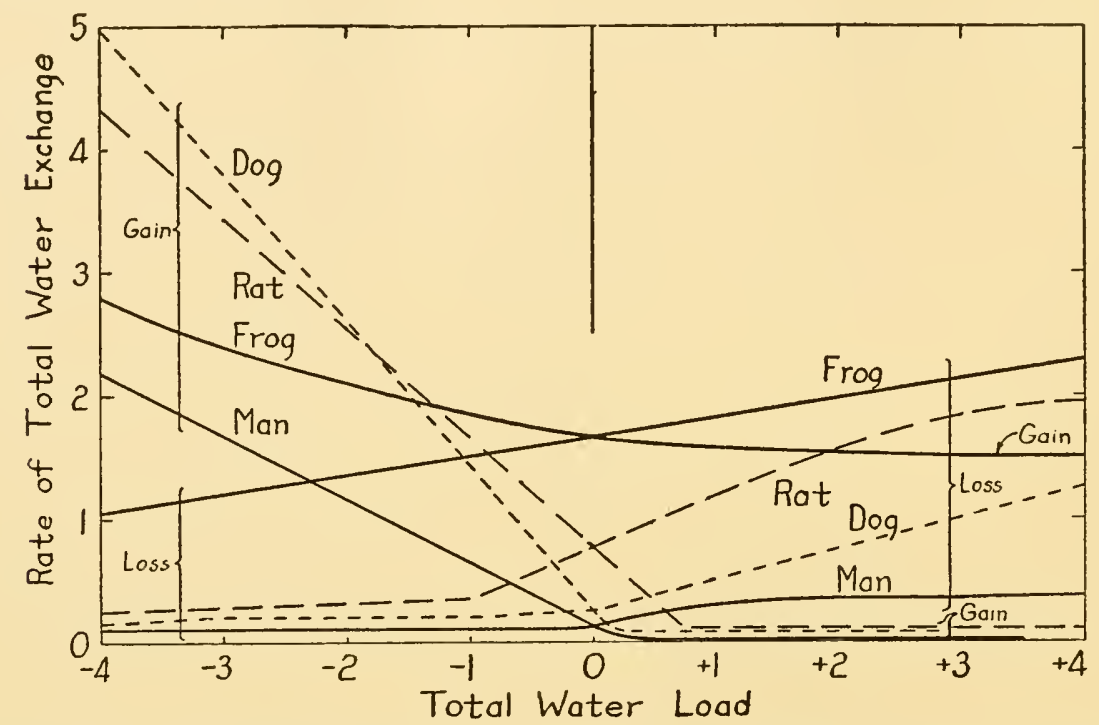

FIG. 108. Equilibration diagrams for 4 species of vertebrates. Rate of total water exchange ( $\%$ of $\mathrm{B}_{0}$ /hour) in the first 1.0 hour of recovery in relation to mean water load $\left(\%\right.$ of $\mathrm{B}_{0}$ ). Dog, from figure 13; Man, from figure 61 ; Frog, from figure 66 (corrected to 1.0 hour); Rat, from figure 73.

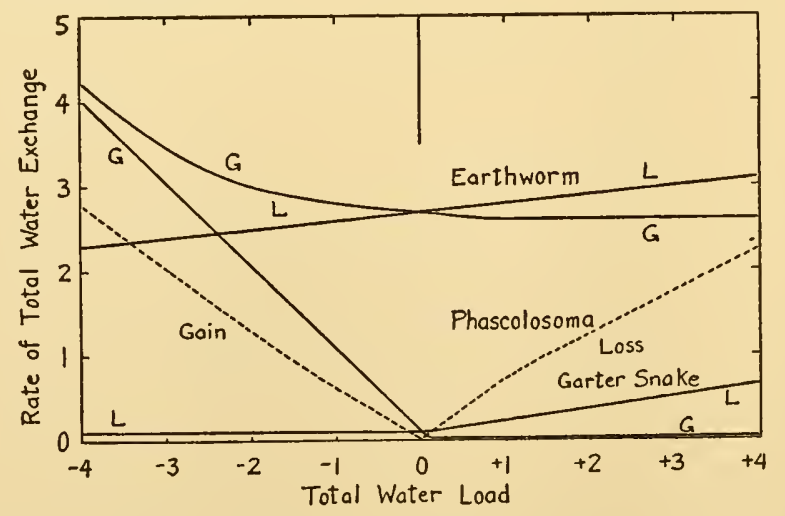

FIG. 109. Equilibration diagrams for 3 species. Rate of total water exchange in the first 1.0 hour of recovery in relation to mean water load. Earthworm, from figure 89; Phascolosoma, from figure 87 (B); Garter snake, from figure 80. 
The positions of equilibrations are indicated on the arbitrary basis of the initial 1.0 hour of adjustment. At 0.1 hour by the clock or during 2 hours by the clock, at other temperatures or during other manipulations, the positions would be otherwise.

Perhaps the general feature of first note is that each of the species studied has a means of adjusting excesses, and a means of adjusting deficits. The two means or paths are usually structur-

TABLE 17

Paths of water exchanges in various genera. $t=$ terrestrial; $s=$ seawater; $r=$ freshwater. Parentheses indicate paths that are available and used only under special conditions. Information from many sources

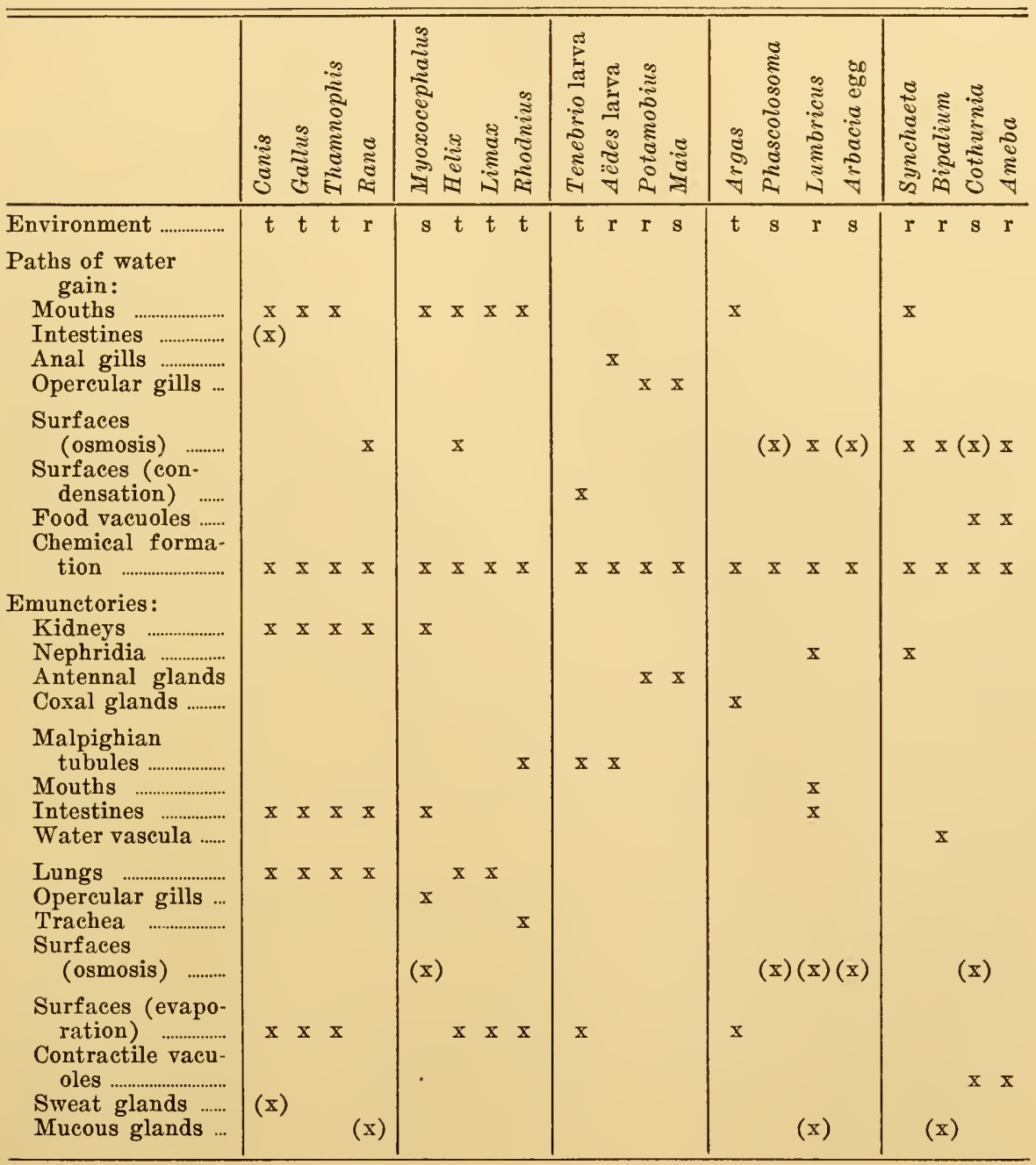


ally distinct, yet functionally they are precisely fitted to one another. The anatomical structures concerned are very diverse over the animal kingdom (table 17). The exchanges accomplished seem appropriate to recovery quite irrespective of the sort of organ provided to carry them on.

By abstraction a generalized equilibration diagram (fig. 110) is derived. So far as I can see, it is general enough to apply to

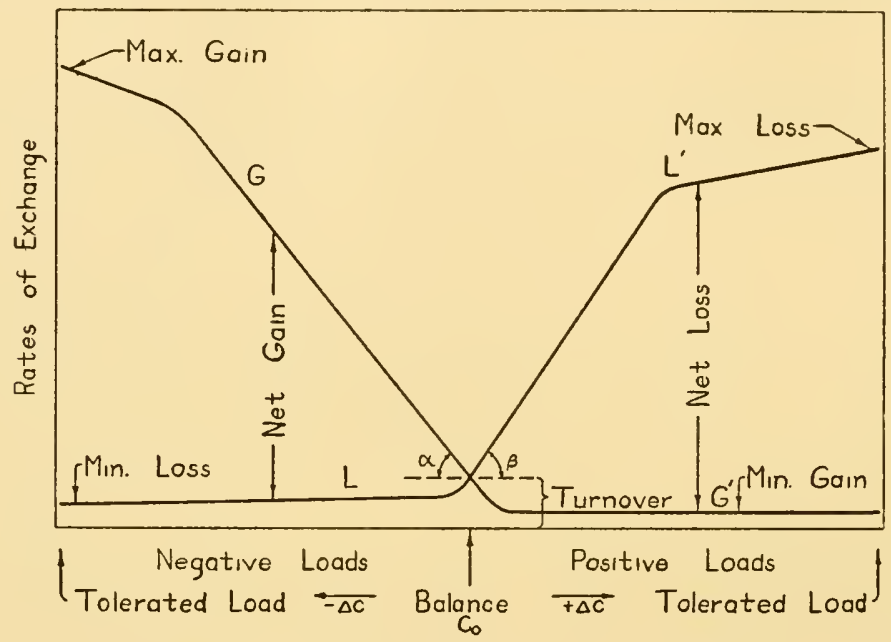

FIG. 110. Generalized equilibration diagram. The definitions of several diverse quantities are shown graphically. Others are defined as follows: $G$ or $G^{\prime}=$ Measured rate of total gain; $L$ or $L^{\prime}=$ Measured rate of total loss; $T=$ Rate of turnover, when $\mathrm{G}=\mathrm{L}$. Min. Loss $=$ the component's measured minimal rate. $\mathrm{G} / \mathrm{T}, \mathrm{L} / \mathrm{T}=$ Augmentation

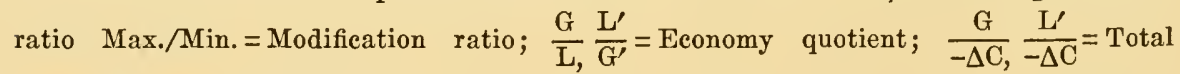
velocity quotient; $\frac{G-L L^{\prime}-G^{\prime}}{-\Delta C, \Delta C,}=$ Net velocity quotient; $\tan \alpha$ or $\tan \beta=\frac{\Delta(G-T)}{-\Delta C}$ or $\frac{\Delta\left(L^{\prime}-\mathrm{T}\right)}{\Delta \mathrm{C}}=$ Total velocity quotient in small departures from balance; $\tan (\alpha+\beta)=$ Net velocity quotient in small departures from balance.

any organism in which gains and losses of water may be separately measured.

Where gains and losses are not separated, the net equilibration diagrams (fig. 111) may be generalized. If the study had been concerned only with parts of organisms, i.e., volumes of tissues and cells, only net equilibration diagrams would have been known. Then the net equilibration diagram would not have been recognized as a partial representation of something that is more completely represented in the total equilibration diagram. 


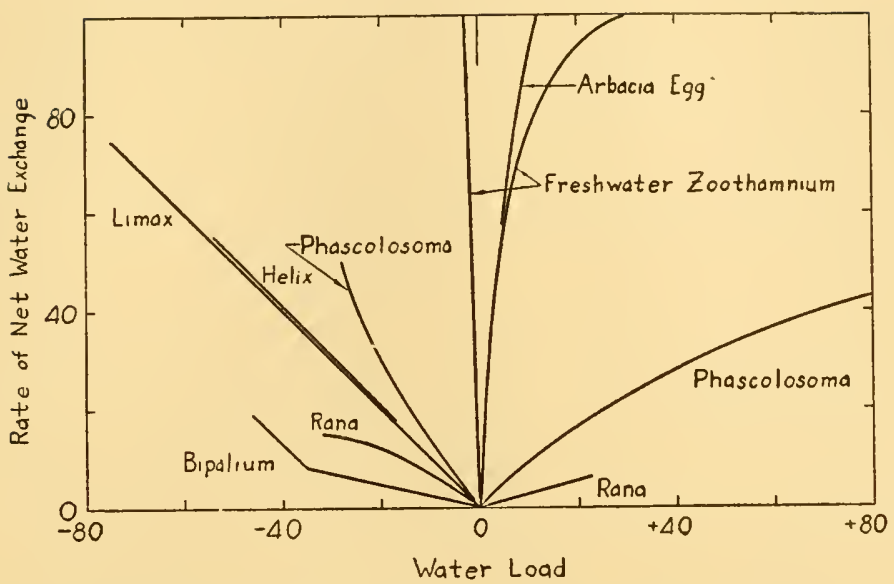

FIG. 111. Rate of net water exchange ( $\%$ of $\mathrm{B}_{0} /$ hour) in relation to water load ( $\%$ of $\mathrm{B}_{0}$ ) in 7 species of animals. The initial 0.07 to 1.0 hour of recovery is represented, except in Limax and Helix for which a period of 24 hours of recovery prevails. Frog, figure 67 ; Limax, figure 82 ; Helix, figure 83; Phascolosoma, figure 87; Bipalium, figure 90; Zoothamnium, figure 92; Arbacia egg (unfertilized), data of Lucke et al. ('31, p. 416).

Some uniformities that occur in all the instances studied are apparent in the general diagram (fig. 110).

(1) Net losses occur in water excesses, net gains in water deficits.

(2) Faster net exchanges accompany larger loads.

(3) Gains are measurably modified in all displacements from balance, but most in excesses.

(4) Losses are modified, as compared with turnover, especially in deficits.

(5) The relative positions of curves for gain and for loss are such that only small ranges of the possible rates of exchange are utilized at usual water contents.

(6) Exchanges follow similar patterns, whatever organs and processes are concerned in the exchanges of the particular species.

(7) At equal positive and negative water loads, recoveries from deficit are more rapid than recoveries from excess.

The equilibration diagram yields a general and concrete notion of physiological adjustments of water content. As the water content of the organism departs from balance, means of restoration work faster and faster in a direction to bring the content toward 
the original. Inevitably, as restoration progresses, the rates of net water exchange decrease until an apparent "resting"' state is reached. This state is an equality of gains and of losses, it is a kinetic equilibrium. Hence the maintenance of constancy on the part of the organism as a whole in the midst of flux, is represented in quantitative terms.

Organisms are continually recovering their water balances; perhaps frogs and earthworms more usually from excesses, mammals and land plants more usually from deficits. The distinction between these two tendencies is related to the environment in which the organisms exist. But both groups are equipped equally to meet the reverse situation, in which frogs are kept from water and dogs are drenched with it. Hence, although on which side of water balance the animal remains, is at times a matter of supply from the customary surroundings, each animal is physiologically prepared for a wider range of conditions and states.

Equilibration need not be limited to water content. Instead of correlating rate of exchange with load, it is equally feasible to correlate rate of change of exchange (acceleration) with rate of exchange. In that manmer the rate of gain or of loss, total or partitioned, appears as a maintained quantity. Whenever the rate has been large, deceleration intervenes toward its recovery; when small, acceleration intervenes. Such a diagram of second (or even third) derivatives also relates itself to the variabilities of rates of exchange, each more extreme rate of water gain or loss being followed more often than not by a rate that is more usual (table 12).

Qualitatively, equilibrations can be classified in a manner that is also not limited by what kinds of organs mediate the exchanges (fig. 112). (a) Some species and most parts have no recognized turnover, depending for recovery from load upon the operation of exchanges that were absent before. (b). In deficits gain may be faster while loss is unchanged; in excesses both may be modified so as to promote net loss. (c) In deficits no modification might occur, rendering recovery unlikely; but that case has not been found. (d) A familiar combination of modifications is shown, the symmetrical analog of case b. (e) In deficits, total loss may increase, but not so much as gain does; allowing possible recovery, but constituting a pattern not yet found. In excesses, total gain increases while loss remains unmodified, thereby adding to load 
and preventing recovery; that also has not been found. (f) Both exchanges are modified in both negative and positive loads in a sense that promotes recovery.

When these combinations are rated according to the number of the possible modifications favorable to recovery that are realized, the maximal is 2,2 , the minimal $-2,-2$. None of the minus modifications are known to occur, and no instances where both gain and loss are unmodified are known. Presumably the organism is doing its utmost to recover when each of the four exchanges is modified in the direction that favors recovery and by a large ratio of aug-

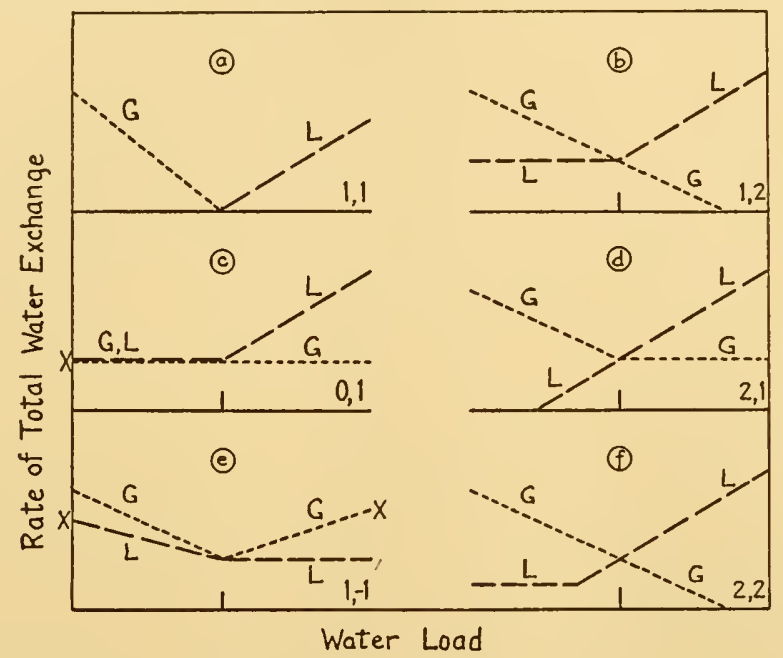

Fig. 112. Schematic representation of some possible types of equilibration diagram. $\mathrm{G}=$ gain, $\mathrm{L}=$ loss. Those marked $\mathrm{X}$ would be inconsistent with recovery, and are not known to occur. The ratings such as $(1,1)$ indicate how many of the total exchanges are modified in favor of recovery.

mentation. Here is a set of patterns that serves to classify all the living units whose water equilibrations are known.

Not all the possible combinations of modified exchanges are shown in fig. 112. No constant association is to be found between the pattern present in deficits and the pattern prevailing in excesses. Moreover, gain may increase more than loss increases, may increase less than loss increases, may decrease more than loss decreases, may decrease less than loss decreases, or may not change; loss likewise; thus allowing 11 conceivable combinations in deficit alone, and 11 more in excess alone. The total possible patterns are 121, therefore. Of them only 25 favor recovery from 
both kinds of loads, and within that number fall all the instances known.

An additional type of equilibration diagram that might be expected to occur in some species is one with a broad range of water contents within which no rapid adjustments occur. There would then be considerable leeway between unallowed excess and unallowed deficit; a stretch of water contents to which the organism is indifferent. Helix seems to correspond to this type, though figure 83 is too coarse to show it; according to Howes and Wells ('34) and previous investigator's it eats every day but ingests water only every several days. Hence large rhythmic fluctuations of water content occur, averaging at daily intervals $\pm 8 \%$ of $\mathrm{B}_{0}$ (table 12 ). Variations of less than $\pm 0.5 \%$ of $B_{0}$ are often found in $\operatorname{dog}$, man, and rabbit, giving rise to neither diuresis nor drinking. Larger excesses usually fail of complete excretion by small margins. The eamel can store much "unrequired" water in its peculiar stomach, but the amount in relation to water load and water exchanges has not been measured (Howell and Gersh, '35).

All this relates to the angles $\alpha$ and $\beta$ in figure 110. These angles measure the change of rate (of gain and of loss respectively) with increasing $\pm \Delta \mathrm{W}$, at the most used parts of the range of possible water contents. Perhaps they measure an irritability of the organism, being the increment of load that calls forth an increment in rate of exchange (response).

I do not conclude that there is any quality very unique or peculiar about the equilibration of water in living organisms. I do conclude that many, and probably all, organisms, organs, and cells have machinery for getting rid of excesses of water or preventing the accumulation of excesses, or both. In fact, with Bernard (see \$4) I doubt whether any organism exists without adjusting its content of water. But some adjust rapidly, and some slowly. Non-living systems such as gelatin blocks, water tanks, city reservoirs, and many others, have equilibrations of water, and would yield diagrams for water exchanges with some of the properties already outlined. Possibly there are constant and significant differences in such systems as contrasted with living ones, so that, for instance, in them recovery does not go to completion after a variety of displacements of water balance. Such distinctions might be valuable in comprehending the properties of both the living and the non-living systems. 


\section{$\S 73$. BEHAVIOR}

Behavior toward aqueous environment shows extraordinary variety; everyone knows that some species of organisms stay in or near water, others shun it. Those animals that have been carefully tested are able to find water more often than not; even visual accompaniments or gravitational features are used for its approximate detection. Other species (bee, fly, spider) sense water by its humidity or its touch and make few mistakes in moving toward it. Water vapor attracts rats, salamanders, wood lice, and some beetles; they frequent moist air still more when they are in water deficit. Other species avoid air that is nearly saturated with moisture (e.g., mealworm beetle). Conditioning is not found to play much role in these preferences. Behavior appropriate for the perservation of water content varies among species from the highly developed sensitivities and responses to water seen in mammals and insects, to the small provision of sensorimotor capabilities in amphibia and earthworms. But even the latter are far from indifferent to water and water exchanges.

\section{\$74. Water CONTENTS ANd tURnovers}

Animals are chiefly water. While that is widely recognized, data that will allow quantitative comparisons among contents of water are here shown (tables 18 and 19). This information can be used (1) to transform body weights to volumes of water, whereupon a number proportional to $\pm \Delta \mathrm{W}$ ( $\Delta \mathrm{W}$ being a fraction of total substance (body weight) present) may be based on water initially contained; (2) to relate water to non-aqueous materials, a ratio especially desirable in those living units where absolute weight or volume camnot be regularly and repeatedly measured; (3) to indicate what deviations may be expected in the proportions of water to non-aqueous materials. Further, it is widely believed that some materials (such as fat) are unrelated to water content, and hence they might be eliminated from consideration by subtracting their weights from both the total and the dry weights.

The specific gravities of the organisms listed vary from about 1.0 to 1.1. In this small range it is usually unnecessary, in view of other inaccuracies, to convert weights to volumes, and vice versa.

Variabilities of weight or volume in one individual maintained in supposed water balance may be compared in a number of species 
(table 12). No uniformity of conditions can be prescribed for all kinds, "natural" environments not often being suitable for obtaining the data required. Man might be best compared at 24-hour intervals, but no one would recommend that interval for Arbacia eggs (Goldforb, '35). Variabilities of weight among individuals are of little use in studies of water relations except as water content is correlated with absolute size (or with age, or other correlatives). In single individuals, high variability is found with high

TABLE 18

Water contents of various species of terrestrial vertebrates when in water balance

\begin{tabular}{|c|c|c|c|c|}
\hline Species & $\begin{array}{c}\text { Number } \\
\text { of } \\
\text { analyses }\end{array}$ & $\frac{100 \text { water }}{\text { wet wt. }}$ & $\begin{array}{l}\text { Mean body wt., } \\
\text { grams }\end{array}$ & Source of data \\
\hline 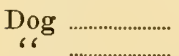 & 3 & $\begin{array}{l}57.3,57.7,62.8 \\
55,64\end{array}$ & $\begin{array}{c}3900,4790,10650 \\
5810,6270\end{array}$ & $\begin{array}{l}\text { Pfeiffer (1887) } \\
\text { Harrison et al. ('36) }\end{array}$ \\
\hline $\operatorname{Man}_{66}$ & 1 & $\begin{array}{l}58.5 \\
65.7\end{array}$ & $\begin{array}{l}69670 \\
61670\end{array}$ & $\begin{array}{l}\text { Bischoff }(1863) \\
\text { Volkmann }(1874)\end{array}$ \\
\hline ، & 2 & $66.0,67.6$ & & Moleschott, quoted by Ger- \\
\hline 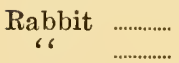 & $\begin{array}{l}2 \\
2\end{array}$ & $\begin{array}{l}63.5,60.3 \\
73.5,73.9\end{array}$ & 1617,2425 & $\begin{array}{l}\text { Pfeiffer } \\
\text { Voit ('29) }\end{array}$ \\
\hline "6 & 3 & $73,75,75$ & 2110 & Harrison et al. \\
\hline Rat ...................... & 2 & 68. & 26 & Lowrey ('13) \\
\hline “ & $\begin{array}{l}2 \\
2\end{array}$ & $\begin{array}{l}65.3 \\
66.8\end{array}$ & $\begin{array}{l}277 \\
355\end{array}$ & $\begin{array}{l}\text { Hatai ('17) } \\
\text { Hamilton and Dewar ('38) }\end{array}$ \\
\hline $\begin{array}{l}\text { Monkey } \\
\text { Cat }\end{array}$ & 2 & $\begin{array}{c}65,72 \\
64,2\end{array}$ & 4040,2870 & $\begin{array}{l}\text { Harrison et al. } \\
\text { Voit }(1866)\end{array}$ \\
\hline Mouse, white & 10 & & & Böhtlingk (1897) \\
\hline “ & 1 & 69. & & Rubner ('08) \\
\hline "6 & 38 & 70 & 2 & Benedict and Lee ('36) \\
\hline Chicken ........... & 3 & $65.2,57.7,55.3$ & $740,2630,3096$ & Pfeiffer \\
\hline Goose ................ & 5 & $71.74 \pm 1$ & 3300 & Voit ('29) \\
\hline Turtle ..................... & 1 & $\overline{7} 7$. & & Rubner ('24) \\
\hline Lizard .................... & 2 & $74.0,70.2$ & & Bezold (1857) \\
\hline & 1 & & & Rubner ('24) \\
\hline
\end{tabular}

rates of turnover and with small body sizes. With the exception of Helix, multicellular species that have been observed maintain their water contents within $\pm 3 \%$ of $\mathrm{B}_{0}$ over a period of 24 hours.

Rates of turnover of water are in each species the total amounts gained and lost again in a unit period of time. Sometimes this quantity is termed water requirement, a recognition that turnover occurs in the maintained state of the organism. Among the diverse species represented (tables 20 and 21) there are great inequalities of physiological factors under which data are obtained. To put dog, cow, rat, and frog, on the same diet, and in the same atmosphere, would be equally unsuitable. While each species is measured (with respect to mean and to variability) in a state where 


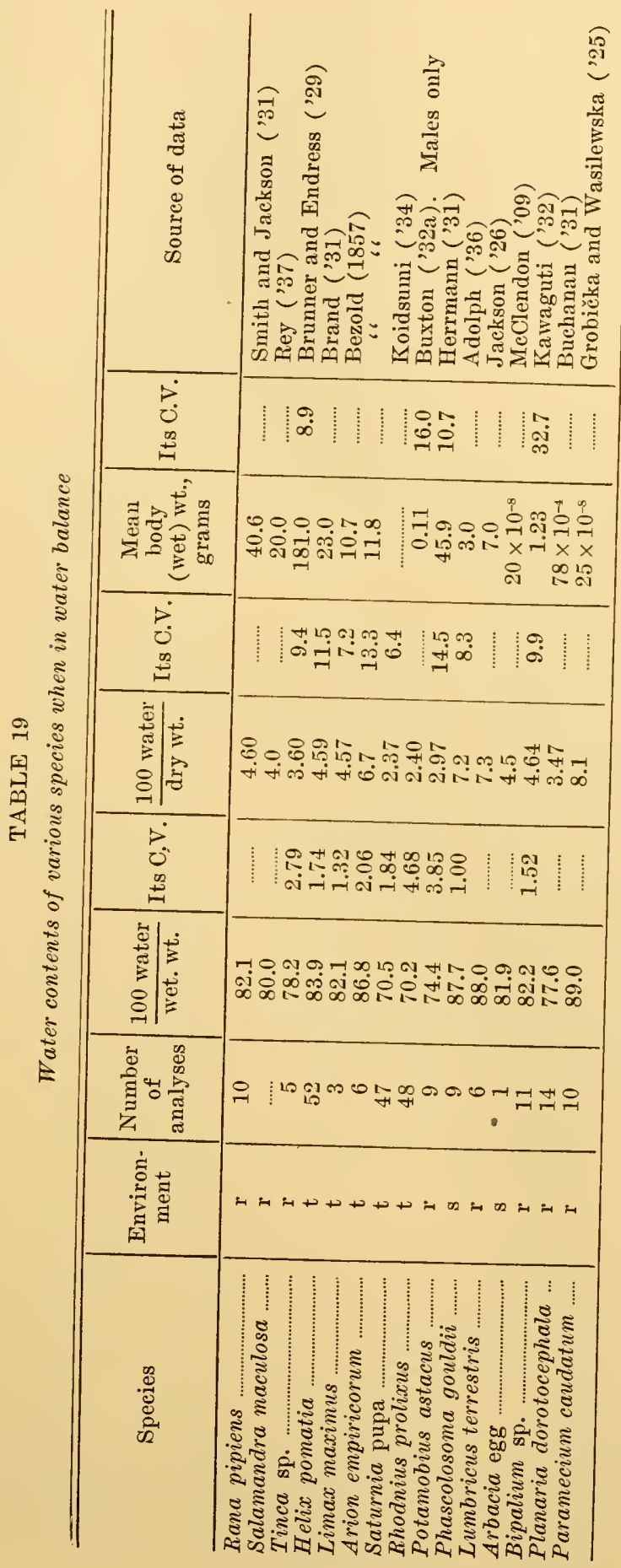




\begin{tabular}{|c|c|c|c|}
\hline 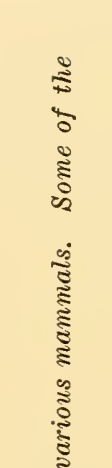 & 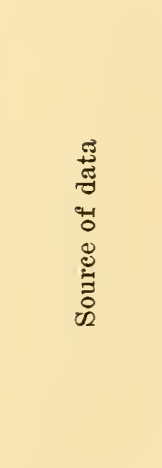 & 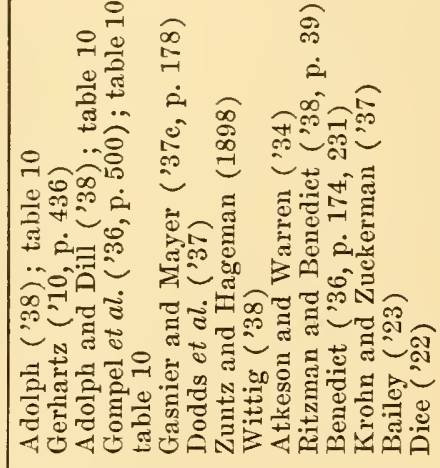 & 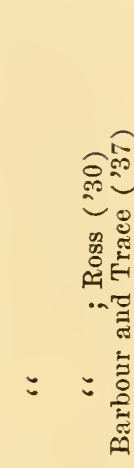 \\
\hline 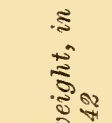 & 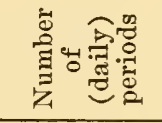 & 웍육오욕육 & 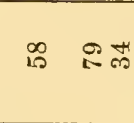 \\
\hline 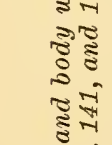 & 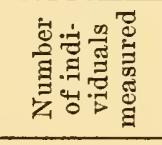 & $0 \pi+\infty \Omega$ & L 100 \\
\hline 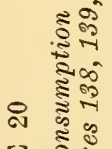 & 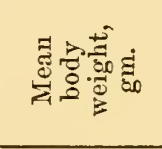 & 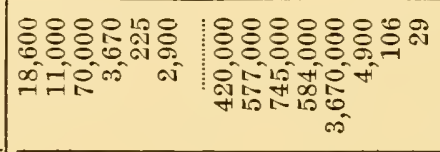 & ㅇำ \\
\hline 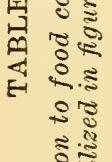 & 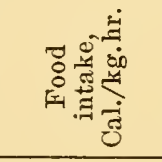 & 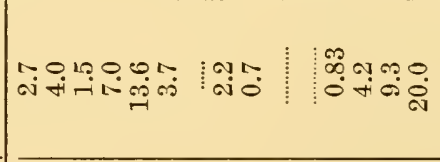 & ํ. \\
\hline 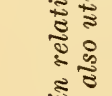 & 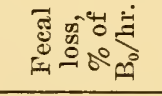 & 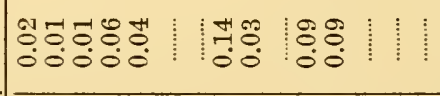 & $\stackrel{\infty}{\circ}$ \\
\hline 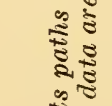 & 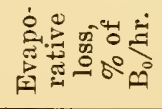 & 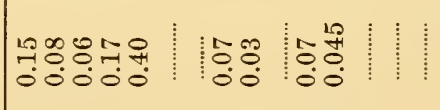 & : \\
\hline $\begin{array}{l}\text { ठे } \\
\text { है }\end{array}$ & 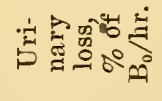 & 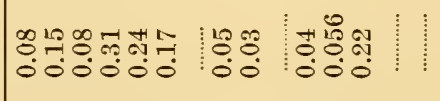 & $\underset{0}{\infty}$ \\
\hline 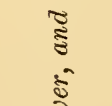 & 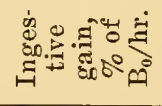 & 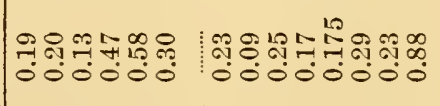 & $\begin{array}{ll}\infty & \text { N } \\
0 & 0 \\
0 & 0\end{array}$ \\
\hline 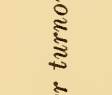 & 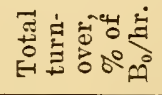 & 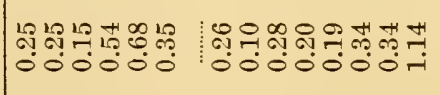 & 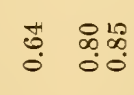 \\
\hline 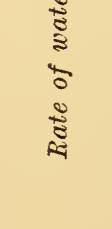 & 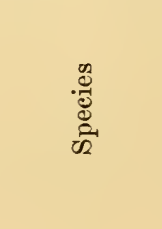 & 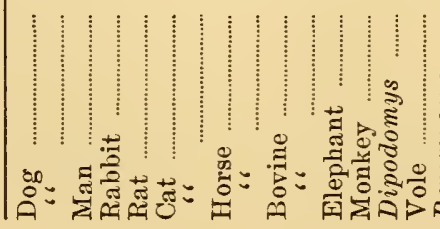 & 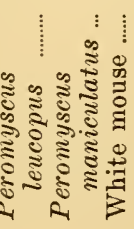 \\
\hline
\end{tabular}




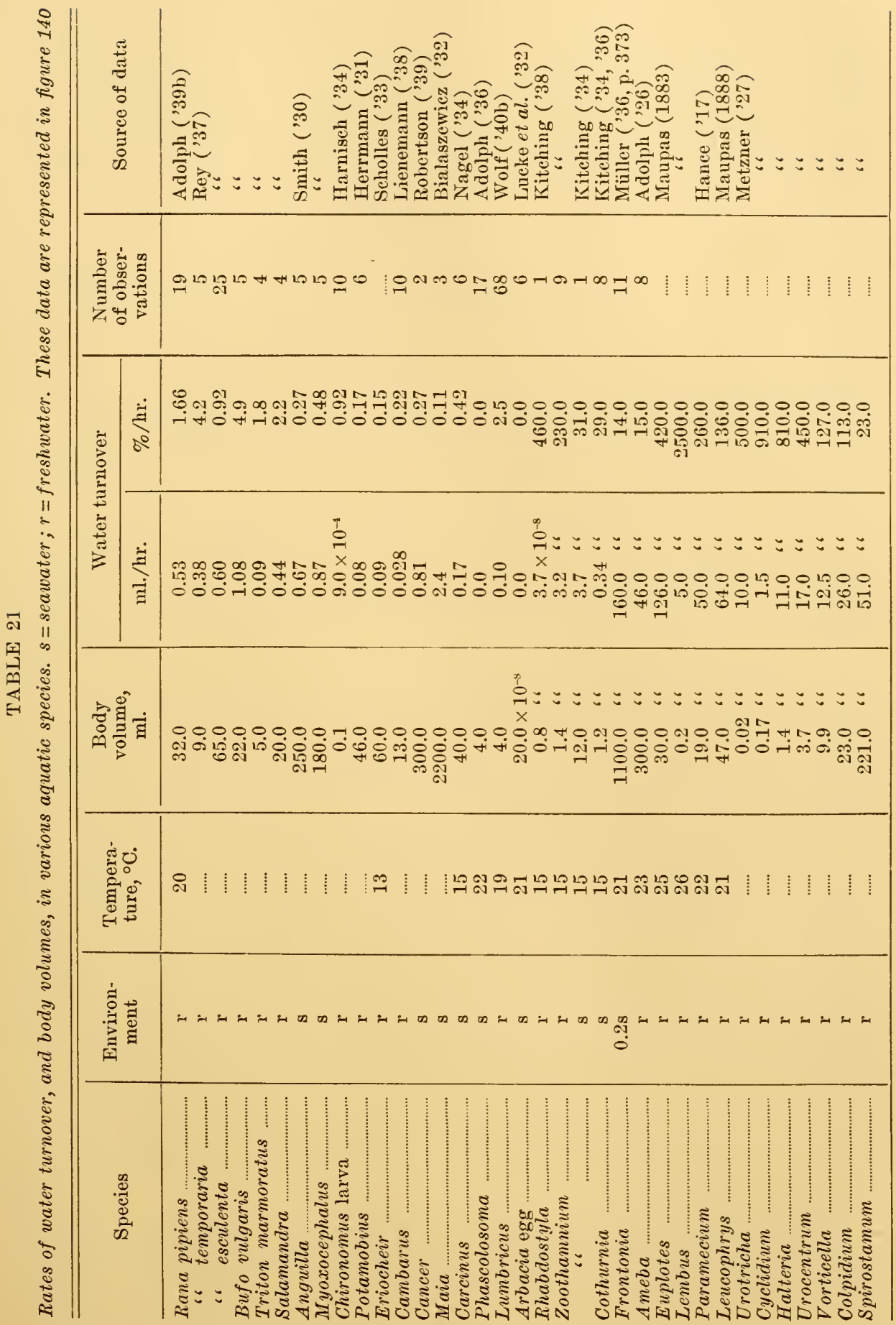


gain of water equals its loss, such a state can be any one of a variety. For instance, the cow may be lactating or not (Atkeson and Warren, '34); the horse may be working' or not ('Zuntz and Hag'emann, 1898); the man may be on a high-protein diet or not (Vozarik, '06). But none of these states makes the mammal's turnover equal to the frog's; only forced drinking does that.

The rates of turnover per unit of body weight are greater in aquatic species than in terrestrial ones; in smaller than in larger kinds; in freshwater than in marine organisms. Among invertebrates, rates of water turnover are strictly known only for some arthropods, protozoa, and the earthworm. Proportional to turnover, but regularly lower, are the rates of loss in weight in terrestrial organisms recently denied access to water. These rates of loss may be substituted for rates of turnover, being considered subminimal values of turnover. In that way values for numerous further species, especially of insects (Buxton, '37a; Gunn, '37), become available. Extremely low rates of turnover are found, as $0.03 \%$ of $\mathrm{B}_{0}$ /hour in mealworm larva, and $0.01 \%$ of $\mathrm{B}_{0}$ /hour in the tortoise (Testudo) of Benedict ('32), rivalling MacDougal's ('12) cactus that lost $0.0004 \%$ of $\mathrm{B}_{0}$ /hour. Granted that the losses are evaporative, it is possible to compare the protections against evaporation enjoyed by these species, and per unit of believed surface area. Then the mealworm larva becomes equal to the tortoise, losing about $0.0003 \mathrm{grams} / \mathrm{square}$ centimeter and hour. These particular species have the lowest known requirement for water.

A deduction from the tenets of physical science would be that any turnover of water occurs only with the transformation of some energy. Otherwise water would move through a circuitous path (through the organism) without fall of potential. Though gain might be passive, loss would require work, or vice versa. Only species without turnover may be suspected of degrading no energy for water exchanges. Yet many have been the attempts to believe that all the processes concerned in water metabolism occur without energy exchange. It is sometimes forgotten that an osomometer also transforms energy in approaching equilibrium by transport of water.

Rates of water turnover (in water balance) may be partitioned among diverse paths (tables 20, 10, and 11). 


\section{\$75. Tolerated LOADS}

What ranges of water contents are compatible with recovery? The equilibration diagrams are constructed for limited increments of water, but most of them presumably could be extended to larger deficits and excesses. For some species the limits of water content that each survives have been measured (table 22). Perhaps the

TABLE 22

Tolerated water loads in diverse species, in $\%$ of $B_{0}$

\begin{tabular}{|c|c|c|c|}
\hline Species & Deficit & Excess & Source of data \\
\hline $\log _{6} \ldots \ldots \ldots \ldots$ & -20 & ........... & Falck and Sel \\
\hline $6^{\circ} \quad \ldots \ldots \ldots \ldots$ & -24 & & Pernice and Sca \\
\hline 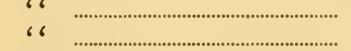 & $\ldots \ldots \ldots .$. & +21 & Falck (1872), by rein \\
\hline Man ..... & $>-10$ & & $\begin{array}{l}\text { Harding and H: } \\
\text { Dennig (1899) }\end{array}$ \\
\hline Rabbit & & +9 & Helwig et al. ('35, '38) \\
\hline Rat " ……........... & -32 & +39 & Pack ('23), Misawa ('27) \\
\hline Rat ..................... & -36 & .......... & New data \\
\hline Cat & -31 & 24 & Czeruy (1894) \\
\hline Guinea pig & $\cdots$ & $\begin{array}{l}+24 \\
+20\end{array}$ & $\underset{66}{\text { Rowntree }(26)}$ \\
\hline & -24 & ............. & Hall ('22) \\
\hline Vole ……….................. & -32 & ......... & “ \\
\hline Deer mouse ......... & -31 & $\ldots \ldots \ldots$ & " \\
\hline Chicken ………...................... & -41 & ......... & Pernice and Scagliosi \\
\hline Pigeon & $\begin{array}{l}-44 \\
-46\end{array}$ & ......... & $\begin{array}{l}\text { Schuchardt (1847) } \\
\text { Falck and Scheffer }\end{array}$ \\
\hline Thamnophis & $>-31$ & $>+18$ & New data, fig. 80 \\
\hline 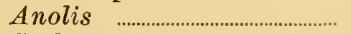 & -46 & 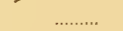 & Hall \\
\hline Sceloporus …………………. & -48 & $\ldots \ldots$ & ، \\
\hline Phrynosoma ………................... & -34 & $\ldots \ldots . .$. & “، \\
\hline 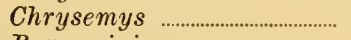 & -33 & .......... & “ \\
\hline Rana pipiens ..................................... & -41 & & $\because 6$ \\
\hline Bufo & -59 & $>+35$ & Adolph ('39b) \\
\hline 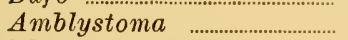 & -47 & ......... & $\begin{array}{l}\text { Rey ('37) } \\
\text { Hall }\end{array}$ \\
\hline Plethodon & -43 & .............. & Caldwell ('25) \\
\hline 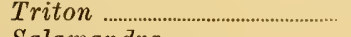 & -51 & $\ldots \ldots \ldots .$. & $\underset{6:}{\operatorname{Rey}}$ \\
\hline $\begin{array}{l}\text { Salamandra } \\
\text { Limax }\end{array}$ & -76 & 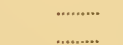 & Kunkel ('16) \\
\hline $\begin{array}{l}\text { Limax } \\
\text { Blatta }\end{array}$ & -42 & $\ldots \cdots \cdots$ & Gunn ('33) \\
\hline 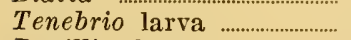 & -52 & .......... & Hall \\
\hline Popillia larva .................... & -40 & ........... & Ludwig ('36) \\
\hline Melanoplus egg .......................... & -33 & .......... & Thompson and Bodine ('36) \\
\hline 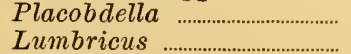 & $\begin{array}{l}-70 \\
-49\end{array}$ & $>+24$ & $\begin{array}{l}\text { Hall } \\
\text { Wolf ('40a) and new data }\end{array}$ \\
\hline 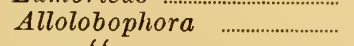 & -60 & ................ & \\
\hline Phascolosoma "………............... & -69 & & Hall \\
\hline 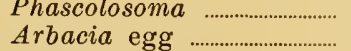 & $\begin{array}{l}-31 \\
-35\end{array}$ & $\begin{array}{l}+98 \\
+155\end{array}$ & $\begin{array}{l}\text { Adolph, fig. } 87 \\
\text { Lucke, fig. } 91\end{array}$ \\
\hline 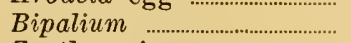 & -46 & & Kawaguti ('32) \\
\hline Zoothamnium ........................... & $>-23$ & $>+39$ & Kitching ('38) \\
\hline 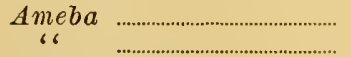 & -48 & +50 & $\begin{array}{l}\text { Mast and Fowler ('38) } \\
\text { Howland and Pollack ('27) }\end{array}$ \\
\hline Muscle of Frog & -35 & ........... & Wolf ('40b) \\
\hline Skin fragment of Frog...... & -43 & $\ldots \ldots \ldots$ & Morosow ('31) \\
\hline Heart "6 "6 ...... & $\begin{array}{l}-70 \\
-75\end{array}$ & $\ldots \ldots \ldots$ & "6 \\
\hline Heart “ & -7 & $\ldots$ & 6 \\
\hline
\end{tabular}


most questionable data are those in water privation; several days, even months, may be occupied to induce the deficit, and food is often refused by desiccated mammals and others, leading to greater deficit of weight but relatively less deficit of water than would occur from lack of water alone.

Diverse increments of water are tolerated by the various species. More factors than the proportion of water to other substances in the body are at stake. Time is one of these; with slow desiccation ( $-40 \%$ of $\mathrm{B}_{0}$ in 120 hours) frogs withstood much more loss than with rapid desiccation ( $-12 \%$ of $\mathrm{B}_{0}$ in 0.7 hour) (Almeida, '26). A thorough study of the numerous factors modifying the survival after deficits and excesses of water would be an extensive investigation. It is noteworthy that dogs are reported to survive the physiologically "crude" procedure of injecting water intravenously almost as well as (repeatedly) ingesting water (Falck, 1872; Rowntree, '23; Chiray et al., '38).

Tolerated loads might alternatively mean those increments limited by some event other than survival and recovery. Tolerances according to the criterion of vomiting, or convulsions, or anorexia, or anatomical lesions, or continuance of diverse functions, are of equal interest though automatically restricted to certain phyla. The difference between tolerance against loading and tolerance against survival is illustrated by the fact that dogs with extreme contents of water may become unable to excrete it rapidly (Harding and Harris, '30); the excretory processes are temporarily depressed, yet after a time the processes may recover and the dogs survive.

The prevention of loads by various means is a part of the regulation of water content to be considered here. A dog often resists the establishment of large excesses by vomiting; that resistance is of great moment in avoiding loads that might not otherwise be tolerated. And the dog resists the occurrence of large deficits by having a body surface that minimizes evaporation. In both cases, prevention accomplishes the same sort of stabilization of water content that recovery (compensation) accomplishes; in both the rates of exchange are the tangible evidences of processes concerned.

\section{\$76. Summary}

Beyond this chapter the formal study of water in animals will no longer be limited to the four sorts of variables and their quan- 
titative relations. Therefore the four may at this point be characterized briefly. They are:

(1) Time (t). Events are followed, beginning either with the time at which water balance is first disturbed or the time at which recovery is permitted. Completion of recovery is marked by restoration of water balance, in which state the rates of gain of water equal the rates of loss of it. Time intervals $(\Delta t)$ are diverse periods of time selected for study, differing not merely in clock units, but also in physiological significance. Initial periods of recovery are particularly distinguished from indifferent (steady) periods and from periods of maximal rates.

(2) Velocity quotients $(1 / \Delta t)$ are the reciprocals of time intervals. They characterize processes of water exchange, being obtained either by dividing rates of exchange by load, or from the analysis of exponential curves of load during recovery.

(3) Water increments $(\Delta W)$. Excesses and deficits of water contained in living unit are measured in diverse ways, often as increments of body weight. Definitions of control content are set up, both in relation to water exchanges and in relation to nonaqueous materials of the body. Diverse means of establishing increments are distinguished. Extreme increments are barely tolerated loads of water, as judged by survival or other criterion. Only moderate increments prevail in ordinary fluctuations of content measured at successive equal intervals of time.

(4) Water exchanges $(\delta \mathrm{W} / \Delta \mathrm{t})$. Gains and losses of water are total, partitional, or net. The economy quotient is the ratio of gains to losses, departing extremely from 1 whenever water balance is lost. The ratios of maximal to minimal rates indicate the modifiability of total exchanges and of their separable paths.

Equilibration diagrams, by comparing rates of exchanges with water contents, indicate the relative responses to diverse displacements from water balance. At balance the turnover rates are characteristic for the species and its physiological state.

During recoveries of water content, rates of exchange are traced in respect to time. For several species and several types of disturbance of water content, numerical comparisons, in each of the diverse dimensions, indicate the activities concerned in recovery.

Time intervals $(\Delta t)$ and the corresponding velocity quotients 
may be classed as follows. Each is measured by clock in relation to physiological events.

$(A)$ Initial interval after increment is imposed, or after recovery begins. It may be the first one hour $\left(\Delta t_{\mathrm{I}}\right)$, or the interval for half the load to be dissipated $\left(\Delta t_{\mathrm{H}}\right)$.

$(B)$ Uniform interval, often in a stationary state $\left(\Delta \mathrm{t}_{\mathrm{S}}\right)$.

(C) Interval containing maximal rate of exchange $\left(\Delta t_{\mathrm{L}}\right)$.

(D) Interval including complete recovery $\left(\Delta \mathrm{t}_{\mathrm{R}}\right)$.

$(E)$ Instantaneous intervals along the time axis $\left(\Delta \mathrm{t}_{\mathrm{M}}\right)$.

Types of increment of water content $(\Delta \mathrm{W})$ may be grouped. Each is measured on one species by one method, such as body weight, liquid volume, ratio water/dry weight.

(A) Net deficits. (a) By water privation. (b) By hypertonic solute. (c) By previous exosmosis. Etc.

$(B)$ Net excesses. Each may follow single or repeated administrations of water (a) By mouth or stomach. (b) By injection : by vein, under skin, into peritoneal cavity. (c) By previous endosmosis. Etc.

(C) Net random changes. (a) Control conditions. (b) Unusual temperatures. (c) Unusual physical exercise. Etc.

$(D)$ Gross exchanges, $\pm \delta \mathrm{W}$ per unit $\Delta \mathrm{t}$. Each may or may not be corrected for control rates. According to paths as follows: (a) Gain: alimentary, metabolic, cutaneous. (b) Loss: urinary, vacuolar, fecal, evaporative. (c) Both: osmotic.

The bases for these classifications are, as in all classifications, those of dimensions and of utility in thought. While $\Delta \mathrm{W}$ and $\Delta \mathrm{t}$ may be varied in infinite types and intervals, only two algebraic signs (deficit, excess) prevail in $\Delta \mathrm{W}$ and only one sign in $\Delta t$. The varieties (types) of $\Delta \mathrm{W}$ and of $\Delta \mathrm{t}$ are not infinite in number but correspond to procedures and conditions for observations and measurements. In other words, types of load and classes of time intervals are treated as discontinuous variables, leaving the four continuous variables to be graded according to their numerical values. Each category could, of course, be subdivided further or in a different fashion. Thus, with respect to urinary loss, one nephron could be studied; with respect to metabolic gain, the water formed from one precursor or in one organ could be measured. In general, while the four sorts of variables are distinguished by their physical dimensions, the subdivisions depend on physical and 
chemical conditions, physiological states, anatomical units, and physiological outcomes.

The number of varieties of water load explicitly mentioned in the dog alone is about 15 (chapter III). The number of varieties of $\Delta t$ considered is about 12 , even though an arbitrary man-made clock is used in obtaining them all. Among 15 kinds of $\Delta \mathrm{TV}$ with 12 kinds of $\Delta t$, a product of 180 indicates the combinations that might be studied if I were concerned merely in rearranging coordinates.

Uniformities among the water relations studied are features common to all the organisms investigated, while quantities limited to alimentary tracts or to osmosis do not apply in organisms devoid of them. In this way the account comes to consist of the materials of general physiology, and only further inquiry will show just how general the present conclusions are. Some of the recognizable features are: (1) The species studied lose water faster when water content is high and gain water faster when content is low. Rates of gain and of loss are equal at one water content (balance), to which the organism recurs after each disturbance and at which alone exchanges rest. (3) Rates of water exchange are correlatives of water content. They are not always functions of the time elapsed since displacement or since recovery began. (4) Increments of water content exceeding 10 per cent of the water already present are tolerated by all species tested. Certain other generalizations are mentioned above in $\$ 72$. 


\section{Chapter $\mathrm{X}$}

\section{SOME OTHER CORRELATIVES OF WATER CONTENT (IN DOG)}

$\$ 77$. The maintenance of water content has now been examined in relation to water exchanges. Ordinarily a gradual turnover of the body's water is occurring; extraordinarily one exchange (in deficit the gain) augments while the other exchange (in deficit the loss) often diminishes. Where separate exchanges are not observed, net exchanges are still of the same character as where simultaneous gain and loss were separately examined; from which I infer that the pattern is the same in both cases but is incompletely visible in one.

Perhaps the limitation of the study to water exchanges narrowed the view as much as it intensified it. Are exchanges the only processes that vary with water content? They seemed to explain how content is maintained and restored; but many other variables may or may not be equally concerned in water regulation. The possibilities can be tested only by broadening the horizon.

Beyond the intensive investigation of the relations among the four sorts of variables within the water-time system, the inquiry might be pushed out in any of a large number of directions. A few topics that will be followed are:

(1) Diverse correlatives of water content or load (chapters $\mathrm{X}-\mathrm{XII})$;

(2) Equilibration and variability of diverse components (chapters XIV-XV);

(3) Interrelations among components in balances and equilibrations (chapter XVII).

These directions depend upon arbitrary choices, and there is at present no evidence that thorough study of any other combinations of variables would be less fruitful. The purposes in mind are to see how precisely water balance and its regulation can be defined; and then to compare many regulations with that of water in order to find how general the features of equilibration, regulatory behavior, and variability may be.

Water content, which has been scrutinized particularly in relation to water exchanges and to time, is equally related to many 
other types of variables. How these many correlatives vary together will be shown by a study in which $\pm \Delta W$ (water load) is kept as one variable throughout, letting one after another of the many physiological quantities that are measured in relation to it, come under review. Each of them is a part of the complex that goes with water load. The study will be designed to show whether anything that changes with water load inside the dog furnishes a key to understanding what prompts recovery in the body as a whole.

Ideally, the water load might be of one type in all divisions of the study. Experiments would be set up with dogs deprived of water as defined in $\$ 13$ and with dogs administered water by stomach as defined in $\$ 12$. Then, within the limits of the stationary state of water load, diverse analyses and measurements would be made. No such ideal program has been carried through; and not from the difficulty of planning it with singleness of purpose, but from the realization that a strictly steady state with respect to all measurable components (e.g., chloride content of body, heat content of body) does not exist. Practical difficulties of other sorts creep in, and for the present it seems sufficient to indicate, by means of the partial data from diverse sources that are available, what relations are known to exist.

First, within one species (dog) several types of changes in water content will be considered. Those correlatives that are peculiar to one type will be detected, and hasty generalizations as to what characterizes all water loads may be avoided.

\section{§78. VOLUMES OF PARTS}

It is not hard to suppose that endless changes occur in diverse parts of a dog with each load of water. Point is given to the search for those changes as soon as it is asked: Where is the excess water deposited, or whence is the deficit withdrawn? Are there depots for water; what does a reserve of water look like? And, are some tissues especially sensitive to water loads? Evidently volumes of tissues have to be measured, and not in relation to the tissue's own exchanges (as in $\$ 58$ ) but in relation to the whole body's water content.

At least three general procedures are suitable for measurement of increments of volume in any one portion of the dog's body. (a) The difference of net weights or volumes of parts secured at 
autopsy (table 23, column 2) from individuals subjected to water loads and from those not loaded. If $\mathrm{F}$ is the fresh weight (wet weight) of the part, then $100\left(\mathrm{~F}_{\mathrm{i}}-\mathrm{F}_{0}\right) / \mathrm{F}_{0}=\Delta \mathrm{V}$ is the change of volume. But the weights of any one organ relative to the control body weights among different autopsied individuals vary (Engels, '04; Stewart, '21; Sato, '30) to a degree that makes many comparisons of organ sizes under diverse water loads of little statistical significance. (b) In one and the same individual, some organ sizes may be mechanically compared before and after water is adminis-

TABLE 23

Volume $(\Delta V)$ and dilution $(\Delta E)$ in various tissues of growing dogs when water-loaded by consuming diets deficient in water. Values in parentheses are clearly insig-

nificant $(P>0.05)$; some others may be also

\begin{tabular}{|c|c|c|c|c|c|c|c|c|c|}
\hline \multirow[t]{2}{*}{ Tissue } & \multicolumn{2}{|c|}{$\begin{array}{l}\text { Falck and } \\
\text { Scheffer } \\
(1854 b)\end{array}$} & \multirow{2}{*}{$\frac{\begin{array}{l}\text { Sehiff } \\
\text { et al. } \\
\left({ }^{\prime} 25\right)\end{array}}{\Delta / D}$} & \multirow{2}{*}{$\begin{array}{l}\text { Garo- } \\
\text { feano } \\
\text { et al. } \\
(' 25)\end{array}$} & \multicolumn{5}{|c|}{ Hamilton and Schwartz ('35) } \\
\hline & $\Delta \mathrm{V}$ & $\Delta / \mathrm{D}$ & & & $\Delta / \mathrm{D}$ & $\Delta / \mathrm{Cl}$ & $\Delta / \mathrm{Na}$ & $\Delta / \mathrm{K}$ & $\Delta /$ base \\
\hline $\begin{array}{r}\text { Nun } \\
\text { ar }\end{array}$ & & & 2 & 1 & 6 & 6 & 6 & 6 & 6 \\
\hline Whole body... & -21 & -19 & $-26(?)$ & -18 & $-20(q)$ & & $\ldots \ldots \ldots$ & $\ldots .$. & $\ldots \ldots \ldots .$. \\
\hline 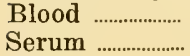 & +8 & -19 & & $\ldots \ldots . .$. & & -19 & $\ldots$ & $\ldots \ldots \ldots .$. & $\begin{array}{r}\cdots \cdots \\
-9\end{array}$ \\
\hline 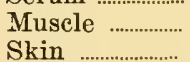 & $\begin{array}{l}-20 \\
-19\end{array}$ & $\begin{array}{l}-11 \\
-24\end{array}$ & -31 & . & $\begin{array}{l}-41 \\
-54\end{array}$ & $\begin{array}{c}(+12) \\
-24\end{array}$ & -7 & $\begin{array}{l}-32 \\
(-27)\end{array}$ & $\begin{array}{l}-19 \\
-32\end{array}$ \\
\hline nn.......... & $\begin{array}{l}-19 \\
+\quad 7\end{array}$ & -11 & & $\ldots \ldots \ldots$ & & & & & \\
\hline$\ldots \ldots \ldots$ & -15 & -2 & -11 & -4 & -1 & -34 & -33 & $(-14)$ & $(-19)$ \\
\hline ............... & +21 & \pm 0 & -14 & $\ldots \ldots \ldots$ & - & -2 & -22 & -1 & -15 \\
\hline $\mathrm{Ki}$ & +4 & -2 & -8 & & -1 & -26 & -37 & \pm 0 & $(-7)$ \\
\hline is ................ & -27 & -12 & $\ldots$ & -15 & .................. & ........................ & $\ldots . . . . . .$. & -..................... & ................. \\
\hline Th & -20 & -2 & $\ldots$ & -12 & ...... & .................. & $\ldots \ldots \ldots$ & ....................... & $\ldots \ldots \ldots$ \\
\hline ysis ... & ............ & $\ldots \ldots \ldots . .$. & ....................... & -29 & .................... & .................. & $\ldots \ldots \ldots$ & ...................... & $\ldots \ldots \ldots$ \\
\hline & & $\ldots \ldots \ldots .$. & ............... & -13 & & .................. & $\ldots \ldots \cdots$ & ................... & $\ldots \ldots \ldots .$. \\
\hline Stomach & -7 & -9 & . & .......... & & 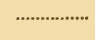 & $\cdots \cdots \cdots$ & 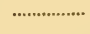 & $\cdots \cdots \cdots$ \\
\hline
\end{tabular}

trated to the whole body, such as spleen (Barcroft et al., '25) and liver (Reid, '29). Ordinarily anesthesia and other conditions are introduced that need to be specified. This procedure has not yet contributed data in those types of water load in which other correlatives were studied in the present investigation. (c) Volumes of distribution may be ascertained repeatedly in one individual. Of particular interest is the congruence of increment in volume of distribution $\left(\Delta \mathrm{V}_{\mathrm{D}}\right)$ with the load of water in the whole body $(\Delta \mathrm{W})$.

Among individuals without water load, two measured volumes (fig. 113) appear to be correlated not only with body size, but relative to body size with one another. As measured, the "plasma" 


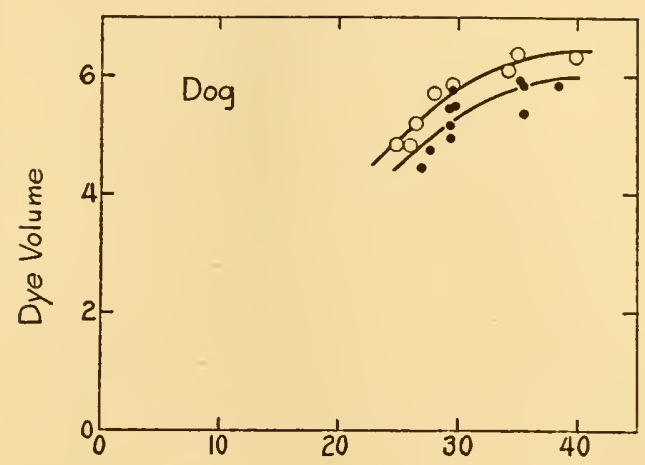

Thiocyanate Volume

Fig. 113. Volume of distribution of the dye T 1824 ("plasma" volume) (\% of body weight) in relation to volume of distribution of thiocyanate ("extracellular" volume) (\% of body weight). Dogs under control conditions; 15 individuals, arerages of 73 measurements. Open circles, males; solid points, females, of which 4 are represented by 2 points each. Data of Gregersen and Stewart ('39).

(T 1824) volume increases with the "extracellular" (thiocyanate) volume. Male individuals have consistently higher ratios than females.

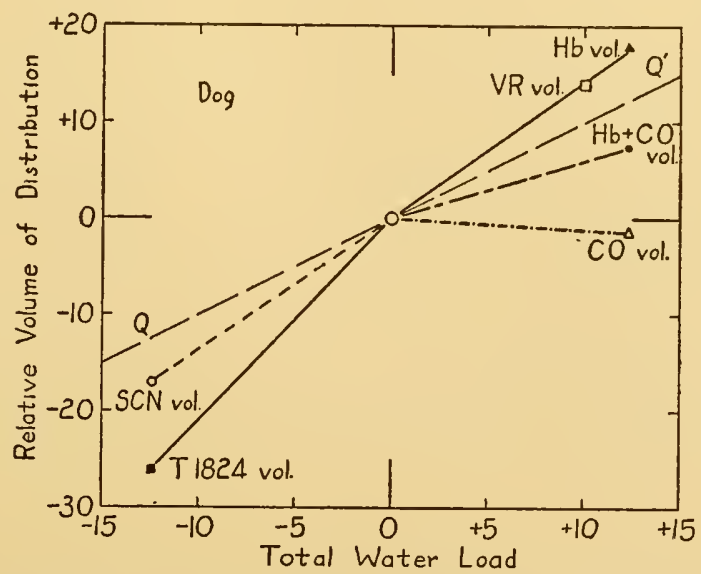

FIG. 114. Increment in volume of distribution (\% of $\left.\mathrm{V}_{0}\right)$ in relation to mean total water load $\left(\%\right.$ of $\left.\mathrm{B}_{0}\right)$. Dog. Line $\mathrm{QQ}^{\prime}=$ load would be equal in partial volume and in whole body. SCN vol.=thiocyanate ("extracellular fluid") volume in 14 individuals deprived of water and food; data of Gregersen and Painter ('39). $T$ 1824 vol.=dye ("plasma") volume in same 14. $V R$ vol=brilliant vital red ("plasma") volume in 10 individuals repeatedly given water by stomach for 8 hours; data of Greene and Rowntree ('27). Hb vol.= dissolved hemoglobin ("plasma") volume in 5 individuals given water by stomach during 1.5 hours; data of Lee, Carrier and Whipple ('22). CO vol. $=$ carbon monoxide ("erythrocyte") volume in same 5 tests. $H b+C O$ vol. $=$ hemoglobin plus CO ("circulating blood") volume in same 5 tests. 
In dogs subjected to either positive or negative water loads the reputed plasma volumes (Hb, VR, T 1824; fig. 114) change by larger fractions than the body as a whole. Unfortunately the water load is not too accurately known in any of the animals represented; but there are additional data (in water deficits) to confirm the conclusion that presumed plasma decreases in volume by more than its proportional share (Korth and Marx, '28; Keith, '22). The volume of "erythrocytes" in circulation ( $\mathrm{CO}$ ), as measured independently by carbon monoxide distribution, does not change appreciably with water" excess; leaving the "combined blood" volume ( $\mathrm{Hb}$ and $\mathrm{CO}$ ) with less load than the whole body. The "extracellular" volume (SCN) in deficit decreases somewhat more than the average water load $(\Delta \mathrm{W})$.

A favorite prediction would be that all volumes of distribution increase and decrease in proportion to the body load of water. So far no one volume is found exactly to fit this notion; the data are not extensive enough to decide for others than those here shown and for $V_{\mathrm{D}}$ of bromide (Brodie et al., '39).

There seems to be no doubt that most of the tissues whose increments of volume have been thus crudely measured, contain extra water when the whole body has an excess, and are depleted of water when the whole is in deficit. The modifications of water content in some tissues are numerically equal to load of the whole, but in other tissues are distinctly greater or less. Plainly the body's load of water is unequally distributed through its parts.

\section{\$79. Water contents (Dilutions) of parts}

To avoid possible confusions, I separate the results based upon volumes measured in a manner capable of determining absolute quantities, from those in which changes of concentration are ascertained.

For these particular tests, excesses of water are produced within 2 or 3 hours, a time too short to allow many chemical contents of the body as a whole to change. The data are all obtained between meals, and diet need not then enter the picture. On the other hand, deficits of water are produced by partial privations lasting many days, as illustrated in the data of Falck and Scheffer (1854b). Two young dogs of the same litter were analyzed (table 23 , column 3 ), one at 76 days of age, the other at 104 days of which the intervening 28 days were spent on a diet of dry "Zwiebach." 
Hence factors of growth in size, diet, time, age and constitution entered into the differences of the two individuals. Each dog was killed, its organs were excised and weighed, and the proportion of water in each organ was ascertained by a particular analytical procedure of drying. The changes of body weight and therefore of total water load ( $\Delta W=-21)$ between "control" and "desiccated" are known; what is the change in each portion of the body?

First it is necessary to decide how the analyzed water contents of the same tissue in each of the two individuals shall be compared. The data known are: wet weight (F), dry weight (D) and water weight $(\mathrm{E}=\mathrm{F}-\mathrm{D})$. The ratio $\mathrm{E} / \mathrm{F}$ is tabulated by Falck and Scheffer ; but $\mathrm{F}$ is itself changing with water privation. The known fraction that is believed to change little or none is D ; or, comparable samples of tissues are those in which $\mathrm{D}_{1}=\mathrm{D}_{0}$. Hence the ratio $\mathrm{E} / \mathrm{D}$ is the basis of comparisons; in order to make its increments comparable to increments of body water $(\Delta W)$ as first defined, it may be corrected to fresh weight and \% of $\mathrm{F}_{0}$ by multiplying by $100 \mathrm{E}_{0} / \mathrm{F}_{0}$.

$$
\frac{100 \mathrm{E}_{0}}{\mathrm{~F}_{0}}\left(\frac{\mathrm{E}_{1}}{\mathrm{D}_{1}}-\frac{\mathrm{E}_{0}}{\mathrm{D}_{0}}\right) / \frac{\mathrm{E}_{0}}{\mathrm{D}_{0}}=100\left(\frac{\mathrm{F}_{1} \mathrm{D}_{0}}{\mathrm{~F}_{0} \mathrm{D}_{1}}-1\right)=\Delta \mathrm{E}
$$

This is the amount of water added to or lost from 100 units of original weight. The increment is all water, however much nonaqueous weight may be included in $\mathrm{F}_{0}$.

The ratio $\Delta \mathrm{E}$ has the same dimensions as $\Delta \mathrm{W}$ and $\Delta \mathrm{V}$, for in both cases the water lost or gained is in per cent of the unit analyzed. Since $\Delta \mathrm{E}$ may be of many types, the type of measurement from which $\Delta \mathrm{E}$ is derived is more specifically designated by the abbreviation $\Delta / \mathrm{D}$, or dilution of dry substance. Both measures, $\Delta \mathrm{V}(=\Delta \mathrm{W}$ in this case) and $\Delta \mathrm{E}$, can be compared for the whole animals of Falck and Scheffer (1854b) :

$\Delta \mathrm{V}=\Delta \mathrm{F} / \mathrm{F}_{0}=-\frac{660.19 \mathrm{gm} .}{3178.72 \mathrm{gm} .}=-20.78 \%$ of $\mathrm{F}_{0}$ (or of $\mathrm{B}_{0}$ in this case) $\Delta \mathrm{E}=\Delta / \mathrm{D}=-\frac{552.78}{3178.72}=-17.39 \%$ of $\mathrm{F}_{0}$ (or of $\mathrm{B}_{0}$ in this case)

The comparison indicates that the dog deprived of water lost nonvolatile materials too, and the two measures of water loss differ (by 18 per cent of their mean). A similar comparison may be made for each tissue mass as weighed and as analyzed (table 23, columns 2 and 3 ).

Measurements of dilution are not limited to analyses by volatili- 
zation of water. Any ratio between a changing fraction and a more fixed fraction, is suitable, and is a type of the general class $\Delta \mathrm{E}$. Thus, instead of total solids (D), the content of chloride ( $\mathrm{Cl}$ ) or of nitrogen $(\mathrm{N})$ or of protein $(\mathrm{Pr})$ may be substituted. The nonprotein fraction need not be ascertained as such, since it is always equal to $(\mathrm{F}-\mathrm{Pr})$. Hence, if $\mathrm{Pr}$ is the per cent of protein by weight, $\Delta \mathrm{E}=\Delta / \operatorname{Pr}=100-\operatorname{Pr}_{0}\left(\frac{100-\operatorname{Pr}_{1}}{\operatorname{Pr}_{1}}-\frac{100-\operatorname{Pr}_{0}}{\operatorname{Pr}_{0}}\right) / \frac{100-\operatorname{Pr}_{0}}{\operatorname{Pr}_{0}}=100\left(\frac{\operatorname{Pr}_{0}}{\operatorname{Pr}_{1}}-1\right)$ A non-chemical measure may equally serve, such as excess specific gravity over that of water, excess refractive index, or increment in depth of color. Then refractometer reading of tissue minus refractometer reading of water $=R I$, a measure of the concentration of non-aqueous constituents, and

$$
\Delta \mathrm{E}=\frac{\Delta}{\mathrm{RI}}=\frac{100}{R \mathrm{I}_{1}}-\left(\frac{100}{\mathrm{RI}_{1}}-\frac{100}{\mathrm{RI}_{0}}\right) / \frac{1}{\mathrm{RI}_{0}}=100\left(\frac{\mathrm{RI}_{0}}{\mathrm{RI}_{1}}-1\right)
$$

From the data of table 23, it is concluded that in water deficit diverse tissues lose water to different extents. While the whole body loses 18 to 20 per cent, the skin, hypophysis, and sometimes muscles, lose more. Most other tissues lose less. The four investigations of tissue dilutions of dry substance $(\Delta / \mathrm{D})$ that are compared, do not show close quantitative agreement. In one investigation (columns 6 to 10) five measurements of dilution were made upon each tissue sample. These also show little agreement, indicating that electrolytes as well as water are redistributed. In each of the latter tests, 4 to 13 days elapsed in water privation; the body weights were not reported in detail, leaving no adequate criterion of body load of water.

Also in water excess, produced in morphinized dogs by infusion of $0.10 \mathrm{M}$ solution of sodium chloride by vein, both relative and absolute increments of water were studied in various tissues (Engels, '04) (see table 29, column 2, §91). The tissues modified more than the average for the whole body are the same as those modified in water deficits.

Often it is supposed that some tissues act as depots, from which water is mobilized in deficits, to which water is brought in excesses (Magnus, '00). How shall a depot be defined? Evidently it has to do with storage of water. Shall I look for a tank (as the camel is said to have), that fills up whenever water content of the body exceeds a certain value and that empties at some lower values (lines $\mathrm{MM}^{\prime}$ or $\mathrm{NN}^{\prime}$ in fig. 115) ? If I do, I find none in the dog. Or, 
perhaps the gates of the depot do not open quite so suddenly and completely (line $\mathrm{OO}^{\prime}$ ). Again, there is none of that sort for water. Maybe any tissue that takes up more water than the average for the body (line $\mathrm{PP}^{\prime}$ ) will be counted as a store. Of that there is evidence, as in the volumes of distribution of SCN, T 1824, and VR in figure 114, and in the tissue dilutions of tables 23 and 29, but no one part of the body seems to stand forth as a distinct store. All tissues share the water load in diverse degrees. If $\Delta \mathrm{V}>\Delta \mathrm{W}$ defines a store, is the tissue whose $\Delta \mathrm{V}<\Delta \mathrm{W}$ an anti-store? It seems to me that for water, at least, the notion of depots is not substantiated sufficiently to use the term; the increments found in each tissue correspond rather to equilibria of partition.

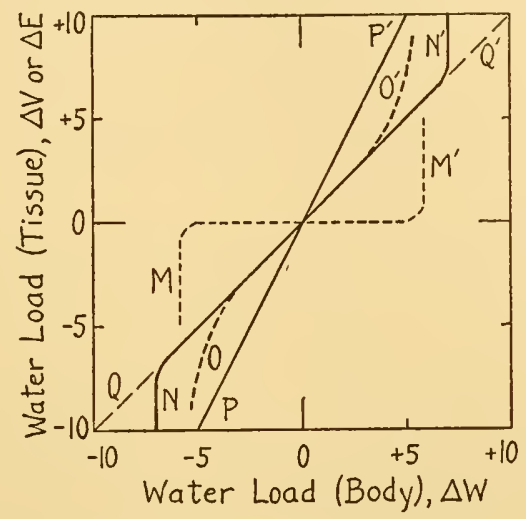

FIG. 115. Diagram of possible relations of water load in a tissue to water load in body. Various lines are described in the text.

In brief, methods are available for ascertaining by how much a given tissue exceeds in water increment the water increment of the whole body. In this way the distribution of water is found to be uneven. Every analyzable unit and every volume of distribution is a compartment, but no very large ratios of partition appear between the units.

\section{$\$ 80$. Dilutions of Blood and plasma}

The circulating blood may play a peculiar role in recoveries of water content in at least two ways. It is undoubtedly the chief vehicle of redistribution and equilibration among tissues. Is it also a messenger whereby kidneys and other tissues become aware that a load of body water exists?

Blood and plasma are analyzed in dogs loaded with excess water 


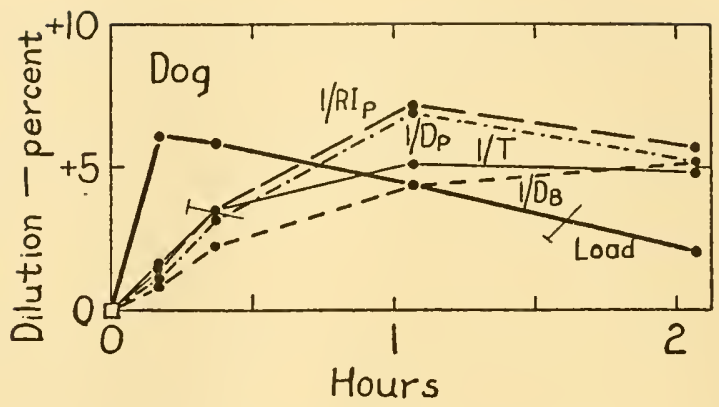

FIG. 116. Increment in dilution (\% of initial) of plasina and blood, and total water load of body ( $\%$ of $\left.B_{0}\right)$, in relation to time after a single ingestion $\left(6.2 \%\right.$ of $\left.\mathbf{B}_{0}\right)$ of water by stomach. Dog. Means of three tests, 2 individuals. Blood was heparinized as drawn: $1 / \mathrm{T}$, increment in ratio of non-erythrocyte volume to erythrocyte volume (hematocrit) ; $1 / \mathrm{Hb}$, increment in reciprocal of hemoglobin concentration (colorimetry of $\mathrm{CO}-\mathrm{Hb}) ; 1 / \mathrm{D}_{\mathrm{B}}$, inerement in ratio of water to dry residue in whole blood. Plasma separated from this blood: $1 / \mathrm{D}_{\mathrm{P}}$, inerement in ratio of water to dry residue; $1 / \mathrm{RI}_{\mathrm{p}}$, increment in reciprocal of refractive index of plasma minus refractive index of water at $17.5^{\circ}$ C. $; 1 / \mathrm{Cl}_{\mathrm{p}}$, increment in reciprocal of ehloride concentration of plasma. New data of Adolph and Kingsley.

by stomach (figs. 116 and 117). Within the first hour after sudden administration, dilutions are not parallel to body load; thereafter they are.

After 1 hour, plasma dilution (fig. 118) is related to body load of water independently of time, and also independently (within the range of variability prevailing) of whether single or repeated administrations of water be used. The latter is remarkable in view

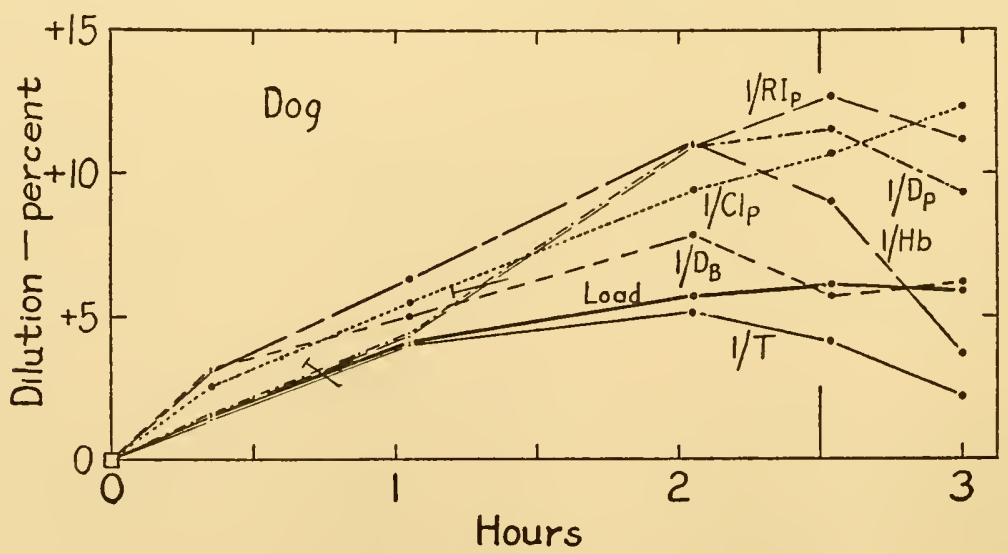

FIG. 117. Increment in dilution of plasma and blood, and total water load of body (\% of initial state), in relation to time after giving the first of 10 portions of water at 0.25 -hour intervals by stomach ( $10 \%$ of $B_{0}$ altogether). Dog. Six tests, 3 individuals. Measures of dilution are the same in figure 116. Data of Adolph and Kingsley. 


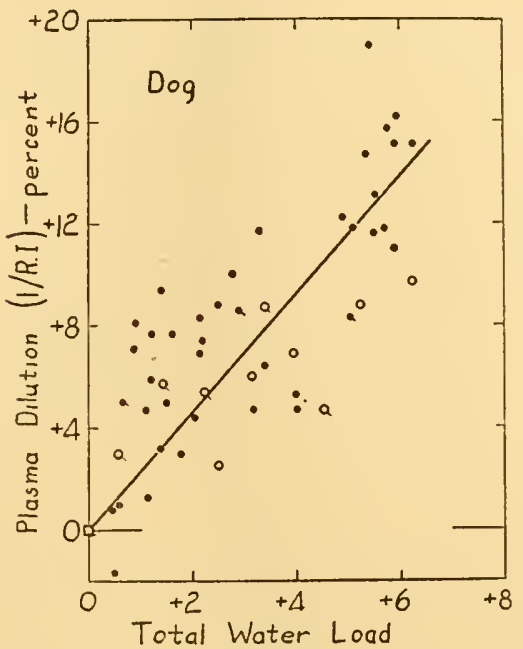

FIG. 118. Plasma dilution, measured as \% increment in $1 / \mathrm{RI}$ as previously defined, in relation to total water load $\left(\%\right.$ of $\left.\mathrm{B}_{0}\right)$. Dog. Individual $\mathrm{C}^{\prime}$ solid points, individual $\mathrm{G}^{\prime}$ open points; 50 observations in 10 tests. All samples were taken at more than 1.0 hour after first administration of water. (The correlation coefficient is +0.66 .) The regression line as drawn indicates that the plasma was diluted 2.31 times as much as the body as a whole. Data of Adolph and Kingsley.

of the somewhat greater rates of urine output that follow single ingestions (figs. 7 and 27 ).

In a similar manner, each of the dilutions measured in samples of blood is correlated with total water load (table 24). The intensity of correlation is indicated by the coefficient $r$, while the mean relationship is indicated by a proportionality, or slope of the regression line, fitted by least squares or other approximate method.

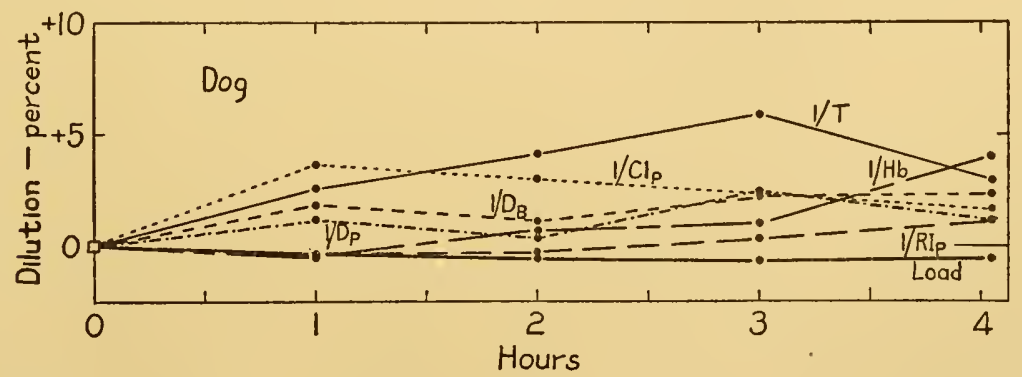

FIG. 119. Increment in dilution of plasma or of blood, and total water load of body $\left(\%\right.$ of $\mathrm{B}_{0}$ ), in relation to time. Dog in control tests without water load. Means of 4 tests on 2 individuals. Measures of dilution as in figure 116. Data of Adolph and Kingsley. 
Both the correlation coefficients and the control samples taken (fig. 119) agree upon the fact that some of the measures of dilution vary in partial independence of water load. These are the measures upon whole blood $(\Delta / \mathrm{T}, \Delta / \mathrm{Hb})$, and plasma chloride $\left(\Delta / \mathrm{Cl}_{\mathrm{p}}\right)$. Other measures in plasma $\left(\Delta / \mathrm{D}_{\mathrm{p}}, \Delta / \mathrm{RI}_{\mathrm{p}}\right)$ vary no more than (C.V.) \pm 3 per cent when body load does not change (fig. 119), and show correlation coefficients greater than +0.5 with modified body loads (table 24). At 1.0-hour intervals the coefficient of difference $(\mathrm{C} \Delta)$ is \pm 1.3 for $\Delta / \mathrm{RI}_{\mathrm{p}}$ and \pm 2.5 for $\Delta / \mathrm{D}_{\mathrm{p}}$. These two are evidently the types of dilution upon which to rely for accurate reflections of body water load.

TABLE 24

Dog in water excess. 2 individuals, 11 tests, 51 observations. New data of Adolph and Kingsley ('40)

\begin{tabular}{|c|c|c|c|c|c|c|}
\hline Covariate, $\Delta \mathrm{E}$ & $\begin{array}{c}\text { C.V. of } \\
21 \text { con- } \\
\text { trols at } \\
\text { 1-hr. } \\
\text { intervals }\end{array}$ & $\begin{array}{l}\text { Mean of } \\
51 \text { incre- } \\
\text { ments } \\
\text { per cent } \\
\text { of mean } \\
\text { control }\end{array}$ & $\begin{array}{l}r \text { with } \\
\text { water } \\
\text { load } \\
\Delta W\end{array}$ & $\begin{array}{c}\text { Regres- } \\
\text { sion, } \\
\Delta \mathrm{E} / \Delta \mathrm{W}\end{array}$ & $\begin{array}{c}r \text { with } \\
\text { plasma } \\
\text { dilution } \\
\Delta / R I\end{array}$ & $\begin{array}{c}\text { Regres- } \\
\text { sion, } \\
\frac{\Delta \mathrm{E}}{\Delta / \mathrm{RI}}\end{array}$ \\
\hline $\begin{array}{l}\text { Total water load, } \Delta W \\
\text { Plasma dilution, } \Delta / R I_{P}\end{array}$ & -0.39 & $+\quad 3.14$ & & & +0.66 & 0.43 \\
\hline $\begin{array}{l}\text { Plasma dilution, } \Delta / \mathrm{RI}_{\mathrm{P}} \\
\text { Plasma dilution } \Delta \mathrm{D}_{\mathrm{P}}\end{array}$ & \pm 2.3 & $\begin{array}{l}+\quad 7.8 \\
+\quad 71\end{array}$ & +0.66 & 2.31 & +0.94 & 103 \\
\hline $\begin{array}{l}\text { Plasma dilution, } \Delta / \mathrm{D}_{\mathrm{P}} \\
\text { Plasma dilution, } \Delta / \mathrm{Cl}_{\mathrm{P}}\end{array}$ & \pm 2.8 & $\begin{array}{l}+\quad 7.1 \\
+\quad 50\end{array}$ & +0 & $\begin{array}{l}2.39 \\
2.54\end{array}$ & $\begin{array}{l}+0.94 \\
+0.61\end{array}$ & 1.03 \\
\hline Blood dilution, $\Delta / \mathrm{D}_{\mathrm{B}} \ldots$ & $\begin{array}{l} \pm \quad 3.8 \\
\pm \quad 2.2\end{array}$ & $\begin{array}{l}+\quad 0.0 \\
+\quad 6.2\end{array}$ & $\begin{array}{l}+0.48 \\
+0.45\end{array}$ & 2.05 & $\begin{array}{l}T .01 \\
+0.83\end{array}$ & 0.81 \\
\hline Blood dilution, $\Delta / \mathrm{Hb} \ldots .$. & \pm 4.8 & +11.9 & $(-0.10)$ & ........... & +0.22 & 1.11 \\
\hline Blood dilution, $\Delta / \mathrm{T}$......... & \pm 5.6 & $+\quad 9.6$ & $(-0.05)$ & & +0.45 & 0.89 \\
\hline Rate of total loss & \pm 31.0 & +507.0 & +0.72 & 127.0 & +0.42 & 51.0 \\
\hline Rate of urinary lc & \pm 26.0 & +1010.0 & +0.66 & 295.0 & +0.48 & 117.0 \\
\hline
\end{tabular}

As in water excess, so also in water deficit the relation of total water load to various blood dilutions is ascertained (figs. 120 and 121). During 3 days of partial water privation the plasma dilution decreases steadily and in parallel to water load (weight) of the whole body. Upon sudden ending of the privation, water is drunk ad libitum (fig. 120), whereupon the amount ingested exceeds the amount lost. When drinking is limited to the amount of weight deficit (fig. 121) the changes of plasma dilution are again in marked contrast to those of fig. 116. (a) At 0.2 hour a slight concentration of plasma regularly occurs. (b) Dilution proceeds much more slowly than without previous deficit. (c) Even at 1.0 hour dilution is scarcely maximal. (d) The plasma dilution is greater after privation than without it, though the same amounts of water are ingested.

Correlations, within these tests of privation and recovery, be- 

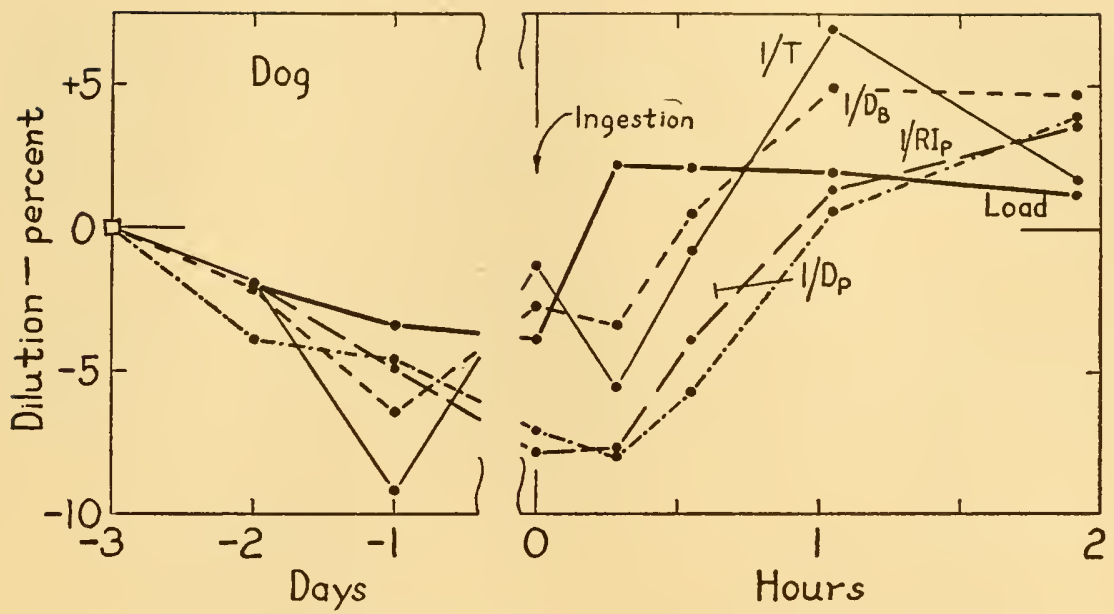

Fig. 120. Increment in dilution of plasma and blood, and total water load of body, in relation to time of water privation ( 3 days), followed by sudden voluntary ingestion of water amounting to about $6 \%$ of $\mathrm{B}_{0}$. Four tests, 2 individuals. Measures of dilution as in figure 116. Data of Adolph and Kingsley.

tween blood dilutions and water load, are less intense (fig. 122). Temporal sequences become important elements, for several days are represented in any one test instead of several hours.

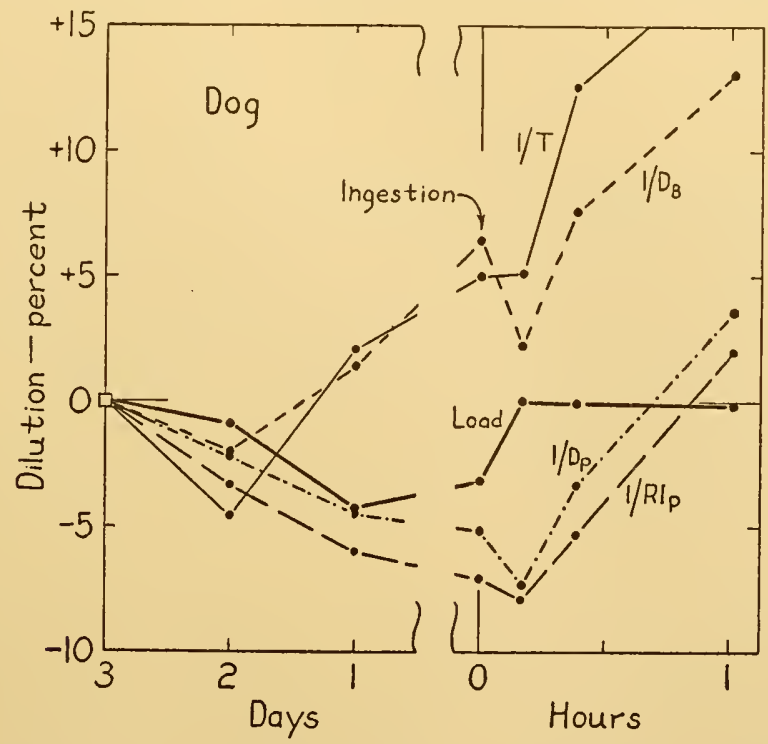

FIG. 121. Increment in dilution of plasma and blood ( $\%$ of initial), and total water load of body ( $\%$ of $\mathrm{B}_{0}$ ), in relation to time during water privation ( 3 days), followed by voluntary water ingestion ( 0.2 hour) of about $3 \%$ of $B_{0}$ of water. Two tests, one individual. Measures of dilution as in figure 116. Data of Adolph and Kingsley. 
The same data might be used to correlate rates of change of plasma dilution during recovery with the dilutions prevailing. Each dilution has the dimensions of relative volume, so that the correlation obtained is a net equilibration diagram. This would resemble, in another type of water load, the relations described in $\S 59$.

That some measures of blood concentration may be converted by computation into others by means of equations or equivalents is generally recognized. A familiar one is the transformation of refractive indices and specific gravities of serum or plasma into pro-

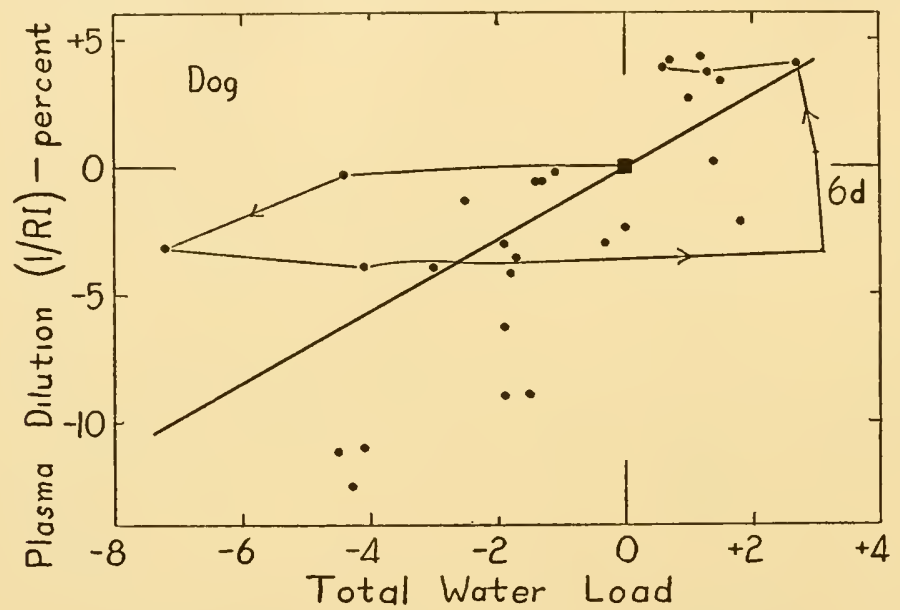

FIG. 122. Increment in plasma dilution, measured as increment in $1 / R I$ ( $\%$ of initial), in relation to total water load $\left(\%\right.$ of $\left.\mathrm{B}_{0}\right)$. Two dogs. The points of test $6 \mathrm{~d}$ are connected; 6 tests in which most water was withdrawn from the diet for 2 or 3 days; then water was suddenly drunk and the blood was sampled repeatedly. Samples taken within one hour of drinking are not ineluded in the correlation; the correlation coefficient of the remainder is +0.68 . Combining this figure with the data of figure 118, the combined correlation coefficient is +0.87 . Data of Adolph and Kingsley.

tein concentrations of serum or plasma (Neuhausen and Rioch, '23; Weech et al., '35). In general the familiar instances of conversion are those in which chemical entities are largely known. It may be recognized just as readily that any one measure of concentration is equivalent to (accompanies) another, providing their coincidence is measured under and limited to prescribed physiological conditions. A case is worked out in figure 123 ; once such a correlation and regression are known, it is quite superfluous for most purposes to measure the same plasma in both ways. The regression line there drawn does not pass through the origin. From this it may 
be inferred that water loads do not merely dilute the solids of plasma in a lump, the increment of refractive index per unit of dry weight differing slightly for various solutes. Rather, diverse constituents are diluted unequally, as is to be expected from the fact that their volumes of distribution are not all alike.

Again, at diverse $+\Delta W$, blood chloride dilutions (fig. 124) and serum electrical conductivities $\left(1 / \mathrm{EC}_{\mathrm{s}}\right.$, fig. 125$)$ are measured, and their relationships are ascertained graphically or by equation; usually $\Delta \mathrm{E}=c \Delta \mathrm{W}$. Such relationships are often termed "empiri-

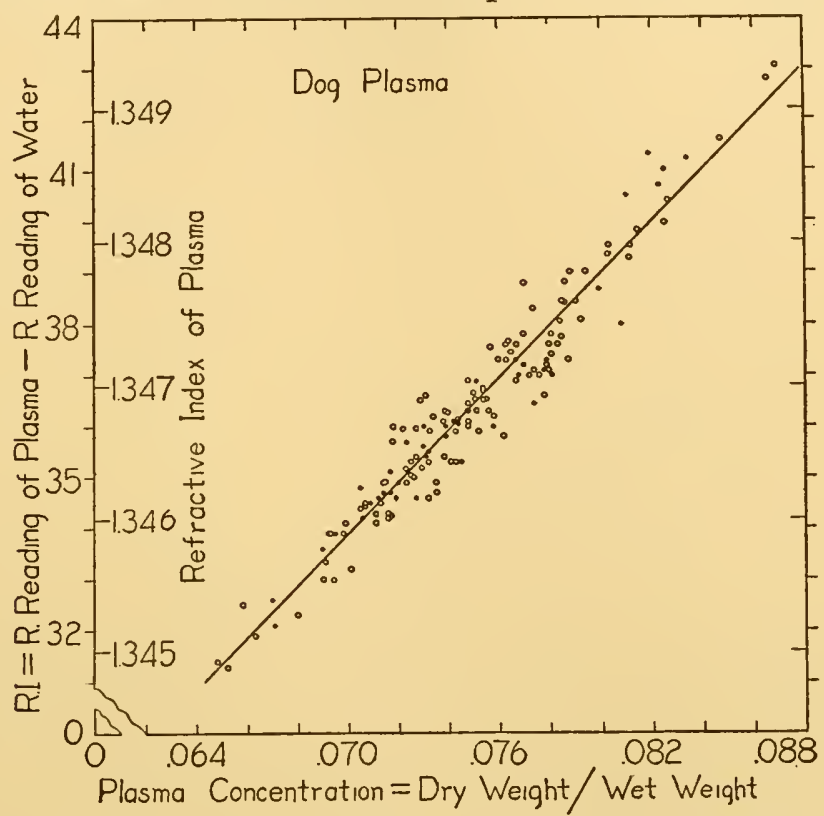

FIG. 123. Refractive index of plasma (RI) in relation to fraction of solids in plasma $(D / F)$. Heparinized plasma of dog. Two individuals (C', open points; $G^{\prime}$, solid points) in 21 tests; given water by stomach, or deprived of water with constant diet, or neither. The ordinates on the outer RI scale, readings for plasma of the dipping refractometer at $17.5^{\circ} \mathrm{C}$. minus the similar reading for distilled water. The correlation coefficient is +0.963 ; the least-squares regression line shown does not pass exactly through the origin. Data of Adolph and Kingsley.

cal"; they are just as valid as "physico-chemical" ones, the chief differences being that they are more difficult to predict, are worked out in vivo instead of in vitro, and sometimes have larger deviations.

This, however, is the sort of correlation that exists among all the quantities that are each separately related to the body water content. Under the specified conditions, variables $\Delta \mathrm{M}, \Delta \mathrm{N}, \Delta \mathrm{P}$, and 
$\Delta Q$ are each correlated with $\Delta \mathrm{T}$. Then, still under these conditions, $\Delta \mathrm{M}$ and $\Delta \mathrm{N}, \Delta \mathrm{P}$ and $\Delta \mathrm{Q}$, etc., also vary in parallel. Conversely, $\Delta \mathrm{M}, \Delta \mathrm{N}, \Delta \mathrm{P}$ and $\Delta \mathrm{Q}$ characterize the state of the organism with respect to water load, and $\Delta \mathrm{W}$ may in turn be found from its correlation with them. All this is implicit in table 24 .

The relation of hemoglobin dilution of the blood to water load of the body as a whole (fig. 124) might be an especially useful one, for having once established the relation, $\Delta / \mathrm{Hb}=1.03 \Delta \mathrm{W}$, data recorded in the past would become available for the study of water loads in which body weights were not measured at suitable intervals, but in which hemoglobin dilution was measured, as was the

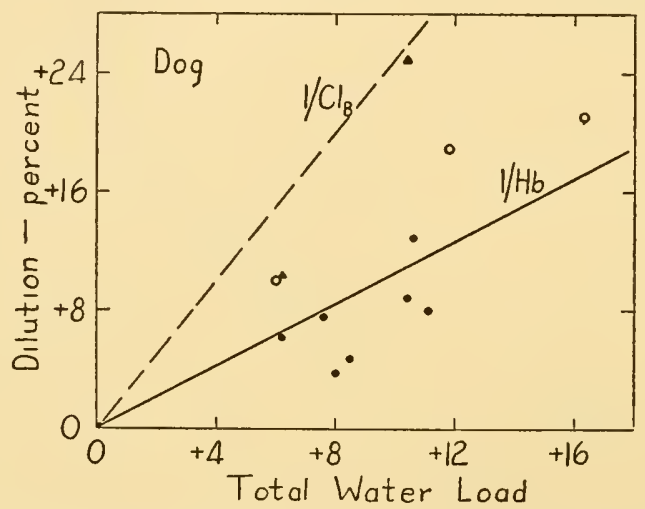

FIG. 124. Increment in dilution of whole blood (\% of initial) in relation to total water load ( $\%$ of $\mathrm{B}_{0}$ ). Dog. Repeated administration of water by stomach. Triangles and dash line, dilution of whole blood chloride, in two individuals of Underhill and Sallick ('25). Circles and solid line, dilution of hemoglobin, in four individuals of Underhill and Sallick and in two individuals of Greene and Rowntree ('27). Occasionally 2 or 3 determinations were made during one test.

case in figure 125. Before further conclusions are drawn, however, it may be recalled that dilution of hemoglobin is in other studies (table 24) not accurately related to water load. Increments in hemoglobin dilution might also be expected to parallel increments in "red cell" volume in water excesses, whereupon it is found (fig. 114) that at $+\Delta W=8$ the volume of distribution of carbon monoxide $\left(V_{D}\right)$ is not significantly different from the $V_{D}$ at $\Delta W=0$.

The changes undergone simultaneously by the various concentrations measured are diverse. At a chosen time, some, as $\Delta / \mathrm{Cl}_{\mathrm{s}}$ (fig. 125), indicate more dilution than the increment in volume of the "plasma" (fig. 114). Others indicate about the same or less dilution than the increment in volume of "whole blood," but not all dilutions measured in whole blood do so. Many plausible ex- 
cuses have been put forward as to why changes of dilution do not parallel changes of volume. Each dilution, like each volume of distribution, is a separate and distinct measure bearing an individual relation to body load of water.

It is possible also to infer how much of each non-aqueous substance (e.g., chloride) leaves or enters the circulating blood (a) by comparison with the dilution of some one (e.g., protein) that is in those circumstances believed not to leave or enter, or (b) by com-

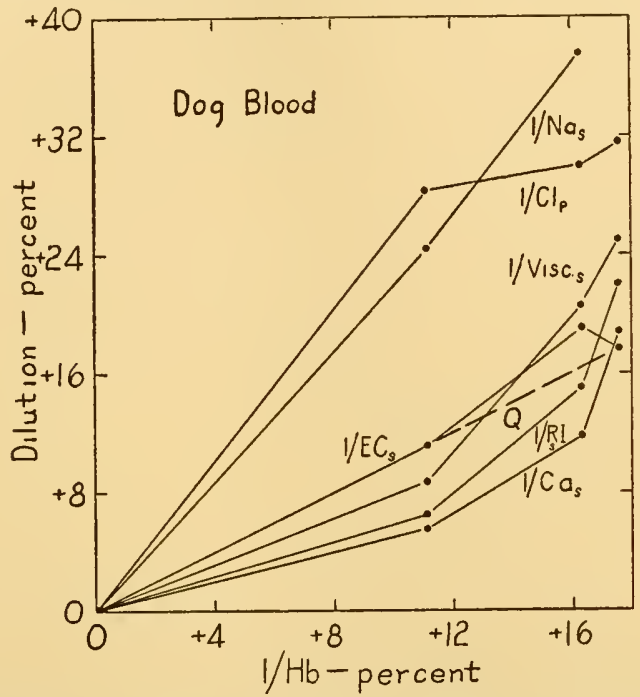

FIG. 125. Increment in dilution of serum or plasma (\% of initial) in relation to simultaneous increment in dilution of hemoglobin in whole blood ( $\%$ of initial). Dog. Since $\Delta \mathrm{Hb}$ is approximately equal to $\Delta \mathrm{W}$ of the whole body (fig. 124), the abscissae here might be considered as total water load. $1 / V$ isc $_{\mathrm{s}}$ is the increment in reciprocal of serum viscosity; $1 / E C_{s}$ is the increment in reciprocal of serum electrical conductivity; etc. $Q$ represents hypothetical equality of dilutions between ordinates and abscissae. Each point is the mean of 2 to 10 (usually 8 ) analyses on as many individuals that were given water repeatedly by stomach. Data of Greene and Rowntree ('27).

parison with the changes in one of the measured volumes of distribution. In the intact body, only such relative estimates are available.

The above studies are confined to water increments by water privation and by administering water by stomach or vein. Comparisons may be obtained in blood and serum of dogs that have been water-loaded by any other means. A favorite type of load that has been investigated is in the state following intestinal obstruction (Haden and Orr, '23). Others are the loss of gastric juice (Gamble and Ross, '25), loss of pancreatic juice (Gamble and McIver, '28), 
and loss of intestinal juice (Herrin, '35). In them also negative dilutions of serum-protein nitrogen, of blood hemoglobin, and of other measures, are found. But the relative values obtained among several sorts of dilution are not like those here reported.

Volumes of distribution of the water load (or should they be termed dilutions of distribution?) allowably may be computed from the increments of some dilution, such as refractive index of plasma (fig. 126). They show that just after a load is administered, time is a large factor, in both positive and negative water loads. After a sufficient period (one hour) the volumes of distribution of water are all much less than 100 per cent of the body (fig. 118); this fact

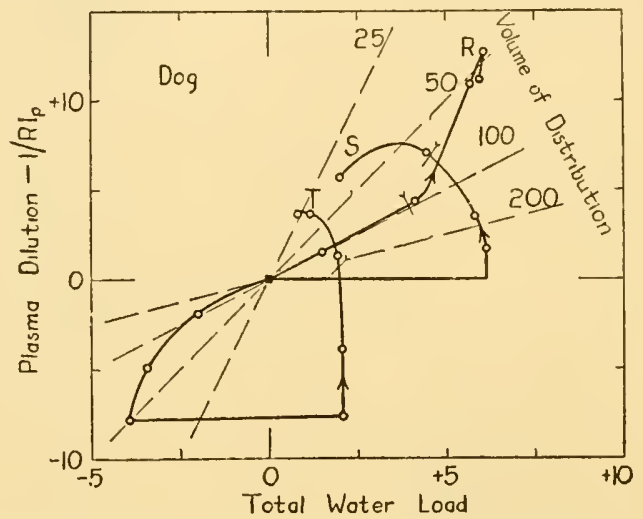

FIG. 126. Increment in dilution of plasma as reciprocal of excess refractive index ( $\%$ of initial) in relation to total water load $\left(\%\right.$ of $\left.\mathrm{B}_{0}\right)$. A supplementary grid shows volumes of distribution of the added water in plasma, as $\%$ of the body weight. The course of simultaneous points is traced in averages of three types of test. One hour after ingestion began is marked $(/)$. $R$, ten successive administrations of water by stomach, 6 tests from figure 117. $S$, single administration of water by stomach, 3 tests from figure 116. $T$, single voluntary ingestion of water by mouth, 4 tests from figure 120.

is confirmed by fewer like data of Abe ('31a) and of Hatafuku ('33a). It indicates again that the plasma undergoes somewhat more change than the body as a whole; the positive increments of water being apparently distributed through a volume less than that occupied by water already present.

In the tests represented in table 24 some nine quantities are measured simultaneously. By correlating these two at a time a fairly complete study is accomplished; though triple and higher multiple correlations still remain open. Of the bi-correlations, only rate of water output in relation to dilution is shown graphically 
(fig. 127). One of the questions at stake is, whether plasma dilution is so closely related to rate of excretion that the dilution could inform the kidneys how much water load exists. The correlation is particularly significant when a wide range of water loads or plasma dilutions is investigated; the coefficient of correlation $(+0.48)$ is higher than for any quantity measured in correlation with rate of excretion, except water load itself. It is not the only

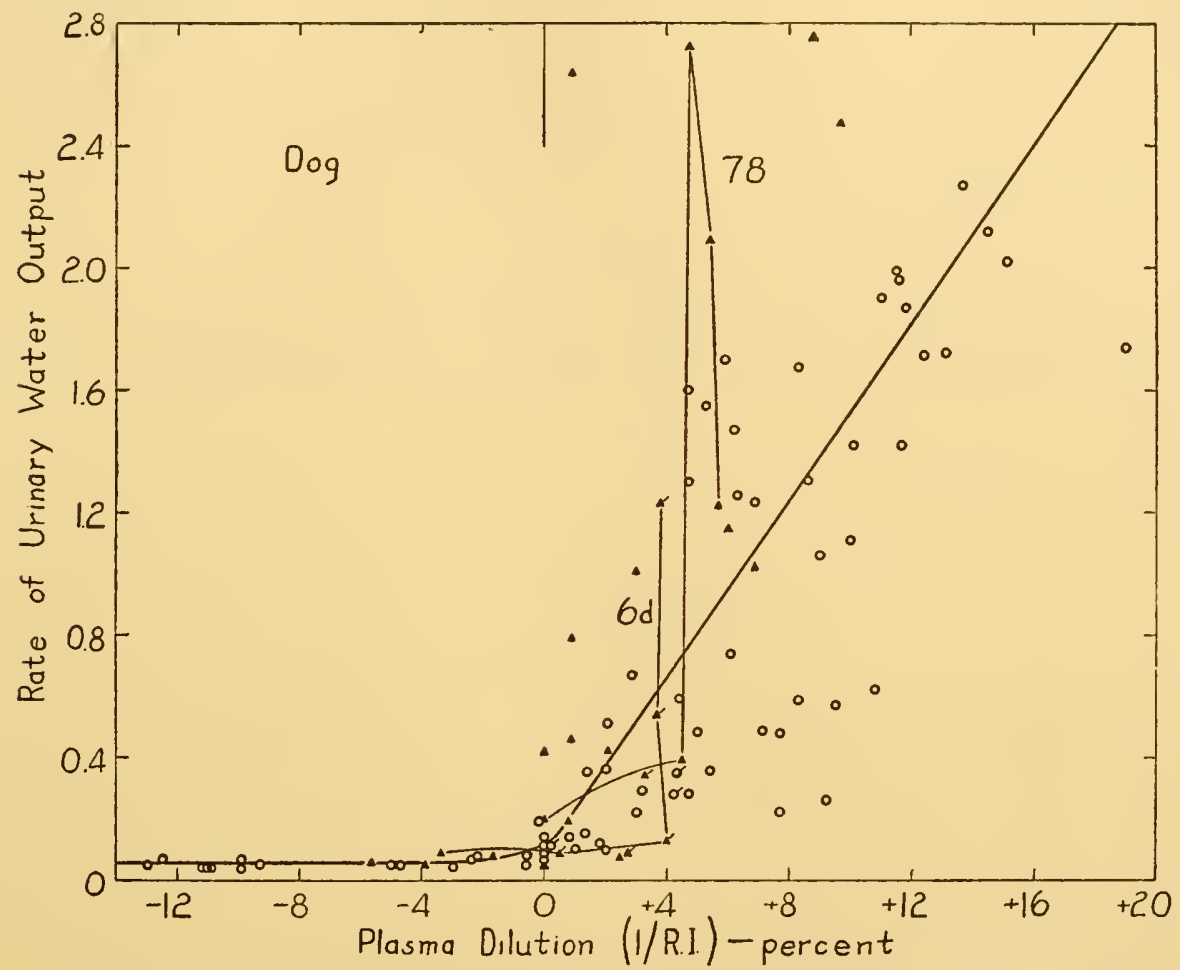

FIg. 127. Rate of urinary water output (\% of $\mathrm{B}_{0} /$ hour) in relation to plasma dilution as $1 /$ RI (\% of initial state). Two individuals $C^{\prime}(O)$ and $G^{\prime}(\Delta)$; the 9 tests of figures 116 and 117 plus 7 more tests in which water was given by stomach, together with the 6 tests of figures 120 and 121 in which water was first denied, are represented. Two of these tests (on $\operatorname{dog} G^{\prime}$ ) have the successive points connected by lines. Excluding the 6 water privation tests, the correlation coefficient in positive loads is +0.48 .

correlation, and to designate plasma dilution as the cause of diuresis would be misleading: To conclude, on the contrary, that "changes of blood concentration and volume are scarcely detectable in diuresis', (Adolph, '30, p. 63), merely because circumstances may be found in which the correlation is poor, is erroneous. To conclude that only a single factor of any kind governs the rate of water excretion may be equally blinding. 
On the whole, correlations between blood dilution and body load of water become apparent when large loads are provided. They are clearest for dilution of total substance in plasma (e.g., refractive index). Many dilutions are colligative, but with slightly diverse ratios. The ratios here recorded indicate volumes of distribution of the water increment amounting to about half the body weight. Further, dilutions are correlated with rates of urinary output of water. Accordingly, blood serves as a distributor, putting all other tissues in an equilibrium of water partition with it, and also furnishes in its dilution a possible stimulus to kidneys, mouth, and other organs, informing them what water content prevails in the body.

\section{\$ 81. Concentrations of URIne}

Urinary concentrations are closely related to the body's water load (fig. 128). Since these relations are widely recognized, they

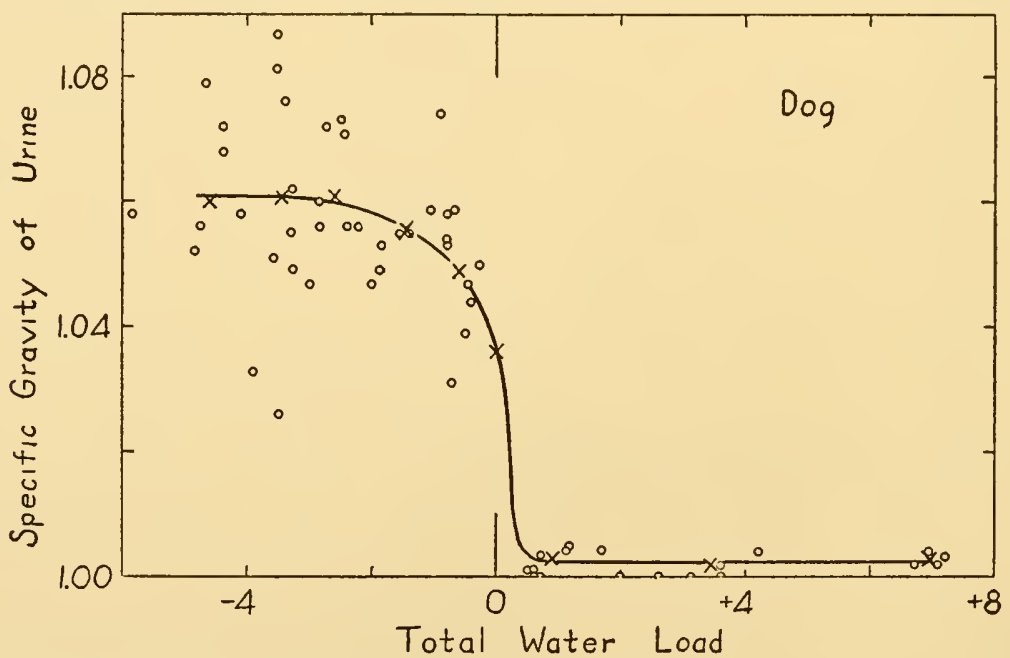

FIG. 128. Specific gravity of urine in relation to total water load (\% of $\mathrm{B}_{0}$ ). Dog, on constant diet. Each dot represents a separate day; in negative loads 3 individuals deprived of water, in positive loads 2 other individuals given repeatedly water by stomach. Each cross represents a mean sp. gr. for a one per cent interval of load. Further data of Adolph ('39a) and of Kingsley.

are likely to be thought of as only qualitative. Actually a single measurement of urinary specific gravity may do more to identify the existence of a water increment than any other one measurement, particularly if other measures are not already controlled by a series of like data on the same individual. Quantitatively the correlation is blurred by the fact that in all positive loads the 
samples of urine vary around one concentration and in all negative loads around another, with a steep transition between. Many other concentrations (chloride, urea) and ratios (chloride/creatinine) of urinary constituents yield equal correlations with water load. Over all, one conclusion emerges, namely, that in water diuresis, rate of output of water augments more than of other substances. In the long run, little else slips out of the body with it; its excretion is highly specific.

\section{\$82. Concentrations of other body fluids}

Digestive juices are collected from fistulas while dogs are subjected to diverse types of body load of water. It is believed that the total particulate (electrolyte) concentrations in most juices are equal to those of blood plasma simultaneously. Some at least of those constituents (e.g., bicarbonate) that increase in concentration in deficits of water, do so in both blood and juice (Herrin, '35). The drained juices, wherever available, therefore serve as automatically extruded samples of analyzable body substance. They are equivalent to any other sort of sample in indicating a part of the distribution of water increments.

\section{§ 83. OTHER COMPOSITIONS}

In the whole body of the dog with water load, few changes in the total amounts of materials other than water have been measured. To ascertain them, the whole body need not be analyzed, for with greater accuracy the increments in content of those materials are ascertained from determinations of intake and of output of them. Nitrogen is said to be characteristically lost in water privation (Straub, 1899; Spiegler, '01). It is uncertain whether the amount lost from the body is related to the duration of the water deficit more than to the extent $(-\Delta W)$ of it; in all cases the negative load of nitrogen $(-\Delta \mathrm{N})$ amounts to less than 1 per cent of the nitrogen in the body. Retentions of nitrogen are sometimes found, especially in short periods of water privation; these are related to the retention of urea and probably of other substances at low rates of water excretion $(\$ 84(4))$. Phosphorus is reported to be retained with nitrogen (Landauer, 1894; Straub, 1899).

Water excess leads to depletion of several measured chemical constituents, as nitrogen, phosphorus, chloride, creatinine, and acid (Heilner, '06; Underhill and Sallick, '25; Greene and Rowntree, 
'27 ; Brull, Poverman and Goffart, '36). The most extreme depletion is that of chloride, which amounts to over 10 per cent of the body's supposed content of it after water equal to half the body weight has passed through the body during one day (Underhill and Sallick). The depletion of some or all constituents may be more closely related to the modified rates of water output than to the water content of the body at some one time.

When water excesses are maintained for months very large deficits of chloride possibly result. Wolff ('35) reported that a 6.3-kg. dog was depleted of 0.65 equivalent of chloride during 157 days of excessive water administration. But I judge, from data of Wier ('40) that a dog of this size ordinarily contains only one-third that much chloride to begin with; hence Wolff's account of chloride exchanges may have had a systematic error.

Is there evidence that in water excess constituents are accumulated in the body or its parts that are not retained in water balance? In water deficit is there any augmented intake of other substances than water? I know of nothing to show clearly that such occurs, though in the shifts of physiological activities that accompany water loads there may easily be some.

Fixed tissues sampled during water loads have been analyzed very infrequently for other constituents than water. Significant depletions in electrolyte contents (relative to content of dry material) of muscle and of skin are demonstrated in water deficit (table 23). If the loss of sodium or of chloride were proportional to that of water, the dilution, which is the ratio of water to electrolyte $(\mathrm{E} / \mathrm{Na})$ would remain constant in the tissue. It appears that no tissue lost an electrolyte in so great proportion as water. In water privation chloride is not lost so extensively from the whole body as from the muscle; hence what chloride may have left the muscle may be in part translocated elsewhere.

The study of compositions might be a mere exercise in correlating, were there not special questions in mind. The chief one is, has the water-loaded body all its non-aqueous constituents in their usual amounts and places, all ready to soak up the water that will be gained or lost in recovery? For the most part, neither lack nor excess of water greatly upsets the dog's content of any other constituent. So there persists a full framework that may serve to indicate when the original water content is restored. 


\section{\$ 84. Correlated metabolisms and behaviors}

Thus far, diverse changes of composition and physical characteristics have been mentioned in relation to the body's water content. Another large group of correlatives are rates of physiological activities; they are set apart from compositions in that they involve intervals of time. The ones considered in the dog concern: (1) outputs of alimentary glands; (2) food intake; (3) energy transformations; (4) renal outputs and clearances; (5) neuromuscular activities. A great many other quantities might be of equal interest if adequate data be correlated concerning them.

(1) The outputs of water in the dog's alimentary glands have been studied in saliva, gastric juice, and bile. Saliva is secreted only in minute amounts ordinarily; in tests some standard stimulus such as pilocarpine or food is given to increase output of saliva. The stimulus is given to the dog once a day (or once an hour) while water privation is decreasing the body water content progressively through several days. Since the changes of body weight are not recorded, rough estimates of water deficit might be made, from the time elapsed and the water missing in the diet. In each series of experiments the saliva is collected from submaxillary glands alone. With each of five stimuli a decrease of salivary output with deficit of water content is found (Crisler, '28; Gregersen, '31; Barron, '32).

At positive increments of water the rates of salivary secretion have been recorded less precisely. With very large administrations of water, "unstimulated" salivation is reported as excessive (Rowntree, '23; Underhill and Sallick, '25). Possibly this production of saliva is correlated with vomiting (Rowntree); it is apparently reinforced by additional stimuli such as spinal irritation or pituitrin (Theobald, '34). The loss of saliva from the mouth may then be almost as effective as the outputs of urine in relieving the $\operatorname{dog}$ of the water load. But these events occur only in certain circumstances and in extreme excesses of water content.

Vomiting is a direct means of refusing water; of decreasing the intake of it. It is probably more useful to regard it so than to classify vomiting as a means of output from the body. I believe it has not been shown that water introduced by a non-alimentary route is appreciably lost through the alimentary tract.

Gastric juice output has been measured in relation to water excesses (Pavlov, '01; Lönnquist, '06; Foster and Lambert, '08; 
etc.). It increases both when food is given, with the water and when not. The increased rate of secretion might be more closely related to the water load either of the body or of the alimentary tract (of the stomach itself). Whether the rate of juice output from the walls of the stomach that are in contact with water given, is the same as from pouch separated from the water, is not certain (Sutherland, '21). While the stomach produces more fluid, it also produces more acid and chloride (Ivy, '18).

Bile output through a fistula is apparently not consistently affected by water excesses (Bidder and Schmidt, 1852, p. 162; Snell and Rowntree, '28). The composition of the bile is not significantly changed.

Intestinal juice, pancreatic juice, buccal mucus, and colonic excretion have probably not been measured in terms of output rates, in water loads such as are being studied here.

It has not been demonstrated that a quantitatively reproducible correlation exists between water load and rate of any one alimentary output. Many physiologists are confident that passage of water into the alimentary tract "stimulates" the output of several juices. Existing data show extreme irregularities of performance, even with uniform procedure. On top of this is the uncertainty as to what numerical water loads (of absorbed water) prevail during and after the administrations and privations of water. The nearest thing to a correlation at present is between salivary output and size of water deficit. One of the motives in obtaining those measurements was the hypothesis that shortage of saliva might be furnishing a tangible signal to other organs and tissues of the dog, leading to compensatory responses. But since shortages of water have been observed in several diverse fluids and tissues, there is less occasion to think of any single one of them as providing a message.

(2) Food intake is greatly reduced in water privation, as was noted in early experiments (e.g., Pernice and Scagliosi, 1895). This is not true on all diets nor at all loads, and quantitative studies are needed. With the anorexia have been correlated qualitative decreases of gastric motility (Rose et al., '31) originally on the assumption that a "direct connection" between them exists. No criterion appears to have been proposed in physiological science for distinguishing a direct connection from an indirect one. Perhaps a useful statement of the situation is that by habitual mental associ- 
ations some physiologists are ready to make "derivations" (sensu Pareto, '35, p. 508) relating anorexia and gastric motility, which they do not make relating gastric motility and water content of the body.

It may be pointed out that going without food is a possible means of conserving water to the body. In extreme water deficits, food that is eaten is actually regurgitated and thus rejected. Without food, less water is excreted, both in urinary and in evaporative paths (Morgulis, '23, p. 133, p. 266) ; and the water already present more nearly suffices to maintain the gradually shifting balance of water.

(3) Energy transformations. In the body as a whole, energy metabolism is most often studied in the particular stages of energy transformation represented by oxygen consumption and by carbon dioxide output. No change in over-all rate was found in 3 hours after placing warm water in the stomach in amounts up to $2 \%$ of $\mathrm{B}_{0}$ $(+2 \Delta \mathrm{W})$ (Bidder and Schmidt, 1852; Rubner, '02; Lusk, '12). But after administering loads of $+10 \Delta \mathrm{W}$ by stomach, Heilner ('07) found increases of 10 and 18 per cent in rate of carbon dioxide elimination.

Rates of oxygen consumption increase only at certain times during water excess, indicating therefore less relation to water load than to interval after introduction. Increases of 20 to 600 per cent may occur for short periods after fluids isotonic with blood are rapidly introduced by vein (Davis, '35), but disappear entirely before the load has been eliminated.

Significant modifications in respiratory quotient with water loads of the type discussed here have, I believe, not been found.

(4) Renal clearances, or ratios of the rate of excretion of any constituent to the concentration of that constituent in blood or in plasma, are greatly modified with water content of the body. For the most part the correlations already made are with rate of urinary water excretion (Austin et al., '21). However, having established the relation between $\Delta \mathrm{W}$ and $\Delta \mathrm{W} / \Delta t$, perhaps choosing the steady state for the comparison, I indicate that the relation of load to the renal clearance (fig. 129) is equally significant with that of rate.

With this information at hand, it next appears possible that the increased output of diverse substances at the same plasma concentration of them can account for the depletions of nitrogen, phos- 


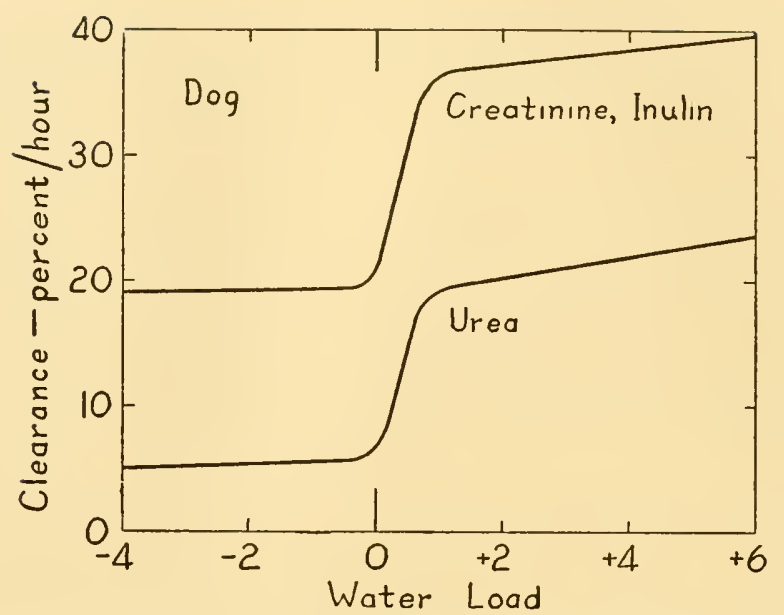

FIG. 129. Renal (urinary) clearance from plasma of creatinine, inulin, and urea ( $\%$ of $\mathrm{B}_{0} /$ hour) in relation to sensible water load $\left(\%\right.$ of $\left.\mathrm{B}_{0}\right)$. Dog. The urea elearance was transformed, by means of figure 26, from a relation to rate of urinary water excretion as averaged in data of Dominguez (' $35, \mathrm{p} .537$ ) to an approximate relation to water load. The ratio of creatinine clearance to urea clearance was then taken from Shannon ('36b, p. 210), and of inulin clearance from Shannon ('35 and '36a).

phorus, and some of the other materials with water excesses, as noted in $\$ 83$.

An actual example of the effects of water excretion upon urea content may be taken from data of Greene and Rowntree ('27). Dog 183 weighed $7.0 \mathrm{~kg}$.; taking 66 per cent of this as the volume of distribution (Painter, '40) of urea, which was $6 \mathrm{mM}$ in whole blood, some 28 millimols were present in the body initially. This dog may have formed 9.3 millimols of urea per hour. If the rate of urea formation and the volume of distribution be constant, but the clearance of urea be augmented, almost doubled at times, during

\section{TABLE 25}

Computation of extra urinary elimination of urea that might occur during maintained water excess in which urea clearance is doubled. Dog

\begin{tabular}{|c|c|c|c|c|}
\hline Hours & $\begin{array}{l}\text { Urea produced, } \\
\text { m. mol./hr. }\end{array}$ & $\begin{array}{l}\text { Urea exereted, } \\
\text { m. mol./hr. }\end{array}$ & $\begin{array}{c}\text { Mean urea } \\
\text { content of body, } \\
\text { m. mol. }\end{array}$ & $\begin{array}{l}\text { Urea content } \\
\text { at end of hour, } \\
\text { m. mol. }\end{array}$ \\
\hline 0 & 9.3 & 9.3 & 28.0 & 28.0 \\
\hline 1 & 9.3 & 15.6 & 23.4 & 21.7 \\
\hline 2 & 9.3 & 13.1 & 19.7 & 17.9 \\
\hline 3 & 9.3 & 11.1 & 16.7 & 16.1 \\
\hline 4 & 9.3 & 10.3 & 15.5 & 15.1 \\
\hline $5 \ldots$ & 9.3 & 9.9 & 14.8 & 14.5 \\
\hline 6 & 9.3 & 9.4 & 14.2 & 14.0 \\
\hline
\end{tabular}


6 hours of copious, uniform water administration, the following values result (table 25). At the end of 6 hours the urea concentration in the blood was actually $3 \mathrm{mM}$, corresponding to a urea content of the whole body of 14 millimols. This agrees with the amount expected (last column) upon the assumptions mentioned. The agreement makes it probable that the rate of urea formation itself is actually unaffected by water excess. Another notable outcome of the computation is that the urea content of the body approaches (at only half the control content, or $\Delta \mathrm{u}-50$ ) a new balance, in which elimination of urea equals production of urea. Chloride in plasma behaved similarly in the same test.

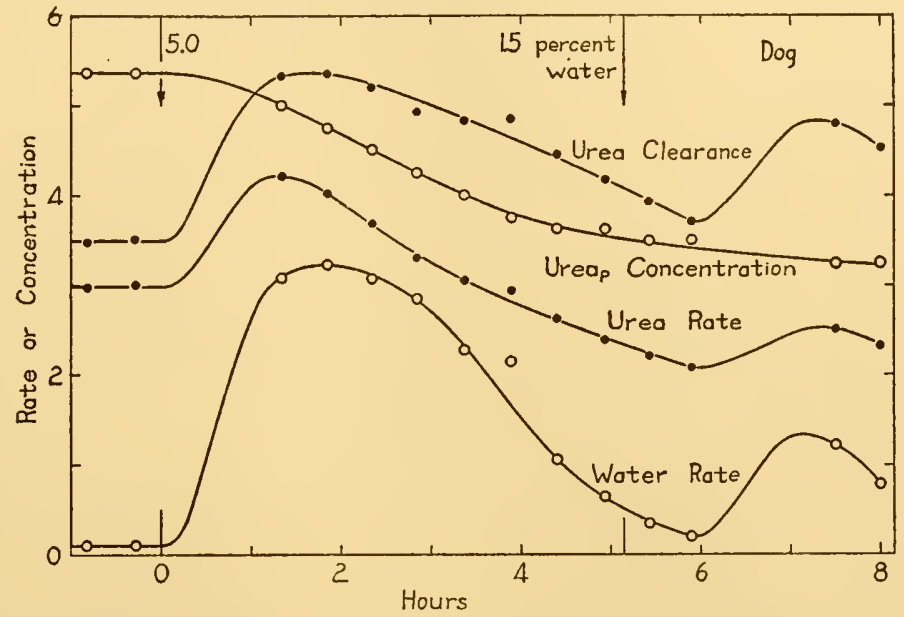

FIG. 130. Rate or concentration (in arbitrary units) in relation to time after water ingestion. One test on $\operatorname{dog} \mathrm{E}$ of Shannon ('36b, p. 208). Water was administered by stomach at zero time $\left(5.0 \%\right.$ of $\left.\mathrm{B}_{0}\right)$ and at 5.15 hours $\left(1.5 \%\right.$ of $\left.\mathrm{B}_{0}\right)$. Urea Clearance $=$ urinary clearance of urea from plasma, in units of $2.0 \%$ of $B_{0} /$ hour. Urea Concentration = concentration of urea in plasma, in units of 0.8 millimols/liter. Urea Rate = rate of urinary urea output, in units of 0.10 millimols/hour. Water Rate $=$ rate of urinary water output, in units of $0.4 \%$ of $\mathrm{B}_{0}$ /hour.

To conclude that the kidneys are responsible for the depletions of solutes in $+\Delta W$ is like blaming the pumping station for the great flow of water from faucets near dinner time. In water deficits the lesser renal clearances by themselves favor retentions of solutes.

The above relations for urea are shown in figure 130 as they change with time during single loadings and unloadings of water. Renal urea clearance there temporarily augments by 50 per cent. Since water excesses are measured ordinarily as sensible loads, the 
rates of urea clearance may bear a different relation during rising water loads than during falling loads. Further, at one load the urinary rates are often higher following sudden accession of water to the body $(\$ 12)$ than during decrease of load.

Rates of excretion of urea (Marshall, '20) and chloride (Kingsley) suddenly augment, when the rate of water excretion augments following ingestion of water. Chloride is eliminated four times as fast as without water ingestion, then its output subsides before water excretion diminishes; as though the sudden increase of water excretion caught the processes of solute excretion napping, and some time were required to exact the separation of each solute from the flood of water. The ratio of solute output to water output is accordingly found to be smaller in proportion as more of each has been just previously excreted.

In brief, while the content and elimination of urea and other substances are being influenced by water content and exchange, the concentrations of many or all substances in blood and other tissues are simultaneously affected. Evidently (1) the changes of concentration noted in section 79 are not solely those of dilution; (2) time factors loom larger than load factors in some of them; (3) other methods than those measuring rate of urea excretion alone are required to ascertain whether the production of urea is affected by water content; similarly for any other component.

(5) Neuromuscular activities have been noted only in qualitative fashion. In extreme positive loads of water the dogs of Rowntree ('23) exhibited muscular twitchings, hyperirritability, asthenia, ataxia, vomiting, coma, convulsions, and death. Each is a type of behavior capable of quantitative study at known water loads; several may hasten the recovery of the animal toward water balance. Individual reflexes and responses are equally susceptible of evaluation, and might be found modified at moderate water loads.

In extreme negative loads, still fewer observations are recorded. Anorexia, vomiting of food, restlessness, ataxia, and death are known. Dyspnea is prominent (Pernice and Scagliosi, 1895) but panting in response to concomitant heat is slower to start (Gregersen, '31; Dontas, '39). All instances in which "thirst drives"' have been induced in dogs may furnish material for the present study. Water load, in turn, is a proper measure of the intensity of these urges or drives to activity. 
Certainly the presence of too much or too little water in the dog disturbs the bodily functions in many measurable ways. From the even tenor of maintenance, departures are visible in the alimentary secretions, food ingestions, energy transformations, renal clearances, and diverse neuromuscular activities. Several of these modifications are distinctly correlated with the suddenness of water loading. Which of them come as shocks and which are compensatory can be decided only in part, when judged according to their promotion of recovery of water content of the whole body.

\section{\$ 85. Quantitative characterizations of Water loads}

Increments in diverse volumes, concentrations, and rates of functioning in particular tissues, now appear correlated with water loads of the whole body. Each of these is evidently interrelated with all the others; and as long as only one variable $(\Delta W)$ is, or possibly two variables ( $\Delta \mathrm{W}$ and $\Delta \mathrm{t}$ ) are, alone "independent," the relations among all the correlatives can with ease be described quantitatively. To do this, tables 23 and 24, and numerous twodimensional graphs such as figure 124, are combined in an alignment chart (fig. 131). The latter emphasizes the concept that no one of the quantities plotted is of more consequence than any other; all are coordinate states or happenings.

To the increments measured might be added the modifications in a variety of further quantities such as pressures (in arteries, cranium, spinal fluid, Rowntree, '26), physical condition of skin, and rectal temperatures (Keith and Whelan, '26).

Each of the modifications mentioned and most of their combinations, may appear in many types of water load. Speaking of man, Underhill and Fisk ('30, p. 348) state that: "Such apparently different chemical conditions as Asiatic cholera, infant diarrheas, intestinal obstruction, influenza, war gas poisoning, and extensive superficial burns, exhibit as a common symptom a marked concentration of the blood."

Measures of blood concentration have been preferred above all others to characterize states of water load. Data concerning them are obtained more easily than concerning any other correlative except body weights that have yet been used. It might be inferred from the above statement that any and every kind of blood concentration is modified. Haden and Orr ('23) say more specifically that "the characteristic features of dehydration are: increased 
blood viscosity, increased plasma non-protein nitrogen, increased protein destruction, decreased oxygen capacity, decreased blood volume." They quote specific researches on each point. But those features are not common to all types of "dehydration." The type that most interested Haden and Orr was the state of upper intestinal obstruction with vomiting and inanition. An example of

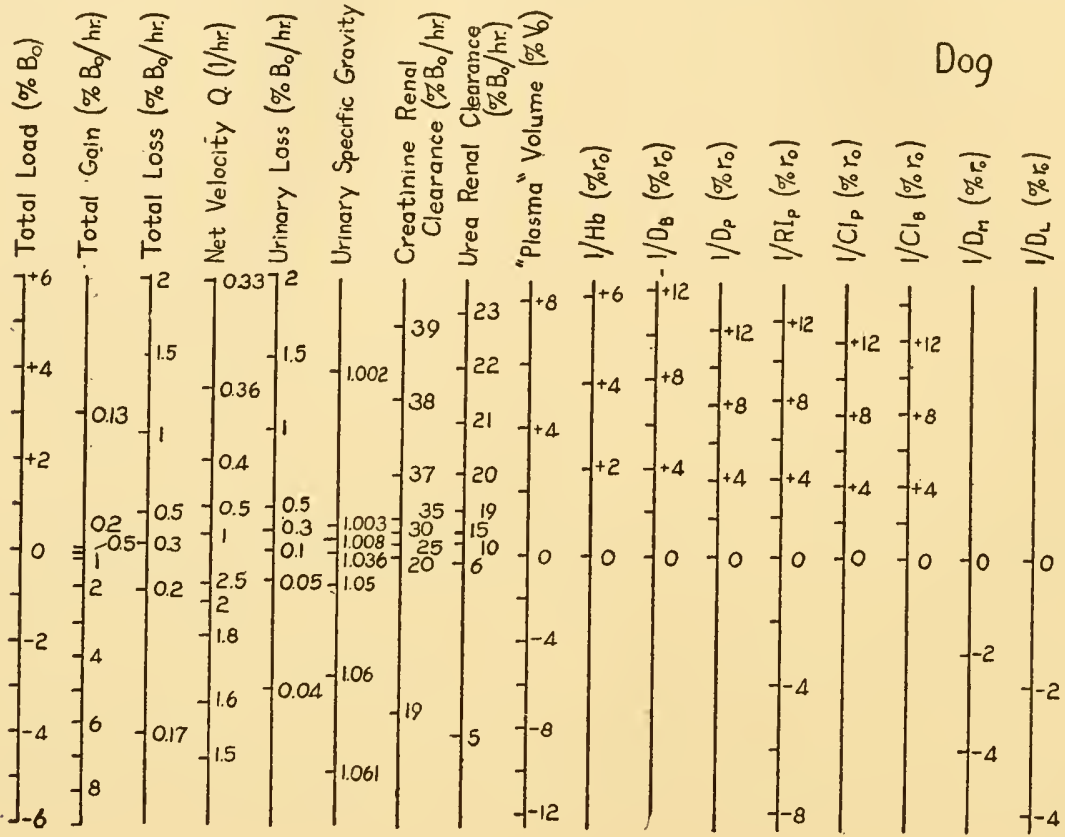

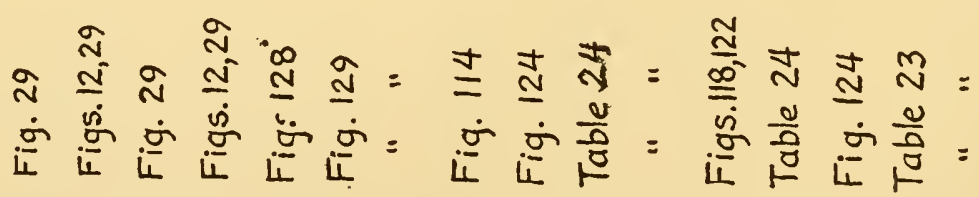

FIG. 131. Alignment chart showing relations among 17 variables. The chart is read along any horizontal parallel. Each of the quantities was measured in relation to total water load of dog in approximately stationary states, as shown in previous charts which are here indicated.

an exception to their supposedly general picture is, that decreased oxygen capacity is not characteristic of deficit by water privation (Flinn and Scott, '23). Any other categorical characterization of all negative water loads is equally incapable of applying to all; some are more useful than others in differentiating among its types, depending upon the variability of the data, the goodness of the correlation, and the ease of measurement. 
By these criteria the modifications under diverse water loads of the dog that at present seem suitable for routine use are: (a) total dry residue of plasma, or any of its colligatives, (b) rate of water exchange and (c) "plasma" volume. It must be stipulated that initial or control measurements be made on the same individual when in water balance; for most quantities vary among individuals enough to make estimations of any but the most extreme loads of water impossible in the living dog.

One combination of modifications represents a water load of specified type and amount. Moreover, by a sieving of the single characters making up the combination, diverse types of load may be distinguished, thus differentiating the water deficit studied by Haden and Orr ('23) or that studied by Gamble and McIver ('28) from that studied by Herrin ('35) or that shown in figure 122.

Having aligned some of the variables correlated with body water content in $\operatorname{dog} \mathrm{s}$, I can evaluate some of the statements that have been made about water content. Frequent has been the use of the terms dehydration, hydration, and the like. As these terms implicitly referred to some one type or any group of types of water load, whatever characterizations are suggested from time to time may now be differentiated.

(1) A view of water balance, proposed particularly by Gamble ('29) is that the water content of the body depends on the total solute present in the body. "Volume is determined by the total quantity of substances in solution. ... The primary event on which the loss of water depends is, namely, a loss of substance." Gamble investigated dehydration by abstracting from dogs various body fluids, particularly pancreatic juice, intestinal juice, and gastric juice. That fact clarifies the meaning of his generalization; the generalization covers depletion of water content by taking away body fuids, each of which contains solutes as well as water. When on the contrary, solutes are not removed, body volume is not proportional to the substances in solution, so that vapor pressure or its colligatives are not constant (fig. 131).

A further view, put forward by Magnus ('00), is that "the blood plasma may be completely sustained, over a considerable period of progressive dehydration, by water and materials derived from interstitial body fluids." (Gamble, '29, p. 912; also Underhill and Fisk, '30, p. 349). Yet in another type of water deficit than theirs the blood plasma decreases in volume (fig. 114) and increases in 
concentration (fig. 122); it even loses more than its proportional share of the water that is missing from the body as a whole. Still it cannot be said that "dehydration is limited to loss of water from the interstitial and from the vascular compartments." In none of the actual measurements available, is it clear that plasma volume or concentration is kept more nearly uniform than any other volume or concentration, nor is the evidence clear that cellular substance is protected from sharing in water deficit.

As the dehydrations and hydrations that are observed most often experimentally, happen to consist in removing or adding extracellular fluid and solutes to the body, investigators have gained the impression that extracellular fluids are more variable than intracellular. When it is inferred that "in dehydration extracellular water is depleted before intracellular water," it is not realized that the more frequent choice of conditions of sodium imbalance has led physiologists to extend the prediction that the whole syndrome will occur even in types of water load where sodium is not much disturbed, such as desiccation by water privation.

(2) Many theories suppose that water content depends upon the dictates of particular nerve cells or endocrine glands. None of the quantitative data that can be correlated in the present study lend more support to such a view than to any other view. Possibly relations of this sort are not expected to be made quantitative. It appears just as likely that any (or all) of the correlations that have been worked out represent crucial means of regulating the body's water content. But there is here no known way of detecting a crucial correlative as contrasted from an indifferent one. Perhaps a physiologist not favoring such theories may nevertheless be grateful to them for having suggested the gathering of data now utilized in descriptions.

\section{§ 86. Summary}

Each of several kinds of physiological modifications is correlated with $\pm \Delta \mathrm{W}$. Further, within the conditions prevailing, these various kinds are correlated with one another. Accordingly, any or all of the above data might be added to an alignment chart of bodily changes, or to a table of characterizations of dogs having diverse loads. Further kinds of physiological activities might be brought into the system of those already correlated, when adequate 
information is available. Such would be rates of blood flow, of pulmonary ventilation, of muscle shortening, of swallowing. As each physiological function is measured, increasing numbers of correlations become possible, for all that have gone before fit with the new data.

At present it is inferred that both positive and negative water loads are distributed beyond the blood; the whole body thus shares in the water. The plasma increases its load by a fraction greater than the remainder of the body does. But I see no evidence that any one "compartment" regularly maintains its volume or concentration while others are sacrificed. No tissues store outstanding proportions of water. From data on volumes and concentrations of tissues it is inferred that electrolytes mostly stay where they are, while water added to or subtracted from the body ultimately distributes itself so that vapor tensions are everywhere nearly equal. If solutes are by manipulation added or subtracted with water, other situations result.

Among the many variables of the organism that are correlated with water load, it seems arbitrary to emphahize any one correlative as constituting either a signal or a director of processes concerned in recovery, though some are of such a nature that they could serve in that capacity, such as increments of plasma dilution and of plasma volume. Modifications in metabolisms, behaviors, and compositions, whether regarded as unavoidable coincidences or as advantageous adjustments, are at the same time characteristics by which diverse degrees and types of water load may be differentiated and classified. 


\section{Chapter XI}

\section{SOME OTHER CORRELATIVES OF WATER CONTENT (IN OTHER SPECIES)}

\$ 87. Many compositions and functions of the dog are modified in water loads; they have now been recounted. Some will next be shown to change similarly in other kinds of animals as well. How nearly are the modifications suffered by various mammals alike at similar water loads? Is there a combination of features that holds in all species? Existing data can decide for man, frog, rabbit, and rat; but for the major portion of the animal kingdom, ignorance of these matters is almost total.

Lest I raise hopes of being able to present a well-filled body of information for even these species, I add at once that the material does not cover much of the same ground in any two of them. On blood of man, for instance, some thirty sorts of changes in concentration with water increments might be described, including the relations of some of them to one another. But no one change was measured often enough to furnish a coefficient of correlation, nor were extreme water loads studied. And, the same kinds of concentration were not measured in any species other than man except dog.

The data proper to this chapter have been analyzed just as carefully as in the previous one, but I refrain from presenting them at full length. The conclusions from them are nevertheless indispensable if general characterizations of states of water load are to be drawn.

\section{§88. MaN}

The object is to find what variables suffer modifications along with water contents in man. Each such variable serves both as an indicator of water load and as a security for its recovery. The information available concerns chiefly volumes of distribution, blood dilutions, compositions, various metabolisms, and specific behaviors.

(1) Volumes. Most of the volumes of distribution $\left(V_{D}\right)$ that have been measured in diverse water contents are "plasma" volumes (brilliant vital red, trypan red; see tables 5 and 6 ). It is 
remarkable that three independent studies (Dresel and Leitner, '29, '31; Gregersen and Bullock, '33; Marx, '35, p. 79) show that in both positive and negative increments, $\Delta \mathrm{V}_{\mathrm{D}}$ is eight times $\Delta \mathrm{W}$; or, only 13 per cent of the body weight takes part in the distribution of the water increment. If consistency of results is a basis for trusting them, these though not numerous are highly trustworthy.

When Dresel and Leitner ('29) investigated the effect of ingesting only $50 \mathrm{ml}$. of water $\left(0.1 \%\right.$ of $\left.\mathrm{B}_{0}\right)$, a volume that does not usually induce diuresis, the "plasma" in four subjects apparently increases by 6.6 per cent, a volume which itself is three times that of the ingested water. It is true that dye volumes are measured with limited accuracy, yet this series differs significantly from controls. Much remains to be learned in the relations of $\Delta V_{D}$ to body load of water, it seems.

"Corpuscular" volumes are, in the three investigations mentioned, modified almost as much as "plasma" volumes insofar as hematocrit estimations indicate the ratios between them. But when persons without spleens were tested, then in excesses of water the total circulating "corpuscular" volumes are no longer modified (Dresel and Leitner, '29). Individuals having certain diseases involving livers or kidneys also show no modifications of "plasma" volumes after drinking water in excess (Oka, '38a).

Few other volumes of distribution have been measured in any type of measured water increment. If the object is to find a qualitative increase in some $V_{D}$ with increase in the body's water content, that has been accomplished. If modifications of volume are to be relied upon as indicators of water deficit and excess, adequate quantitative correlations must be made.

(2) Dilutions. The weights and the water contents of human tissues are known from autopsy materials, but not their changes with water increments. With respect to dilutions of blood constituents, some few quantitative relations can be made out from data confined to three types of water increment, namely: single ingestions by mouth, continued ingestions by mouth, deficits by privation. It is readily recognized that other types of increment in man yield other results, as was the case in dog.

The time courses of modifications in dilution have been reported several times for chloride of whole blood in water excesses (Priestley, '21; Marx, '26; Dresel and Leitner, '29; Farkas, '32; Smirk, '33). The diversity, both in amount of dilution and in temporal 
sequence, among the mean results obtained by the several investigators, is greater than the diversity found by any one of them. A similar diversity in the degree and in the duration of dilution appears for dry residue and hemoglobin of whole blood; and for refractive index, evaporative tendency, and electrical conductivity of serum (data reviewed by Marx, '35; Rominger, '20; Abe, '31c). That most constituents which are modified suffer positive dilution in some tests, is the conclusion that may be drawn. But the magnitudes of these changes are by no means reproducible. Most of the changes at one time or another exceed the increment that would be expected if the water drunk were equally distributed through all the body water already present. This statement extends to water too, judging from meagre data reported by Marx ('35, p. 79) and Gregersen and Bullock ('35).

A very prompt decrease of the erythrocyte "count" may be found in arterial blood after sudden water ingestion. When $0.3 \%$ of $\mathrm{B}_{0}$ of water is given by duodenal tube, the maximal dilution is reached in only 0.05 hour. When $0.7 \%$ of $B_{0}$ is given by mouth, in contrast, the maximum is reached in 0.15 hour. In both cases the increment in dilution is of the order of 20 per cent, and disappears again in about 0.25 hour; a second dilution coming on only after 0.50 hour (Klein and Nonnenbruch, '30). Doubtless extended study of the time relations of the blood and tissue changes would reveal many points of physiological refinement and of individual diversity.

In steady states of water increment it has not been demonstrated whether dilutions of blood also remain stationary. The amounts of change in various constituents again bear no constant proportion to one another (Farkas, '32). Those that change, uniformly show positive dilution, and in most cases indicate very small volumes of distribution of the excessive water contained in the body at the time.

A number of distinct differences in the courses and amounts of change in blood dilutions are observed in pathological individuals. Some persons with diseases involving injuries of the hypophysis show either no or a greatly delayed dilution of the dry substance or the hemoglobin of the blood after drinking the usual $2 \%$ of $\mathrm{B}_{0}$ of water (Kiss, '27; Marx, '28). Those with certain liver injuries show very prompt and extreme dilution of dry substance (Kiss). Possibly a useful scheme of differential diagnosis could be worked 
out for many lesions from changes of blood dilution following the "water tests" ; but the evidence now indicates that most differences hold only in means, and hence (as usual) repeated and multiple tests are required clearly to demonstrate diseased states.

In body fluids other than blood, little information is available concerning concentrations, except in urine. It would be possible to go into detail for some dozen constituents of urine as excreted during $\pm \Delta W$. When urinary rates (and $+\Delta W$ ) increase, total substances (Krüger, '37) and those individually measured all tend to be reciprocally diluted. Or, their rate of excretion changes only 1 to 4 fold while the water excreted is modified 25 fold. In steady states of water excretion the reciprocal relation is less dependent on time, and can be described precisely for chloride (Farkas, '32, Kingsley).

In general, the dilutions undergone by many constituents of the blood and plasma confirm the measurements of volumes in showing that the plasma takes a large share of the body's water increment, a conclusion that may or may not conflict with conceptions based on the "plausible" assumption that osmotic pressure of fixed constituents controls the distribution of added or subtracted water. I imagine that some of the data represented are not reproducible enough to justify the effort expended in correlating them; but there seems to be almost no way of foretelling which they are. So long as statistically significant numbers of measurements, and their probable errors, were not the fashion in physiology, adequate data were not often provided. Today they serve at least to indicate the sorts of quantitative information that are needed for the description in which I am interested. I am not certain that any of the plasma dilutions are significantly different in man from their homologs in dog.

(3) Compositions. Changes of composition of the body as a whole may be measured in metabolic experiments in which water contents are varied while the diet is otherwise constant. The reproducibility of such retentions and depletions, and the role of time in them, may be again questioned, since large numbers have not been done. Among three electrolytes measured by Wiley and Wiley ('33), water privation leads to increased sodium content, either increased or unmodified chloride content, and decreased potassium content. At no time does the deficit of any one (in per cent of content) equal the deficit of water. Each returns to nearly the original value when water balance is restored. The relative 
proportion of the two cations also is modified, sodium becoming a larger fraction of the body's electrolytes.

These relations may further serve to demonstrate that one constituent (water) may not be varied alone. Though water be the only "intentional" variable, a host of other components automatically change their amounts with it, and not in equal proportions.

Other components (properties) of the body may be measured statically in relation to water content; but few suitable data concern them. These are various pressures (vascular, cerebrospinal, intraocular), temperatures (rectal, surface), and plasticities (of eyeballs, skin). Excitability to electrical stimulation through the skin (chronaxie) increases in the first hour after water is ingested (Achelis, '30). No one quantity can at present be said with certainty to be independent of water load.

(4) Metabolisms. Rates at which diverse processes occur are likewise modified with water contents.

Oxygen consumption and carbon dioxide production are probably significantly accelerated by water excesses. No regular changes in respiratory quotient have been found. The increases of rate of oxygen consumption precede the augmented rates of water excretion (Jarisch and Liljestrand, '27; Lublin, '29; Grollman, '29), and may be concerned rather in the activities of ingestion and absorption and in the excitement of the procedure than in maintenance of water contents. Lequime ('40) found no modification of rate of oxygen consumption.

Rates of volume flow of blood (cardiac output) are likewise said to be increased by an average of 20 per cent. The indirect methods used to measure them (absorption of nitrous oxide or of acetylene), and the variability among paired controls, suggest that some reservations be kept about them. Frequencies of heart beat regularly decrease after water ingestion.

Various types of catabolism have been supposed to be modified by positive water increments, from the facts of faster elimination of nitrogen, phosphorus, urea, or chloride in urine (e.g., Orr, '14). Once these faster eliminations are corrected for the faster water excretions (in the manner shown in table 25), the supposed increases in catabolism no longer appear. Unknown factors of analogous sorts may be suspected in almost any process that is being studied. So long as the conclusion is drawn that the rate of nitrogen excretion as measured by analyses of urine, or the absorp- 
tion of acetylene as administered by Grollman's procedure, are increased during water excess, the conclusions in this form will stand. But if it be inferred that nitrogen is liberated from protein faster, or that the blood passes through the heart faster, then when other methods and other corrections and other factors receive recognition, the conclusions will need restatement.

Among alimentary secretions, particular attention has been given to measurements of salivary flow (Cannon, '18; Gantt, '29; Winsor, '30; Gregersen and Bullock, '31). In most of them the deficits of water in the body are not recorded, and for present purposes are merely approximated from statements of the times elapsed in producing the state of desiccation. By each of the arbitrary procedures chosen, the rate of flow decreases with increasing deficits of water content. It may not be that, by extrapolation, the rate increases with water excesses; that needs investigation, but happened not to interest those who wanted to relate the water supply of the mouth to the sensation called "thirst."

A few estimates of Baird et al. ('24) and of others indicate rapid secretion of gastric juice following water ingestion $(+\Delta \mathrm{W})$.

Rates of renal excretion of numerous substances are modified with water excesses; a few are unaffected between urinary outputs of 0.04 and $0.4 \%$ of $\mathrm{B}_{0}$ /hour (e.g., creatinine (Marshall, '20; Krüger, '37)). The two most abundant substances in human urine are chloride (Kingsley) and urea (Marshall, '20); the time elapsed since water load is established is an important factor in the elimination of both of them. The relations between solutes and water in excretion serve to characterize the excretory processes in man; no differences from dog have been recognized.

Clearances through the kidneys are well known to be correlated with rates of water excretion (urea, Austin et al., '21) and hence with water content. Ordinarily clearances are measured after excessive administration of the substance whose clearance is being studied. Within limits, the copious excretion of the substance itself modifies the rate of water excretion (i.e., solute diuresis). Again, each substance cleared has its own quantitative relation to water load (Smith, '37). Man could be distinguished from dog. solely by the several influences of water content (augmentations) upon clearances.

Behaviors of diverse sorts have been qualitatively reported in extreme water loads. Large excesses are accompanied by muscu- 
lar twitchings, restlessness, chills, vomiting, dyspnea, asthenia, sweating, diarrhea, incoordination of movements, stupor, aphasia, dilatation of pupils, opisthotonus, convulsions, unconsciousness, and death (Marx, '35; Helwig et al., '35, '38; Barahal, '38). These ill-defined terms refer not merely to "symptoms," but also to states of the organism. Certain units of the broad syndrome actually relieve the body of water, particularly vomiting, dyspnea, sweating, and diarrhea. Other units may be viewed as parts of an inevitable complex, whether of unavoidable sequelae, or of the organism's attempts to avoid excesses and recover from them.

Prolonged drinking of about $15 \%$ of $\mathrm{B}_{0}$ of water per day increases the urge to drink (Regnier, '16; Kunstmann, '33). After 8 to 127 days the subjects often awoke at night and got out of bed to get water. That the drinking opposes recovery of water content can be surmised; it may still be the shortest way to total balance.

In water deficits another set of behaviors appears (King, 1878; McGee, '06), as observed in travellers lost in the hot desert. They are: inability to swallow, dimness of vision, partial deafness, vertigo, delirium, incoordination of movement, emotional irritability, dreams of eating and drinking, and illusions of lakes and running streams. Restlessness, tormenting sensations, and mental confusion prevail. Each word stands for a long story of gruelling human experience. Some attempt has been made to classify these manifestations according to the degree of deficit at which they occur, with emphasis upon those incident in extreme loads. All of them are susceptible of quantitative study by refined methods under controlled conditions and in known states of water load; I have faith that quantitative relations will mean more than qualitative characterizations.

(5) Summary comment. Most of the data reported were originally obtained upon the hypothesis that some one change in the body would be found to initiate the responses of the organism to the load of water. With respect to water excess, Nonnenbruch ('24) concludes that: "No regular changes are known that occur in the body after drinking one liter of water and regulate the elimination of it." I think that is not a fair statement of the situation now. Often the changes found are less regular than was hoped; that does not modify their statistical significance. The known changes may take part in regulating excretion but only on a par with any other concomitant features. No sign from heaven is likely to point out one key that unlocks the eliminatory activities. 
With respect to water deficit, whimsical choice has emphasized the dryness of the mouth. It is said (Cannon, '32, p. 62) that a sensation of "thirst is due to local dryness of the mouth" and acts "as automatic stimuli to make certain that the reserves of water are maintained." No method of measuring the sensations as such has been devised, nor of evaluating the dryness. The salivary flow to the mouth has been measured; since it does not alone control the dryness of the mouth, it itself is not regarded as a regulator. The theory that a single governor exists for a physiological activity does not appear now to be substantiated. Moreover, there is no means of recognizing a regulator even if examined. A specific volley of nerve impulses or an isolatable extract are possible links in a chain, or elements in a complex. Repeatedly it is found that many factors vary simultaneously; each one is as central as any other, and only by convenience of thought is one exalted above another. Hence the emphasis may be put on the interrelations of the diverse variables and the degrees to which the correlations hold in repeated tests, in diverse individuals, in diverse species, and in diverse types of water increment. Thus characterizations and mutual relations are established, all with respect to the one variable, water content.

The foregoing sorts of data should make it possible to design methods for identifying diverse states of water content in man. One task is to differentiate various types of $\pm \Delta \mathrm{W}$, a second task is to choose indices by which to estimate the degree of increment or deviation from water balance, a third is to differentiate control states from pathological states. The serious lack of reproducible measurements makes these tasks at present more virtual than real.

(1) In spite of all the "dehydrations"' that have been studied, and the liters of solutions that have been swallowed, it is at present difficult to identify the specific procedure of water loading, from measurements of compositions and metabolisms alone. The clearest case is to find whether or not solutes (chloride) were added or subtracted along with water, by analyses of their proportions in urine and in plasma.

(2) Suitable indices of increment in water content are increments in dilutions of urine and of plasma, rates of salivary flow, and augmentations of urea clearance. Dryness of lips or edema of skin are qualitative only.

(3) Increments in volumes and in dilutions, especially of plasma 
(not whole blood), are demonstrable as averages, while any single individual or determination may fail to register any. Every increment involves one measurement in a control state and a second measurement in a loaded state. The ideal diagnostic procedure is one that does not need the first, for rarely is the diseased individual available then. Hence a great variability must be recognized in control states, and a greater one is loaded states; and only the coarsest increments of water content can be recognized with high probability from the specific gravity or refractive index of plasma. More reliance can be placed in simultaneous determination of a volume, a plasma concentration, a rate of salivary flow, a metabolism, a clearance, and a rate of water exchange. Then the role of variability in each is mutually minimized; the characterization of a state of water load is laborious yet certain.

\section{\$ 89. FroG}

Frogs differ from mammals in containing higher proportions by weight of water, and probably much larger and more variable volumes of interstitial fluid. The distributions of excesses and deficits of water may therefore prove to be very different in them. For present purposes all species of Rana are treated together; nearly all the measurements at hand were made upon frogs suddenly killed and sampled.

Volumes of tissues are compared in control and in partially desiccated individuals, each being measured as a fraction of the original body weight (table 26 ). That the losses represent depletion of water alone was ascertained in a third group of frogs that

TABLE 26

Distribution of water losses among tissues of 10 frogs (mean $B_{0}, 43.57$ gm.) desiccated by-42.7 per cent of $B_{0}$ and separately analyzed. Data of Smith and Jackson ('31)

\begin{tabular}{|c|c|c|c|c|c|c|}
\hline \multirow{2}{*}{ Tissue } & \multirow{2}{*}{$\begin{array}{c}\% \text { of } \\
\text { body in } \\
\text { controls }\end{array}$} & \multirow{2}{*}{$\begin{array}{c}\% \text { of } \\
\text { tissue } \\
\text { weight } \\
\text { lost, } \Delta V\end{array}$} & \multirow{2}{*}{$\begin{array}{c}\% \text { of } \\
\text { whole } \\
\text { body's } \\
\text { loss }\end{array}$} & \multicolumn{2}{|c|}{$\begin{array}{l}\text { \% water found by } \\
\text { analysis, } 100 \mathrm{E} / \mathrm{F}\end{array}$} & \multirow{2}{*}{$\begin{array}{c}\text { \% water } \\
\text { lost, } \\
\Delta / \mathrm{D}\end{array}$} \\
\hline & & & & Control & $\begin{array}{l}\text { Desic- } \\
\text { cated }\end{array}$ & \\
\hline Whole body & 100.0 & -42.7 & 100.0 & 82.14 & 87.40 & -43.5 \\
\hline 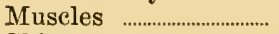 & 38.0 & -30.5 & 27.1 & 82.15 & 74.52 & -30.0 \\
\hline Skin ............ & 13.3 & -41.2 & 12.3 & 81.05 & 70.84 & -35.0 \\
\hline Skeleton & 12.0 & -4.2 & 0.7 & 58.13 & 57.94 & -0.4 \\
\hline Liver ....... & 3.81 & -57.7 & 4.1 & 76.08 & 72.10 & -14.4 \\
\hline Stomach + intestine & 1.42 & -30.0 & 1.1 & 85.00 & 75.21 & -39.6 \\
\hline Blood ................. & 3.27 & -80.8 & 5.7 & 88.97 & 77.33 & -51.3 \\
\hline "Lymph spaces" ... & 23.0 & -85.1 & 45.8 & ............... & $\ldots \ldots \ldots \ldots$ & ........... \\
\hline
\end{tabular}


were replaced in water; they fully recovered their water contents before analysis. Volume of collectable blood, whether measured by washout (Gürber, 1889) or by drainage (Smith and Jackson, '31), decreases relatively more than the partially desiccated body as a whole. When the same tissues manifest both losses of weight and changes of composition (dilution of dry residue), it is possible to compare the increments of tissue volume as found by the two independent methods (table 26, columns 3 and 7 ); actually they yield similar values in all organs except liver.

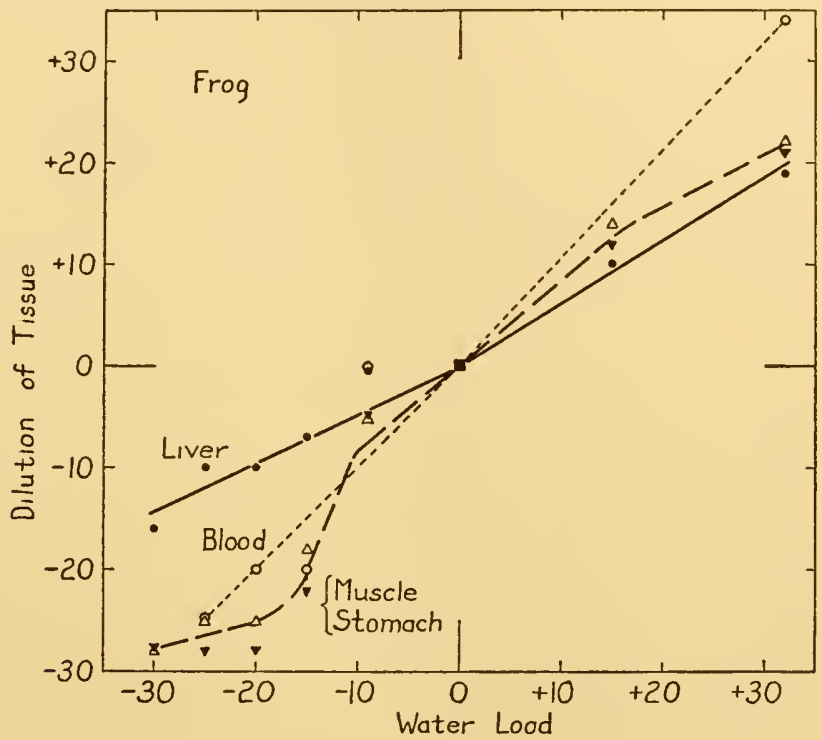

FIG. 132. Increment in dilution of tissue ( $\%$ of control) in relation to water load (\% of $\mathrm{B}_{0}$ ). Frog. Dilutions are measured by ascertaining dry residue in weighed samples, whence $\Delta \mathrm{E}=100\left(\mathrm{D}_{\mathrm{a}} / \mathrm{D}_{1}\right)-100$. In negative loads each point is the average of 4 individuals of Rana esculenta (Rey, '37, p. 1120); in positive loads each point is the average of 12 individuals of Rana pipiens (new data).

It is evident that in extreme desiccation the interstitial fluid (= the non-collectable juice) of the frog suffers greatest reduction. The blood is next greatest. Perhaps the circulating tissues of the body, therefore, change most. Other measures of dilution are modified similarly to dry residue (specific gravity, enumeration of blood erythrocytes, Durig, '01, p. 414). In general (fig. 132 and table 27) the relative dilutions differ among various tissues at similar water loads of the body. If there are any depots for water, they are the blood and lymph. Since with water load the dilutions 
of various tissues are modified disproportionately, some inkling of their increased functional incompatibility can be visualized.

In cases where individual frogs are analyzed, it is found that tissues of one kind vary among them no more than in mammals. Both in deficits and in excesses the blood shows the largest variation among individuals.

The distribution of water loads can be partially visualized, revealing characterizations sufficient to identify a precise state of water load (fig. 132) even if the total water load had not been measured. Thus, the mean dilution of muscle of $+22 \Delta \mathrm{E}$ corresponds to a mean increment in body of $+32 \Delta \mathrm{W}$. Using the rela-

TABLE 27

Increments in dilutions of tissues, in per cent of $E_{0}$, in frogs having diverse water loads. Coefficients of variation (C.V.) are $\sigma /$ control dilutions. $\Delta / S=$ reciprocal of increment in specific gravity

\begin{tabular}{|c|c|c|c|c|c|c|c|c|c|}
\hline \multirow[t]{2}{*}{ Tissue } & \multicolumn{2}{|c|}{$\begin{array}{c}\text { Durig ( ' } 01 \text {, } \\
\text { p. } 420 \\
\text { p. 416) }\end{array}$} & \multicolumn{2}{|c|}{ Ueki ('24) } & \multirow{2}{*}{$\frac{\begin{array}{c}\text { Iizuka } \\
(' 26)\end{array}}{\Delta / D}$} & \multicolumn{2}{|c|}{$\begin{array}{l}\text { Heller } \\
(' 30)\end{array}$} & \multicolumn{2}{|c|}{$\begin{array}{l}\text { Adolph (new } \\
\text { data) }\end{array}$} \\
\hline & $\Delta / \mathrm{D}$ & $\Delta / \mathrm{S}$ & $\Delta / \mathrm{D}$ & (C.V.) & & $\Delta / \mathrm{D}$ & $\Delta / \mathrm{D}$ & $\Delta / \mathrm{D}$ & (C.V.) \\
\hline Number of analyses & 3 & & 8 & & 5 & 14 & 16 & 12 & \\
\hline Whole body & -26 & $(-28)$ & -19 & \pm 5 & -23 & -10 & -24 & +32 & \pm 7 \\
\hline Blood & & -53 & -27 & \pm 15 & -39 & & 0 & +34 & \pm 39 \\
\hline $\begin{array}{c}\text { Muscle, gastroc. ........... } \\
\text { adduc. }\end{array}$ & $\begin{array}{r}-42 \\
\ldots \ldots . .\end{array}$ & $\begin{array}{l}-31 \\
-27\end{array}$ & $\begin{array}{l}-19 \\
-19\end{array}$ & $\begin{array}{l} \pm 7 \\
\pm 7\end{array}$ & $\begin{array}{l}\cdots . . . \\
\cdots \cdots . .\end{array}$ & $\begin{array}{r}-9 \\
\ldots \ldots\end{array}$ & -24 & +22 & \pm 11 \\
\hline " tib. ant. ............. & $\ldots . . .$. & $\ldots . .$. & $\ldots . . .$. & $\ldots . .$. & & ....... & ....... & +19 & \pm 11 \\
\hline $\begin{array}{l}\text { Leg } \\
\text { Liver }\end{array}$ & -20 & -18 & $+\cdots$ & +9 & $\begin{array}{l}-23 \\
-14\end{array}$ & -11 & -11 & +19 & \pm 19 \\
\hline Stomach & $\ldots . .$. & $\ldots . . .$. & -18 & \pm 7 & $\ldots . .$. & $\ldots . .$. & $\ldots . .$. & +21 & \pm 13 \\
\hline Skin & ....... & ....... & -8 & \pm 19 & ....... & ....... & ....... & ....... & $\cdots \cdots$ \\
\hline
\end{tabular}

tion conversely, I find that a body of $+27 \Delta \mathrm{W}$ furnishes muscles of $+19 \Delta \mathrm{E}$, as it actually did in the study of recovery from water excess by isolated muscles (fig. 98).

The frog, therefore, differs from $\mathrm{dog}$ and man in the greater distensibility of its extracellular fluid volume. Each correlative of water load is, however, just as invariably related with water content in frog as in mammal.

\section{$\S 90$. RABBIT}

The compositions of various tissues are measured in water excesses under two conditions : during life in biopsy samples of blood and of some other tissues excised under local anesthesia, and at sudden death. Two types of water excess prevailed; the single administration of water by stomach, and the repeated administra- 
tion by stomach until about $25 \%$ of $\mathrm{B}_{0}$ had been retained and convulsions began (table 28). In a few similar tests of Gomöri and Molnar ('32), the rabbits weighed $+34 \%$ of $\mathrm{B}_{0}$ by the time convulsions began; correspondingly, their tissues showed greater dilution. While it is also possible to include data of Gomöri and Molnar on other rabbits given large amounts of water with pituitary extract until convulsions began (at $+31 \%$ of $\left.\mathrm{B}_{0}\right)$, I judge it advisable to preserve the distinction between the two types until the water load with pituitrin is shown to be indistinguishable from

TABLE 28

Dilutions $(\triangle E)$ of rabbit tissues, in per cent of $E_{0}$, after water loads were established in the whole body. Values in parentheses are those clearly not significant

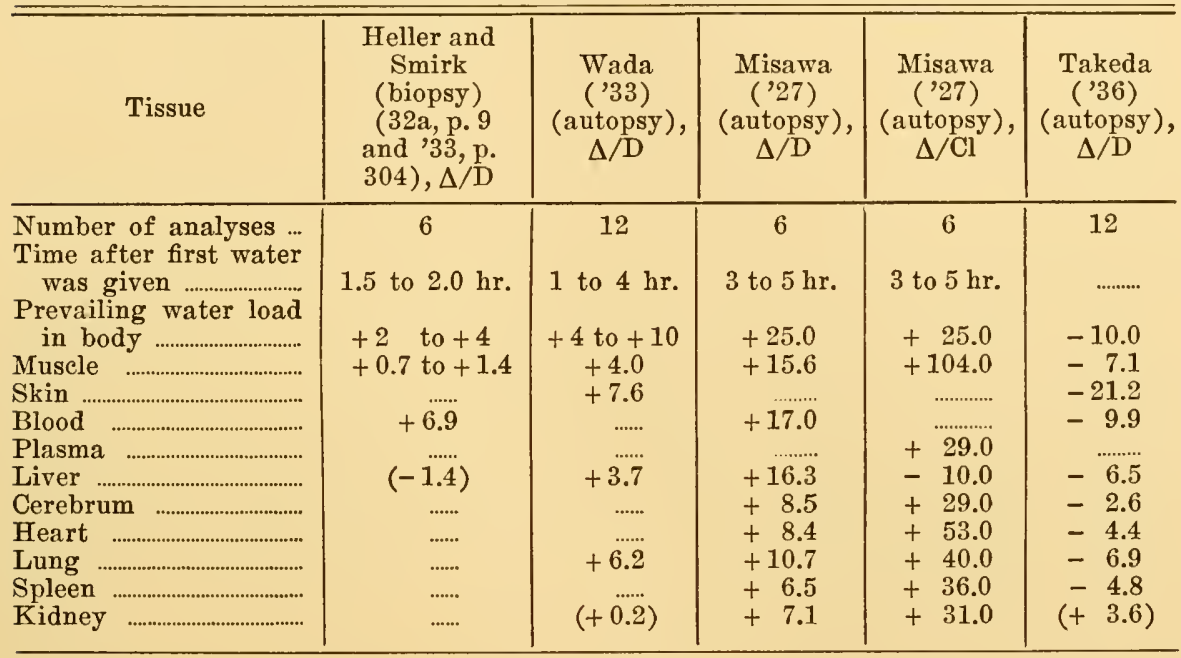

that without the pituitrin. In $d o g$ and in man the two types are already known to be not identical, even aside from rates of water exchange.

Among the tissues, blood manifests larger increments of water content than the average tissue. Only blood and skin appear as possible known depots for water. Muscle, liver, lung, and others are modified significantly less both in positive and in negative water loads. This is far from supporting the supposition repeatedly expressed (Underhill and Fisk, '30) that "the tissues act as reservoirs to maintain the water content of the blood at a constant level through the removal of excess fluid from the blood in hydremia and the release of fluid to the blood in anhydremia." Without computation of $\Delta \mathrm{E}$, it is difficult to appreciate how small an inerement of 
water in blood is equivalent to a large load of water in the whole body.

Among metabolic events, water content brings large changes in clearances of several solutes through the kidneys (Kaplan and Smith, '35). Rates of excretion of chloride, sodium, calcium, inorganic phosphate, and nitrogen increased 4 to 11 fold during 4 hours of continued water administration (Misawa, '27). Some of the chloride came from heart, lung, and spleen according to the analyses represented in table 26, column 5. In contrast, excretion of magnesium did not change and output of potassium decreased. The decrement of sodium and the retention of potassium in the body are opposed to results reported for man $(\$ 88(3))$ at smaller $+\Delta W$. Whatever the relations of chemical components to water in the rabbit, it is evidently impossible to suppose they all behave alike in the presence of water excesses. Planned attempts to identify changes in the contents of each of these electrolytes in six tissues of rabbits in positive water loads revealed no consistently significant modifications in any (Wada, '33); more samples at fewer times would perhaps have yielded significant results.

The rabbit appears to be characterized in water loads by large increments in dilution of blood and skin, and small modifications of muscle, as compared with dog. Whatever this means in terms of tissue functioning, it indicates that when the experimenter discovers that a rabbit is more convenient than a dog in providing suitable quantities of materials for analytical procedures, he does not virtually analyze a dog, or vice versa.

\section{$\S 91 . R_{A T}$}

Tissue compositions in this species change during absorption of a dose of water from the alimentary tract (Heller and Smirk, '32a, p. 16). Dilutions, as measured from dry residues, indicate that the partition of water between liver and leg muscle is uniform, but the first tissue is diluted more than the whole body, the other tissue receives less water than its proportional share (table 29, column 5).

Young rats were subjected to prolonged restriction of water intake by Jackson and Smith ('31b). Just enough water to maintain a body weight of 60 grams was allowed at all ages. When autopsied, all the tissues tested contain somewhat less water relative to their dry content, than control rats of the same age (but greater size), or than rats of the same size prevented from growing 
TABLE 29

Increments in dilutions $(\Delta E)$ of tissues, in per cent of $E_{0}$, computed from analyses of dry residues $(\Delta / D)$, in various species

\begin{tabular}{|c|c|c|c|c|c|c|c|}
\hline \multirow{2}{*}{ Tissue } & \multicolumn{4}{|c|}{ Excesses } & \multicolumn{3}{|c|}{ Deficits } \\
\hline & Dog & Frog & Rabbit & Rat & Dog & Frog & Rabbit \\
\hline 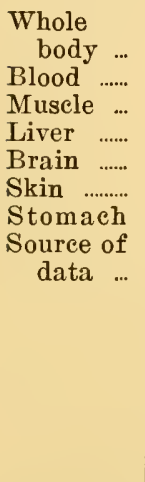 & $\begin{array}{l}+12 \\
+9 \\
+17 \\
+9 \\
+9 \\
+12 \\
\ldots . . . \\
\text { Engels } \\
\text { ('04, p. } \\
356) ; \\
0.6 \% \\
\text { NaCl by } \\
\text { vein }\end{array}$ & $\begin{array}{c}+32 \\
+34 \\
+21 \\
+19 \\
\ldots \ldots . . \\
\ldots \ldots . \\
+21 \\
\text { table } 27 \\
\text { water } \\
\text { by peri- } \\
\text { toneum }\end{array}$ & $\begin{array}{l}+25 \\
+17 \\
+16 \\
+16 \\
+9 \\
\ldots . . . \\
\ldots . . . \\
\text { table } \\
28 ; \text { re- } \\
\text { peated } \\
\text { water } \\
\text { by } \\
\text { stomach }\end{array}$ & $\begin{array}{c}+5 \\
\quad \ldots . . . \\
+4 \\
+9 \\
\ldots . . . \\
\ldots . . . \\
\ldots . . . \\
\text { Heller } \\
\text { and } \\
\text { Smirk } \\
\text { ('32a, } \\
\text { p. 16); } \\
\text { water } \\
\text { by } \\
\text { stomach }\end{array}$ & $\begin{array}{c}-20 \\
-19 \\
-41 \\
-16 \\
-15 \\
-54 \\
-9 \\
\text { table } 23\end{array}$ & $\begin{array}{c}-43 \\
-51 \\
-30 \\
-14 \\
-13 \\
-35 \\
-40 \\
\text { table } 26\end{array}$ & $\begin{array}{l}-10 \\
-10 \\
=7 \\
=7 \\
=3 \\
-21 \\
+\quad 2 \\
\text { table } 28\end{array}$ \\
\hline
\end{tabular}

by restriction of food intake. The long period of time that elapsed during water restriction marks a type of water deficit unlike that by more temporaly water restriction. Water here appears to have the same role as any other indispensable food; its continued deficit in the body automatically inhibits the taking of all other foods (anorexia), with inevitable deficit of total substance in the body. That is a restatement of the above facts in the language of increments.

\section{\$92. Comparisons}

Other species than mammals and frogs have been studied only in isolated respects. A few observations of tissue concentrations exist upon pigeons (Schuchart, 1847; Falck and Scheffer, 1854a) and chickens (Pernice and Scagliose, 1895; Korr, '39). Measurements of oxygen consumption and carbon dioxide production in desiccation have been extended to several insects and molluses (Caldwell, '25; Thompson and Bodine, '36). Only by confusing diverse types of water load could a considerable body of comparative data be aligned. Among the meagre materials already mentioned, what uniformities and what contrasts are found?

What measurements could conceivably be made upon all kinds of organisms whose water contents are modified? None would be suitable that depend on morphological similarity; hence besides 
total water exchanges, total contents of diverse substances, rates of various total metabolisms, and some behaviors are available. In large animals and man, total contents have not been analyzed in diverse $\pm \Delta W$. Metabolisms also are very likely to be studied by methods not strictly comparable in diverse species. The actual data cover only two sorts of measurements in several species : dilutions of dry residue in particular organs, and rates of oxygen consumption.

With respect to rates of oxygen consumption, the only apparent uniformity is that found by Caldwell ('25, '31), in animals subjected to progressive evaporation and privation of water; namely that oxygen is consumed in moderate deficits of water slightly more rapidly than in controls, and in more extreme deficits equally or less rapidly than in controls. The definition of "moderate" and "extreme" is numerically diverse in the several species, and even so, exceptions have already been found. It is not implied that the increased rate of oxygen consumption is independent of increased muscular movements, nor that the movements are or are not "voluntary."

In comparisons among existing data, some interpolation is required to relate the data to some common increment of water content. An experimental increment of water that seems heroic for man appears to investigators so small as to be hardly worth studying in frog or worm. Of course, anyone is free to decide that body weight is not the most desirable basis for computing increments; but objections attach to any other possible basis, I believe.

Apparently the leading motive in the study of changes in blood after forced water ingestion was the picture that a modified blood circulating through the kidneys may excite them to specific activities. Extended investigation in man suggested that "the elaboration of urine is not or at least not solely dictated by the condition of the blood" (Farkas, '32). Part of the lack of parallelism between blood and excretion is that demonstrable blood dilution precedes the increase in rate of urinary output (Rioch, '27). Discovering this, some investigators relinquish this picture and the studies it suggests, while others modify the picture to suit the facts, as is readily done. Other notions initiated other studies. The supposition that the organism spends energy to eliminate the excess water, led to measurements of oxygen consumption in body (and in kidneys); the theory that the kidneys were informed of water 
excesses by hormonal or nervous pathways led to searches for hormones in blood, brain, kidneys, and urine. I think it helpful to suppose that all the modifications identified are equally significant parts of a many-sided physiological state.

In a study of physiology in the most general sense, it is useless to compare individual itssues, for none of them is common to all animals. Hence tissue comparisons are automatically limited to mammals, or to vertebrates, or to some one or few phyla. Organs having the same name may not be phylogenetically homologous even within the phylum (as, the several types of kidneys of vertebrates). Tissues of the same name may be compounded of diverse elements in extremely different proportions (as, the skin of vertebrates). Since the same name represents a variety of physiological as well as morphological units, the significance of numerical comparisons of water in tissues is accordingly limited.

The local distribution of water excesses and deficits, characterizing various species, concerns not merely the relative dilution of the tissue, but also the proportion of the whole body that it represents. When the absolute increments of water in each tissue are added together, a sum equivalent to the total body load $(\Delta W)$ is obtained. Approximately it is found that the same kinds of tissues take the bulk of increment both in excess and in deficit (table 29). They are blood, muscle, and skin, the most abundant tissues. Among species there are clear differences, muscle playing its largest role in dog, skin in all species. In reality all tissues are, in small part, reservoirs of diverse size and distensibility. The order in which relative dilutions occur are usually (table 29) : skin, blood, muscle, stomach, liver, brain.

In water shortage does the volume or dilution of the blood plasma (the "fluid matrix" of cells) change less than the volume or concentration of the body as a whole (Gamble, Underhill)? It may be remarked that constancy of medium in which living cells are working can be preserved only at the expense of constancy of cells. I find no evidence that blood has less than its share of water load, either in dog or in man, in frog or in rabbit, and I know of no positive evidence in any other animal. In the frog's water deficits, where the data are adequate, half the tissues lose less than the average, half lose more, and the blood loses most of any. In dog, man, and rabbit, the blood volume is changed as much as or more than the body as a whole. Contrary conclusions might be arrived at by 
(1) showing that another type of water increment has other water distributions, as indeed has been partially established in dog, or (2) using some other measure of volume or concentration than those underlying the present conclusion.

Among metabolic rates, alimentary secretions are qualitatively modified alike in $\mathrm{dog}$ and in man, but quantitatively the data are not accurately enough controlled for comparisons. Clearances are augmented on different scales in dog, man, and rabbit. Rates of excretion appear to vary similarly with $\pm \Delta \mathrm{W}$ in $\mathrm{dog}$ and in man.

I think it would be possible to distinguish a herd of ten individuals of some one species from any of the other five species of mammals discussed, by studying their physiological states in water excess or in water deficit. With the inclusion of still more species, probably the single distinctions would become blurred by intermediate conditions of composition and metabolism, but the combinations would not. What measurements would be chosen first, in order to differentiate among species? Present evidence would point to : urinary rate and drinking rate, dilution of dry residue of plasma, urea clearance through kidneys, and volume of distribution of vital red. Of course, preference for these measurements and criteria depends partly on the fact that they have already been used; better methods are probably awaiting discovery. Outside of vertebrates there are few criteria for distinguishing species by their responses to water increments, for modifications of rates of oxygen consumption in positive water loads have alone been tested to the point where marked correlation is known to exist.

In my opinion not all, perhaps none, of the modifications accompanying water loads are "accidents." Some or all are parts of the process of recovery, spring boards by which equilibration takes off. But, neither my predecessors nor I am able to decide which are the ones that contribute toward recovery, and which not. Recovery of water content has not been shown to be so rapid or so complete without them. 


\section{Chapter XII}

\section{FURTHER CORRELATIVES OF WATER CONTENT AND EXCHANGES}

$\$ 93$. The correlatives of body water that have been examined are only some of the many possible ones. A few other factors involved in water exchanges may now be considered. How are they related to maintenance and to equilibrations of water content?

\section{\$ 94. Possible forces}

Very often there is a strong urge to add to the study outlined, the step of inferring an "underlying" force in each water distribution and water exchange. Without the thought that something underlying can be investigated, it is said, there is no incentive to obtain data. An incentive may well be indispensable to investigation; but I find scant evidence that all the forces concerned in an exchange are ever identified. Thus, "osmotic" pressure may be inferred to be operative in some cases, from relations between concentration gradients and rates of water exchange. But other sorts of forces, both known and unknown to physicists, may be simultaneously present, yet described also by the very equations derived on the assumption that only "osmotic" pressure prevails. In brief, forces tending to restore water content may be differentiated in part, and summed to equal the net force present. The categories are labels by which a physiologist thinks about movements of water in terms of another science, and remembers and predicts what happens under specified conditions. Several instances may be cited.

(1) Forces concerned in water exchanges of many aquatic organisms are supposed to be chiefly differences of osmotic pressure between living unit and medium. For eggs of Arbacia, diverse media affect rates of exchange roughly in proportion to their relative concentrations (fig. 91). A physiologist interested in labelling forces then chooses whether (a) to define osmotic pressure as the total of all forces that are proportional to concentration, or (b) to assert that osmotic pressure probably represents a majority of the forces at work in moving water and regulating its exchanges. In either case what is accomplished is to classify forces according 
to the arbitrary criteria devised and used in the science of dynamics. Some forces are proportional to rate of movement or exchange, others to acceleration. The assignment of forces to classes may be a source of satisfaction, but there is nothing ultimate in the identification.

A diagram of water equilibration in Arbacia egg or in Phascolosoma (figs. 91 and 87 ) virtually relates initial concentration of body with rate of osmosis. That relation is very readily visualized by those accustomed to juxtaposing a force and a flow, perhaps as partial cause and effect. By extension, water equilibrations in dog and earthworm may be viewed as representing a force and a flow; in them, however, no causal relation between force and flow has been implied. Often people refuse to believe that data go together (are correlated) unless they be shown some "mechanism" connecting them, in terms with which they are already familiar. But biological science does not have to wait until categories of forces can be differentiated and until intermediate steps become familiar; a correlation in itself represents biological processes in which much other interest can be found.

Analyzing further the concept of osmotic pressure, I believe no physicist pretends to have demonstrated a "mechanism," in his sense, by which osmotic pressure is effective in moving water. The concept is of the same order as that of gravitation; mathematical relations of masses and the distances between them, sometimes loosely termed "forces of attraction," have been worked out, but no one presumes to identify the invisible threads by which gravity pulls. Osmotic pressure, however, like gravitational pressure, is a familiar phenomenon. This familiarity, coupled with its fertility in predictions, may account for the feeling of satisfaction that comes to those who find mathematical relations in water exchanges of a sort that fit the generalizations derived from phenomena termed osmotic pressure.

(2) With each volume increment are correlated diverse modifications other than those in rates of volume change. Thus, accompanying the increments of "plasma" volume in hemorrhage and in transfusion (fig. 96), numerous characteristic changes in blood (and other parts of organisms) of concentrations, physical properties, metabolisms, and many others, have been found. Various attempts have been made to arrange those changes known to occur with hemorrhage into "causal" chains. But each arranger pic- 
tures a different series (Meek and Eyster, '21; Blalock, '27; Gesell et al., '30). With more connecting arrows, and tipped at both ends, a description of interrelations results that may help to visualize the number of relations present, for it suggests the equal importance of many coordinated variables. In the absence of criteria by which to decide which of these are "directly" related to the restoration of blood volume, all appear equally concerned in what goes on. Changes in "blood" pressure are often believed to have a special relation to changes of "blood" volume, since it is a "hydrostatic" pressure having the dimensions of a force that may move fluids. That the "capillary blood" pressure minus mean extravascular pressure may ordinarily balance the effective "osmotic" pressure between blood and extravascular spaces (Starling, 1896) has been a useful hypothesis. Adding inferences, it is supposed that physiologically effective gradients of osmotic pressure are modified (in parallel with mean capillary blood pressure) and at balance always equal the "colloid osmotic" pressure as arbitrarily measured in vitro.

A quantitative relation between capillary blood pressure and rate of water exchange obtains for single minute blood vessels in data of Landis ('27). How to fit the measured net "colloid osmotic" pressures with the rates of passage of fluid is unknown; it is believed and hoped that the net rate will be zero when this pressure equals the net capillary blood pressure. These "forces" might even be plotted in a diagram that is analogous to an equilibration diagram, showing forces in place of rates. But its descriptive value, showing how blood volume is equilibrated in an average capillary, is little at present, for the inferences involved in its construction seem to me more presumptuous than when rates of fluid exchange are correlated with increment of blood volume.

(3) While the hypothesis of Starling is conceived as applying to the maintenance of blood volume, the adjustment of tissue cell volumes is quite generally supposed to occur at rates proportional to the difference of "total osmotic" pressure between inside and outside. No assurance is at hand that either all this or only this force is at work.

Within extravascular (interstitial) compartments it is commonly believed that effective "osmotic" pressure and effective "hydrostatic" pressure are both lower than inside blood vessels. "Capillarity" and other "surface" forces have occasionally been 
imagined to be considerable. The effective osmotic pressure of this common medium might be great toward one kind of cell, less toward another. Possible diversity of properties among cell types thus begins to receive recognition.

For terrestrial vertebrate individuals, more diverse forces are suggested, as those operative in kidneys and in the neuromuscular apparatus of intake. For cutaneous tissues of frogs, "electrostatic" forces have been imagined to compete against "osmotic" pressure in intake. Often it is implied that single cells, or worms, or some other category of living units, are incapable of performing exchanges for which mammals use special organs. That view fails to recognize the fact that water is exchanged and adjusted without such organs.

So there are theories as to the physical types of forces for several kinds of units in which water exchanges have been observed. And it is probable that recognized categories can be found to supply suggestions for each of the very many living units that have not been yet observed. In general, it is doubtful whether any one type of force is more prevalent in the water exchanges of all varities of cells than it is among all varieties of emunctories. There are plenty of cells even in dogs that are not protected by an "internal medium" from extreme changes of concentration about them; constancy of volume in them may require compensations and adjustments managed as intrinsically as in bacteria. There are also assertions that known varieties of forces taken together are insufficient to explain the phenomena found, and I believe such assertions are just as helpful and as helpless as those to the contrary.

In practice, a force is no more ultimate than a rate. Both are equally implicated in regulations, and whatever superiority as a working hypothesis a force might have over a rate, its descriptive value is distinctly inferior. Until it is demonstrated that an "underlying" force is more than a parameter in an equation, is a phenomenon measurable in its own right, the understanding of water exchanges in organisms is not limited to instances in which the nature of a force can be recognized.

\section{\$95. Permeability}

Rates of water exchange are often ascertained under conditions where quantities are combined in dimensions equivalent to "perme- 
ability" to water. This is accomplished by finding the volume of water exchanged per unit of time, the area of surface believed effective in the exchange, and the gradient of a measurable or supposed pressure under which the water moves. Very often permeability is termed such only when "osmotic" pressure is believed to be the only force acting. How far does permeability to water represent the exchanges concerned in equilibration of water content?

In the investigation here pursued, diverse factors have been correlated with the exchanges of water as measured. The area of surface of exchange, and the gradient of known pressure existing, may be regarded as two such factors taken at random. For water, the rate of exchange $\delta \mathrm{W} / \Delta t=h \times \mathrm{S} \times \Delta P$. The area of surface exchanging is $\mathrm{S}$, the gradient of effective pressure is $\Delta P$, and the permeability coefficient is $h$. Automatically the instances in which $h$ can be computed are those where $S$ and $\Delta P$ are known in addition to the rates of exchange. The accident whereby these two enter into the definition of a familiar physical "constant" does not, I think, make them different from others. I believe that any set of factors may be grouped into equations and coefficients which when dubbed and investigated will be enlightening.

Permeabilities, like other coefficients, are useful in that they stand for ratios of two or more measurable factors. They are useful even when they are not constant. A coefficient of any sort comes into "good" standing when it succeeds in cancelling factors or variables; it is confusing when its implications are taken to represent more than a coefficient.

Above I found it useful to eliminate body mass and volume of water from certain equations by putting $\delta \mathrm{W} / \Delta \mathrm{t} \div \Delta \mathrm{W}=1 / \Delta \mathrm{t}$. This bit of algebra facilitates comparisons of rates $(\delta \mathrm{W} / \Delta \mathrm{t})$ among diverse increments of water $(\Delta T W)$ exchanging with diversely sized and specific organisms or parts. The permeability coefficient represents a similar attempt to eliminate the two further factors, surface area, and gradient of at least one of the forces believed concerned in the exchange of water. The coefficient happens to have the dimensions: $\frac{\text { mass }}{\text { area } X \text { force } X \text { time }}$ or $\frac{\text { distance }}{\text { force } X \text { time }}$ thereby allowing numerical comparison of diverse sizes and species of organisms, but is no "better" than any other ratio relating other dimensions. 
In order that an effective "osmotic" pressure gradient shall be proportional to a concentration gradient, some solutes either do not penetrate the surface of the living unit, or do so very slowly as compared to the water. How fast any solutes pass is not always ascertained, and sometimes perhaps cannot be wholly ascertained, thus giving rise to permeability coefficients bearing diverse tentative definitions.

In a study of any permeability in which "osmotic" pressure is believed to be a factor, two further assumptions are introduced. (1) The concentration inside, if measured at all, is measured as one of the colligative properties of a hypothetical solution prevailing in the organism. (2) The "effective" volume of this solution contained within the organism or tissue is not the entire volume of the body. It is usually estimated by finding the relative volumes when organisms or cells are believed to have concentrations equal to their media. It is the volume of distribution of an increment of water. Then the osmotically effective volume is the measured volume (V) minus the "non-solvent" volume $(b)$. In some instances $b$ occupies about the same space as the total solids of the tissue, or the fats plus proteins of the tissue, or the cells of the tissue. There is no predictable relationship of $b$ to any one colligative that holds for a variety of organisms and tissues, and "independent'. methods of measuring it (e.g., Leitch, '34, '36) deal with diverse definitions of $b$.

Permeability coefficients are often expected to be constant. But as I scan the values reported for water in living tissues I find, first, that few of them have been tested under many conditions, and second, that all which have been so tested are not constant. Choosing dissimilar conditions within one species at a time, I find the following significant contrasts of coefficients :

(a) Different in two directions with like gradient of concentrations (differential permeability). Phascolosoma; leucocyte of rabbit.

(b) Different with time. Phascolosoma in exosmosis; Echinometra egg.

(c) Different in media of diverse ranges of concentrations but of supposedly equal gradients of concentration. Ceratocephale egg.

(d) Different in media containing diverse non-penetrating solutes. Arbacia egg in various calcium concentrations. 
(e) Different with physiological condition of organism. Arbacia egg "injured" by heat; at diverse ages.

With the numerous criteria and conditions under which permeability has been studied in mind, values of $h$ in diverse species may be compared, perhaps by means of the partial list tabulated by Lucke et al. ('39). The highest are for erythrocytes (of man, $3.0 \mu$ /atmosphere minute) and for Phascolosoma (2.1). Actually many permeability coefficients fall in a restricted band of values lying between 3 and 0.1. In freshwater organisms ( $A m e b a)$, values are as low as 0.02. Finally there are numerous aquatic organisms such as certain insects, and fish eggs, that are nearly impervious to water, and for which indeed exact permeability coefficients are not known.

In precise comparisons the reciprocal of permeability, the reluctivity, is a useful coefficient. It may be thought of as representing the resistance to water exchange per unit of exchanging surface.

Permeability coefficients have scarcely been computed for animals that are continually taking in water through body surfaces in the stationary state of turnover. Such are frog ( $\$ 37)$ and earthworm $(\$ 49)$. The frog takes in 0.53 milliliters per hour (table $21)$ through a surface of 120 square centimeters. The "osmotic" pressure of the blood is 0.24 osmolar (Adolph, '27c, p. 329), whence the permeability is $0.14 \mu$ /atmosphere minute. Having ascertained that "osmotic" pressure inside the water-loaded body is approximately proportional to fraction of dry substance $\mathrm{D} / \mathrm{B}_{0}$, hence to $1 /\left(B_{0}+\Delta W\right)$, and observing figure 66 , I conclude that the permeability is constant in positive water loads but increases several fold in negative loads, as though in deficits osmosis were less opposed. The earthworm takes in 0.10 milliliters per hour (table 21) through a surface of 24 square centimeters, its body fluid being 0.16 osmolar (Adolph, '27, p. 56). The permeability is $0.20 \mu$ /atmosphere minute, presumably increasing 5 fold or more in extreme water deficits. The protozoa of $\$ 52$ and $\$ 54$ might be similarly treated if the internal osmotic pressures of their substance were known by more. than guesses. If, conversely, a supposedly universal value for the permeability to water be ascertained, perhaps from the rate of shrinkage upon entering a hypertonic medium, then from the rate of turnover the internal "osmotic" pressure may be crudely predicted (Kitching, '36). 
In the end, it is rather arbitrary to segregate under the term permeability certain classes of water exchange, namely those in which the investigator thinks he can see and estimate the force that operates and the surface of exchange. Where thickness of boundary is also known, separate classes would be studied under the term diffusion. Where the area of exchanging surface is unknown or invisible, another related coefficient may be used, $i=\delta \mathrm{W} /(\Delta \mathrm{t} X$ $\Delta \mathrm{P})$. Avoiding the factor of area, this "coefficient of osmotic flow" $(i=h \times \mathrm{S})$ also avoids complications connected with the presence or absence of convection behind the boundary, such as by the blood stream; for the coefficient may include any such factors (Bohr, '09, p. 251). Where the pressure under which exchanges occur is unknown, it is sufficient to measure the rate of exchange, $R_{\mathrm{w}}=\Delta \mathrm{W} / \Delta \mathrm{t}$; hence $\mathrm{R}_{\mathrm{w}}=h \times \mathrm{S} \times \Delta \mathrm{P}$.

It is apparent that the arranging and ordering of experimentally measured values of water exchanges reduces itself to ascertaining other possible quantities with which to correlate the measurements of rates. Some of these may be combined, reducing several variables to one; occasionally several variables yield a constant, furnishing a useful and economical description.

Whether or not it is worthwhile to compare water exchanges through alimentary tracts, kidneys, and antennal glands with those through surface membranes of supposedly less differentiated sorts, is a matter of opinion. The "pressures" involved in securing, swallowing, and absorbing a drink of water in a mammal would require precise definition, and for the present they might be regarded as incommensurate with the pressures under which fluid crosses the walls of blood capillaries. Were someone to label them " "psychic pressures," physiologists would try to avoid measuring water intakes for many decades, I suppose. Yet a water deficit, particularly if considered as a concentration increment, is proportional to something that has the dimensions of pressure and might be put into the equation by which the coefficient $i$ is computed.

In brief, coefficients of permeability and of osmotic flow combine the values for rates of water transfer with factors of pressure gradient. They may advisedly be defined so as to be independent of any particular mode of transport such as diffusion, of any particular force such as osmotic pressure, and of any implication to constancy. While those coefficients may be compared for only a few organisms and parts under specified conditions, rates of water 
exchange and velocity quotients can be measured upon many more organisms or parts. In chosen conditions and materials, permeability coefficients, coefficients of osmotic flow, or velocity quotients, or all of them, may prove to be constant with time, or with concentration gradients, or with water increments. Economy of numerical evaluation is achieved by discovering the conditions within which constancy prevails ; in other circumstances, quantitative comparisons are still allowed by use of the same coefficients.

Where the factors of pressure gradient and of surface area of exchange are known, the "water-time" system of four variables is expanded into a system of six variables. If this "water-timegradient" system were to be studied exhaustively, $\left[(n-1)^{2}+\right.$ $(n-1)] / 2$ or 15 combinations of coordinates (taken 2 at a time) would be obtained instead of the mere 6 combinations of the restricted system.

The uniformities among diverse organisms in which net water is passing across an external boundary to and from a liquid medium (osmosis) are roughly as follows: (1) Upon transfer to a medium of higher concentration than the original medium, water loss is faster. (2) Simultaneous exchanges of solutes modify the rates of exchange of water. (3) Rates of water exchange are influenced by temperature, specific ions and molecules, orientation of concentration gradient, "non-solvent"' components of organisms, durations of exposure, and other recognizable factors. (4) Zero water exchange may occur without equality of osmolar concentrations inside and outside, as is characteristic of many organisms in fresh water which nevertheless are permeable to water. (5) Modifications of rates of water exchange with water load are not described by a single permeability coefficient in any species. It may be concluded that equilibration of water content involves more factors than those customarily recognized in the one equation of permeability.

\section{\$96. BoDy SIZE, AGE}

In all the data presented in this investigation, water contents and exchanges were measured relative to body weight. Up to the present point an implicit trust has been placed in this basis, when comparing diverse individuals of one species and of different species. I now inquire what influences this basis has on the relations found both within and among species, and what consequences lead 
from it. And I want to know whether equilibration of water changes with age independently of body size.

If body weight had been disregarded, contents and exchanges of water would have been measured in grams or milliliters. No change in the forms or shapes of the graphs representing a single species would be introduced with the new numerical scales, however, for load $( \pm \Delta W)$ was in every instance correlated with time or with rate $(\delta \mathrm{W} / \Delta \mathrm{t})$. In the latter case, increment of water appeared in both coordinates, and hence any changes of scales would be proportional in the two coordinates. In many data more than one individual of a species was represented; this would have been inadvisable when load was measured in grams if the individuals differed in body weight by more than perhaps 10 per cent. Hence, most of the data would have been separate for each body size.

Another measure of size, such as a length, skin surface area, kidney weight, blood volume, or alimentary tract area, might have been substituted. Any or all of these are, of course, equally admissible. Some cannot be measured during life; others can be measured but with much less accuracy than body weight. Very often the new measure may be inferred from body weight through prepared correlations; this is the case with skin surface area, a measure of size that has enjoyed vogue in recent physiology. Is area especially related to rates of water exchange? Equations and constants are already available, in most species considered here, for transforming body weights to surface areas. Surfaces are roughly power functions of body weights; and many of the exponents, found empirically, approximate the value $2 / 3$. Inaccuracies inherent in those correlations are avoided by relating water exchanges directly to logarithms of weight; and then the implication is not so strong that surface area or length or weight itself has any particular connection, except by convenience of thought and availability of data.

(1) Within the species. Relations between rates of water turnover and body weight are established in two species of mammals. In units of centimeter, gram and hour, the young rat shows rates of ingestion of preformed water (in turnover) such that (fig. 133) $\mathrm{R}=0.041 \mathrm{~B}^{0.60-0.63}$; while believed surface area (Lee, '29) $\mathrm{S}=12.5 \mathrm{~B}^{0.60}$. When the equations are combined, $\mathrm{R}=0.0033 \mathrm{~S}$; or, in one hour 1/300 milliliter of water is ingested for each square centimeter of body surface. 


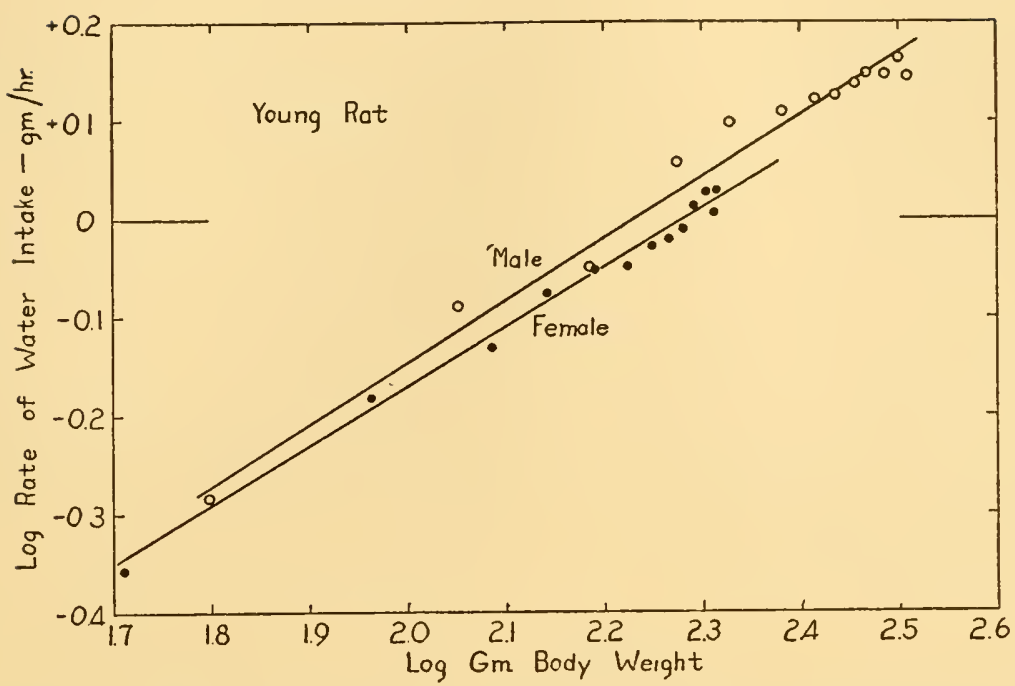

FIG. 133. Intake of free water in relation to body weight. Measurements during every 10 days in 24 male and 27 female white rats from 35 to 155 days of age. For males the line corresponds to the equation: $R_{\mathrm{w}}=0.039 \mathrm{~B}^{0.83}$; for females, $R_{\mathrm{w}}=0.043$ $\mathrm{B}^{0.60}$. Data of Richter and Brailey ('29).

Similarly, the young bovine shows rates of ingestion of preformed water such that (fig. 134) $R=0.000119 \mathrm{~B}^{1.30}$; while believed surface area (Brody et al. '28, p. 29) $\mathrm{S}=0.31 \mathrm{~B}^{0.59}$. In this case the excellent correlation is a temporary and transitional one, for at

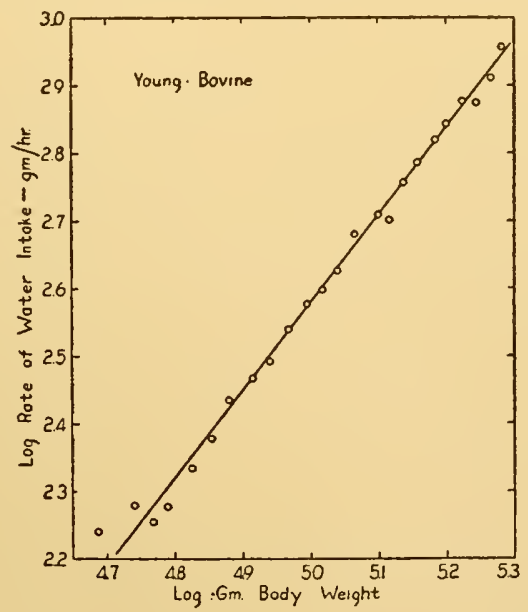

Fig. 134. Intake of water as such in relation to body weight. Weekly estimates in 18 to 12 calves on a diet chiefly of milk, from 3 to 26 weeks of age. The line corresponds to the equation: $R_{w}=0.000119 \mathrm{~B}^{1.30}$. Data of Atkeson, Warren and Anderson ('34, p. 252). 
later ages water consumption becomes progressively more nearly proportional to $\mathrm{B}^{2 / 3}$, as food consumption does (Brüggemann, '38). Or, according to an allometric equation of relative growth $R=a B^{b}$, $\mathrm{b}$ decreases progressively with age to a stable value of 0.67 .

The data for bovine represent an early stage in the development of the individual. Those for rat are distinctly late in the life history. The data for bovine caution against basing a broad and attractive generalization upon the data for rat.

The individuals measured differ not only in body weight, but in age and all that goes with it. Only arbitrarily is weight used above as abscissae, instead of age or total food intake or total bulk of alimentary flora and fauna.

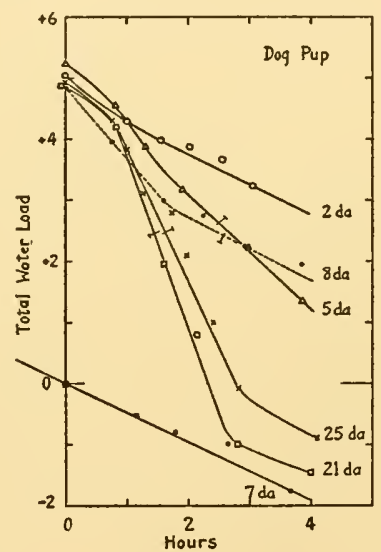

FIg. 135. Course of total water load $\left(\%\right.$ of $\left.\mathrm{B}_{0}\right)$, in a young dog $\mathrm{Ha}$ at the diverse ages indicated. About $5 \%$ of $\mathrm{B}_{0}$ is given by stomach tube on each of 5 days. At ages less than 21 days, each point is the body weight just after urine is drawn by bladder puncture; thereafter the bladder is emptied spontaneously. Rapid excretion of the load is acquired between the 8 th and 21st day. Also the specific gravity of the urine becomes less, and becomes so earlier, after the 8th day. New data.

In dogs a marked change with age in the recovery from water excess can be traced. Water diuresis is not perfected at birth; instead, urinary water output is little faster under positive water load than in water balance. What slow diuresis can be found lasts many hours after water is administered. This fact allows the study of what a dog is like without much equilibration of water content. Rapid water diuresis appears 5 to 21 days after birth (fig. 135), at which time the adult response is fully developed. While the time of acquirement of water diuresis differs slightly among litters of pups, and even in the individuals of one litter (fig. 136), there is no doubt of its finality. Body size or state of nutri- 
tion plays no special role in the time of appearance of the adult response to water load.

It seems possible to locate the water between the time it is put into the pup's stomach and the time it is slowly lost. The stomach is empty one hour after administration; the intestine is no fuller at that time than in control individuals. Only slight volumes of fluid are found in the peritoneal cavity. The blood plasma is diluted for at least 3 hours. I conclude that the water is absorbed quickly, is retained throughout the body, and eliminated only very slowly. This would mean that the pup in the first two weeks of life is rather dependent upon receiving nutrients in proportions

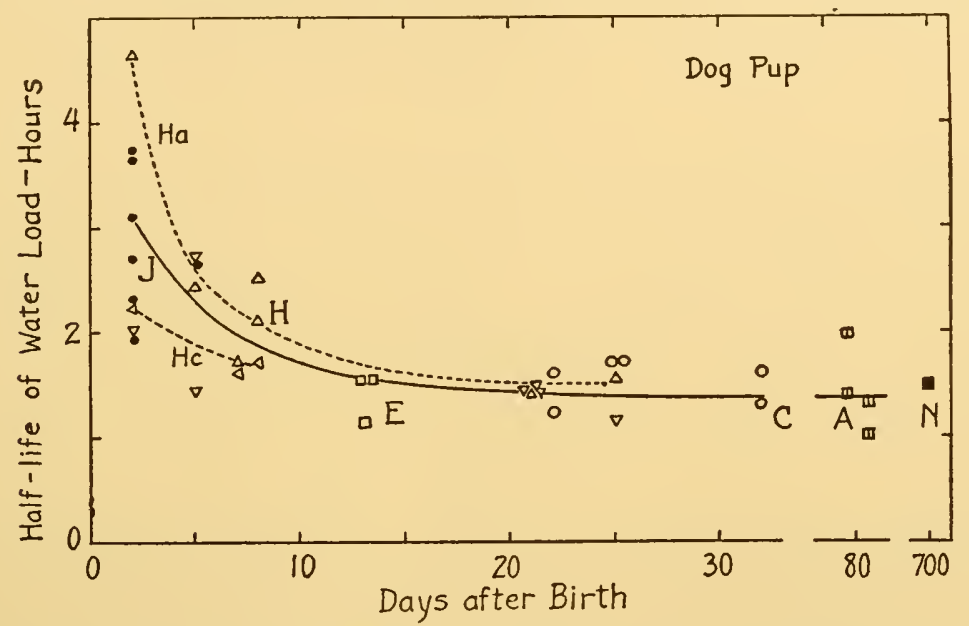

Fig. 136. Duration of the half-life of total water load in relation to age. Nineteen young dogs of 5 litters, and adults $(N)$. Solid line represents the mean for all litters, dotted lines represent 2 separate individuals of litter $\mathrm{H}$. New data.

and amounts determined by some agency outside itself; regulations of water and other substances develop before weaning is possible.

Similarly, water drinking is not demonstrable at birth. A very young pup that takes milk from a bottle does not take water from a bottle, indicating that the two ingesta are distinguished. The newborn is not induced to take water by depriving it of water for three hours, nor by administering a concentrated salt solution. The first drinking of water as such I found at 15 days of age. So, compensations by diuresis and by drinking appear at about the same age. At first the drinking is slow; and the correspondence between the water deficit and the amount taken voluntarily is not close at 15 days of age, while at 35 days of age, hence before as well as after weaning, the adult speed and accuracy have been acquired. 
Equilibration diagrams, then, change as the pup develops (fig. 137). At birth, turnover of water (per unit of body weight) is double the adult's, and in water loads the modifications in rates of exchange are small. With surprising suddenness, rates both of gain in deficit and of loss in excess attain high velocity quotients. Only upon a few days of the pup's life can intermediate stages be found. Thereafter, the time required for recovery from water load is independent of body size. Turnover, however, slowly diminishes (as the ratio of surface to mass decreases). The transition to compensatory processes makes clear how inadequate is the provision for adjustments in the newborn pup; and, by contrast,

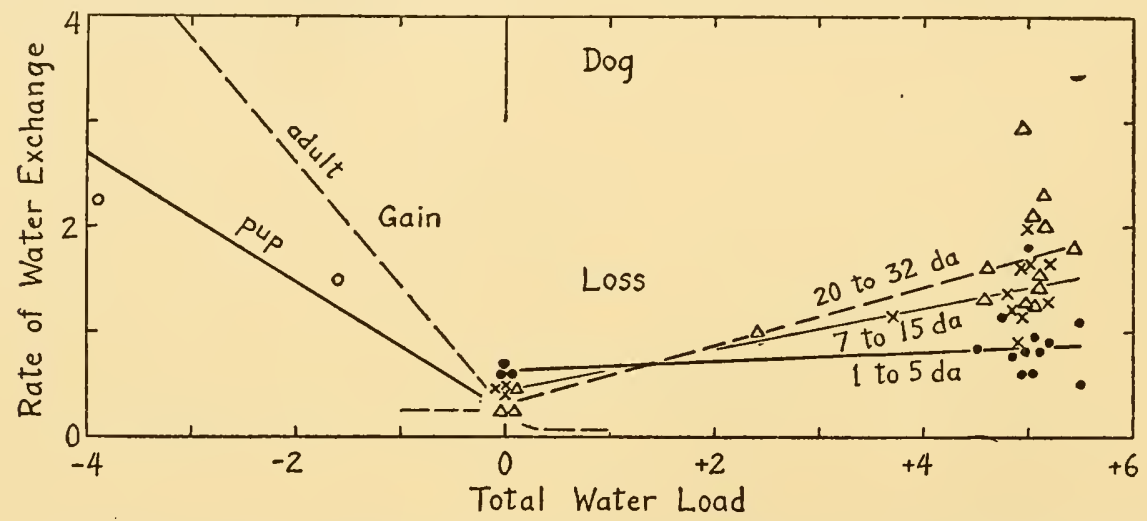

FIG. 137. Rate of total water exchange ( $\%$ of $\mathrm{B}_{0} /$ hour) in relation to administered water load $\left(\%\right.$ of $\mathrm{B}_{0}$ ). Equilibration diagrams. Young dogs in the first 1.0 hour of recovery. In excesses, pups up to 15 days of age had their bladders emptied by puncture through the body wall; at later ages micturition was spontaneous. Solid points represent newborns at 1 to 5 days of age, erosses those 7 to 15 days of age, and open points those at 16 to 32 days of age. New data. Adult dogs may be compared from figure 13.

emphasizes what the pattern of equilibration enjoyed by the adult accomplishes.

In man within the first days of life the rate of water turnover increases daily (Drossel, '29). The rate of urinary water output progresses from $0.02 \%$ of $\mathrm{B}_{0}$ /hour in the first day, to $0.24 \%$ of $\mathrm{B}_{0}$ /hour in the sixth day (Gundobin, '21, p. 363). Of course this change is related to the interruption of nutrition at the metamorphosis of birth, and the gradual increase in intake of food (and water) during the first week. Throughout the first year the rate of urinary output subsequently diminishes in relation to body weight, being nearly proportional to $\mathrm{B}^{0.7}$, when judged from Gundobin's data. 
When excessive water is ingested by the human infant the proportion of it returned by renal diuresis is small in the first months (Ohlmann, '20; Lasch, '22). It is not certain that the transformation of compensatory adjustment is as sudden as in the dog. Only after 6 months of age in man is the return as prompt and complete as in adults. There is some evidence that at 5 to 10 years of age the urine excreted within 3 hours markedly exceeds the water given, which is not the case in adults (fig. 49). In these measurements, a number of unrecognized differences other than age may exist; also, differences such as size of ingestion, or promptness and ease of micturition, may be legitimately included in a single variable.

In frogs (Rana esculenta), where metamorphosis and respiratory modifications are proceeding apace (table 30 ), age is less

TABLE 30

Rates of water turnover at diverse sizes and ages in Rana temporaria at $18^{\circ}$ to $22^{\circ} \mathrm{C}$. Anus and mouth were ligated for 3 to 5 hours. Data of Rey ('3\%, p. 1132)

\begin{tabular}{|c|c|c|c|c|c|}
\hline \multirow[b]{2}{*}{ Stage } & \multirow[b]{2}{*}{$\begin{array}{l}\text { Number } \\
\text { of tests }\end{array}$} & \multirow[b]{2}{*}{$\begin{array}{l}\text { Mean body } \\
\text { weight, } \\
\text { gm. }\end{array}$} & \multirow[b]{2}{*}{$\begin{array}{l}\text { Mean body } \\
\text { surface, } \\
\text { em. }^{2}\end{array}$} & \multicolumn{2}{|c|}{ Rate of water intake } \\
\hline & & & & $\begin{array}{c}\text { per unit } \\
\text { weight, } \\
\% / \mathrm{hr} \text {. }\end{array}$ & $\begin{array}{l}\text { per unit } \\
\text { surface, } \\
\mathrm{cm} . / \mathrm{hr} \text {. }\end{array}$ \\
\hline 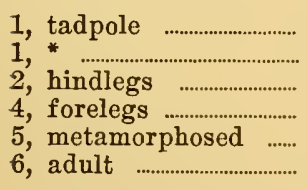 & $\begin{array}{r}5 \\
5 \\
3 \\
3 \\
3 \\
25\end{array}$ & $\begin{array}{r}0.70 \\
0.64 \\
1.97 \\
1.94 \\
1.92 \\
65.0\end{array}$ & $\begin{array}{r}5.5 \\
5.3 \\
11.0 \\
13.5 \\
12.2 \\
130.0\end{array}$ & $\begin{array}{l}1.5 \\
3.1 \\
1.4 \\
2.9 \\
2.8 \\
0.92\end{array}$ & $\begin{array}{l}0.0018 \\
0.0037 \\
0.0022 \\
0.0033 \\
00039 \\
0.0047\end{array}$ \\
\hline
\end{tabular}

* Mouth not ligated.

correlated with body size; no single rule is apparent in the rates of water turnover recorded. Physiological metamorphoses inevitably accompany aging in many or all species.

Based on body weight, the turnovers decrease with age in dog, man, and rat, but not in bovine and frog. In water increments the rates of intake or output, the time relations of exchanges, and the velocity quotients also show diverse modifications at different ages. This fact may adequately prevent anyone from viewing regulations as fixed properties of unchanging organisms.

(2) Among species. Correlations with body weight furnish a wide opportunity for comparisons among species. Data upon rates of water exchange are available in mammals of very diverse sizes for (a) total water intake (or turnover) in water balance and (b) maximal water intake after hypophysial injuries (fig. 138) ; (c) 
urinary water output in ordinary water balance, and (d) maximal water output in water diuresis (fig. 139). Large numbers of measurements have gone into the determinations of means or maxima in each category. In a previous chapter these values of rates of water exchange are considered as species differences without reference to body size. Here they are found to be related with body weight according to allometric equations.

The conclusions indicated among mammalian species are: (1) Total intake is roughly proportional to $\mathrm{B}^{0.9}$. (2) Maximal intake

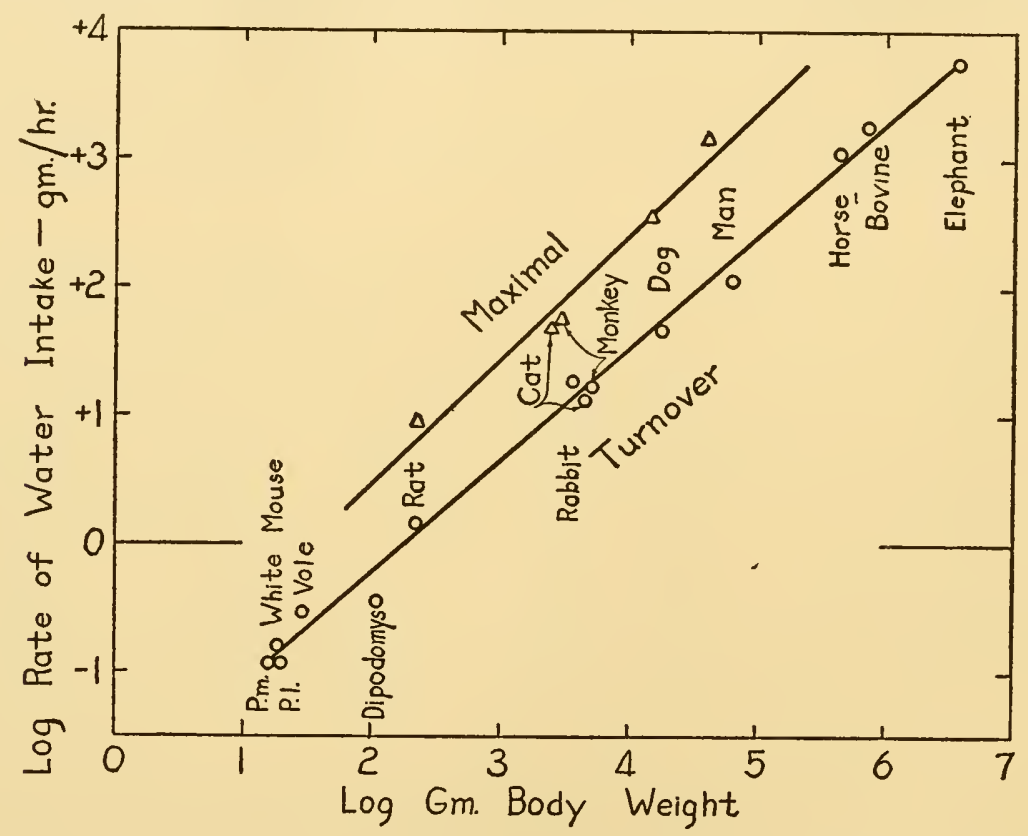

FIG. 138. Rate of total water intake in relation to body weight among diverse species of mammals. Lower line, mean turnover rates in control conditions (from table 20): $R_{\mathrm{TO}}=0.010 \mathrm{~B}^{0.88}$. Upper line, maximal rates after injuries of hypophysis (from table 13): $R_{\max }=0.033 \mathrm{~B}^{0.97}$.

is nearly proportional to $\mathrm{B}^{1.0}$ (Richter, '38). (3) Ordinary urinary output is proportional to $\mathrm{B}^{0.8}$. (4) Maximal urinary output is nearly proportional to $\mathrm{B}^{0.8}$. (5) The ratio of ordinary total intake (or equal output) to urinary output, ranges from 2 for small species to 5 for large ones. (6) The maximal urinary rates equal 32 to 20 times the ordinary rates, the augmentation ratio being less in small animals which have turnovers already large in proportion to body weight. ( 7 ) Maximal intakes after hypophysial injuries, and maximal outputs after water ingestion are nearly equal. 
Maximal intake rates equal 5 to 17 times the turnover rates (= augmentation ratios).

These correlations might be used to predict by interpolation the probable rates of water exchange in other species. I do not recommend prediction as a substitute for measurements of them. It is apparent that time of recovery from water load is independent of body size; rat and man remove loads equally rapidly.

Data upon water exchange in other classes and phyla of terrestrial organisms apparently are not available, with a few excep-

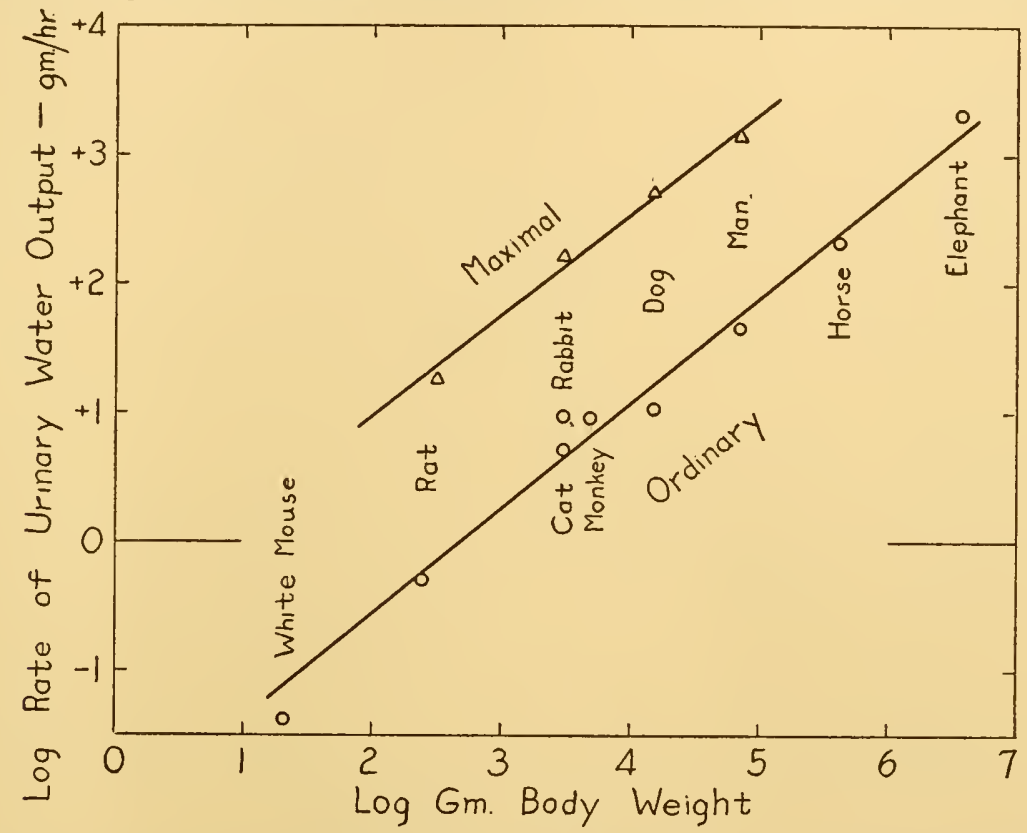

FIG. 139. Rate of urinary water output in relation to body weight among diverse species of mammals. Ordinary rates are in water balance (from table 28): $R_{\text {TO }}=$ $0.0064 \mathrm{~B} 0.82$. Maximal rates are during continued forced administration of water by stomach (from table 13): $\mathrm{R}_{\max }=0.26 \mathrm{~B}^{0.78}$.

tions as pigeon (Falck and Scheffer, 1854a) and chicken (Korr; Hester et al., '40). Among aquatic animals (fig. 140) rates of turnover are proportional to $\mathrm{B}^{0.75}$ and maximal rates to $\mathrm{B}^{0.66}$. This confirms the more approximate conclusion of Pütter ('26) that water exchanges are proportional to body surface area. At equal body weights the turnover rates of these aquatic animals are roughly identical with those of small mammals of figure 138 .

A rule enunciated by Richet (see Morgulis, '23, p. 91) is that the intensity of vital functions depends directly upon the size of the 
organism. I presume that an intensity of the sort meant is measured by the rate of water exchange; and there the rule holds. Of course, when exchanges are measured as fractions of the body weight per unit of time, all the rates diminish with greater body size; actually, they vary as some power of weight that is less than 1 . Since all measures of size are correlated with others, any one of them that can be accurately and conveniently measured is preferred for use. Usually that one is body weight.

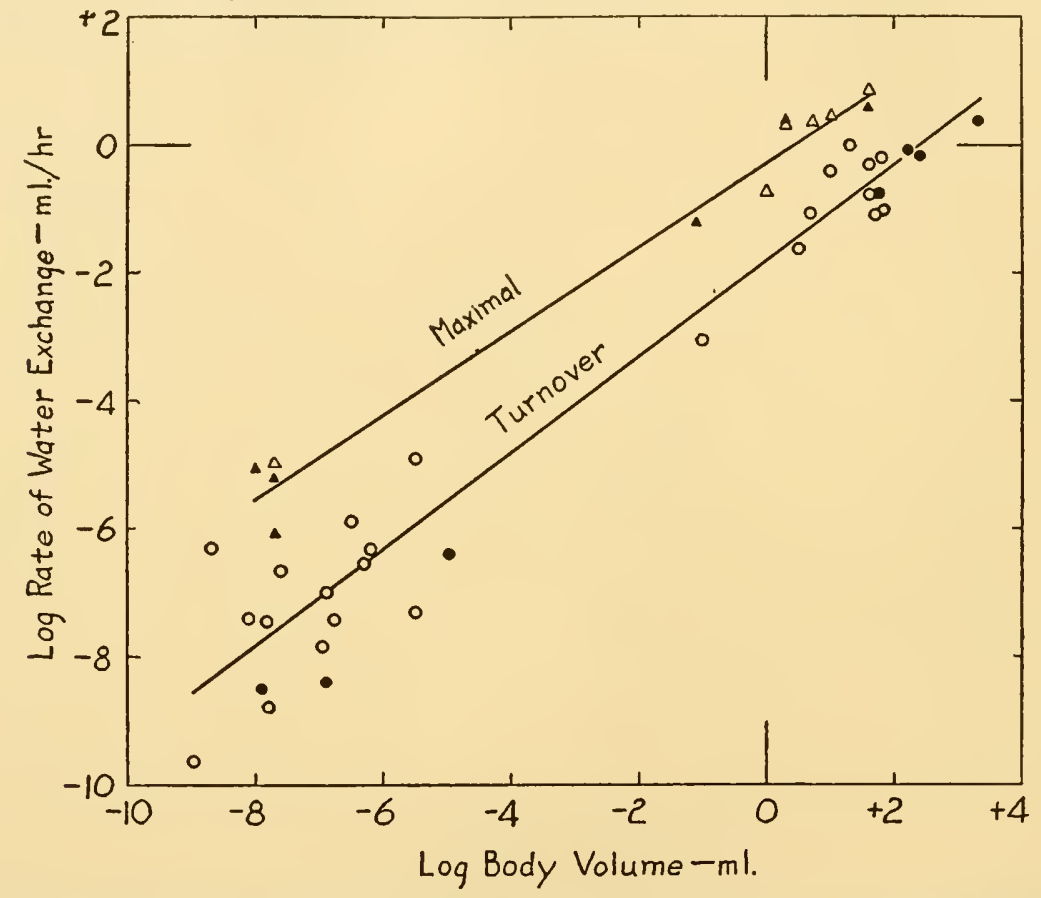

FIG. 140. Rate of water exchange in relation to body volume among many species of aquatic animals. Both scales are logarithms of either $\mathrm{ml}$. or $\mathrm{ml} . / \mathrm{hr}$. Maximal rates (from table 13) are either for output in positive loads (solid triangles) or for intake in negative loads (open triangles): $R_{\max }=0.50 \mathrm{~B} 0.66$. Turnover rates (from table 21) are either for marine species (solid circles) or for freshwater species (open circles): $R_{\text {TO }}=0.016 B^{0.75}$.

(3) Additional features. Special structures and shapes, such as the ears of the rabbit and the wings of the bat, may modify the correlations of rates of water exchange with size among species, as in every other relation of body size. No attempt is made here to sieve out the partial correlations of water exchanges with pulmonary ventilation rates, kidney weights, oxygen consumption rates, and a host of other quantities. Two or three more selected variables will, however, be followed. 
(a) It is recognized that the exchanges of water as described depend upon absorbing and excreting surfaces, conveying and circulating bodies, coordinating pathways, and many other parts. The presence of probable oversize (factor of safety) of each, is itself related to body size among species. Rapid movement of internal fluids is often absent in small species, such as eggs, certain worms, and sone sessile organisms; without convection, the movement of water is often presumed to be limited by processes of molecular "diffusion." With convection inside the body, the integument may be so thick and immobile as to limit osmosis. In general, how large might an organism be before osmosis through its permeable tissues would limit its water intake or output? Computations might be predicated; the only experiments seem to be on the frog (of 32 grams weight). When blood circulation was suddenly stopped, water output ceased, but intake of water from a dilute salt solution was unchanged in rate (Adolph, '31b). When stopped ("death") by previous desiccation, even maximal water intake was undiminished in initial rate (fig. 66).

(b) Among terrestrial animals it is widely recognized that turnover of water is directly related to turnover of food. Sometimes it is specified that the nitrogenous fraction of the food is a correlative (Babcock, '12); at other times that rate of energy transformation is important. In mammals most water is regularly drunk with or following food ingestion.

For mammals the rate of water intake during daily or longer periods is correlated with the potential energy of the food taken during the same periods. The ratio of the two increases with body size (fig. 141) ; possibly the ratio should be between the logarithms of the two intakes, making this relation also allometric. The two species having least ratios are inhabitants of deserts. Since water formed by oxidation is nearly the same per potential Calorie of all foodstuffs, the diversity among species is wholly in the portion of the water ingested as such.

(c) For many of the same species of mammals the water output may be partitioned by paths (fig. 142), the total output being taken as equal to the intake. The fractions put out in fecal, urinary, and evaporative channels are those indicated. The evaporative fraction is nearly constant per unit of potential energy at all body weights; this is often spoken of as the proportionality of rate of vaporization to rate of energy transformation. Its range of vari- 
ation is apparently as large in one species as among species. In small species nearly all water turned over is therefore expended in evaporation. Most of the diversity with body size is in the urinary fraction. Fecal outputs become large only in large species; these species are ruminants, and all ruminants are large. Conversely, few species not ruminants are eventually available for further comparison within that range of body sizes. Man happens (according to fig. 142) to put out the largest fraction of water loss as urinary, being at a size between the energy evaporators and the roughage eliminators.

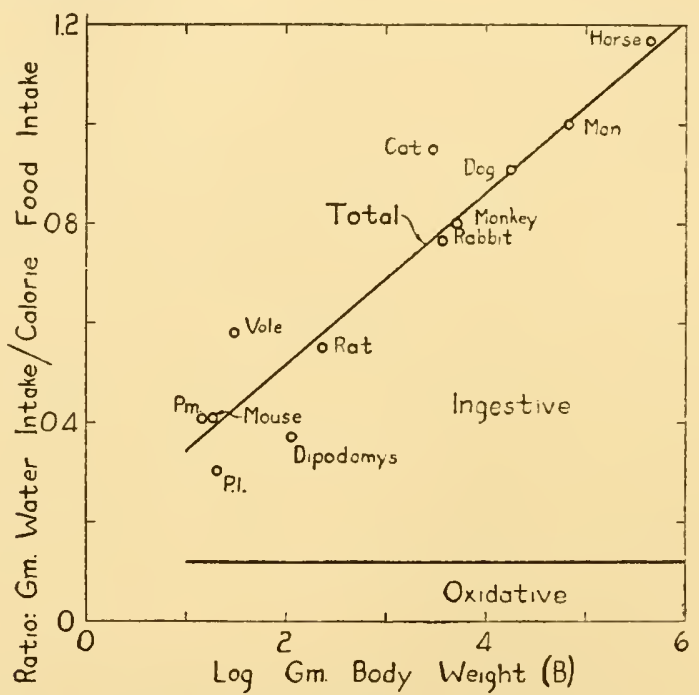

FIG. 141. Total quantity of water ingested and formed for each Calorie of food ingested (and a suggested partition of it) in relation to body weight among diverse species of mammals. The line corresponds to the empirical equation, gm./Cal. $=0.17+$ $0.173 \log$ B. The data are from table 20. The elephant consumes about $2.3 \mathrm{gm} . / \mathrm{Cal}$. according to Benedict ('36); this point (not shown) would be far above the line.

Figure 138 relates rate of water exchange to body weight alone. These are species that have turnovers; it is only an inference that species not yet measured will fit, within the present range of variations. Figure 141 includes rate of energy exchange, as well as rate of water exchange and body weight. This is a mutual relation among three quantities; there is no evidence that one of the three sets the pace for other one or two. It would be possible to add further correlatives and in the end to build up an inclusive description of many relations to water exchanges among mammals, and among some other restricted groups of animals.

In this brief fashion I have tried to present certain materials 
of ontogenetic physiology and of comparative physiology of water exchanges. Size and age are convenient correlatives of rates of exchange, for each brings all its other correlatives into the picture. Clearly, at birth equilibration of water content is not, in dog or man, of adult dimensions; it is transformed later into the recognized pattern of the adult. Regressions of size with rates of water exchanges in turnover and in maximal compensations, both total

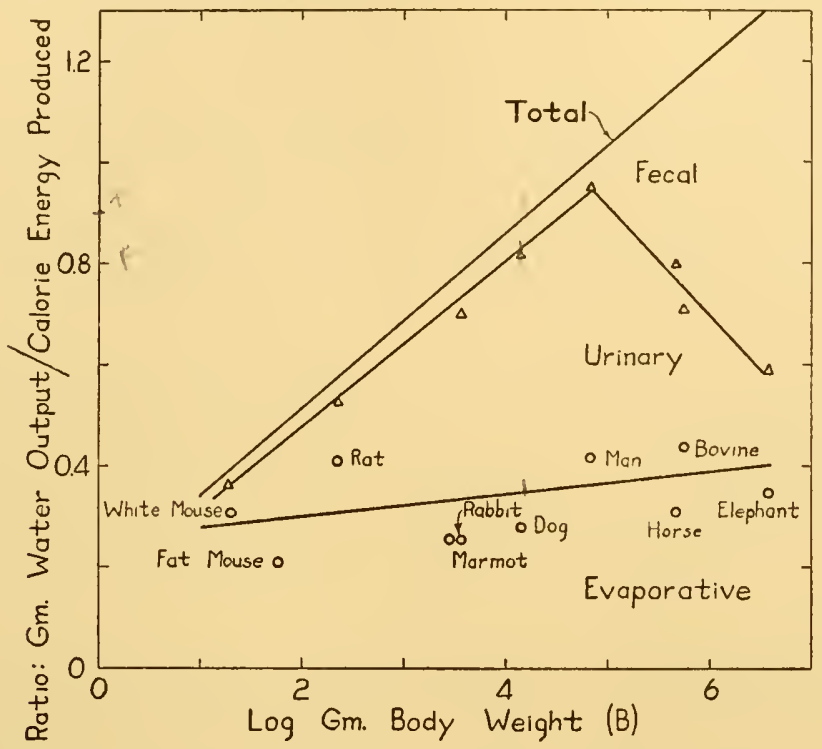

Fig. 142. Partition of rates of water output by paths, for various species of mammals, relative to rates of energy transformation. Each species is represented at its body weight. Total output (turnover) is taken as equal to the total intake of figure 141. Partition is relative to the total water loss, as listed in table 16 and in additional data of Benedict ('38).

and partitioned, turn out to have the same sorts of significance as regressions of size and age with energy exchanges. Many data fit allometric equations of relative magnitudes. Presence or absence of internal convection, and types of metabolism of nitrogen, energy, and roughage, are partial correlatives that may be distinguished in the relations of water exchanges among species.

\section{$\$ 97$. Temperature}

Are the water exchanges as measured also influenced by body temperatures that prevailed? In "warm-blooded" animals, increases of body temperature above the usual are accompanied by enormous increases of rates of total water exchange. Considera- 
tion of these may be deferred to a later chapter (XIV). In "coldblooded" animals, rates of water exchanges may be studied over ranges of as much as $35^{\circ} \mathrm{C}$. With respect to turnover, data on frogs show "temperature coefficients" for ten degrees C. up to 2.6 (Krause, '28). Whether maximal rates of intake or of output in water loads increase proportionally would be interesting to know.

Season makes a difference in turnover rates at equal temperatures, according to DeHaan and Bakker ('24), winter frogs showing half the rates of summer frogs. This may involve many differences of metabolism that have not yet been correlated. It may be remarked that analyzable water contents of frogs also vary with temperature and with season (Donaldson and Schoemaker, '00; Gaule, '01), corresponding to upward shifts of equilibrated body weight $\left(\mathrm{B}_{0}\right)$ at temperatures below $9^{\circ} \mathrm{C}$. (Adolph, '27a ; Rey, '38, p. 1113).

Rates of water exchange were compared at five temperatures in Arbacia eggs (Lucke et al., '31). For swelling in 60 per cent sea water, the rate of entrance of water and the permeability coefficient increased 2.7 fold for ten degrees C. rise in temperature. For shrinking (recovery) the same factor was 2.4. This means that the equilibration diagram (fig. 91) increases its ordinates in that proportion.

If close comparisons of water relations among species are to be made, a single body temperature might be chosen for many species, but no one temperature would be suitable for all. Warm-blooded animals automatically fix the temperature of the body; cold-blooded animals are subject to conditions imposed by the investigator within limits. Whatever temperature is imposed is inevitably open to objection on some other grounds. Hence it may be explicitly stated that most of the quantitative comparisons of water exchanges would be different at other temperatures from the ones represented, and that a particular relation between exchanges and temperature characterizes each species.

\section{§98. RACES}

Do racial differences also matter? Closely related species or subspecies of animals frequent diverse environments, foods, and climates. Attempts have been made to relate whatever racial diversities prevail to the rates of water intakes in deer-mice. Comparisons may here be limited to simultaneous tests under identical 
conditions (table 31). It turns out that two species of deer-mice differ significantly in voluntary water intake, though reared in the laboratory upon constant diet. The difference was 32 per cent of the mean water intake, and thrice the probable error of each mean. Two subspecies of either species, however, drank like amounts of water. Such a difference between species calls attention to but one of the large number of "constitutional" factors related to water exchanges in any class of animals. So far as the meager facts indi-

TABLE 31

Rates of intake of free water compared in five subspecies of deer mice (Peromyscus). Paired tests between and within species. All individuals were bred in captivity. Data of Ross ('30)

\begin{tabular}{|c|c|c|c|c|c|c|}
\hline \multirow{2}{*}{ Subspecies } & \multirow{2}{*}{$\begin{array}{l}\text { Number } \\
\text { of indi- } \\
\text { viduals }\end{array}$} & \multicolumn{4}{|c|}{ Water intake in $\%$ of $B_{0}$ /hour } & \multirow{2}{*}{ Mean } \\
\hline & & Pair 1 & Pair 2 & Pair 3 & Pair 4 & \\
\hline P. m. s. ................... & 28,24 & 0.73 & & 0.86 & ........ & 0.79 \\
\hline P. m. g. & 29 & & 0.67 & & $\ldots$ & 0.67 \\
\hline P. m.r. & & & 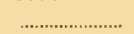 & 0.83 & & 0.83 \\
\hline P.e.e. & 27,20 & 0.52 & & & 0.45 & 0.48 \\
\hline$P . e . f$. & 28,21 & & 0.49 & & 0.49 & 0.49 \\
\hline Difference & ................. & 0.21 & 0.18 & 0.03 & 0.04 & 0.28 \\
\hline Probability ............. & ....................... & 0.00002 & 0.000001 & 0.05 & 0.05 & 0.000000 \\
\hline No. of tests .......... & ................. & 5 & 6 & 5 & 4 & 5 to 10 \\
\hline
\end{tabular}

cate, races and subspecies (as classified on the usual criteria) are not sufficiently different physiologically to exchange diverse amounts of water in turnover.

\section{§99. Summary}

It turns out to be possible to deal with many variables either separately or together, when all relate to some one or two variables $(\delta \mathrm{W}, \Delta \mathrm{W} / \Delta \mathrm{t})$ that are kept continually in view. The procedure is to classify correlatives according to dimensions and in other arbitrary ways. Further, within the classes certain factors are mutually exclusive (e.g., two diets, two atmospheres), and hence each of those could be considered independently of its alternatives.

No basis is found for believing that variables not given special consideration are qualitatively different from those mentioned. For the most part the variables studied were thought of earlier in the development of physiological science, or were recorded handier. So far as I can judge, size of body, or rate of nitrogen turnover, is just as "intimately" related to rate of water exchange as is refractive index of blood plasma, or body water content itself. But the 
roles of the former in recovery from water loads is not so easily hypothesized. Each variable also has interrelations with all the others. There is no evidence that an organism is physiologically compartmented to the extent that water exchanges are independent of functions that do not have the name "water" attached to them or implied in them.

In chapter IX and previous ones I attempted to limit the number of variables considered, to those classified as constituting the time-water system. There, other variables were recognized only in order to label the conditions of measurement, or else to lay them aside for future consideration. In chapters $X$ to XII certain of the other variables are introduced in the form of correlatives of that first system. The study of correlatives may serve to interrelate many or all the known measurements that are recognizably concerned with water in animals.

Forces of several physical varieties are undoubtedly concerned in the water exchanges of animals. The difficulties of measuring them with assurance of completeness has so far prevented the construction from them of the analog of an equilibration diagram. Grouped factors in water exchange, such as permeability, are within limits useful in representing comparative water exchanges. Body size and age, and their correlatives, serve as backgrounds against which to view the developments of functions of exchange and compensation within the species, and the comparisons among diverse kinds of animals. Factors of both environment (e.g., temperature) and heredity distinguish the manners in which maintenance and adjustment of water content are achieved. 


\section{Chapter XIII}

\section{WATER BALANCES AND EXCHANGES. RECAPITULATIONS}

$\S 100$. The materials thus far presented, though serving special interests, may also be considered preliminary to more general conclusions now to be drawn. These conclusions are partially limited by the voluntary choices of variables and the more involuntary availability of data. But within these limitations a variety of comparisons and a considerable number of inductions may be made. This chapter, in fact, attempts to systematize the materials in such a way as to provide a summarized picture of the physiological regulation of water content in animals. Were the study limited to water relations, the presentation might close with it.

\section{$\$ 101$. Classification of variables}

The scheme followed up to this point may be viewed in terms of variables, classed as follows, largely according to their dimensions. Separate divisions are maintained for the quantities most stressed; numerous distinguishable sorts are lumped together in the other divisions $(c f . \S 5)$.

(1) Rates of water exchange or volume changes $\left(R_{\mathrm{w}}\right.$ or $\left.R_{\mathrm{v}}\right)$.

(2) Living units; species, parts of organisms (U).

(3) Paths of exchange of water or volume ( $p)$.

(4) Changes in properties of whole or of parts $(\mathrm{s})$.

(5a) Types of load of water or volume (f).

(5b) Amounts of water content or volume ( $W$ or $V$ ), or load $(\Delta \mathrm{W}$ or $\Delta \mathrm{V})$.

(6) Time and time intervals ( $\mathrm{t}$ or $\Delta \mathrm{t}$ ).

(7) Velocity quotients for water volume $(k)$.

(8) Changes in metabolism and in behavior (M).

(9) Forces, permeability, size, age $(\mathrm{N})$.

First I describe how each of the divisions named, when related to others in the list, forms part of the picture of regulation. When the picture is completed, the order in which factors are considered will have had very little influence upon the relations, comparisons, and uniformities found.

The sorts of variables mentioned may be grouped into continuous and discontinuous factors. For, quantities such as water con- 
tent and time may be divided into infinitesimal gradations; while paths of exchange and types of displacement are more limited in number and kind, and are usually thought of as qualitatively diverse. So, (2) to (5a) are at least in part discontinuous variables, while $(5 \mathrm{~b})$ to $(9)$ are usually quantitative factors. Some belong in both groups. Thus, changes in properties or in age are continuous with respect to each component or individual but discontinuous with respect to the many individuals, constituents, and tissues in which they appear. In the numerous cases of this sort, other classifications might be profitably explored.

Again, some of the variables that can be fixed by an experimenter are often thought of as independent; those that brook no external selection may be termed dependent. Then (5a) to (6) are usually chosen at the will of the observer, and (7) to (9) follow from the physiological constitution of the organism. Though the distinction is not a biological one, this grouping of variables is useful in that it facilitates quantitative treatment of the coordinated properties. By the methods of nomography, it is simpler to represent on paper any number of dependent variables, provided the number of socalled independent variables is reduced to one or two, than to deal with a greater number of the latter. Accordingly, fixing (2) and (6) so that they do not vary, (5b) and many other quantities can be represented (fig. 131). And in a succession of such nomograms, several selected types of displacement or of conditions for recovery (5a) and ages (9) might be portrayed. In this way an epitome is obtained that describes quantitatively a physiological pattern.

Having already discussed (section 11) the interrelations of the four variables of the "water-time" system, (1) (5b) (6) (7), and treating at least two of the four as dependent variables, I can now show some of the relations among the remaining six sorts of variables. In the list of variables there are many possible subdivisions; of these some are mutually exclusive, and may be contrasted, while others are duplicate ways of classifying.

The object in the following section is to indicate general features and contrasts, and not just to represent the numerous transformations of coordinates that could be made. Each sub-heading emphasizes a single one of the variables named.

\section{$\$ 102$. SCopes of the Classes of variables}

(1) Rates of water exchange $\left(\mathrm{R}_{\mathrm{w}}\right)$ during recoveries may be 
grouped into total, partial, and net. They are either gains or losses.

Acceleration of intake or output (table 14) might be a measure of the readiness with which the living unit responds to loads. Wherever latent periods are absent, accelerations are, of course, enormous. In other instances (all of which are water outputs) accelerations are less, and occupy varying periods of time up to 2 hours, whether gross or net exchanges be considered.

Decelerations fall into similar classes, for rapid decelerations usually follow rapid and prompt accelerations; where accelerations are less, decelerations are also less (table 14).

Maximal rates of exchange are, within limits, direct functions of water increments. Actually, few species show any limits to the increase of net intake or of net output. It might be said that the "bottle necks" of water exchange, if any exist, are never completely crowded.

Equilibration diagrams for water $(\delta \mathrm{W} / \Delta \mathrm{t}$ at various $\pm \Delta \mathrm{W})$ are of one type, provided maximal rates of exchange at any one load are chosen for correlation.

(2) Species and parts (U). For present purposes it is often sufficient to group species as is done by taxonomists, and parts as is done by anatomists. Wherever physiological similarities appear, however, alternative bases for classifying are indicated. Thus, among insect species, larvae often belong to utterly different categories with respect to water balances and exchanges from either embryos or adults.

Innumerable bases could be found for lumping individuals, or organs, or cells, measurements upon which shall be averaged. At present many body builds, many muscles, many red blood cells are studied as though they constituted a homogeneous population, in spite of the fact that in the future, distinctions between gastrocnemii and tibiales antici will probably become significant. In the same arbitrary way, I separate parts in situ from parts isolated. Fifty years ago it was usual to mix observations on anesthetized rabbits and on unanesthetized men. Tomorrow, correlations may be further limited to one age, litter mates, one individual, one diet, one cage.

(3) Paths of water exchange (p) may first be identified anatomically (table 21). In many species and even phyla none is known. None appears to be separately distinguishable in the exchanges of 
most parts of organisms, other than the general surfaces of those parts, be they lymph vessels, connective-tissue sheaths, or nuclear membranes. But for cerebrospinal fluid of dog and man (Weed, '38), intraocular fluid (Robertson, '39), and other units, it is generally believed that special surfaces of one-way exchange exist.

Where paths have been identified it is possible to make contributory studies upon specific organs. Thus, the kidneys are often believed by vertebrate physiologists to offer an understanding of many aspects of water regulation. Possibly some information concerning the elimination of water excesses as responses to water loads will eventually come from their separate study; little that relates to loads is recognizable now in analyses of renal function. Ordinary turnover rates are not often regarded as properties of particular organs; is the distinction between diuresis and normal output made by organisms or by physiologists?

(4) Changes of properties ( $\mathrm{s}$ ) with water loads are as numerous as the physical and chemical procedures for measuring them. Very many contributions of biophysics and biochemistry furnish data for this particular sort of physiological investigation. Contents, pressures, volumes, and dilutions may be distinguished. Actually most measurements of composition now available concern the distributions of water loads within organisms. No doubt a great many properties that have not been measured, also change with water increment; often they were not measured because no theory was conceived to indicate that there might be a particular connection.

(5a) Types of water increment imposed by the experimenter have diverse consequences. Classification of types might recognize: environment for recovery, diet, procedure for loading, path of loading, stationary loads.

Diverse tolerances, time relations, recovery quotients, and paths are found in the responses to various types. A tentative conclusion is that time relations are the most variable features of the responses, among factors so far considered. For ultimately recovery occurs, and largely by the use of some single path of exchange, in response to most types of water load.

(5b) Water contents and various volumes, modified experimentally, are evaluated relative to initial or control weight or volume of distribution $(\Delta \mathrm{W}$ or $\Delta \mathrm{V})$. In most cases the changes in weight or volume, or in water content relative to analyzed dry 
content, are rapid enough so that other changes of composition are much smaller than those of water.

(6) Time (t) is an extremely large factor in determining the state of the organism with respect to water (fig. 106). The curves of change in water content after displacement have in common the trend toward $\mathrm{W}_{0}$; but the time scales of these changes are very diverse. Initial, steady, maximal, and fractional hours are distinguished.

"Latent" periods are absent in all water intakes (table 14). They are also absent in water outputs by blood of rabbit, by Phascolosoma, and by Arbacia eggs, living units in which exchanges occur across the entire surface. Where present, these periods are hypothesized to represent delays (a) of translocation, (b) of arousing responses to water load, (c) of paths or processes of exchange, or $(d)$ of development of some unmeasured mediate quantity with which the measured load is correlated.

(7) Velocity quotients (k) are computed from rates and increments $(\delta \mathrm{W} / \Delta \mathrm{t} \div \Delta \mathrm{W})$, or from the exponential curves of water load in time. Often the quotients for net exchanges are nearly uniform over a wide range of water increments, at some selected time after the increment had been imposed. The values of the guotient are various in diverse tissues and species (table 16). All values found at maximal rates are above 0.3 /hour, except in water excess of the snake. This means that recovery of water content at balance occurs in all those species in 0.1 to 4 hours; no load lasts appreciably longer, as would be the case in some organism indifferent to water or unprovided with means of detecting and correcting aberrant water contents.

(8) Changes of metabolisms (MI), or of rates with which processes go on in the organism, furnish numerous correlations with water content. Of these, clearances through vertebrate kidneys have received most study; next in quantity of information are total rates of oxygen consumption. Studies of neuro-muscular behavior name thirst as an urge that appears in negative water loads, but furnish few measurements of the activities that go with it. Convulsions, vomiting, and anorexia have not been quantitatively correlated. Among some one or few activities of organisms, such as clearance, excretion, chemical transformation, and movement, it is useful to find in what range of water increments, in what types of water load, and in what conditions they attain particular rates. 
Then, so far as the one kinetic variable (water exchange) is concerned, all those increments and conditions look quantitatively alike. It may seem fantastic to homologize all the conditions under which a diuresis of a certain magnitude occurs, or all those under which a 3 per cent increase in dilution of plasma exists; yet just this sort of common response is a basis for analyzing physiological phenomena, I think.

(9) Other correlatives (N) such as size, forces, age, sex, permeability, of the living unit each show quantitative relationships capable of extensive study. Most large species are terrestrial, but it is possible to select equally sized organisms in fresh water, in sea water, and on land; both their turnover rates for water and their maximal rates of water exchange are then found to be but weakly related to type of environment. For the most part, rates of water exchanges are in proportion to powers of body weight varying from $\mathrm{B}^{0.6}$ to $\mathrm{B}^{1.0}$.

\section{$\S 103$. Interrelations OF THE VARIABLES}

If variables are chosen so that not more than one of them need be treated as independent, the interrelations among many may be represented in a single diagram (fig. 131). In similar diagrams the quantitative differences among species and tissues may turn out to be almost infinite; these differences serve to characterize each living unit. Uniformities are equally evident; it is easier to state these uniformities as contours in diagrams than to put them in words. A nomenclature might then be invented by which to designate the shapes of the contours, similar to that used for classifying finger-prints. When the pattern of interrelations shall be known for a number of species, comparison of quantities and classification of qualitative combinations after the manner of $\$ 72$ can be carried out. But whereas there only three variables (load, gain, loss) entered into 25 qualitatively different combinations, here seventeen variables may yield thousands of relative types.

So long as investigation consisted in measuring the simultaneous changes of any two factors, an enormous number of studies $\left(2^{n-1}\right)$ would be made, since the number $(n)$ of quantities and conditions seems semi-infinite. Even in $2^{(n-1)}$ papers the interrelations in water exchanges would scarcely have been touched, for the number of combinations of three variables at a time turns out to be about $3^{(n-2)}$, etc. Temporarily, investigators gain satisfaction from finding qualitative answers, from making two measurements at a 
time, and from inferring that what is true in rabbit holds equally in man. In time, however, the wishful reasonings by which the particular pairs of variables were chosen will probably disappear, and the remaining $(n-2)$ quantities become conspicuous by the absence of data concerning them.

While figure 131 furnishes an exact description of the water relations in one kind of organism under one set of conditions, the scheme of description is general, for it applies to any organism, group, or parts. From many such descriptions the range of values over which the quantities vary may be found. No other test of the generality of correlations with water load seems to exist.

\section{$\oint 104$. Procedures or steps used}

The first step in any investigation is to select kinds of observations or measurements that are to be made; very often, of course, that is done subconsciously. The second step, grouping those kinds into classes of variables, seems to be more successful if done with all the consciousness that can be brought to bear. Thereafter, the correlations among classes of variables are made in the light of whatever hypotheses prompted the investigation, largely by standardized methods. A set of measurements, tests, and conditions are chosen; they are classified; and the inevitable coincidences among them are ascertained. Any desired degree of completeness in characterizations may be attained, such that finer and finer contrasts can be found among diverse species or individuals or states.

The specific steps in arriving at this stage of physiological description of water relations may be set down as follows:

(1) Select by some reproducible criterion a group of individuals or parts for experimental tests (U).

(2) Select one or more modes of imposing water excesses or deficits in graded amounts (f). Fix the conditions for maintenance of the individuals and for recovery from the increments.

(3) Follow in time ( $t$ ) the changes of water content or volume (W or V), and hence the rates at which these changes occur $\left(R_{\mathrm{W}}\right)$. Ascertain their deviations.

(4) If desired, identify and measure separately the exchanges of water or volume in particular paths of gain or of disposal $(p)$.

(5) If desired, identify and measure separately other changes in the organism ( $\mathrm{s}, \mathrm{M}, \mathrm{N})$ at diverse water increments and times; changes of composition, structure, partition, rates, and frequencies 
of diverse processes, behaviors, and others. Ascertain their deviations.

(6) Set down the quantitative changes that are measured and their interrelations, remembering that water content is the quantity whose maintenance is to be examined.

(7) Note the unique and the general characteristics of each system (group of variables) described.

(8) If desired, find whether some of the changes vary progressively with repeated imposition of the same water increment (acclimatization, facilitation).

There is nothing very peculiar about this procedure; it is a particularized and explicit form of any plan of experiment. It emphasizes the fact that materials and conditions are selected, the precision of time and of quantities, and the notion that what the experimenter does is much the same, regardless of the hypotheses that he entertains. In a primitive stage of physiology, an investigator of water relations might stop with stage (3) or (4) or (5), and might be content with a qualitative answer to a single question. At present, the most complete quantitative description is required to answer any comprehensive question concerning water relations, forming a durable part of a maturer science of physiology. In the future, still further steps may be added.

\section{$\$ 105$. Outline of water Relations}

Using the specific materials in earlier chapters, I can now sketch the investigation of water maintenance and its correlatives without the details. This recapitulation in general terms allows emphasis on uniformities found among many instances, and disregard of elements that vary with species or parts, and with conditions.

At the outset, water increment or load was defined as the difference of water content in the organism or part in two physiological states, namely, test and control. In general, control states were arbitrarily chosen, but a further definition of them was found in the existence of water balance (equality of intake and output rates) in what are believed to be standard, usual, resting, or occasionally basal, situations. Sometimes the biologist has difficulty in choosing "natural" conditions for an organism or its isolated parts, but be can usually define the conditions existing.

With water increment were correlated diverse variables that had been measured simultaneously or in other relation with it. Of 
these the rate of water exchange was most frequently ascertained; it seemed to be specially related (by virtue of dimensions and common component) to water increment and content. Sometimes the rate of exchange was known only as net flow; at other times both gain and loss were simultaneously measured, and either in total or in paths. The symmetry of gain as contrasted with loss was stressed.

Water content was readily disturbed under controlled and measurable conditions by two general procedures: (a) stopping the continual gain or the continual loss, (b) imposing extra gain or loss. Each load was initiated either gradually or suddenly, and then either released from further interference or partially continued, and often in such a manner that an approximately stationary state of water increment prevailed. Accordingly, the several rates of exchange might remain stationary within chosen limits of time; otherwise the temporarily modified rates of water exchange were measured.

It turned out that net rates of water exchange were markedly different at diverse water increments. Within the limits compatible with life, net gains appeared in negative increments (water deficits) and net losses in positive increments. Hence the exchange was always of a type that dispelled the increment, thereby accomplishing recovery, and compensating for the increment of water in the animal. Net exchanges were zero solely when intake equalled output, hence at water balance. At other contents high intakes accompanied low outputs, and vice versa; gains and losses increased together only in what were believed to be "forced" situations.

When organisms or their parts were allowed to recover, the time courses of water exchanges were followed. Sometimes the initial rates of exchanges were the most rapid ones; at other times delays occurred. As the increment diminished with time, the rates of exchange were modified according to the relation of increment to rate in steady states. Eventually the net rates decreased to zero, at which time it could be said that recovery ceased. The return of water up to this time was then comparable with the increment originally present.

Diverse types of water increment were produced by various means, recognizing specified conditions under which recovery occurred. In general, increments were absolute (such as are usually measured by changes of weight or volume), or relative to designated constituents (such as are usually measured by changes of a concentration). The same increment might be absolutely negative and relatively positive. 
Behavior toward water in environments was of two sorts: certain animals were able to locate water, usually without seeing it; and certain animals frequented moist air instead of dry. In both sorts, the preference for water or moist air was exaggerated when the body was in water deficit. Statistically significant preferences for environments that tend to diminish water losses and favor water gain thus play a role in the maintenance of water content. Not all species are known to show evidence of preference for water in environment; some may accept whatever comes, just as tissues and some parasites in situ do.

Variabilities of water content and of water exchanges measured the net results of regulation. One individual undergoes continual fluctuation of each, but the narrow or wide limits within which it varies indicated how sensitive the system of organism plus environment is toward increments of water. Frequencies of reversals, precisions of rates, and durations of movements, served to characterize the processes of maintenance.

So long as the study of water was confined to the factors of increment and time, variables of four classes of dimensions were dealt with : content $(\Delta W)$, exchange $(\delta W / \Delta t)$, time $(t)$, and velocity quotient $(1 / \Delta t)$. For each species and set of conditions, certain numerical characterizations among these four were selected, which by their uniform relations allowed many comparisons. These were:

Rates; turnover, initial, stationary, minimal, and maximal (tables 10, 13, 15, 21).

Economy quotients (table 9).

Modification ratios (table 11).

Augmentation ratios (table 11).

Tolerance curves (fig. 106).

Tolerated loads (table 22).

Variabilities; successive, individual, within species (table 12). Acceleration and decelerations of exchanges (table 14).

Periods; latent, recovery, to maximal rates (table 14 ).

Half-life (any fractional-life) of load ( $\$ 7$, table 32).

Precisions of turnover $(\$ 23)$.

Completenesses of recovery or return $(\$ 7)$.

Partitions of exchange (tables 10, 11, 20, 17).

Equilibration diagrams (fig. 110).

Usefulness of comparisons made according to these criteria is illustrated in the various tables. In general the comparisons drawn 
were among (a) species, individuals, tissues, and cells; (b) types and agents of increment and conditions of recovery; and (c) paths of water exchange. The preferred characterizations were such as were demonstrated to apply to very diverse sorts of living units, almost regardless of their special structures and functions. Less general criteria were available when additional correlatives were taken into consideration. Thus, all organisms exhibit water exchanges after a water increment is imposed; but only some exhibit exchange through integumentary paths, or in proportion to exposed area.

TABLE 32

Adjustments of water load. $G=$ gain, $L=$ loss

\begin{tabular}{|c|c|c|c|c|}
\hline \multirow{2}{*}{ Species } & \multicolumn{2}{|c|}{ Net gain in defieit } & \multicolumn{2}{|c|}{ Net loss in excess } \\
\hline & $\begin{array}{l}\text { Hours for } \\
\text { half return }\end{array}$ & Modifications & $\begin{array}{l}\text { Hours for } \\
\text { half return }\end{array}$ & Modifications \\
\hline 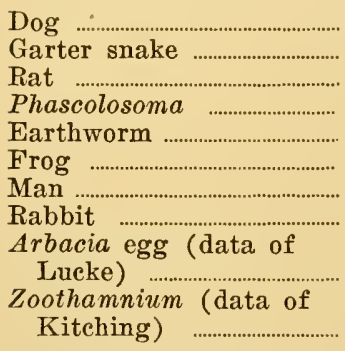 & $\begin{array}{l}0.02 \\
0.04 \\
0.15 \\
0.4 \\
1.0 \\
1.4 \\
1.6 \\
0.04 \\
0.1 \\
0.02\end{array}$ & $\begin{array}{l}\text { G, (L) } \\
\text { G } \\
\text { G, L } \\
\text { G } \\
\text { G, L } \\
\text { G, L } \\
\text { G, (L) } \\
\text { G, L } \\
\text { G } \\
\text { G, L }\end{array}$ & $\begin{array}{l}1.5 \\
4.0 \\
1.8 \\
1.5 \\
1.6 \\
2.3 \\
2.1 \\
1.8 \\
0.04 \\
0.2\end{array}$ & $\begin{array}{cc}\text { G, } & \text { L } \\
(G), & \text { L } \\
\text { G, } & \text { L } \\
\text { L } \\
\text { L } \\
\text { L } \\
\text { G, } \\
\text { G, } \\
\text { L } \\
\\
\text { L } \\
\text { L }\end{array}$ \\
\hline
\end{tabular}

Within one class of animals showing structural similarities, e.g., vertebrates, water increments are accompanied by characteristic changes in particular tissues. In vertebrates, some of the modifications found are in:

(4a) Tissue volumes, volumes of distribution;

(4b) Tissue concentrations;

(4c) Amounts of components in tissue and body;

(4d) Pressures within tissues.

(8a) Rates of exchange of components;

(8b) Rates of transformation of energy;

(8c) Rates of circulation of blood and other fluids;

(8d) Behaviors and other physiological activities;

(8e) Responses to excitations, agents.

The diverse sorts of modifications in tissues coincident with increment in a single variable $(\Delta \mathrm{W})$ constitute a whole pattern of strains or displacements; the organism is then out of kilter until recovery 
has occurred. Of these correlatives all appeared to be equally significant, for no means was found of distinguishing direct ties or nexuses from others. All quantitative relations therefore equally characterized the water relations of an organism or its parts, and whatever was neglected through convenience or ignorance left the description of the physiological relations incomplete by that much. Possibly all of the changes are resistances to further increment and promoters of recovery.

A considerable number of modifications in composition and metabolism were actually and quantitatively correlated with water increment $( \pm \Delta \mathrm{W})$; extensive data were for the dog, represented in figure 131. In that correlation, $\pm \Delta \mathrm{TW}$ has the arbitrary status of an independent variable; but the relations are reciprocal among all the quantities present.

Finally, the rôle of forces in water exchanges, of bodily dimensions, of developmental ages, and of other factors of heredity and environment were considered. Each category emphasized particular aspects of the adjustments of water content.

\section{$\$ 106$. UnIFORMiTIES}

The qualitative results arising from the investigation outlined are indicated by the specific conclusions now set forth. Each is an induction from data already presented; whenever exceptions to the statements made shall turn up, they will be noteworthy and useful.

(1) The living units studied respond to each change of water content by (among other things) modifying their rates of water exchange.

(2) The modified exchange is of such a sign that excesses are eliminated and deficits are paid off, when conditions allow.

(3) Upper and lower limits of water content exist, compatible with continued life and observed activity; outside these tolerated loads, recovery of water balance usually does not occur.

(4) The variability of water content of one individual in standard conditions is related to the amount of modification of exchange rates that occurs per unit increment of water content (fig. 47).

(5) Among differentiated chanmels or paths of water exchange in any species, gain by only one path and loss by only one path is markedly modified with water load (table 10).

(6) In no case is water found to be manufactured from other: chemical substances at faster rates in response to water deficits. 
(7) Time relations and rates of recovery may be similar even though paths and structures employed are diverse (fig. 111).

(8) Rates of water exchange are faster with larger increments than with moderate ones. But the total times occupied in recovery (and the velocity quotients) are nearly independent of increment.

(9) The amounts of water returned before the control rates of exchange again prevail, do not exceed the water loads administered (figs. 1 and 49), when compared with equally timed control individuals.

(10) Recovery is faster, and often more prompt, in negative increments of water than in positive (fig. 106).

(11) Species or units that eliminate excesses rapidly, also provide for restoring deficits rapidly. Though diverse organs be concerned in gain and in loss, the two are proportioned to each other.

(12) Tolerance curves are usually such that net exchanges are most rapid in the early portions of recovery, diminishing as loads are discharged. Most loads are therefore exponential with time.

(13) High rates of turnover accompany high variabilities of water content at successive times (table 12).

(14) Intake by mammals is usually more variable in periods of hours than in periods of days. Output, however, is equally variable in periods of diverse durations (table 12).

(15) No one kind of change other than augmentation of water exchanges, is known to occur in all organisms studied, in invariable correlation with increments of water content.

(16) But in vertebrates, for instance, certain changes of composition, rates of other exchanges, activities and behaviors are found to accompany water increments under specified conditions (table 29).

(17) Maximal rates of urinary output are nearly proportional to body weight, among the species of vertebrates studies (table 13).

(18) Evaporative loss is a larger fraction of total loss in small mammals than in large ones. Oxidative gain is a larger fraction of total gain in small mammals than in large ones.

(19) Rates of exchange of water are among many animals proportional to the 0.6 to 1.0 power of body weight (figs. 133 to 140).

(20) In mammals in which turnovers in water balance have been measured, the maximal rates of intake or of output observed (fig. 138) are 20 to 30 times the rates of turnover in the respective individuals. 
(21) Animals frequent environments that furnish water to them, or that minimize loss of water. Both behaviors are exaggerated in water deficit.

(22) In water increments of the whole body, plasma is diluted twice as much as the whole body (dog). Whole blood dilutions are less certainly correlatives of load.

(23) Volumes of distribution of several injected distribuends also change markedly more than does the whole body (fig. 114).

(24) Most tissues analyzed share in the distribution of excesses and deficits of water, at least in four species (table 29). In diverse species no one tissue preserves its water content more consistently than others. No outstanding depots of reserve water have been identified.

(25) Hence very many living units, whole individuals, their parts and aggregates, of diverse species, may be studied with respect to water loads; and similar patterns are found in their maintenances and recoveries of content.

These conclusions are arrived at from correlations which are explicit here. Further tests of their generality lies in additional information. At present none is known to me that contradicts any one conclusion. But generalizations are always provisional; it is not improbable that organisms exist which fail to fit some of the specifications of those studied thus far. It does seem to me improbable that any species of organisms exists whose individuals are wholly without means of adjusting their water contents; and regulation of this function may be one of the many requisites for survival.

Quantitative conclusions do not lend themselves to statement in words; they can be fully reviewed only by reexamining the diagrams and tables.

\section{$\$ 107$. Diversities}

Comparisons among species and among parts of organisms that were modified in water loads, revealed features of difference that are not known to be mere corollaries of structural difference:

(1) In frog, earthworm and ciliate (in fresh water) the rates of water gain are no less in water excesses than in turnover.

(2) Some whole aquatic animals (Phascolosoma, Arbacia egg) manifest no turnover of water; in them recoveries of water content are accomplished through exchanges of novel sorts, instead of through quantitative modifications in paths already operating. 
(3) Many diversities are related with body size, age, surface of exchange, possible forces of exchange, structural differentiations, and other features of the units whose content is being equilibrated.

(4) Though paths of water exchange differ, no one kind of path is regularly (inherently) slower or faster than other kinds, or its exchanges less or more accurately related to water load.

(5) Species and living units may be arranged in various series, depending, for instance, on velocity quotients in recoveries. For gains the order of rates is : dog, snake, rat, plasma, Phascolosoma, earthworm, man, frog, muscle. For losses : plasma, dog, rat, earthworm, Phascolosoma, man, frog, muscle, snake (figs. 106 and 101).

Such series are often qualified by the fact that water excess was produced in each unit (dog, plasma, frog, and muscle) by slightly different procedures (administration by stomach, injection of citrated blood by vein, injection by peritoneum). Further restriction in type of load, however, would not permit comparison of whole rabbit with its plasma or its leucocytes; even injection or previous osmosis does not allow, in diverse units, identity of loading. As in all scientific investigations, tentative comparisons are drawn, provisional generalizations are obtained, without assurance that another generation of physiologists will find as much satisfaction in these particular relations as in some others.

The above diversities among species are the materials for study of the comparative physiology of water relations. All the quantitative materials of tables 8 to 16 are the outcome of that study, which can scarcely be summarized more briefly. Equilibration diagrams, having a uniform plan, fall into classes according as one or two kinds of exchanges are modified in positive and in negative load (fig. 112). Their quantitative study tells how much modification in each kind occurs, perhaps as evaluated by augmentation ratios.

Rough comparisons of water exchanges have been made in the past, but those with which I am familiar do not appear justified. "The amphibian is unable to preserve his water content . . . independent of that of his outer world," says Cannon ('32, p. 283). That is no distinction of amphibia or of aquatic animals, for all species need access to water at some times. Even amphibia do not wait for water to come, but seek it (Czeloth, '30), specific behaviors being indispensable means of preserving water content. "The urine output of most dogs is less readily increased on water ingestion that is the case with man," imply White and Findley ('37b, p. 747). 
That is contrary to data presented above. And so for further statements, including some that I have made.

There is no evident limit to the variety of ways in which living units adjust their water contents. Varied though the compensations and behaviors contributing to water adjustment are, the outstanding fact is that every unit that has been investigated gives evidence of special activities, one of which is modification of water exchanges, that result in maintaining water content more constant than when those activities are experimentally prevented. The apparent relations among those activities and modifications, both qualitatively and quantitatively, is set forth in a manner that coordinates the materials of the comparative physiology of water.

\section{\$108. Agents and types of load}

A great many agents regularly modify water relations, whether they are widely recognized as "hydrators" and "dehydrators," or not. The measurable water increments $(\Delta \mathrm{W}$ and $\Delta \mathrm{V})$ observed in animals under the influences of some few of these agents were already studied. But other agents abound, and many of them would scarcely be proclaimed as producers of $\Delta \mathrm{TV}$ or $\Delta \mathrm{V}$. Examples are: diuretics, diaphoretics, cathartics, blood substitutes, secretagogs, hot atmospheres, and low oxygen tensions.

Whatever designations such agents may have, a common property is to influence water content or volume and water exchanges. Hence the modifications in the body and its parts at any water load may be compared quantitatively in the same manner as above. The effects of several agents may thus be characterized with respect to any and all the modifications of volumes, concentrations, and metabolisms that can be measured.

Something of this sort has been done for pituitary extracts, for instance. With respect to water output after water administration, the times to maximal rate of urinary output, or the times for eliminating half the water increment (mid-excretion point, index of diuresis, half-life) are compared (Burn, '31; Heller and Urban, '35; Martin and Herrlich, '39). Equally useful in those assays would he any of the parameters listed in $\$ 105$.

Particularized tests of the organism's status with respect to water may be useful in the comparison of all conceivable agents, whether those who make the tests are interested in the agents or in the organisms. Such would be: (1) Total water output, or total 
intake, in 1,2 , or 4 hours. (2) Volume of distribution of the dye T 1824 at 1 hour. (3) Clearance of inulin or urea through kidneys at 1 hour. (4) Specific gravity of urine at 1 hour. (5) Rate of flow of saliva at 1 hour. Most of these tests mean more if the same individuals are similarly measured in control periods.

Or, the problem might be to test the "efficacy" of blood substitutes. For that purpose, measurements of $\delta \mathrm{V} / \Delta \mathrm{t}$ after the infusion of standard volumes of each may be selected. In order to characterize further the maintenance of blood volume, I set up the hypothesis that the responses to subsequent hemorrhag'e have more to do with blood volume than the responses to physical exercise have. Hence, the investigator of physiological patterns compromises between (a) being overwhelmed by the semi-infinite number of measurements that could characterize them, and (b) using hypotheses and other factors of convenience to select a few quantities that can be measured with accuracy enough to describe the peculiar pattern of the organism's states and responses. A substitute is ideally a complete replacement for the naturally occurring material. For blood, the substitute is such that among other things it becomes subject to at least some of the regulations and maintenances that preserved the original volume. Only in that way does it continue to fit into the living unit of the blood and the living unit of the whole body.

While the influences of agents and conditions can be studied by means of tests on single individuals or their parts, comparisons of those influences among species and parts seem to me to require general modes of characterization. Where feasible, permeabilities and reluctivities may be computed under specified conditions; these take into account the areas serving for water exchange and the pressures effective in moving the water. Where areas are unknown, other coefficients may serve. Where pressures are unknown, rates of water exchange may be compared at like water increments. "Like" water increments may be those having equal ratios to body weight (as used in this study) or having any other common denominator that someone selects.

The investigation of agents and types of water load therefore reduces itself to the repetition of one or many arbitrarily selected tests. But knowledge that the test is one aspect of a pattern of regulatory modifications of the organism, aids considerably in understanding what the agent does to the organism. It is enhancing or inhibiting some identifiable portion of the living unit's adjustments of water content. 


\section{\$109. General theories of water constancy}

A number of theories have been proposed concerning water in animals, as they have concerning every other aspect of organisms that has been observed, aiming to picture in one way or another the maintenance of constant water contents. (a) One theory states that forces related to protein constituents of tissues hold water there. "Absorption of water by muscle is determined in the main by the state of the colloids contained in the muscle" (Fischer, '10, p. 74). (b) Another suggests that the water content of tissues is governed by the ratio of cholesterol to phospholipid in them (Mayer and Schaeffer, '14). (c) Again, hydrostatic pressures and partial osmotic pressures may be balanced when water is distributed equitably between a unit of tissue and its surroundings (Starling, 1896; Schade, '27). (d) Or, perhaps the total quantities of solute present in the whole body dictate the quantity of water held (Gamble, '29).

Emphasizing water exchanges, (e) Rowntree ('22) said, "The total output of water is determined by the total intake," and further, "The need of the body for water is determined largely by environment and metabolism." (f) Reversing the terms, Richter ('38) stated, "Maximum intake may be determined by the maximum capacity of the kidneys," while "it would seem likely that the maximum output is determined by the total fluid capacity of all the cellular spaces of the body." (g) Very often it is supposed that the kidneys watch over the water content of the body, while nothing is said concerning organisms that have no kidneys. These last two hypotheses seem to have been framed to apply to certain mammals. Explicitly limited is (h) the inference of Babcock ('12) that "the water requirement of mature animals that excrete urea, when at rest, depends chiefly upon the amount of digestible protein consumed." (i) Adolph ('33) thought the requirement of mammals might be predicted by adding together the factors of nitrogen, salt, and energy metabolisms. These last two ignore the general experience that some other investigator will experimentally raise another factor to first magnitude, requiring another statement to express the relationship.

In general, all these and many other views may partially correspond to facts, but not in the manner hypothesized, for all are aspects of a large picture that I believe is one of numerous interrelations. There is much evidence that no factor $\mathrm{D}$ is invariably correlated with water content $W$, and much that many factors $\mathrm{E}, \mathrm{F}, \mathrm{G}$, 
etc., are often correlatives. Any of the above theories may be useful as working hypotheses; they have suggested experiments and measurements. But to suppose that a complete and permanent understanding of water regulation will be obtained by pushing the responsibility for regulation of $\mathrm{A}$ upon $\mathrm{B}$ which in turn needs regulation by $\mathrm{C}$, and so ad infinitum, is encouraging an endless game of shuffling the factors. All theories actually take the form of making variable $A$ dependent upon variable $B$. Sooner or later some other investigator suggests that B depends on A. Evidently no one theory satisfies either the data or the investigators. Eventually there arise one or more inferences concerning each of the many relations that are likely to be established.

I know of no theory specifying the forces concerned in water maintenance that applies to all organisms and living units. I share the opinion that forces balance across the boundaries of all living units in stationary states. I too recognize partial similarities between some of the sorts of forces present in living units and those in non-living systems. I see little probability of identifying all the forces present and assigning a proportionate role to each; or of separating forces concerned in water balance from those related to other components. And I see no way of feeling completely satified even though categories are assigned to each force present.

The theory that all organisms are set to adjust their water contents by modifying their rates of water exchanges, appears to me to be as valuable an inference as any. For the cases investigated it has changed from a theory to a rule. The pattern in which the organisms are set, and the quantitative features of their adjustments, are revealed by measuring further the modifications of composition, of metabolism, of behavior, and of variability that coincide with diverse water increments of the organism.

Very possibly there is no ultimate "determinant" of water content, short of the whole living organism and its environment; for anything less is a very partial account of the adjustments concerned. If so, then whatever all living units have in common, all water regulations also have in common. In other respects very diverse relations may be shown, and no theory general enough to preclude other theories is to be expected.

\section{$\$ 110$. Summary}

The course of the investigation may be described as follows. At the start, particular procedures (especially the emphasis on 
description) were selected. Then data concerning water increments and exchanges in the dog were studied intensively, and particular correlations between them were specified. After like data in other species were added, comparisons among them were drawn, together with a number of generalizations. Further, it became evident that parts of organisms, even single cells, could be treated in the same manner as whole individuals; for each unit preserved and recovered volume. The variable labelled water increment was kept throughout, while diverse changes of compositions, metabolisms, and other physiological activities were correlated with it. Finally some general properties of organisms having increments of water or volume were enumerated and quantitatively compared.

It might be asked: why was the maintenance of water in the whole body studied, instead of maintenance of water in the blood plasma, whose role as the "internal medium" of the organism has been emphasized since Bernard (1859, p. 42) first pointed it out? I think it is clear that exchanges by gains and losses, behaviors toward environments, successive variabilities of content, and correlated events, are capable of much more complete and accurate study on the body as a whole. For, water could be measured as it went in and out, it and other metabolites could be located, sensory phenomena could be studied, and fluctuations from hour to hour could be evaluated for the whole; whereas for the plasma alone little quantitative accuracy has been achieved and few exchanges are known. These are the advantages that carried the investigation of water regulation beyond the stage at which Bernard left it.

Many more properties of water-loaded organisms will come to light when further observations are added, for no one investigation is likely to exhaust the possible interrelations. The pattern of interrelated quantities that has been ascertained serves provisionally to describe the regulation of water content in the organisms studied. Once it is known what features are characteristic when water content is regulated, it becomes worthwhile to see whether other components of organisms are regulated in similar ways. 
Part B

REGULATIONS OF SEVERAL COMPONENTS AND IN GENERAL 



\section{Chapter XIV}

\section{HEAT}

\section{$\S 111$. INTRODUCTION}

The investigation pursued thus far has presented, both in detail and in general, the physiological relations of water and of certain volumes. The relations are such as are known to be implicated in the maintenances of water content and exchanges in living units. The patterns found might be peculiar to water, or else some features might be common to many components. To find which, it is necessary to study other physiological components, and in considerable variety. To this end, the term component is not limited to chemical entities, but is extended to include all sorts of quantitative properties of living units. Perhaps anything that can be measured in the organism may be treated in a fashion analogous to that in which water and volume were treated. That too can be ascertained only by trying.

The investigation now becomes an inquiry into maintenance and recovery of various functions of organisms, and ultimately of the interrelations among maintenances. The components chosen for intensive study (chapter XV) may or may not turn out to be a random sample of the very large number that can ultimately be studied. Even though future physiology may discover types of regulation not yet observed, the relations described herewith prevail in the situations named.

Certain choices could be exercised among the data available. All the components might be selected for one species (dog), or for one type of production of increments' (exercise by running), or for one path of exchange (urinary). Instead I propose to utilize data that seem to me most adequate, distributed quite at random in these and other respects. In the end the study yields a partial picture of a block of physiological patterns that operate together in one individual.

Heat is the first component to be considered, and the species chiefly studied are man and rabbit. How are heat exchanges and other properties modified when a man is warmer or cooler than usual? What is usual? 


\section{\$112. Maintenance in Man}

The recognition of constancy of heat content of the human body is almost universal. Perhaps this content is measured, as body temperature, oftener than content of any other component.

How are heat exchanges related to heat content? This might be answered by procedures familiar in the study of air-conditioning of a house. Observations of the thermostatic arrangements are alone not sufficient; the behavior of the installed apparatus in the house must be examined. The whole house would itself be exposed to diverse external conditions when performance is being described. In the human body a similar study is a desirable preliminary to characterizing a state of fever, of hypothalamic lesion, or of heat stroke.

That heat gain in the long run equals heat loss may be regarded as a prediction of the first and second "laws" of energy. None the less, it was accepted as a singular victory for thermodynamics when Rubner (1894) in the dog', and Atwater (see Lusk, '33, p. 120) in man, ascertained that the oxygen consumed and the carbon dioxide produced in 24 hours of metabolic transformations actually yield energy equivalent to the amount of heat collected in a calorimeter.

The known course of energy transfer predicts that heat is scarcely transformed into any other kind of energy without the presence of large differences of temperature. Of all forms of energy, therefore, the chances of completely measuring heat output are greater than of measuring other outputs. Turnover of heat in man amounts to about $1 \mathrm{Cal} . / \mathrm{kg}$. hr. in usual circumstances. Further, a huge number of factors have been identified as having some influence upon it.

The balanced state of the body is any one in which rate of heat gain equals rate of heat loss. Though the actual heat content of the body might be unusual, rates of both total exchanges are then correspondingly greater than basal ones, and an unusual balance prevails. Hence it is possible to distinguish a usual balance from others.

Usual balances are characterized by the presence of those rectal or oral temperatures found in a random population. The variabilities of these temperatures at a uniform time of day in a given set of conditions (table 33) are indicated by standard deviations, 


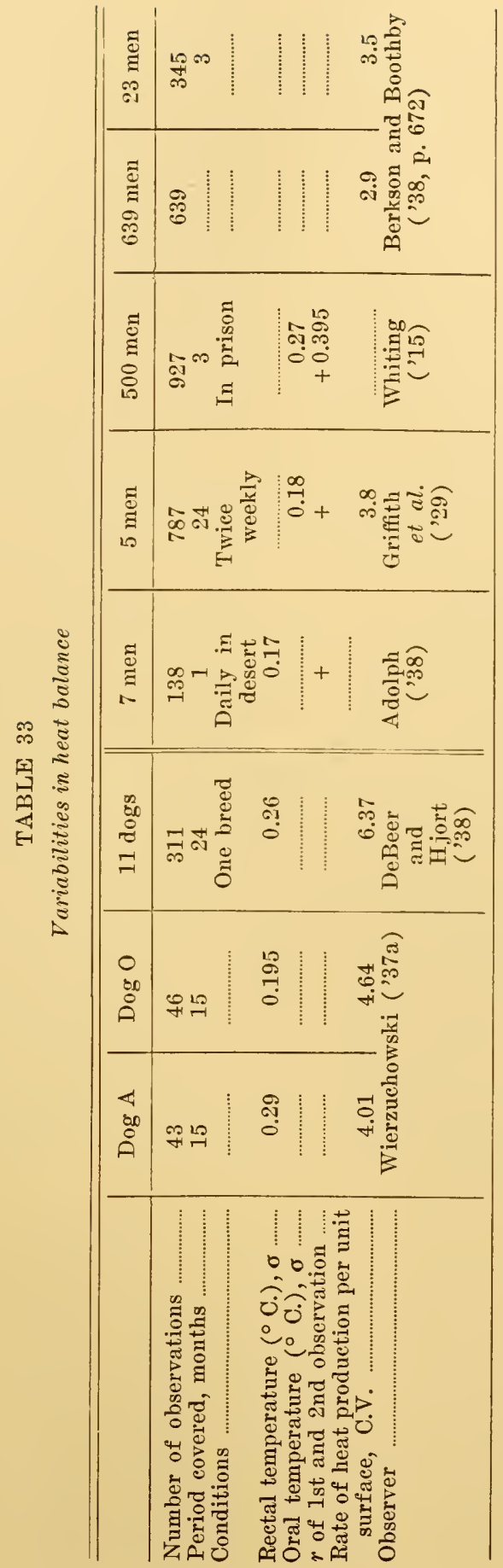


in both dogs and men, of about $\pm 0.20^{\circ} \mathrm{C}$. It is well known that a daily cycle shows changes of more than this. If the individual were exposed to random conditions instead of selected ones, greater or smaller variations might be found. In ascertaining a man's bodr temperature one rirtually asks whether his body is keeping within two or three times the standard deriation for the population. If not, two further questions arise: is he out of balance, or, is he maintaining an unusual (non-basal) balance? Both questions can be answered either by repeated temperature measurements that indicate whether net gains or losses of heat are occurring, or by complete calorimetry of both heat production and heat loss. It is amazing that few human individuals have been discovered who consistently differ in rectal temperature from the mean of all individuals. But the hourly rariations in one individual are greater (standard difference \pm 0.13 to $\pm 0.28^{\circ}$ C.) in newborns than in adults $\left( \pm 0.10^{\circ} \mathrm{C}\right.$.) (Raudnitz, 1S8S).

It mar be noted that in one individual on different dars the rate of heat production under "basal" conditions fluctuates by coefficients of variation of \pm 3.5 per cent (table 33 ). Among individuals of one body weight and height the coefficient is \pm 2.9 per cent (Berkson and Boothbr, '38), values which show that unknown correlatives are insignificant in subjects selected as "normal."

The maintenance of constant heat content may depend upon either (a) continual equality between gain and loss, or (b) successive compensations of one by the other. If gain and loss differ by more than the errors of measurement, within periods of (say) one hour or less, it is natural to ask, which of the exchanges has the more uniform rate, and, does the uniformity of rate indicate a less pliable process? Quantitative answer's do not appear to exist for man, but will be given below for rabbit.

\section{$\S 113$. Heat loads (Max)}

Departures from the usual balanced state are produced by accidental or deliberate changes in body and surroundings. The initiation of heat deficits by cold media was systematically studied by Lefèrre ('11). A man is placed in a water calorimeter (his p. 94 ) or in a wind calorimeter (p. 103), and the rates of sensible heat loss (non-evaporative loss) are ascertained (table 34 ). In water, as much as 5.7 Calories/kilogram of body weight are lost in 0.2 hour. During the exposure the rate rapidly diminishes until a sta- 
tionary rate of loss, $21.7 \mathrm{Cal} . / \mathrm{kg}$. hr., prevails. This represents an increase (augmentation ratio) of about 22 times the control rate of heat loss.

The resistance to heat loss might be computed in arbitrary ways ; in any case it changes during the loading. By a usual definition the resistance is the reciprocal of rate of loss per unit temperature difference between inside and outside. Lefèvre (p. 498) used as "coefficient of resistance" the ratio of heat production to heat loss, but this is the definition of the economy quotient $(\$ 13)$.

TABLE 34

Rates of heat loss during loading, Cal./kg. hr., in various species; indicating how quickly deficits of heat may be imposed. Data of Lefèvre (1897)

\begin{tabular}{|c|c|c|c|c|c|c|c|c|}
\hline \multirow{2}{*}{ Species } & \multirow{2}{*}{$\begin{array}{l}\text { Body } \\
\text { weight, } \\
\text { kg. }\end{array}$} & \multicolumn{2}{|c|}{$\begin{array}{l}\text { Rate in water } \\
\text { at }+5^{\circ} \mathrm{C} .\end{array}$} & \multicolumn{3}{|c|}{$\begin{array}{l}\text { Extreme rate in other } \\
\text { conditions }\end{array}$} & \multirow{2}{*}{$\begin{array}{c}\text { Rate of } \\
\text { turn- } \\
\text { over }\end{array}$} & \multirow{2}{*}{\begin{tabular}{|} 
Maximal \\
augmen- \\
tation \\
ratio, \\
station- \\
ary
\end{tabular}} \\
\hline & & $\begin{array}{l}\text { First } \\
\text { minute }\end{array}$ & $\begin{array}{c}\text { Sta- } \\
\text { tionary }\end{array}$ & Conditions & $\begin{array}{l}\text { First } \\
\text { minute }\end{array}$ & $\begin{array}{c}\text { Sta- } \\
\text { tionary }\end{array}$ & & \\
\hline Dog .......... & 8.0 & 72 & 25 & $+1^{\circ}$ C. a & & 6. & 2.0 & 12 \\
\hline Man ................. & 64.5 & 97 & 21 & $+5^{\circ}$ C. air & & 4.4 & & 22 \\
\hline $\begin{array}{l}\text { Monkey ... } \\
\text { Pi } \alpha\end{array}$ & 2.7 & 169 & 50 & $-8^{\circ}$ C. water & 267 & 82.0 & 4.0 & 20 \\
\hline small ... & 4.2 & 255 & 63 & $+5^{\circ}$ C. air & & 8. & 4.0 & 16 \\
\hline $\begin{array}{l}\text { Rabbit } \\
\text { Guinea }\end{array}$ & 4.5 & 51 & 17.4 & $-18^{\circ}$ C. water & 96 & 33.0 & 3.4 & 10 \\
\hline pig ...... & 0.7 & 174 & 67.0 & & $\cdots$ & $\ldots \ldots \ldots . .$. & 3.9 & 17 \\
\hline Chicken ... & 2.5 & 77 & & & $\ldots \ldots \ldots \ldots$ & ........... & 3.0 & 12 \\
\hline Duck .............. & 2.3 & 72 & 31.0 & 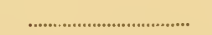 & ............ & ........... & 3.0 & 10 \\
\hline
\end{tabular}

Negative heat loads of other types are produced by rectal irrigation, or by drinking ice-water, or by surrounding the body with ice. All are like baths in eliciting local deficits of heat that are later distributed.

Positive heat loads are rapidly acquired in physical exercise, diathermy, intense radiation, stoppage of heat losses, and other circumstances.

For accurate measurement of the heat load, not only the total heat lost but also the total heat produced during transition is ascertained, for in any transitional state the load is the difference between the integrated gain and the integrated loss. Alternatively, heat load is ascertained as the product of increment in tissue temperature and of heat capacity. In the whole man, various temperatures appear in diverse portions of the body, and approximations of their distribution (Burton, '35) are necessary to evaluate the heat content of the body. This measurement is less practical 
than the first, both during periods of rapid change, and especially where one volume of tissue (superficial) is being cooled while another volume (deep) is not being cooled.

Acclimatizations have been recognized, whereby exposure to given conditions yields smaller loads after repetitions than before. Changes of body heat content are thereby resisted. A man immersed in water at $5^{\circ} \mathrm{C}$. for a few seconds subsequently diminished the rectal temperature by $1.0^{\circ} \mathrm{C}$. After 3 months of training, twice as long an exposure secured less decrease of rectal temperature $\left(0.1\right.$ to $0.7^{\circ}$ C. $)$. Less heat was also lost in the later exposures (Lefèvre, 1894). In periods of physical exercise in hot atmospheres, progressively faster losses of latent heat occurred, with smaller accumulations of heat in the body, during the first ten days of life in the desert (Adolph, '38). Even basal heat production appears to become slightly slower every summer (Gessler, '25), when the organism partially transforms itself from a frequent regulator for deficits to a more frequent regulator for excesses. All these are acclimatizations.

It might be possible to derive an "absolute" value for the heat content of man; this would ideally be the total heat lost in cooling to absolute zero, a determination not likely to be made. Such a value would be useful in comparing heat increments with increments of other components, for all changes of heat content might then be fractions of the content that prevails during heat balance. Some value for this content (in Cal. $/ \mathrm{kg}$.) could be assumed, such as $309^{\circ} \times 0.83 \times \mathrm{B}_{0}$, the coefficient 0.83 being the mean specific heat capacity of human tissues, and $309^{\circ} \mathrm{K}$. the usual average temperature of the body. Assumptions concerning heat capacities may if desired be avoided in studies solely of net exchanges, since the relations here considered hold equally if $\Delta \mathrm{T}$ (inerement of mean temperature) is substituted for $\Delta \mathrm{H}$ (increment of heat content per unit of body weight). Rates of change of either local or integrated tissue temperature $(\delta \mathrm{T} / \Delta \mathrm{t})$ then replace the rates of heat exchange $(\delta \mathrm{H} / \Delta \mathrm{t})$.

In brief, heat loads may be estimated with almost the accuracy of water loads. Special circumstances of heat production and heat distribution are recognized as opposing the experimental establishment of either deficit or excess. The oppositions and the sharp discomforts incident to heat loads are evidences of the narrow range within which heat content is usually preserved. 


\section{\$114. Recoveries (MAN)}

In tests now to be considered, excesses of heat develop during physical exercise in hot atmospheres. Thereafter the net rates of heat loss are studied during later recovery indoors, where no solar radiation prevails and the air is cooler (fig. 143). In the first 0.3 hour the net loss is slow, heat production probably decelerating gradually; thereafter load diminishes with time up to 1.0 hour. In other tests, deficits develop during the drinking of ice-water, and other adjustments are occurring initially (fig. 143).

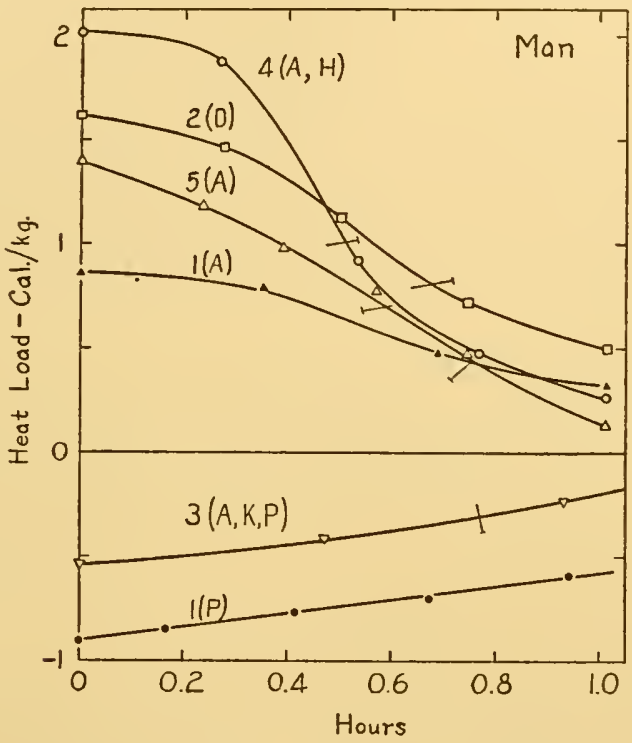

Fig. 143. Heat load (increment in Calories per kilogram of $\mathrm{B}_{0}$ ) in relation to time during recovery. Heat loads (increment of rectal temperature $\times 0.83$ ) were imposed either by walking in the hot desert or by drinking ice water; recovery consisted in resting indoors in an air temperature of 30 to $31^{\circ} \mathrm{C}$. Several tests that started with about the same load are averaged, the number of tests included being indicated. Five subjects (A, D, H, K, P). Further data of Adolph ('38), and of Pinson and Adolph ('42).

The rates of net exchange are clearly related to the diverse heat loads (fig. 144), gain of heat augmenting in deficits and loss augmenting in excesses.

In still other tests total exchanges are measured instead of net exchanges. After moderate cooling (fig. 145) the rate of heat production is no greater than before; shivering is usually absent. In this and similar tests, therefore, recovery occurs by temporary diminution in rate of heat loss. I draw the same conclusion from 


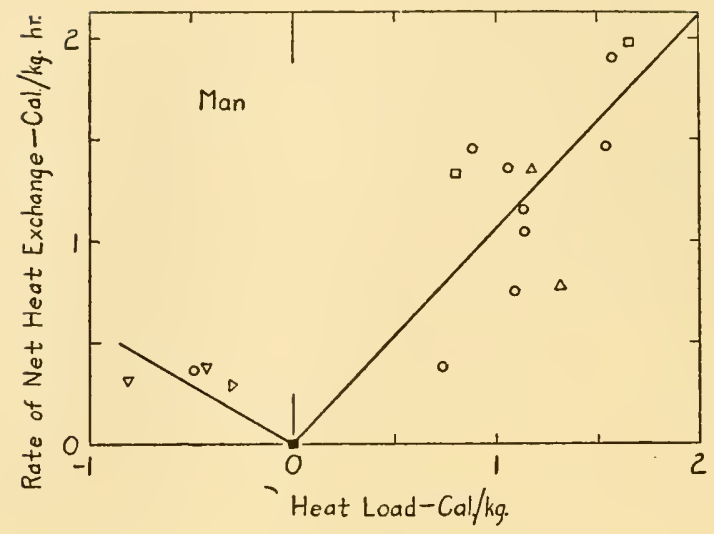

FIG. 144. Rate of heat loss in relation to mean heat load. Net heat equilibration diagram. Man. Three individuals of 75 to $77 \mathrm{~kg}$. in first 0.5 hour of rest indoors, after physical exercise outdoors in the hot desert; or 3 individuals in the third 0.5 hour after drinking ice water. In positive loads, both coordinates were computed from changes of rectal temperature alone $(\times 0.83)$, it being shown that surface temperatures changed little. Further data of tests represented in figure 143.

tests with smaller heat deficits made by Cannon et al. ('26). In the conditions prevailing, the diminished rate of loss accompanies lower surface temperatures, less sensible loss, and less loss by vaporization. In other subjects, or in the same subjects diversely

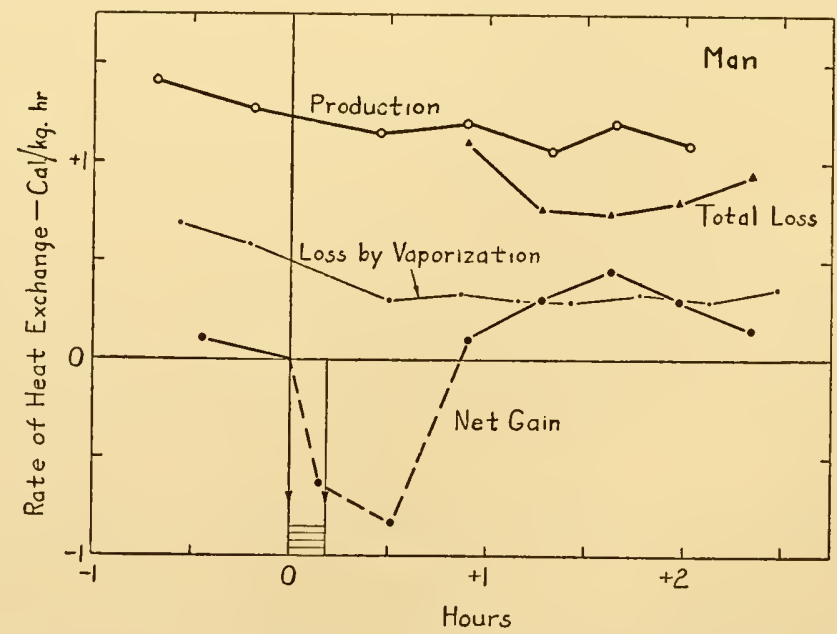

FIG. 145. Rates of heat exchanges in relation to time after ice water was drunk (Man, subject $P$, one test). Production was computed from rate of oxygen consumption, vaporization (latent loss) from uncorrected rate of insensible weight loss, and net gain from rate of change in mean body temperature using Burton's ('35) coefficients. The drink, taken during 0.2 hour, imposed a heat debt of $0.74 \mathrm{Cal} . / \mathrm{kg}$. Total loss is gain by production minus net gain. New data of Pinson and Adolph ('42). 
acclimatized or merely in another atmosphere, the mode of recovery might be quite different. That the production of heat may augment in such heat deficits may be computed from earlier data collected by Yagloglou ('24). The loads coincident with each rate of heat gain there reported may be estimated from (1) the fact that rectal temperature did not change more than $0.3^{\circ} \mathrm{C} .,(2)$ the correlation between effective temperature and skin temperature, and $(3)$ the computation of mean body temperature according to Burton's ('35) method.

Whereas in heat deficits the heat loss is ascertained from heat production minus net gain of heat content, in heat excesses the heat loss happens itself to be measured (fig. 146) and heat production is found by subtraction.

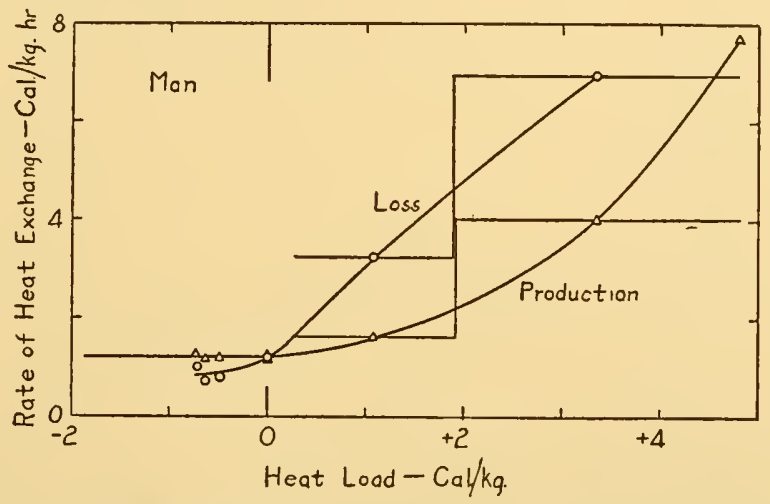

FIG. 146. Rate of heat exchange in relation to heat load. Total heat equilibration diagram. In the range of $+\Delta H$, a single experiment is represented, on a man after heat accumulated from indoor exercise. Loads or heat storages were estimated from rectal temperatures alone. Heat output was the sum of vaporization (from weight loss), radiation (from radiometric sampling), and convection (estimated) during periods of 0.32 hour; heat production was estimated from output plus storage; all by Hardy et al. ('38, p. 489) whose data are plotted. In the range of $-\Delta H$, the data are new ones of Pinson and Adolph and represent recoveries after drinking ice water. An extreme limit of $-\Delta \mathrm{H}$ is designated for heat production from data of Winslow et al. ('37, p. 8).

The properties of the equilibration diagram for heat (fig. 146) are similar to those of the diagram for water. Total gain exceeds total loss in deficits, and is less than total loss in excesses. Only at one point (zero load) are gains and losses equal. In the diagram, the net losses in excesses are much faster than the net gains in equal deficits.

Often the measurement of heat production (gain) is termed indirect calorimetry, of heat loss direct calorimetry. The word 
calorimetry, being in common, tends to confuse two separate measurements; it is clear that only in heat balance, and on the average, does one equal the other. It may also be emphasized that gain is more than that by oxygen utilization when radiations or diathermy are received by the body.

Change in rate of heat production is sometimes termed "chemical" regulation of body heat, while change in rate of heat loss is called "physical" regulation. Often both designations are limited to changes occurring under particular circumstances. The terms actually identify the two directions of the physiological heat exchange with two recognized varieties of energy transfer.

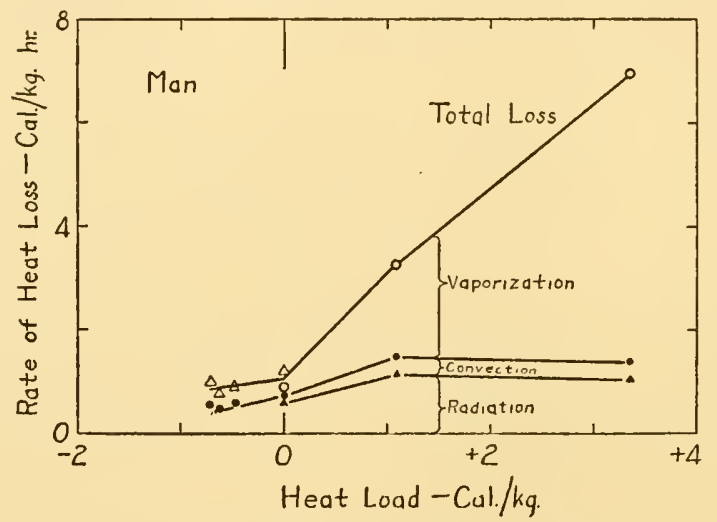

FIG. 147. Rate of total heat loss, and its partition, in relation to heat load during recovery. Vaporization is latent loss; eonvection plus radiation is sensible loss. The positive load was previously ereated by physical exereise indoors. The same single experiment of Hardy et al. ('38), as in figure 146. The negative load resulted from drinking cold water one hour previously; Pinson and Adolph ('42).

"Physical" compensation of heat content occurs in the diminution of loss rate in heat deficits as well as in increase of loss rate in heat excesses. But "chemical" compensation of heat content in man appears to be insignificant or sometimes non-existent in either direction, as in the particular data cited here. These facts become emphatic upon contrasting heat regulation with other regulations.

Within one hour all recoveries from heat excesses are 70 per cent complete. The values of net velocity quotients for heat $(1 / \Delta t)$ fall between 0.7 and 1.2/hour. Recoveries from deficits are the slower, even in the interval of time when maximal quotients are found. Often the body rewarms without extra production of heat; heat is simply lost more slowly. Probably certain environmental conditions could be found in which the relative rates of recoveries would be inverse to those here represented. 
Partitions of heat losses among the several paths are ascertained in more elaborate studies (figs. 145 and 147). Loss by vaporization is computed as 0.57 Calories for each kilogram of water evaporated; the water evaporated, in turn, equals 90 to 100 per cent of the diminution in body weight. The partitions among paths change enormously with heat content of the body, loss by vaporization showing the largest modification ratio of any path. The maximal rate of vaporization exchanges about $13 \mathrm{Cal} . / \mathrm{kg}$. hr. (Adolph and Dill, '38); vaporization reaches a minimal rate near

TABLE 35

Rates of heat exchange, Cal./kg. hr. Man, in diverse states

\begin{tabular}{|c|c|c|c|c|c|c|}
\hline Path & $\begin{array}{l}\text { Mean } \\
\text { rate }\end{array}$ & $\underset{\text { rate }}{\text { Maximal }}$ & $\underset{\text { rate }}{\text { Minimal }}$ & $\begin{array}{l}\text { Modi- } \\
\text { fication } \\
\text { ratio }\end{array}$ & $\begin{array}{l}\text { Augmen- } \\
\text { tation } \\
\text { ratio }\end{array}$ & $\begin{array}{c}\text { Conditions for extreme } \\
\text { rates }\end{array}$ \\
\hline \multirow{2}{*}{$\begin{array}{l}\text { Vaporization } \\
\text { loss }\end{array}$} & 0.3 & 12.8 & $\ldots \ldots$ & 128 & 43 & Walking, in desert \\
\hline & $\ldots . .$. & $\ldots \ldots$ & 0.1 & 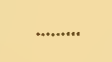 & 0.3 & Sitting, in hot moist air \\
\hline \multirow[t]{2}{*}{ Sensible loss } & 0.7 & 21.7 & ....... & $\propto$ & 31 & $\begin{array}{l}\text { Sitting, in cold water } \\
\text { (Lefèvre, 1897) }\end{array}$ \\
\hline & $\ldots . .$. & $\ldots \ldots \ldots$ & 0 & ........... & 0 & Sitting, in hot air (Win- \\
\hline Total loss & 1.0 & 21.7 & 0.1 & $\begin{array}{l}217 \\
\ldots \ldots \ldots\end{array}$ & $\begin{array}{c}22 \\
0.1\end{array}$ & $\begin{array}{l}\text { Sitting, in cold water } \\
\text { Sitting, in hot moist air }\end{array}$ \\
\hline \multirow{3}{*}{$\begin{array}{l}\text { Oxidative } \\
\text { gain }\end{array}$} & & & & & & Running (Robinson et al., \\
\hline & 1.0 & 16.0 & $\ldots \ldots$ & 20 & & $\begin{array}{l}\text { '37) } \\
\text { Rowing (Henderson et al. }\end{array}$ \\
\hline & $\ldots . . .$. & $\ldots \ldots \ldots$ & 0.8 & $\ldots \ldots \ldots$ & 0.8 & Lying, basal \\
\hline
\end{tabular}

heat balance and does not decrease further in utmost conservation of heat.

Extremes of augmentation that have been observed (in stationary states) are shown in table 35 . The modification ratio of heat gain in man is less than that of heat loss, and less than that of each path of loss. There is evidence that gain may become much more responsive to heat deficits with acclimatization to cold environments in individuals who are continually and variously exposed. Ordinarily behavior and choice of environment are, especially in heat deficits, more effective in securing recoveries than any of the modifications of exchanges yet measured.

Some conclusions that might be drawn are : (1) Rate of net heat exchange during recovery is more rapid the greater the load. (2) Within a limited range of loads, rates of heat loss are modified 
more than rates of heat production. Rates of production do not much vary with those loads that prevailed, in the particular individuals studied at physical "rest." (3) Recoveries from deficits are slower than recoveries from equal excesses. Speculating, I imagine that recoveries from deficits might "cost" more if stored potential energy were expended in faster heat production. Rates of loss by three separable paths of heat loss (vaporization, convection plus conduction, radiation) are all increased in positive head loads. (5) But quantitatively, vaporization plays the greatest role in modifying the rates of heat exchange. (6) Roughly the partitions among paths indicate the order in which various cooling devices are turned on and off.

Each of these conclusions is analogous to one drawn for water exchanges and equilibration in man, heat replacing water in the relations examined. In a word, heat content is recovered in the presence of any heat load, by modifications in appropriate directions of those exchanges that are already operating. No one path of exchange is alone concerned, but "physical" compensations (losses) are more greatly modified than "chemical" ones (gains).

\section{\$ 115. Heat Exchanges of RabBit}

(1) Maintenance of heat content in the rabbit is such that, according to Gasnier and Mayer ('34) the rectal temperature does not ordinarily change as much as $0.1^{\circ} \mathrm{C}$. within one hour. Exchanges of heat during turnover may be analyzed according to paths. In periods of 0.25 hour in a calorimeter, rates of three heat losses (fig. 148) varied by amounts roughly proportional to their mean magnitudes.

Is it possible that gain of heat is steady while loss of heat fluctuates, the two being equal only on the average? That is a question asked by Gasnier and Mayer ('34). They did not measure total heat gain in short successive periods, and no suitable data on variability of rate of gain seem to exist for the rabbit. Assuming that heat production is constant within the deviation of its measurements, they drew the tentative induction that loss hunts about (in the sense of a mechanical governor) to keep pace with gain. The theory is that a rate that varies little (gain) is setting the pace for a rate that varies much (loss); one is inflexible while the other takes care of discrepancies between them. The little flywheel (loss) does more clumsily but fitly what the big flywheel (gain) 


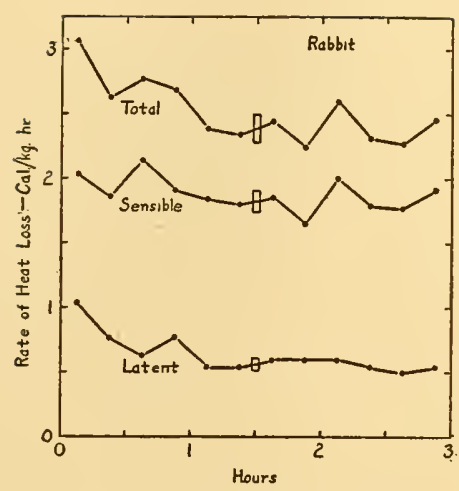

FIG. 148. Rate of heat loss in successive periods of 0.25 -hour. One rabbit in a calorimeter at $23^{\circ} \mathrm{C}$. Sensible loss is the heat picked up by the calorimeter; latent loss the vaporization equivalent of the moisture picked up by the air. Mean differences between successive periods are represented by the small rectangles; they vary only between 8 and 13 per cent of their means. Data of Gasnier and Mayer ('34, p. 220).

does more smoothly but independently. These inferences are susceptible of further investigation; at present the conclusion might be that: using small variability as one criterion of independence and large variability of dependence, then heat gain appears to be the more independent variable.

By the same criterion, and to a greater degree, losses by vaporization which fluctuate by \pm 14 per cent (table 36 ), contrast with sensible losses which are relatively stationary, varying \pm 5 per cent. Perhaps the former is compensating the ineptitudes of the latter.

From the same data for rates of heat exchange, it is possible to find whether the differences between successive half-hours and

TABLE 36

$\nabla$ ariabilities (coefficients of difference, $C \Delta$ ) in rates of heat exchange, at intervals upon single days. Rabbit. Data of Gasnier and Mayer

('34, p. 153, 204, 206, 218, 220)

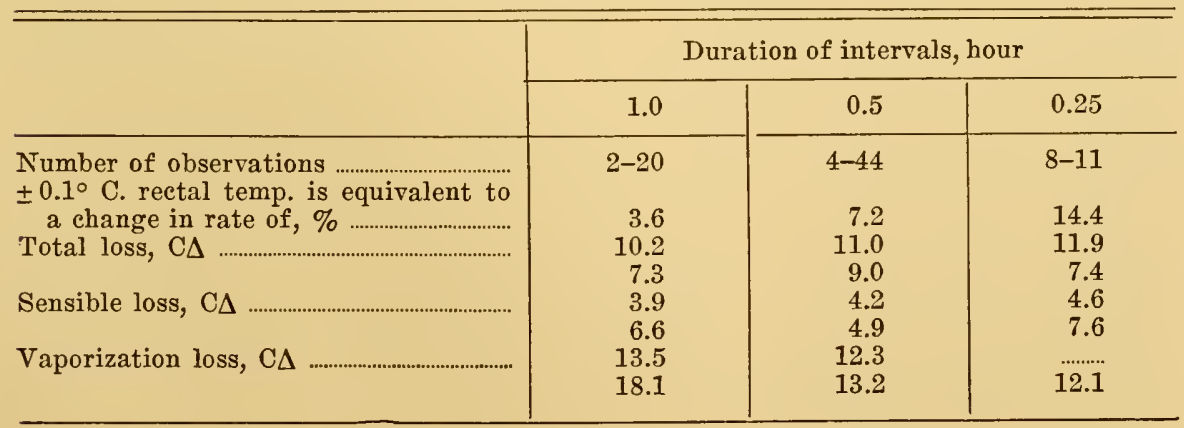


hours are greater or less than between successive quarter-hours (table 36). Since no systematic diversity appears in the coefficients of difference with various time intervals, it may be concluded that (a) constancy of these measurements is not increased in the longer periods, and (b) inertia-like fluctuations (runs and gaps) do not last longer than 0.25 hour.

Extending the determinations of loss by vaporization to still shorter times, Gasnier and Mayer ('34, p. 186) estimated the losses of body weight (latent heat losses) within successive periods whose duration averaged about 0.01 hour. In these the variability was very much greater. Although the method of measurement was satisfactory in the absence of the rabbit, it is possible that the

TABLE 37

Rates of heat loss, Cal./kg. hr., in dry air at $18^{\circ} \mathrm{C}$. Rabbits in diverse states. Data of Gasnier and Mayer ('34)

\begin{tabular}{|c|c|c|c|c|c|c|}
\hline Path & $\begin{array}{l}\text { Mean } \\
\text { rate }\end{array}$ & $\begin{array}{c}\text { Maximal } \\
\text { rate }\end{array}$ & $\begin{array}{c}\text { Minimal } \\
\text { rate }\end{array}$ & $\begin{array}{l}\text { Modi- } \\
\text { fication } \\
\text { ratio }\end{array}$ & $\begin{array}{l}\text { Augmen } \\
\text { tation } \\
\text { ratio }\end{array}$ & State \\
\hline $\begin{array}{l}\text { Vaporization } \\
\text { loss }\end{array}$ & $\begin{array}{c}0.58 \\
\ldots \ldots \ldots . . .\end{array}$ & $\begin{array}{l}1.80 \\
\ldots \ldots \ldots . . .\end{array}$ & 0.31 & $\begin{array}{c}6 \\
\ldots \ldots . .\end{array}$ & $\begin{array}{l}3.1 \\
0.5\end{array}$ & $\begin{array}{l}\text { Methylene blue injected } \\
\text { After morphine, and ice } \\
\text { bath }\end{array}$ \\
\hline Sensible loss & $\begin{array}{l}2.73 \\
\cdots \cdots \cdots\end{array}$ & $\begin{array}{l}5.60 \\
\ldots \ldots \ldots . .\end{array}$ & 0.16 & $\begin{array}{l}35 \\
\ldots \ldots . .\end{array}$ & $\begin{array}{l}2.1 \\
0.06\end{array}$ & $\begin{array}{l}\text { Dinitrophenol injected } \\
\text { After morphine, and ice } \\
\text { bath }\end{array}$ \\
\hline Total loss & $\begin{array}{l}3.31 \\
\ldots \ldots \ldots . .\end{array}$ & $\begin{array}{l}6.60 \\
\ldots \ldots \ldots\end{array}$ & 0.51 & $\begin{array}{l}13 \\
\cdots \cdots . .\end{array}$ & $\begin{array}{l}2.0 \\
0.15\end{array}$ & $\begin{array}{l}\text { Dinitrophenol injected } \\
\text { After morphine, and ice } \\
\text { bath }\end{array}$ \\
\hline
\end{tabular}

recoils of the animal upon the automatic balance used were sufficient to create irregularities in apparent rate of loss, and I draw no conclusions from the data.

(2) Heat loads are established by means, such as ice baths, similar to those in man. Stationary rates of loss during loading may be as high as $33 \mathrm{Cal./kg.} \mathrm{hr.} \mathrm{(table} 34$ ). This, however, represents a smaller augmentation ratio than obtained in man, since the rate of turnover is in rabbits several times that in man (for equal body masses). Positive heat loads are established by injecting dinitrophenol or methylene blue along with a narcotic.

During recoveries from established loads (table 37), two paths of heat loss may be compared. In extreme conditions, sensible losses are almost as greatly modified as are latent losses. Possibly there is a contrast here with man (table 35 ), where vaporization plays a more predominant role. 
Diverse heat deficits are represented by particular rates of exchanges in the course of a single recovery (fig. 149), each load being computed from rectal temperature. In calories, heat gain (production) is modified most; relative to turnover, heat loss changes most. Each path of exchange being modified in a manner appropriate to the recovery of heat content, the spread between heat gain and heat loss is large, showing economy ratios of over 3 .

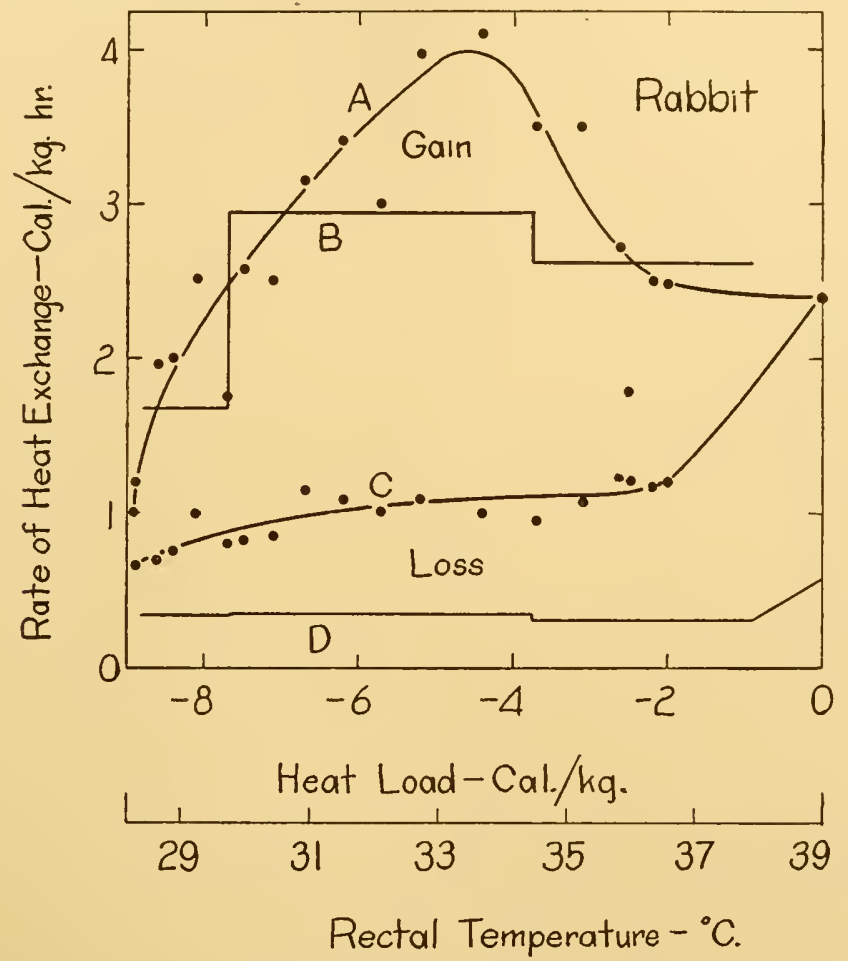

Fig. 149. Rate of heat exchange in relation to heat load (adult normandy rabbit 4). The data are from a single test, the individual being previously cooled by narcotization and immersion in ice water while encased in a rubber sack. Rewarming started at a rectal temperature of $28.7^{\circ} \mathrm{C}$. when put into a calorimeter at $20^{\circ} \mathrm{C}$. Heat exchanges are in Cal./kg. hr. A, heat gain computed in short periods as heat loss + heat retention; $\mathrm{B}$, heat gain computed from oxygen consumption; $\mathrm{C}$, heat loss measured by direct calorimetry; D, heat loss by vaporization of water. Heat load in Cal. $/ \mathrm{kg}$. is computed from rectal temperature minus $39^{\circ} \mathrm{C} . \times 0.83$. Data of Gasnier and Mayer ('35, p. 156).

The net heat exchanges in relation to heat load may be represented in a partial equilibration diagram similar to that in man and other animals (fig. 150), but covering a much larger range of negative loads. 
At very low body temperatures and loads, it was observed, the rates of all heat exchanges are absolutely slower than at intermediate ones. This may possibly represent a zone of loads extremely embarrassing to the narcotized organism; indeed, in slightly different circumstances recovery might not occur.

The development of heat equilibration during infancy may also be viewed in the rabbit (fig. 151). It seems to me that the significance of equilibration is best appreciated by comparison of the usual organism with an organism partially divested of regulations.

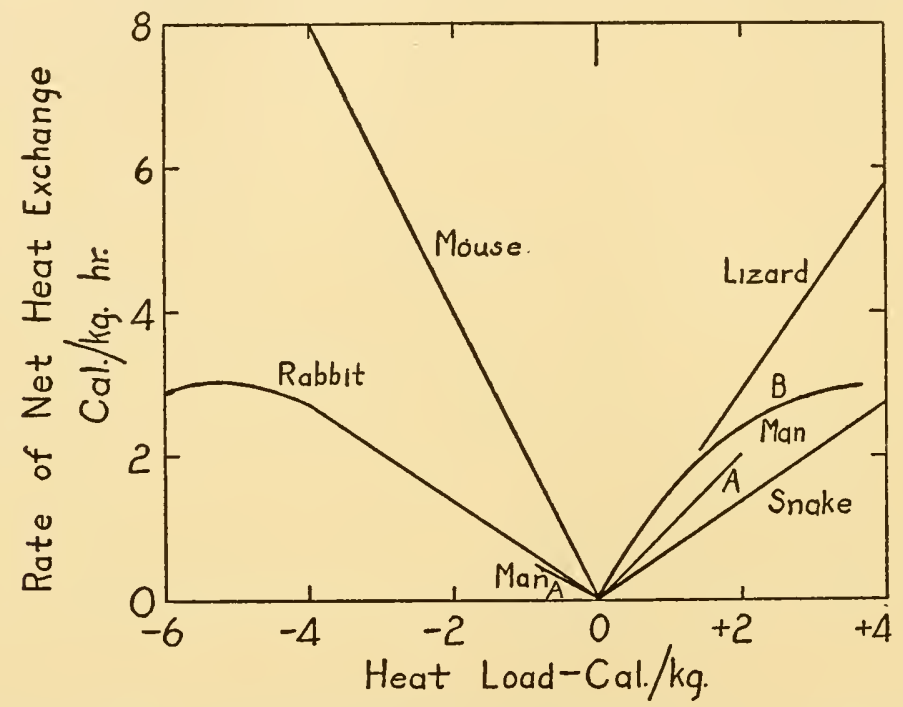

FIG. 150. Rate of net heat exchange in relation to heat load in several species of vertebrates. Comparison of net heat equilibration diagrams. Man, A, data taken from figure 144, and B, data from figure 146; Rabbit, data from figure 149; Mouse, Snake (Boa) and Lizard (Heloderma), data from figure 152.

Such is the infant. The gradual acquirement of the compensatory responses to load then outlines in a vivid manner what the stabilization means in the life of the individual. The high rate of heat production of the newborn animal is not greatly increased in heat deficits (Giaja, '25b). Shivering develops at about 4 days after birth (Ginglinger and Kayser, '29). Before that age, recovery appears to depend on the automatic decrease of heat losses in a suitable environment, selected by the parent or by the experimenter, rather than upon any great modification of functions in the body.

Older individuals economize on energy turnover, and with it 
acquire a moderate reserve of available augmentation in heat production which assists in compensating negative heat loads. But even in adults the range of "chemical regulation" is an augmentation ratio of less than 2. Loss, therefore, is the more flexible in rabbit as well as in man.

Although rabbits with positive heat loads were studied to some extent (Terroine and Trautman, '27; Mayer and Nichita, '29), the periods of measurement were too long to allow accurate correlation of heat exchanges with heat loads during recovery. Both heat pro-

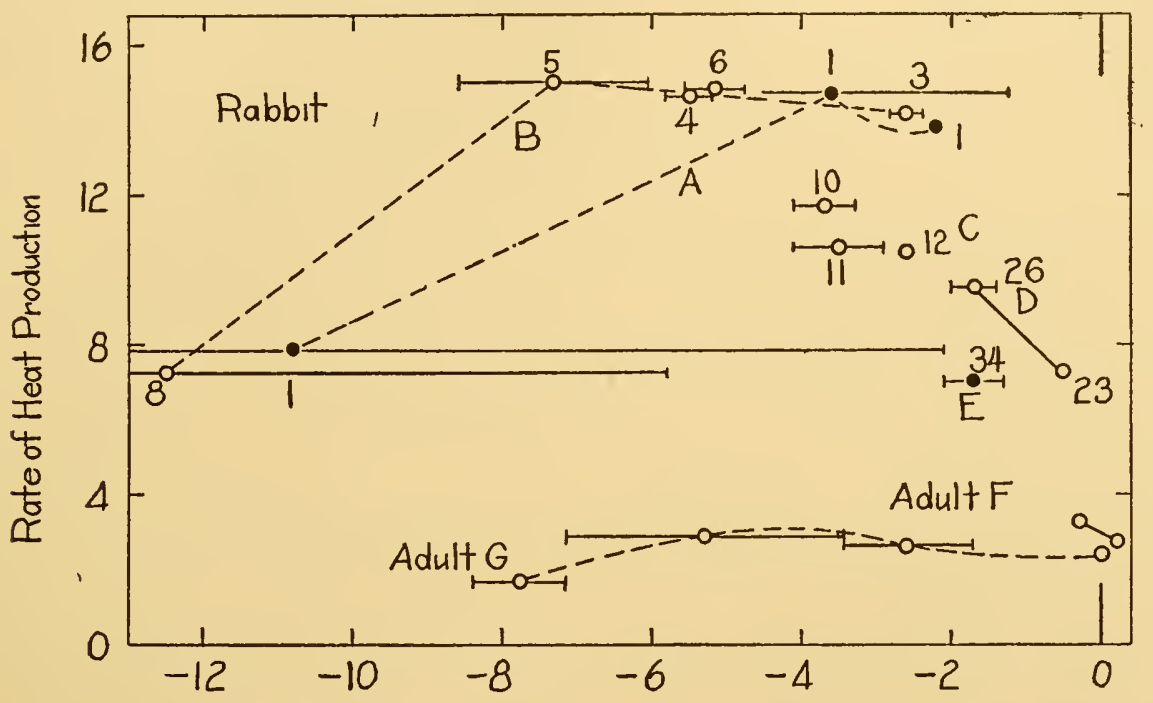

Heat Load

Fig. 151. Rate of heat production (Cal./kg. hr.) in relation to heat load (Cal./kg.) in rabbits of diverse ages. Each point represents several individuals that cooled during the 2 to 3 hours required for the measurement of oxygen consumption, except in group $\mathrm{G}$ where rewarming was proceeding. Points are roughly connected in successive age groups designated A to F. Ages are numbered in days after birth; from data of Ginglinger and Kayser ('29, p. 740). Additional adult curve (G) from figure 149 (data of Gasnier and Mayer, '35). Horizontal lines indicate range of heat loads prevailing during the measurements, as computed from rectal temperatures $\left(\mathrm{T}^{\circ}\right)$ alone; $\left(\mathrm{T}^{\circ}-39^{\circ}\right)$ $\times 0.83$.

duction and heat loss are augmented in heat excesses brought about by confinement in warm air.

Thus, although the turnover of heat in rabbit is thrice as rapid as in man, no marked differences are known in the variability of heat content and of heat exchanges, nor in the modifications of heat exchanges in the presence of heat loads. But in rabbit variations 
of exchanges are more fully known, and extreme deficits of heat have been examined. Heat regulations are acquired after birth in the rabbit, neither gain nor loss being subject to effective selfmodification at birth.

\section{\$116. Comparisons among SpeCies}

The properties of the equilibration diagram for heat in man and rabbit having been established, a few quantitative diversities in other species and a few general features may be mentioned. In

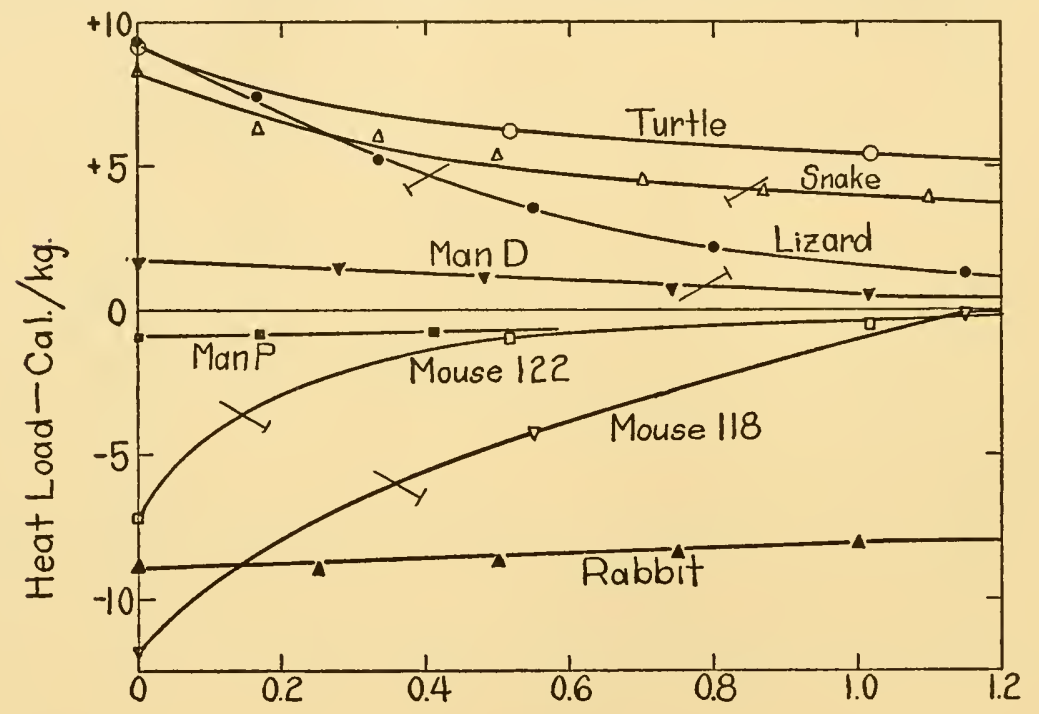

Hours

FIG. 152. Course of heat load during recovery, in various species of vertebrates. Recovery began at zero time, after heating or cooling by various means. Heat load= $\circ$ C. difference from control $\times 0.83$. Turtle, Gopherus, $2.4 \mathrm{~kg}$., new data. Snake, Boa, 6 kg., data of Benedict ('32, p. 68). Lizard, Heloderma, (Gila monster), 1.2 kg., new data. Man D, Homo, $77 \mathrm{~kg}$. from figure 143. Man P, $77 \mathrm{~kg}$. new data. Mouse 122, $0.040 \mathrm{~kg}$, and Mouse 118, $0.023 \mathrm{~kg}$., Mus; data of Chevillard ('35, p. 1046). Rabbit, Lepus, data of Gasnier and Mayer ('35); cf. figure 149.

the actual tests an individual during loading accumulates heat or cold faster or slower than usual; thereafter initial or control conditions are restored, allowing heat exchanges to proceed as they will (fig. 152). The selection of control conditions by experimenters is arbitrary; tests which allow the animal to select its environment from among a number available (Fraenkel and Gunn, '40) are to be recommended. Certainly environmental conditions affect heat exchange, just as they do water exchange. Since no 
method is possible of doing away with the environment, the organism's provisions for dealing with heat loads are, I believe, manifested most clearly when the enviromment is uniform throughout one series of tests. In a more extensive inquiry, many quantitative relations would be established for one organism recovering in each of many environments. In daily life the organism is continually shifting its rates of heat exchange to cope with complicating circumstances as they arise.

Most organisms (poikilothermic) attain temperatures approximating those of the environment or parallel to them, and often they show curves of cooling or warming partially similar to those of inanimate objects (fig. 152). In others (homeothermic) the net exchanges are sometimes opposite in direction to that toward customary inorganic equilibrium. In poikilothermic species rates of heat exchanges are still of consequence in maintaining a gradient of heat.

Terroine and Trautmann ('27) ascertained the relations between rates of heat production and air temperatures in each of 12 homeothermic species. Each showed a minimal rate at some intermediate ("neutral") temperature. The rates could now be related to heat loads if data upon mean body temperatures had also been obtained. Each of those 12 species has its own relation between augmentation of heat production and increment of temperature, some species being more responsive than others to equal heat increments. That might be a basis for visualizing a phylogenetic gradation of regulations.

Maximal rates of heat production in heat loads divided by minimal rates are ratios of modification. Under the name of "metabolisme du sommet," they were found by Giaja ('25a) to be 3.2 to 4.2 in the mouse and four other species, but by Herrington ('40) only about 2.2. Even in monotremes, in which the heat contents are irregular, augmentations of that order are found in low air temperatures (Martin, '02). It may be said that the monotremes' inexact maintenances of body temperature are not the outcome of mere inability to modify their heat productions.

Portions of further equilibration diagrams might be established for other species, particularly the dog. (Wada et al., '35; Hemingway, '38; Shelley and Hemingway, '40) and the mouse. The latter species recovers from temperature deficits more rapidly than larger mammals (fig. 152). During recovery, heat is produced by 
oxidation much faster than in heat turnover, and heat is lost markedly slower (Chevillard, '35, p. 1046). It is said that heat may be lost faster in the dead mouse than in the living one (Pincus et al., '33). Control of the mouse over its temperature develops during the first ten postnatal days (Pembrey, 1895; Sumner, '13; Ginglinger and Kayser, '29). In the 2-day-old mouse compensatory heat productions and their accompanying muscular movements (Pincus et al., '33; Stier, '33), are maximal in environments of $22^{\circ} \mathrm{C}$.; in still cooler ones the recovery becomes slower and less successful, just as in the rabbit (fig. 149). Guinea pigs and some other species, in contrast, are at birth fully armed with compensations for heat increments.

Coefficients may be computed and compared in order to characterize the convection systems, heating systems, and refrigerating systems of animals. They are convenient parameters of conduction, permeability, emissivity, vaporization, radiation, and other quantities. Some of these coefficients are constant under limited conditions. But the so-called physical and chemical processes involved are by no means fully identified; so far as I can ascertain, there is no relation between the constancy of a parameter of heat exchange and the fact that an imputed process in it has a name.

All the methods of comparing water exchanges appear to apply also to the study of heat exchanges. Among species of diverse body sizes and shapes, rates of heat production in turnover may be set forth (Lambert and Teissier, '27; Benedict, '38, p. 131), and related to supposed body surface area and to many other quantities. Velocity quotients, modification ratios, economy quotients, tolerated loads, tolerance curves (fig. 152) and equilibration diagrams (fig. 150), are all applicable for comparisons. These quotients, ratios and diagrams would be required for thorough characterizations of modifications in thermal adjustments of animals such as are produced in diverse lesions of nervous tissues (Brooks, '35; Clark et al., '39) and many other states. Moreover these quantities in heat exchanges are directly comparable with the corresponding quantities in water exchanges and other exchanges (see table 40 ), for most have the same dimensions.

Differentiations may be made between increments of heat and changes of heat balance. For, balance having been defined as a point where gains equal losses, all the criteria of water balance apply to heat balance. Heat load $(\Delta \mathrm{H})$ without change of heat 
balance $\left(\mathrm{H}_{0}\right)$ does not stay constant in any circumstances that allow of recovery. In practice, endless arguments arise as to whether in a state of hyperthermia (fever) or hypothermia (1) the "thermostat" of the organism has changed its setting or (2) there is an aberrant imbalance between gain and loss of heat. Once criteria such as those suggested have been adopted, the question can be answered by calorimetry in every instance. By speculation, one chooses between ( $1 a$ ) assuming the existence of a second regulator to "reset the thermostat," which presumably means there are two or more superimposed thermostats, and $(1 b)$ regarding a shift of heat balance as a concatenated member of a large group of interacting quantities.

Enough has been set forth to indicate that investigation of heat regulation yields the same sorts of quantitative relations as does water regulation. In particular, the tolerance curve and the equilibration diagram relate the rates at which restoration occurs to each increment of heat load. Evidently this sort of physiological pattern is not limited to biochemical entities such as water that have particular kinds of absorptive and emunctory organs at their disposal. Heat is an entity of a different category, with entirely different arrangements physically, anatomically, and physiologically concerned in its exchange. When similar patterns emerge among supposedly dissimilar processes, it becomes profitable to take the tentative view that diverse kinetic equilibria can be studied independently of the specific forms of machinery present.

\section{$\$ 117$. Summary}

Maintenance and recovery of mean body temperatures (heat contents) may be described by means of relations of heat exchanges to heat contents. Partial data exist for man, rabbit, mouse, and some other species. Variability of heat content may be viewed as a resultant of the variabilities of heat gains and heat losses. There are slight indications that heat losses may be more concerned in compensating for the unsuitabilities of heat gains than vice versa. Further, losses by vaporization may through successive modifications compensate for ordinary fluctuations in sensible losses, and vice versa.

Equilibration of heat content may be characterized in the same ways as the air-conditioning of a building that has an installed thermostatic equipment. Variabilities in diverse conditions, and 
modifiabilities in diverse states of heat load, are described. Maturing of the animal's heating and cooling system during early postnatal life, and changes of the system after experimental lesions, may be similarly characterized.

Diversities among species are related to body size, supposed phylogeny of temperature maintenance, the expenditure of water for cooling, and the use of oxidations for warming. Quantitative differences consist in the relative modifications elicited in heat gains and heat losses by a given load of heat.

The relations of heat in certain animals may be described in the same terms as the relations of water. But whereas mature mammals and birds manifest fixed patterns of compensation and behavior toward heat, many other animals are indifferent to heat until extremes are reached, whereupon they shun environments that impose those extreme increments of heat content. Both kinds are successful in preserving heat content, but those with the narrowest deviations of content (homeotherms) also modify a larger number of interrelated variables in accordance with content. 


\section{Chapter XV}

\section{DIVERSE COMPONENTS}

\section{\$118. Total substance}

A variety of properties and constituents of organisms are now to be considered with respect to maintenance. Total bulk of the body is one. Are deficits of body substances compensated by increased intakes, or by decreased eliminations, or by both? In studying the maintenance of total body weight, all substances, including water, are measured in the exchanges.

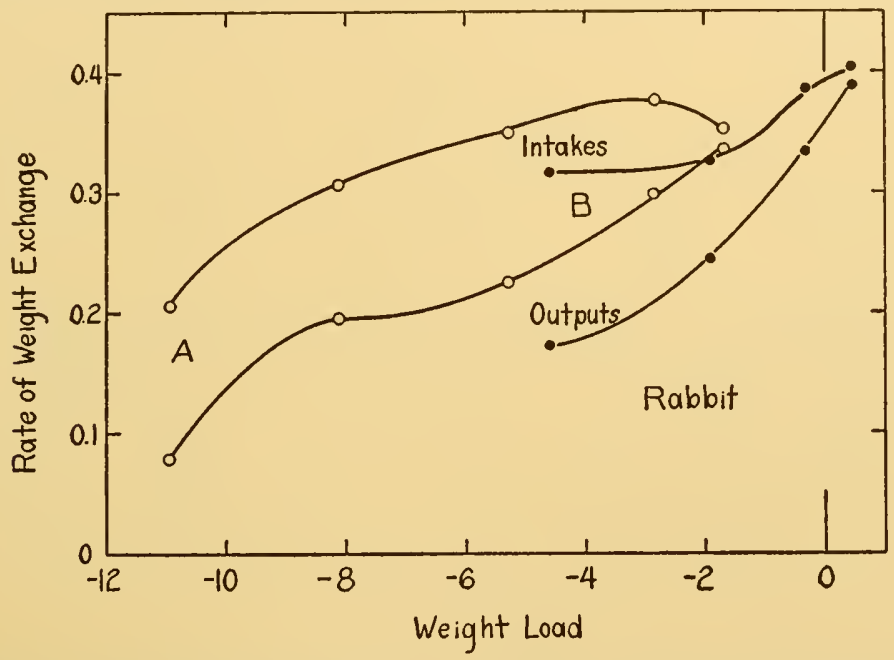

FIG. 153. Rate of weight exchange ( $\%$ of $B_{0} /$ hour) in relation to weight load ( $\%$ of $B_{0}$ ). Rabbit. Two deficits of body weight were previously produced by partial (A) or complete (B) privation of food while water was available. Each point represents one day during progressive recovery of 6 or 8 rabbits that were allowed a uniform food $a d$ libitum for 0.25 of the day. Rate of output was computed as rate of intake minus rate of net gain in weight. Data of Maclagan ('37).

Data for the rabbit record total intakes, and relate the intakes to increments of weight. Elimination rates are computed from intakes minus weight increments (fig. 153). Deficits are established by complete or partial abstinence from food (either including water or not), excesses are established by overfeeding or forced intake.

The relations shown are far from familiar to all who eat. Recovery from deficit in the rabbit may occur not by augmentation of 
intake but by suppression of output. However, in other tests, moderately increased rates of intake are recorded by Maclagan ('37). In another species, the dog, augmentations of intake occur after each deficit (fig. 154) ; in still another, the rat, food missed is never made up (fig. 155).

Recoveries of net substance may be ascertained from body weight alone (fig. 156). In all four species reported, the curves are exponential and asymptotic. The velocity quotients are here in-

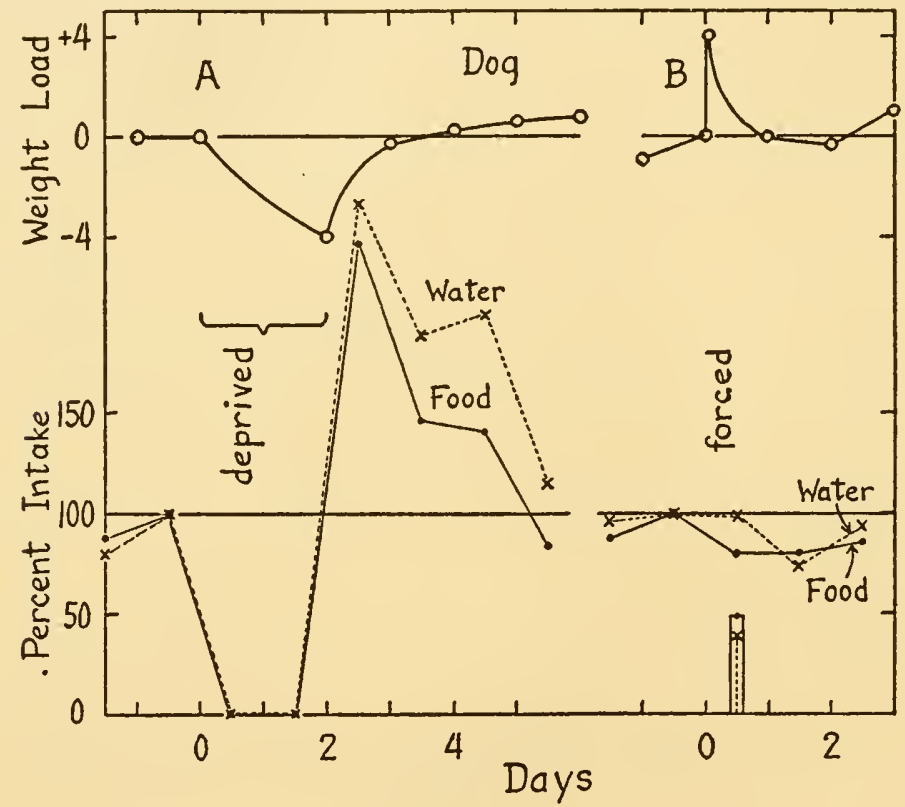

FIG. 154. Increment of body weight ( $\%$ of $B_{0}$ ), and relative rates of intake, in relation to time during and after (A) total privation for 48 hours, (B) forced intake by stomach. Dogs were allowed unlimited dry food and water except during the 48 hours. Amounts forced are indicated in block at lower right. Four tests on 3 individuals (A) and five tests on 2 individuals (B). New data.

verse to the initial (administered) loads, and there is nothing to indicate that they vary from species to species.

Even when fed limited amounts of a constant diet, animals gain body weight faster in the initial part of recovery from food deficit than later, this gain corresponding to the greater retention (or the lesser elimination) of substance (dog, Morgulis, '28). Hence recovery involves here, and in other known instances, decreased rates of elimination; anabolism proceeds with less wastage.

During a single meal the rate of ad libitum food intake of the rat 
(fig. 157) is proportional to the amount still to be eaten. The diminishing rate also corresponds to a weight deficit that diminishes exponentially with time. Rather similar data exist for cats that lapped up their food (Bousfield, '33).

The maximal loads possible in privation of food vary with initial states of the individual, rates of depletion, and other factors. Deficits of body weight that are barely tolerated probably do not differ significantly among the mammalian species that have been

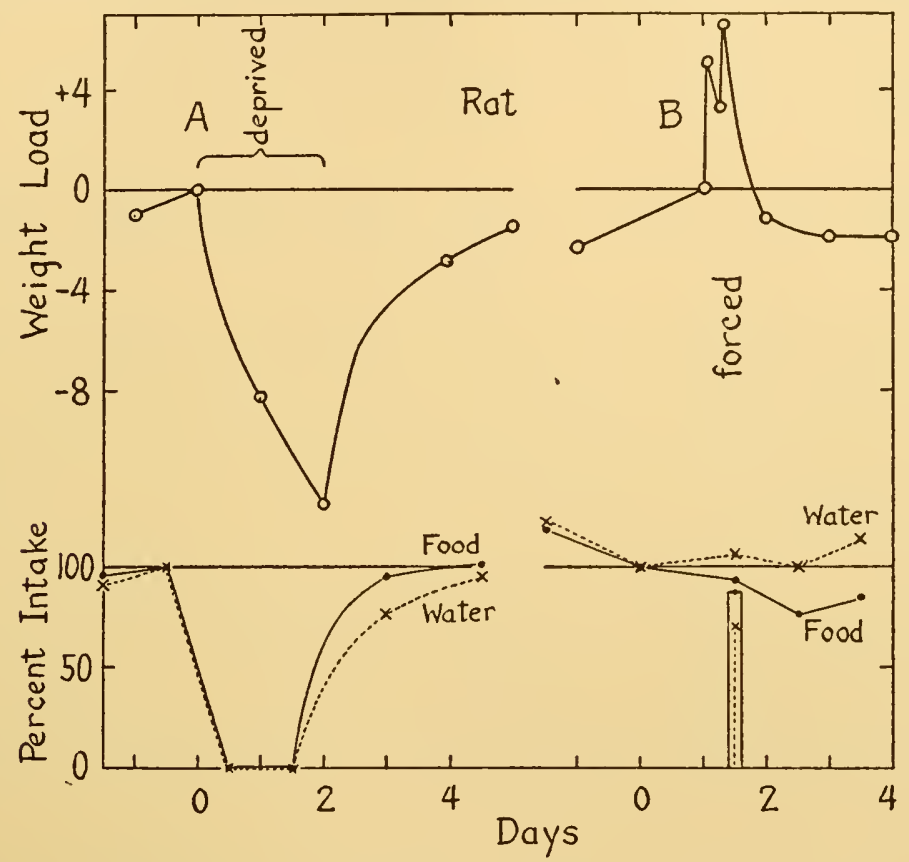

Fig. 155. Increment of body weight ( $\%$ of $\mathrm{B}_{0}$ ), and relative rates of intake, in relation to time after (A) total privation for 48 hours, (B) forced intake by stomach. Rats were allowed unlimited food (dried milk) and water except during the 48 hours. Amounts forced are indicated in block at lower right. Seven tests on 7 individuals (A) and five tests on 5 individuals (B). New data. In $A$ the rats consumed no more food or water per day after deprivation than before deprivation; the dogs (fig. 154) did.

studied. Extreme loads are : dog, $-63 \%$ of $\mathrm{B}_{0}$ (Howe et al., '12); cat, - 54 (Sedlmair, 1899) ; rabbit, -49 (Rubner, 1881); and man, - 45 (Luciani, 1890).

Immature rats appear to withstand prolonged partial feeding. that limits their weight. Subsequent recovery, if allowed at early ages, occurs with faster net gain in weight, yet with slower intake of food, than in control individuals (Jackson, '37). 


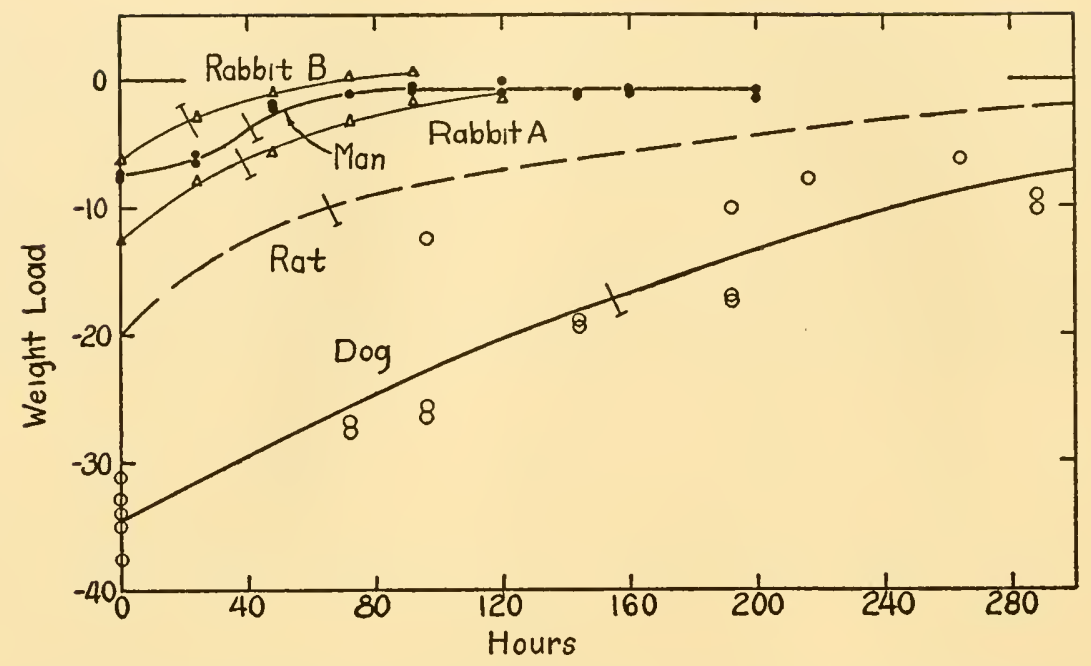

FIG. 156. Body weight load $\left(\%\right.$ of $\left.B_{0}\right)$ in relation to time, during recovery in 4 species of mammals. All were previously deprived of food but allowed water; during recovery, food was allowed generously but was probably artifically limited in some respect in each experiment. $\perp=$ half of load returned. Man, 2 individuals of Howe, Mattill, and Hawk ('11). Dog, 5 individuals of Morgulis ('28 and '29). B and A, rabbit, 8 and 6 individuals of Maclagan ('37) as in figure 153. Rat, 11 individuals of Hitcheock ('26, p. 216).

Forced overfeeding has been less frequently studied. The rates of recovery from it have now been ascertained. It is known that combustions as well as excretions proceed more rapidly (specific dynamic action, luxus consumption). Food is often refused (figs. 154 and 155) and absorption is said to be less complete. I find no

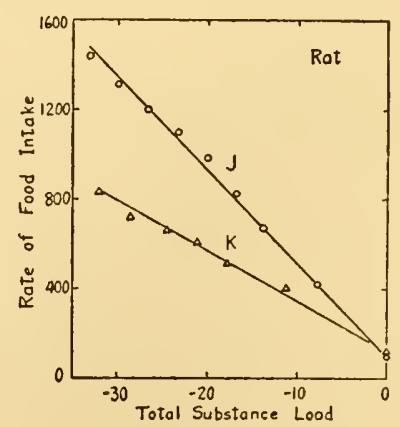

FIG. 157. Rate of intake of total substance or food ( $\%$ of $B_{0} /$ hour) in relation to load of total substance or body weight $\left(\%\right.$ of $\left.B_{0}\right)$. Rate of intake is given in terms of the number of pellets of food eaten when the rat pressed a lever to obtain each one. $\mathrm{J}$, mean of $8 \mathrm{rats}, \mathrm{K}$, mean of $5 \mathrm{rats}$, that were almost completely deprived of food until the initial 1.0 hour in which the test was made. Data of Skinner ('38, p. 397). 
measurements of food intakes after prolonged food excesses, nor precise information upon the tolerated loads attained by overfeeding. Altogether, however, the form of the equilibration diagram is the same for total substance (fig. 158) as it is for water.

At zero load, turnover rates of total substance show small variability. Illustrative data are for the rat, in which species the amount of food consumed varies in daily periods only 10 per cent of the mean. It is sometimes said that food intake is habitual, and this expresses in one phrase the fact that many undefined factors are present in maintenance of every species, and recognizes a gov-

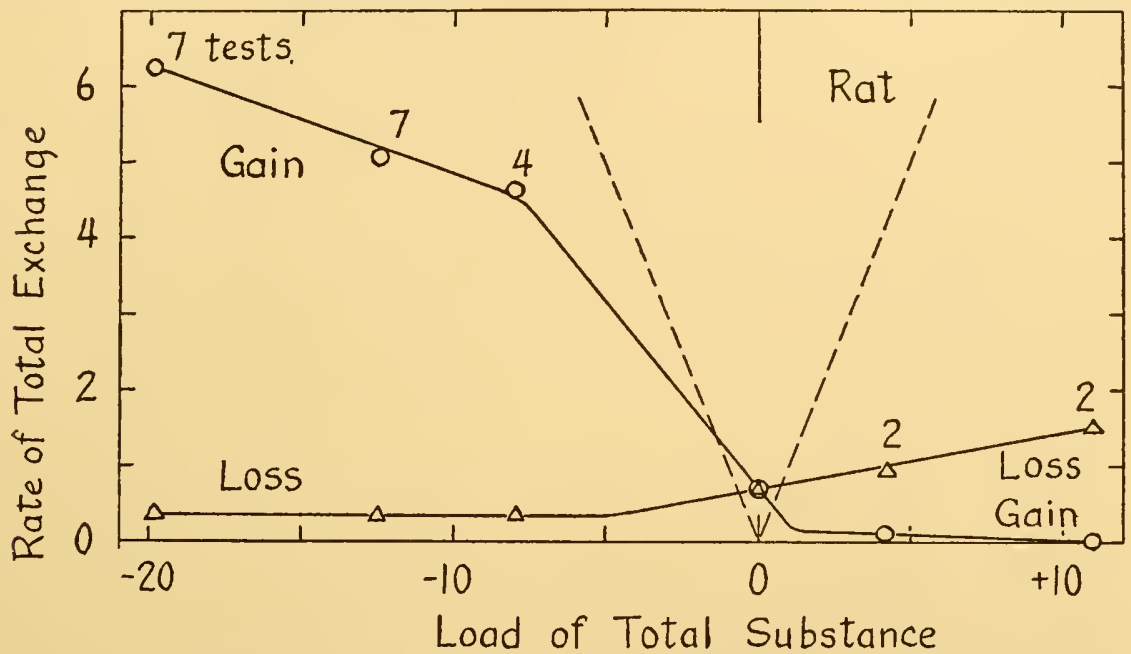

Fig. 158. Initial rate of exchange of total substance (\% of $\mathrm{B}_{0}$ in first 1.0 hour) in relation to imposed load of total substance (body weight) ( $\%$ of $\left.B_{0}\right)$. Rats were deprived of food and water for $0,1,2$, or 4 days, or given excess of food and some water on one day; then were allowed to recover. Most of the exchange is water. At the dash line, exchange during the hour would equal load. New data.

ernor-like action in all of them. The epithet habitual does not recognize the actual fact that a rat is not fooled into continuance of eating when food has already been introduced by stomach or peritoneum.

It is well known that in deficits approximate balances of intake and of output may be struck that are much smaller than turnover at zero load. Such rates may be visualized in figure 153. But it may not be assumed that load is stationary if a rate of intake is artificially adjusted to equal an expected rate of output. For in that state, rate of output may reach some other lower value. Rather, the situation in both deficits and excesses is delineated (for 
the $\operatorname{dog}$ ) in figure 159. Total energy is measured in place of total substance; the two are equivalent, since no qualitative choice of foods is allowed. Intake, which is controlled by the experimenter, varies from 2 to $5 \mathrm{Cal} . / \mathrm{kg}$. hr., without much discrepancy between it and output. Outside these rough limits, in low intakes the output is at a constant minimal rate; while in high intakes, the dog continues to eat all the food given, and retains a considerable proportion instead of expending it. In contrast to water, storage of component is a prominent feature, modification of output playing a smaller but also significant part in adjustment.

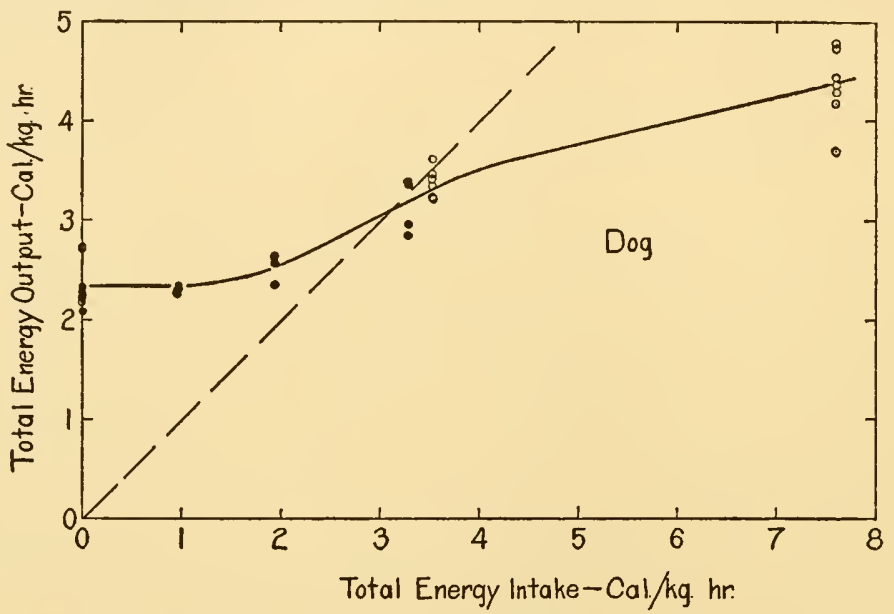

Fig. 159. Rate of total energy output in relation to rate of total energy intake. Meat was given in varying amounts to a dog kept in a calorimeter at $20^{\circ}$ to $30^{\circ} \mathrm{C}$. Each point represents a period of one day; individual II closed circles, individual III open circles. Along the dash line output would equal intake. Data of Rubner ('02, p. 109 solid points, p. 115 open points).

Duration of each allowed rate of intake influences the output. For carbon (fig. 160) the intake and output are proportional to the total substances. During each day that an intake greater than the turnover of former maintenance is fed, the output increases until finally the output becomes equal to the intake. Given time, therefore, a new balance of exchanges is struck, and a new content of total body substance appears to be maintained. This fact accounts for much of the confusion prevailing as to how the content of total substance (or of total potential energy) is maintained. Particularly in the dog, there often seems to be a discrepancy between the amount ingested and the amount "required." The answer is that minimal maintenance is a balance, within the accuracy of usual 
measurement; while ad libitum maintenance is also a balance, more expensive to hold. Many days are required to demonstrate a balance at either, after a transition of intakes is allowed. A considerable range of carbon contents is thereby permitted, such as was not found for water or heat.

For nitrogen, one part of the story is very similar (fig. 160), namely, the gradual adjustment of output to equal intake. But in contrast to carbon exchange, when nitrogen intake ceases, then its output becomes very low. Hence, over a wide range of intake rates for nitrogen, output rates approximately equal them (fig. 161).

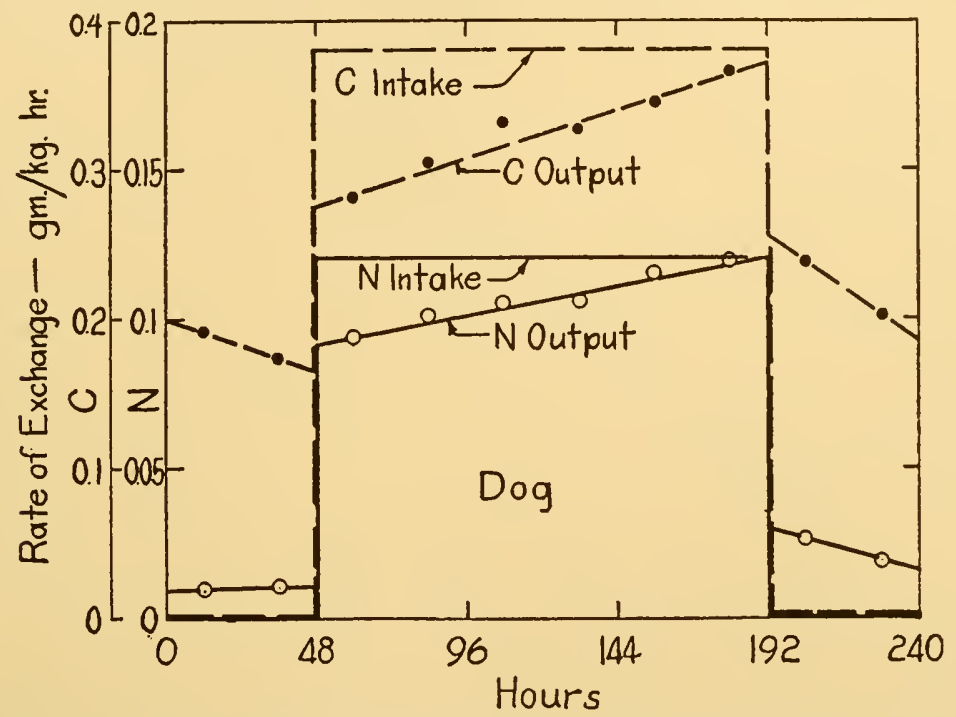

Fig. 160. Rate of exchange of nitrogen and of carbon in successive days. A dog of $5.86 \mathrm{~kg}$. was deprived of food for 2 days, then fed an excessive quantity of meat (500 gm.) each day for 6 days, then starved for 2 days. Rates of outputs gradually adjusted toward the rates of intake. Data of Rubner ('02, p. 246).

Yet, exact balances occur at only one point for each individual. The fraction of any excess nitrogen taken in that is actually stored is small, and the rate of unstoring it during deficient intake is small, as compared with carbon or with total energy. Variability of content is least for that component in which modifiability of exchange is greatest. Where constancy of output is maintained (energy), storage is provided, with wide range of contents.

Very many studies, especially of nitrogen metabolism, have ignored the pre-existing content of nitrogen in the body, and worked entirely with rates of exchange (fig. 161). For example, Voit and Korkunoff (1895) tried to find the minimal intake of meat for 
balance. Starting with $0.015 \mathrm{gm} . \mathrm{N} / \mathrm{kg}$. hr., they gave slightly more on successive davs until output was no longer greater than intake. But meanwhile the nitrogen content of the bodr was being depleted; the deficit so obtained may or may not have been serious; and acclimatization of output (as in fig. 160) may have been slow or fast. Balance finally occurred at $0.028 \mathrm{gm}$. N/ kg. hr. (C, fig. 161).

Hence in nitrogen exchange, the time factor is large, for weeks may be required to establish stationary rates at one bodily content.

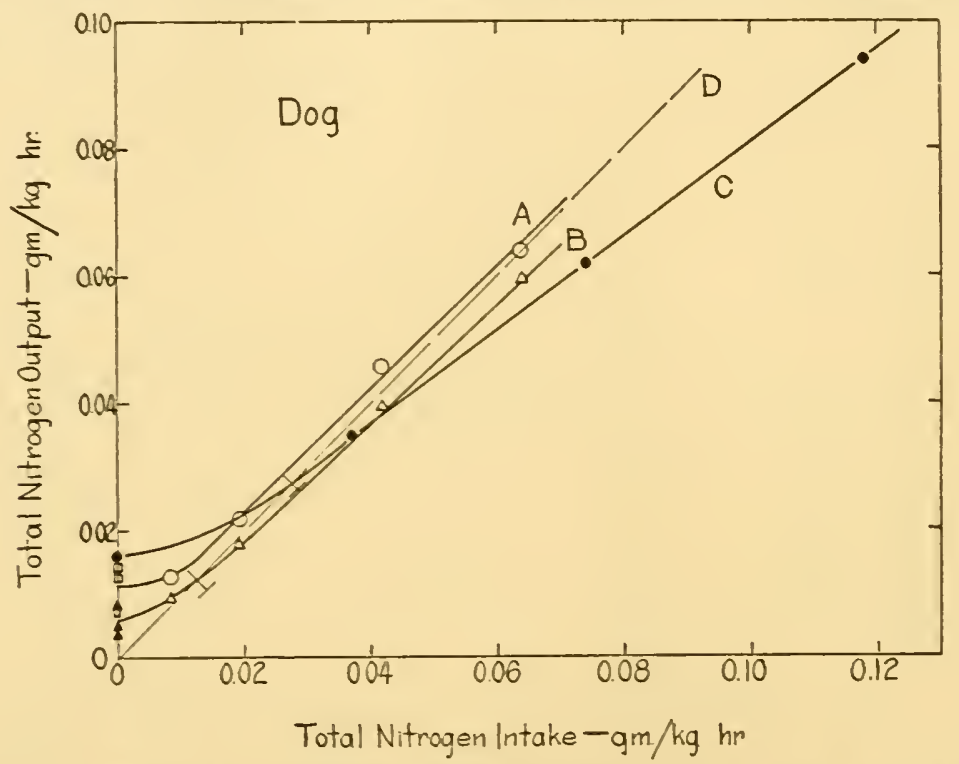

FIG. 161. Rate of total nitrogen output in relation to rate of total nitrogen intake. Points of nitrogen balance, indicated by 1 , fall on line $\mathrm{D}$, equality. Solid circles (curve C) dog II of Rubner ('02), cf. figure 159, fed meat onlr. Squares and solid triangles, 3 dogs of Michaud ('09). Open circles and triangles, dog of Toit and Korkunoff (1595); curre B (triangles) indicate exchanges when fat and carbohrdrate supplements are giren in large amounts, the difference from curre A (circles) being "protein sparing." Periods rary from 1 to 14 dars at direrse points.

Sereral ordinary criteria of balance may be satisfied at any of many nitrogen contents, for approximate equality of intake with output occurs in a great range of contents. Hence the emphasis is placed on the minimal nitrogen turnover, the "practical or physiological minimum." This is distinct from output in deficits of nitrogen ("experimental minimum"). Actually nitrogen intake is much more variable than water intake; the range of approximate balances that can be forced to occur for water, do frequently occur for nitrogen. In dogs there appear to be no measurements of rolun- 
tary protein intake independent of total intake, and in most practical situations there is little opportunity for an animal to select nitrogen intake independently of total energ. intake.

With all this in mind, it is scarcely worthwhile to believe that present data rield more than a sketch (fig. 162) of a critically derived equilibration diagram for nitrogen.

The fact has been established that certain particular substances are selected for ingestion by rats when these substances are depleted. Such substances are water (Richter, "36) and ritamin B (Richter et al., '3ib, "33b). The increased "demand" for calcium following parathroidectomy (Richter and Echert, "3ia) is mani-

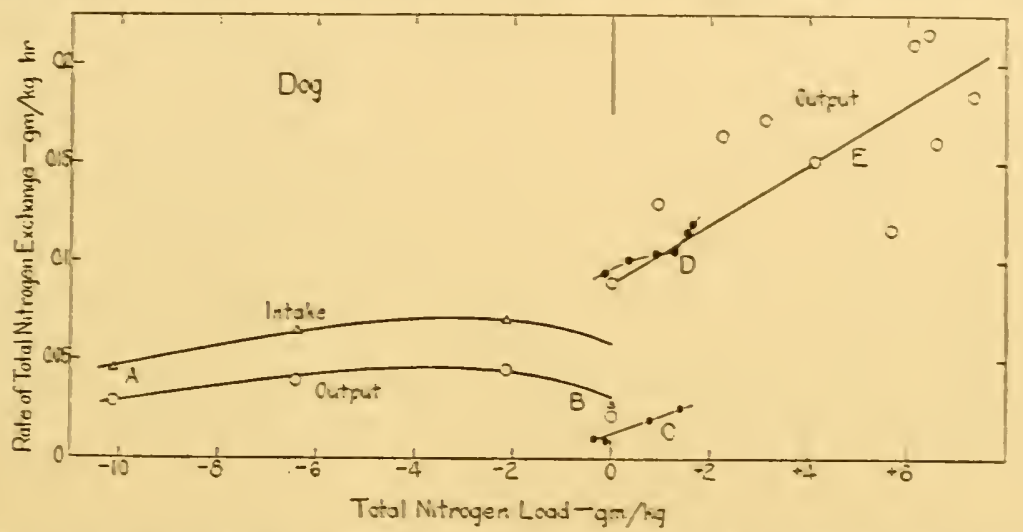

FIG. 162. Rate of total nitrogen exebange in relation to total nitrogen load. Ni. trogen equilibration diagram. Dog. Only the quantity of whole diet was raried in each indiridual, not the constitutents of the food. which differed in $A, B$ from those in $D, E$. A, four weeks of recorery following partial priration of an adequately constituted diet for nine weeks, indiridual of $13.94 \mathrm{~kg}$. of Morgulis ('23, p. 2606). B, prerious balance of same individual. C, output in dail periods of no food intake, indiridual of $5.86 \mathrm{~kg}$. of Rubner ( 02, p. $2 \div 6$ ). D, output in suceessire dars of excessire food intake, same indiridual. $E$, output in suceessire days of excessire food intake, dog III of $1.1 \mathrm{~kg}$. of Rubner ( 02 , p. 113).

fested by 3 to 6 fold increases in calcium lactate ingestion. Sodium chloride is taken 6 times as rapidly as usual after adrenalectom? (Richter and Eckert, :3Sa) and during pregnancy (Barelare and Richter, '38). Specific "appetites" of this sort have hardly been measured in other species. Although in everydar life the animal can do little to unmix its foods, upon the specific appetites appears to depend the maintenance of particular chemical constituents of every indiridual.

Excesses of food lead to faster recoreries of some constituents 
of it and slower of others. Hence recorery of total substance depends on what particular materials are ingested. In a mixture, water is eliminated most rapidly when in a certain excess relatire to other materials; nitrogen next, chloride next, and carbon last. Other substances could be intercalated in this list. Each component has a relocity quotient characteristic of it, and these quotients may be investigated either in ingestions of more or less pure components, or in mixtures. In general, also, direrse quotients are found for one component with each mixture, and this is a matter for further study.

It is a plain fact that every organism tends to have a constant amount of material in it. In some protozoa accession of material leads to reproduction of more indiriduals (Adolph, '31a), restoring each to a size near the mode. In mammals, accession leads to faster disposal, at rates differing for each constituent (energr, carbon, nitrogen, water). Depriration of material leads to extraordinary efforts to obtain it, and especially to obtain the rery constituents that are lacking. Whereas the buman individual sometimes relies upon the bath-room scales to guide his rate of intake, organisms generally temper their exchanges so as to maintain a uniform bods weight without any visible measuring derice.

\section{\$119. GLUcose IN DOG}

One of the first components for which a maintenance of constancy was realized to exist (Bernard, 1855; Chaureau, 1856) was sugar. Deprivation of carbohydrate did not much decrease the blood's concentration of sugar; feeding nothing but sugar, even infusing a solution of it, did not much increase it. The identification of glycogen as one of the forms of storage for carbohrdrate led to the dim realization that reversible equilibria buffer the changes in actual glucose content of the body. From this prototype of reactions through which excesses and deficits tend to be absorbed, has grown the knowledge of diverse paths of disposal for sugars, as well as for innumerable other components both chemical and phrsical.

To obtain quantitative data upon unanesthetized dog, two types of glucose increment are used; excesses are produced by continuous intravenous infusion of glucose, deficits by previous intravenous injection of insulin. The glucose content of the body as a whole is computed on the assumption that the rolume of distribution of glu- 
cose is $50 \%$ of $\mathrm{B}_{0}$ (Wierzuchowski, '36) under all the conditions measured. Equally informing, and perhaps more direct, would be to correlate the concentration of reducing substances found in whole blood with the rate of its change; then, however, the partition of disposals would require further assumptions before common dimensions would be shared.

Glucose was infused at constant rates, fire of which are represented in figure 163. At the smaller rates, loss equals gain after

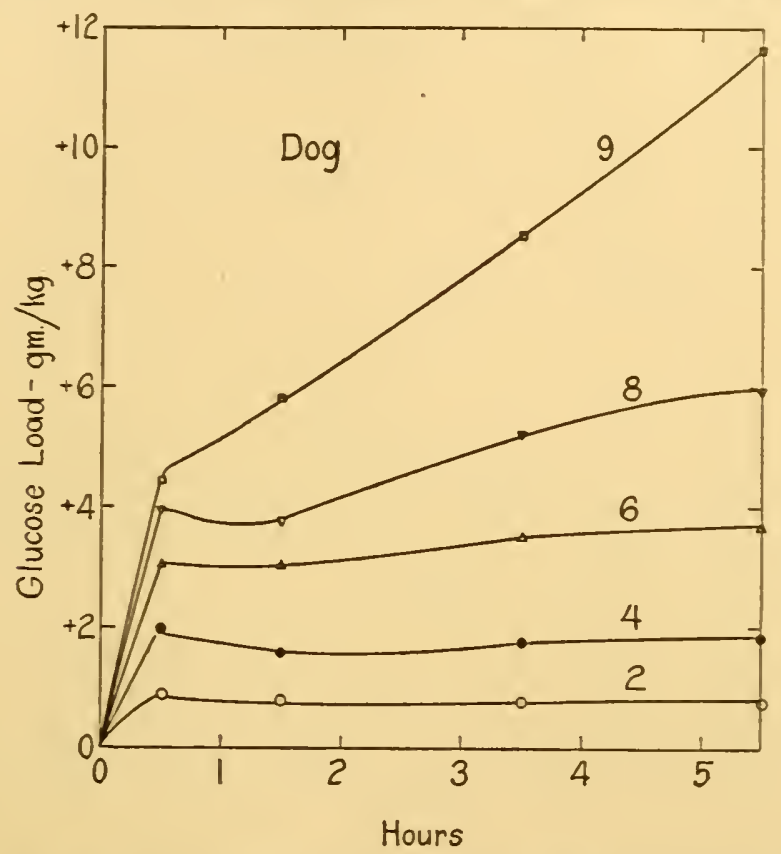

FIG. 163. Glucose load (increment in gm. of glucose/kilogram of $\mathrm{B}_{0}$ ) in relation to time. Glucose was infused intrarenously for 6 hours at constant rates that are indicated (in $\mathrm{gm} . / \mathrm{kg}$. hr.) by numerals on each curre. Loads are computed from blood concentrations assuming a uniform rolume of distribution of $50 \%$ of $\mathrm{B}_{0}$. Data of Wierzuchomski ('36, p. 322).

the first hour, so that loads are stationary. But at higher rates glucose accumulates. The rate of total loss (disposal) of glucose remains steady after the first hour (fig. 161); but at infusion rates of 8 and $9 \mathrm{gm} . / \mathrm{kg}$. hr. the rate of loss does not equal the rate of gain. The loss is partly in urine (fig. 165), though almost none is so lost at the lower rates of administration.

When rates of loss are correlated with loads (fig. 166), it is evident that output attains a limiting rate when $8 \mathrm{gm} . / \mathrm{kg}$. hr. are 


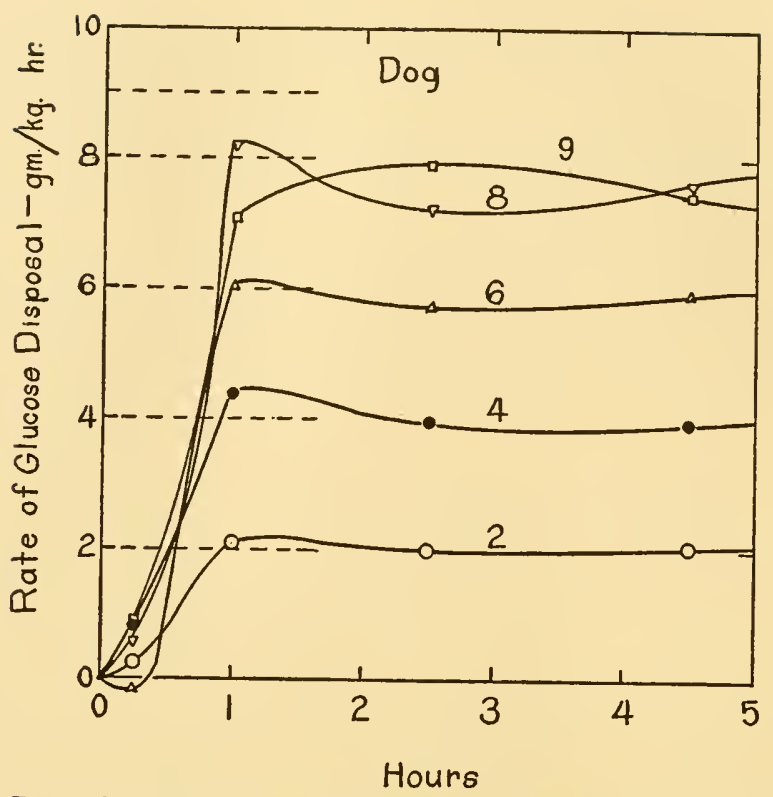

Fig. 164. Rate of glucose disposal in relation to time. Infusions continued during 6 hours at rates that are indicated by the dotted lines and by the numerals, in $\mathrm{gm} . / \mathrm{kg}$. hr. Rates are inferred from changes of blood sugar concentrations during periods of $\frac{1}{2}$ to 2 hours, each rate being indicated at the middle of the period. Same data of Wierzuchowski ('36, p. 322) as in figure 163.

infused. This corresponds to a load of $7 \mathrm{gm} . / \mathrm{kg}$., and at lower loads the rate of loss is nearly proportional to load.

In excesses, three paths of disposal are measured: amounts excreted, amounts oxidized, and amounts disappearing but not ex-

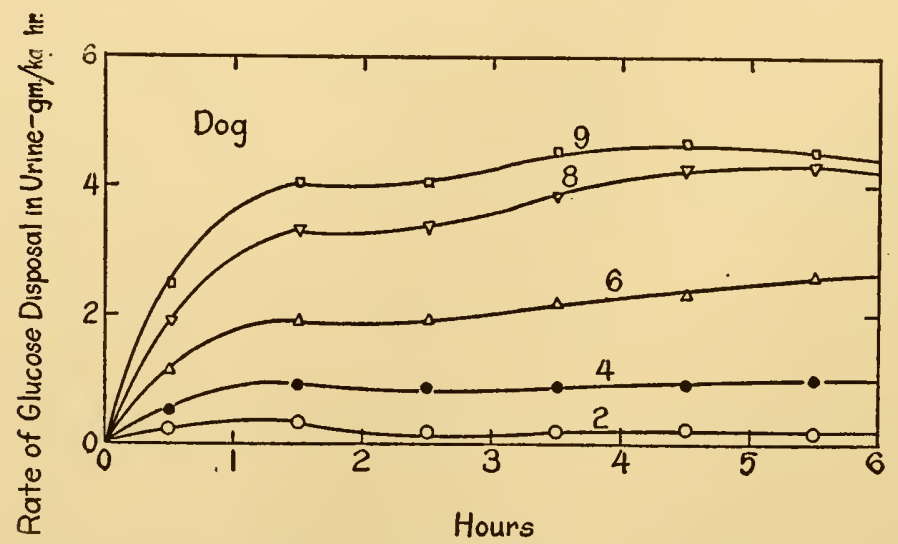

Fig. 165. Rate of excretion of glucose in urine, in relation to time. Glucose was infused continuously at rates indicated by numerals, in $\mathrm{gm} . / \mathrm{kg}$. hr. Data of Wierzuchowski ('36, p. 314). 
creted or oxidized (fig. 166). The proportions represented by these paths differ enormously at various glucose increments, representing quantitatively facts that are loosely known. At small increments practically all glucose is transformed, presumably to other forms of carbohydrate. While the rates of synthesis and oxidation are limited to about $3.6 \mathrm{gm} . / \mathrm{kg}$. hr., excretion is not limited but augments in proportion to the glucose load. One might envisage difficult situations for the organism if (a) there were no excretion of glucose, (b) there were excretion of glucose at small loads (= no threshold), or (c) combustion of the extra sugar were not carried

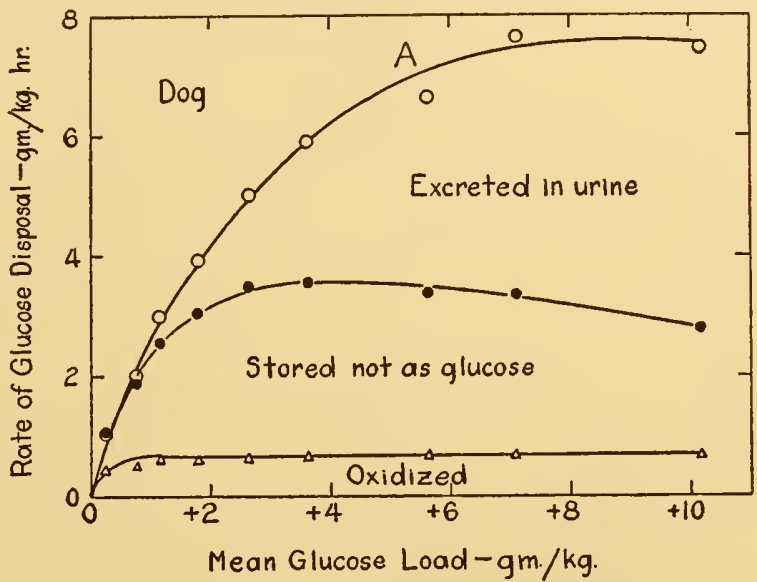

FIG. 166. Rate of net glucose disposal, and its partition, in relation to mean glucose load in approximately steady states of glucose excess. Dog. Glucose was injected intravenously at constant rates during 6 hours. The last 3 hours were taken in each test, 29 or 48 tests being included in the 9 means. Glucose load was computed from blood sugar concentrations and $a V_{D}$ of $50 \%$ of $B_{0}$. Hence an increment of $1 \mathrm{gm} . / \mathrm{kg}$. or $0.1 \%$ of $\mathrm{B}_{0}=2 \mathrm{gm}$./liter of whole blood; and since the initial content was 0.97 $\mathrm{gm}$. $/$ liter of blood, $1 \mathrm{gm} . / \mathrm{kg}$. or 5.55 millimols $/ \mathrm{kg} .=206 \mathrm{per}$ cent of the control content $\left(\mathrm{G}_{0}\right)$. Data of Wierzuchowski ('36, pp. 314, 322, 327; '37b, p. 153).

out. As it is, there are arrangements for meeting exceedingly large influxes of glucose, and it has often been remarked that the organism has reserve means of meeting situations so extreme that they may have never occurred in non-experimental situations. How extreme these are, may be realized from the statement (Trimble et al., '33) that glucose is ordinarily absorbed from the alimentary tract at a rate of only $1 \mathrm{gm} . / \mathrm{kg}$. hr. In Wierzuchowski's experiment, the dog speeds up its rates of disposal of glucose even to the point where excretion of it is faster than removal of it by synthesis.

Total exchanges and turnovers are subject to small uncertain- 
ties as to whether all the oxygen apportioned to carbohydrate represents glucose burned. In addition, whatever the portion of glucose that passes to carbon dioxide, much more glucose may be continuously formed and transformed in many processes of intermediary metabolism, and the turnover of glucose recognized for the whole body may be but a small part of the sums of all the local formations and transformations of this chemical compound. The dog's turnover has been estimated at $0.25 \mathrm{gm} . / \mathrm{kg}$. hr. (Reid, '36), and is the same in depancreatized individuals.

Glucose content, at least of the blood, is less variable than contents of many other components (table 38). According to data of

TABLE 38

Sugar concentrations in blood under standard conditions. Each observation was made on a separate day

\begin{tabular}{|c|c|c|c|c|c|}
\hline Species & $\begin{array}{l}\text { Number of } \\
\text { individuals }\end{array}$ & $\begin{array}{l}\text { Number of } \\
\text { observations }\end{array}$ & $\begin{array}{l}\text { Mean, } \\
\text { gm./1. }\end{array}$ & C.V., & Source of data \\
\hline Dog & 4 & 48 & 0.97 & 6.2 & Wierzuchowski ('37b) \\
\hline Man & $\begin{array}{r}1 \\
23 \\
23 \\
141\end{array}$ & $\begin{array}{l}44 \\
5 \text { each } \\
23 \\
141\end{array}$ & $\begin{array}{l}0.94 \\
0.94 \\
0.94 \\
0.97\end{array}$ & $\begin{array}{l}4.6 \\
4.7 \\
6.4 \\
7.2\end{array}$ & $\begin{array}{l}\text { Okey and Robb ('25) } \\
\text { "6 } \\
\text { Pierce and Scott ('28) }\end{array}$ \\
\hline Rabbit & $\begin{array}{r}100 \\
27 \\
. \\
-\ldots . . .\end{array}$ & $\begin{array}{r}157 \\
100 \\
85 \\
1000\end{array}$ & $\begin{array}{l}1.16 \\
1.05 \\
1.18 \\
1.24\end{array}$ & $\begin{array}{l}10.5 \\
16.1 \\
18.2 \\
11.5\end{array}$ & $\begin{array}{l}\text { Eadie ('23) } \\
\text { Clough et al. ('23) } \\
\text { Scott and Ford ('23) } \\
\text { Scott ('27) }\end{array}$ \\
\hline
\end{tabular}

Wierzuchowski ('37b) almost no variations are detectable in a dog at hourly intervals, nor even at the close of a day of loading and unloading of glucose. Adrenalectomy does not increase the variation of blood sugar concentration from day to day (data of Zucker and Berg, '37). Among species, the rabbit (table 38) in contrast to dog and man, shows higher content and greater variability. That contrast represents a difference in maintenance; yet heretofore rabbit and dog have been studied indiscriminately.

The data yield a glucose-time system of four variables $(\Delta \mathrm{C}$, $\delta \mathrm{C} / \Delta \mathrm{t}, \mathrm{t}, 1 / \Delta \mathrm{t})$. At diverse loads and rates of loading, characteristic latent periods, times to maximal rates, and initial rates of disappearance are found (figs. 163 and 164), all of which are comparable to the temporal characteristics of the dog's water-time system with continued administrations $(\$ 12)$. The velocity quotients $(1 / \Delta t)$ for the total glucose exchange (fig. 167), are more constant with time in slow infusions than in rapid. Evidently after the first 


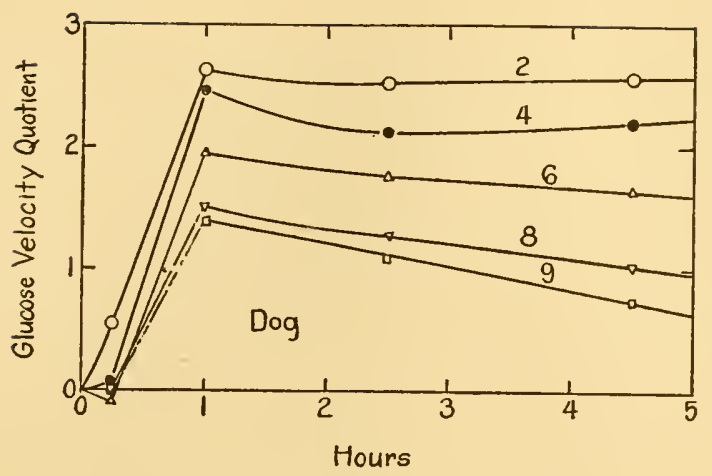

FIG. 167. Glucose velocity quotient (1/hour), in relation to time after continuous infusion of glucose began, at rates indicated by numerals (in $\mathrm{gm} . / \mathrm{kg}$. hr.). Data of Wierzuchowski ('36), from figures 163 and 164.

hour the state is a steady one (fig. 168), the velocity quotient for total glucose disposal is independent of time; and this quotient diminishes progressively with load to a much greater extent than the velocity quotient for water disposal.

While rates of excretion of glucose increase with loads (fig. 166), the rates of synthesis and of oxidation are independent of loads above $2 \mathrm{gm} . / \mathrm{kg}$. of body weight. The patterns of these paths stand in sharp contrast; for here are two paths for disposal (rate of synthesis, rate of oxidation) that do not vary with load, while another (renal excretion) is mainly dependent on it. The latter is such that clearance from blood, and velocity quotient, are approxi-

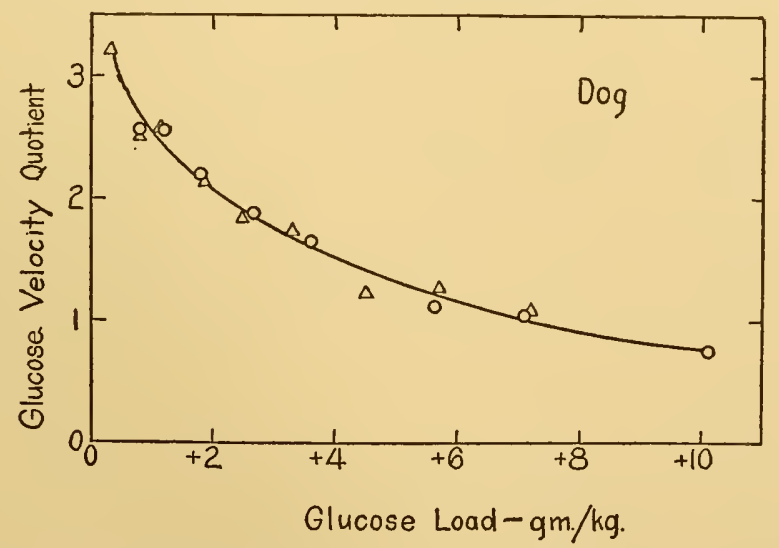

FIG. 168. Glucose velocity quotient (1/hour) for net disposal, in relation to glucose load. Dog. The triangles represent the period 1.5 to 3.5 hours, and the circles 3.5 to 5.5 hours after continuous glucose infusion began. Same data of Wierzuchowski ('36). 
mately constant at all loads above $1 \mathrm{gm} . / \mathrm{kg}$. That load corresponds to what was once termed the "assimilation limit."

Some parallel data have been obtained by the entirely independent method of analyzing total carbohydrate in the body (fig. 169). Many points of technical difference may be noted between this set of tests and the previous ones; this set nevertheless indicates the slowness of the exchanges of total carbohydrate, especially with excretion blocked, as compared with those of glucose alone. Removal of pancreas does not limit the "ability" to utilize carbohydrate, but limits those high rates of utilization to greater loads.

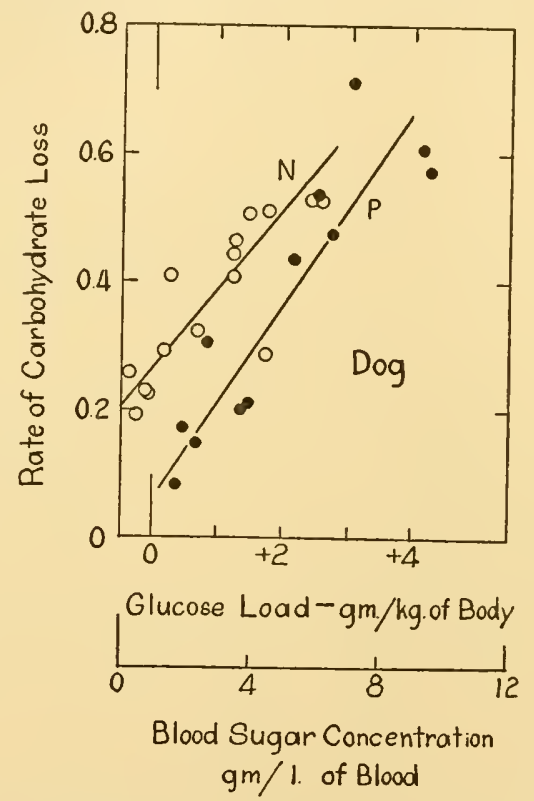

Fig. 169. Rate of earbohydrate loss (in gm./kg. hr.) in relation to mean glueose load. Dog under nembutal anesthesia after "evisceration," and privation of food for 3 previous days. Glucose was infused by vein continuously for 2 hours before the test began and during 4 hours in which the earbohydrate disposal was being measured. Each point represents one individual, samples of whose tissues were analyzed before and after the 4-hour period. Note that ordinate scale is ten times that of figure 166. Here the loss is probably all by oxidation. $\mathrm{N}$, control; $\mathrm{P}$, pancreas also removed. Data of Soskin and Levine ('37).

Deficits of glucose are induced by injections of insulin (fig. 170). Thereafter glucose is gained (net) and more slowly as zero load is approached. Absence of medullary portions of the adrenal glands interferes but little with the course of recovery from deficits of glucose. The slower exchange after high doses of insulin may be accredited to persisting action of this agent in tending to remove 
glucose. Possibly the curves would lose their convexity if the persisting action were discounted.

Taken together, figures 166, A, and 170, A, constitute a diagram of net equilibration. But the scales of the latter figure must be reduced to one-twentieth their present size in order to match those of the former figure. The slopes near zero load are about equal in the two curves, meaning that small excesses and small deficits are adjusted with equal speeds. Only, the range of deficits that is tolerated (and chemically possible) is very small compared with the range of excesses.

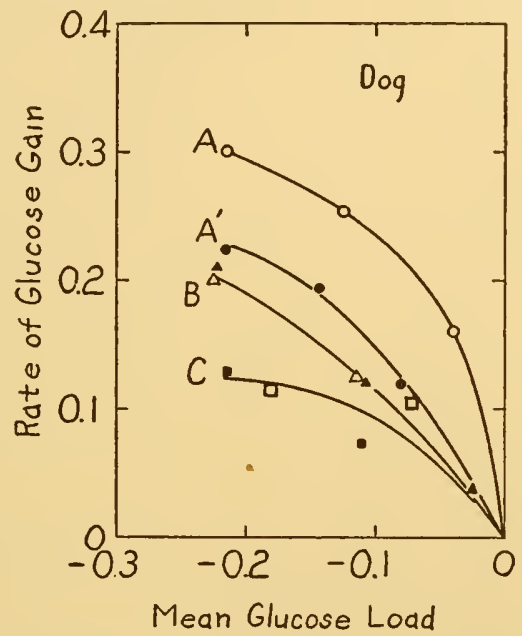

FIG. 170. Rate of net glucose gain (gm./kg. hr.) in relation to mean glucose load (gm. $/ \mathrm{kg}$. ), as found in the blood of dogs previously subjected to intravenous injections of insulin. A and $\mathrm{A}^{\prime}, 17$ and 12 tests with 0.1 unit insulin $/ \mathrm{kg} . \mathrm{B}, 5$ and 8 tests with 0.5 unit/kg. C, 11 and 15 tests with 1.0 unit $/ \mathrm{kg}$. Solid points, bilaterally medullectomized; open points, unoperated. Data of Zucker and Berg ('37, p. 541).

If total exchanges of glucose were estimated instead of net exchanges, the ordinate at zero load would be increased by 0.25 gm./kg. hr. It is unknown how total glucose gain varies in positive loads, and total loss varies in negative loads. Whereas water, heat, nitrogen, and carbon are probably lost only by modifications of their rates of elimination from the dog's body, the specific component glucose is disposed of by chemical transformation, a process which also makes its total loss difficult to measure. Similarly, in deficits the rates of net glucose gain reported might barely result from diminutions of total loss without any increases of total gain.

Had the blood been chosen for study in place of the whole body, 
rates of recovery in steady states of excess of blood glucose might be correlated, complete data for which are available. From other sources, recoveries after single administrations would be represented by a series of "glucose tolerance"' tests. For them, a known amount of glucose is suddenly injected into the circulating blood, after which the blood's concentration of sugar is measured at successive times. Such tests in dogs show that (1) disappearance of glucose is absolutely faster with larger doses, (2) disappearance rates diminish with time and with concomitant decrease of increment, (3) disappearance rates are modified by removal of liver, pancreas, or adrenals, by anesthesia, insulin, phlorizin, renal ligation, and numerous other factors (Goldstein et al., '32).

Many devices have been used to compare glucose tolerance curves. Rates of recession, slopes of return, hyper-glycemic areas, peak effects, and terminal levels, are all of service to the graphically minded. "Insulin sensitivity curves" may equally be termed tolerance curves in glucose deficits, to indicate that they differ in component and sign from tolerance curves for other substances. Finding now that "tolerance tests" are particular instances of recoveries, all the rate and time factors and ratios found useful for water ( $\$ 105)$ may be applied to glucose.

Glucose, therefore, exhibits net equilibration of content both in stationary states and temporary states. Excesses are dissipated by several paths, and the proportion lost by each path varies with the load. Deficits and excesses of equal magnitude are returned at about the same rates. Promptness of recovery as exhibited in tolerance curves in positive and negative loads is matched by the smallness of variabilities of content found in single individuals and in populations.

\section{$\$ 120$. Carbon Dioxide in maN}

Carbon dioxide (plus bicarbonate, etc.) may be thought of as a constituent of the body as a whole. Exchanges of carbon dioxide in mammals represent chiefly losses by elimination through lung alveoli, competing with gains by internal production. Increments in the carbon dioxide content of the body are roughly estimated from the extra carbon dioxide that has been eliminated (a) in forced breathing and (b) in recovery from equilibrium with high tensions of inspired $\mathrm{CO}_{2}$. It is found that 0.2 hour or more is required in man to attain a new stationary content of this substance, the net retention or elimination approaching some asymptote. 
The equilibration diagram for carbon dioxide (fig. 171) shows enormous responses to increased contents. Neither in increased nor in decreased contents is there a known change in rate of internal (chemical) production. Recovery from deficit is limited to suppression of loss alone. This fact was described by Haldane and Poulton ('08); after forced breathing, apnea persists until the

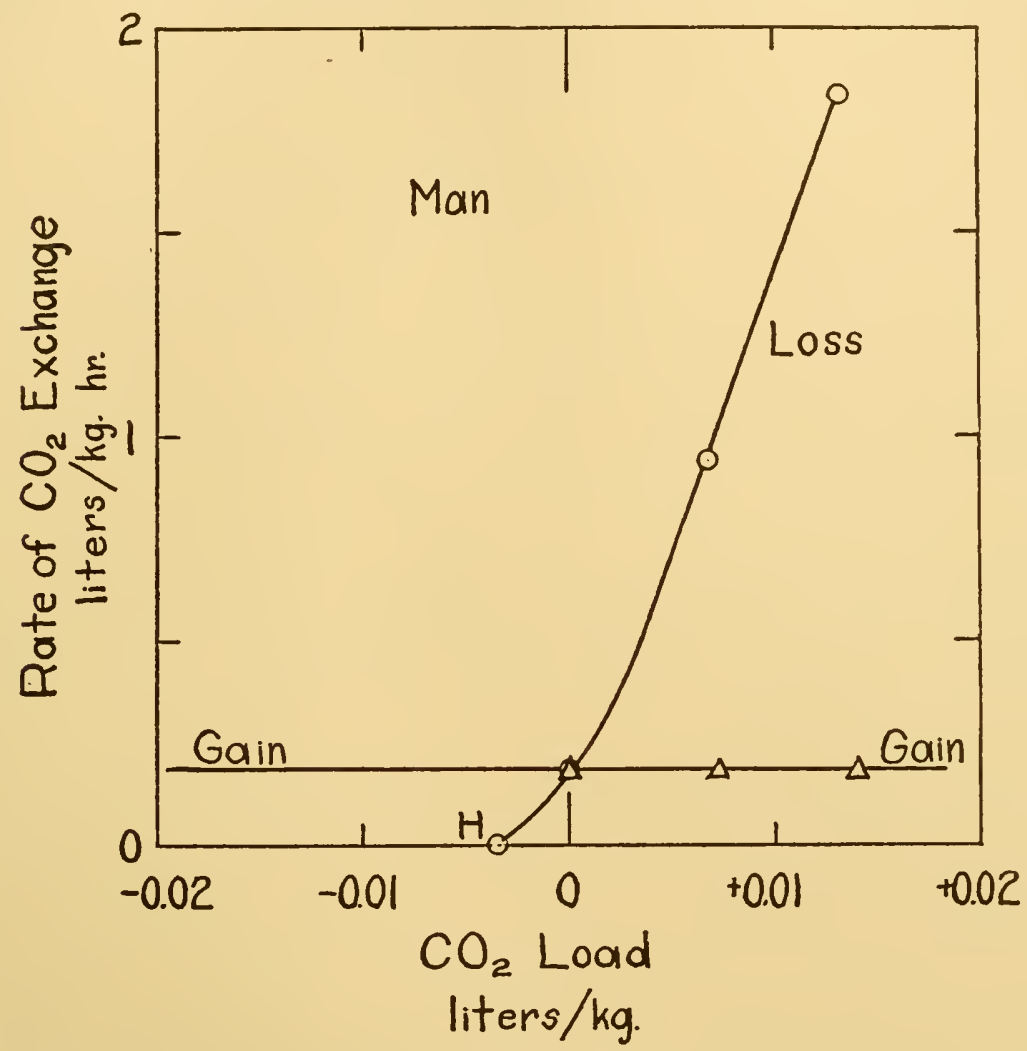

Frg. 171. Rate of carbon dioxide exchange in relation to load of carbon dioxide. Man in steady states of load. Excesses were produced by inspiring uniform mixtures of carbon dioxide and air; deficits by overbreathing for periods of time during which alveolar and expired airs were collected. Gain is by metabolic production; loss is by expired air. Loads are in liters of $\mathrm{CO}_{2} / \mathrm{kg}$. of $\mathrm{B}_{0}$; rates in liters of $\mathrm{CO}_{2} / \mathrm{kg}$. hr. Data in negative loads are from Liljestrand ('16); in positive loads from Campbell et al. ('14); point $\mathrm{H}$ from Haldane and Poulton ('08).

mean alveolar tension of carbon dioxide is slightly less than the usual (the apnea point of Nielsen, '36).

To estimate the content of carbon dioxide in the body, reliance may be placed in chemical analyses of cat and dog (Irving et al., '32). Roughly 1.06 liters $/ \mathrm{kg}$. are found at balance, and loads 
might be computed relative to that. A large part of the body's total carbon dioxide is fixed in bones; this portion is not measurably touched when increments of \pm 75 per cent of the usual alveolar tension of carbon dioxide are experimentally imposed (Irving). If the remainder of the body's carbon dioxide rapidly interchanges with blood, the volume of distribution of increments is roughly $70 \%$ of $\mathrm{B}_{0}$; this value is also that found for the distribution of added bicarbonate (Palmer and Van Slyke, '17). In anesthetized cats breathing mixtures of air and 7.5 per cent carbon dioxide, Shaw ('26) found $0.0017 \mathrm{l} . / \mathrm{kg}$. of whole body retained for $1 \mathrm{~mm}$. $\Delta \mathrm{p}_{\mathrm{CO}_{2}}$ in blood, while blood alone had an increment of 0.00331 ./liter (or $\mathrm{V}_{\mathrm{D}}=52 \%$ of $\mathrm{B}_{0}$ ).

Returning to man, I believe that in the range of carbon dioxide contents near balance, the dissociation of carbon dioxide in the body is proportional to that of blood (in other words, that the volume of distribution of increments is constant), and that an increment of $1 \mathrm{~mm}$. of mercury tension of blood carbon dioxide $\left(\Delta \mathrm{p}_{\mathrm{CO}_{2}}\right)$ equals an increment of 0.0031 liter $/ \mathrm{kg}$. of body ( 0.7 times an increment of 0.0045 l./liter of blood), or about 0.3 per cent of all the carbon dioxide present. The value 0.0031 resembles the 0.0021 to 0.0028 1./kg. for each mm. $\Delta \mathrm{p}_{\mathrm{CO}_{2}}$ derived by Liljestrand ('16) and from selected data of Adolph et al. ('29) and of Nielsen ('36); it is the value used in figure 171.

Should it turn out that much uncertainty is introduced into figure 171 by Liljestrand's assumptions (a) that carbon dioxide content of the body is proportional to change in alveolar tension of carbon dioxide, and (b) that production of carbon dioxide in tissues is independent of load, other coordinates could be substituted. Instead of the whole body, the blood alone may have its contents of free or of total carbon dioxide ascertained, and the net rates of exchange may be computed from serial analyses of samples from circulating blood. Instead of carbon dioxide output, rates of total ventilation or of alveolar ventilation may be correlated with carbon dioxide tension in alveoli (as Campbell et al., '14, and others, have already done). It is well known that under chosen conditions the rate of carbon dioxide elimination is precisely proportional to (alveolar) ventilation rate (Douglas and Priestley, '24). In other words, the concentration of carbon dioxide in expired air or in air drawn from alveoli is uniform. But the high correlation between ventilation rate and carbon dioxide tension does not, as always, exclude many other factors from being related to ventilation rate. 
The enormous change in rate of carbon dioxide output through the lungs with each small change in load or tension of carbon dioxide, is the feature that led Haldane ('17) to emphasize this component as the example par excellence of a regulated quantity. Its constancy was evident, once alveolar air sampling and analysis were perfected; indeed, qualitative tests alone are sufficient to demonstrate the responses to change of $\mathrm{p}_{\mathrm{CO}_{2}}$. By relating now the rates of elimination and of production to the loads of $\mathrm{CO}_{2}$ in the body, I can compare the sensitivity of this regulation (as measured by net velocity quotient) with corresponding sensitivities to other components $(\$ 136)$. The believed insensitivity to carbon dioxide exhibited by diving mammals and very many other living units, may eventually be compared by means of the same parameters.

\section{\$121. OXYGEN IN MAN}

Oxygen, too, is easier to discuss in qualitative terms than quantitative. Excesses of oxygen are difficult to impose beyond the lung's, but deficits occur both in deprivation and in increased oxygen utilization. At the conclusion of a period of oxygen deprivation, oxygen intake is measured, indicating how rapidly net oxygen is added to the body in the first few seconds of recovery. Without physical exercise the rate of oxygen consumption may increase tenfold, as in dinitrophenol administration to dogs (Hall et al., '33). It is believed that small deficits of oxygen then prevail and induce the augmented intake.

As oxygen is consumed during recovery, the oxygen deficit or debt is paid off at initial rates that are indicated in figure 172. The rate of gain of oxygen is temporarily very large, while the rate of recognized combustion of it is immediately smaller than during the exercise. Thereby, the net oxygen content of the body is restored.

Rate of oxygen intake is related to oxygen load, but not independently of time (fig. 172). Or, deceleration of oxygen intake following exercise is correlated with time as well as with deficit or debt. No guarantee exists at present that other types of oxygen load are related with oxygen exchanges like the one here described.

The oxygen content of the human body in oxygen balance is variously estimated (exclusive of the lungs) at 0.7 to 1.5 liters or 0.01 to 0.02 liters $/ \mathrm{kg}$. (Irving, '34). This is based on the belief that very little molecular oxygen exists outside of that combined with hemoglobin. Such a quantity of oxygen is sufficient to carry on usual combustions for only 3 to 6 minutes at rest. 
It is apparent that oxygen deficits, computed in this way, easily exceed the actual oxygen contained in the body. Or, to restore the body to oxygen balance, 10 or 20 times its resting content may be required. This is an instance of a "subzero" increment. Such is to be expected in instances where the component measured can be substituted by or manufactured from some other components. It might have been found for glucose if the total glucose that transformed to something else had been measured following insulin injection, instead of the net deficit of glucose. Or, if glucose deficit

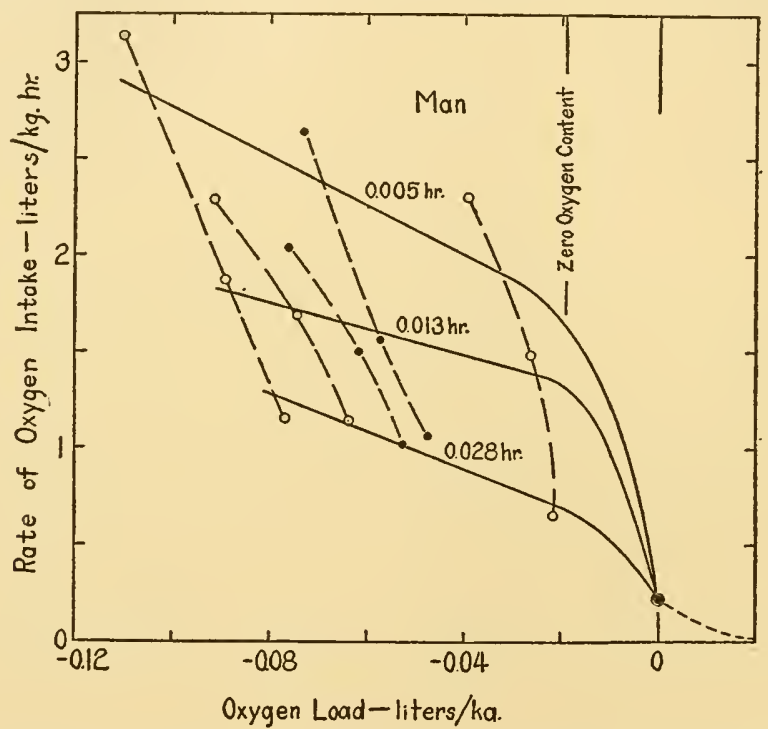

FIG. 172. Rate of oxygen intake (absorption) in relation to oxygen load. Rates were measured in periods of 0.008 to 0.010 hour following violent physical exercise, 3 successive periods being measured in each of 5 tests on 2 subjects. Rates are in liters of oxygen (at NTP)/kilogram of $B_{0}$ and hour; loads (debts) in liters of oxygen/kilogram. Data of Hill and Lupton ('23, p. 146) and Hill, Long and Lupton ('24, p. $467)$.

is accompanied by increased metabolism of other substances, then the restoration of each of them to its initial state might be part of the glucose recovery, and all the restoration may be prerequisite to the complete disappearance of the glucose deficit.

Surprisingly little excess of oxygen is ever contained in the human body, even when in a chamber of compressed oxygen. Since the blood absorbs practically no more oxygen than when in ordinary air, great excesses are automatically prevented (except in the air in the lungs). Whatever small excesses do occur, as when oxy- 
gen is breathed instead of air, or oxygen gas is injected, certainly are quickly lost by utilization almost in situ.

Tolerance curves for rate of oxygen intake, and recovery of rate of oxygen intake, are quantitatively represented in many data: e.g., Margaria et al. ('33, '34), Hansen ('34), Herxheimer ('35), 1ljin-Kakujeff ('36, see fig. 176 below), and Szwejkowska ('38).

It may be noted that in the present investigation, the terms deficit and debt are used interchangeably. Whether the deficit as computed from the oxyg'en intake during acceleration subtracted from the oxygen intake after acceleration has ceased, is the same as the deficit (debt) as computed from the oxygen intake during deceleration minus the oxygen intake after deceleration, is of no consequence in the relations here discussed. That question becomes of consequence when someone is concerned whether chemical combustions are permanently suppressed when the body economizes under scarcity of oxygen.

If some other correlative of oxygen content is substituted for it, as arterial oxygen concentration, or inspired air tension of oxygen, the oxygen intakes also may be correlated with steady states of deficit. Thus, for instance, a relation is established between ventilation rate and oxygen load; this correlation is of interest in that ventilation rates increase much only in those oxygen tensions less than half of the usual ones in atmospheric air.

Comparison of figures 171 and 172 indicates the partially reciprocal relations of carbon dioxide and oxygen. The same breathing that brings oxygen into the lungs also bails out carbon dioxide. But more oxygen in the lungs does not add significantly to the oxygen content of the body as a whole. For carbon dioxide, on the other hand, the ventilation of the Iungs itself looks almost like a limiting process to its content. Oxygen and carbon dioxide are each able to arouse more alveolar ventilation, but only in ranges of tensions or contents that usually do not occur together. Further, heat excesses, increased breathing (panting) modifies but little the content of either. Adjustments of oxygen load are prevented from interfering with adjustments of carbon dioxide content by the agency of hemoglobin in blood, and panting is harmless as long as it refills dead space. There was a time when no one predicted either that excess oxygen would arouse no response of breathing or that carbon dioxide deficit would be compensated. The relative influence upon alveolar ventilation of equal increments of tension in 
oxygen and in carbon dioxide, over diverse ranges of tension, had to be found by measurement. If community of path were a complete epitome of physiological study of outputs, the individuality of eliminations of each component would be ignored. Instead, it is now observed that means are available of adjusting several single components without interfering beyond a limited small amount with other components that exchange through the same path.

In brief, the maintenance of oxygen tensions in diverse tissues of the human body is the equivalent of keeping the body's oxygen content constant. Deficits of a few milligrams of oxygen do not persist when recovery is possible, and payment of oxygen debt is a process rivalled in velocity of exchange of substance only by removal of carbon dioxide excess. Paths of exchange for both are pulmonary, as though this path is capable of faster exchanges (relative to load) than any other. For oxygen the faster recovery is in deficits, for carbon dioxide the faster recovery is in excesses; and much may be inferred concerning the appropriateness of each of those modifications.

\section{\$122. Lactate In MaN}

Deficit of oxygen is at the present day considered to be in close connection with excess of lactate. By no means all types of oxygen shortage are accompanied by measurable lactate accumulation, and the relations found in physical exercise might equally be considered an exceptional type of oxygen deficit, rather than the rule.

Lactate may be injected by vein, and the recovery thereafter may be followed by the usual technique of tolerance curves (fig. 173). In man no measurements of lactate content of the whole body at the several times appear feasible; hence the procedure (already used in glucose equilibration) is followed of ascertaining the concentrations of lactate in whole blood. In the recovery almost no lactate is excreted; and one-fifth or less of the amount administered is represented by extra consumption of oxygen. The remainder seems to be transformed into other sorts of carbohydrate.

What the relation may be, at various times during recovery, between concentration of lactate in blood and content of lactate in the body as a whole, is open to surmise. One guess is (Margaria et al., '35) that if equilibrium were established, the volume of distribution would be constant at $90 \%$ of $\mathrm{B}_{0}$. Figure 173 does not support this estimate in that type of load. 
The concentration in the blood being taken as such, whatever its relation to that in the whole body, I relate the rate of disappearance to load (fig. 174). In the first half-hour, the disposal seems to be nearly proportional to the mean concentration present. The ratio of net rate to load (velocity quotient) that prevails in recovery from autogenous lactate of physical exercise (see also Newman et al., '37), has the same value as in recovery from injected lactate.

In each unit of time during recovery, the amount of lactate dissipated may be compared with the amount of oxygen consumed

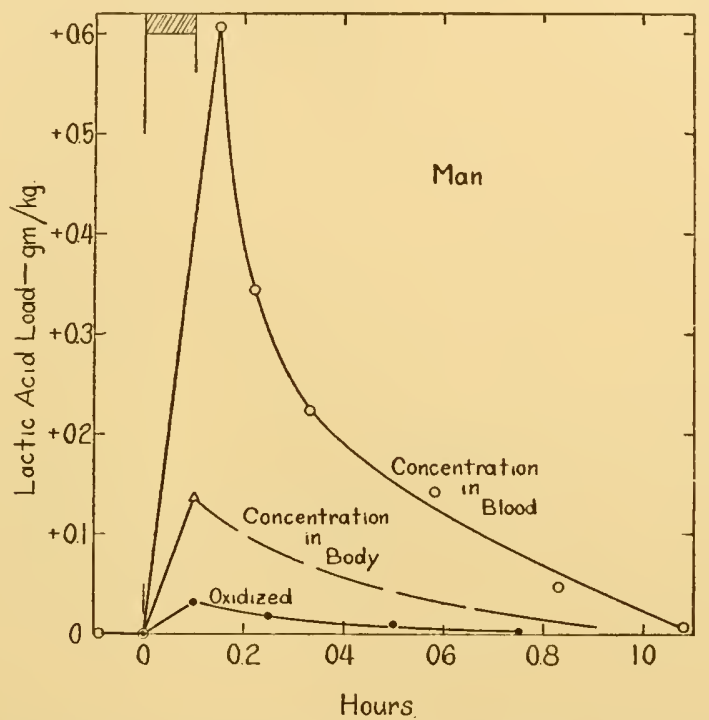

FIG. 173. Lactic acid load in relation to time. Man. Sodium lactate is injected by vein during the initial 0.1 hour. The excess lactic acid present in the blood is the upper curve. Excess oxygen consumption indicates the total amount of lactate oxidized. The 12 individuals are assumed to weigh $70 \mathrm{~kg}$. each. On this basis the concentration in the blood is much greater than in the body. Data of Dietrich and Zeyen ('32).

(fig. 173, and Margaria et al., '33, etc.). Ratios of the two rates are here far from constant; possibly constancy cannot be expected in the presence of rapidly changing contents. While lactate disposal is at approximately the same rate after exercise and after injection, the oxygen consumption after exercise is in higher proportion and preponderantly earlier.

No information seems to be available to indicate separable paths of recovery from deficit of lactate. Recovery of the whole body may be by slow accumulation, such as occurred for carbon dioxide, or it may involve faster production. Nothing appears to be known 
about the rate of lactate turnover, only net rates of exchange having been ascertained. Lactate thus represents the many substances that enter metabolism, undergo disposal, and are suitable for the study of temporal relations in equilibration of content.

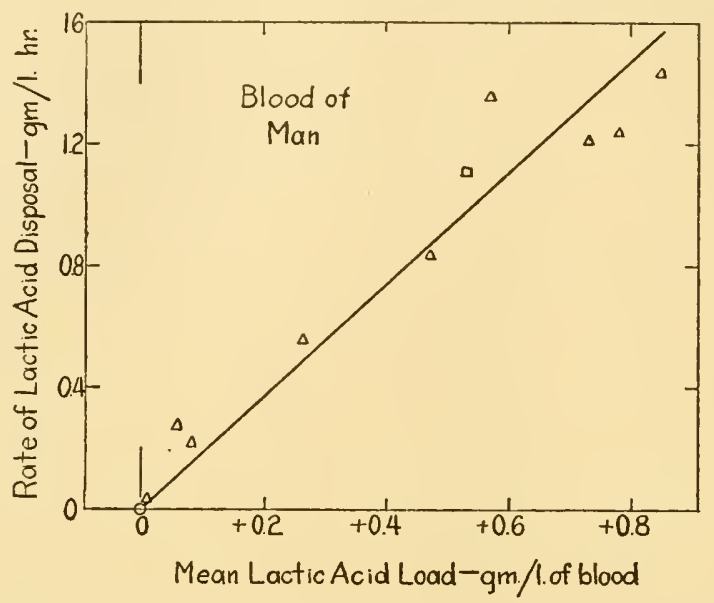

FIG. 174. Rate of lactate disposal in relation to lactate load. Man. Disposal is measured by disappearance from the blood during the first 0.5 hour of recovery. Triangles; one individual studied by Margaria et al. ('33). Evidence is adduced for the belief that $1 \mathrm{gm}$. lactic acid/liter of blood equals $0.9 \mathrm{gm}$. or 10 millimols lactic acid $/ \mathrm{kg}$. of $\mathrm{B}_{0}$. Square, mean of the tests with injected lactate by Dietrich and Zeyen ('32) shown in figure 173. The line of ordinates is drawn at zero lactate in blood.

\section{\$123. Summary}

A great number of bodily components may be studied in a manner similar to those that have been exhibited. The number is by no means limited to chemical constituents, as is thoroughly shown by the study of heat. Some of the same data as are used for heat could be used to illustrate the equilibration of the component body temperature as such. Portions of the data for total substance are available as equivalents of total potential energy content and its various fractions. Other data not mentioned may contribute to the study of body weight or surface, total concentration, specific gravity, osmotic pressure, electrical capacity, electrical charge, and many other measurable quantities. That the components named all have physical and chemical terms, merely expresses the fact that properties of living units depend upon non-biological instruments for their measurement.

Sometimes the content of the component in the body, or its in- 
crement (load), may be inferred from analyses of one tissue such as blood. Thus, the content of oxygen might be estimated from arterial oxygen concentration. In the case of heat the exchanges of the whole body were actually so ascertained from local temperatures. Several assumptions underlie any such procedures, especially the assumption of a roughly constant volume of distribution of the component under consideration. For exact work it is desirable to study by some independent method the limitations under which this volume is constant or known. One of the most severe limitations is probably time. Any sudden increment itself upsets the relative concentrations among tissues; and only either by measurement in numerous tissues or by knowledge of the whole load in the body, can the regression of the concentration in the samples with the load of the whole body be ascertained.

With greater certainty, the rates of exchange and the loads may be kept in terms of concentration of the one tissue sampled. This is what is ordinarily done in the study of glucose "tolerance" in mammals. And so it may be done for any conceivable component. It is a procedure available for the study in single individuals of components that are transformed within the body; for total exchanges of such components cannot be measured by their elimination alone.

When, therefore, glucose equilibration was studied from the point of view of the whole organism, the same data were also referable to the blood concentration of glucose. I elected to put this component in terms of the whole body in view of the fact that various paths of disposal are measured for the body as a whole. I elected, however, to consider the exchanges of lactate in the blood alone, since disposal was not separately measured, and in spite of the fact that removal of diverse portions of the body from intercourse with the blood is known to modify greatly the rates of exchange by the blood.

The definition of components is guided ordinarily by convenience of measurement. Concentrations are very often proportional to contents, and reciprocal to volumes of distribution. Each may receive separate definition on occasion. Glucose, for instance, is more accurately termed "total reduction of X's reagent under prescribed conditions of analysis"; for, use of $Y$ 's procedure may redefine it. Whether "combined" substances shall be lumped with "free" materials in one component is a matter of convenience. Even water is variously fractionated by analytical methods into 
"bound," "non-solvent," and the like, depending upon the many procedures of measurement. However, for inherently related components an increment of one is inevitably an increment of another. Oxygen content may include the oxygen of oxyhemoglobin, may or may not be represented by change of valence, may or may not comprise the securities for its deficit. Each component is a bank holding, and often it is, I imagine, the part of physiological security to have little of it in cash.

I find that the components for which I have presented quantitative data are the very ones that have in the past been most frequently mentioned as objectives of regulation. Bernard (1878) specifically named water, heat, oxygen, hemoglobin, and acid. Henderson ('17) added nitrogen, carbon, glucose, and others. The number of components is almost infinite, since those not ordinarily present in the organism (drugs, electromotive forces, parasites) are also unloaded after they have gained access to it. Whereas the fact of constancy in the content of each component has been stated, and the organs by which mammals modify exchanges of it have been examined, the possibility that the quantitative relations might be similar among water, heat, and carbon dioxide has not been previously explored. It turns out that a remarkable parallelism exists among the patterns of compensation of all. That fact allows comparisons of the processes concerned in adjustments of each component in common terms. But first, some properties that are ordinarily identified with parts of the body instead of with the organism as a whole will be mentioned.

\section{\$124. Heart Frequency}

Arbitrarily and tentatively, many physiological functions (components) and their increments are assigned to particular organs or tissues. Frequency of heart beat is named in connection with the visible location of movement in the heart, though very many other parts of the body are concerned in making possible, controlling, and maintaining this activity (component). The situation is similar to that for water, which is distributed through all tissues, though when its volume or concentration in the plasma is measured, its regulation is said to concern the plasma alone. Among components to be considered in relation to topographical units of the body, some concern known organs, others particular tissues, and still others single cells and their parts. Parts and their functions 
may be studied either in situ or isolated. The examples here chosen give preference to "non-chemical" components.

In man, heart frequency is perhaps the physiological quantity most often measured. Enormous numbers of circumstances are loosely known to disturb it; yet the quantitative tolerance curves describing its modifications are few except in physical exercise.

Recoveries occur in very short periods of time; therefore data are selected in which frequencies are counted in intervals of 0.3 minutes. Choosing recovery from physical exercise and from depressor stimulation (fig. 175), I relate the decelerations and accelerations of heart beat to the increments in frequencies (loads)

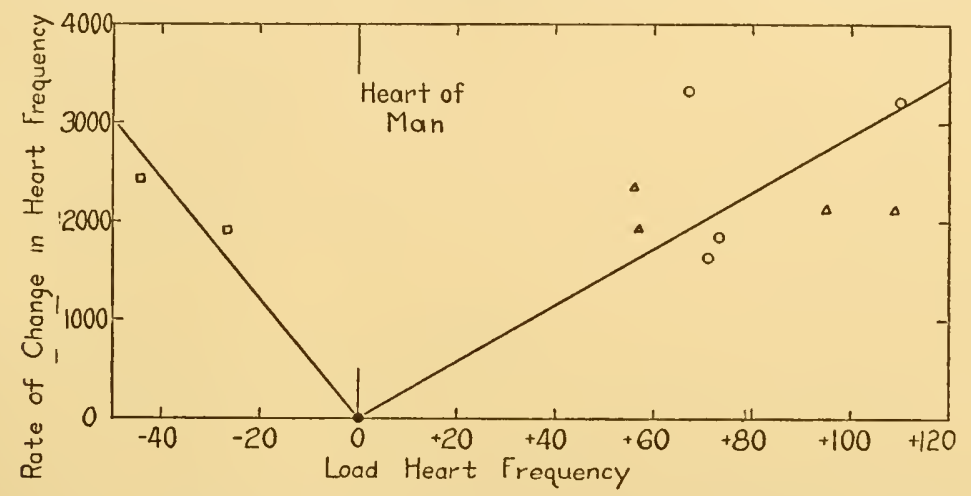

FIG. 175. Acceleration and deceleration of heart beat in relation to mean frequency of beat. Man. Decelerations are computed as decreases of frequency during the first 0.005 hour (18 to 20 seconds) of recovery at the completion of physical exercise (running). Accelerations are during the first 20 seconds after release of pressure on the region of the carotid sinus. Inerements and rates of change are all in per eent of the resting frequencies that prevailed before exercise or compression began. Squares, 2 hypertensive individuals of Mies ('32). Triangles, 4 groups of 8 to 12 tests each in 12 individuals; data of Cotton and Dill ('35). Cireles, 4 groups of 11 to 15 tests each on 26 individuals; 11 of whom were trained runners; data of Dill and Brouha ('37, pp. 13 to 16 ).

during those initial intervals. A net equilibration diagram results.

As was done for other components, those who made the observations presented the data as found under conditions named, without stopping to test what nerves or endocrine organs could be blocked without interfering in the recovery. Possibly faster recoveries will be found under some other conditions; and it can always be supposed that recovery for the heart begins at some time other than at the cessation of leg-running or at the release of pressure on the neck. 


\section{\$125. Arterial BLOOD PREsSURe}

The arrangements in mammalian organisms whereby constancy of arterial pressure is maintained, have been studied in considerable detail and are widely known. Certain "insensitivities" in the piezostatic arrangements are required to prevent the variations of pressure during each cardiac cycle from inducing large modifications of mean tension of blood vessels at each diastole. Automatically a decrease of pressure lasting more than one heart beat raises the frequency of muscular contractions in blood vessel walls ; and conversely.

I select as conditions for recovery from excess of systolic arterial pressure (as commonly measured) the sequelae of physical exercise (fig. 176), and for recovery from deficit the sequelae of pressure upon the neck and carotid sinus (Mies, '32; Weiss and Baker, '33). The pressure is changing at a net rate, and tells nothing as to whether the heart and the various arterioles are working at "cross purposes" or not.

The velocity quotients of recovery are in figure 176 about $24 /$ hour, and in two tests of Mies about 34/hour; utilizing in both series of data the first 0.01 hour after the "stimulus" ceased. Recovery of systolic arterial pressures in man from certain other types of load, also appears to be at about this rate.

Diastolic arterial pressures show opposite changes to those of systolic in the same exercise (fig. 176). Their recoveries are of approximately the same velocity quotient, however. The same holds true in the recoveries of arterial pressures that follow change of posture (Ogden et al., '38).

A few data for recovery of mean arterial pressure following nerve stimulations in anesthetized cats (Bayliss, '15, p. 691) indicate velocity quotients, both in excesses and deficits, of 250 to 700/hour. Following hemorrhage, the recovery of mean arterial pressure in similar animals is less rapid (Brooks, '35).

Since the intra-arterial pressure varies cyclically with each heart beat, it is possible to study also the rate of pressure change within a single cycle. From analyses of rapid recordings it is shown (Hamilton and Woodbury, '37) that during diastole the fall of pressure is approximately exponential with time. The same curve, with equal velocity quotient, is obtained upon suddenly occluding an artery and observing the continued fall of pressure within its peripheral segment (Dow and Hamilton, '39). It may be said that 
arterial pressure has a turnover, being gained and lost during each heart beat. Superimposed upon that exchange of pressure is the rate at which the pressure (systolic, diastolic, mean) increases or decreases while recovering from each increment above or below the usual.

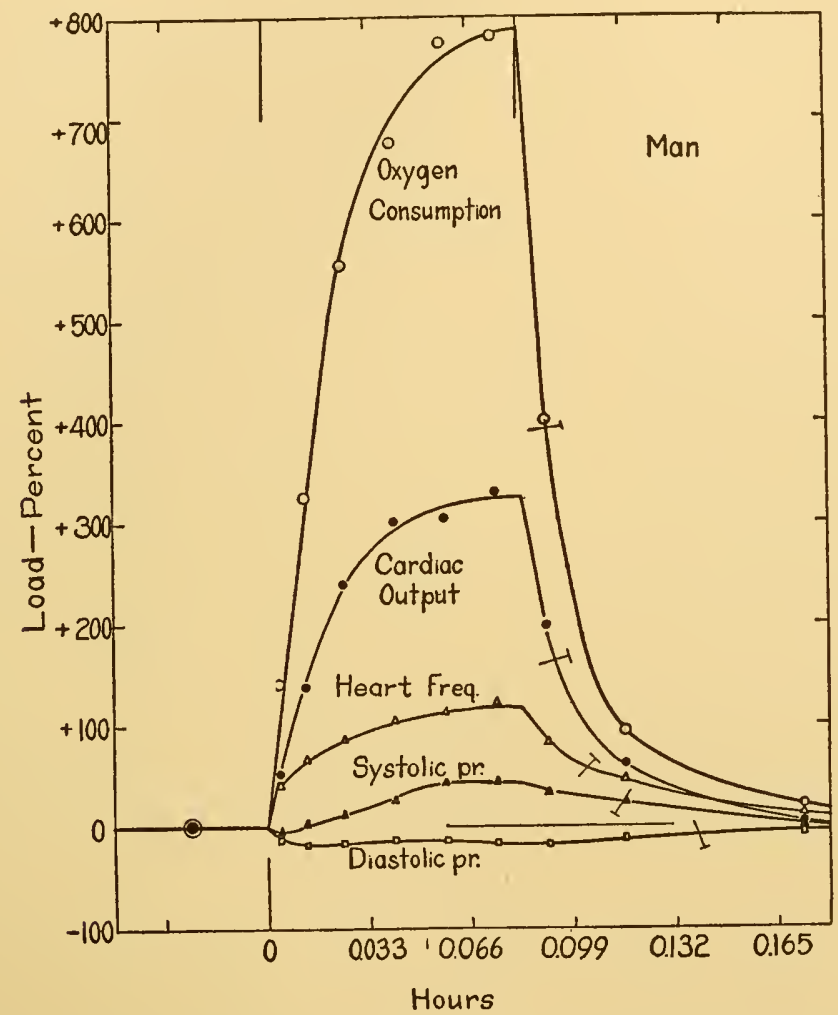

Fic. 176. Loads of various components during physical exercise and recovery, in relation to time. Man. One individual working on a stationary bicycle at the rate of 46,000 kg.m./hour. Recovery began at 0.083 hour. In the five quantities measured, each load is expressed in per cent of the value in the fore-period or control state. The systolic and diastolic pressures were estimated in the brachial artery. The "cardiac output" was inferred from the rate of uptake of nitrous oxide in the lungs. Data of Iljin-Kakujeff ('36).

\section{\$126. Volume FLOW OF BLOOD}

A flow may be equilibrated in the same fashion as a pressure or a frequency or a chemical entity. For the circulation as a whole, presumed "cardiac output" (rate of volume flow of blood) may be measured by any of the approximate methods. By a nitrous oxide method in man (rate of absorption of $\mathrm{N}_{2} \mathrm{O}$ in the lungs), the recov- 
ery of cardiac output (fig. 176) after a certain intensity of exercise, appears to occur in the first half minute with a velocity quotient of 75/hour. Hence the tolerance curve for presumed cardiac output or rate of total blood flow, is similar to that for heart frequency and to that for systolic arterial pressure.

When cardiac output is divided by frequency of heart beats, values for "stroke volume" are obtained. "Stroke volume" shows approximately the same deceleration as the above quantities. Or, by multiplying cardiac output by oxygen delivered per heart beat ("oxygen pulse"), values for rate of oxygen consumption are obtained. Altogether, three more quantities are thus added to the group of recoveries measured in the one experiment of figure 176 .

Constancy of convection is secured by a variety of self-induced modifications in the vascular apparatus. Failure of blood or of some of its components to arrive in the usual amount in a given tissue mass, is followed by conpensatory increases of blood flow ("reactive hyperemia"). These are adequately seen in the sequelae of partial arterial occlusion (Rein and Schneider, '37). Flow of blood in excess of the mean rate also appears to be compensated equally promptly; perhaps the regulation is one of sufficiency rather than of opposition to excess.

The same investigators ('30, p. 264) were actually able to demonstrate that during acceleration of flow to a muscle that was stimulated by electrical shocks to appropriate nerves in an anesthetized $\mathrm{dog}$, the arterial inflow exceeds the venous outflow, and during deceleration vice versa. Hence a charge or load of blood fills the widened blood vessels as long as the greater blood flow lasts. Recovery (deceleration) of local flow, like the recovery of so many diverse components, is then related to this measured load or excess.

\section{$\$ 127$. Regeneration of tissue}

(1) Tadpole tail. Experiment consists in removal of diverse amounts of tissue, after which the rate of tissue replacement may be measured. From the large number of studies in which partial information has been gained, I select the data of Zeleny ('16) upon the tails of tadpoles of Rana clamitans. Amounts of bodily material removed are ascertained in terms of linear length, the whole tail's length being taken as 100 per cent. The tissues replaced may or may not have the same proportions, compositions, or anatomical elements as those removed. 


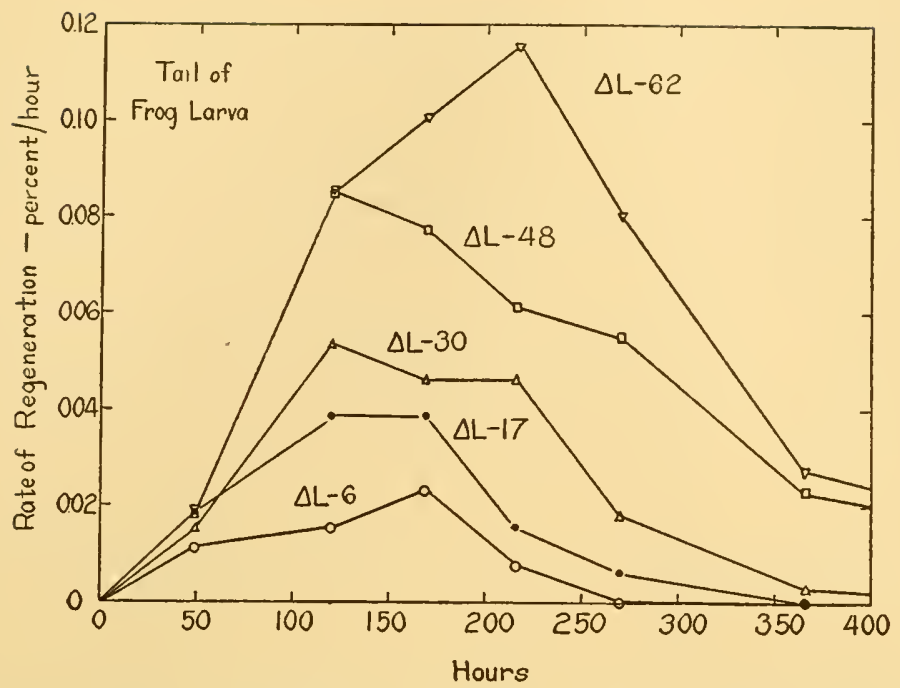

FIG. 177. Rate of regeneration in relation to time. Tail of tadpole of Rana clamitans. At zero time lengths of tail $(\Delta \mathrm{L})$ were eut off, varying from 6 to 62 per cent of the original tail length. In successive periods of 2 to 6 days' duration the rates of replacement of tail length were measured, as \% of the original tail length/hour. Data of Zeleny ('16, p. 120).

Regeneration (fig. 177) in course of time accelerates, and later decelerates. In whatever interval the rates of net gain be compared (fig. 178), the construction of new tissue is faster when the amount missing is greater. The processes end sooner when the

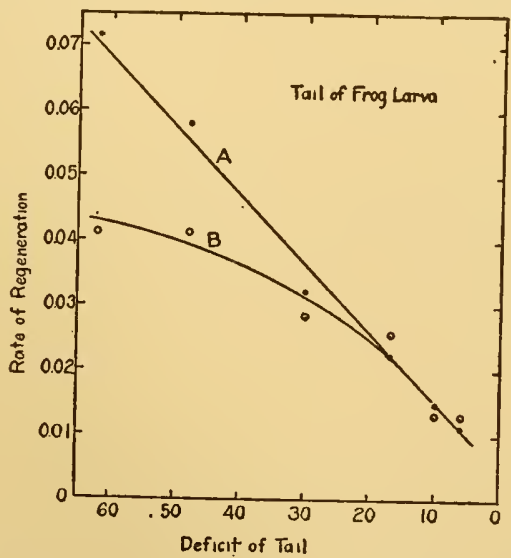

FIG. 178. Rate of regeneration in relation to mean deficit of tissue $(\Delta L)$. Length of tail of tadpole of Rana clamitans. The initial deficit was created by cutting off from 6 to $62 \%$ of the original length of tail. The mean rates of regeneration in length, in \% of the original length/hour, are computed from initial periods of two durations; $A, 6$ days; B, 12.5 days. Same data of Zeleny ('16, p. 88). 
removal is small (fig. 177); but the whole return is in no instance equal to the initial deficit.

By transformation of coordinates a half of an equilibration diagram is obtained (fig. 178). The curves may be convex, concave, or straight depending upon the stage of recovery. But at all stages the rates of tissue construction are of lower order of magnitude than those encountered in any recovery that has been thus far considered. Every biologist knows that regeneration is a slower process than recovery of water content after privation. It is now

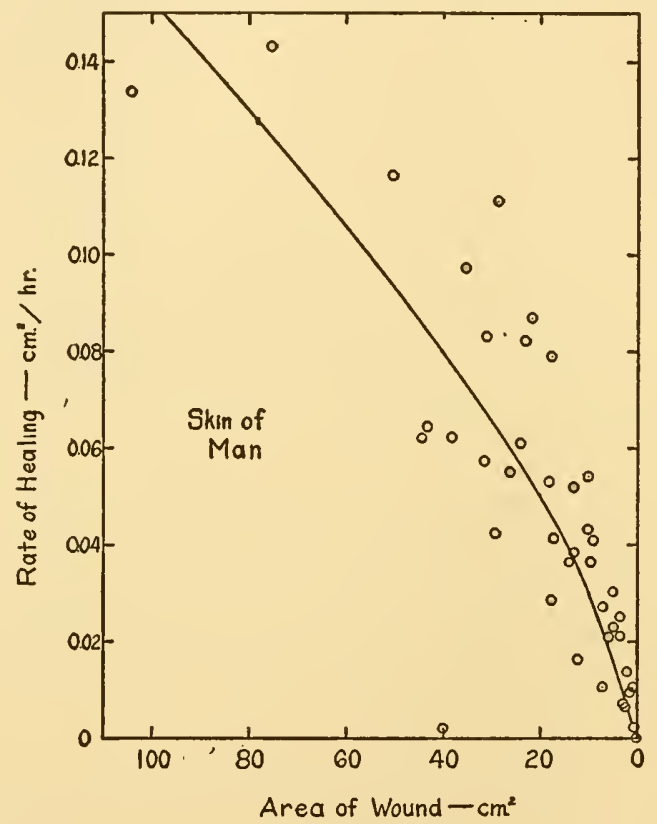

FIG. 179. Rate of scar-formation in skin in relation to mean area yet to be covered. Man. Measurements of area of wounds were made every 96 hours, and the first two intervals recorded in each of 20 individuals recovering from war wounds are indicated. Ages 20 to 40 years; no diversity with age was apparent in the data as plotted. The line corresponds to the equation $R_{\mathrm{H}}=0.006 \mathrm{~S}$ 0.7. Data of Carrel and Hartmann ('16) and Noüy ('16a and '16b).

possible to compare them quantitatively; adult Rana shows maximal velocity quotients for water replacement (fig. 70 ) of $0.7 /$ hour, while larval Rana shows maximal quotients for tail replacement of 0.0014 /hour. The factor of contrast is about 500 .

(2) Human skin. In man, parallel data measure the regeneration of wounded tissues. The wounds studied involved more tissue than could properly be called skin; the amount of closure was the quantity actually measured, and not all closure was by formation 
of new tissue. The relation of rate of healing to area of wound (fig. 179) is such as to suggest that the healing is proportional to the linear periphery of the wound. In that edge, new tissue proliferates and contraction of old tissue occurs.

In this tissue, cicatrization is a process much slower than recovery from stretching or compression, for instance. But compared to replacement of some other human tissues such as cartilage, the velocity quotient of only 0.003 /hour seems large.

(3) Rat viscera. In like manner the rate of hypertrophy of diverse organs of the rat after excision of the symmetrically paired ones may be measured (Addis and Lew, '40). Four of those structural units, namely kidney, adrenal, ovary, and testis, are replaced in a few days to the extent of 56 to 70 per cent of the weight excised; others such as prostrate and uterine horn are not appreciably restored.

Formation of more tissue, therefore, however it be measured, is faster after the amount of tissue already present has been diminished. The opposite case, in which excess of tissue is transplanted into place, has yet to be studied. What is often thought of as a variety of anatomical adjustment is thus measured in a manner comparable to any of the other components that have been examined.

\section{\$128. Excitabllity of Isolated nerve}

But one example is chosen from the study of isolated tissues. It could be also studied, though with possibly diverse results, upon similar tissues in situ.

Conduction of an impulse along a nerve renders the tissue temporarily unresponsive to further stimulation. Recovery of excitability may be studied by finding what intensity of stimulus (electrical potential) is needed in order that a second impulse be conducted. The deficit of excitability is total (load $=-100$ per cent) just after the first impulse; the measurement of the reciprocal of potential required identifies how much deficit exists at diverse times thereafter. In a chosen set of conditions the sciatic nerve trunks of frogs recovered half their excitability to electrical stimulation in $21 \times 10^{-8}$ hour ( 0.75 milliseconds). Volleys of impulses conducted at this time are also accompanied by half of the usual action potential (Graham, '35). It is possible that the average axone suffers the same modifications as the nerve trunk. 
During the course of a single recovery of electrical excitability, the net rate of change is, according to Blair ('36, p. 65), expressed by the equation $\delta Q / \Delta t=k \Delta Q$, in which $Q$ is the reciprocal of the potential required to excite in the control state. This $k$ is the velocity quotient as defined above; in frog nerve, as in many other tissues, $k$ is constant. For his frog nerve in conditions chosen, the velocity quotient has the value 16,000,000/hour; half-life $=0.45$ milliseconds. With other electrodes and other conditions, $k$ would be somewhat different. On the whole, these are recoveries that occur at enormously faster rates than any mentioned above.

By another method it is possible to produce varying initial deficits of excitability. At one locality of the isolated nerve first an inadequate stimulus is given, followed at a stated interval by one now just adequate to gauge the excitability of that locality. In that manner only the first portions of recovery are evaluated, as was done for many other components.

Much of the special descriptions given to excitability, and to recovery from it, may be recognized as particular forms of relations that are as general as equilibration itself. Whether the special features are more than quantitatively different from other maintenance remains, I think, to be ascertained.

\section{§ 129. OTHER DATA}

Numerous materials are available for the quantitative study of recovery processes, though none seem to be recorded exhaustively. I imagine there is no need to exhibit further examples in detail. But I have examined a sufficient number to give me the impression that a great portion of quantitative physiology could be represented in the form of equilibration diagrams and tolerance curves.

Some additions to the repertoire already exhibited, which I have visualized to date, are:

(1) Clearances, whether of plasma, blood, or other tissue; whether renal, hepatic, or in any other path; whether labelled as such or not. In each case a rate of loss (disposal) or of gain (accretion) is correlated with a concentration present. Many clearances in dog and in man are reviewed by H. W. Smith ('37). Other "disappearance curves" are for dyes (H. P. Smith, '25), galactose (Bollman et al., '35), borate (Rost, '03; Michaelis and Maass, '07), ethyl alcohol (Ewing, '40), propylene glycol (New- 
man and Lehman '37), osmotic pressure (Wettendorff, '01), and host of others, in dog; for radioactive sodium (Hamilton and Stone '37), acid (Haldane, '21), and those shown in table 43, in man.

(2) Regains in various tissues after depletions. Plasma proteins (H. P. Smith et al., '20; Stanbury et al., '36), hemoglobin (Whipple et al., '25) and radioactive iron (Hahn et al., '38) in $\operatorname{dog}$; red blood corpuscles (Schiödt, '38) in man. Absorption of sodium or of chloride through the body surface was found in frogs or goldfish only in marked deficits of each separately (Krogh, '39). That is the physiological equivalent of the equally specific ingestive selection of sodium chloride from among other salts in solution by adrenalectomized rats (Richter and Eckert, '38a).

(3) Tolerance curves, whether named such or not, and absorptions, penetrations, and accumulations.

(4) "Growth curves," which may be regarded as regains of size from deficits. Each young individual is an incomplete or deficient organism that constantly tends towards adult dimensions. The farther from mature weight, the faster does growth usually occur, within limits. All the equations that have been employed to represent the data of growth may thus be viewed as particular tolerance curves, as instances of correlation between rate (growth) and load (deficit of final size). No qualitative distinction is apparent between usual growth and the great gains of body weight that occur after retardation by deficient diets or by privations of food ( $\$ 118)$.

(5) Adjustment of electrical polarization and other potential differences in isolated muscle or nerve or skin, as after oxygen privation; dark and light adaptation in the eye of man (Hecht et al., '38); recovery of posture in man after being jostled; viscosity of the interior of Ameba (Angerer, '36) ; shortening and lengthening in muscle after adequate stimulus. Partial data exist for recoveries of body surface, acetone, epinephrine, lead, methyl alcohol, sulfur, amino acids, and specific gravity. Every biologist is familiar with many not named, even though he has come to associate "regulation" only with some restricted few components or features of particular organisms. A study of any component may be planned in analogy to those presented above, for all components have loads and exchanges.

\section{$\$ 130$. Summary}

The fact that none of the components mentioned failed to show 
relations between exchanges and loads similar to those already indicated, leads to the tentative conclusion that the methods of study and the relations are general ones. Instead, therefore, of regarding the study of water exchanges as an isolated field of physiology, I see in it and each other "field" a special case of very widespread relations having many features in common. He who learns how to deal with data for heat exchanges discovers forms of relations that indicate directly what data will be suitable for arterial pressures or glucose. Where analogies were sought, comparisons according to dimensions become feasible.

What selection may have gone into the inclusions and exclusions required to report these materials? So far as I am aware, the only stipulation was that data exist correlating net rates of exchange of the component with the relative content of the same component. I have not rejected any data that seemed adequate in number to yield a decisive correlation. There are plenty of zero correlations, meaning that the component is not regulated or recovered. It seems probable that data constituting conclusive exceptions (negative correlations of net rate and load) do not exist; yet whether or not they will later be found cannot be predicted.

Further, I gain the impression that no organism would exist indefinitely in which recoveries did not occur of most of the components that are regularly represented in it. There are components for which absence or slowness of recovery is compatible with survival, such as lead in man, uric acid in many kinds of insect pupae, low temperatures in poikilothermic animals, or amputated leg in man. The generalizations made might be alternatively phrased so as to exclude these and similar instances from expectation of recovery, though there is no general rule for knowing what those instances will be before they are tested.

The general induction is that recovery from deficits occurs by increase of gain over loss, and recovery from excesses by increase of loss over gain. Nothing is said about whether recovery of another component regularly occurs or not. If the present investigation were limited to extracting qualitative statements of that sort from biological observations, the results might seem inconsequential indeed. But since gains and losses are capable of quantitative comparisons, it is evident that a pattern has been found by which many components, and perhaps all organisms and their parts, may be studied. 


\section{Chapter XVI}

\section{UNIFORMITIES AND COMPARISONS AMONG COMPONENTS}

\section{\$131. VARIABILITIES}

The materials contained in the last two chapters lead to highly general considerations. How much of the approach suggested in chapter I has proven useful in dealing with the regulations of diverse components? What general rules are to be derived with respect to rates of exchanges? Which of the rules contribute to the understanding of physiological maintenances?

The measurement of how much a given physiological property fluctuates under defined conditions allows several kinds of comparisons. The variability of content of one or many components may be studied in a single individual, in two or more states, in random individuals of a race or species, or in various periods of time. Each, it seems to me, represents the resultant of organismal and other processes that are maintaining the living unit.

Upon investigating single individuals, a series of standard deviations of content or of exchange are found. Once a mean control content of the component is measured or its mean rate of exchange is established, deviations may be converted into coefficients of variation. The several quantities studied may then be arranged to form a series of increasing variabilities (table 39). Those maintained most accurately are quantities such as body weight that possess a supposedly large inertia, but equally may be

TABLE 39

Coefficients of variation during 15 to 24 months in dogs under standard conditions

\begin{tabular}{|c|c|c|c|}
\hline & Indiv. A & Indiv. $O$ & 11 indiv. \\
\hline 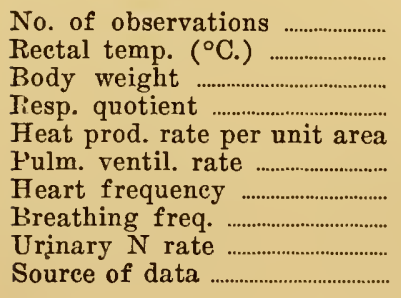 & $\begin{array}{l}43 \\
0.77 \\
1.33 \\
1.25 \\
4.01 \\
8.33 \\
\ldots \ldots \\
12.8 \\
24.9 \\
\text { Wierzu }\end{array}$ & \begin{tabular}{c}
46 \\
0.52 \\
1.32 \\
2.22 \\
4.64 \\
5.79 \\
\hdashline .3. \\
8.33 \\
18.0 \\
$($ ' $37 \mathrm{~b})$
\end{tabular} & $\begin{array}{c}281 \\
0.69 \\
\ldots \ldots \ldots \ldots . . \\
\ldots \ldots \ldots . . \\
6.37 \\
\ldots \ldots . . \\
10.9 \\
18.5 \\
\ldots \ldots \ldots \ldots \\
\text { DeBeer and Hort ('38) }\end{array}$ \\
\hline
\end{tabular}


quantities of no visible mechanical inertia such as respiratory quotient.

Data concerning variation are capable of representing what the organism does to exert control of content, whether or not this control employs recognizably special forces or organs. It may be that the rectal temperature of a snake would be, under like conditions, just as constant as was that of $\operatorname{dog} A$; the snake perhaps "does" nothing internally to preserve its temperature, while the dog does; instead the snake may "select" its environment to accomplish the same end. Further knowledge of that is a matter for observation. What is now in hand is the study of many components, preferably measured simultaneously; both the organism and the conditions available entering into the control of each. The dog or man is to be described as found, leaving aside the dog put under selected loads, and the dog placed successively under two or more recognizably different conditions.

Among the components in any organism, those found to vary little while exchanging continuously, may be defined thereby as components whose constancy is usually of more consequence to the individual than others. Generally the organism is exerting a type of behavior or of process that diminishes any positive or negative load, restoring the content toward the mean.

Another method of stating this general conclusion is that of Gasnier and Mayer ('38, p. 119). Each property or component occurs with such values (contents) as are arrayed in a frequency curve. Those values which are rare (of low frequency) are evidently prevented from occurring by a resultant of whatever processes operate to avoid them. Possibly the "effort" made to avoid these values is inverse to their frequency. Therefore the frequency curve that is epitomized by the coefficient of variation (and by the coefficient of difference) becomes by definition the measure of all that discourages extreme contents.

Variabilities apply to rates of exchange as well as to loads. As in loads, variability between successive periods may depend on the length of the period. This is the case in rates of urinary water loss in dogs under control conditions; the mean difference is less for 0.25-hour periods than for 0.5-hour periods or for 1.0-hour periods (fig. 45). This fact indicates that something prevents large short-time fluctuations; it is evidence of an "inertial" gov- 
ernor. In other exchanges there may be no significant change of standard difference with length of period. This is the case in total heat losses (table 36 ) ; it may suggest the absence of a stabilizing "inertia" for rate that outlasts the shortest period studied. Or, there may be larger differences between shor't periods than between longer ones, as in the cases of rates of ingestive water intake by dog or man (table 12) and of superficial blood flow (Burton and Taylor, '40). This feature characterizes some discontinuous process, as drinking or vasoconstriction, which makes the gain of water or loss of heat periodic.

Further relations may be found for variabilities by comparing the loads with the rates of turnover of the same component. A variation of rectal temperature of \pm 0.77 per cent of $37^{\circ} \mathrm{C}$. (A, table 39) is equivalent to a standard deviation of $\pm 0.29^{\circ} \mathrm{C}$. or a heat content of \pm 0.24 Calorie/kilogram of body weight. In the interval of one week between determinations of rectal temperature, at least 200 Calories/kilogram have been gained and lost (turned over) by the dog's body; for the whole interval of time, therefore, gain equals loss with a precision of about 1 part in 1000 . Or, considering the variation of the dog's body weight, I note that while $1.33 \%$ of $\mathrm{B}_{0}$ is gained or lost (net), about $40 \%$ of the total substance $\mathrm{B}_{0}$ is gained and lost (total) in one week's time; an accuracy of 1 part in 30. Here are in figures the knowledge that many have in the rough, that body temperature is more constant than body weight, relative to the whole quantities of heat and of body substance (food and water) with which the metabolic processes deal. Additional species may be compared in table 12 .

How much augmentation of rates occurs, on the average, at the loads corresponding to borderline variations? The coefficients of variation and of difference are by definition such that about twothirds of instances measured fall within their latitude. What change in rate of exchange has on the average accompanied this much load? Such comparison (fig. 47) serves to link the equilibration diagrams with the respective variabilities. For water and for heat the net rates of exchange at the $\odot$ of load are one-tenth to onefourth of the turnover rates. Should this ratio prove to be nearly constant, it also follows that, when all net equilibration diagrams are put into a uniform proportion between ordinates and abscissae, the steeper the curves (or the greater the value of the net velocity 
quotients) near balance, the smaller the variability. Data are at present not sufficient to decide how widespread this relationship may be.

What is the normal state of an organism, a term that everyone uses but no one defines? Every biologist has a concept of the norm; precise meaning's can only be quantitative ones, I believe. Heretofore I have spoken of normal individuals or states as being the controls that are compared with others in which some load prevails. They are considered to have zero load of whatever component is being measured. On the basis of variations I am able to affirm further what latitude may be expected in their content of any one or several components. A single measurement may be far from the mean, but among a number of measurements the content is represented by the standard deviation of the observations. The deviation thus helps to define the normal state and aids in characterizing the organism in the same manner that listing the conditions of environment or of observation does.

But whereas variability could be measured under numerous conditions, the normal state is also that in which measurable kinetic equilibrium of a specified component prevails, that is, where gain of it equals loss. It is a particular stationary state of common occurrence. However, the turnovers also vary more or less, for it is rarely possible to demonstrate that gain exactly equals loss; errors of measurement, the "hunting" of supposed governors, and the durations of the period in which the exchanges are measured, all being factors. Hence the normal state varies by a precision $(\S 23)$ as well as by a content.

As usual, then, it is found that the normal state or norm has no meaning except as it is defined. It can never be given an absolute or complete definition, but approximations are obtained by listing conditions, states, contents, exchanges, numbers and kinds of individuals, and variabilities of each. The norm for any component reflects preponderantly those very events in organisms that constitute maintenance of physiological state.

\section{\$132. Behavior and maintenance}

The study of animal behavior, which is sometimes regarded as mere tabulation of what responses are elicited by each kind of stimulus, also contributes to understanding maintenances. A man at rest finds himself cold. He, perhaps subconsciously, moves to 
a warmer environment, or adds clothing; both are behaviors that result in preserving usual contents of heat in his body. The fact that the man has means of compensating the heat deficit by faster heat production and slower heat loss than usual, does not limit him to reliance upon those means. The behaviors are "reactions that minister to the preservation of the individual" (Hobbes, 1647). Until that rough conclusion is reached, the reactions manifested by animals are just a miscellaneous collection of sensitivities; after it has been comprehended, the reactions seem anything but random.

" Speaking generally, behavior regulation of body temperature in poikilotherms takes the form of a locomotory reaction. If an insect is too cold, its easiest way of getting warmer is to move to a warmer place" (Fraenkel and Gunn, '40). That is the kind of behavior that has also been observed in the maintenance of such components as total substance, glucose, carbon dioxide, water, and oxygen.

Very often it is said that the animal becomes "restless" or "uneasy" in an environment unfavorable to its continuance. This statement appears to be explanatory, in the absence of further information as to what sense organs are activated, what tissues, what communicating systems, what effector organs. Once those facts are known, they too are partially explanatory. When further it is found that the content of water is more constant in the animal that stays in high humidity, this finding, it seems to me, explains something which the identification of anatomical factors does not; namely, that the particular behavior practiced, out of all the behaviors made possible by those structures, results in greater constancy of water content. To state that greater constancy accompanies one behavior and lesser another, is a correlation of the same order as to state that greater constancy goes with a particular kind of skin, or a particular manner and rate of excretion.

It is then a task of the student of regulations to find how widespread that correlation is. So far, data are insufficient to allow any great generalization. Listing the studies that I happen to know concerning behavior toward water, moisture, food, constituents of food, and heat, I gain the strong impression that most preferences for environment promote constancy of content of those components; quite independently of my guess that animals would hardly have survived if they did not frequent environments that are "favorable" in those respects. 
The most interesting correlation in behavior is, I think, the modification of behavior with load of component. The animal deprived of food does things to find it that the well-fed animal does not. Such a correlation was found for water content and atmospheric humidity in rat and in cockroach $(\$ 43$ and $\$ 47)$. It means that preference for moisture is greater in water deficits, even as drinking is faster in water deficits.

Very often it is said that an animal in water deficit has "drive" or "thirst drive." That term denotes a hypothetical state of the animal, and connotes a virtual force that expends itself in seeking water. It is comparable to inferring that a force of "osmotic" pressure moves water into the body, and if I were to call it " $\mathrm{drink}$ ing pressure" no fault could be found. But what is directly measured is not drive, but either water deficit or water drinking. The intermediate term can be dispensed with, and water load be used to describe the physiological state.

In brief, behaviors that diminish losses or enhance gains in defieits, and those that enhance losses or diminish gains in excesses, protect against inconstancies. Rarely is any one behavior continuously operative, so long as many components are being preserved by a single individual. But, however modified by conditioning or intelligence, appropriate behaviors are found widely in organisms that observably respond to environments.

\section{$\$ 133$. Sequences IN TIME}

By comparisons and inductions from data such as figure 176, sequences of load are found to fall into a generalized tolerance diagram (A, fig. 180). The scale of times as well as of loads varies enormously for diverse components, and somewhat for diverse species. Five periods or phases may be distinguished, in each of which a different physiological state (load) prevails.

(I) Control or initial state, in which load is zero.

(II) Transitional or loading state, in which an increment or load is gained under the influence of component, agent, or conditions.

(III) Stationary state, in which load is relatively uniform; often conditions are constant.

(IV) Recovery or unloading state, in which load is lost.

(V) New control state, in which recovery is completed or nearly so; it may or may not differ from the initial control state $I$. 
State $I$ is characterized by the mean content $\left(\mathrm{C}_{0}\right)$ and by its variability $\left(\sigma_{\mathrm{c}}\right)$. Descriptions of conditions that prevail enable the measurements to be reproduced.

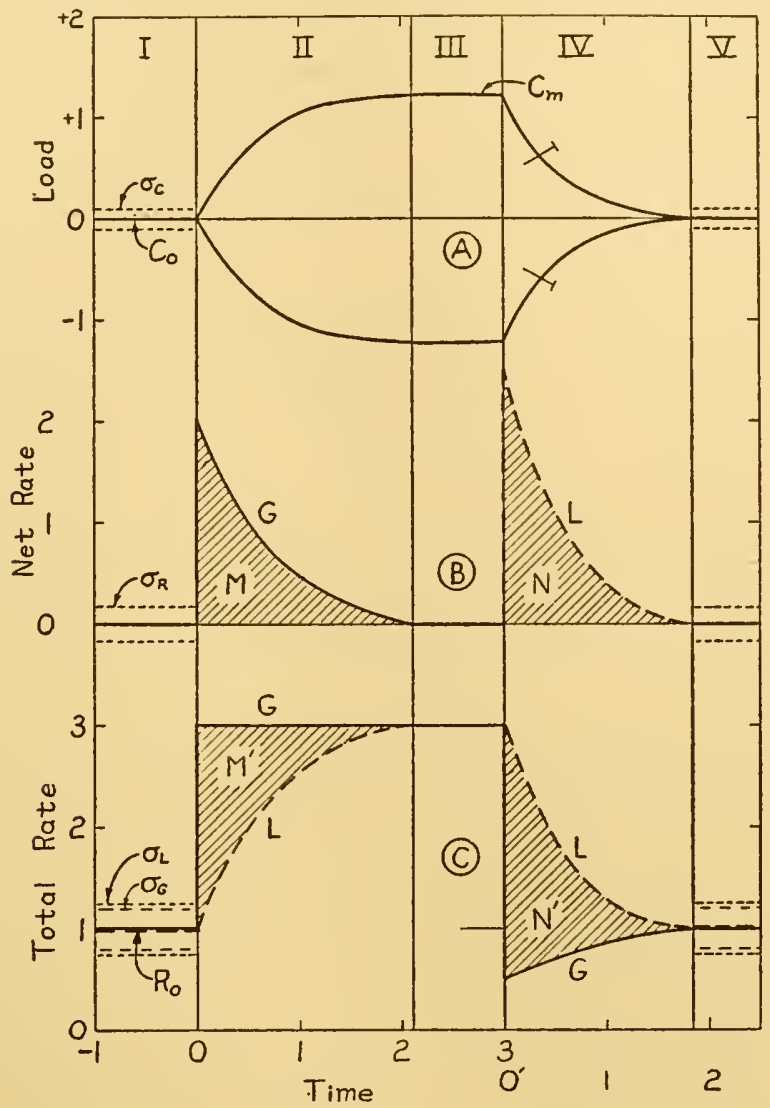

FIG. 180. Diagrammatic representation of physiological states (in load and in rate of exchange) in relation to time. An agent impinges at time $O$ and ceases to act at time $\mathrm{O}^{\prime}$. Five periods, representing five physiological states are distinguished: I, control or balanced; II, initiating or loading; III, stationary or loaded; IV, recovery; V, second control or recovered or balanced or final. Rates given represent gains $(G)$ and losses $(L)$ in positive loads; in negative loads $G$ and $L$ would be interchanged. Variabilities of load $(\sigma c)$ from the control mean $\left(\mathrm{C}_{0}\right)$, and of rates $(\sigma R, \sigma G, \sigma L)$ from the control means $\left(R_{0}, G_{0}, L_{0}\right)$ are indicated. Areas $M=N=M^{\prime}=N^{\prime}$ are each equal to $\mathrm{C}_{\mathrm{m}}$, the maximal or stationary load. Portions of this curve are observable in the actual data of figures $21,23,143,163,164,160,176,106$, and others.

State II is related to the initial and the stationary states, very often being represented by an exponential curve connecting the two. Its duration is sometimes longer when the stationary load is greater. In some respects it represents the resultant of what the 
agents and conditions do to the organism and of what the organism does in response. Partial generalized treatments of state II were given by Widmark and Tandberg ('24) and Burton ('39).

State III may not appear in a given instance, for the agents or conditions that induced a load may be removed before it appears. Constancy of load means that rate of gain remains equal to rate of loss, and often state III is identified from rates of exchange. Otherwise the stationary character may be judged solely from the constancy of content.

State IV is the portion of the sequence that has been studied most extensively in this investigation. It seems to represent most often what the organism does to adjust contents. Sometimes its slopes are the reverse of those in state II. Very often the agents that induced the load persist with diminishing effects for long periods of time (as when drugs wear off or atmospheres gradually cool), in which case recovery can hardly be regarded as an unencumbered activity of the organism. Sometimes there is no way of knowing how long agents persist (as when epinephrine is injected), any more than of knowing exactly when agents not followed by immediate consequences have become effective (as when water is ingested). In all instances the investigator may choose a time that can be recognized reproducibly and count hours from it, leaving to anyone the "interpretation" of intervening events.

State V is sometimes known only as an asymptote; sometimes it is assumed to be identical with the zero load of state I; sometimes it is acknowledged or demonstrated to be different from state I.

Very many physiological sequences are incomplete approaches to hundreds of successive stationary states; these may be regarded as states IIId, Vc, etc., perhaps being distinguished only by whether the load has just increased (III) or decreased (V). Thus, an increment of blood volume, or a load rate of oxygen consumption in a gastrocnemius muscle (Keller et al., '30) varies enormously during each muscular contraction. Transitions and recoveries probably succeed one another with each posture and each movement; and an asymptotic state is rarely reached. Data illustrating the loads and rates involved during transitions from one stationary state to another have been worked out with respect to rate of oxygen consumption in human exercise (Hansen, '34; Szwejkowska, 
'38). Anyone can within a few minutes construct a curve (like A, fig. 180) for heart frequencies in man.

With respect to rates of exchange, the sequences are bound up with those of load. The same five states may be distinguished in the exchange diagram ( $\mathrm{B}$ and $\mathrm{C}$, fig. 180 ) whether net rates, total rates, or certain partial rates are measured. In instances in which gains and losses have been measured, one exceeds the other during. any transitional state and the converse relation holds during the recovery state. Moreover, since net rate integrated with respect to time equals load, the areas of difference between total gain and total loss are equivalent to maximal load, and very often are equal to one another $(M=N)$. When $M$ does not equal $N$, it is pertinent to inquire in what identifiable respects state $\mathrm{V}$ differs from state I.

Further consideration may be given to recovery states. Rates of recovery are rates of decrease of load. Some of the loads studied follow exponential equations of the type $\Delta \mathrm{C}=a e^{-k t}$, as was early shown by Michaelis ('07). Differentiating with respect to time, $\delta \mathrm{C} / \Delta \mathrm{t}=\mathrm{R}=-a k e^{-k t}(\S 71)$.

The load throughout the five states might be analogous to the height of a ball thrown upward. The height attained depends on several distinct factors; the fall (recovery) exhibits accelerations free of the force of the throw. In ballistics the usual trajectory is a parabola; in physiological tolerance curves it is often two exponentials.

Certainly the relation between load and time is not an invariable one for diverse components, nor for one component in many species. No augmentation of exchange may be evident for 0.3 hour (water excess in $\operatorname{dog}$ ), or the load may be proportional to time (ethyl alcohol in dog), or no recovery may be measurable (amputated leg in dog). The last part of recovery may take as long as the individual lives; the first part may be infinitely slow or fast.

In practice, recoveries are compared quantitatively either in a uniform fraction of the recovery $(\Delta \mathrm{C} / a)$ or within an arbitrary interval of time during the recovery $(\Delta t)$. With each set of data it is therefore necessary to designate the value of $1 / a$ or of $\Delta t$ that is used. Very often the half-life $\left(t_{\mathrm{q}}\right)$ of the load $(a=2)$ is a suitable means of comparison, and $k=\ln 0.5 / t_{\mathrm{q}}$. However, when, as is often the case, the entire curve of loads during recovery is known, 
and it is exponential, then the value of $1 / \Delta t=k$ is independent of the fraction $1 / a$ or the interval $\Delta t$ used.

Figure 180 describes, therefore, the relations to time in any disturbance and recovery. A story concerning a particular component may be planned by measuring those quantities that will fit it. Conversely, all components may be compared by means of the number's found for each of the curves and parameters represented. They seem to describe adjustments of any physiological components.

\section{$\S 134$. LOADS}

A load is defined as the deficit or excess of any measurable component in a living unit, relative to its content in a control state. A component is, in turn, any property of an organism that is susceptible of measurement. What are the several methods of measuring loads?

(a) Taking the organism or its part as it happens to be, the investigator keeps a complete account of gains and losses while the individual passes from state I to state III; and later from state III to state $V$. At the end he decides whether state $V$ equals state $I$, whether net gain or loss throughout state II equals net loss or gain throughout state IV, and whether some of the component escaped measurement by having been transformed. At whatever times gains and losses are ascertained, the coincident loads and rates are known.

(b) By an appropriate kind of measurement that presumably does not interfere with the physiological state, repeated determinations of load are obtained. Examples of the kinds of measurement that are available are: (1) Body weight or volume or length as an indication either of total substance or of water content, depending upon the conditions in which the measurement is applied. Determinations upon small tissue samples by a variety of physical and chemical and serological procedures, with or without either estimates of volumes of distribution or assumptions that volumes of distribution remain constant. (3) Introduction in vivo of foreign indicators, either physical, chemical, or serological, usually with subsequent sampling of tissue for analysis.

The changes of load with time being known, the successive rates of net exchange may be computed. Or, the changes of load with time and the rate of total gain being known, the rate of total loss 
may be computed, or vice versa; for, the difference between total gain and net exchange is the total loss.

It may be induced from equilibration diagrams that at two diverse loads, the same rate of exchange would not be expected to occur. Yet it is sometimes assumed that a measurement of rate of net loss, for instance of nitrogen during nitrogen deficit, is a measurement of turnover in balance.

Wherever positive loads have been investigated, negative loads may be expected to be of equal interest. The history of physiology shows, however, that intensive studies of changes in one have not often led to inquiries into the paired state. This omission indicates that other considerations than quantitative symmetry of function have guided investigations.

The range of loads observed in the study of each component is limited. Occasionally little experimental effort is put into obtaining extreme states (tolerated loads), while in some instances the production of injury (irreversible change) in the organism is approached. The criterion of irreversibility is open to many differences of judgment or end-point; perhaps the criterion of "death" (judged by a chosen independent test) within a certain number of hours from the time at which the load is imposed, is a generally useful measure of tolerated load. Each component may then be varied within the range stretching from mean viability in negative load to mean viability in positive load.

The magnitude of load in the stationary state or at the maximum is as much a function of the rate of recovery as of the agent. impinging. For, the maximal load is a sum of gain and of loss, and since rate of loss usually emulates rate of gain, it is often the case that no acceleration of gaining will long outdistance losing. An analogy is throwing a ball vertically; acceleration of gravity becomes equal to acceleration of lift at a very finite height.

Overshooting of the content characteristic of balance, did not regularly occur during recovery in any of the components here reported (cf. Burton, '39). In quantitative comparisons of diverse components a problem is to find commensurate coordinates, for, both loads and rates are measured in a variety of units (table 40). For certain specific comparisons, common units are available; calories, grams, chemical equivalents, weights, volumes; these represent appropriate particular equivalences of energy or of substance. For other purposes it is preferable to record every load 


\begin{tabular}{|c|c|c|c|}
\hline 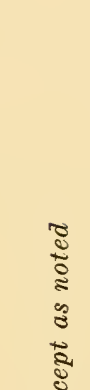 & 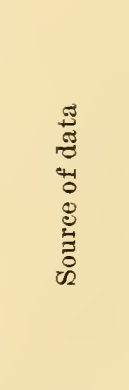 & 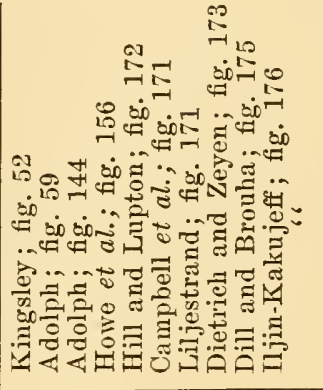 & 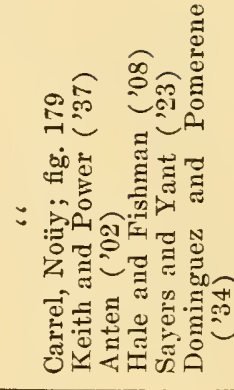 \\
\hline 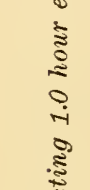 & 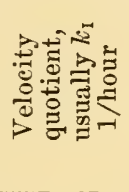 & 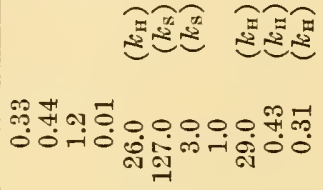 & 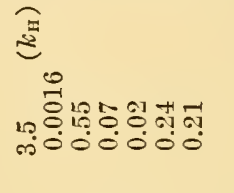 \\
\hline 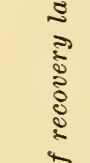 & 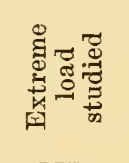 & 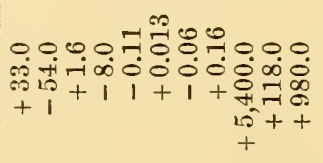 & 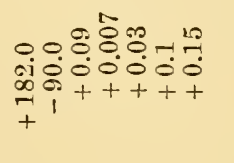 \\
\hline 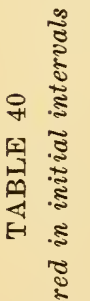 & 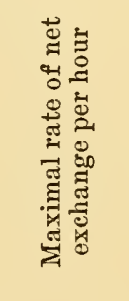 & 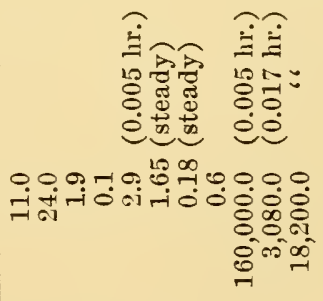 & 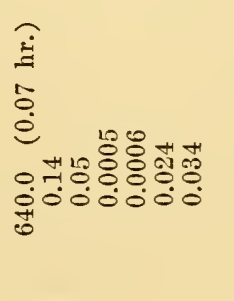 \\
\hline $\begin{array}{l}\text { ङ } \\
\Xi \\
\vdots \\
\text { है }\end{array}$ & 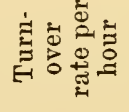 & क. & : \\
\hline 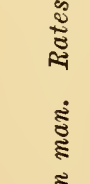 & 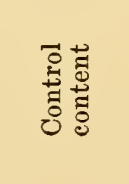 & 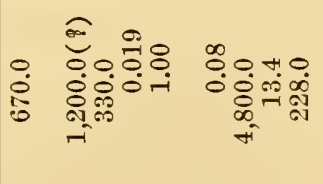 & 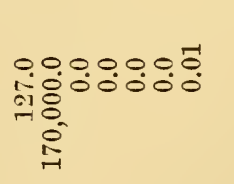 \\
\hline 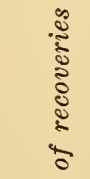 & 总 & 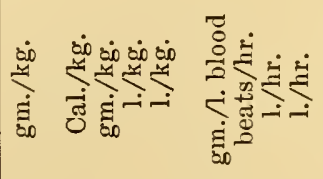 & 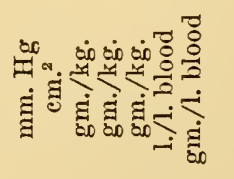 \\
\hline ت্ & 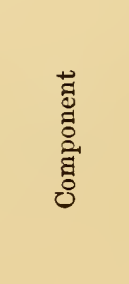 & 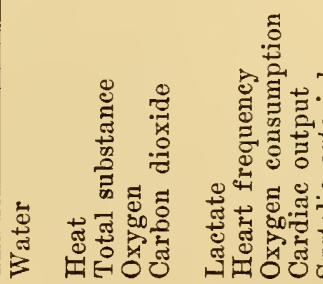 & 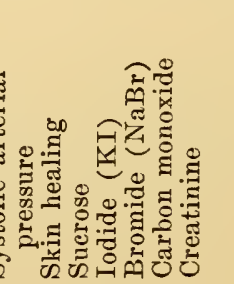 \\
\hline
\end{tabular}


as a fraction of the body's control content. But this is difficult to accomplish for heat, temperatures, pressures, and electrical potentials, which either have no physiological zero or are regularly absent from the body. If fraction of control content could be used universally, it would have the advantage that every conceivable component, regardless of its physical dimensions, could be expressed in it.

Loads might also be equated in units of variability $(0)$ or in units of daily or hourly differences (CD). This, the "beta" method, would have the advantage of being independent of all physical dimensions, and the great disadvantage of requiring a special study of variability before any comparisons could be made.

Could lethal extremes be used to equate loads to a common scale? The difficulties here are: (1) the physical scales would differ from the physiological scales within the range of positive loads as compared with the range of negative loads; (2) the scales would differ for two types of loading of the same component, one of which killed at a less point on the physical scale; (3) no lethal load exists for some components; (4) the scale of loads would be revised with each statistical investigation of tolerated maximum; (5) in man the lethal limit could often not be determined, and in elephants would be rather expensive. On the whole, I see no probability of finding a biological means of making commensurate loads of all types and components.

Loads from which recoveries occur evidently represent debts and credits in the organism. For some, equivalents are known that act as security for their discharge (phosphocreatine for oxygen, hydrogen ions for carbon dioxide, osmotic pressure for water). It is unnecessary to limit the measurement of debts and securities to chemical or any other variety of components. The security may be divided among many forms at one time. The combined states and activities of the organism represent this security, as evinced in everything that changes during recovery.

\section{$\S 135$. RATES OF EXCHANGE}

Rates of physiological activity are comparable for various components wherever the components have similar dimensions or known equivalences. Sometimes dimensions themselves can be transformed with high assurance of accuracy, as in the loss of heat (cal./kg. hr.) by evaporation of water ( $\%$ of $B_{0} / \mathrm{hr}$.), when the latent heat of vaporization is believed to be known. 
The recital of the rates of turnover of diverse components, as in table 40, may be of interest in certain comnections. Each mean rate, and the variability of each rate, characterizes the individual and the species. It establishes norms against which any unusual individual or an individual in any new physiological state may be compared, in much the manner that physical anthropology and clinical medicine practice.

It is not always realized that most components concerning whose gains and losses little is known, still have turnovers. Constant potential, pressure, or concentration probably represents continual decay and replacement, though no prediction may be safe for all components. Prothrombin in dog's plasma decays after the plasma is isolated from the body; in fact no organic compound in wet killed tissue is known to last indefinitely. Often intermittency of content furnishes a means of estimating turnover rates. Thus, arterial blood pressure falls during each diastole; this fall multiplied by the heart frequency, is the turnover of pressure. And so minimal values of the turnover of excitability in heart, peristalsis in gut, tension in single muscle fiber, and lift during walking are ascertained. In general, measurements of turnover depend upon one of the following methods: (a) flow across a boundary, (b) difference in flows to and fro (arteriovenous differences), (c) accumulation of marked materials, and (d) intermittent contents. All methods treat the rate as though stationary over some period of time.

In the study of regulations of any one component the several rates of exchange relative to turnover rates are indicated by augmentation ratios and modification ratios; they indicate the latitudes over which rates of exchange respond. The maximal rates of exchange express capacities with which each organism is endowed. In particular, it turns out that for most components (table 40) the maximal rate of gain is similar to the maximal rate of loss.

For a few components, economy quotients (ratio of total gain to total loss) are known. At extreme loads it is the rule that economy quotients are far from 1. A ratio similar to the economy quotient was proposed by Verworn (1898, p. 487), termed "biotonus," representing the rate of assimilation or anabolism relative to the rate of dissimilation or catabolism. Metabolic equilibrium prevailed only when the ratio was 1 . This limited form of econ- 
omy quotient was not illustrated by quantitative data, and states in which it varies were not ascertained.

The most efficacious recovery might be defined as that which exists when the economy quotient becomes infinity or zero; in which case either loss or gain is completely suppressed, and net exchange equals total exchange. That rarely occurs, and with some excuse. Perhaps it can be supposed that complete suppression appears only where cessation of exchange can be managed without interfering with any other component (as, when ingestive gain of water ceases in the dog's positive water load). But for one exchange to concern only one component is the exception; further physiological properties are modified by the continued production of heat in positive heat loads, and by the continued gain of carbon dioxide in positive loads of carbon dioxide. Other cases of unforeseen interrelations give rise to more elaborate and less widely accepted excuses, with little descriptive foundation, such as could be given for the continued intake of water by the frog in positive water loads. To illustrate how unforeseen combinations arouse unwitting excuses, I quote a careful observer. "The Corixidae are air breathing," says Krogh ('39, p. 118), "and one would expect their integuments to be practically water impermeable. Is it possible that they take up water and salt in such quantity with the food that an osmotic regulation becomes necessary?" In this one expression of surprise it is implied that oxygen exchange is highly correlated with water exchange through any one surface, that impermeability is less trouble than exchange, that every property of an organism has fitness, and that a physiologist knows what to expect more often than not. Yet no biologist mixes his data and his "derivations" to a less degree; most scramble them more.

Not only is it difficult to discover a rate of exchange that corrects one component without affecting others; it is also apparent that the relative rates of exchange among components are compatible one with another. Heat loss by evaporation would be impossible in a steady state (fig. 48) if water gain were slower than water loss by this path. Even decelerations fit together; a man would readily undercool (fig. 143) if his water and heat loss by vaporization did not decelerate before the heat load was entirely dissipated.

Components that are loaded slowly also recover (unload) slowly in most instances. Thus, rectal temperature and heart beat 
in exercise (man), lactic acid and oxygen in exercise (man), and excitability and lactic acid in stimulation of nerve (frog) constitute pairs that move at very different rates, but in nearly the same ratio in both directions. Either reversed processes or matched "governors" may be at work in the two states (II and IV) of the organism. Actually the processes usually do not occur in the same paths, for example the gain and the loss of water. A difficulty in phrasing a more general induction is that the maximum attained by the load often is itself bound up with compensation and recovery, the asymptote of loading (state III) being already an expression of rapidity of recovery.

Intermittency of rate occurs in a great many components (limb movement, sleep, reproduction), and for the most part it is ignored in their study. The dog accomplishes by intermittent ingestion of water exactly what the frog accomplishes by nearly continuous imbibition of water. Intermittency is a convenience to the organism in permitting it to go about other activities which preclude the exchanges in question. The dog devotes perhaps 0.5 per cent of its life to drinking, 2 per cent to eating, 1 per cent to micturition, 30 per cent to sleeping, and so forth. Segregation of each allows greater variety of undertaking in all the remainder of the time.

Rates of recovery in organisms are modified by many agents and conditions. Loss of water, gain of heat, and loss of lactate, are all accelerated by moderate physical exercise in man. Glucose removal by dog is changed by previous glucose administration, by deprivation of adrenal glands, of pancreas, and of liver. Carbon monoxide is removed faster when oxygen, or oxygen + carbon dioxide, is breathed. Results of experiments, treatments, and therapies may be suitably measured, of course, as modifications of loads (tolerance curves) and of rates (exchange curves). It appears to be exceptional, as was said, to find agents that modify one component only.

Rates of exchange show acclimatizations. These are progressive changes in rates incident at given loads, that appear after continued or repeated loadings. Time is required to bring them forth. Thus, repeated administrations of water to a dog result in greater rates of urinary output at the same water excess (Kingsley). The rate of heat loss increases upon successive days of exposures to hot atmospheres (Adolph and Dill, '38). Less chloride is lost through the skin in sweat upon successive exposures to hot atmospheres 
(Dill et al., '38). The exchanges and velocity quotients are not, it seems, permanently fixed when the load first appears; a sort of conditioning is apparent, using that term either as an ecological or a psychological one. Occasionally such progression with time is termed adaptation, or accommodation; each term has its implications which no one wishes to extend to all phenomena in which repetition has an effect. Here is evidence that patterns of maintenance are not all preformed; it is possible that exchanges of many components are changed with experience superimposed upon initial endowment.

In general, most components of organisms appear to be provided with outlets that pump faster in repletion, and with intakes that pump faster in depletion. Each of many physiological functions has flood controls and drought controls. Turnover is absent for those components whose content is zero, and for relatively few others. Where continuous turnover occurs, machinery for recovery is already in motion; recovery is achieved "by modifying the speed of a continuous process', (Cannon, '32, p. 181).

\section{$\$ 136$. VELOCITY QUOTIENTS}

Instead of regarding each component and its compensations as discrete, I can next regard them as coexisting in one individual. I can then compare the adjustments of diverse components, and not merely in terms of what tissues handle them, but also quantitatively, and chiefly by means of velocity quotients. Velocity quotients have the same dimensions as constants of chemical reactions, and wherever they are constant with time, correspond to reactions of the "first order," whether or not chemical transformations are involved. Their numerical values indicate how many times over, a load of the given amount would be disposed of in one hour. Diverse components in one species (table 40) of which the extreme loads studied are less than the tolerated loads, show an enormous range of values of this quotient. In man the extreme quotients differ by a factor of $10^{5}$; more extreme quotients may be found in still other components, as would be apparent if the recovery of excitability in nerve $(16,000,000 / \mathrm{hr}$., $\$ 128)$ were included in the comparison.

Grading components according to the velocity quotients shown in their adjustment, I note (table 41 ) that respiratory exchanges and heart frequency recover most rapidly. The elimination of 
certain electrolytes and the replacement of excised tissues are the slowest processes. Net exchanges of heat and of water are about equally provided for. About the same order among components is represented in the quotients available for two species.

Physiologists know that it takes longer to recover one's nitrogen balance than to recover one's breath; that an excess of bromide may last for weeks and an excess of lead for years; while an excess of $\mathrm{CO}_{2}$ lasts for a few seconds and a deficit of excitability for a millisecond. In poorly defined terms it could be said that the fastest adjustments are the most urgent; and for some of the corresponding components there is evidence that loads of them interfere

TABLE 41

Rates of recoveries in dog and in man. Data from tables 42 and 40 . Velocity quotient $=$ rate $/$ load $=1 /$ hour

\begin{tabular}{|c|c|c|c|}
\hline Component & Net exchange & $\underset{k_{\mathrm{H}}}{1 / \Delta \mathrm{t} \text { in }} \operatorname{dog}$ & $1 / \Delta \mathrm{t} \operatorname{in}_{k_{\mathrm{H}}} \operatorname{man}$ \\
\hline 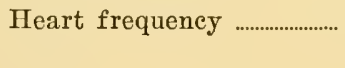 & $\begin{array}{l}\text { Gain } \\
\text { Loss }\end{array}$ & $\begin{array}{r}90.0 \\
330.0\end{array}$ & $\begin{array}{l}60.0 \\
29.0\end{array}$ \\
\hline Water ....... & $\begin{array}{l}\text { Gain } \\
\text { Loss }\end{array}$ & $\begin{array}{l}33.0 \\
0.45\end{array}$ & $\begin{array}{l}0.44 \\
0.33\end{array}$ \\
\hline Lactate & Loss & 1.6 & 1.0 \\
\hline 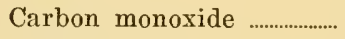 & Loss & 0.55 & 0.24 \\
\hline Creatinine & Loss & 0.45 & 0.21 \\
\hline Total substance & Gain & 0.004 & 0.01 \\
\hline
\end{tabular}

readily with many other processes. Stated more accurately, the velocity quotient is a suitable measure of the promptness with which recoveries occur.

The data also indicate a relation between velocity quotient and tolerated load. Attainable loads of carbon dioxide and of oxygen are very small compared to water in terms of molecules, grams, or other usual dimensions. For those two components a small increment in molecules is a large increment in per cent of the content at balance, however. Again, the ratios of turnover to content (table 40) are very large for those components having high velocity quotients (oxygen $=12$ ) ; this ratio may prove to stand in close proportion to the quotient. It may mean that high sensitivity to increment accompanies large change in relative concentration, such a change being secured by having the content small. Many exhaustive studies are required to furnish the data from which such correlations can be adequately and broadly drawn.

When quotients are considered in relation to loads, several 
varieties may be distinguished. The velocity quotient may relate rates to total loads (incremental $k$ ), or limit itself to any specified range of loads (limited $k$ ). Its rates may represent the total, the net, or the partial exchange; partial exchanges being as numerous as the measurable paths for the exchanges. Diverse time intervals are concerned wherever stationary states are not studied. Of course the varieties and sizes of the living units compared by the quotient are as large as taxonomy. As in all the relations so far discussed, therefore, there is nothing universal about

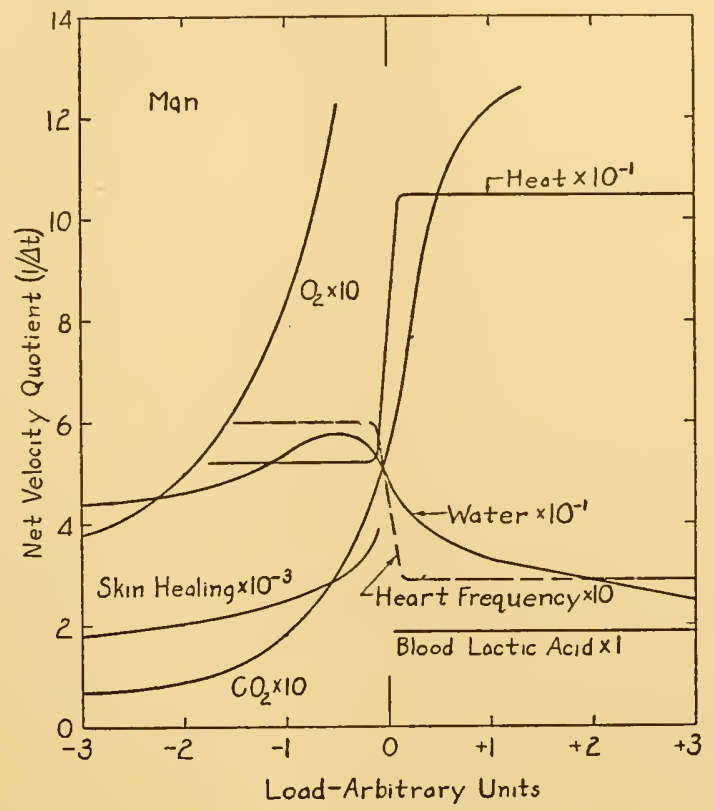

FIG. 181. Velocity quotient (1/hour) of net exchanges in relation to load. Man. The loads are in diverse units, the abscissae being scale divisions upon the graphs from which these data are derived. Water, first 1.0 hour of recovery, figure 61 . Heat, first 0.5 hour of recovery, figure 144. Oxygen, first 0.005 hour of recovery, figure 172. Carbon dioxide, steady state, figure 171. Lactic acid in blood, first 0.5 hour of recovery, figure 174. Heart frequency, first 0.005 hour of recovery, figure 175. Skin healing, 96-hour periods of recovery, figure 179 .

velocity quotients; they are means of comparison among particular distinguishable functions.

Total velocity quotients, where known, vary with load, usually approaching infinity at small loads and approaching stable values at large loads. Even net velocity quotients are for many components not constant at diverse loads; hence some of the comparisons made are arbitrary in so far as the loads present are unequal or 
incommensurate. Wherever commensurate, it is feasible to compare quotients at the numerically same load. Frequently the quotient happens to change but little (less than twice) throughout the range of loads investigated (fig. 181). Often a sharp shift in the value of $k$ occurs at $\mathrm{C}_{0}$. When the range of loads is limited to either positive ones or negative ones, no trends greater than a factor of 10 are actually found in net quotients.

The reciprocal of velocity quotient has the dimensions of resistance, for the load may be classed as a potential and the rate as a flow. When $k$ is constant with load, then, it appears that there is no greater resistance to the exchanges under large loads than under small loads. As for other parameters, the term resistance as here used may have little except its dimensions in common with the term as used in other sciences.

TABLE 42

Initial rates of recovery in dog. Velocity quotient, $k_{H},=1 /$ hour $=$ $0.693 /$ half-life in hours

\begin{tabular}{|c|c|c|c|c|}
\hline Component & $\begin{array}{c}\text { Net } \\
\text { excliange }\end{array}$ & Distributee & $\begin{array}{l}\text { Velocity } \\
\text { quotient }\end{array}$ & Source of data \\
\hline Heart frequeney .......... & Loss & ........ & 330.0 & $\begin{array}{l}\text { Brouha et al. ('36); Dill } \\
\text { et al. ('32) }\end{array}$ \\
\hline Water & $\begin{array}{l}\text { Gain } \\
\text { Loss }\end{array}$ & $\underset{66}{\text { Body }}$ & $\begin{array}{l}33.0 \\
0.45\end{array}$ & $\begin{array}{l}\text { Adolph; fig. } 10 \\
\text { Kingsley; fig. } 22\end{array}$ \\
\hline Calcium ………………….... & Loss & Serum & 3.0 & Freeman ('36) \\
\hline Lactate ……………….............. & "6 & Blood & 1.6 & Riegel ('27) ; Hahn ('33) \\
\hline Glucose & Gain & “6 & $\begin{array}{l}1.5 \\
1.0\end{array}$ & $\begin{array}{l}\text { Wierzuchowski ; fig. } 166 \\
\text { Zucker and Berg; fig. } 170\end{array}$ \\
\hline $\begin{array}{l}\text { Carbon monoxide ....... } \\
\text { Galactose }\end{array}$ & Loss & “، & $\begin{array}{l}0.55 \\
0.50\end{array}$ & $\begin{array}{l}\text { Stadie et al. ('25) } \\
\text { Bollman et al. ('35) }\end{array}$ \\
\hline 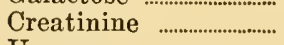 & "، & Body & 0.45 & Dominguez et al. ('35) \\
\hline $\begin{array}{l}\text { Urea } \\
\text { Propylene glycol ....... }\end{array}$ & “ & Blood & $\begin{array}{l}0.20 \\
0.10\end{array}$ & $\begin{array}{l}\text { Marshall and Davis ('14) } \\
\text { Newman and Lehman } \\
\text { ('37) }\end{array}$ \\
\hline Ethyl alcohol & ، & ، & 0.06 & ( 6 \\
\hline Brilliant vital red ....... & “6 & "، & 0.05 & Smith ('25) \\
\hline Plasma protein ............. & Gain & Plasma & 0.02 & Stanbury et al. ('36) \\
\hline$\underset{6}{\text { Total nitrogen }}$ & $\begin{array}{l}\text { Loss } \\
\text { Gain }\end{array}$ & Body & $\begin{array}{l}0.04 \\
0.01\end{array}$ & Rubner ; fig. 162 \\
\hline 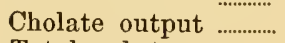 & Gain & Bile flow & 0.014 & Berman et al. ('41) \\
\hline Total substance .......... & Gain & Body & 0.004 & Morgulis; fig. 156 \\
\hline
\end{tabular}

If velocity quotients are considered in relation to time, $k$ is constant whenever an exponential equation represents the data: $\mathrm{C}=a e^{-k t}$. In such instances the rate of exchange is at all times proportional to load $(\mathrm{R} / \Delta \mathrm{C}=k)$. When $k$ is not constant with time, values are compared within some particular interval. This may or may not be a clock interval; it is possible to compare $k$ in the first half of each recovery, or first $1 /$ eth, or first $1 / 10$ th. 
If the half-life $\left(\mathrm{t}_{\mathrm{q}}\right)$ be ascertained, then $k=\ln 0.5 / \mathrm{t}_{\mathrm{q}}=0.693 / \mathrm{t}_{\mathrm{q}}$. That happens to be the manner in which the quotients shown in table 42 are obtained.

Hence the quotient indicates how much of the load has been dissipated in the interval chosen; its reciprocal indicates how long a time is required for completion (return) of a chosen portion of the task (load) incumbent on the organism.

\section{\$137. Paths of exchange}

Among diverse components it is possible to compare all those exchanged through any one particular channel, whether emunctory, synthesis, or alimentation. Here an approach is made to organ physiology, a science which seems at best to recognize a small part of the relations involved. Paths can rarely be distinguished for exchanges other than those by the body as a whole; separable paths of exchanges by parts of organisms are almost unknown except for certain types of chemical transformations.

Anatomically distinguishable paths (table 43) are of two kinds:

TABLE 43

Some paths prominently concerned in the exchanges of several components in dog and man. Gain $(G)$ and loss $(L)$. Those paths in which modification is known to occur with LOAD of the component in question are in italics

\begin{tabular}{|c|c|c|c|c|c|}
\hline Component & $\begin{array}{l}\text { Rectal } \\
\text { (fecal) }\end{array}$ & $\begin{array}{l}\text { Respiratory } \\
\text { (pulmonary) }\end{array}$ & $\begin{array}{l}\text { Excretory } \\
\text { (urinary) }\end{array}$ & $\begin{array}{l}\text { Synthethic } \\
\text { (chemical) }\end{array}$ & $\begin{array}{l}\text { Alimentary } \\
\text { (ingestive) }\end{array}$ \\
\hline $\begin{array}{l}\text { Water } \\
\text { Heat }\end{array}$ & L (G) & \multirow{5}{*}{$\begin{array}{l}\mathrm{L} \\
L \\
\mathrm{~L} \\
L \\
\stackrel{L}{L} \underset{(G)}{G}\end{array}$} & & $\begin{array}{l}\mathrm{G} \\
G,(\mathrm{~L})\end{array}$ & $G,(\mathrm{~L})$ \\
\hline $\begin{array}{l}\text { Total substance ...... } \\
\text { Glucose }\end{array}$ & & & $L$ & & $G$ \\
\hline $\begin{array}{l}\text { Glucose } \\
\text { Carbon dioxide ....... }\end{array}$ & (G) & & $\begin{array}{c}L \\
(\mathrm{~L})\end{array}$ & $\begin{array}{l}G,(\mathrm{~L}) \\
G\end{array}$ & \\
\hline 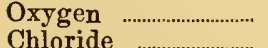 & (L) & & ( & & $G$ (I) \\
\hline Lactate & & & (L) & $G, \quad L$ & 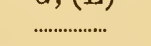 \\
\hline
\end{tabular}

One-way paths (renal, most alimentary) allow only losses or only gains. Two-way paths (pulmonary, chemical) regularly exchange certain components in both directions; however, a particular component ordinarily moves in only one direction. Reciprocating action allows certain economies of movement (breathing), and is inherent in respiration and in some chemical transformations. So far as is known the kidneys of mammals do not reciprocate, except if a loss of water is defined as a gain of total concentration, or the like. In contrast, the alimentary apparatus serves for losses by excretions, regurgitations, and salivations. Conversely, each path 
of exchange is characterized by its role in respect to each component.

Exchanges might also be classified as reversible and irreversible. In a strict sense an irreversible process is one from which there is no recovery. When a man loses a leg, replacement does not occur; yet there is some healing and there are certain functional compensations. In a second sense many water exchanges are termed irreversible, for what goes in through the mouth ordinarily comes out through other organs. Yet these organs are so coordinated that many "errors" of intake are accurately adjusted by rates of output, and vice versa. Few indeed are the instances where chemical reversibility regularly operates in living organisms; yet whatever happens is reversed so far as the whole individual is concerned, for loss of any component is the reverse of its gain. To distinguish a "biological" reversibility is hardly necessary, since the living unit simply combines processes or rates of activity that the dead unit does not use. But, perhaps most physiological phenomena are just peculiar combinations.

Organisms' gains or losses of particular components at measurable rates, occur by processes that are not understood sufficiently to allow them to be classified with much finality among varieties of forces or energies recognized in physics or in chemistry; as diffusion, conduction, convection, radiation, chemical transformation, synthesis and decomposition. This statement is not at variance, I believe, with the fact that the major efforts of biologists of a generation have been exerted in the hope of securing such identifications. Consideration of the whole body, on the other hand, instead of portions of it such as the blood plasma or the kidneys, seems to be the key to measuring the relations in which tissues, organs, and individuals may be compared.

Some rates of recoveries are probably limited by patently mechanical events, such as recovery of posture in swaying, recovery of limb position in running or boxing, movement of food out of the alimentary tract, change of lung volume. Similarly, in any vibratory or pendular movement the recovery may be restricted by a "natural" frequency. Such limitations are generally identified from comparisons with dead units as models. They emphasize to me merely that anatomical or microphysical provisions are sometimes as crucial in the organism's life as factors that are less familiar. 
Some physiologists would say that recovery is "of course" rapid for those components that are handled by organs of large proportions such as organs of respiration. This view regards anatomy not only as separate from physiology but antecedent to it. Equally, the ample organ is caring for those components for which rapid elimination or absorption often arises. Again, in one organ such as kidney there is at one time rapid elimination of one component and slow elimination of another. No one knows whether ample provision for eliminating $J_{1}$ invokes hypertrophic function with respect to any $\mathrm{J}_{2}$. $\mathrm{Or}$, leaving organs aside, there is provision for the rapid production of glucose within a dog and not of raffinose. Shall the organism be said to lack inventiveness toward making the latter substance, or shall the actual provision be regarded as sufficient and any greater provision as encumbering? Questions of this sort lead to no decisive answers; only the description of the rates found while the investigator asks the questions, corresponds to the facts known.

A problem of the organism is how to regulate the most components, each in the largest range of rates, with the least machinery. Were a separate machine (organ) present for each, the burden of rarely active tissues would be enormous. By multiplying the uses of one structural unit, economy of body is greater. It was once widely supposed that one "organ" served only one function, though I presume one function might include its dealing with many components.

The kidneys are examples of organs that regularly excrete scores of components, and each in an independent or nearly independent clearance. How the independence of one component from another is secured, no one has ascertained. To some small extent histological differentiation is correlated with diversity of the components handled, e.g., glucose is absorbed in proximal tubules of frog, chloride in distal tubules. But there are not so many visible differentiations as components. In fact, unforeseen (foreign) components (e.g., diodrast or phenol red in dog) are handled just as specifically as usual ones. Recovery from excesses of a hundred components occurs through one and the same kidney.

"In a living organ we are dealing with something of which the functions, if we speak of functions, are endless, since the activities are endless, constantly seeming to grow in number as we investigate further. Its true function, to the eye of a physiologist, 
is to maintain these endless activities in balance with the endless activities of other organs, and not merely to perform one specified action"' (Haldane, '17, p. 85).

Where paths are still less visibly specialized, as in capillary surfaces and cell surfaces, it is said that "permeability" controls exchanges. It is often inferred that $(a)$ permeability is fixed, and that $(b)$ one surface limits all components. Neither of these inferences seems at present justified by adequate data. Rather, there is evidence that exchange of the one component in deficit or excess is modified without modification of exchange of many other components. This fact points to equilibration of the same sort as in whole organisms. It means that $(a)$ conditions for exchange (permeability) are not fixed, $(b)$ permeability is not just a limiting: factor, $(c)$ permeability is not all one function, and $(d)$ permeability probably is not vested in an ordinarily pictured monolayer.

I find no evidence upon which to base a conclusion that disposals by storage or by chemical tranformation are faster than or slower than disposals by elimination or by translocation. Even in the same species supposedly identical paths do very dissimilar things. Each component still requires study in its own right; no rules have been uncovered by which the rates of exchange may be predicted.

For almost any one of the components that have been investigated it is possible to distinguish two or more paths of simultaneous exchange. In those same components, however, only one path varies its rate of exchange with any one load. The impression might be gained that the constant exchange (turnover) is by unavoidable paths; whereas the particular path that interferes least with other components is the sole department of adjustment, suiting its rate of specific activity to the special task before it. In the case of glucose (dog) three paths of adjustment were distinguished, each of which varied its rates of exchange over restricted ranges of load, and no two were alike. Or, three disposals came into operation in diverse proportions; synthesis, oxidation, and excretion. There is probable utility to the organism in this precise arrangement of functions and in the exact overlapping by which they share with one another and reinforce one another. The competition among the paths of an organism is a quantitative ordering of activities so that excretion does not seize that which synthesis will preserve, and vice versa. 


\section{$\S 138$. TXPES OF LOADING}

The means by which increments are produced in organisms might merit an amount of study equal to that here put upon the recoveries from increments. Indeed much effort in current physiology goes into the search for methods and agents by which particular components may be experimentally disturbed. The means (types) are in part peculiar to each class of organisms, and therein define its properties. Increments of many, perhaps all, components are to some extent avoided or resisted by organisms; and one study that might be made, though not attempted here, is to find how few components suffer increment from each agent.

An organism in balance is defined as one that maintains constant, within measured limits, the contents of one or more specified components. Persons who speak of positive and negative balances, would in my terminology speak of positive and negative increments, or retentions and depletions. Similarly, those who speak of "levels" of intake would mean rates of intake or of turnover. "Levels" of concentration or of composition are, on the other hand, usually contents of the component specified.

Increments or loads arise incidentally in numbers of situations in which the organism finds itself. Often loading is the organism's reaction to recognizable stimuli, often not. Descriptively speaking, positive loads follow (a) forced gains at rates exceeding the rates of loss, (b) inhibited losses at rates smaller than the rates of gain, or (c) both. Negative loads follow the inverse conditions; but where no turnover is present only (a) is possible. For some components only (b) is feasible.

The load is maintained in a stationary state when forced gain is equated with concurrent loss, or vice versa. Data of physiological significance are obtained chiefly when the organism is free to manage at least one of the two exchanges; ultimately it then exhibits the load and rate characteristic of State III (fig. 180). When the experimenter decides the rates of both gain and loss, the organism exhibits nothing but a changing load. When the experimenter decides neither the intake nor the output, the organism enter State IV and recovers (at characteristic rates).

For some components the rate of some exchange in State IV is not measurably different from the rate of its free exchange in State III, at any one load. No method is apparent of predicting for which components or under which conditions this holds true. There 
is no evidence that exchanges in the stationary state are any more or less characteristic of the organism's physiological constitution than the exchanges in any other states. But they have the great advantage, in common with exchanges in State I, of being independent of chronology or nearly so.

For many a component it has been observed that the more of it the body has, the more of it is steadily exchanged. Or, within limits $\mathrm{R}$ is positively correlated with $\mathrm{C}$. Independently investigators rediscovered this for total substance, total energy, nitrogen, water, and carbon dioxide. Surprise would have been saved in $(n-1)$ instances had there been any inductions concerning equilibrations in steady states.

The rich organism is sometimes (a) one that has much, sometimes (b) one that has credit, and sometimes (c) one that spends much, either by high turnover or by temporarily reducing its content. All these meanings are disentangled by relating $\delta \mathrm{C} / \Delta \mathrm{t}$ with $\Delta \mathrm{C}$ and $\mathrm{C}$.

The loadings and unloadings of diverse components that have been studied in one species have not all been measured in the same individual, nor simultaneously, nor under like conditions. Hence it is by no means certain that they are comparable. Comparability becomes most certain by simultaneous measurements in the same individual (chapter XVII) of diverse components and of their exchanges. Then the question arises whether component $J_{1}$ behaves the same when $J_{2}$ is also modified, and when agent $Y_{2}$ is substituted for $Y_{1}$.

So a study of loading and recovery consists in mensuration of a group of concurrent changes. The changes found characterize the agent inducing the load, and the organism reacting. The distinctions among agents and among modified properties replace the customary generalizations from isolated observations made, without benefit of clocks, upon diverse species and under random conditions.

\section{\$139. Comparison of Kinetic parameters}

It is desirable at this point to compare several terms that are currently employed in designating the exchanges of components by organisms and their parts. Names used for these quantities are metabolic rate, clearance, accumulation rate, invasion coefficient, permeability coefficient, velocity quotient. All are kinetic quan- 
tities of recognized dimensions; each has its connotations. What usefulness has each in describing phenomena of the sorts that are dealt with in this investigation?

Metabolic rate is ordinarily the number of molecules or quanta appearing or disappearing in a unit of living material per unit of time $\left(\mathrm{L}^{3} \mathrm{~L}^{-3} \mathrm{~T}^{-1}\right.$, or $\left.\varepsilon \mathrm{L}^{-3} \mathrm{~T}^{-1}\right)$. Instead of a unit volume or mass $\mathrm{L}^{-3}$, a unit of surface $\mathrm{L}^{-2}$ is often used, and occasionally a unit of length $\mathrm{L}^{-1}$, an individual, a population, a cell. In practice, metabolic rate is sometimes limited to processes that involve recognized chemical transformations; occasionally it means only rate of oxygen consumption. Originally, of course, it had little quantitative connotation. Often it is regarded as applying chiefly in stationary states.

Clearance started from the specific definition: "the volume of blood which one minute's excretion of urine suffices to clear of urea" (Möller et al., '28). The volume flow $\left(\mathrm{L}^{3} \mathrm{~T}^{-1}\right)$ is a virtual one and not a visible one, as is more evident in the older equivalent, urea excretory ratio: urea excreted in one hour's urine/urea found in one volume of blood. Usage already extends the term clearance to other volumes than blood (e.g., plasma, volume of distribution, body) to other intervals of time than minutes (e.g., hour), to other disposals than excretion (e.g., chemical conversion), to other paths than urinary (e.g., hepatic, unknown) and to other substances than urea (e.g., creatinine). Hence investigators now speak of plasma clearance, galactose clearance, renal clearance, complete clearance, filtration clearance, tubular clearance. The original definition is no longer a sure guide to the meanings. Sometimes clearance is referred to a unit of supposed body surface; accordingly the dimensions are often $\mathrm{L}^{3} \mathrm{~L}^{-2} \mathrm{~T}^{-1}$, which overlap those for metabolic rate. In supposition clearances occur in stationary states; in practice they are measured as often in states of unloading. Originally clearance was distinguished by the fact that no overall chemical transformation but only translocation was known to be involved, the same substance being measured in urine and in blood. But more recently it is permissible to measure merely the rate of disappearance from blood (e.g., Bollman et al., '35), really a rate of decrement in concentration, and to call this $\left(\mathrm{L}^{3} \mathrm{~T}^{-1}\right)$ a clearance without recognizing the path of exchange. It is evident that no unanimity of usage prevails, even to the extent of preserving dimensions other than the factor $\mathrm{T}^{-1}$. Clearance is nearly always a 
rate of emptying, and that limitation necessitates a separate term for rate of gain.

Accumulation rate is such a term. Ordinarily it is related to body mass and has the dimensions $\mathrm{L}^{3} \mathrm{~L}^{-3} \mathrm{~T}^{-1}$, but frequently it too is referred to unit of believed surface of exchange. Occasionally it is restricted to net exchanges, and to movement against a gradient of concentration.

Invasion coefficient (Bohr, 1899) was devised to express the changes of phase (partitions) involved in intake of oxygen and output (evasion) of carbon dioxide. It recognizes the differences of partial pressures existing at two ends of a gradient, and includes ostensibly more processes than diffusion. It is the number of molecules passing down the gradient of partial pressure per unit of surface and of time $\left(\mathrm{L}^{3} \mathrm{~L}^{-2} \mathrm{P}^{-1} \mathrm{~T}^{-1}\right)$. Certainly the terms invasion and evasion have emphasized the direction more than any other factor of exchange.

Permeability coefficient is usually defined so as to apply only to such movements of substance or heat along a gradient of pressure or concentration as are induced by the gradient itself. But it also recognizes the surface area of exchanges $\left(\mathrm{L}^{3} \mathrm{~L}^{-2} \mathrm{P}^{-1} \mathrm{~T}^{-1}\right)$. Sometimes the coefficient $(h)$ and time $(\mathrm{T})$ are lumped together into a "minute number." Wherever the thickness over which the gradient extends is also measurable, a coefficient of diffusion (Fick, 1855) may be computed $\left(\mathrm{L}^{3} \mathrm{~L}^{-2} \mathrm{~L}^{1} \mathrm{P}^{-1} \mathrm{~T}^{-1}\right)$. A coefficient of osmosis may be kept separate to designate the molecular movement of water.

Velocity quotient has been used here in connection with phenomena of excretion. In all but name it has long been current in connection with changes of excitability (time constant), rate of relative growth (increment of size per present size per unit of time) and certain other physiological events (velocity constant). Possibly its virtual use is coeval with the expression of biological phenomena by exponential equations. It is the change of quantity in a given time per unit of quantity present $\left(\mathrm{L}^{3} \mathrm{~L}^{-3} \mathrm{~T}^{-1}\right)$, or, by extension, any $\mathrm{T}^{-1}$. It may be computed from any of the above parameters of exchange, and often vice versa.

Recovery rate has had no previous distinct vogue, though often employed qualitatively. It might be defined as the net rate of exchange relative to the load $\left(\mathrm{L}^{3} \mathrm{~L}^{-3} \mathrm{~T}^{-1}\right)$. While the load is diminishing it is not equivalent to metabolic rate, but relates the exchange to the load of the component being exchanged, and not to the 
organism's mass as such; and is not concerned in stationary states. It is identical with a net velocity quotient in any State IV.

This list of kinetic parameters that have already been used in biological work might be extended indefinitely. There may be no iron-clad objection to the formulation of quotients for each new dimension and component that can be studied. But, two practical problems arise. First, a new name or letter is customarily used to express relations found in each phenomenon analyzed; later it is usual for the phenomenon to gain membership in a much wider class. Then the parameter corresponding to it loses the original and special meaning. Seemingly this endlessly arising situation may be avoided by noting the dimensions of each parameter used and trying to use only one name for each combination of dimensions. That is very difficult to do consistently in one's own investigations and still more difficult to plan for in advance in a democratic world. Second, every analysis of biological phenomena involves connotations as well as quantities. Is it more useful to unite clearance and metabolic rate under one term, or to maintain a biological separation that has no algebraic justification? Both views have their merits, and in diverse connections each solution is useful, even in one investigation.

Whereas an engineer would speak of Flow $_{1}\left(\rho_{1}\right)$, Flow $2\left(\rho_{2}\right)$, Flow $_{3}\left(\rho_{3}\right) \ldots$, physiologists are prone to speak of excretion rate $(\mathrm{A})$, utilization rate $(\mathrm{B})$, distribution rate $(\mathrm{C})$ and disposal rate $(\mathrm{A}+\mathrm{B}+\mathrm{C})$. The latter list bristles with implications, implications that unfortunately differ for each reader.

Not being a reformer, I am content to point out the situation. Every observation calls forth a desire to distinguish it from every other and a desire to identify it with others. The observer strikes some sort of a compromise. At any one stage of a science, phenomena are on the whole being lumped as their identities become emphatic, and split as current needs indicate. Common dimensions are supposedly prerequisite for lumping, but do not compel it.

\section{$\$ 140$. Changes in tissues, other metabolisms, etc.}

The data presented in this investigation illustrate the widely recognized fact that any physiological state involves some modifications in a number of components. Excess of glucose makes itself felt in heat production, circulatory changes, lactate metabolism, lipid deposition, shifts of electrolytes, water deposition, and water 
excretion. Conversely, the multifarious contents and exchanges of any one component are manifested in loads of many other components. Hence it is possible to compare, for instance, the rate of water excretion or ingestion in relation to diverse loads of each of several components $(\$ 80)$. Various "reactions" of compensation and defense are commonly recognized in pathological science (Bloom, '40), each of them representing' a load or a rate that accompanies some particular type of disturbance of usual content.

Several parts of one organism are simultaneously recovering from the load of the whole with respect, for instance, to water or to glucose. Every cell, tissue, organ, and organism has its own recovery rate; and comparisons among the simultaneously observed units may be as extensive as the data. Whereas blood and liver may be alike in rates of return of water load, they may be enormously diverse with respect to glucose exchange. Their diversities express in quantitative fashion those divisions of labor that have been the objectives of much study. For, "special functions in special tissues" has been a mainstay of physiological concepts since Galen.

\section{\$141. SPecies}

Data such as have been presented in previous chapters furnish materials for physiological characterization of individuals, species, and classes of animals. They also extend to cells and organs. What are some of the manners in which they may be arranged to this end?

It was found practical ( $\$ 70)$ to distinguish numerous species, in respect to a single component, by their rates of turnover, their rates of maximal exchange, etc. With data now at hand for several components in one species, further differentiation is in order. A list of components and exchanges such as is shown in table 40 characterizes, I judge, only one species. Certainly the list for man differs significantly from that for dog (table 42). I believe the relative quantities can suffice to show the difference, independently of the absolute or physical units there employed.

Equilibration diagrams serve as epitomes of most of what is known concerning regulation of each component, and a book of such diagrams might be composed for one species. Or, adopting a uniform arrangement and set of scales for all the materials, several components may be put in direct comparison in one dia- 
gram. Thus data are available for water (fig. 29) nitrogen (fig. 162) and glucose (figs. 166 and 170) in the dog. Taken together (fig. 182), these net equilibration diagrams furnish a beginning of a physiological description of the species, for they deal with quantitative activities that occur in response to each component's displacement. In an anatomical description, the initial study might be the observation of four legs and a head; later the relative sizes and growths of these parts are accurately ascertained. No less significant, I believe, are the relative magnitudes of the physiological processes that occur in one body. Plenty of "reasons"

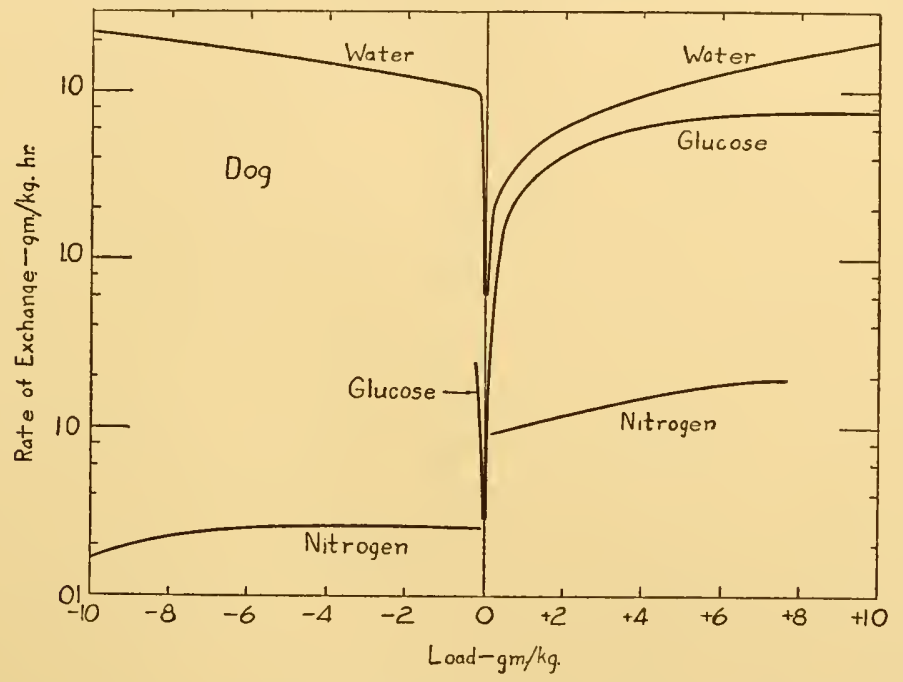

FIG. 182. Rate of net exchange plotted on a logarithmic scale, in relation to load, for three components. Dog. All are gains in deficits and losses in excesses. All rates are measured in steady states. Loads are arbitrarily compared by weight/kilogram of $\mathrm{B}_{0}$. Data are from figures 29 (water), 166 and 170 (glucose), and 162 (nitrogen).

might be hypothesized why an excess of nitrogen "should" be eliminated less readily than an equal excess of water or of glucose; none are set forth now. Here are the facts; these are the scales upon which the dog handles the three components. Modification ratios, augmentation ratios, maximal rates, economy quotients, and velocity quotients all characterize the component as well as the species. Quantitative differences among components are discerned, independently of whatever machinery aids recovery of each in the body.

Certain comparisons can be made by denominating initial loads as 100 per cent (fig. 183), and following their subsequent history. 
That plan makes evident the relative time scales in which recoveries operate. Sometimes it is possible to limit comparisons to the shapes of tolerance curves, leaving the loads in whatever arbitrary units are convenient for covering the range of known data. Recovery depends upon net exchange, for no amount of increased intake would overcome equally augmented output. Increments of some components are disposed of with great haste (oxygen,

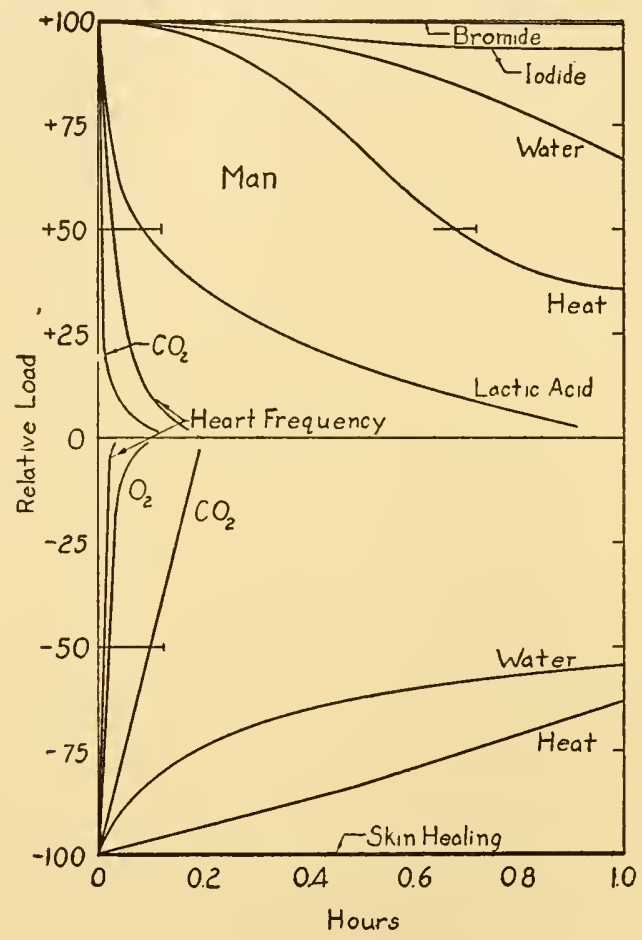

FIG. 183. Relative load in relation to time for nine diverse components. Man. Bromide, data of Hale and Fishman ('08). Iodide, data of Anten ('02). Water, from figure 51 (A) and figure $56(\mathrm{P})$. Heat, from figure 143 . Lactic acid in blood, from figure 173. Heart frequency, same data as are represented in figure 176. Carbon dioxide, approximate data of Adolph et al. ('29); and of Liljestrand ('16), cf. figure 171 . Oxygen, from figure 176. Skin healing, data of Carrel and Hartmann ('16), cf. figure 179.

carbon dioxide), others almost not at all (fat, calcium, lead). Correspondingly the content of carbon dioxide is exceedingly uniform, that of fat not. Hence adjustments of diverse components are to be classed according as the responses to loads, expressed perhaps as velocity quotients (tables 42 and 40 ), are fast or slow. Lists of such quotients give the order in which recoveries appear 
to the organism (table 41), the order being nearly the same in two mammalian species. Among anatomically more diverse species it is probable that somewhat greater physiological differences also will appear.

When the compensatory equilibrations in an organ and in a whole individual are compared, it will probably be found that the latter has (1) adjustments to more components, and (2) greater independence of adjustments among the several components. Adequately to test (1) is difficult within a human lifetime. Statement (2) has not been rigorously tested. If the man receives water with any known combination or quantity of solutes, contents of water and solute are eventually restored. But no one organ in situ is known to recover fully without the whole body recovering. And no isolated organ so recovers, to present knowledge. Here are outlined two of the many quantitative methods of comparing regulations in the organism and in its parts.

It is a temptation to suppose that the compensations of the whole body are prerequisite to the recoveries of each compartment in it. For instance, the maintenance of body $\mathrm{B}$ might enable maintenance of "extracellular" volume $\mathrm{V}$, it in turn enables maintenance of cell $\mathrm{L}$, and it in turn enables maintenance within it of Golgi body $\mathrm{N}$. The dog regulates heat content in $B$, but such regulation is unknown for any $\mathrm{V}$ or $\mathrm{L}$ of the dog's body. On the other hand, the properties by which $\mathrm{N}$, L, or $\mathrm{V}$ exchange water with their surroundings may be just as prerequisite to B's water maintenance as the reverse. All the units are coordinate, so far as I perceive, each of them regulating its components in its own sphere and fashion; there is no tenable distinction between prerequisites and postrequisites.

Comparative physiology evaluates the differences among species, and the same data that manifested the uniformities serve also to indicate the contrasts among living units. Quantitative differences need not depend on anatomical or chemical differentiations, and may be finer distinctions than they. Whereas biochemical tables list the compositions, potentials, and catalysts of several species, physiological tables comprise their rates of change in selected states. Comparisons have in the past usually been limited to those between two species and in respect to one component in one load. Evidently any scheme that coordinates the information of numerically diverse loads, and is capable of serving for all spe- 
cies, extends the field of work. Anyone may derive new views and concepts in comparative physiology by listing the accuracies of regulation (variability of content), or the modifications of exchange, of some component in several species.

\section{§ 142. Equilibration diagrams}

Having found equilibration diagrams of great use in representing what is known of regulations by compensation, I summarize their characteristics. They are constructed as follows:

(a) The increments of content were ascertained for some component in a living unit. These increments were termed loads $(\Delta \mathrm{C})$.

(b) The content of the component in the same or another individual in a control state was taken as zero load $\left(\mathrm{C}_{0}\right)$.

(c) The rates of gain and of $\operatorname{loss}(\delta \mathrm{C} / \Delta \mathrm{t})$ of the same component were ascertained at diverse loads.

(d) The units employed in measuring $\mathrm{C}$ were either sensibly identical or believed similar in loads $(\Delta \mathrm{C})$ and in rates $(\delta \mathrm{C} / \Delta \mathrm{t})$ of one component.

(e) When rates were proportioned to loads, velocity quotients $(1 / \Delta t)$ were obtained that were independent of the dimensions of $\mathrm{C}$.

(f) The diagram correlating rates with loads was constructed in a uniform fashion for several chemical constituents, energies, forces, and frequencies of diverse organisms.

(g) Quantitative comparisons were facilitated among many components with respect to the relative rates and velocity quotients found.

The generalized equilibration diagram (fig. 110) has the following features:

(h) $\mathrm{G}$ (gain) $>\mathrm{L}$ (loss) whenever $\Delta \mathrm{C}$ is negative, and $\mathrm{L}>\mathrm{G}$ whenever $\Delta \mathrm{C}$ is positive. Each is of a nature that tends to restore $\Delta \mathrm{C}$ to zero.

(i) At balance $\mathrm{G}=\mathrm{L}$. This is found to occur in those states that were chosen as controls or zero loads.

(j) At other loads than zero, either $G$ has another magnitude, or $\mathrm{L}$ has another magnitude, or both.

(k) The rate of net exchange increases as load increases.

(1) The slopes $(1 / \Delta t)$ of the lines correlating net rates with loads near the zero load, measure the velocity with which any small load that accumulates is again lost from the organism.

(m) The equilibration diagram represents a symmetry of proc- 
esses that restore the organism after negative and after positive displacements from balance. It represents also a contrast of total gains and of total losses at any one load.

I think the physiological importance of the features found to prevail among all equilibration diagrams is best exhibited by supposing that the diagram turned out differently:

(n) It might have occurred that neither $\mathrm{L}$ nor $\mathrm{G}$ varied with load. Then one value of $\mathrm{C}$ would be as likely to occur as any other ; no value would persist nor be approached in recovery, except temporarily and by chance.

(o) L might vary, but equal $\mathrm{G}$ at all loads, with the same consequence.

(p) In negative loads $L$ might exceed $G$, and in positive loads vice versa. Then recovery would be impossible, for the content would move farther from $\mathrm{C}_{0}$. These possibilities were named in $\$ 72$.

(q) L might vary, but equal $\mathrm{G}$ at more than one load in the same individual. Then if once sufficiently displaced in content, a new balance would be maintained.

(r) L and G might vary at random and independently, never being coordinated. Then recovery would be wholly left to chance, like an animal in an insoluble maze.

It is apparent that there are numerous possible arrangements in the organism that would not automatically lead to recovery. That these other arrangements are not found, indicates to me that the relations represented are of consequence to the organisms concerned, that probably the species would not be here without arrangements that are successful and that insure survival.

It was mentioned $(\$ 72)$ that total exchanges $G$ and $L$ might each be classed into 11 types of modification in relation to load. Together, 121 combinations of the two seemed possible; 96 were judged incompatible with recovery. Of the remaining 25 combinations only a few were found for the component water, but several have turned up in the study of other components. Reviewing the equilibration diagrams that have been presented, I find 6 of the 25 in actual operation, not counting fragments. These 6 are represented in figures $13,61,72,146,171$ and 172 . When any other type of equilibration diagram shall be found, it will offer an interesting contrast with these.

It is possible to suggest a few incidental features of physiology 
that may have discouraged the previous realization of quantitative equilibrations: (1) Until recently, long periods of time were used in measuring metabolisms and other rates of activity. Hence recovery of balance was often completed before the measurement of exchange was made. (2) Conditions of balance were preferred; turnover and minima were ascertained first. (3) Loads were measured chiefly in blood. But the rates of exchanges by blood were too rapid to measure in some instances; it was difficult to assume that volume of blood was constant; and only net rates of change in concentration were ascertainable. (4) It was supposed that all the separable processes of exchange needed to be labelled before description of the exchanges could be undertaken. For the most part, however, the materials were at hand, and a mere shift of inquiry finds new relations present among them.

The specification of conditions for study, of course, suggests innumerable further studies by these procedures. Equilibrations would be concerned with all the capacities of living units to combat excesses and to make up deficiencies. In a general way several biological sciences are mainly concerned with just these; particularly toxicology, pathology, ecology, and physiology. One of the imputed virtues of a scheme like that of the equilibration diagram might be that one investigator need no longer be a specialist on one component. Rather, having studied the relations of $J_{1}$ in one living unit $\mathrm{U}_{1}$, he is now qualified to tackle any $J$ in any $U$ with which he is interested in becoming familiar. He may revel in any quantitative relations and not be confined to a single component.

What quantities (components) in organisms are susceptible of study by equilibration diagrams? I should predict that any measurable characteristic of any living unit may be correlated with the rate at which the same characteristic changes. Seemingly any procedure for quantitative measurement, any organism, and any clock, are all that are requisite to the investigation of an equilibration. The devices or procedures employed are not limited to those familiar in any one field of biology or of non-biological science. Wherever there is a definable system, changes occur in it whenever displacements or increments of component occur.

$\S 143$. Summary of the variables studied

For any one component, some nine sorts of quantities were investigated. These were roughly classified ( $\$ 101)$ into three dis- 
continuous variables: living unit, type of loading, and path of exchange; and six continuous variables: load, time, rate of exchange, velocity quotient, change in tissue (volume, dilution, amount) and change in activity (energy transformation, behavior). A useful summary of these variables is their definition, which may now be succinct, together with references to the foregoing use of certain ones.

Living unit, $\mathrm{U}$, the portion of organisms under actual observation or measurement.

Component, $\mathrm{J}$, any measurable property of the living unit.

Content, C, amount of component present in the living unit.

Load, $\Delta \mathrm{C}$, increment of content;

Excess, $+\Delta \mathrm{C}$; Deficit, $-\Delta \mathrm{C}$ (fig. 110).

Balance, $\mathrm{C}_{0}$, when $\mathrm{C}=0$.

Time, $\mathrm{t}$, hours by the solar clock.

Rate of exchange, $\mathrm{R}$, rate of transfer or change of component, $\delta \mathrm{C} / \Delta \mathrm{t}$.

Gain, G; Loss, L.

Total, net, partitioned.

Loading, numerical increase of $\pm \Delta \mathrm{C}$.

Recovery $=$ Unloading, numerical decrease of $\pm \Delta \mathrm{C}$.

Recovery rate, net $\mathrm{R}$ during unloading.

Turnover $=$ Turnover rate, $\mathrm{R}_{0}$, or $\mathrm{R}$ at $\mathrm{C}_{0}$, when $\mathrm{L}=\mathrm{G}$.

Tolerance, sequence of loads.

Velocity quotient, $k, 1 / \Delta \mathrm{t}, \mathrm{R} / \Delta \mathrm{C}$.

Augmentation ratio, $\mathrm{R}_{\mathrm{m}} / \mathrm{R}_{0}$ where $\mathrm{R}_{\mathrm{m}}$ is measured total rate, usually maximal or minimal.

Modification ratio, $\mathrm{R}_{\text {max. }} / \mathrm{R}_{\mathrm{m} 1 \mathrm{n}}$.

Economy quotient, G/L.

Physiological state $=$ State, combination of contents in the living unit, with emphasis on the one or more contents measured.

Condition, any environmental situation of $U$ believed to be reproducible.

Stationary state, any state in which $\mathrm{C}$ does not change with time, hence $\mathrm{G}=\mathrm{L}$.

Kinetic equilibrium $=$ Normal, stationary state in which $\mathrm{C}_{0}$ and $\mathrm{R}_{0}$ are present.

Equilibration, group of relations of exchange to content, or rate to load.

Body mass, B, measured weight or volume of $\mathrm{U}$. 
Volume, $\mathrm{V}$, any volume in $\mathrm{U}$ measured by a specified procedure. Usually a volume of distribution $\left(V_{D}\right)$ of a distribuend added to or subtracted from $\mathrm{U}$.

Dilution, E, reciprocal of concentration of some substance or physical colligative found in some part of $U$ that was analyzed.

It may be piously hoped that few inferences will be drawn from the terms used in this nomenclature, that would add to the meanings conferred by the methods of measurement as actually used. In so far as each term is an abstraction, it is only so by virtue of the fact that similar measurements were employed for similar data. If these actual methods be kept in view, it may be more difficult than otherwise to wander far into metaphysics from the phenomena and relations so described.

\section{\$144. General summary}

All the quantitative correlations in this investigation fall into a very few types. Each type includes those correlatives having certain dimensions; hence to summarize them it is only necessary to refer to the nine classes of variables $(\$ 101)$. Taken two at a time the correlations would fall into 36 types. In actuality those utilized were more limited than this; in nearly every case either time or load was one correlative; hence, at most, 15 types prevailed. A few of these correlations were common enough to merit special names; these were:

Load vs. time, the tolerance diagram. $\Delta \mathrm{C} \propto \mathrm{t} . \mathrm{Ex}-$ amples, figures 180 (A), 1.

Rate of exchange vs. time, the exchange diagram. $\mathrm{R} \propto \mathrm{t}$. Examples, figures 180 (B), 2.

Rate of exchange vs. load, the equilibration diagram.

$R \propto \Delta \mathrm{C}$. Examples, figures 110, 13 .

Particular portions of tolerance curves, or curves for particular components, are currently designated by special names. Thus, there are insulin sensitivity curves, contraction curves, elimination curves, accumulation curves, and penetration curves. Similarly, particular kinds of exchange curves are diuresis curves, excretion curves, and age curves.

It seems to me that by the classification of those and other variables, and of those and other correlations, into a few types, a huge mass of data assumes manageable proportions. Instead of a be- 
wildering miscellany the material becomes ordered and useful. Considered in this way all that has been presented may be viewed as requisite to comprehensions of organisms. Thereupon it becomes possible to derive general statements concerning what organisms do to maintain their constant properties.

It is clear that regulations have to do with what I have defined as contents (properties) of the organism. Content of each component varies within the limits fixed by the processes of its maintenance. Departures from this norm are forestalled by behaviors whereby the animal frequents environments that favor the norm, and by compensations whereby exchanges are modified in a direction to restore the norm. Some processes are ready at all times to prevent or to correct departures of every component. A separate structure is not available for performing each such duty. All the processes have patterms that are similar in many features that have been mentioned; in addition each exhibits quantitative differences peculiar to it. The species is physiologically characterized by that particular combination of these quantitative features which it embodies. 


\section{Chapter XVII}

\section{INTERRELATIONS AMONG COMPONENTS}

$\S 145$. Up to this point I have studied each component as though it were independent of others. Though an organism evidently has many components under regulation, each could be examined separately. Had that not been possible, but little progress in their analysis would have been made by the correlation of so few variables as were actually recognized.

The more closely a physiologist examines what is going on in an animal, the more he realizes that any single component is not receiving the organism's whole "attention." To find mutual influences at work, he may measure adjustments of two or more components that are simultaneously disturbed. No new materials might be needed to show coincident recoveries among several components, for some have already been presented in the form of correlatives of water load $(\$ 80, \S 85)$. There, a tendency possibly prevailed to regard water increment as an independent variable and other components as dependent. A more explicit study of several components upon an equal footing, and the exchanges of each, may therefore serve to indicate what a bag of interrelations the organism is.

Data appropriate to the study of great numbers of simultaneous properties and events are not difficult to record, for tables may fully represent them. The desire to visualize more and more relations among them, however, drives me to explore additional methods of representation. The same sort of urge that compels an investigator to seek more data, here plunges him into a search for multiple relations.

\section{$\$ 146$. HeAT LOAD AND WATER LOAD IN MAN}

Everyone knows that a quick way to get into negative water load is to overheat. Similarly a convenient way to get into negative heat load is to drink ice water. These two sorts of experimental situation may be considered in detail.

Transitions from heat balance to considerable positive heat loads occur when a man walks for an hour in the hot desert (Adolph, '38, p. 492). During walking (transitional state), as heat 
accumulates in the body, water leaves the body; this I represent by plotting the heat load against the simultaneous water load (fig. $184, \mathrm{~J})$. At the end of the period of walking, the man sits at rest in a cooler atmosphere indoors; recovery of heat content begins a few minutes after the exercise and exposure to solar radiation cease. Later, recovery of water content begins, for now the subject drinks cold water as rapidly or as slowly as he desires it. In this period both heat load and water load are diminished, so that the initial or balanced state of the man is approached.

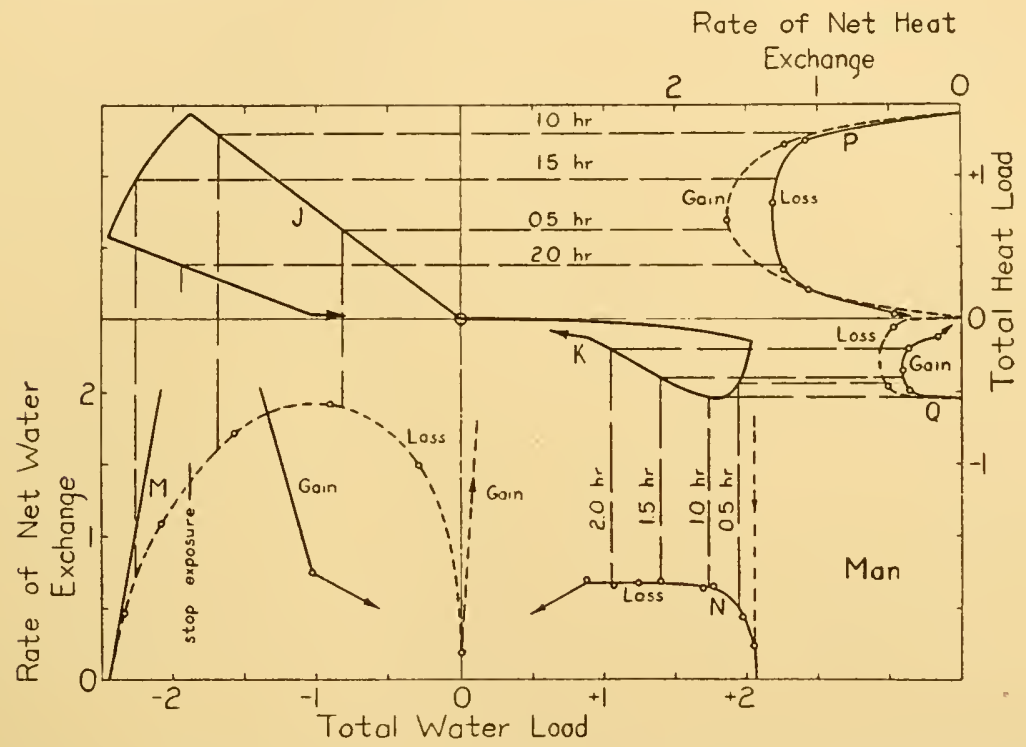

FIG. 184. Heat load (Cal./kg. of body weight) in relation to water load ( $\%$ of $\mathrm{B}_{0}$ ). Man. The data are arranged to show the simultaneous courses of both loads (and of the net rates of exchange, per hour, of heat and water) during loading and recovery. Exposure in the desert conferred negative water load and positive heat load (two tests on 1 subject are averaged); ingestion of ice water conferred positive water load and negative heat load ( 3 tests on 3 subjects are averaged). In all tests, recovery occurred in air of about $31^{\circ} \mathrm{C}$. Each point is the mean for a period lasting 0.37 hour. Further data of Adolph ('38) and of Pinson and Adolph ('42).

At every stage of the tests, the rates of net exchange both of heat and of water are, of course, known. When those rates are each related with the loads of the same component (fig. 184, M and $\mathrm{P})$, portions of two simultaneous equilibration diagrams are obtained. Anyone may trace corresponding points on the two separate curves by following various dash lines from one equilibration curve to the other. In this manner the relations among four 
simultaneous quantities, two loads and two rates of exchange, are established.

I now consider the second sort of experiment. Negative heat loads together with positive water loads follow the drinking of ice water (fig. 145). Recoveries of both components simultaneously are thereafter accomplished while sitting. Relating heat load with water load (K, fig. 184), I observe that the load of heat as estimated increases after the load of water begins to diminish. But later the load of heat diminishes the more rapidly of the two (as judged by the fraction of the load disposed of in a unit of time), so that heat balance is at the end of 2 hours more nearly restored than water balance; the same fact appears in $J$ also. At every stage of $K$ the rates of net exchange of heat and of water are known; indeed the total rates also were measured (fig. 145) but are not plotted here. The two exchanges, when each related to loads of their own component, yield two equilibration diagrams ( $\mathrm{N}$ and $\mathrm{Q}$ ).

Thus two of the four possible combinations of positive and negative loads of two components are worked out; the other two an experimenter could secure by appropriate arrangements, such as ingesting hot water to make both loads positive, and injecting pilocarpine to make both loads negative. Further, each administered load might be varied in diverse tests, particularly in relative magnitudes of the two components, each yielding data for a "loop" diagram. While the development of loadings might be regarded as controlled to a considerable extent by the experimenter, the recoveries are not, in so far as they freely proceed in a uniform environment.

What comparisons of simultaneous quantities are facilitated by the type of diagram shown in fig. 184? The following may be mentioned: (1) loads of two components, (2) positive and negative loads of each component, (3) time relations of loading and unloading, (4) rates of net exchange of two components, (5) rates of loading and of recovery at one load, and ( 6 ) four quantities ascertained at one time ( 2 loads and 2 rates).

Quantitative relations, in this as in every other diagram, increase in clearness as time is spent in working with them. The number of data contained in this figure is no greater than in a table, but more relations are shown than would be explicit by nongraphical methods. The four quantities recorded do not just 
"happen" to be simultaneous; rather their simultaneity manifests the quantitative interrelations among them. Meanwhile the specific communications by which they are related remain unidentified.

What is learned from simultaneous increments of two components that was not found in each component separately (figs. 61 and 144)?

(1) Recovery of water content is slower after excess of ice water than after excess of warm water (data of Pinson and Adolph, '42).

(2) Recovery from deficit of water content is faster after the subject is overheated than after he is only deprived of water (fig. 58).

(3) Maximal rates of total water loss during exercise in a hot atmosphere (loading) are greater than those in positive water load without exercise (unloading).

(4) Recoveries of water take longer than recoveries of heat, in both of the combinations tested. Or, heat velocity quotients are greater in positive heat loads than in negative heat loads, they are greater than water velocity quotients in negative water loads, and in positive water loads.

(5) During exercise in the hot desert (loading) the velocity quotient for water loss approximately equals that for heat gain. A corollary of this fact is that line $J$ is straight, water loss being proportional to heat gain during the exercise. It is strictly not characteristic of all exercise tests in the desert nor elsewhere.

(6) Rapid water loss (sweating) accompanies a large heat load and a rapid heat loss. Even if no one knew that vaporization of water is always accompanied by a loss of heat, the establishment of this relation would be just as significant, upon the criterion of description. The "willingness" of the organism to contract a water debt when in positive heat load seems to me of the same order as the "willingness" to absorb the cold as well as the water from the alimentary tract into which ice water is put. The constitution of the organism and of the universe makes them equally inevitable, I believe.

How reciprocal is the correlation between heat exchanges and water exchanges in the course of daily life? Negative water load develops where positive heat load prevails, but usually not vice versa, for negative heat load often induces negative water load too 
(cold diuresis). It may be that physiologists are merely more familiar with the instances in which heat load precedes water debt; for it is easy enough to arrange for the inverse to happen by depleting water, then administering a pyretic drug to develop a positive heat load or pilocarpine to develop a negative heat load. Here it may be observed how arbitrary is the distinction between what the organism does during loading of its "own free will" and what is ordinarily regarded as forced upon it.

Each interrelation of water and beat is quantitatively characteristic of the organism. Those oxidative chemical reactions that produce $0.12 \mathrm{gm}$. of water with each calorie of heat, depend upon the constitution of the organism just as much as do the activities whereby during loading in the desert $13 \mathrm{gm}$. of water are lost while each calorie of heat is gained (line $J$ ). The only difference I discern is that the former ratio is less readily modified by conditions than the latter; it is more often seen in vitro. Actually a rather constant relation was found (Adolph, '38, p. 494) between heat retained and water debited. If each of these ratios had a name, chosen as acceptably as "respiratory quotient," the relation would seem real to more individuals. When a quotient is bandied about, discussed, and abbreviated, it takes on an individual meaning.

Numerous other data relate water loads (various body weights) with simultaneous heat loads (various body temperatures). Tests of McConnell and Yagloglou ('25) may be used to trace the first half of curve $J$ during exercise indoors in still air or in moving air of diverse bumidities. Data of Winslow et al. ('37) and of Hardy and Soderstrom ('38) cover some of the same and many other atmospheric conditions. Each set describes what happens in the human body when heat accumulates and water depletes. Unfortunate it seems that those observers did not investigate recovery states.

In brief, two components may be experimentally loaded at one time. Then both during loading and during recovery the relative changes in their increments and their exchanges may be compared. In examples analyzed the increments are (1) negative heat and positive water, and (2) positive heat and negative water. Equivalences in rates of their exchanges indicate certain patterns by which ane component is preferentially treated or functionally linked in others. 


\section{\$147. Several components DURing Physical EXERCISE (MAN)}

In many researches a number of physiological functions have been measured during and after physical exercise. A small series of such measurements has been analyzed above (fig. 176); it may be used for further correlations (fig. 185).

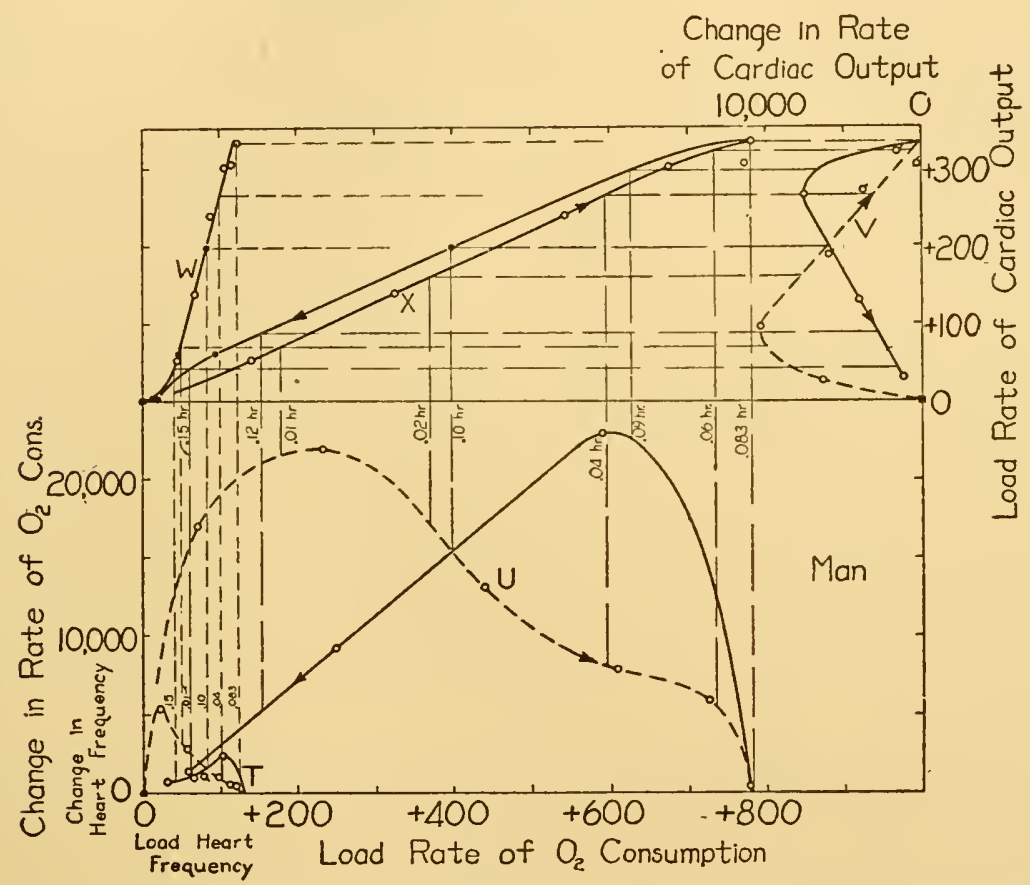

Fig. 185. Load in the rate of cardiac output in relation to load $(a)$ in the heart frequency and $(b)$ in the rate of oxygen consumption; during and after physical exereise. Man. The simultaneous movements of two loads at a time are shown in the upper left diagrams. Below are two equilibration diagrams for quantities $(a)$ and $(b)$; to the right, one equilibration diagram for quantity $(c)$; dash lines during gains, solid lines during losses (recoveries). Corresponding points are connected at diverse intervals of time. All loads are $\%$ of the initial rate of frequency, all changes in rate or frequency are in $\%$ of the initial rate or frequency/hour. The data are those of IljinKakujeff ('36) in figure 176.

Three selected components are here interrelated two at a time; rate of oxygen consumption, heart frequency, and rate of cardiac output. No objection has been found $(\$ 72)$ to treating rates of activities as components; since rates are now loads, accelerations are their rates of net exchange. It happens that in these three components all the loads are positive. 
At simultaneous points rate of cardiac output is correlated with heart frequency (W, fig. 185). The points during exercise (increasing loads) and the points after exercise (decreasing loads) fall upon a single line $W$. The changes in rate of cardiac output (V) and in heart frequency ( $\mathrm{T}$ ) therefore form similar halves of two net equilibration diagrams. Both in transitional and in recovery states the initial changes are the fastest.

When cardiac output is correlated with oxygen consumption $(\mathrm{X})$, the points fall along a very narrow loop. The shape of the loop is an indication of the great deceleration of oxygen consumption at the beginning of recovery (curve U). Again the initial changes of state are the fastest.

The similarities among the three components are much greater than anyone would anticipate in figure 176. Not only the loads in relation to time, but the loads in relation to one another and in relation to their rates of change are of one pattern. But the magnitudes are very different when computed, as all of them are, in per cent of the rate or frequency in the control state. Possibly relative scales that would make the magnitudes equal are justified; further utility in such an equalization would be found if it turned out that in a variety of kinds and intensities of exercise and of other circumstances, uniform proportions prevailed among the three components.

Figure 185 shows that cardiac output changes 3.9 times as much as heart frequency. Or, for each 1.0 per cent of increment in heart beat 3.9 per cent of additional blood circulates. This is the slope of line W. Similarly the slope of line $\mathrm{X}$ is such that cardiac output changes 0.44 times as much oxygen consumption. And, oxygen consumption augments 8.9 times as much as heart frequency.

The three components are, indeed, commonly recognized to be related to one another by the fact that their ratios have names: (a) oxygen consumption/cardiac output = arterio-venous oxygen difference; (b) oxygen consumption/heart frequency $=$ oxygen pulse; (c) cardiac output/heart frequency $=$ stroke volume. Each of these ratios of absolute units varies somewhat during the exercise, since none of the three regressions is exactly linear and through the origin.

The acceleration in exchange of the three components are similar, within about 15 per cent, for uniform fractions of the loadings; that is, the three dash lines of figure 185 are superimposable if 
the maximal loads present in each are brought to a common scale. But the decelerations are probably significantly diverse; upon the superimposed scales the deceleration of oxygen consumption is greatest and of heart frequency is least.

Altogether, 6 variables are represented in figure 185, and simultaneous points of all 6 may be found connected by light dash lines. Among varying numbers of variables from 2 to 6 at one time, the total number of combinations is 57 ; or among 2 at a time is 15 . Whatever features are evident now that were not noticed in figure 176, are brought out solely by the transformations of coordinates.

Many other quantities have been studied during and after physical exercise. Indeed, the same tests that have just been analyzed furnish data for systolic and diastolic arterial pressures (fig. 176). Each of these is very different from the other 3 components; for both show negative loads at some portion of the test; and none of the 7 additional bicorrelations of each with other single loads forms a line having a narrow loop resembling $\mathrm{X}$ or $\mathrm{W}$.

When other data concerning physical exercise are examined, the conditions and states and individuals concerned are, of course, different from the above. Selecting two familiar components whose rates of change contrast greatly, I correlate heat loads with simultaneous values of heart frequencies, using data of Christensen ('31). Two rates of work are represented in figure 186. In $\mathrm{S}$ a stationary state with respect to heart frequency is maintained during the interval from 0.2 to 1.0 hours after work began, but not with respect to rectal temperature or to heat load. In $R$, what looked at first ( 0.1 to $0.5 \mathrm{hr}$.) like a stationary state of heart frequency, later proved distinctly unsteady. Both tests show approximately rectangular loops correlating the two loads, two of the four corners being at the start and the stop of exercise. A partial diagram of this variety was used by Dill et al. ('31, p. 512). The rates of loading and unloading contrast sharply for the two components. But each rate follows a similar course in loading and in unloading. Heart frequency augments and diminishes suddenly at start and stop, so that most of its transition and its recovery is completed in 0.05 hour. Heat load changes almost uniformly with time throughout the test, requiring about as long for its recovery as for its loading, and never reaching a stationary state.

All the components that have been studied in chapter XV, except those of tissue replacement, have been at some time or other 
measured during and after exercise in man. Each could enter a series of interrelations of the sort indicated. To insure a basis for comparisons it is desirable, of course, to secure all the measurements during one bout of exercise, or during a series of reproducible bouts. In the end, economy of representation of the data could be secured by constructing alignment charts : one chart for loads, one for rates of change, one for velocity quotients, or one for all combined. The components considered would include water, heat, total substance, potential energy, external work, glucose, oxygen, carbon dioxide, lactate, chloride, nitrogen, and as many

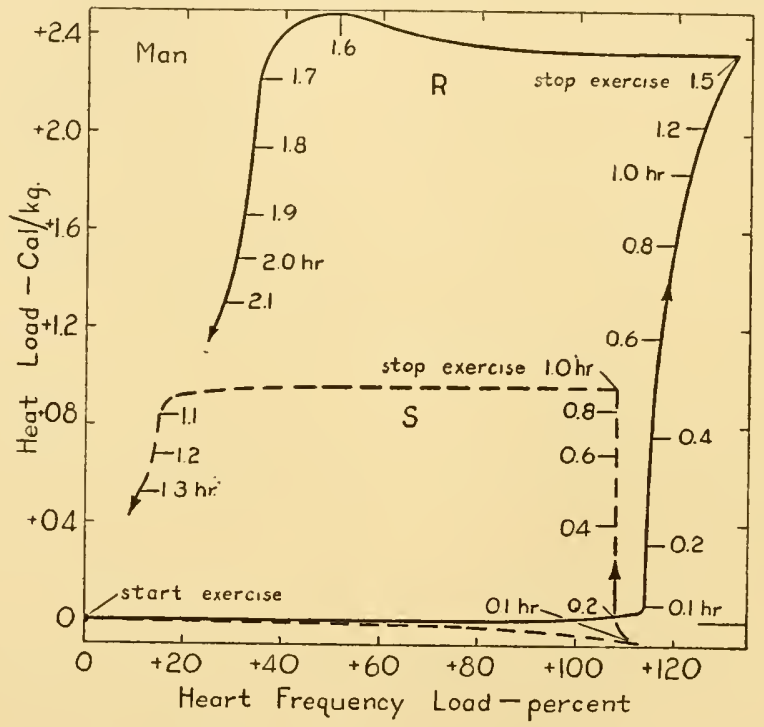

FIG. 186. Heat load (Cal./kg.) in relation to heart frequency load (\% of initial frequency) during and after exercise. Man, subject M.N., $72 \mathrm{~kg}$., working on stationary bicycle. R, $86,000 \mathrm{~kg} . \mathrm{m}$./hour external work for 1.50 hours; $\mathrm{S}, 58,000 \mathrm{~kg} . \mathrm{m} . /$ hour for 1.00 hour. Heart frequency and rectal temperature were originally read every minute. Heat load is computed as increment of rectal temperature $\times 0.83$. Data of Christensen ('31, pp. 463 and 464).

others as could be handled. The limitations to the investigation would not be in securing the data but in grasping the interrelations present, for the number of correlations even when taken only two at a time, increases as $\left[(n-1)^{2}+(n-1)\right] / 2$, or $\left(n^{2}-n\right) / 2$. The reward of the investigation would be a picture of how the organism coordinates a number of simultaneous processes in a combination that would snarl up many a man-managed factory indefinitely. 
Physical exercise is, in brief, an example of physiological activity that involves numerous components. Two groups of them, examined with respect to loads and net rates during loading and during recovery, are found to be characteristically intercorrelated both in cases where nexuses are recognized and in others. Recovery in each is relative to recovery in the remainder.

\section{\$148. Some other COMPONEnts}

In the past the physiological interrelations among components have been studied chiefly: (a) where one chemical compound is formed from others, $(b)$ where cations and anions balance, or diverse cations replace one another, (c) where energy is transformed from one kind to another. The studies may equally well be extended to components where "there is no reason to expect" mutual associations. As soon as "empirical" correlations have been found, plenty of "reason" will usually be forthcoming. Many physiologists object to the correlation of data except in the light of some theory. To write $A=f(B)$ is, they say, useless unless $f$ is either a constant or a believed causal connection. But it is probable that any one relation, usually a partial correlation, between any $\mathrm{A}$ and any $\mathrm{B}$, is eventually worthwhile for someone to investigate. When he feels the urge to find how $\mathrm{A}$ is related to $\mathrm{B}$, that urge can be legitimately satisfied. Indeed, the manners and forces of interactions in organisms seem to be so various that I believe physiology cannot afford to wait for non-empirical hypotheses or visible "connections" to precede measurement. For, meanwhile physiology would be limited by the contents of other sciences. As an instance, relying on the knowledge of chemistry, no one has been able to guess yet how much water is retained in mammalian livers of any species for each gram of carbohydrate (glycogen) loaded.

All correlations, then, are equally valid, according to any general criterion that has been proposed, other than opinion. $A=f(C)$ has no more statistical significance than $A=f(B)$, though it may have a higher coefficient of correlation, or carry smaller probable error, or be known to occur in more species. Only by personal judgments and attractive hypotheses is a greater intimacy assigned to one relation than to another. I doubt whether it is any contribution to physiology to say that "all measurements of the state of the body with respect to water must be relatively 
empirical until the mechanisms of regulation are understood" (Adolph, '33, p. 349). Evidently a non-statistical kind of significance is referred to in the statement of C. Y. Cannon et al. ('32) that "If these results could be explained or predicted, reasoning from a physiological basis, they would be highly significant. Since no good physiological explanation can be made, the level of significance secured is only great enough to warrant further test." Wishing to find in what manner the activities of the organism are knit together, I do not think it desirable to suppress knowledge of any relation that comes to light.

Were no other connections visible, body size alone would require that water content, water turnover, heat exchange, total substance, heart frequency, and many other quantities are related in the way they have been ascertained to be. They need no common organ of exchange or common physical characteristic to fix them at those particular rates. In attempting to "explain" those observed correlations, one theory suggests one connection where a hundred probably exist. Experience teaches, I think, that biological relations usually have too many factors to allow identification of predominant or causal ones even if such exist.

Having decided that any two kinds of measurement made simultaneously upon one living unit may be usefully correlated, I might now analyze any of a semi-infinite variety of investigations. It seems appropriate to mention several that concern the component water, with which the present work started. Each will illustrate a system of variables manifesting an interrelation among components that are being simultaneously handled by a living unit.

(1) In the dog, the continuous infusion of $1.1 \mathrm{M}$ solution of glucose induced marked losses of water (experiments of Wierzuchowski, '36). The urinary outputs of water and of glucose were measured during 6 hour's of infusion, when for each gram of glucose retained about 24 grams of water were depleted. At the same time exchanges of oxygen, carbon dioxide and some others were ascertained, allowing interrelations to be established among $18 \mathrm{com}-$ ponents.

(2) In the dog in water loads, measurements of concentrations of diverse variables in blood have been presented (figures 126 and 117). Each variable may be designated as a distinct component, and in reality the data already used in other connections are now available for comparisons among components. Indeed, table 24 
quantitatively correlates 7 components that were measured simultaneously. These interrelate the state of and what is happening in the whole with the state of its parts. In the same manner equilibrations also might be represented, as they exist simultaneously in diverse parts of one organism. They would indicate the competitive rates of recovery among the parts.

(3) In man, water was withdrawn from the diet to varying extents, and in daily periods the net exchanges of seven electrolytes were measured (Wiley and Wiley, '33). Conversely, to constant diet and water intake each of four salts in equivalent amounts was added in a separate period (Wiley, Wiley and Waller, '33). Diagrams may be constructed by plotting the body's load of one against the load of another, upon which each period traces a different loop. It was found that deficit of water leads to deficit of electrolytes, and excess of electrolytes leads to excess of water, a relation that is, however, only qualitatively reciprocal.

In other tests on man, water and varying amounts of sodium chloride were taken by mouth either together or separately (Adolph, '21). The load and the rate of exchange, of water and of chloride, depended on the relative times and amounts in which they arrived in the body. Water might be depleted by salt excess when intake of water was denied. Evidently interrelations need not be limited to simultaneous states but may relate what follows with what precedes. Very many speculations suggest how electrolytes may be related among themselves and to water in the human body; planned experiments can test all of them quantitatively.

In a third set of tests on man, sodium chloride was withdrawn from the diet, while the exchanges of water and several electrolytes were measured (Goodall and Joslin, '08; Stöhr, '34; McCance, '36, '37). Deficit of sodium led to deficit of water and of some electrolytes; numerous correlations are possible that are limited by the fact that each individual was tested differently. Going further, if the researches of Tuteur ('10), Wiley, and others had been planned to allow correlation with these latter ones, the same amount of manipulative effort would have produced results capable of a high degree of synthesis.

There is no great trick to finding interrelations among components; most researches in physiology that are at all quantitative, ascertain data suitable for the sort of study here illustrated. A point to be emphasized is that a constant proportionality, or an 
equation that relates two quantities under a variety of circumstances, is not needed before the interrelations may be analyzed and represented as aspects of the organism's functioning. Countless are the circumstances in which heart frequencies have been measured in man. All of these circumstances are associated with change in some other measurable component, and often with known loads of many components. Each load is a "factor" in heart frequency; whether each is a separate factor is a matter for definition and for further study of relations. Hidden in the data of physiology are, I believe, hundreds of clear correlations that have not even been surmised, which nevertheless indicate how components are related in the animal body.

The physiologist may take advantage of the generalization that every bodily function (component) is related to nearly every other. Keys et al. ('38) wished to select persons who would acclimatize advantageously to high altitudes. They, therefore, made eight random measurements upon each of ten men; later took those men to the mountains for some weeks, and found which were able to do most physical and mental work there. Correlations between the initial measurements and the later performances revealed what combinations of physiological characteristics were best suited to life in the mountains. The important feature is that any set of tests may serve as a basis for discovering a given physiological aptitude. No one need know what constituent processes are at stake in the particular acclimatization; "blind" trials upon random individuals will furnish the information needed for extensive predictions.

In the end, physiology has a set of relations similar to what anatomy has in "Cuvier's Law." Cuvier (1827, p. 83) realized that he could pick out bones belonging to one species of animal from a mixture, and predict the conformation of the jaw and teeth from that of the claws. "Every organized being forms a peculiar system of its own, all the parts of which mutually correspond." Similar statement is frequently possible in physiology and in functional pathology, as has long been shown in medical practice. Just as a hypertrophied heart often accompanies unusually high arterial blood pressure, so deficit of salt often accompanies modified function of adrenal glands. To say that one can see no "connection" between what these small glands are metabolizing and what the whole body is metabolizing may be correct; but that statement blurs an interrelation, which holds even though in some bodily 
states the glands are dispensable. Again, the rapid turnover of water in a frog, in quantitative contrast to that in a man, accompanies formation of urine always hypotonic to the blood plasma, immersion in fresh water, and absorbent skin. The frog's normal state depends upon them all, and the impression is gained that any other combination of properties would be incompatible.

Interrelations and interactions among components that are under increment are properly connoted by the term syndrome. This term has the present advantage of emphasizing the total nature of the physiological state instead of some supposed mechanical relation among parts or components.

In general, interrelations among components may be established and appreciated without knowledge of the lines of mutual communication or influence. Interrelations are the substance of physiological science. Several illustrations serve to indicate that the unforeseen character of the relations is so common as to be no barrier to the acceptance of all correlations that are statistically significant. Compatibility among properties is visible in every maintenance and recovery of those properties.

\section{\$149. General features}

In the data and interrelations here presented, certain characteristics may be discerned. What particular and what general conclusions may be drawn? How may the methods illustrated furnish a synthetic picture of the physiological patterns of the organism?

(1) First some characteristics of the diagrams here employed may be mentioned. Diagrams were constructed in which load of one component was plotted against load of another component (figures 184, 185, 186). (a) The broader the "loop" (ratio of its smallest diameter to its largest diameter) the greater is the difference in rates of net exchange of the two loads. Scales might be proportioned for this purpose by taking maximal loads as equal coordinate units.

(b) When no "loop" is present, correlation of the two loads is very high; the correlation is then almost independent of time.

(c) The departures from an oblique line connecting the points of initial state and of stationary or extreme state, are often equal upon the two sides of it. This symmetry means that loading and unloading of the two components bear similar relations to one another. 
(d) In equilibration diagrams, the greater the difference in rates of loading and of unloading, at any one load, the more is time a correlative and the less is load a correlative, of the rates of exchange.

(e) Recovery in one component does not prevent recoveries in others, though it may diminish their rates. Discharge of one load does not often create large loads of another component. Were any one component predominant it might easily be that all sorts of disturbances (loads) would arise in an uncompromising restoration of that component to zero load. Perhaps such easy compatibility manifests niceties of coordination among the handlings of diverse components. Conflicts may continually arise : obtaining water may preclude cooling off, fleeing may inhibit excreting water, mobilizing glucose may involve destroying water. The solution of those conflicts $i s$ the organization of the organism.

Conceivably, interrelations are of two sorts. In one, $\mathrm{C}_{0}$ is fixed for all components; that is the case in the data presented. In the other, $\mathrm{C}_{0}$ changes for one or more of the components. The difficulties of distinguishing whether $\mathrm{C}_{0}$ changes or not, lie in the effort to define $\mathrm{C}_{0}$, not in the record of what happens. If it be said that final content $\left(\mathrm{C}_{f}\right)$ after $f$ hours is the criterion of recovery without change in $\mathrm{C}_{0}$, then $\mathrm{C}_{\mathrm{f}}=\mathrm{C}_{0}$ is the sign of it. If temporarily gain equals loss at a content other than the original $\mathrm{C}_{0}$, then that is the criterion that $\mathrm{C}_{0}$ is not fixed. Such distinctions are required only when words are to be used to summarize the relations among components.

(2) One question that has not yet been explicitly asked is: how specific is the interrelation between the component that is loaded, and the modification of exchanges of the very same component? The only adequate answer is to be derived, I believe, by a correlation of the following sort. Set down as abscissae all components whose loads are to be tested, and set down as ordinates all components whose modifications of exchang'es are to be observed. See what exchanges occur, and by how much each is modified, over a wide range of loads of each component. To do this adequately, a diagram showing net exchange of each $y$ against load of each $x$ is constructed in each box of the chart. It turns out, for the components listed in chapter XV, that exchange of no "foreign" component is modified as much as is the component that is under increment. For a remarkably high proportion of components, con- 
siderable modification appear's only in increments of $y$ itself. The relation between load and corrective exchange is therefore unique and specific; the very component that is present in unusual amounts is the one whose exchanges are most modified. The existence of that specificity was guessed in the first place; now that it rests explicitly upon a considerable array of facts, its non-random character is more evident.

(3) Having found that certain features become easy to grasp in diagrams of interrelations (load of one component plotted against load of another component), how can I formulate multiple correlations? Or is it necessary to stop with two or three components at a time? One step might be to represent an additional component in a 3 -dimensional diagram.

In another direction it is possible to proceed by combining components. Wherever two or more components have the same dimensions, their combination may be a sum or a mean; the values obtained, however, preserve the definitions given to each of the components included. Where the dimensions differ, restricted progress is possible. (a) The components may be redefined to make them commensurate in terms of control contents of each; in terms of standard deviations of each; in terms of lethal loads of each; in terms of selected coefficients of physical or physiological equivalences $(\$ 134)$. (b) A combined load may be represented as a multidimensional sum or product, e.g., $x \mathrm{gm} . / \mathrm{kg}$. plus $y$ cal. $/ \mathrm{kg}$. plus $z$ atmospheres; or $v$ gm. $\times$ cal. $/ \mathrm{kg}$. $\times$ atm. (c) Tables or alignment charts may represent an unlimited number of components even though each of them be visibly a separable variable.

In particular, I believe all the changes measured at any moment during one bout of exercise may be counted up so as to represent the total combined state of the organism. Instead of the two components represented in fig. 185, some 20 or 30 separable components might enter into one quantitative statement of a physiological state at a chosen moment.

Combined components actually occur in every science. For certain of them names exist. Any solution is such; cardiac output (heart frequency $X$ stroke output) is another. Water plus certain solutes is plasma; plus heat is warm plasma; plus blood cells is whole blood. So "biological" terms as well as non-biological ones are often known whereby to designate combinations of properties. For numerous other combinations terms will be forthcoming when 
the study of relations is undertaken. Indeed, it is not necessary to recognize whether or not a component is compounded of other elements. A quotient or a subtrahend may be regarded as a combination in this general sense, equally with a multiplicand or any other $\mathrm{f}\left(\mathrm{J}_{1}, J_{2}\right)$. A correlative of two or more recognized components sometimes answers the definition of a resultant, and usually of an "emergent."

Not only may several loads in an organism be considered as a combination, but their simultaneous evaluation constitutes a statement of net physiological state. The various modifications thus assess the organism as a whole, as well as they assess individual components. The extent to which this estimate can now be carried is not great; more needs to be known concerning physiological ratios of each component. For certain components, physical and physiological equivalents are established: $1 \mathrm{gm}$. of water vaporized $=0.58$ Calories absorbed $; 1 \mathrm{gm}$. of fat burned $=9$ Calories of heat produced; 1 calorie transformed $=<0.25$ calorie of external work. Many other equivalents are more limited in their applicability, as their physiological ratios vary of tener with circumstances. The assets of the organism in any two net states have been in fact but very partially compared.

I believe physiology cannot avoid regarding many combined components as one net component. The emergent of one combination becomes in turn one of several elements in the next combination, and this process may go on indefinitely. Permeability, excitation, and respiration are names of such multiple combinations. Or, in a plane or solid figure the manner in which the state of the organism moves hither and yon might be represented. I could use more than three dimensions if I could grasp the simultaneous meaning of more coordinates. Familiarity and practice with such numbers of variables might conceivably cultivate such a grasp.

In many correlations it is customary and convenient to distinguish independent variables from dependent ones. The distinction is created by the experimenter or recorder of relations, and, so far as I see, has no counterpart in the organism. Arbitrarily the investigator selects certain conditions for the organism, and for the time being these are described as independent variables. But in another kind of test the organism may select its own quantities of each same variable, in accord with its physiological states. Arbitrary definitions that are required for the representation of results 
are not to be attributed to the components represented. He who creates variables in a separate order of independence is one who also terms those variables "causes." Only by virtue of having tested some variables as if independent, does it appear justified to imagine they are causes.

Methods of representing many physiological variables are now very primitive, and I hope that more promising ones will be found than the crude diagrams herewith suggested. The organism is apparently not limited in performance by human inadequacy in recording the multitude of its relations.

(4) Another approach to interactions of physiological properties is as follows. A great variety of loads and conditions might be studied in their interrelations if a measure of "value" is selected. Familiar examples are the commercial yields of agricultural crops (Fisher, '37). The monetary value of yields equal in weight varies from time to time with economic situations; similarly any scale of physiological value varies with the appraiser. Diverse measures of value emphasize man-power, longevity, enjoyment of leisure, meat or milk production, piece work, mental stability, physical fitness, or any other better or worse defined criterion. Medicine and surgery have an almost singular measure of value in the survival and comfort of the patient.

In Arbeitsphysiologie, to operate machines of certain specifications, measurable muscular forces and energies are expended in particular manners. Physiologists choose criteria as to what are the immediate and ultimate effects of the work upon the human organism. Not content with rate of oxygen consumption, it is usual to measure heart frequency, cardiac output, arterial pressures, coordination and speed of movement, reaction times, effects of giving sugar, effects of rest periods, effects of room temperatures, and many other "factors." Multiple indices are devised whereby each measurement and test takes its share in rating the individual.

Some scientists have a tradition of "varying one factor at a time" ; others vary many simultaneously. Those who have considered the matter quantitatively point out that the latter "arrangement possesses two advantages over experiments involving only single factors: (i) Greater efficiency .... and, (ii) Greater comprehensiveness" (Fisher, '37, p. 110). "If the investigator, in these circumstances, confines his attention to any single factor, we 
may infer either that he is the unfortunate victim of a doctrinaire theory as to how experimentation should proceed, or that the time, material or equipment at his disposal is too limited to allow him to give attention to more than one narrow aspect of his problem", (Fisher, '37, p. 101).

Adopting some components earlier discussed, an investigator of athletic accomplishment might choose ten loads for simultaneous study, as follows :

(1) Water load, 0.

(2) Water load, $+0.5 \%$ of $\mathrm{B}_{0}$.

(3) Heat load, +0.2 Cal. $/ \mathrm{kg}$.

(4) Heat load, + 0.5 Cal./kg.

(5) Glucose load, 0.

(6) Glucose load, $-0.1 \mathrm{gm} . / \mathrm{kg}$.

(7) Glucose load, + $0.2 \mathrm{gm} . / \mathrm{kg}$.

(8) Heart frequency, +20 per cent of resting, induced by epinephrine.

(9) Heart frequency, +40 per cent of resting.

(10) Heart frequency, +60 per cent of resting.

Of the different combinations of the $n$ loads taken 2 at a time, in this case 45,8 are mutually exclusive by definition, so that 37 experiments at least are to be performed. In each state the human subject runs 1 kilometer in the shortest time he can; these times are correlated with each of the ten loads. For a more complete answer, enough repetitions will be made to establish the statistical significance of each combined physiological state in the performance of running. This is the explicit form, in the language of increment, of myriads of investigations.

In general, to evaluate $n$ states characterized by diverse simultaneous loads, a plan is set up in which $n(n-1)(n-2) \ldots / 1$. 2.3... total combinations are studied. States that represent two or more loads belonging to one component are precluded; leaving in the above example 133 experiments. In each the investigator ascertains the time required to run, and thereafter applies multiple correlation, or some other technique of measuring association, to the results, finally deriving a value for each of the combinations of components.

The usual scheme of combining loads is the Latin square, which arranges all the possible combinations in a certain sequence that 
secures maximal randomness. Many physiologists consider any such formal plan a discouragement to their sentiments and pleasure in work. At the same time it may be realized that "as the art of experimentation advances the principles should become clear by virtue of which this planning and designing achieve their purpose" (Fisher, '37, p. 9). The design of experiments now looms large in physiology as in other sciences.

It may be said that the analysis of simultaneous interrelations among components exposes quantitative competitions during recoveries. The load of each component modifies exchanges of itself more than exchanges of other components. Components are found combined in multiple ways, each combination constituting an entity that may still be represented as a single component. All components and combinations may be compared in terms of their effect upon some common index of "value." All enter into an account of the actual physiological state of the living unit.

\section{\$150. Meanings of interrelations}

So far it might be supposed that the study of related components is simply a matter of trying to represent a number of events that "by chance" are simultaneous. But it was noted early $(\$ 80)$ that any change in one component inevitably leads to changes in other components. It may now be inferred that this extensive association is a basis of recovery and of physiological organization.

Numerous are the instances in which one component does not change without modification of others. If water is removed from the organism, either solutes are removed too or osmotic pressure is increased. If the heart beats more frequently, either the cardiac output increases or the stroke output decreases. It might be possible to dissociate any two given components in the organism, but I infer that during life one given component is rarely if ever dissociated from all others. I draw the induction that many components are interrelated or tied to one another. This mutiplicity of connections has the effect of anchoring each component; whenever the component is disturbed (loaded) the resistance to change of the other components is met.

A conception reached is that of a large stress-strain system. Displacement of any content puts under strain not only the component itself but all the components that are associated with it. In addition, other components serve to locate the zero load of the 
first one, for, the fact is that the original zero load is again precisely located after recovery. I infer it is only the quantities (contents) that are not changed that are able to "inform" the processes of recovery how much to return. If any component were independent of all others, probably no means would be left the living unit of "knowing" where balance lies.

Undulations of diverse components similar in time are also evidence of interdependences. Such rhythms may be "spontaneous" or induced. The interrelations are made known by the demonstration of what components take part in the one rhythm. When first observed the undulations are usually regarded as random, later they are said to be physiological. Often, I believe, rhythmical changes are stabilizing something; perhaps even concerned in maintaining constant a function that itself shows no periodicity, as in the uniform flow of blood with intermittent heart beats or uniform flow of heat with fluctuations in peripheral blood flow of man in every minute (Burton and Taylor, '40).

The facts lead to the general proposition that probably no one physiological function can be different from what it is without many others being different. Each change involves a "network" of many unforeseen changes. To find a new equilibration or balance for one component means securing some arrangement for bringing all the others to new balances too. The shift of $\mathrm{C}_{0}$ might be sudden and complete, as in some components at birth; or progressive, as during ontogeny or acclimatization.

A living unit may be viewed as such a system of unexceptionally related quantities. The components are each subject to certain ties and relationships in space and time. The existence of interdependencies among them is a matter of fact; that all components have interrelations can never be demonstrated except with extrapolation; that, further, these interrelations are the visible elements of recovery and of stability probably remains a hypothesis.

Such a view may be compared with the implicit supposition of many physiologists that an organism is a conglomerate of chiefly uncorrelated happenings. For each function it is assumed there is a regulator or key or master lever. All or any components might then be at designated loads, at one time or separately or in any combination. This supposition could be supported by finding some load of a component that shows high constancy unaccompanied by changes of other compounds (after exhaustive search). 
Observation of uniformities indicates, on the contrary, that correlated happenings are found in most instances where they have been looked for. That realization is altering the course of physiological science.

I conclude that where recovery and constancy of a component occur, there are interrelations with other components. The equilibrated organism is probably one whose $n$ components are all associated in such a way that loadings of even a considerable number will not destroy the "memory" of that net state of the organism, to which all properties trend when recoveries intervene.

I conclude that any two or more simultaneous changes in components, when correlated, show evidence of inherent interrelations. Examples such as water content and heat content, or various properties in physical exercise, furnish coefficients and ratios of preferential change. Diagrams that correlate several components, and resultants that combine components, are of practical use in dealing with numbers of physiological variables. All those variables enter into one general account of the pattern of the organism and the means by which its properties are fixed. 


\section{Chapter XVIII}

\section{CHOOSING PHYSIOLOGICAL VARIABLES}

$\$ 151$. As the facts and relations have accumulated in this investigation, certain rules for their selection gradually appeared. I was not consciously formulating such rules; rather the materials themselves seemed to move into place, and I then began to wonder by what tokens they gravitated. Given the objective of finding regulatory processes of kinds that could prevail in all animals, the steps by which that search was accomplished became partly automatic. Now that the objective is achieved, I believe it is worth while to turn aside long enough to examine the general import of these rules of procedure.

This is not a treatise on methods. Yet, every scientist has methods. They are equally effective and decisive in the substance of his results, whether or not he recognizes and classifies them. Methods are usually gained by unconscious experience and by the examples of others, almost never by precept.

Scientists find strange those procedures they do not habitually use. Sometimes suspicions are aroused by them. To understand all investigations, it is necessary to admit that ways of doing things other than those sanctioned by custom may be justified, even be successful. For, to limit the solution of inquiries to the sort of contribution to science for which tastes are already cultivated would be to exclude all efforts to see beyond the limitations of present outlook.

\section{\$152. Procedures}

How did this investigation actually proceed? Though most of the steps taken were unforeseen, there was a pattern among them $(\$ 104)$. What ones of the procedures might illustrate general usages in scientific work? Would they be useful in planning investigations, in so far as investigations are explicitly planned?

A basis of this inquiry consisted in distinguishing among physiological variables. Each variable was some quantity that could be identified in a defined and reproducible manner. Since the identity of the quantity depended upon the operations by which it was measured, each variable had dimensions that in part at once offered a 
practical classification of it among its fellows. Thus, there were loads $(\Delta \mathrm{W}, \Delta \mathrm{C})$, each distinct with respect to component, species, living unit, and other particulars. There were rates of exchange $(\delta \mathrm{W} / \Delta \mathrm{t}, \delta \mathrm{C} / \Delta \mathrm{t}$ ), each distinct (in addition) with respect to paths of exchange and unit of time involved. There were components, living units, analyzable tissues, modes of chemical analysis, rates of physiological activity, volumes, concentrations, various ratios. Any quantity that could be adequately defined and classified might appear on the docket of data available.

Among these data, further choices were made, taking two variables at a time from among those quantities that had been measured simultaneously. The choices were guided by the desire to learn how exchanges are concerned in maintenances of content. At first, loads and rates were correlated; $\Delta \mathrm{W}$ was related to $\delta \mathrm{W} / \Delta \mathrm{t}$, $\Delta \mathrm{C}$ to $\delta \mathrm{C} / \Delta \mathrm{t}$. Later, $\Delta \mathrm{W}$ was successively related to each of many varieties of $\Delta \mathrm{E}, \Delta \mathrm{G}, \Delta \mathrm{H}$, etc.; to $\delta \mathrm{C} / \Delta \mathrm{t}, \delta \mathrm{G} / \Delta \mathrm{t}, \delta \mathrm{H} / \Delta \mathrm{t}$, etc. The variables and the relations among them having been classified, living units were compared within one class of relations after another.

Without indicating many of its subdivisions, the whole course of the investigation is represented by the following outline:

$J_{1}$. Component Water (and Volume). Chapters II to XIII.

$\mathrm{M}_{1}$. The four variables. Chapters II to IX.

$\theta_{1}$. Species Dog. Chapters II to IV.

$\phi_{1}$. Type of loading $\Delta W_{5}$. $\$ 7$ to $\$ 8$.

$N_{1}$. Individuals $B$. Figures 1 to 6 .

$g_{1}$. Tolerance diagram. Figures 1 and 4 .

$p_{1}$. Urinary exchanges. Figure 4 .

$\mathrm{p}_{2}$. Other paths. Figure 15.

$g_{2}$. Other bi-correlations. Figures $2,6,19,17$.

$\mathrm{N}_{2}$. Other individuals. Figures 35 , etc.

$\phi_{2}$. Other types of loading. Chapter III.

$\phi_{3}$. Preference among environments. $\$ 20$.

$\phi_{4}$. Variability. Chapter IV.

$\theta_{2}$. Other species. Chapters V to VII.

$\theta_{3}$. Parts of organisms. Chapter VIII.

$\mathrm{M}_{2}$. Other groups of variables. Chapters X to XII.

$\mathrm{J}_{2}$. Other components. Chapters XIV to XVII.

For certain purposes it would have been valuable to designate the precise type of each content or exchange, thereby differenti- 
ating it meticulously, as for example $\delta \mathrm{W}_{5} \theta_{1} \mathrm{~N}_{4} \mathrm{p}_{3} / \Delta \mathrm{t}_{2}$. I felt that the distinctions did not at present warrant that ideal exactitude.

Among the variables little distinction was made between independent and dependent variables, or between stimuli and responses. Yet in any specific experimental arrangement it was usually possible to designate (provisionally) certain variables as subject to conditions that the investigator can dictate more easily. I note further that breathing frequencies and drinking frequencies were as useful in the investigation as heart frequencies, in spite of the fact that the former are more often susceptible of "voluntary" control in man. States of recovery were emphasized, they being relatively free of the experimenter's influences. Such emphasis represents the fact that I had hypothesized how regulations could be studied; it guided my search for explanatory relationships.

Among components, various physical and chemical classifications were used. Among paths, and tissues, various anatomical names were also employed. In so far as physiology is a comparison of phenomena and magnitudes, it appears destined to have little peculiar and specific terminology of its own for single components, but to reserve its language for relations and combinations.

I believe the above outline of this inquiry is typical of any comprehensive research. Some one variable (deviation of content) is to be followed through a variety of physiological facts. Some one (component) is set up as a major division. Some group (the four variables) defines the data to be correlated first. And the process of definition and delimitation is followed down to the last detail. Another arrangement might reveal just as much about regulations, and perhaps something different about them; any other arrangement would shuffle the same and similar classes into different relative ranks. All this is done either implicitly or explicitly. in any design of experiments.

\section{\$153. General features}

Certain modes of progressing, from data to correlative data, having been employed, the sort of scheme represented in this progression now appears "logical" in certain respects. Some of these respects were unforeseen.

(1) More relations are established by keeping one factor present through many correlations. Thus $\mathrm{A} \propto \mathrm{B}, \mathrm{A} \propto \mathrm{C}, \mathrm{A} \propto \mathrm{D}$, etc. But since $\mathrm{A}$ is common to all, $\mathrm{B} \propto \mathrm{C}, \mathrm{B} \propto \mathrm{D}, \mathrm{C} \propto \mathrm{D}$ can also be correlated, 
for all were measured simultaneously or virtually so. With six variables 15 bi-correlations are established, and with $n$ variables $\left(n^{2}-n\right) / 2$ correlations taken two at a time. But if, as is empirically done in "random" physiology, the procedure had been $\mathrm{A} \propto \mathrm{B}, \mathrm{C} \propto \mathrm{D}, \mathrm{E} \propto \mathrm{F}$, etc., then with six variables only 3 correlations are established. The same number of data then yield only $3 / 15$ as many relations taken two at a time, or in general $1 /(n-1)$ as many.

(2) Among more than two variables at a time, all quantities measured either actually or virtually simultaneously are interrelated. But methods of representing them had to be selected; those used are contour charts (fig. 19), tables (table 24), and parallel alignment charts (fig. 131). Other techniques of partial correlation and multiple correlation might be applied (Fisher, '38). Further procedures as devised will aid in the comprehension and evaluation of large numbers of coexisting quantities; need for them grew during the inquiry.

(3) Very often $\mathrm{A} \propto \mathrm{B}$, and $\mathrm{A}^{\prime} \propto \mathrm{C}$ are studied, under the impression that $A$ and $A^{\prime}$ are the same, perhaps because they have the same name. Thus, both $\mathrm{A}$ and $\mathrm{A}^{\prime}$ may represent dehydration of dog, or excitation of nerve, or osmotic pressure of plasma. But as already seen, there are many unidentical dehydrations, or nerve trunks, or osmotic pressures. So it was necessary to define carefully and provisionally.

Sometimes the data are averages of statistical groups that later or in the light of other relations require splitting into new categories. At times a rough value obtained under unrecorded conditions or by crude methods is useful ; later new refinements are indicated, or non-uniformities of conditions are recognized. Supposedly homogeneous material turns out to be heterogeneous. One of the tasks was to define diverse types of water load; there proved to be scores of them (chapter III). To differentiate amphibia from mammals, a few data on water exchanges will suffice; but to distinguish rabbits from dogs with respect to water equilibrations, numerous factors may be necessary. So, continual revision and refinement of data proceeds as occasion arises.

(4) Among the measurements of particular variables, some are on diverse species, others at diverse temperatures, others by diverse paths. From them are found (a) what relations persist among the data, what uniformities exist. From them (b) the diversities are 
ascertained. So it is possible (a) to induce general relations and (b) to distinguish and classify peculiar characteristics of living units and of environments. Definition and classification are giant sieves by which it is possible to cull out uniformities and differences.

(5) The correlations examined lead to certain broader inductions that are rigorously derived; "Induction is demonstration by recurrence,' said Poincaré ('13, p. 40). Many physiologists express mild interest in having syntheses among grouped facts carried out in their science, but think it impractical to do so by exact methods. I believe that the methods used here and elsewhere are capable of great rigor and extension; that sound and accurate synthesis by quantitative description has been demonstrated. Nothing spurious is added to the facts through exploration of any number of relations, in so far as the language used is descriptive.

(6) Choices of variable in further researches are facilitated by the conceptual scheme used here, as by any other. The multiple relations of each variable are already explicit. New observations are classified, and patterns of relation to other variables are suggested.

(7) From the conclusion that enormous numbers of factors in an organism are interrelated, two corollaries follow: (a) The number of physiological studies of two variables at a time is almost infinite. (b) Most such studies show positive or negative correlations, not zero correlations. This expresses the enormous "fruitfulness" of physiological research, since its "success" is widely taken to depend on finding positive or negative correlations. I believe it is misleading to suppose that there is anything unique in the correlations found, or that one choice of variables was any cleverer than other possible choices; or to intimate there is not a semi-infinite number of other relations just as meaningful if they had been described instead, whether found by help of or without help of a plausible hypothesis. To that statement much exception will be taken, and it remains to be ascertained whether the relations that have been recorded in a century, concerning water in mammals, for instance, are more informing to a beginning student than an equal number of relations that were not recorded.

In a sentence, the general procedures that may be of use in any physiological investigation consist in : finding many correlatives of one or few variables, developing methods of recording relations, 
distinguishing among increments supposed to be similar, ascertaining uniformities and diversities of relations, demonstrating which forms of relations combine frequently, schematizing relations into comprehensive concepts, and avoiding the conclusion that correlations known are more crucial to the organism than those unknown.

\section{\$ 154. Description}

The general investigation here pursued serves to illustrate the fact that concrete results are gained by this particular sort of study of quantitative physiological variables. It may be recalled, parenthetically, that none of the procedures are novel. What do I see in the use of the procedures mentioned? Is this book an exercise in arithmetic, or is it a part of physiology to which no other approach is known?

By one definition, all biology is the representation of whatever orders and relations are to be found in vital phenomena. Observations and measurements require coordination, for facts are probably meaningless to man except as they are related with other facts. To comprehend the relations requires some scheme; the difficulty is to have a scheme without confusing the procedure with the findings ultimately represented. Of course, "nature" is not limited by one or many schemes, it may be guessed, nor bounded by the number of dimensions portrayable in human record or thought.

The study and representation of such order and relations may be included in the term quantitative descriptive physiology. By its scrupulous definition of variables it indicates plans, and maybe the only one, by which relations are found, comprehended, and conceived. Pareto ('34, p. 178) speaks of mathematics as "the one method so far discovered for dealing with interdependencies."

It may be said that science constructs maps of one portion or another of phenomena. All maps are inaccurate, corrections are continually required. "Empirical" observation stores instances; description makes maps. Where no map exists an explorer has completely blind choice of where to go, and in an early stage of a science it seems almost fortuitous what observations are made next. But later, in proceeding from one observation to another, various mental gymnastics ("logics") are introduced to suggest connections. I believe quantitative description is the known means of coordinating those particular bodies of data that show how an 
organism is integrated. The data of biology are for the use of all travelers; anyone may select materials, as I have, that belong on certain quadrangles. New combinations of variables are made, whereupon general features emerge.

At first sight it might seem easy to use published data for a new investigation. I have not found it so. Ideally, hard-won materials might be rescued from the oblivion into which the hypotheses that accompanied them have fallen. Rare indeed is the record of a research that is complete enough to serve for other ends. Often, too, it takes more time to set down the limitations of an unfamiliar technique than to carry out new measurements. The data recorded are already selected in view of one conclusion, and very commonly the omission of one or two more correlatives defeats the new objective. So he who revels in details and relations can rarely be supplied, to his exacting requirements, from sources other than his own tests.

The accomplishments of mapping are illustrated in another science, meteorology. The prolonged study of many maps led to the recognition of intricate combinations of quantities that now together foretell tomorrow's weather. The first weather maps foretold nothing, for it took years for Brandes to devise them, and weeks for Galton to receive and record the information embodied in them (Geddes, '30, pp. 7 and 8). The choice of variables was arbitrary and depended on the recording instruments available. Facility in constructing and in grasping the weather's characteristics came with long practice and observation. Quantitative physiology appears now to be clumsily discerning similarly elementary combinations.

The individual who knows how to divide and multiply numbers can get along in many situations. But he who uses algebra can deal with larger and more complicated relations, while he who uses calculus is equipped to deal with multifarious and involved phenomena. In the end the biologist chooses whether to ignore the multiplicity of relations or to do whatever is now possible toward understanding them. It has been suggested by others that description (correlation) becomes more important as the system studied is more heterogeneous and complex; I suppose nearly every vital function is part of such a system.

Already physiology has used quantitative description. The multiple relationships in blood as modified during respiratory ex- 
change have been studied intensively (Henderson, '28). Though often not regarded as such, this study is stated by its author to be description. The special conditions in blood (physical heterogeneity, static equilibrium, chemical components) have been supposed by many to constitute a special case; however, while peculiar features are to be emphasized in any single investigation, no one need suppose that the methods there employed are by any magic limited to blood.

The multitudinous measurements of rates of oxygen consumption are now commonly recognized as descriptive. In the past certain hypotheses concerning the seats (locations) of regulators were set up. Many are the notions that have been vigorously flouted (Lusk, '33). I gather that these guesses have largely been forgotten now (Benedict, '38), and that the search for uniformities and variabilities and comparisons in the light of hypotheses of relationships, sufficiently satisfies those who give their efforts to this field of quantitative physiology.

Unforunately for human endeavors, there is no way of proving that one procedure is "better" than another. No one will know except by arbitrarily weighed results whether quantitative description was more economical of or enthusing to investigative effort than the search for causal relations or any other procedure. And, of course, each person will weigh differently the results of any search to which his predilections have brought him.

It is amusing to contemplate what advance in description of water balance would have materialized if all the effort that has been expended in gathering data concerning water in animals had somehow been coordinated. It is coordinated, but in small bits only. To coordinate it on a large scale would require that numerous investigators agree on the precise procedure by which water relations are to be varied (treating content as an independent variable in experimental studies). It might be supposed that no two persons would be likely to have a sufficient intensity of interest in the one physiological system. But it might equally be supposed that the mutual gain in correlative material to be realized would even enhance the interests. It is impossible to foresee what net modification in imaginative or incentive possibilities would ensue. In any such effort a preliminary period of exploratory indirection might prevail ; as soon as rough data are available, a detailed tentative plan matures. Perhaps the freedom of which all investigators 
are justly proud does allow of voluntary superplanning when a common design is widely recognized to be possible.

It is said of certain work that it is "mere" description. It is implied that description is less worthy of time and attention than material interwoven with hypotheses of some other sort. It is even said that description is "undigested," as though without hypothesis of singular nexus there is no thought or work. Unique nexuses between two variables $A$ and $B$ are rarely found; to imagine they are there stimulates interest; yet that sort of interest has usually, I gather, no value to the next generation of scientists. In so far as such interest pervades the materials of physiology, it is an ephemeral one.

Hypotheses that are alternative possibilities are sometimes said to be confusing. Yet equally well two hypotheses may be regarded as more fruitful than one. It may rather be an advance, in practice, to mention more than one hypothesis in each relation, in order not to limit the outlook or to make any one hypothesis seem too serious. For, a hypothesis is a personal aid to comprehension and a product of imagination, and there has been invented no means of ascertaining how many hypotheses will be fitted by the same data. Reasoning by exclusion hence is perhaps never conclusive.

Evidently there are diverse grades of description, and to recommend or to condemn all equally shows no acumen. "Procedure by construction is useful when it manifests something beside the juxtaposition" (Poincaré, '13, p. 41). Even the merest description involves much more thought in the making than is manifest in the final record. While the conclusions generally lack the embellishment of theories, in fact those who have worked by descriptive methods know that rich ideas (hypotheses) initiated inquiry, selected the measurements, and suggested the maps constructed. The arrangements of materials confer the significances to human view; further hypotheses are needed for successive rearrangements. But, so far as human weaknesses allow, the hypotheses are in descriptive work eliminated from the final product of investigation, and in no case are the cornerstones of it. Vanity hates to strike out the charming hypotheses that enticed one into the investigation.

If there be a hypothesis involved in the representation on paper of two simultaneously observed quantities, then this hypothesis is equally inherent in any and all recording of data. If correlation 
be a hypothesis, it is the minimum, others being added thereto when the data are given any "interpretation," or designated as having some particular kind of comnection with one another. I believe descriptive methods make it easier to distinguish hypotheses from facts. It even seems possible that "Description is the method of most use in biology" (Just, '39, p. 10). Certainly "description by no means opposes experiment, it calls for it."

Occasionally it is said that descriptive propositions have no human interests. Actually, however, men (e.g., Galileo) suffered martyrdom for them; whether the earth or the sun was the spatial center of rotation of the other, was ascertained by quantitative description. The fact, when demonstrated, precipitated great conflicts of men's sentiments and concepts, for plain equations of relations aroused intense feelings.

I have tried to make clear that an explanation of physiological regulations consists in the rules according to which organisms maintain constancies. Some scientists think explanations can only come from imagining hidden "mechanisms"; others find abstractions from relations among data more illuminating. It is important not to suppose that either procedure has a monopoly; and important not to expect the first kind to be used in a work devoted to the second. No one has, I believe, really found mechanisms of regulations of a causal nature such as are often subsumed. Until they are found, explanations of regulations rest upon those concrete forms of physiological relations in organism which have been here described.

\section{§ 155. Other MLDES OF PROCEDURE}

An understanding of the place of quantitative description may be furthered by comparison of how physiologists behave with description in view and of how they behave with other kinds of thinking in its place.

Hypotheses are intellectual means of avoiding randomness in inquiries, of building up interest, and possibly of increasing the chances of finding correlations other than zero. Some of the common hypotheses of relation among biological data may be classified as follows : mechanism, cause, purpose, origin, analogy, intermediaries, interaction. These terms overlap, and are diversely understood among physiologists that use them.

In choosing the direction in which he shall progress from one 
phenomenon to others, the investigator entertains various sentiments, hunches, and hypotheses. Emphatically there are many ways of consciously (as well as unconsciously) visualizing nexuses between a first observed fact and a second. Investigators are not limited to tracing origins, or to imagining causes, or to seeking analogies or to following communication lines; many paths of progression exist other than those postulated. Visualizations of nexuses are important chiefly in moving the mind along, in inducing the investigator to look for something; seldom are they indispensable in the final contribution.

Of mechanisms in the restricted sense, just as much information appears to be given by descriptive physiology as by other hypotheses of relation. For, mechanically minded physiologists emphasize, it appears, the chemical intermediaries, the anatomical elements, and the physical categories that are correlatives in each body of relations. In the broader sense, all correlations indicate mechanisms (categories), and are equally explanatory.

Of causes there is regularly more hope than realization. One relation is exalted while the rest are ignored. An engineer does not designate any one part or one process of a machine as the cause of its functioning; he regards most or all of its parts and arrangements as indispensable. Physiologists say they regard a living machine similarly, and behave quite otherwise. When one part of a machine is missing or damaged, its replacement, if leading to restoration, may be spoken of as the cause of the dysfunction. So there may be causes of failure but hardly causes of success. "Our grand foible turns out to be ... the facile and hasty reference of natural phenomena to mystical bogus entities, or to emotionally associated and whimsically selected 'causes' (Wheeler, '28b, p. xxiv).

"In studying the interaction between oxygen and carbonic acid, it is of the first importance not to regard the change in one substance as cause and the change in the other as effect. If we think of our terms mathematically as variables and functions, the difficulty does not arise. This error is an example of one of the most familiar and one of the most natural of fallacies and it was responsible for the long delay in reaching an understanding of carbonic acid transport"' (Henderson, '28, p. 82).

To suppose that a physiological regulation is managed by a director or a lever is probably in the same category as to decide 
what controls the rainfall. The doctrine of anatomical, biochemical and mechanical controls is an ancient one, at least as old as the location of emotion in the heart or thinking in the liver. Four humours have been succeeded by sixteen endocrines; the heart and the liver by the adrenals and the brain. The present generation laughs at only the humours. But the supposition is the same in all, and has scarcely faded with what I believe are successive disappointments. For many physiologists, concrete thinking may be difficult without, and may profitably employ, pictures of machinery. But to suppose that the distribution of blood in a man is dictated by a head office seems to me as unlikely a view as to suppose that a school of fishes has an appointed leader.

"Many will discover in themselves a longing for mechanical explanation which has all the tenacity of original sin. . . . It is easy to see how the demand for this sort of explanation has its origin in the enormous preponderance of the mechanical in our physical experience. But nevertheless just as the old monk struggled on to subdue the flesh, so must the physicist struggle to subdue this sometimes irresistible, but perfectly unjustifiable desire" (Bridgman, '27, p. 47). "A law of science, however wide its scope, does not go beyond a statement of the relationship or of the cause of these facts. Now that mind of man is so constituted that this iguorance of causes is to it a constant source of irritation; we are almost resistlessly tempted to pass beyond the mere statement of law to erecting a theory of the reality that underlies the law" (More, '13, p. 194).

Of purpose there is no clear evidence in the study of regulations. Many will insist that a pattern of equilibration bears the same evidence of intention as a watch. Others will equally feel no such inference. For, though equilibration may represent how an animal recovers its content, it tells nothing of why it does so.

Of origin of an innate pattern of regulations there is no information, of course. Such a pattern may be coeval with the first animal, and those who require to know where the first physiological regulation arose can do nothing to retrieve a situation of which there is no record. In the individual the development of constancy, of equilibration, and of appropriate behavior has been described for particular components. There is evidence that specific regulations are acquired.

Scientists wish to generalize before sufficient data have been 
obtained to warrant generalization. Sometimes the earliest general statement proves to be correct, within limits; often not. Since the limits are not stated, the generalization is not an inductive conclusion but a hypothesis. Hypotheses in their place are useful; those that are "reasonable" are often less reliable than those that are free of wishful thinking. In biology, however, one need not subscribe to a conclusion without knowing how much is opinion, and upon what and how many observations it is based.

Premature generalizations might ultimately be divided into those that later prove to be widely true, and those that prove to be true only in a minority of instances. Often arises the human question, which was the discovery? the first vague guess, or a later specific guess, or one of the several tests of whether the formulation was true or not? The first was one guess that was later found to correspond with the facts under limited circumstances, among other guesses that were later discredited since they were either untestable, or never tested, or found by test to be untenable. The second was also an inference or jump beyond that which had been demonstrated. Of the tests each was a step in mapmaking. "It is much easier to discover than to see when the cover is off," remarked Thoreau (1863, p. 71).

If physiology is to be more than a body of qualitative facts about which are built up projected or extrapolated aphorisms, physiologists will engage in searching out, by critical induction, conclusions that are epitomes of the observed facts and relations. Data will be at a premium, and perhaps no one will be a compleat physiologist who does not furnish quantitative relations with his observations. "Truths are fecund only if bound together'" (Poincaré, '13, p. 279). Far-reaching procedures of synthesis, combination, consolidation, will probably take precedence over the search for keyfactors and analogies.

A different opinion is clearly expressed thus: "Of the two types of formulation consciously employed that which is purely statistical is unsatisfying, since the constants it provides are dimensionless and arbitrary; expressions derived from physical theory have the enormous advantage that the character of their contained constant immediately gives basis for concrete experimental tests. Without such tests these equations may also remain arbitrary", (Pincus and Crozier, '29). I am not convinced that in any single instance in biology, nor in any other science, the character of the 
constants has never been more than that of parameters in equations. It may be satisfying to suppose that they are more, and it gives an impetus for experimental tests if those suppositions are followed through. But the satisfaction is no part of the structure of ascertainable facts and relations, nor is the realization of predictions a proof of their theoretical basis. For there are usually more possibilities than have been imagined. The satisfaction gained by one experimenter may be unwelcome to another; probably whatever is permanent in science will be limited to the "purely statistical." Mathematical language has for most men the fewest implied notions and fancies.

Some physiologists suppose that they can distinguish a "direct" connection between two phenomena from an "indirect" or incidental one; a "primary" or "fundamental" or "essential" factor or nexus from a "secondary" one. But no rule is given for making this distinction. Evidently a "direct" nexus is one that agrees with certain hypotheses, or with certain types of hypotheses. Perhaps expertness is even signalized by insensitiveness towards the assumptions implicit in its own field.

Some do not think phenomena exist until a plausible "theory" (of physicochemical mechanism) is found for them. Thus, they find it unbelievable that dogs drink water in amounts approximately equal to their deficits of water, or that rats select foods containing vitamin $\mathrm{A}$ when vitamin $\mathrm{A}$ is depleted, even though measurements have been reliably presented. They say, before we accept those correlations show us what physical and anatomical features are concerned, "how" the animals make the choices. They limit their nexuses to particular sorts, and quantitative relations seem to them to leave these phenomena as "isolated facts" of doubtful existence.

I hope it is understood that the broad statements of this chapter are simply the projections of experience and inference. The rigorous inductive procedures of previous chapters have now been supplemented by extrapolations whose force is of the same magnitude as those of persons holding other opinions. These extrapolations issue from experiences with those procedures that have been emphasized in this investigation.

So, present day physiology is exposed to the distinct, not mutually exclusive, possibilities; (a) That several methods of discovery and of investigating relations among phenomena will be 
equally "fruitful." (b) That some procedures are incapable of the accomplishments made by others. (c) That recognition of the validity and effectiveness of additional general methods in physiology need not reduce but rather reinforce the solidity and assuredness achieved by use of those procedures already employed.

\section{§ 156. Summary}

It requires re-emphasis that correlations alone may be highly enlightening, without reference to causes or mechanisms, or even to possible purposes or origins. Biometry has evolved on the interests inherent in correlations; its lessons have usually been limited to a particular subject content instead of being used freely in other fields of biology. "Physiometry" might supplement much of present-day physiology.

Both specific outlines of the present investigation and general methods of choosing variables and relations are reviewed. It is useful, in multiplying relations among phenomena, to keep selected variables present through more than one study. Uniformities and patterns of relation may then be reached by inductions.

Will scientists continue to add knowledge at faster and faster rates without having plans for coordinating and assimilating what they add? For physiology, some of the plans here reviewed are available. In particular the mapping of coordinated physiological components, and the prolonged search of these maps for uniformities of relations, may become a major activity of those interested in living units.

General methods and procedures are of use both in planning investigations and in reporting them. They help clearly to segregate hypotheses and opinions. Descriptive physiology is the study of multiple relations among phenomena, expressed in language that is freed to the utmost of hypotheses. So far, description furnishes the only tested explanation of physiological regulations; it tells what the net processes of regulation accomplish. This fact suggests that the definition, classification, and correlation of selected variables may constitute a method to be used in various fields. It can indicate how each activity of organisms is actually performed. 


\section{Chapter XIX}

\section{PHYSIOLOGICAL REGULATIONS}

\section{\$157. Meanings of Regulation}

While the entire study pursued has been concerned with physiological regulations, no refined definition of the term has been attempted. Rather, concrete notions of what happens to perpetuate the properties of organisms have been allowed to accumulate. Many connotations and implications commonly attached to the term regulation have thereby been left behind. Some of the charms of the word Regulations are, perhaps, displaced by specific correlations among physiological quantities. It remains to be decided whether the series of relations that was ascertained to exist among selected quantitative data is of the sort meant by biologists who discuss self-regulation.

Definite notions of what are now called regulations in organisms have been familiar since at least 580 в.с. "The preservation of health consists in an accurate adjustment of forces (irovouiav $\tau \hat{\omega} \nu \delta \nu \nu a \mu \epsilon \omega \nu$, balance of tendencies)," wrote Alcmaeon the physician of Crotona (Diels, '03, p. 107). "The ascendency ( of any one of the forces leads to sickness, . . . while health resides

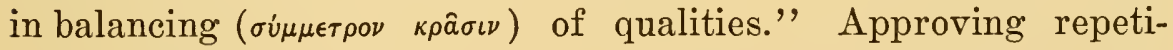
tions of that idea recur, as in Plato (Phaedo, verse $\Gamma 36$ ); and the Hippocratic doctrines of physical constitution or maintenance

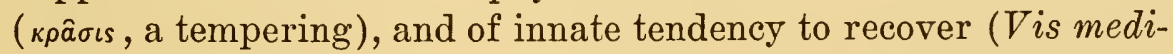
catrix naturae) are said to be corollaries of it (see Brock, '29, p. 6, $9,103)$. The restatements of these doctrines in the medical sciences of the centuries up to ours have been reviewed by Neuburger ('26).

A modern definition of regulation has been offered as follows: "We shall understand by regulation any occurrence or group of occurrences in a living organism after any disturbance of its organization or normal functional state, and which leads to a reappearance of this organization or this state, or at least to a certain approach thereto"' (Driesch, '08, p. 166).

In these and other concepts I gather that regulation is either (a) the processes and events that favor preservation or recovery 
of constitution and function, or (b) the provision of the states and properties (regularities) that characterize the living individual, or both. In other words, physiological regulations are an outcome of the combinations of properties possessed by the living unit in its environment, and seem to be such as to maintain and restore states of balance as I defined them. The definitions and statements quoted are, it appears to me, inexact notions concerning those fixities in the midst of flux which the variabilities of components and the equilibration diagrams more specifically and exactly describe. It seems clear that the very relations included in these particular abstractions have been quantitatively represented in this investigation. So long as their recognition depends only upon their manifestations (loads, rates, and reactions), there is nothing indefinable or incomprehensible about regulations.

The word regulation has been current in physiology at least since 1870, with a meaning not very different from that expressed here. Often it was used in connection with the striking constancy of body temperature. In a separate field of biology it was used to characterize the adjustments of body form during regeneration of tissues. The broad implications of the word were set forth by Driesch ('01), who actually classified organic regulations, though in ways that now seem useless.

Like other students of biology, I was repeatedly told that regulations were important. What they were and how they operated was far from apparent. Gradually they acquired meaning, not as inferences from metaphysics, but as hazy inductions from biological experience. More recently I observed data concerning balances, variabilities, recoveries, and behaviors that manifest a common bearing upon the maintenance of properties. Bit by bit I have come to realize that this pattern of maintenance is probably what my preceptors were talking about. But, as a result of my backwardness I have an unforeseen advantage over them, for I build my abstractions about equilibration diagrams and frequency diagrams, while they used words. My concept is in quantitative and dimensional form, theirs was, I infer, less definitely founded.

According to my notion, a physiological regulation is not the intangible director of what goes on in an organism, but is the specific pattern of certain correlations (coordinations) operative during life. A regulation is the constellation of activities whereby some property or component of the organism is self-maintained. 
There are plenty of other notions about regulations. Very often a process is said to be regulatory only when an investigator thinks it is advantageous to the organism. Or again, regulation is such when states or changes occur that do not usually occur in dead or inanimate systems. Very often it is stipulated that "active" adjustments or that energy-consuming maintenances be involved. There are many phenomena, commonly said to be unregulated, in which objects warm up in a cool environment, as when water and sulfuric acid mix; while there are many others, still said to be regulated, in which objects merely cool in a cool environment, even as a man cools off after exercise. The term regulation is in that case reserved for unexpected phenomena. I believe that on further analysis none of these definitions is practical or operational. Illdefined usages of the word regulation dangerously enforce the urge to coin a new one.

Regulation, being an aspect of all phenomena concermed with maintenance and recovery, may consist in (1) resistance to loading, (2) preference among conditions, especially by shunning some, and (3) compensation or correction of loads. In general, it might be counted more clever for the organism (1) to be impervious to certain components, and (2) to avoid those components that are deleterious, instead of having (3) to compensate for disturbances.

A man resists the creation of heat deficit or excess by clothing himself appropriately, prefers air-conditioning, and compensates a deficit or excess with circulatory modifications. A turtle prefers moderate air temperatures, and compensates (by modification of process) only an excess of heat. An earthworm only selects its environmental temperature, avoiding extremes. The actual effectiveness of a single means of response to heat is indicated by the wide distribution of earthworms.

The roles of these three kinds of maintenance among diverse organisms may be grasped by table 44 . The grouping of several components may suggest that many vicissitudes are to be continually met by each individual. Each species has a diverse armamentarium for coping with them. Very often one sort of activity, such as locomotion, partially serves to cope with an enormous number of components. Like swallowing, it is a general form of response that in specific form is keyed to each of many loads, and perhaps in addition takes blanket care of newly-encountered and unspecified ones. 
Whatever the specific manners in which each functional state of a living unit is preserved, each state is definable in no uncertain terms. All processes that are found to contribute to or detract from this preservation are concerned in regulations. Perhaps most or all that happens in organisms will be found to be such.

TABLE 44

Means of maintaining constancies with respect to several components in 4 species. Symbols \pm refers to positive and negative loads. Further details concerning corrections are listed in table 32

\begin{tabular}{|c|c|c|c|c|}
\hline Species & Water & Heat & Carbon dioxide & Oxygen \\
\hline Man & $\begin{array}{l}\text { Corrects, + and -, } \\
\text { fig. } 60 \\
\text { Avoids, + and - } \\
\text { Resists, + and - }\end{array}$ & $\begin{array}{l}\text { Corrects, + and -, } \\
\text { fig. } 202 \\
\text { Avoids, + and - } \\
\text { Resists, - }\end{array}$ & 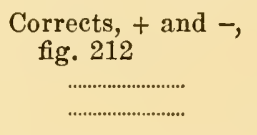 & 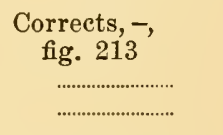 \\
\hline Frog & $\begin{array}{l}\text { Corrects, + and -, } \\
\text { fig. } 86 \\
\text { Avoids, - } \\
\text { Resists, +, } \\
\text { Adolph ('31b) } \\
\text { No resistance, -, } \\
\text { Adolph ('32) }\end{array}$ & $\begin{array}{l}\text { No correction } \\
\text { Avoids, } 40^{\circ}, \\
\text { Morgan ('22) }\end{array}$ & 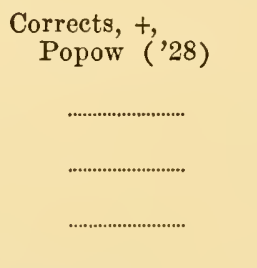 & $\begin{array}{l}\text { Corrects, }- \text {, } \\
\text { Wolvekamp } \\
\text { ('34) }\end{array}$ \\
\hline $\begin{array}{l}\text { Earth- } \\
\text { worm }\end{array}$ & $\begin{array}{l}\text { Corrects, -, } \\
\text { fig. } 107 \\
\text { Avoids, -, } \\
\text { Wolf ('38a) }\end{array}$ & $\begin{array}{l}\text { No correction } \\
\text { Avoids, } 27.5^{\circ} \text {, } \\
\text { Wolf (' } 38 \mathrm{~b})\end{array}$ & $\begin{array}{l}\text { Corrects, }{ }^{+}, \\
\text {Voigt ('32) }\end{array}$ & 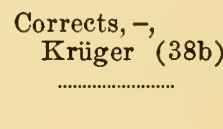 \\
\hline Ameba & $\begin{array}{l}\text { Corrects, +, } \\
\text { section } 114 \\
\text { Resists, t, } \\
\text { Müller ('36) }\end{array}$ & $\begin{array}{l}\text { No correction } \\
\text { Avoids, } 35^{\circ}, \\
\quad \text { Verworn, p. } 452\end{array}$ & $\ldots$ & $\ldots \ldots \ldots$ \\
\hline
\end{tabular}

\section{§ 158. Classification}

Further understanding of regulations may be gained by attempting to classify them in additional ways. As in the past, meaningless (insoluble) propositions may be avoided by appealing in each classification to the procedures of measurement.

(1) Each component found in an organism, and every procedure of measuring it, might be said to identify a regulation toward whose maintenance or avoidance the organism is working. To the organism each component would perhaps hardly seem to be a discrete entity; to the investigator there is no more direct way of dealing with a regulation than by measuring the corresponding component. Since measurement of whatever is being regulated implies dimensions, components themselves may be classified accordingly. No cognizance is here taken of possible physical proc- 
esses of regulation, nor of anatomical structures, except as they also define the living units studied. Chemical constitutions may be used to define certain components; tests and instruments to define others. For each component the various latitudes, compensatory rates, and other correlatives may be listed as characteristic of the organisms tested.

(2) Regulations by equilibration may be grouped according to the nature and sign of the exchanges aroused at every increment of content. The feature named in each group is an element in the patterns distinguished earlier ( $\$ 72$ and $\S 142$ ). Among many components, one organism (dog) exhibits:

(a) Indifference. Rate of exchange is independent of load within limits; e.g., deficit of the element lead. This is a lack of compensation.

(b) Avoidance. Exchange is minimized by diminishing the gain; e.g., water excess (fig. 14).

(c) Conservation. Exchange is minimized by diminishing the loss; e.g., water deficit (fig. 14).

(d) Dissipation. Loss appears where none was present in balance; e.g., carbon monoxide excess (table 41).

(e) Faster dissipation. Loss is hastened over that in balance; e.g., water excess (fig. 14).

(f) Accretion. Gain appears where none was present in balance; e.g., deficit of skin (fig. 179).

(g) Faster accretion. Gain is hastened over that in balance; e.g., water deficit (fig. 14).

Each of these categories recognizes a direction of exchange (gain or loss) and a change in rate (increase or decrease). In very many loads two of these activities proceed simultaneously. Where only net exchanges are known, this classification is of no avail.

An example indicating the sorts of modification concerned in recoveries is heat in man (fig. 145). It was formerly supposed that heat deficits are corrected by increased heat production in oxidations, some or all of the increase being related to muscular movements of shivering. Under many circumstances both increased production and decreased loss are indeed realized. But under the conditions of the present test no augmentation of heat production is found; net heat is solely gained by decreased rate of heat loss. 
The parts played in recovery by the gains and the losses appear to me to represent one common denominator in the interests of all physiologists.

(3) Paths of exchange may be classified. Such categories as synthesis, absorption, and excretion might be recognized. But endless difficulties would arise as to whether hydrochloric acid is synthesized or results from a local change of hydrogen ion concentration; or whether hemorrhage is excretion or decomposition. For a limited variety of species the structures concerned as paths may furnish divisions, but in general, redistributions and transformations do not commonly pass over defined morphological routes.

(4) Attempts to compare components according to the tolerated load of each can hardly be recommended at present. If I were able to choose one criterion of survival and one unit for all loads that are to be compared, this classification might be carried out quantitatively (table 40). There is no implication that a large permitted load is "better" for the organism than a small one; that is a matter for further study in the light of each selected criterion.

(5) Quantitative classification might depend on accuracy of preservation of constancy for diverse components. Then values of C.V. or of $\mathrm{C} \Delta$. for content of each component would be ascertained in particular ranges of conditions (as in tables 12 and 39). The time periods and physiological states chosen are strikingly arbitrary.

(6) Rates of net recovery might be compared (table 40). Occasionally a single component would fall into very different classes in diverse species, upon this criterion.

I prefer to handle regulations by a combination of classifications (2) and (6). An evaluation of recoveries by those methods condenses much biological information. I find it very unsatisfactory to classify regulations according to chemical, physical, and anatomical categories. Anatomical considerations are of no help in protozoa. Those considerations put similar components into diverse classes in organisms of different structure. They separate for one component the gains from the losses, and one path of gain from another. Such categories detract from the characterization of adjustments and their kinetic aspects, and I think immediate progress in unravelling regulations depends in part upon ignoring them. 


\section{\$ 159. Measures of Regulation}

Qualitative aspects of regulations are of great variety, and a high proportion of current physiological investigation contributes bits of information concerning them. Quantitative measures of what goes on in organisms to secure and ensure uniformity of physiological state appear to be of only three sorts at present, all of which were abundantly illustrated.

(a) Variability of content of component. In any one of many possible sets of conditions the latitude of loads found is ascertained. Some coefficients that have been employed above are: (1) standard deviation and coefficient of variation, (2) root mean square of differences and coefficient of difference, and (3) frequency of occurrence.

TABLE 45

Specifications of regulation of water in dog

\begin{tabular}{|c|c|c|c|}
\hline Parameter & $\begin{array}{l}0.25 \text {-hourly } \\
\text { intervals }\end{array}$ & $\begin{array}{l}\text { 1.0-hourly } \\
\text { intervals }\end{array}$ & $\begin{array}{l}\text { 24-hourly } \\
\text { intervals }\end{array}$ \\
\hline $\begin{array}{l}\text { Load, } \mathrm{C} \Delta \\
\text { Intake, } \mathrm{C} \Delta \\
\text { Output, } \mathrm{C} \Delta \\
\text { Frequency of } \Delta \mathrm{W} \pm 0.2 \text { or more } \\
\text { Turnover rate, } \% \text { of } \mathrm{B}_{0} \\
\text { In stationary state } \\
\text { Net velocity quotient, } \mathrm{k}_{\mathrm{l}}, 1 \text { hour } \\
\text { Economy quotient } \\
\text { Augmentation ratio } \\
\text { Recovery in first } 1.0 \text { hour, } \% \text { of } \mathrm{B}_{0}\end{array}$ & $\begin{array}{c}0.024 \\
180.0 \\
19.0 \\
\ldots \cdots \cdots \cdots \cdots \cdots \cdots \\
\text { When } \Delta \mathrm{W}=+\cdots \\
0.37 \\
0.08 \\
0.3,3.3 \\
0.50\end{array}$ & $\begin{array}{c}0.098 \\
75.0 \\
16.0 \\
0.15 \\
0.25 \\
\text { When } \Delta \mathrm{W}=-2 \\
1.7 \\
18.0 \\
14.0,0.8 \\
2.00\end{array}$ & 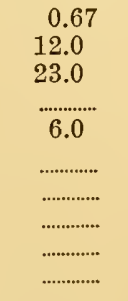 \\
\hline
\end{tabular}

(b) Rates of exchange of the component loaded. These may be studied in relation to diverse loads in the form of an equilibration diagram. Or, by abbreviation, various parameters of the exchanges may be studied, such as: (1) velocity quotient, (2) economy quotient, and (3) augmentation ratio. Provisions for minimizing exchanges (resistance to and prevention of them) are prominent.

(c) Preference for environments that tend to preserve or restore the contents of component.

Proceeding with specifications in the way an engineer does, a sample appraisal of regulation with respect to one component (water) for one species (dog) would be as shown in table 45. Any single specification indicates something concerning the regularity of the component and of its exchanges; all of them together make the characterization seem as complete as is allowed by available 
methods. An equilibration diagram to which variability of content has been added, represents most of that information in one frame (fig. 47). It is probably meaningless to bewail the fact that not everything known about the dog's water relations is represented in the one table or diagram.

Comparison among several components (table 40) with respect to any or all measures of regulation reveals the wide variety of values found. Ultimately a table many times as large as table 45 might be drawn up, based upon observations made simultaneously in respect to $n$ components.

Stability or stabilization is a term used qualitatively in some of the same connotations as regulation (LeDantec, '10). Arguments as to whether "living matter" is more or less stable than nonliving, are avoided by comparing specific measurements of particular components.

An ordinary definition is that stability is the reciprocal of a variability $(1 / 0)$, therefore of a load $( \pm 1 / \Delta C)$ or a rate $(\Delta t / \delta C)$. Another quantity related to stability is the steepness of the slope in the net equilibration diagram; this is rate/load and has already been recognized as net velocity quotient $(1 / \Delta t)$. Both kinds are shown in fig. 47 ; they are not commensurate.

"What characterizes a living being is without doubt first that it is at each instant the seat of a flux of matter and energy, but above all that in spite of this flux and due to it, it remains constant, or rather that it maintains itself similar to itself" (Gasnier and Mayer, '39, p. 146). That statement carries its own designation of measures that will describe regulation.

\section{\$ 160 . Kinetic equilibrium}

Figure 47 exhibits two sorts of minima into which the organism may be said to fall in respect to a component. One is the reciprocal of frequency of occurrence, which represents the outcome of all the "difficulties" in the way of having any other load than zero; the other is equilibration, the net rates of exchange that are aroused when the content departs from $\mathrm{C}_{0}$. Both curves present the picture of a groove into which the organism gravitates and in which it tends to remain. The trough of this groove is at balance of the component. It represents a kinetic equilibrium, since exchanges are known (for many components) to be still going on; but positive and negative exchanges are equal, so that the net rate 
is zero. To refer to the trough as a locus of dynamic equilibrium would add the inference that forces also have there a resultant of zero, which is probable, but for them no measurement is usually available.

Maintenance and return to a mean position is illustrated, both mechanically and physiologically, by tonus distribution in limb muscles and tendons of man. The resting position of standing erect means approximately equal lever tensions exist in antagonistic muscles + gravity. Any inequality or movement is ultimately recovered from by pull back to initial posture. This maintenance represents a pattern of rates and forces in the body. It by no means depends on gravitational forces alone any more than it depends upon muscular forces alone. Here is an instance in which actually more of the forces have been measured than of the rates of transformation involved in their manifestation, and whatever satisfaction lies in the study of forces rather than in the study of rates of exchange is here available. A grand panorama of neuromuscular patterns is visible in that distribution of both forces and rates, and dynamic and kinetic equilibrations might here be worked out side by side.

It has long been recognized that relations resembling equilibrations occur in non-living systems. A general statement is the theorem of LeChatelier ('84; '88, p. 48). Derived at first from thermochemical studies, "if the solubility of a gas increases with diminution of temperature, then its solution will take place with the development of heat," the hypothesis now includes many other phenomena. In current expression, the generalization is that "If a change occurs in one of the factors determining a condition of equilibrium, the equilibrium shifts in such a way as to tend to annul the effect of the change"' (Taylor, '24, p. 307). Change is specified, and rates of reaction are the usual measurements that illustrate it. While the phenomena studied are those of heterogeneous systems, it is possibly misleading to extend the theorem itself to living units that are exchanging with their environments. The environment is in that case part of the system to be observed, and metastable equilibria, reflexes, growth, and week-long recoveries complicate the picture. Some day it may prove valuable to have one theorem, or even one induction, for all those systems.

There is regularly one value or range of values of each component toward which the organism or species is indifferent, as, one 
tissue temperature in each part of a man. That might justify designation of an equilibrium as a complacency (Raup, '26). Apparently the particular content at which the human organism is in balance recurs just as inevitably as it does in any other sort of system at equilibrium. Other organisms, as the turtle, are indifferent toward a wide range of temperatures. Turtles modify their coefficients of heat exchange only at high positive loads, and not at others; in addition they exhibit behaviors of shunning extreme temperatures that are effective in preventing extreme loads.

Maintenance is probably less commonly a cessation of activities of the organism (static) than of recovering their usual rates after displacement (kinetic). Measurements of rate therefore describe the equilibrium of the organism; what it tends toward rather than what it keeps. The contrasts between rates during displacement or load and the rates at equilibrium are the very features that emphasize regulation. The terms "homeostasis"' (Cannon, '32), "complacency" (Raup), and others, possibly imply a static system. Any other new term such as "homeokinesis" will in its turn become inadequate as concreteness is gained; hence my determination to get along with older terms such as equilibration (Spencer) and regulation (Bernard). Moreover, the quantitative aspect of regulation will hardly be adequately represented by any word.

\section{$\S 161$. Multiple Regulations}

It seems as though the number of components regulated and the types of their loading is almost limitless. For, not only can new components be investigated, but components can be subdivided and grouped; and the rates of rates (accelerations), and even changes in acceleration, can be added. The conditions as well as the physiological states may differ in a semi-infinite variety of combinations to yield new types of increment for each measured component. Certainly the distinctions among types and varieties cannot be ignored, and one way of limiting the study in practice is to agree on arbitrarily standard conditions and species. Another is to study many recoveries simultaneously in a single individual, leading to an understanding of multiple regulations.

Regulation of some particular component is isolated only as a scientific tour de force, the isolation and definition usually depending on methods of measurement and on conditions imposed upon the organism. For the complete description of a regulation, the 
whole functioning organism and its ancestors would be described in all possible aspects. But approximate description is facilitated by selected circumscription. In this way the breathing of man was studied as an isolated activity, so long as control rates of pulmonary ventilation showed variabilities less than the rates that were found after disturbances. In this way the respiratory changes in blood were considered by themselves. The isolation is ordinarily accomplished by defining distinctive conditions so that the correlatives that are significant are either recognized or controlled. Acumen in biology is often in the recognition of highly correlated variables and the neglect of others.

When a biologist studies a "closed" system he may profitably remember that the act of limiting the independent variables also limits the manifestations of regulations. Thus, the study of the respiratory cycle in blood limits the regulations to those components that change during this cycle. Limitation is desirable for many reasons; but only on comparing additional states are the regulations exhibited in wider variety.

In general, each variable and condition brings to light certain aspects of regulations. Identity and similarity of components is in the end judged by arbitrary criteria of resemblance in the quantitative manifestations observed (e.g., rates of exchange) and in the procedures used in measuring them.

Continuance of life itself is not always a correlative of recovery. Frogs that were desiccated by $32 \%$ of $\mathrm{B}_{0}$ or more, often died (fig. $66)$; but whether or not they died, water was regained equally fast. Hence death did not depend on inability rapidly to regain water content of the body as a whole. A deficit of water that exceeded the tolerated water load (as judged by survival) did not indicate inability to gain water faster than it was lost. Conversely, recovery toward water balance did not mean recovery of all functions required for survival; and there was no fast relation between the kind of deprivation to which the animal was subjected and the kind of recovery that was required for survival. Other functions than ability to gain net water content were concurrently upset, so that what was described as regulation of water content was insufficient to insure survival.

Someone can always assert: the organism is not "primarily" regulating water content, but is adjusting the concentration or the permeability. How does he tell? There appears to be no criterion 
for what is primary to an organism. Actually each adjustment is part of several or many equilibrations, even as rate of water exchange was found to vary in relation to loads of heat, glucose, and chloride as well as of water. All exchanges that occur are equally parts of the study of physiological constitutions.

On the whole, large numbers of loads and rates are continually compatible among themselves. Evidently one recovery does not ordinarily proceed in disregard of others. That is a description of observed phenomena, whatever implications of fitness be suspected in the plain statement of it.

\section{$\$ 162$. Signs and TeSTS}

An equilibration diagram is an orderly description of regulation in some component, of a completeness that has so far been attained for few components. Yet if their relation to the whole diagram is understood, much can be inferred from a few signs and tests. What is the relation of signs and tests to the diagram as a whole?

In clinical use a sign (of disease) is but poorly distinguished from a symptom; often the two words are synonymous. In terms of the definitions formulated in this investigation, a sign is a load; a content of some component observably different (qualitatively or quantitatively) from that in control individuals or states.

No clinician regards "seriously" those signs or symptoms that also occur physiologically and from which recovery is rapid. Rapid is here defined according to the rate at which each component recovers in usual individuals. Every clinician has a rough idea of the mean rate of recovery from each such displacement, even though in most instances those rates and their variations have not been accurately recorded. In the present terminology, he implicitly estimates loads or states, and rates of recovery from them. No one worries about an increment of rectal temperature in man after muscular effort or exposure to hot atmospheres that has a half-life of less than 1.0 hour; nor about an arterial blood pressure double the usual that lasts for 0.1 hour after a mountain climb. The persistence of a sign indicates that ordinary recovery is delayed or inoperative, though eventually unloading may occur. Meanwhile an excess (load) of heart frequency may be borne for years, but a deficit (load) of oxygen may be instantly fatal. 
Signs may be considered in general as either qualitative, or else quantitative abbreviations (portions) of the relations shown in the equilibration diagram. The total data of equilibration can rarely be measured at one sitting; on that account if on no other, simplified and conventional observations are substituted. The fact that the substitution was made without formal comprehension of the multiple and quantitative interrelations, is an illustration of the usual manner in which science proceeds. Almost never does a well-rounded description spring full-formed to light.

Tests are sequelae of disturbances artificially introduced. Not being satisfied with measurement of whatever loads and rates occur spontaneously, the physiologist or physician prods the organism to see how certain loads and rates are modified.

TABLE 46

Some signs and tests commonly used in appraisal of certain regulated components of man

\begin{tabular}{l|l|l}
\hline \hline \multicolumn{1}{c|}{ Component } & \multicolumn{1}{|c}{ Sign } & \multicolumn{1}{c}{ Test } \\
\hline Water & Relative body weight & Water-ingestion \\
Total substance & Puffed skin & Pitting of skin \\
Heat & Body weight/height & Alimentary utilization \\
Heart frequency & Rectal temperature & Sweating \\
Pressure in arteries & Pulse count & Exercise index \\
Glucose & Systolic sign & Exercise index \\
Posture & Cone. in blood & Tolerance \\
Leucocytes in blood & Romberg & Tait-McNally \\
Acid & White cell count & Leucocytic reaction \\
Oxygen & Alveolar $\mathrm{CO}_{2}$ tension & Bicarbonate ingestion \\
& Cyanosis & Oxygen inhalation \\
\hline
\end{tabular}

The general form of tests is the tolerance curve (A, fig. 180). By some one type of procedure or agent, a measurable load is imposed and the subsequent changes are followed with respect to time. Occasionally the observer differentiates with respect to time, obtaining a net exchange curve (B). Rarely indeed are the complete rates of total gain and the complete rates of total loss (C) recorded. Of course, in many components it is quite sufficient for most purposes to observe merely the maximal load, or to demonstrate that recovery is sensibly complete within 0.1 hour, or the like. I note no exception to the guess that every sign and every test can be represented as a point in a diagram or a curve of the forms shown in figures 110 and 180.

In physiological as in clinical appraisal of an individual, each sign and test partially represents the regulation of a particular set 
of components. Some procedures frequently used to characterize men are listed in table 46 . One estimate of the utility of these signs and tests would be to compare the numbers of determinations of each made in a hospital per bed per year. A large experience, and multifarious beliefs, are epitomized in the mass employment of each of them.

A physiological abnormality or a disease may be viewed as a simultaneous array of continued loads in many components. The displacements from balances are multiple. It is even possible that with respect to each single component the state might be regarded as within the physiological range, whence the simultaneous combination constitutes the disease. The "underlying" states so often mentioned are in my opinion the interrelations themselves. Of course, any one component appears loaded in numerous diseases. Differential diagnosis applies a dichotomous key to the combinations among the various components commonly measured or tested.

The practice of observing each component probably developed independently of every other, and in parallel with the development of instruments and human needs. Now that the quantitative characteristics common to many components are partially known, shall each load be described in a diverse and traditional way, or shall it be acknowledged, for instance, that all tests are tolerance curves? Shall it further be recognized that common scales, such as percentage of $\mathrm{C}_{0}$, can be used for most components? I am not concerned with reform or advocacy; my present obligation is to point out that the means of schematizing (perhaps simplifying) these matters of everyday physiology and nosology are available, and that some of their uniformities have been quantitatively ascertained.

Knowledge of the multiple interrelations among loads and components is some indication of the complexities inherent in therapeutics. Therapy can be regarded as the removal of loads. But removal of one load may enhance others; this is the limitation of "rational" therapy, for no physiologist or clinician comprehends how many components and what loads of each are concerned. Every sign suggests a treatment, but no assurance of recovery in all respects is obtained except by trial. It is widely understood that what seems most "indirect" is sometimes the shortest way to 
cure. All this might be put into quantitative terms, as corollaries of the above study of physiological interrelations. Further, it can be realized how infinitely small is the chance of finding a therapeutic agent that shall correct one load without disturbing other components; this statement corresponds to the discrediting of symptomatic treatments.

A task of medicine may be pictured, even as Hippocrates pictured it, as the encouragement and assistance of physiological recoveries and the prevention of loadings. Popularly it is supposed that physiology contributes to this art chiefly by furnishing "wiring diagrams" to show the connections between one organ and another, with arrows leading hither and yon. From that the clinician is expected to guess which wires to cut or mend. I believe that physiology fulfills a larger function by furnishing its story of equilibrations through exchanges, and its detailed picture of interrelations. From those it is possible to rationalize the particular combination of signs, the possibility of correctives, and the prognosis of inherent self-managements. From those it is also possible by simplified tests to plot the state of the individual and to measure his progress toward or from balance.

\section{$\$ 163$. Summary}

Regulation corresponds to certain of the correlations (interrelations) that are here investigated. Whether one likes this term or some other or none, the associations of rates of exchange with particular excesses and deficits recur in many organisms in uniform patterns of a sort epitomized by it. Realization of the constant features of these patterns aids in assimilating and comprehending diverse data of physiology and applied biology. Various notions about health and disease are found to be particular instances of these general properties.

The concept of regulation is here not something added to the observation of phenomena, but is a generalized statement of uniformities in the relations among them. Instead of being a luxurious superfluity in biology, a metabiology, I believe that study of certain data compels the recognition of relations conveniently denoted by the word regulation, in the same sense that it compels the recognition of individuality, coordination, life, equilibrium, or other forms of relation. 
Preservation of function, and restoration of it after disturbance, characterize most of the physiological quantities that have been measured. Each equilibration appears as inevitable. No estimate is attempted of the number of components whose preservation (within certain limits of accuracies or stabilities) is requisite to the continued existence of any particular living unit. It is plain that all components exist compatibly and recover after disturbances in the midst of a community of regulations. 


\section{Chapter XX \\ SOME SPECULATIONS CONCERNING REGULATIONS}

$\$ 164$. In previous chapters I have tried to limit the account of regulations to data, to relations, and to inductions from them. The conclusions are drawn, I hope, in such a manner that they need not be accepted without examination of their foundations. Still more general statements may be possible, and undoubtedly relations have unwittingly been established that in the future can be recognized as inductions.

Here and there statements have been introduced, as suggestions, that are not regarded as rigorous inductions; these have been labelled as possibilities or beliefs or opinions, wherever I was conscious of their nature. Further, every statement contains uncertainties; if in nothing else, then in its being general, or incompletely delimited. Induction $\mathrm{Y}$ derived from four instances and no exceptions may be some day found to have more exceptions than instances; ideally the general statement would not overreach the instances. In practice that is cumbersome; and for convenience, but later often by oversight, the qualifications are omitted. Hence it is always possible that what seems to one person a well-founded induction seems to another person mere extrapolation; agreement between them might be reached by common assent to some one criterion of probability.

Having enough facts and relations to furnish an account of regulations without resort to speculations, does not mean that I have no products of imagination, nor that I do not enjoy speculating. Most of my speculating was done before this investigation began to materialize. A preference for recording hypotheses corresponds to a scientific taste that probably owes a considerable portion of its vogue to lack of desired information. Those whose tastes are ruined by the strong flavoring of conclusions by hypotheses, may on that account not enjoy the absence from this inquiry of hypotheses of a usual sort. Those who revel in relations among detailed and numerical facts may feel quite otherwise. Exercise in description is just as fascinating as stretching toward imagery, if one tries it and likes it. 
Very often speculations are not distinguished from conclusions. Here they are separated in some degree, and what relations are lost by the separations may be compensated in part by the alternative segregations.

\section{$\S 165$. Distinctions between generalizations}

AND THEORIES

Occasionally an induction is rigorously arrived at, yet no one, least of all its author, can trace the steps in its path and the facts that support it. Then the induction appears as a guess, undistinguished from all the other speculations with which scientific work is littered. It, like any theory, awaits the clear recording of its foundations and evidences, before its provisional nature can be dispelled. Perhaps that was the case with the generalization called physiological regulation, in the mind of Bernard, or of Pflüger, or of Haldane; but I cannot prove it.

In an age in which novelty is often the criterion of scientific value, it is necessary to point out that most inductions are built upon data already familiar. The broader or more general correlation, however, is now accounted boresome, because it reconsiders data either long familiar or forgotten. It is even implied that old material is merely being reclothed in new words and symbols; this is a leering characterization of the inductive process. The inductive scientist values the uniform relations among the facts more than he values the decent burial heretofore accorded to the facts. No doubt the descriptive physiologist also finds some satisfaction in the novelties (a) of discovering that separate phenomena resemble or relate to one another, and (b) of ascertaining the intensities of diverse correlations. He it is who is producing the generalizations of physiology.

For comparison with the generalization that organisms have specific means of physiological self-maintenance, it is useful to ask: what are the most widespread features of animal function? What are some of the other broad generalizations of physiology? It seems plain that they are not the statements of compartmented physiology, where muscle knows nothing of gland or blood. My guess is that they are the rules that organisms have stationary states (Lavoisier), quantal activities (Aristotle, Liebig), correlation among activities (Galen), and non-contradiction with inorganic systems (Erasistratus, Wöhler, J. R. Mayer). It seems a waste 
of effort to rediscover the existence of such rules in connection with every component of metabolism and with every organ. The existence of self-recovery after displacement from balance seems to me to be one such generalization, and coordinate with the other rules mentioned.

One very special feature of regulations seems to make the generalization about their prevalence in all organisms and all components unusually probable. The geneticist often uses animals in which certain genes for study are linked to "lethal genes"; then all surviving individuals will be automatically free of the linked genes. I feel a similar assurance that physiological components present in an organism are regulated. Organisms without a certain number of regulations would not survive, and survival per se is a measure of the efficiency of regulations. This view yields the prediction that regulations exist in every organism, for every organism has some constant physiological properties.

Biologists have fears of deductive work, of specific prediction from a generalization, based probably on the experience of numerous failures. With the increase of quantitative biological knowledge, relations become more nearly adequate for certain sorts of deductions, and in particular (1) interpolation may usually be trusted in quantitative correlations, and (2) it is allowable to correlate $\mathrm{A}$ and $\mathrm{C}$, when each is known under identical conditions to be correlated with $\mathrm{B}$. The abuse of these limitations has led to disappointment; the method is none the less valid. Deduction is useful when it recognizes that it is provisional and statistical until the tests are made by which it will become historical.

So induction leads to general rules among similar phenomena. And deduction leads to predictions that can be applied to new tests of the rules. Free play of imagination also creeps into every set of relations examined; this is "seduction." Certain of those speculations and theories are now set down.

\section{$\$ 166$. Maxima and minima}

(1) Minima. Rates of exchange might be thought to be ideally zero. The organism would conserve what it has and waste no energy in acquiring more. That would be maintenance by isolation. The organism perhaps makes "sacrifices" to its non-isolation; perhaps it leaks; perhaps it is cheaper to find new than to save old. So there are nitrogen minima, water minima, energy minima, heat 
minima, and all the rest. Each is a rate of loss or of gain measured at the load that most favors conservation.

Is there ever "minimal work" in the sense of the well-known theorem of mechanies? There it is postulated that the sum of the energies expended tends to be the least that can accomplish the work performed. Perhaps the question cannot be answered in organisms so long as chemical and other processes appear inseparable from mechanical ones. No rigorous solution is likely at present and in a long future, for plainly, identification of minimal work requires a knowledge of all transformations that are going on. In the organism this probably means recoguizing all the correlatives of a given exchange. It was noted, for instance, that water exchange is concerned not only in water equilibration but in heat, solute, and synthetic exchanges. Many of its relations are unknown even qualitatively and others may hardly be measured quantitatively; and so it is for such other components as have been widely studied. To circumscribe the system would defeat the research. Were enough factors considered to bring the result that the work performed was approximately minimal, the outcome would be believed; if the result were not approximately minimal, the investigator would cast around for other modifying factors. That is mere toying with the rules of thermodynamics. There is always the guess that death would be cheaper than maintenance.

(2) Capacities. Equilibrations reveal highest rates of exchange far above the minimal; the uniformity found is that high and low rates of total gain appear "spontaneously," each in loads of opposite sign. The maximal rates roughly indicate the extent of provisions for recoveries. Doubtless these "capacities" for increase in exchanges could be classified as showing anatomical factors, chemical factors, muscular factors, and the like. One of the favorite methods of physiology is to embarrass the living unit by interfering with what goes on. Indeed, experimentation is largely an inquiry into the vulnerabilities whereby loads can be imposed and recoveries be modified. Thus, rates are compared after removal of pancreas, privation of food, cutting of nerves, introduction of agents, or believed inhibition of enzymes. Occasionally an "interference" is found that enhances the rates of particular exchanges; often it is inferred too that this confers an "advantage" that the organism did not have before. I imagine the organism is 
concerned to adjust the relative rates of a huge number of exchanges and contents, not in trying to have the biggest of anything.

The so-called capacities for exchange are far from being fixed. They are one end of a quantitative continuum, ordinarily appearing at the tolerated (maximal) load. They as well as the rest of the continuum are modified as the sequels of past influences (acclimatization, conditioning). Each capacity and provision for modification is itself a component, probably capable of being studied by the methods outlined.

(3) Limiting factors. It is sometimes supposed that organisms would be well off to have every component that may be utilized available to them. But organisms exist by no such ideal of security; having everything is often itself a limitation.

Each "limiting factor," if such there be, presumably corresponds to a load in the organism. The response of the organism tends to reduce that load, by conservation through diminished loss and by accretion through selective gain. It looks as though equilibration is generally set to minimize the limitations imposed by any factor.

One supposition is that limiting factors always prevail in an organism. Rate of physical exercise is said to be limited sometimes by mechanical movement or by cardiac output; rate of oxygen consumption by enzyme concentration. Limiting factors may be defined to include relations of all sorts among components, and the faith may be held that one of the known components is the one that matters. But when the view is expressed that the limiting factor can be identified, that factor becomes a key process, sometimes a cause. Thus, rats died in an average of $\mathrm{X}$ days when deprived of all alimentary materials (Jackson, '25, pp. 98 to 116). When allowed water alone, rats died in an average of $\mathrm{Y}$ days. Since $\mathrm{Y}$ exceeds $\mathrm{X}$, water seemed crucial. This neglects the fact that many other components could be substituted for water, and in many of those instances $\mathrm{Z}$ exceeds $\mathrm{X}$. It spoils the implications of the key when almost any component will take its place. There is no demonstration in those tests that any of the effective components was qualitatively distinctive in controlling survival, so far as I can see.

In general, a limiting factor is a hypothetical relation that is entertained so long as few components, and none of them quantitatively, have been studied. The more exhaustive the studies, the less any single relation is likely to be emphasized. 
(4) Factors of safety. In a broad way, a factor of safety appears to refer to any provision that increases survival (Meltzer, '07). In engineering parlance it is a ratio between that which occurs and that which could occur without destruction. In a living unit it might compare either maximum and minimum (as in modification ratio), or content at tolerated load and content at zero load. All equilibration diagrams express factors of safety and furnish quantitative data corresponding to each definition, since each component is represented both in rates and in loads. No doubt every physiological property is insured by factors of safety of some sort.

Speculations concerning maxima, minima, capacities, safety, and economy bear strong metaphysical connotations at the same time that they bear the stamp of mathematical exactitude. The connotations largely disappear when one has the data in quantitative form. For then a minimum means no more than a point on a curve, and a factor is a defined relation between two points. The speculations are uncalled for as soon as definitions are set up, and data are obtained to correspond to those definitions.

\section{$\S 167$. Origins of Equilibrations}

Means of expressing certain physiological patterns (equilibrations) having been found, it is a temptation to examine the patterns successively in the many connections that anatomical patterns are commonly regarded. Among those aspects, the ontogeny and phylogeny of function have received a share of speculation; perhaps the present quantitative data suggest further ones.

In ontogeny the clearest data seem to be those of heat equilibration. In rabbit (fig. 151), mouse (Pembrey, 1895), and wren (Kendeigh, '39) a series of progressive changes appears in the equilibration diagram for heat. At birth nearly the same rates of heat production and coefficients of heat loss prevail at all body temperatures (heat contents) in those species. But gradually the rate of heat production "differentiates" so as to correlate negatively with heat content, and the rate of loss to correlate positively. No doubt a great variety of sensory and coordinative links take part in this differentiation.

The individual organism starts life in a relatively isolated state. Partial isolation is what the parent organism provides; it tides the organism over the period when its functions are being acquired. 
Control (regulation) is attained over each of the bodily components one after another. In what order do the regulations perfect themselves? Over which components are regulations simultaneously gained? How does the order differ in diverse species? Can earlier acquirement of some specific one be induced by disturbances of content of that particular component? All those questions regard functions as anything but fixed. They contrast the same organism before and after regulations are operating.

Much inquiry will be required to map the development of various regulations in germ cells, embryos, and young and senescent organisms. One speculation is that the development of any one equilibration is rarely if ever independent of others; that equilibrations are not discrete, except by definition.

Not all regulations arise anew in each individual. Some are continuous from parent to offspring, e.g., energy transformation, osmotic pressure. Those that later unfold to view might be, of course, performed or not, and it would be a paradox if the existence or non-existence of physiological Anlagen having no measurable characteristics could ever be decided. The suggestion of Haldane ('17), that processes by which the organism successfully evades or opposes influence which have not fallen within the previous experience of the species, are created at the moment when occasion arises rather than being in latent reserve, can probably never be fully tested.

In phylogeny few actual data can be cited, and little is agreed as to which living animals represent physiological types more ancestral than others. My guess is that the equilibrations of most chosen components have been evolved repeatedly by many historically distinct groups of organisms. Hence either the equilibrations are polyphyletic, or the accepted structural characters on which taxonomy is usually based are polyphyletic, or both. Of course, many equilibrations of functions may be expected to have evolved together, and with structures and with environments. The dog without equilibration of glucose would be as inconsistent with the rest of a dog, as the Arbacia egg with temperature balance at some point other than that of its environment.

The composition (state) of the body is easily guessed to be the emergent of the simultaneous equilibrations of all its components. Conversely, the familiar data of biochemical compositions might be taken to represent first rough correlations among diverse equilibrations. 
Probably all equilibrations have undergone evolution. Whether they evolved by selection, by orthogenesis, by mutation, by foresight, or by all of them, can be imagined without much limitation by fact. Believing that regulations are indispensable, I think some are as old as any form of life. Since there is almost no paleontology of function, one theory will be as plausible as another. I would argue for polyphyletic origins, trial and selection of responses, and resourceful variations.

If I were an animal without heat regulation I think I would try keeping cool by acquiring stepwise some modest arrangements for refrigeration. But I would not stake my water content very far to do it, for, my ancestors had not. I would install a thermostatted heater only after the cooler was working efficiently. At first I would be content with a range of $\pm 20^{\circ} \mathrm{C}$. in body temperature, later of less and less until I got down to about $\pm 0.5^{\circ} \mathrm{C}$. and then I would lose interest in securing greater accuracy unless some other activity began "demanding" further refinement as an aid in accomplishing its work. I would count a heater as economical only if with it I could go places where fuel would be cheap enough to justify it; my ancestors had sat in the sun, or burrowed in the ground, or insulated themselves instead. Actually I presume that I would have no foresight sufficient to count the cost or to weigh the specific outcome; but maybe there is some subconscious way of inferring the whole outcome from trials. Every added regulation would involve a liability of having something go wrong, of requiring upkeep. Perhaps that is why I do not fix carbon dioxide and then reduce it to cool myself. Whether I could discover cheaper means of refrigerating or not would help to decide whether I would maintain $37^{\circ} \mathrm{C}$. or $27^{\circ} \mathrm{C}$. Maybe I could experiment with that after I had the machinery for both cooling and heating, finding which of the two was "easier," and which upset my other components least. If I stopped to picture all the "optimal" velocities that would clamor for recognition, I would never reach a decision.

While I imagine I could have lived for millions of years without doing very much to adjust my body temperature, by the subterfuge of not going to places too hot or too cool, I would have little but subterfuge at first to help govern my water content. But gradually I would explore into ways of retarding my water losses, and of repelling water ingress; I would try to accomplish both at once. I might then find some excuse for setting aside one area of my sur- 
face for output, hoping thereby to quantitate my elimination and to adjust its rate to my water content. Later $I$ would set aside another area of surface for intake; then I could afford to make the remaining surface impervious to water if opportunity ever arose. I would laugh at any one whe thought he could find out why I had done all that. But I would be interested in asking how it is done, even though there were no chance of taking my machinery apart without stopping it all.

In some such vein the imagination can go on replacing the unswerving history of organisms by anthropomorphic argument.

\section{§ 168 . Fordes in BIOLOGICAL EQUILIBRIA}

Forces are ordinarily classified in terms of the procedures for their physical measurement. Their identification in living processes is usually circumstantial, the potential as found being ascertained to be sufficient to push, but never known to be that which does push.

The data presented in this work might be supposed to contribute more to physiology if the rates of exchange were translated into equivalent forces. By habit and assumption, to decide that an event in an organism can be analogous to a process that has a dynamical magnitude and name, appears to many biologists to be something accomplished, however indirect the inference. I take the view that the rates, actually measured, lead to fewer illusions than do those circumstantial equivalents. It is easy to obscure the relations among facts by translating them into imagery so alluring that fiction is taken for reality. When the poetry is later rejected, with or without the expenditure of much labor to separate out the materials upon which it was built, the materials also are often lost or discredited.

Forces are suggested when the component being equilibrated is water; and it would be a usual guess that osmotic pressures are concerned in exchanges. For Arbacia eggs most persons would agree; for frogs some would agree in part; for whole dogs probably no one would agree. The quantitative estimation of those forces would be subject to still less agreement. Intake of water by mouth in dogs happens to be known to involve nerves and muscles that can no longer be described as exhibiting only forces of osmotic pressure, while in frogs intake is scarcely known to involve more than the skin, which corresponds in some respects to the pictured membrane 
of an osmotic system. Output of water in both dogs and frogs is generally believed to utilize hydrostatic pressures furnished by cardiac muscle, and certainly other processes that at times oppose osmotic pressures. The physical and chemical forces, processes, and energies concerned in exchanges of water through kidneys (aglomerular, pronephric, etc.) are known to be diverse among species. In addition, all the species and living units that have no kidneys may not be forgotten. To delay the study of water exchanges until the physicochemical nature of these processes is known seems to me unwarranted; it even seems doubtful that such a millenial reward will ever arrive.

Rates are what biologists observe; forces are what biologists usually think they would like to measure. In practice, expenditures of force are rarely directly measurable, and generally there is no way of ascertaining the worth of what has been hypothesized concerning them. Hence I emphasize that, instead of dynamic equilibria and dynamic changes, I am studying kinetic equilibria and exchanges.

Wherever a believed force is operating at a known rate, the ratio : force/rate, is a resistance. Much could be made of such resistances with respect to the possible control of regulatory events. By speculation, the living unit could be conceived as a large series of resistances so arranged that each rate is adjusted by releasing it to diverse extents. If it be supposed, conversely, that resistance is constant in a variety of circumstances, then rates are proportional to forces. In equilibrations, the stress, proportional to load, is accompanied by a strain (force, rate) that accomplishes recovery.

There is a belief that physiology can be approached only in proportion as physics and chemistry are perfected and understood. Physiology is even regarded as poorly developed physics and careless chemistry. A contrasted belief is that physiology is busy inquiring into relations, the relations being in combinations that mostly do not occur in non-living systems. While some methods, apparatus, and symbols may be common to several sciences, biology can also be independent of them, except to the extent of its reciprocal influence upon sciences of inorganic systems. Actually, of course, most investigators bring facts and relations to light without much respect to their beliefs about forces or vital processes, and fortunately no formal division arises between orthodoxy and unorthodoxy. 


\section{§ 169. Spencer's views}

A chief difference between what has been hypothesized in the past and what has been induced here with regard to regulations, may be stated as a contrast between the study of forces and the study of rates. Noting the difficulty of measuring the forces concerned in compensatory exchanges, I ascertained the rates concerned in them. Actually, speculations in the past about adjustments and recoveries have to do with forces, both specifically and generally. To illustrate, I quote the relevant view of Herbert Spencer (1867, pp. 435, 462, 386) :

This equilibration between the functions of an organism and the actions in its environment, may be either direct or indirect. The new incident force may either immediately eall forth some counteracting foree, and its concomitant structural change; or it may be eventually balanced by some otherwise produced change of function and structure. . . . Direct equilibration is that process eurrently known as adaptation. . . . Here we further find, that this limit towards which any such organic change advances, in the species as in the individual, is a new moving equilibrium adjusted to the new arrangement of external forces. . . . The only new incident forces which ean work the changes of function and structure required to bring any animal or plant into equilibrium with them, are such incident forees as operate on this animal or plant, either continuously or frequently. They must be capable of appreciably changing that set of complex rhythmical actions and reactions constituting the life of the organism; and yet must not usually produce perturbations that are fatal.

Life itself is the maintenance of a moving equilibrium between inner and outer actions-the continuous adjustment of internal relations to external relations; or the maintenance of a correspondence between the forces to which an organism is subject and the forees which it evolves. For if the preservation of life is the preservation of such a moving equilibrium, it becomes a corollary that these changes which enable a species to live under altered conditions, are changes towards equilibrium with the altered conditions.

Raising a limb causes a simultaneous shifting of the eentre of gravity, and such altered tensions and pressures throughout the body as re-adjust the disturbed balance. Passage of liquid into or out of a tissue, implies some excess of force in one direction there at work; and ceases only when the force so diminishes or the counter-forces so increase that the excess disappears. A nervous discharge is reflected and re-reflected from part to part, until it has all been used up in the re-arrangements produced-equilibrated by the reactions called out.

Spencer thus specifies that forces become operative in each equilibration; and that two sorts of equilibration, direct and indirect, may be distinguished. Direct is what is usually meant by biological adaptation. Adaptation is variation in a feature or property of an organism in relation with its state or with the environment; certainly both forces and exchanges fall under that denomination. In state $G$ the organism has properties $g_{1}, g_{2}$, etc.; in state $H$ it has properties $h_{1}, h_{2}$, etc. which are more advantageous 
than $g_{1}, g_{2}$, etc. would be. Leaving the assumption of advantage out of account, I suggest that adaptation is the pattern of modifications already noted in equilibrations, and that those interrelations are directly measured by loads, rates, and their correlatives.

Experimentally it might be possible to set up criteria by which to test whether state $I$, and all that goes with it, more often leads to recovery, or to survival, than state $G$; or whether the combination of $g_{1}, g_{2}$, etc. with $H$ would favor it. Another criterion of survival, or some entirely different criterion, often gives a different answer.

Indirect equilibration, according to Spencer, is gradual variation in a property of the organism or genotype while it is meeting situations that are unlike, in intensity or recurrence, those to which it formerly was exposed. I suppose one could hardly distinguish where the changes coincident with the first equilibration ended and those coincident with the second began. When a modification occurs in a future generation, it too is measured as a rate of exchange and not as a force.

Driesch ('08), also, attempted to distinguish between regulations that are already prepared (adaptedness) and regulations that come into existence when state $H$ arises. I find no criterion of preparedness that is concise. He also suggests that functional disturbances are distinct from structural disturbances, a classification that I find difficult to use. I suggest that only quantitative categories are practical, particularly when like Driesch one is trying to separate the abnormal from the normal.

The differentiation between direct and indirect equilibration perhaps corresponds to the diversities of physiological and correlated ecological patterns encountered in the deer mice of Ross (see $\$ 98$ ). Rates of water turnover and preferences of environment were significantly different in two species, but not in two subspecies of either one of them. Conditioning, even lasting more than one generation, did not abolish the difference. The correlations were of a sort that were thought to favor survival in each of the environments ordinarily selected. The environmental equalization evidently induced no shift in water turnover, for that was stabilized by internal interactions (direct equilibration) to a degree that allowed of no external influence.

Every equilibration, even an unprepared one, represents provisions for contingencies. Whether it also is a prevision can only 
be imagined. Whether it is optimal, beneficial, wise, beautiful, fit, or purposeful can be argued and defined. All those concepts might enter into speculations concerning origins and modifications of physiological regulations.

\section{\$170. Survival and DEATH}

Finding in 14 diverse species a single type of diagram for net water equilibration $(\$ 72)$ instead of several possible types of diagram that are never found, might be used as statistical evidence of one kind of fitness to survive. There are no strong indications of how the fitness of recoveries came about in the course of phylogeny. Fitness might be treated as another correlative; then all the components that are characteristically related (interwoven) with one another are also correlated with this one property of fitness. That the very component which is loaded should also be returned (unloaded), is anything but a random relation of two quantities. Such a fit specificity is observed repeatedly; there is nothing about it to be glossed over or ashamed of. It suggests that the organism would not be here if means of establishing such relations had not been "invented" for each of many components. Appropriateness of the same order is involved in LeChatelier's rule ( $\$ 160)$; the very factor that has undergone change is the one that tends to be annulled in the physico-chemical system. In both non-living and living systems the recognition of the fitness when so defined is descriptive.

Fitness might be treated as a variable that is particularly correlated with survival. Recovery and equilibration in their turn are also correlated with survival in the case of most components, for most displacements (loads) are recovered from during life. The two correlatives of survival are, therefore, correlated one with the other; by such a truism biological interrelations are epitomized. There are exceptions to the universal tendency to recover: missing appendages, benign carcinomas, obesity, and deposited lead are all compatible with survival. Perhaps even in each of these instances there are augmentations of exchange in unrecognized correlatives that tend toward recovery. Subsidiary and contributory "causes" of death take on another meaning when regarded as correlatives of physiological equilibrations.

Death may be viewed from equilibration diagrams in the following ways. (a) Some one or more components reaches a posi- 
tive load at which rate of loss is no longer in excess of rate of gain, or a negative load at which gain is not greater than loss. This is another way of stating that recovery does not occur. (b) Or, one or more components lose their ties with the remainder of the interrelations. Then individual components are incompatible with the surviving state of other components. Quantitative data do not seem to exist by which to differentiate these possibilities. But a person who investigates methods of killing vermin does nothing other than find the limits to the behavior and equilibration that preserve the vermin from each agent that he tests.

The acquisition of equilibration is a "mechanism" of fitness, perhaps. It enables the organism that acquires it to survive, conferring upon it the advantages that Darwin attributed to the fittest individuals. Indeed, regulation as measured in this investigation is perhaps the only criterion known which shows physiological character's to be related to the "success" of the species.

I can imagine further that equilibrations and their factors can be acquired, possibly through processes of conditioning such as are recognized in animal behavior. If an advantageous relation can be experimentally established between load and exchange of the same component, then unusual or artificial advantages can be presented to fool an animal into forming a relation of load to some exchange that later will be disadvantageous. Maybe equilibrations are not fixed, and physiological patterns are labile enough to be manipulated by experimenters.

Everyone can discover some exchanges or behaviors that he judges to be inappropriate for recovery or for survival. He will have a hard time demonstrating that they are deleterious; for, any other exchange or behavior might be incompatible with some more crucial recovery. Load of $J_{1}$ leads to modified exchanges of $J_{2}$ and $J_{3}$, and $I$ rarely feel able to ascertain whether those correlations are appropriate or not. But the exchange of $J_{1}$ itself $I$ can evaluate for every component; it alone I am sure is a measure of recovery, without further ado. All appropriateness outside that correlation of $\Delta J_{1}$ with $\delta J_{1} / \Delta t$ is at present speculation.

\section{$\S 171$. Integrations}

The fact is that diverse functions get along together in one living unit. Some persons suppose there is a head-office for coordination of them all. Formerly it was believed to be a center in the 
nervous system, now it is a master gland, tomorrow it will again be an entelechy. A nerve or gland may be a pathway or mediator of physiological events, but is not known to initiate them. Perhaps all regulators are, so far as organisms are concerned, equally metaphysical.

The resort to such metaphysical agencies has been shown to be worse than useless in our dealings with the inorganic world and it is difficult to see how they can be of any greater service in understanding the organic. The tenderminded may still delight in assuming their intervention in the development and maintenance of unicellular and multicellular organisms, whose integration is so exceedingly complicated and opaque that we are probably still centuries removed from any adequate understanding of their functional composition (Wheeler, '28a, p. 40).

Anyone who begins to comprehend the magnitude of the organism's "task" of simultaneously "caring for" all components is likely to use anthropomorphic language. Very many imagined schemes are built on the supposition: how could I manage all those adjustments if I were the engineer? And endless are the speculations that can be achieved therefrom.

Spencer (1866, p. 61) and Haldane ('17, p. 26) recognized that "coordination is inherent in physiological activity." Some think such a statement avoids the issue because it does not specify a "mechanism." As far as I can see, it states what is known, and recognizes the existence of integration without beclouding the phenomena with fancies. There is hardly any further issue to be faced unless it is shown that integration can be separated from functioning, that the whole is different from the combination of its parts and relations among them. For, "integration itself is a process of equilibration', (Henderson).

\section{\$172. LIST OF THEORIES}

A host of extrapolations and possible extensions of notions previously mentioned are suggested by the material of earlier chapters. They cannot be derived by pure induction; indeed some probably never can be so "proven." A few are: (1) Theory of necessary regulations. All living units regulate (maintain) some components (properties); none survive without phenomena that can be described as equilibrations.

(2) Theory of interdependencies of regulations. No component is regulated (equilibrated) independently of all others.

(3) Theory of requirements. Rates of turnover of any one 
component depend upon the contents and rates of exchange of other components.

(4) Theory of conservation. Rates of turnover are usually near the minimum compatible with indefinitely continued function.

(5) Theory of intermittencies. Long-time regulations, governor-like in action, influence intermittent exchanges (such as alimentation and sexual expression). The average rate over a long period of time is thus uniform, though composed of discrete periods of quick activity.

(6) Theory of relative modifications. In one individual or species, the exchanges of each component are modified so that they remain compatible with one another over wide ranges of possible loads. (Thus, water loss by vaporization does not overshoot the requirements of coincident heat loss.)

(7) Theory of velocities. For one component, diverse species tend to have modifications of rates that bear the same proportions to the load. Or, velocity quotients are rather similar in various animals.

(8) Theory of time scales. Single components and groups of components tend among many species to unload in relatively similar times. Comparative physiology may be built upon the quantitative diversities of time scales and their correlatives.

(9) Theory of exponential recoveries. A majority of velocity quotients $(1 / \Delta t)$ are roughly constant over wide ranges of loads, times, rates, and past treatments. This is especially noteworthy where diverse physical, chemical, and anatomical elements and processes are known or believed to be concerned in the exchanges.

(10) Theory of accommodation. Rates of exchanges are sometimes greater in response to great accelerations and decelerations of load than they are at stationary loads of the same magnitude.

(11) Theory of physiological magnitudes. Loads and exchanges may be studied and compared without reference to physical and chemical categories in which constituent processes may be classified. Similar loads, rates, and correlations constitute like physiological attributes, within the limits described by their dimensions.

(12) Theory of physiological purpose. "All the vital mechanisms, varied as they are, have only one object, that of preserving constant the conditions of life in the internal environment" (Bernard, 1878, p. 121). 
(13) Theory of substitution. "Free" existence, to use Bernard's term, is attained in the course of evolution by the substitution of compensations for preservative behaviors alone. Regulations by compensations allow the organism to occupy environments that otherwise would limit its range.

Postulates of the sorts suggested may be multiplied indefinitely. The phenomena upon which they rest are the everyday observations of physiologists. The isolated facts mean little individually, but in selected correlations can mean much. Limitations in physiology are probably both in producing fertile suggestions of relationship, and in critically amassing data from which each relationship may at long last be induced.

Had any of these broad theorems been substituted for the limited inductions previously made, the outcome of the investigation would have far surpassed the scope of the materials studied. It might have been implied that, in place of $\operatorname{dog}$ B, "protoplasm" was studied; in place of heat, "physical quantities"; in place of equilibrations or correlations, "vital organization." The use of general terms is only by inference a substitute for the use of enough data to see what the varieties as well as the uniformities in their relations may be. Where special physiology ends, and how general is general physiology, are matters of opinion and arbitrary definition.

\section{$\$ 173$. Summary}

Speculations that are related to the physiological phenomena above investigated, propose specific inquiries, such as: (a) a frog ought to drink fast after water denial ( $\$ 37$ ), (b) aglomerular kidneys must be incapable of water diuresis (toadfish, figure 122a), (c) maybe a dog does not waste injected sugar until his stores are full (fig. 209), (d) I guess a man will be able to excrete urine faster than to excrete sweat. Each speculation frames a question that can be answered by test; definite queries were asked in profusion before and during the course of the investigation, and nearly all of them have been withheld from the record. All are framed so that if state $I_{1}$ is substituted for state $I_{0}$, and $i$ is measured before and after the substitution, a quantitative answer is found.

Other speculations are non-specific. They have the form: physiological state $I$ is related to factors (components) $J, K, L$, etc., while factors $M, N, O$, etc. may be ignored. Very often factor $J$ 
alone is named as correlative, and if investigation stops with it, a special role may be imputed to it. Or, supposition is reinforced with colorful language, such as that a living unit is like a dynamo, or a paint mill, or a gelatin block. Or, spice of associations suggests that all living things are dilute water. Most of those statements can be tested, and definitions, criteria, and experiments designed accordingly. But the charm of the speculation is occasionally lost when tests are undertaken.

Finally there are speculations which seem predestined to be fruitless. Origins, purposes, and entelechies will probably never be demonstrated in the way that rates of exchange and their correlations have been. But that is a speculation, too. 


\section{Chapter XXI}

\section{CONCLUSIONS}

$\$ 174$. Physiological regulations have now been described in quantitative terms, thereby gaining a foundation in facts and relations. Regulations are simply those relations according to which the properties of organisms persist. The organisms that were studied seem to have themselves argued the manner in which they are kept constant. I will try here to recount the general features that have turned up among the specific details already provided.

\section{§175. Abstract of the investigation}

I wanted to study how a given property or content of an organism is preserved during life. How constant the content is, could be ascertained by observing what successive numerical values it has, and ultimately expressing those values by some statistic of scatter. What happens to restore it after it has been disturbed from its usual value, could be found by measuring the rates of its change or exchange, especially during recovery. How the organism forestalls the occurrence of disturbances, could be learned by seeing how often the organism frequents an environment that either minimizes or promotes exchanges, instead of some other environment.

I first tried to find how the content of water in the whole body of the dog is maintained. How long does recovery from a higher or a lower amount than usual take? I measured not only content and its change in relation to time, but also all exchanges of water (gains and losses) in relation to increments (loads) in content of water. It turned out that gain and loss are equal at only one content (balance). In all excesses losses are faster than gains, in all deficits gains are faster than losses; thereby balance is recovered. Variability of water content I ascertained by weighing the dog at successive equal intervals of time. Content is corrected periodically by the dog's ingestion of water, and more gradually by the dog's excretion of urine $(\$ 25)$.

Then I looked at other species. Man, frog, rat, rabbit, earthworm, and ameba, each use some particular rate and kind of water transfer to compensate for water loads (equilibration). Several that were tested, notably the rat, showed preference for moist 
atmospheres when in water deficit; that preference tended to maintain the water content, too. The amount of water in the body fluctuated to a degree characteristic of each species. For the understanding of all those regulations, only measurements of water content, water exchanges, and time were required.

A diverse type of water increment resulted from each experimental procedure that induced the increment. There were dehydrations by privation of water, by injection of sucrose, by drainage of pancreatic juice, by exosmosis. Some of the types kept the animal in a stationary state of water increment. Diverse shifts of water balance itself also were noted, indicating that from day to day the total water content might be maintained at progressively different amounts.

Not only whole organisms from man to ameba, but as well diverse parts of organisms from blood volume to single cell, manifested similar regulations. Each equilibrated its usual content according to a common pattern, but by a variety of paths of exchange. Comparisons of rates of exchange, modifications in those rates with water increments, variabilities of content, and preferences for diverse aqueous environments, distinguished quantitatively each sort of living unit.

Further understanding of water regulations was sought in variables other than water increment, water exchange, and time. Simultaneous compositions of parts (tissues), volumes of parts, body sizes, and metabolisns of many kinds, were accordingly correlated with those variables. Inkling's were thereby obtained concerning the intermediary processes of water exchange, and the numerous relations of water that are strained by the one stress of water increment. Each increment of water could in fact be characterized by the contents and exchanges of a whole set of its correlative components. Various methods were worked out of representing these numerous correlations; each species and part was thereby differentiated numerically within a pattern that was common to all.

Altogether, a detailed description of water relations of living units resulted. Only those relations that seemed implicated in processes of regulation were included, but they were many (chapter XIII). Water content appeared to be fixed by virtue of all the other constant properties of the organism; the many fixed the point to which the one would return after each disturbance.

Using analogous measurements and correlations, I could now 
study other components (constituents, properties, activities) of organisms and their parts. Heat, total substance, glucose, carbon dioxide, heart frequency, blood flow and others, were summarily examined, chiefly in $\mathrm{dog}$ and man. Each had a variability and an equilibration; and for several the organisms manifested appropriate behavior toward environments that contained them or influenced them. The patterns of regulation were common to all components.

Thereupon the several components of one organism could be compared, with respect to variability, tolerance, equilibration, modifications of exchange, paths of exchange, and specificity of exchange. In this study the physiologist did not appear to be limited to the chemical or other nature of each component; for, the parameters used in comparisons were not expressed in physicochemical units. Rather, each parameter was a quantitative relation concerning regulation; hence was a physiological characterization of the organism as observed.

Once several components had been studied, simultaneous handlings of components by the organism could be examined. Their interrelations seemed to manifest the compatibilities and preferences in the contents and exchanges of each. Thus, frequency of heart beat is after a disturbance stabilized within 0.1 hour, heat content only after 2.0 hours. But their recoveries are not independent; rather the organism is constituted in such a manner that every component falls into an order relative to every other. Most components have some tie, known or unknown, with one another; their exchanges especially are not wholly independent; and the associations among them are evidenced by the interrelated increments and rates of recovery.

In all the above investigation, procedures of general interest were being used, their descriptive nature constituting one approach to the study of general, special, and comparative physiologies. Historically the elements of those methods have been widely employed; they alone seem to have revealed the general features of what is meant by physiological regulations. Speculations about all the phenomena visualized in the several components that were studied, and about the general features of regulation, could lead to extensions of the investigation in a variety of directions. For the present, regulations seem to have become concretely real in the quantitative relations by virtue of which they are here analyzed. 
This general survey of the investigation indicates the outcome only to the extent that words can embody it; its numerical features remain in tabular and graphical forms. How could it be otherwise in animals that teem with numbers?

\section{$\$ 176$. Equilibrations}

Some points in the physiological pattern of adjustments may be emphasized. The study of water relations of dog allowed the selection of three quantities: deficit or excess (load) in the whole body; rate of gain and loss (exchange); and time. Reproducible types of load were chosen, and occasionally the paths of exchange were distinguished from one another (partitioned). Rates of net exchange increase with loads; their correlation was termed equilibration $(\$ 9)$. Other species of animals, and finally organs, cells, nuclei, and populations were found suitable for the same sort of study. The general features common to the interrelations of the few variables mainly treated were then formulated $(\$ 72)$.

Comparable variables (loads, rates of exchange, and times) were selected concerning other components of organisms. These components were heat, glucose, oxygen, carbon dioxide, lactate; frequency of heart beat, hydrostatic pressure of blood, excitability of nerve, and some others. Regulation existed for each component studied; the uniformities and quantitative contrasts among the components were ascertained $(\$ 142)$. The forms of time relations and equilibrations were now general (fig. 180, fig. 110). Equilibration diagrams also furnished a ready and exact means of classifying the compensatory processes by which animals recover their normal contents $(\$ 72, \S 142, \S 178)$.

Components simultaneously equilibrating in one individual were interrelated. Combinations of components could be treated as resultant and emergent components. Thereupon it was inferred that the whole organism might be compounded of such interrelations, each load and equilibration being compatible with many others. Though methods of correlation seemed adequate to the task of identifying the relations, synthesis of variables was limited by ability to comprehend the multiplicity of them. Thus any one equilibration, representing regulation of a single component, is one member in an extensive network of mutual dependencies. These were thought to constitute multiple kinetic equilibria that characterize a physiological unit while it is maintaining its properties. 
The study of the interrelations of many equilibrations thus led to a general concept of a living unit, for each individual may be viewed as composed physiologically of a great many connected and inseparable regulations. Thus it turns out that the study is of any living unit as a whole. Even where "parts" were named, as distributees or as paths, the part was playing its role in relation to the economy of the unit; it, in turn, was related to its environment and to other living units. I think the above specific picture of functional continuities both describes and explains the correction of disturbances in physiological properties.

\section{§ 177. Alternative studies of the same material}

Throughout the course of the investigation it was apparent that the same data and relations might be used for a variety of monographs other than the one centering on regulations.

(1) A customary limitation would be to consider that one component, such as water and its metabolism, furnishes a complete story in itself (chapters II and III).

(2) Or, one species, such as dog, or man, could be studied with respect to many components and their exchanges (tables 40,42).

(3) Or, any other living unit, such as a city, a muscle, or an erythrocyte, could be described by the kinds of correlations its components enter (chapter VIII).

(4) A survey of comparative physiology could be based upon the quantitative differences in the same few components among many species $(\$ 98, \S 107, \S 141)$.

(5) A research in organ physiology would characterize the paths of the exchanges measured $(\$ 137)$. The kidneys of one or many vertebrate species, or the guts of all animals, might thus be described in terms of quantitative exchanges of components.

(6) One type of agent or environment might be studied as related to diverse components, in one or many species $(\$ 108, \S 138)$. Thus physical exercise of one or many kinds, or low oxygen tensions, or sulfanilamide, or age, or temperature could be charted; uniformities and differences in the agent's relations to diverse physiological components are thereby ascertained.

(7) Application of the knowledge about regulations might be directed toward specific objectives $(\$ 162)$. Devising of clinical tests of function, finding therapies, discovering insecticides, defining the "normal," improving physical performance, all require knowledge either explicitly or implicitly of regulations. 
In general, two or three variables and their mutual relations can be comprehended at one time. Any two or three of the quantities mentioned above: components, exchanges, time, paths, species, parts, tissues, metabolisms, or velocity quotients, or any other variable that anyone selects, may be the features about which an account is built. The number of such accounts is only limited by the minuteness of subdivision made in defining each variable, a minuteness that is often an asset.

The number of "dependent" variables, and the number of "degrees of freedom," in an organism can probably never be considered finite. Even if someone were able to demonstrate that all had been found, it would still be possible to redefine some, and subdivide others.

Since all these studies were touched upon in the course of the investigation as carried out, their multiplicity dispels any notion that the methods used are peculiar to the specific system of variables dealt with, or to the search for regulations. The interrelations to be ascertained appear to be limited only by human capacities to compute them and comprehend them. There probably are not many short-cuts to understanding the organism.

\section{\$178. Contributions made}

What particular aspects of regulations were emphasized, and what uniformities have been ascertained by inductions from the quantitative data presented above?

(1) The relations of a bodily component were compared in diverse species of animals, particularly with reference to its content, exchanges, time factors, and changes of coincident physiological properties.

(2) Variabilities of content indicated both the usual limits of content, and the intensities of processes that opposed variations of it.

(3) Displacements and disturbances of content beyond the usual, made possible the study of rates of recovery, the kinetics of physiological adjustments. Increments of content were usually exponential with time.

(4) Correlations of rates of exchanges with contents of the same component (equilibration diagrams) portrayed the parts played by gains and by losses in the regain of usual states in the organism. 
(5) The velocity quotient (rate/load, or 1/hour), a generalized clearance, served to express in one dimension the exchanges of all components.

(6) Not only many species and individuals, but diverse living units (parts of organisms), were quantitatively compared in respect to rates of turnover, equilibration diagrams (or portions of them), preference for particular environments, and variabilities of content; whether or not like structures and transformations occurred in those units.

(7) The rate of gain of a component was closely correlated with the rate of its loss. The magnitudes of net rates of loss and of gain were often similar at numerically equal positive and negative loads of one component.

(8) Augmentations of net exchange were sometimes secured by modification of total gain, sometimes by modification of total loss, sometimes by both, and in diverse proportions. These differences gave a qualitative basis for the classification of equilibration diagrams.

(9) The balance of content was usually struck near the minimal rates (of both gain and loss) that were found under any circumstances.

(10) Certain types of study could be viewed as applications of the substantiated theorems of regulation. Signs of disturbance, tests of function, therapy, toxicity, acclimatization, conditioning, and many other phenomena, are corollaries of the general description of an organism's attempts to maintain its functions.

(11) Interrelations among components were investigated. Simultaneous contents and events appeared to represent the relative regulations of diverse components in stationary states and in recovery.

(12) Multiple equilibrations as indicated in the study of simultaneous equilibration diagrams (fig. 185)' manifested the quantitative physiological patterns concerned in maintenance of constancy of the compounded functions of organisms.

\$179. Conclusions concerning Physiological constancies

I suggest the following as the most concrete findings of the investigation.

(1) The variability of any one quantity or component in the organism is limited, under given conditions. 
(2) Exchanges that tend to restore this quantity toward its mean become faster when displacements (loads) occur.

(3) Net rates of exchange are usually proportional to coincident loads, however diverse the physicochemical kinds of processes concerned in the exchanges.

(4) Hence a pattern common to regulations is found in the several quantitative investigations of : (a) frequency of occurrence of loads in standard states, (b) rates of exchange in steady states, and (c) rates of exchange in recoveries.

(5) Upon the precise coordination in the living unit between rate of gain of a component and rate of loss of the same component (e.g., fig. 15) appeared to depend the constancy and accuracy of content of that component.

(6) Finite durations of load and limited accelerations of exchanges are implicit in each initiatory, steady, and recovery state.

(7) Recoveries occur at different rates for each component; the maximal rates in the same individual differ enormously for diverse components, no matter in what units each is compared.

(8) Recoveries may be compared as velocity quotients $(1 / \Delta t)$, which may differ by factors of $10^{5}$ among diverse components (table 40).

(9) The study of each component with respect to either the frequency of occurrence, of the rate of exchange as correlated with load, or the environment frequented, characterizes quantitatively one property toward which the organism is self-maintaining.

(10) The pattern of regulations is not fixed in the individual, but develops during ontogeny, and undergoes various acclimatizations and conditioning:s.

\section{§ 180. Retrospect}

To me the study of physiological regulations is as impelling as any other sort of investigation is to others. I have tried to convey that feeling, lest anyone think the inquiry superfluous. Diversity of investigators leads to variety of endeavor, in physiology as elsewhere; those who desire to study regulations, sooner or later find suitable means of doing so, other than metaphysical ones. Among these means I envisage numerous further developments and refinements in quantitative description of whatever happenings are related to maintenance and recovery of components in organisms. 
I have attempted to formulate, and to represent quantitatively, many notions related to regulations and metabolisms that are vaguely and partially present in the minds of all physiologists. In these formulations, much may seem meaningless to the reader until he independently searches for consistent patterns in whatever organisms and components he is interested. Perhaps he too, realizing that each aspect of physiology is not a separate study, will help to describe the organism as it now seems to be, looking for many kinds of meaning in its being that way. To him it will be apparent that all components and their preservation together are the concern of the organism.

As I examine the constancy of one after another of the properties of an individual, I realize how rarely the organism is defeated in preserving its constitution. Though those several properties seem individually difficult enough for the organism to manage, the mutual compatibility among many or all is still more amazing. Yet, the mutuality itself may be the secret of the organism's selfpreservation; so long as a thousand properties are of consequence, no one of them can fail to find its balance in the combination. Though the representation of those relations upon paper may seem clumsy, the innate pattern of the organism can only by contrast seem elegant. For, the continuity of function and content is the organism so far as the physiologist knows it.

The investigation started with the question: What do animals do to maintain their physiological constitutions and activities? Detailed researches were required to answer it in the partial manner shown. I believe the answer as given adequately represents what can be done by methods and materials now at hand. In a sentence it is that: Animals preserve their constitutions and activities like themselves, within the limits of variation that characterize the normal, either by preventing disturbances from occurring, or by compensating for each actual and incipient departure from normal. Every organism and every component has its own peculiar equipment and its own correlated processes for doing this, but all are similar in net action. The processes automatically adjust the content or property to the characteristic norm, being provided in every case both specifically and adequately. The physiological provisions are such as can be classified, compared, and assessed. That account seems to me to make regulations real as health and inevitable as life. 


\section{REFERENCES}

Abe, S. 1931a. Tohoku Jour. Exp. Med. 17, 174-218.

Abe, S. 1931b. Tohoku Jour. Exp. Med. 17, 412-427.

Abe, S. 1931e. Tohoku Jour. Exp. Med. 17, 429-479.

Achelis, J. D. 1930. Arch. ges. Physiol. 226, 212-238.

Addis, T., and Lew, W. 1940. Jour. Exp. Med. 71, 325-333.

Adolph, E. F. 1921. Jour. Physiol. 55, 114-132.

Adolph, E. F. 1926 . Jour. Exp. Zool. 44, 355-381.

Adolph, E. F. 1927a. Jour. Exp. Zool. 47, 1-30.

Adolph, E. F. 1927b. Jour. Exp. Zool. 47, 31-62.

Adolph, E. F. 1927e. Jour. Exp. Zool. 49, 321-351.

Adolph, E. F. 1930. Quart. Rev. Biol. 5, 51-67.

Adolph, E. F. 1931a. The regulation of size as illustrated in unicellular organisms. Thomas, springfield. $233 \mathrm{pp}$.

Adolph, E. F. 1931b. Amer. Jour. Physiol. 96, 569-586.

Adolph, E. F. $\quad$ 1932. Biol. Bull. 62, 112-125.

Adolph, E. F. 1933. Physiol. Rev. 13, 336-371.

Adolph, E. F. 1936a. Amer. Jour. Physiol. 117, 366-379.

Adolph, E. F. 1936b. Jour. Cell. Comp. Physiol. 9, 117-135.

Adolph, E. F. 1938. Amer. Jour. Physiol. 123, 486-499.

Adolph, E. F. 1939a. Amer. Jour. Physiol. 125, 75-86.

Adolph, E. F. 1939b. Ann. physiol. 15, 353-367.

Adolph, E. F. 1942 . Federation Proc. 1, 1-2.

Adolph, E. F. New data.

Adolph, E. F., and Dill, D. B. 1938. Amer. Jour. Physiol. 123, 369-378.

Adolph, E. F., and Kingsley, H. D. 1940. Amer. Jour. Physiol. 128, P298-P299.

Adolph, E. F., Nance, F. D., and Shiling, M. S. 1929. Amer. Jour. Physiol. 87, 532-541.

Alemaeon. In: H. Diels, Die Fragmente der Vorsokratiker. Weidmann, Berlin. 1903. 601 S., S. 107

Almeida, M. O. 1926. Jour. physiol. path. gén. 24, 243-249.

Angerer, C. A. 1936. Jour. Cell. Comp. Physiol. 8, 329-345.

Anten, H. 1902. Arch. exp. Path. Pharm. 48, 331-355.

Atkeson, F. W., and Warren, T. R. 1934. Jour. Dairy Sci. 17, 265-277.

Atkeson, F. W., Warren, T. R., and Anderson, G. C. 1934. Jour. Dairy Sei. 17, 249-256.

Austin, J. H., Stillman, E., and Van Slyke, D. D. 1921. Jour. Biol. Chem. 46, 91-112.

Babcock, S. M. 1912. Univ. Wis. Agr. Exp. Sta., Res. Bull. No. 22, 87-181.

Bailey, V. 1923. Sei. Mo. 17, 66-86.

Baird, M. M., Campbell, J. M. H., and Hern, J. R. B. 1924. Guys Hosp. Rep. 74, 23-54.

Baldes, E. J., and Smirk, F. H. 1934. Jour. Physiol. 82, 62-74.

Barahal, H. S. 1938. Psychiatr. Quart. 12, 767-771.

Barbour, H. G., and Trace, J. 1937. Amer. Jour. Physiol. 118, 77-86.

Barcroft, J., Harris, H. A., Orahovats, D., and Weiss, R. 1925. Jour. Physiol. 60, 443456.

Barelare, B. Jr., and Richter, C. P. 1938. Amer. Jour. Physiol. 121, 185-188.

Barron, L. E. 1932. Amer. Jour. Physiol. 100, 559-563.

Bastert, C. 1929. Z. vergl. Physiol. 9, 212-258.

Bayliss, W. M. 1915. Principles of general physiology. Longmans, London. 850 pp. 
Beck, L. V., and Shapiro, H. 1936. Proc. Soc. Exp. Biol. Med. 34, 170-172.

Bellows, R. T. 1939. Amer. Jour. Physiol. 125, 87-97.

Bellows, R. T., and Van Wagenen, W. P. 1938. Jour. Nerv. Ment. Dis. 88, 417-473.

Benedict, F. G. 1932. Carn. Inst. Wash. Publ. No. 425, 539 pp.

Benedict, F. G. 1936. Carn. Inst. Wash. Publ. No. 474, 302 pp.

Benedict, F. G. 1938. Carn. Inst. Wash. Publ. No. 503, 215 pp.

Benedict, F. G., and Lee, R. C. 1936. Ann. physiol. 12, 983-1064.

Berkson, J., and Boothby, W. M. 1938. Amer. Jour. Physiol. 121, 669-683.

Berman, A. L., Snapp, E., Ivy, A. C., and Atkinson, A. J. 1941. Amer. Jour. Physiol. 131, 776-782.

Bernard, C. 1855. Leçons de physiologie expérimentale appliquée à la médicine. Baillière, Paris.

Bernard, C. 1859. Leçons sur les propriétés physiologiques et les altérations pathologiques des liquides de l'organisme. Baillière, Paris.

Bernard, C. 1878. Leçons sur les phénomènes de la vie communs aux animax et aux végétaux. Baillière, Paris.

Best, F., und Cohnheim, O. 1910. Z. physiol. Chem. 69, 113-116, 117-119.

Bezold, A. v. 1857. Z. wiss. Zool. 8, 487-524.

Bialaszewicz, K. 1932. Arch. int. physiol. 35, 98-124.

Bidder, F., and Schmidt, C. 1852. Verdauungssaefte und der Stoffwechsel. Reyher, Mitau und Leipzig. $413 \mathrm{~S}$.

Bischoff, E. 1863. Z. rat. Med. [3], 20, 75-118.

Blair, H. A. 1936. Cold Spring Harbor Symp. Quant. Biol. 4, 63-72.

Blalock, A. 1927. Arch. Surg. 15, 762-798.

Bloom, W. 1940. Amer. Nat. $74,129-147$.

Blumenthal, H. 1935. Z. Morphol. Ökol. Tiere, 29, 667-719.

Bohr, C. 1899. Ann. Physik 68, 500-525.

Bohr, C. 1909. Skand. Arch. Physiol. 22, 221-280.

Böhtlingk, R. R. 1897. Arch. sci. biol., Petersburg, 5, 395-416. Quoted from Morgulis ('23).

Bollman, J. L., Mann, F. C., and Power, M. H. 1935. Amer. Jour. Physiol. 111, 483-491.

Bonsmann, M. R. 1930a. Arch. exp. Path. Pharm. 156, 145-159.

Bonsmann, M. R. 1930b. Arch. exp. Path. Pharm. 156, 160-175.

Bousfield, W. A. 1933. Jour. Gen. Psychol. 8, 446-453.

Boycott, A. E., and Douglas, C. G. 1909. Jour. Path. Bact. 13, 414-428.

Boyd, E. M., and Dingwall, M. Jr. 1939. Jour. Physiol. 95, 501-507.

Boyd, E. M., and Garand, N. D. 1940. Amer. Jour. Physiol. 130, 403-409.

Boyd, E. M., Garand, N. D., and Livesey, R. J. 1939. Quart. Jour. Pharm. 12, 19-29.

Brand, T. v. 1931. Z. vergl. Physiol. 14, 200-264.

Bridgman, P. W. 1927. The logic of modern physics. Macmillan, New York. 228 pp.

Brock, A. J. 1929. Greek medicine. Dent, London. 256 pp.

Brodie, B. B., Brand, E., and Leshin, S. 1939. Jour. Biol. Chem. 130, 555-563.

Brody, S., Comfort, J. E., and Matthews, J. S. 1928. Univ. Missouri Agr. Exp. Sta., Res. Bull. No. 115.

Brooks, C. M. 1935. Amer. Jour. Physiol. 114, 30-39.

Brouha, L., Cannon, W. B., and Dill, D. B. 1936 . Jour. Physiol. 87, 345-359.

Brues, A. M., and Masters, C. T. 1936. Amer. Jour. Cancer 28, 324-333.

Brüggemann, H. 1938. Zentrabl. Agrikulturchem. Abt. B. Tierernähr. 10, 296-384.

Brull, L., Poverman, R., et Goffart, H. Arch. int. physiol. 43, 238-252.

Brunner, C., und Endress, H. 1929. Z. Biol. 89, 85-113.

Buchanan, J. W. 1931. Biol. Bull. 60, 309-326.

Burgess, W. W., Harvey, A. M., and Marshall, E. K. Jr. $\quad$ 1933. Jour. Pharm. 49, 237-249. 
Burn, J. H. 1931. Quart. Jour. Pharm. 4, 517-529.

Burton, A. C. 1935. Jour. Nutr. 9, 261-280.

Burton, A. C. 1939. Jour. Cell. Comp. Physiol. 14, 327-349.

Burton, A. C., and Taylor, R. M. 1940. Amer. Jour. Physiol. 129, 565-577.

Butler, V., Beard, J. W., and Blalock, A. 1931. Areh. Surg. 23, 848-854.

Buxton, P. A. 1932a. Parasitol. 24, 429-439.

Buxton, P. A. 1932b. Biol. Rev. 7, 275-320.

Caldwell, G. T. $\quad$ 1925. Biol. Bull. 48, 259-273.

Caldwell, G. T. $1931 . \quad$ Physiol. Zool. 4, 324-359.

Calvin, D. B., Smith, A. H., and Mendel, L. B. 1933. Amer. Jour. Physiol. 105, 135-145.

Campbell, J. M. H., Douglas, C. G., and Hobson, F. G. 1914. Jour. Physiol. 48, 303-316.

Canuon, C. Y., Hansen, E. N., and O'Neal, J. R. 1932. Iowa Agr. Exp. Sta., Bull. No. $292,102-114$.

Cannon, W. B. 1918. Proc. Roy. Soc. 90B, 283-301.

Cannon, W. B. 1932. The wisdom of the body. Norton, New York. 312 pp.

Cannon, W. B., Querido, A., Britton, S. W., and Bright, E. M. 1926. Amer. Jour. Physiol. $79,466-507$.

Carpenter, T. M., and Fox, E. L. 1930. Jour. Nutr. 2, 359-374.

Carrel, A., and Hartmann, A. 1916. Jour. Exp. Med. 24, 429-450.

Chambers, R., and Reznikoff, P. 1926. Jour. Gen. Physiol. 8, 369-401.

Chanutin, A., Smith, A. H., and Mendel, L. B. 1924. Amer. Jour. Physiol. 68, 444-460.

Chauveau, A. 1856. Compt. rend. Acad. Sei. 42, 1008-1012.

Chevillard, L. 1935. Ann. physiol, 11, 1015-1054.

Chiray, M, Justin-Besanȩon, L., Debray, C., et Lacour, M. 1938. Bull. Aead. Med. Paris $121,416-421$.

Christ, W. 1926. Klin. Woch. 5, 2113-2114.

Christensen, E. H. 1931. Arbeitsphysiol. 4, 453-469.

Churney, L. 1941. Cytology, Geneties and Evolution. Univ. Penna., Philadelphia. 113128.

Clark, G., Magoun, H. W., and Ranson, S. W. 1939. Jour. Neurophysiol. 2, 61-80.

Clough, H. D., Allen, R. S., and Root, E. W. Jr. 1923. Amer. Jour. Physiol. 66, 461-466.

Cook, S. F., and Seott, K. G. 1932. Biol. Bull. 63, 505-512.

Cotton, F. S., and Dill, D. B. 1935. Amer. Jour. Physiol. 111, 554-556.

Crandall, L. A. Jr., and Anderson, M. X. 1934. Amer. Jour. Dig. Dis. Nutr. 1, 126-131.

Crandall, L. A. Jr., and Roberts, G. M. 1936. Amer. Jour. Physiol. 117, 318-322.

Crisler, G. 1928. Amer. Jour. Pliysiol. 85, 324-331.

Cuvier, G. 1827. Essay on the theory of the earth. Transl. by R. Jameson. 5th ed. Edinburgh, Blackwood. $550 \mathrm{pp}$.

Czeloth, H. 1930. Z. vergl. Physiol. 13, 74-163.

Czerny, A. 1894. Arch. exp. Path. Pharm. 34, 268-280.

Darrow, D. C., and Yannet, H. 1935. Jour. Clin. Inves. 14, 266-275.

Davis, H. A. 1935. Proc. Soc. Exp. Biol. Med. 33, 242-246.

Davis, H. A., and Dragstedt, L. R. 1935. Amer. Jour. Physiol. 113, 193-199.

Davis, J. G., and Slater, W. K. 1928. Biochem. J. 22, 338-343.

Davson, H., and Danielli, J. F. 1938. Biochem. J. 32, 991-1001.

DeBeer, E. J., and H.jort, A. M. 1938. Amer. Jour. Plyysiol. 124, 517-523.

DeHaan, J., and Bakker, A. 1924. Jour. Physiol. 59, 129-137.

Dennig, A. 1898. Z. diätet. physik. Therap. 1, 281-299.

Dennig, A. 1899. Z. diätet. physik. Therap. 2, 292-323.

Dice, L. R. 1922. Ecology 3, 29-47. 
Dietrich, S., und Zeyen, M. 1932. Z. klin. Med. 120, 517-548.

Dill, D. B., Bock, A. V., and Edwards, H. T. 1933. Amer. Jour. Physiol. 104, 36-43.

Dill, D. B., et Brouha, L. 1937. Travail humain 5, 1-17.

Dill, D. B., Edwards, H. T., Bauer, P. S., and Levenson, E. J. 1931. Arbeitsphysiol. 4, 508-518.

Dill, D. B., Edwards, H. T., and Talbott, J. H. 1932. Jour. Physiol. 77, 49-62.

Dill, D. B., Hall, F. G., and Edwards, H. T. 1938. Amer. Jour. Physiol. 123, 412-419.

Ditmars, R. L. 1937. The making of a scientist. Macmillan, New York. $258 \mathrm{pp}$.

Dodds, E. C., Noble, R. L., and Williams, P. C. 1937. Jour. Physiol. 91, 202-211.

Dominguez, R. 1934. Proc. Soc. Exp. Biol. Med. 31, 1146-1149.

Dominguez, R. 1935. Amer. Jour. Physiol. 112, 529-544.

Dominguez, R., Goldblatt, H., and Pomerene, E. 1935. Amer. Jour. Physiol. 114, 240254.

Dominguez, R., Goldblatt, H., and Pomerene, E. 1937. Amer. Jour. Physiol. 119, 429438.

Dominguez, R., and Pomerene, E. 1934. Jour. Biol. Chem. 104, 449-471.

Donaldson, H. H., and Schoemaker, D. M. 1900. Jour. Comp. Neurol. 10, 109-132.

Dontas, S. 1939. Arch. ges. physiol. 241, 612-629.

Douglas, C. G. 1910. Jour. Physiol. 40, 472-479.

Douglas, C. G., and Priestley, J. G. 1924. Jour. Physiol. 59, 30-36.

Dow, P., and Hamilton, W. F. 1939. Amer. Jour. Physiol. 127, 785-792.

Drabkin, D. L., and Shilkret, H. 1927. Amer. Jour. Physiol. 83, 141-146.

Dresel, K., und Leitner, Z. 1929. Z. klin. Med. 111, 394-419.

Driesch, H. 1901. Die organischen Regulationen. Engelmann, Leipzig. $228 \mathrm{~S}$.

Driesch, H. 1908. The science and philosophy of the organism. Black, London. 2 vols.

Drossel. 1929. Arch. Kinderhk. 88, 30-40.

Durig, A. 1901. Arch. ges. Physiol. 85, 401-504.

Eadie, G. S. 1923. Amer. Jour. Physiol. 63, 513-519.

Eimer, K. 1926. Arch. ges. Physiol. 212, 781-786.

Engels, W. 1904. Arch. exp. Path. Pharm. 51, 346-360.

Ewing, P. L. 1940. Quart. Jour. Stud. Ale. 1, 483-500.

Falck, C. P. 1852. Arch. physiol. Heilk. 11, 125-140, 754-772.

Falck, C. P., und Scheffer, T. 1854a. Arch. physiol. Heilk. 13, 61-73.

Falek, C. P., und Seheffer, T. 1854b. Arch. physiol. Heilk. 13, 508-522.

Falck, F. A. 1872. Z. Biol. 8, 388-443.

Falck, F. A. 1873. Z. Biol. 9, 171-242.

Farkas, G. 1932. Areh. ges. Physiol. 230, 76-93.

Ferber, R. H. 1860 . Arch. Heilkunde 1, 244-250.

Fick, A. 1855. Pogg. Ann. 94, 59-86.

Fischer, M. H. 1910. Oedema. Wiley, New York. 209 pp.

Fisher, R. A. 1938. Statistical methods for research workers. 7th ed. Oliver and Boyd, Edinburgh. $356 \mathrm{pp}$.

Fisher, R. A. 1937. The design of experiments. 2nd ed. Oliver and Boyd, Edinburgh. $260 \mathrm{pp}$.

Flinn, F. B., and Scott, E. L. 1923. Amer. Jour. Physiol. 66, 191-208.

Foster, N. B., and Lambert, A. V. S. 1908. Jour. Exp. Med. 10, 820-843.

Fraenkel, G. S., and Gunn, D. L. 1940. The orientation of animals. Clarendon, Oxford. $352 \mathrm{pp}$.

Freeman, N. E., and Wallace, W. M. 1938. Amer. Jour. Physiol. 124, 791-799.

Freeman, S. 1936. Amer. Jour. Physiol. 115, 701-705. 
Frey, E. 1907. Arch. ges. Physiol. 120, 117-136.

Friedlich, A., Holman, C. B., and Forster, R. P. 1940. Bull. Mt. Desert Is. Biol. Lab., 1940, 24-26.

Fukuda, T. R. 1935. Jour. Cell. Comp. Physiol. 7, 301-312.

Gamble, J. L. 1929. New Eng. Jour. Med. 201, 909-917.

Gamble, J. L., and MeIver, M. A. 1928. Jour. Exp. Med. 48, 859-869.

Gamble, J. L., McKhann, C. F., Butler, A. M., and Tuthill, E. 1934. Amer. Jour. Physiol. $109,137-154$.

Gamble, J. L., Putnam, M. C., and MeKhann, C. F. 1929. Amer. Jour. Physiol. 88, 571580.

Gamble, J. L., and Ross, S. G. 1925. Jour. Clin. Inves. 1, 403-423.

Gantt, W. H. 1929. Amer. Jour. Dis. Child. 37, 1125-1127.

Garofeano, M., Lazar, N., et Derevici, M. 1925. Compt. rend. Soc. Biol. 92, 731-732.

Gasnier, A., Gompel, M., Hamon, F., et Mayer, A. 1932. Ann. physiol. 8, 870-890.

Gasnier, A., et Mayer, A. 1934. Ann. physiol, 10, 147-236.

Gasnier, A., et Mayer, A. 1935. Ann. physiol. 11, 143-161.

Gasnier, A., et Mayer, A. 1937a. Ann. plyysiol. 13, 111-130.

Gasnier, A., et Mayer, A. 1937b. Ann. physiol. 13, 131-143.

Gasnier, A., et Mayer, A. 1937e. Ann. physiol. 13, 175-182.

Gasnier, A., et Mayer, A. 1938. Ann. physiol. 14, 101-121.

Gasnier, A., et Mayer, A. 1939. Ann. physiol. 15, 145-214.

Gaule, J. 1901. Areh. ges. Physiol. 87, 472-537.

Geddes, A. E. M. 1930. Meteorology. Blackie, London and Glasgow. 392 pp.

Gerhartz, H. 1910. Arch. ges. Physiol. 133, 397-499.

Gerritzen, F. 1936. Acta med. scand. 89, 101-123.

Gesell, R., Krueger, H., Gorham, G., and Bernthal, T. 1930. Amer. Jour. Physiol. 94; $365-386$.

Gessler, H. 1925. Arch. ges. Physiol. 207, 370-375.

Giaja, J. 1925a. Ann. physiol. 1, 596-627.

Giaja, J. 1925b. Ann. physiol. 1, 628-634.

Gibson, J. G. 2nd, and Evans, W. A. Jr. 1937a. Jour. Clin. Inves. 16, 301-316.

Gibson, J. G. 2nd, and Evans, W. A. Jr. 1937b. Jour. Clin. Inves. 16, 317-328.

Gibson, J. G. 2nd, Keeley, J. L., and Pijoan, M. 1938. Amer. Jour. Physiol. 121, 800806.

Gilman, A. 1937. Amer. Jour. Physiol. 120, 323-328.

Ginglinger, A., et Kayser, C. 1929. Ann. physiol. 5, 710-758.

Goldfarb, A. J. 1935. Biol. Bull. 68, 180-190.

Goldstein, L. A., Tatelbaum, A. J., Ehre, S., and Murlin, J. R. 1932. Amer. Jour. Physiol. 101, 166-184.

Gömöri, P., und Molnar, S. 1932. Arch. exp. Path. Pharm. 167, 459-468.

Gompel, M., Hamon, F., et Mayer, A. 1936. Ann. physiol. 12, 471-503.

Goodall, H. W., and Joslin, E. P. 1908. Trans. Assoc. Amer. Physicians, 23, 92-106.

Graham, H. T. 1935. Amer. Jour. Physiol. 111, 452-465.

Greene, C. H., and Rowntree, L. G. 1927. Amer. Jour. Physiol. 80, 209-229.

Greene, J. A., and Luce, R. P. 1931. Jour. Nutr. 4, 371-378.

Gregersen, M. I. 1931. Amer. Jour. Physiol. 97, 107-116.

Gregersen, M. I. 1938. The distribution and regulation of water in the body. In: MacLeod's Physiology in modern medicine. 8th. ed. Mosby, St. Louis. Part VIII.

Gregersen, M. I., and Bullock, L. T. 1933. Amer. Jour. Physiol. 105, 39.

Gregersen, M. I., and Painter, E. 1939. Amer. Jour. Physiol. 126, P511-P512.

Gregersen, M. I., and Stewart, J. D. 1939. Amer. Jour. Physiol. 125, 142-152. 
Gregory, R. A., and Lee, D. H. K. 1936. Jour. Physiol. 86, 204-218.

Griffith, F. R. Jr., Pucher, G. W., Brownell, K. A., Klein, J. D., and Carmer, M. E. 1929. Amer. Jour. Physiol. 89, 555-583.

Grobicka, J., et Wasilewska, J. 1925. Trav. Inst. Nencki (Varsovie), Lab. Physiol. 3, i, $1-23$.

Grollman, A. 1929. Amer. Jour. Physiol. 89, 157-163.

Gundobin, N. P. 1921. Transl. S. Rubinstein. Die Besonderheiten des Kindesalters. Berlin. $592 \mathrm{~S}$.

Gunn, D. L. 1933. Jour. Exp. Biol. 10, 274-285.

Gunn, D. L. 1937. Jour. Exp. Biol. 14, 178-186.

Gunn, D. L., and Cosway, C. A. 1938. Jour. Exp. Biol. 15, 555-563.

Gürber, A. 1889. Arch. Physiol. 1889, 83-95.

Haden, R. L., and Orr, T. G. 1923 . Jour. Exp. Med. 38, 55-71.

Haden, R. L., and Orr, T. G. 1929. Jour. Exp. Med. 49, 945-953.

Hahn, P. F. 1933. Jour. Biol. Chem, 101, 29-34.

Hahn, P. F., Bale, W. F., Lawrence, E. O., and Whipple, G. H. 1939. Jour. Exp. Med. $69,739-753$.

Haldane, J. B. S. 1921 . Jour. Physiol. 55, 265-275.

Haldane, J. S. 1917. Orgauism and environment as illustrated by the physiology of breathing. Yale Univ., New Haven. $138 \mathrm{pp}$.

Haldane, J. S., and Poulton, E. P. 1908. Jour. Physiol. 37, 390-407.

Haldane, J. S., and Priestley, J. G. 1916. Jour. Physiol. 50, 296-303.

Hale, W., and Fishman, C. 1908. Amer. Jour. Physiol. 22, 32-42.

Hall, F. G. 1922. Biol. Bull. 42, 31-51.

Hall, J. F. Jr., and McClure, G. S. 1936. Amer. Jour. Physiol. 115, 670-678.

Hall, V. E., Field J. 2nd., Sahyun, M., Cutting, W. C., and Tainter, M. L. 1933. Amer. Jour. Physiol. 106, 432-440.

Hamilton, B., and Dewar, M. M. 1938. Growth $2,13-23$.

Hamilton, B., and Schwartz, R. 1935. Jour. Biol. Chem. 109, 745-753.

Hamilton, J. G., and Stone, R. S. 1937. Proc. Soc. Exp. Biol. Med. 35, 595-598.

Hance, R. T. 1917. Jour. Exp. Zool. 23, 287-333.

Hansen, E. 1934. Arbeitsphysiol. 8, 151-171.

Harding, V. J., and Harris, L. J. 1930. Trans. Roy. Soc. Canada [3], sect. 5, 24, 101110.

Hardy, J. D., Milhorat, A. T., and DuBois, E. F. 1938 . Jour. Nutr. 16, 477-492.

Hardy, J. D., and Soderstrom, G. F. 1938. Jour. Nutr. 16, 493-510.

Harnisch, O. 1934. Z. vergl. Physiol. 21, 281-295.

Harrison, H. E., Darrow, D. C., and Yannet, H. 1936. Jour. Biol. Chem. 113, 515-529.

Hashimoto, M. 1914. Arch. exp. Path. Pharm. 76, 367-400.

Hatafuku, J. 1933a. Tohoku Jour. Exp. Med. 21, 13-42.

Hatafuku, J. 1933b. Tohoku Jour. Exp. Med. 21, 43-76.

Hatai, S. 1917. Amer. Jour. Anat. 21, 23-37.

Hecht, S., Haig, C., and Chase, A. M. 1937. Jour. Gen. Physiol. 20, 831-850.

Heilner, E. 1906. Z. Biol. 47, 538-561.

Heilner, E. 1907. Z. Biol. 49, 373-391.

Heller, H., and Smirk, F. H. 1932a. Jour. Physiol. 76, 1-22.

Heller, H., and Smirk, F. H. 1932b. Jour. Physiol. 76, 23-38.

Heller, H., and Urban, F. F. 1935. Jour. Physiol. 85, 502-518.

Heller, J. 1930. Arch. exp. Path. Pharm. 157, 286-297.

Helmholz, H. F., and Bollman, J. L. 1940. Jour. Lab. Clin. Med. 25, 1180-1187.

Helwig, F. C., Sehutz, C. B., and Curry, D. E. 1935. Jour. Amer. Med. Assoc. 104, 15691575 . 
Helwig, T. C., Schutz, C. B., and Kuhn, H. P. 1938. Jour. Amer. Med. Assoc. 110, 644645.

Hemingway, A. 1938. Amer. Jour. Physiol. 121, 747-754.

Hemingway, A. 1940. Amer. Jour. Physiol. 128, 736-746.

Henderson, L. J. 1917. The order of nature. Harvard Univ., Cambridge. 234 pp.

Henderson, L. J. 1928. Blood, a study in general pliysiology. Yale Univ., New Haven. $397 \mathrm{pp}$.

Henderson, Y., and Haggard, H. W. 1925. Amer. Jour. Physiol. 72, 264-282.

Herrin, R. C. 1935. Jour. Biol. Chem. 108, 547-562.

Herrington, L. P. 1940. Amer. Jour. Physiol. 129, 123-139.

Herrmann, F. 1931. Z. vergl. Physiol. 14, 479-524.

Herxheimer, H. 1935. Arbeitsphysiol. 8, 801-806.

Hester, H. R., Essex, H. E., and Mann, F. C. 1940. Amer. Jour. Physiol. 128, 592-602. Hill, A. V., Long, C. N. H., and Lupton, H. 1924. Proc. Roy Soc. 96B, 438-475.

Hill, A. V., and Lupton, H. 1923. Quart. Jour. Med. 16, 135-171.

Hippocrates: Ancient medicine. Transl. by W. H. S. Jones; in Loeb Classical Library: Hippocrates, vol. 1. Heinemann, London. 1923. 1-64.

Hirsch, G. C. 1937. Areh. exp. Zellf. 19, 385-404.

Hitcheock, F. A. 1926. Amer. Jour. Physiol. 79, 206-217.

Hobbes, T. 1647. Elements of philosophy. Part IV, chap. 25, section 12.

Hoitink, A. W. J. H. 1939. Arch. néerl. physiol. 24, 169-193.

Holtz, F., and Brand, T. v. 1940. Biol. Bull. 79, 423-431.

Howe, P. E., Mattill, H. A., and Hawk, P. B. 1911. Jour. Amer. Chem. Soc. 33, 568-598.

Howe, P. E., Mattill, H. A., and Hawk, P. B. 1912. Jour. Biol. Chem. 11, 103-127.

Howell, A. B., and Gersh, I. 1935. Jour. Mammalogy 16, 1-9.

Howes, N. H. 1940. Jour. Exp. Biol. 17, 128-138.

Howes, N. H., and G. P. Wells. 1934. Jour. Exp. Biol. 11, 327-343.

Howland, R. B., and Pollack, H. 1927. Jour. Exp. Zool. 48, 441-458.

Huber, B. 1924. Jahrb. wiss. Bot. 64, 1-20.

Iizuka, N. 1926. Ann. physiol. 2, 310-328.

Iljin-Kakujeff, B. J. 1936. Arbeitsphysiol. 9, 138-146.

Irving, L. 1934. Sci. Mo. 38, 422-428.

Irving, L., Foster, H. C., and Ferguson, J. K. W. 1932. Jour. Biol. Chem. 95, 95-113.

Ivy, A. C. 1918. Amer. Jour. Physiol. 46, 420-442.

Jackson, C. M. 1925. The effects of inanition and malnutrition upon growth and structure. Blakiston, Philadelphia. $616 \mathrm{pp}$.

Jackson, C. M. 1926. Proc. Soc. Exp. Biol. Med. 23, 500-504.

Jackson, C. M. 1937. Anat. Rec. 68, 371-381.

Jackson, C. M., and Smith, V. D. E. 1931. Amer. Jour. Physiol. 97, 146-153.

Jarisch, A., und Liljestrand, G. 1927. Skand. Arch. Physiol. 51, 235-248.

Just, E. E. 1939. The biology of the cell surface. Blakiston, Philadelphia. $392 \mathrm{pp.}$

Kaltwasser, J. 1938. Protopl. 29, 498-535.

Kamada, T. 1936. Jour. Fac. Sci. Tokyo Imp. Univ. 4, 215-219.

Kaplan, B. I., and Smith, H. W. 1935. Amer. Jour. Physiol. 113, 354-360.

Kaunitz, H. 1937. Biochem. Z. 293, 142-156.

Kawaguti, S. 1932. Mém. Fac. Sci. Taihoku Imp. Univ., i, Zoology, 7, 39-55.

Keith, N. M. 1922. Amer. Jour. Physiol. 59, 452-453.

Keith, N. M. 1924. Amer. Jour. Physiol. 68, 80-96.

Keith, N. M., and Power, M. H. 1937. Amer. Jour. Physiol. 120, 203-211. 
Keith, N. M., and Whelan, M. 1926. Amer. Jour. Physiol. 77, 688-702.

Keller, C. J., Loeser, A., und Rein, H. 1930. Z. Biol. 90, 260-298.

Kendeigh, S. C. 1939. Jour. Exp. Zool. 82, 419-438.

Kennedy, J. S. 1937. Jour. Exp. Biol. 14, 187-197.

Kermack, W. O., and MeKendriek, A. G. 1937. Proc. Roy. Soc. Edinb. 57, 228-240.

Keys, A., Matthews, B. H. C., Forbes, W. H., and MeFarland, R. A. 1938. Proc. Roy. Soc. London, 126, 1-29.

King, J. H. 1878. Amer. Jour. Med. Sci. 75, 404-408.

Kingsley, H. D., and Adolph, E. F. New data.

Kitching, J. A. 1934. Jour. Exp. Biol. 11, 364-381.

Kitching, J. A. 1936. Jour. Exp. Biol. 13, 11-27.

Kitching, J. A. 1938. Jour. Exp. Biol. 15, 143-151.

Klein, O., und Nonnenbruch, W. 1930. Z. klin. Med. 112, 568-583.

Kleitman, N. 1927. Amer. Jour. Physiol. 81, 336-340.

Klisiecki, A., Pickford, M., Rothschild, P., and Verney, E. B. 1933a. Proc. Roy. Soc. $112 \mathrm{~B}, 496-521$.

Klisiecki, A., Pickford, M., Rothschild, P., and Verney, E. B. 1933b. Proc. Roy. Soc. $112 \mathrm{~B}, 521-547$.

Knight, R. C. 1917. Ann. Bot. 31, 221-240.

Koidsumi, K. 1934. Mém. Fac. Sci. Taihoku Imp. Univ., i, Entomology, 12, 41-79.

Koizumi, T. 1932. Sei. Repts. Tohoku Imp. Univ. (4) Biol. 7, 259-311.

Kolb, L. C. 1939. Amer. Jour. Physiol. 128, 195-201.

Korr, I. M. 1939. Jour. Cell. Comp. Physiol. 13, 175-193.

Korth, C., und Marx, H. 1928. Arch. exp. Path. Pharm. 136, 120-128.

Krause, F. 1928. Z. Biol. 87, 167-174.

Krijgsman, B. J. 1930. Z. vergl. Physiol. 11, 702-729.

Krogh, A. 1939. Osmotic regulation in aquatic animals. Univ. Press, Cambridge. $242 \mathrm{pp}$.

Krohn, P. L., and Zuckerman, S. 1937. Jour. Physiol. 88, 369-387.

Krüger, F. v. 1937. Z. ges. exp. Med. 101, 666-682.

Krüger, F. 1938. Zool. Anz. 11, Suppl., 141-146.

Künkel, K. 1916. Zur Biologie der Lungenschnecken. Winter, Heidelberg. $440 \mathrm{~S}$.

Kunstmann. 1933. Arch, exp. Path. Pharm. 170, 701-718.

Kunz, H., und Molitor, H. 1927. Arch. exp. Path. Pharm. 121, 342-357.

Lambert, R., et Teissier, G. 1927. Ann. physiol. 3, 212-246.

Landauer, A. 1894. Ungarisches Arch. Med. 3, 136-188; Maly Jahresbericht, 24, 531535.

Landis, E. M. 1927. Amer. Jour. Physiol. 82, 217-238.

Landis, E. M., and Gibbon, J. H. Jr. 1933. Jour. Clin. Inv. 12, 105-138.

Lasch, W. 1922. Z. Kinderhk, 36, 42-57.

Lavietes, P. H., Bourdillon, J., and Klinghoffer, K. A. $1936 . \quad$ Jour. Clin. Inves. 15, 261268.

LeChatelier, H. 1884. Compt. rend. Acad. Sci. 99, 786-789.

LeChatelier, H. 1888. Recherches expérimentales et théoriques sur les équilibres chimiques. Dunod, Paris. $225 \mathrm{pp}$.

LeDantec, F. 1910. La stabilité de la vie. Alean, Paris. $300 \mathrm{pp}$.

Lee, F. W., Carrier, E. B., and Whipple, G. H. 1922. Amer. Jour. Physiol. 61, 149-158.

Lee, M. O. 1929. Amer. Jour. Physiol. 89, 24-33.

Lefèvre, J. 1894. Compt. rend. Soc. Biol. 46, 516-519.

Lefèvre, J. 1897. Arch. de physiol. [5], 9, 7-20, 317-332, 758-763, 802-809.

Lefèvre, J. 1911. Chaleur animale et bioénergétique. Masson, Paris. 1107 pp. 
Leitch, J. L. 1931. Univ. Calif. Publ. Zool. 36, 127-140.

Leitch, J. L. 1934. Jour. Cell. Comp. Physiol. 4, 457-473.

Leiteh, J. L. 1936. Carn. Inst. Wash. Publ. No. 475, 53-70.

Lequime, J. 1940. Acta med. seand., suppl. 107, 224 pp.

Lester, H. M. O., and Lloyd, L. 1928. Bull. Entom. Res. 19, 39-60.

Lewis, W. H. 1931. Bull. Johns Hopkins Hosp. 49, 17-27.

Lienemann, L. J. 1938. Jour. Cell. Comp. Physiol. 11, 149-161.

Liljestrand, G. 1916. Skaud. Arch. Physiol. 33, 153-182.

Loeb, R. F., Atchley, D. W., Benedict, E. M., and Leland, J. 1933. Jour. Exp. Med. 57, 775-792.

Lowrey, L. G. 1913. Anat. Rec. 7, 143-168.

Lönnquist, B. 1906. Skand. Arch. Physiol. 18, 194-262.

Lublin, A. 1929. Z. klin. Med. 109, 371-388.

Luciani, L. 1890. Das Hungern. Studien und Experimente am Menschen. Voss, Hamburg. $239 \mathrm{~S}$.

Lueke, B., Hartline, H. K., and MeCutcheon, M. 1931. Jour. Gen. Physiol. 14, 405-419.

Lucke, B., Hartline, H. K., and Ricea, R. A. 1939. Jour. Cell. Comp. Physiol. 14, 237252.

Lucke, B., and McCutcheon, M. 1927. Jour. Gen. Physiol. 10, 665-670.

Lucke, B., and McCutcheon, M. 1932. Physiol. Rev. 12, 68-139.

Ludwig, D. 1936. Physiol. Zool. 9, 27-42.

Lusk, G. 1912. Jour. Biol. Chem. 13, 27-47.

Lusk, G. 1933. Clio medica: Nutrition. Hoeber, New York. 142 pp.

MacDougal, D. T. 1912 . Ann. Bot. 26, 71-93.

Maclagan, N. F. 1937. Jour. Physiol, 90, 385-394.

MeCance, R. A. 1936. Proc. Roy. Soc. 119B, 245-268.

MeCance, R. A., and Widdowson, E. M. 1937. Jour. Physiol. 91, 222-231.

McClendon, J. F. 1909. Amer. Jour. Physiol. 23, 460-466.

MeConnell, W. J., and Yagloglou, C. P. 1925. Jour. Amer. Soc. Heat.-Vent. Engin. 31, $35-57$.

McCutcheon, M., and Lucke, B. 1927. Jour. Gen. Physiol. 10, 659-664.

McGee, W. J. 1906. Interstate Med. Jour. 13, 279-300.

Magnus, R. 1900. Arch. exp. Path. Pharm. 44, 68-126, 1900.

Magnus-Levy, A. 1910. Biochem. Z. 24, 363-380.

Maluf, N. S. R. 1940. Jour. Cell. Comp. Physiol. 16, 175-187.

Margaria, R., and Edwards, H. T. 1934. Amer. Jour. Physiol. 108, 341-348.

Margaria, R., Edwards, H. T., and Dill, D. B. 1933. Amer. Jour. Physiol. 106, 689-715.

Marshall, E. K. Jr. 1920. Jour. Pharm. 16, 141-154.

Marshall, E. K. Jr., and Davis, D. M. 1914. Jour. Biol. Chem. 18, 53-80.

Martin, C. J. 1902. Phil. Trans. 195B, 1-37.

Martin, S. J., and Herrlich, H. C. 1939. Proc. Soc. Exp. Biol. Med. 42, 451-454.

Marx, H. 1926. Deut. Arch. klin. Med. 152, 354-370.

Marx, H. 1928. Deut. Arch. klin. Med. 158, 149-172.

Marx, H. 1935. Der Wasserhaushalt des gesunden und kranken Mensehen. Springer, Berlin. $335 \mathrm{~S}$.

Mast, S. O., and Fowler, C. 1935. Jour. Cell. Comp. Physiol. 6, 151-167.

Mast, S. O., and Fowler, C. 1938. Biol. Bull. 74, 297-305.

Maupas, E. 1883. Arch. zool. éxp. et gén. [2], 1, 427-664.

Maupas, E. 1888. Arch. zool. éxp. et gén. [2], 6, 165-277.

Maximov, N. A. 1929. The plant in relation to water. A study of the physiological basis of drought resistance. Allen and Unwin, London. $451 \mathrm{pp}$. 
Mayer, A. 1901. Essai sur la soif. Ses causes et son mécanisme. Alcan, Paris. 170 pp. Mayer, A., et Nichita, G. 1929. Ann. physiol. 5, 774-841.

Mayer, A., et Schaeffer, G. 1914. Jour. physiol. path. gén. 16, 23-38.

Meek, W. J., and Eyster, J. A. E. 1921. Amer. Jour. Physiol. 56, 1-15.

Mellanby, K. 1938. Parasitol. 30, 392-402.

Meltzer, S. J. 1907. Jour. Amer. Med. Assn. 48, 655-664.

Melville, K. I. 1936 . Jour. Physiol. 87, 129-143.

Metzner, P. 1927. Tabulae Biol. 4, 490-496.

Michaelis, L., und Maass, T. A. 1907. Biochem. Z. 5, 1-5.

Michaud, L. 1909. Z. physiol. Chem. 59, 405-491.

Mies, H. 1932 . Z. klin. Med. 120, 613-627.

Misawa, H. 1927. Jap. Jour. Med. Sci., VIII. Int. Med. 1, 355-383.

Molitor, H. 1926. Biochem. Z. 172, 379-391.

Möller, E., McIntosh, J. F., and Van Slyke, D. D. Jour. Clin. Inv. 6, 427-465.

More, P. E. 1913. The drift of romanticism. Houghton Mifflin, Boston. $302 \mathrm{pp}$.

Morgan, A. H. 1922. Jour. Exp. Zool. 35, 83-114.

Morgulis, S. 1923. Fasting and undernutrition. Dutton, New York. 407 pp.

Morgulis, S. 1928. Jour. Biol. Chem. 77, 627-645.

Morgulis, S. 1929. Jour. Biol. Chem. 83, 299-310.

Moritz. 1901. Z. Biol. 42, 565-611.

Morosow, B. D. 1931. Arch. exp. Zellf. 10, 157-173.

Müller, R. 1936. Arch. Protistenk. 87, 345-382.

Nagaoka, H. 1936. Jap. Jour. Med. Sci., III. Biophysics 4, 199-211.

Nagel, H. 1934. Z. vergl. Physiol. 21 468-491.

Neuhausen, B. S., and Rioch, D. M. 1923. Jour. Biol. Chem. 55, 353-356.

Neuburger, M. 1926. Die Lehre von der Heilkraft der Natur im Wandel der Zeiten. Enke, Stuttgart.

Newburgh, L. H., and Johnston, M. W. 1934. Jour. Nutr. 7, 107-116.

Newman, E. V., Dill, D. B., Edwards, H. T., and Webster, F. A. 1937. Amer. Jour. Physiol. 118, 457-462.

Newman, H. W., and Lehman, A. J. 1937. Proc. Soc. Exp. Biol. Med. 35, 601-603.

Nielsen, M. 1936. Skand. Arch. Physiol. 74, suppl. 10, 87-208.

Nonnenbruch, W. 1924. Ergeb. inn. Med. 26, 119-206.

Noüy, P. L. du 1916a. Jour. Exp. Med. $24,451-460$.

Noüy, P. L. du 1916b. Jour. Exp. Med. 24, 461-470.

Ogden, E., Shock, N. W., and Heck, K. 1938. Quart. Jour. Exp. Physiol. 28, 341-348.

Ohlmann, J. 1920. Z. Kinderhk. 26, 291-303.

Oka, H. 1938a. Tohoku Jour. Exp. Med. 32, 133-162.

Oka, H. 1938b. Tohoku Jour. Exp. Med. 32, 356-373.

Okey, R., and Robb, E. I. 1925. Jour. Biol. Chem. 65, 165-186.

Onozaki, N. 1935. Tohoku Jour. Exp. Med. 25, 1-13.

Orr, J. B. 1914. Biochem. Jour. 8, 530-540.

Pack, G. T. 1923. Amer. Jour. Physiol. 65, 346-349.

Painter, E. E. 1940. Amer. Jour. Physiol. 129, 744-755.

Palmer, W. W., and Van Slyke, D. D. 1917. Jour. Biol. Chem. 32, 499-507.

Pareto, V. 1935. The mind and society. Harcourt Brace, New York. 4 vols.

Parry, A. A. 1936. Jour. Cell. Comp. Physiol. 8, 277-287.

Pavlov, I. P. 1910. Transl. Thompson, W. H. The work of the digestive glands. 2nd. Engl. ed. Griffin, London. 266 pp. 
Pembrey, M. S. 1895. Jour. Physiol. 18, 363-379.

Pernice, B., und Scagliosi, G. 1895. Arch. path. Anat. 139, 155-184.

Petrilli, A. Unpublished data.

Pfeiffer, L. 1887. Z. Biol. 23, 340-380.

Pflüger, E. 1877. Arch. ges. Physiol. 15, 57-103.

Pickford, M. 1936. Jour. Physiol. 87, 291-297.

Pielou, D. P. 1940. Jour. Exp. Biol. 17, 295-306.

Pielou, D. P., and Gunn, D. L. 1940. Jour. Exp. Biol. 17, 286-294.

Pierce, H. F., and Scott, E. L. 1928. Arch. Int. Med. 41, 586-600.

Pincus, G., and Crozier, W. J. 1929. Proc. Nat. Acad. Sci. 15, 581-586.

Pincus, G., Sterne, G. D., and Enzmann, E. 1933. Proe. Nat. Acad. Sci. 19, 729-733.

Pinson, E. A., and Adolph, E. F. 1942. Amer. Jour. Physiol. 136, 105-114.

Pinson, E. A., and Wills, J. H. Unpublished data.

Pisek, A., und Berger, E. 1938. Planta 28, 124-155.

Plato: Phaedo. Transl. Jowett, B. 1892. 3rd. ed. 2, 240.

Poincare, H. 1913. The foundations of science. Science Press, New York. 553 pp.

Popow, N. A., und Wagner, L. B. 1928. Z. vergl. Physiol. 8, 89-98.

Priestley, J. G. 1921. Jour. Physiol. 55, 305-318.

Pütter, A. 1926. Die Drei-Drüsentheorie der Harnbereitung. Springer, Berlin. 173 S.

Ragan, C., Ferrebee, J. W., Phyfe, P., Atchley, D. W., and Loeb, R. F. 1940. Amer. Jour. Physiol. 131, 73-78.

Raudnitz, R. W. 1888. Z. Biol. 24, 423-552.

Raup, R. B. 1926. Complacency. The foundation of human behavior. Macmillan, New York. $201 \mathrm{pp}$.

Regnier, A. 1916. Z. exp. Path. Ther. 18, 139-164.

Reid, C. 1936. Jour. Physiol. 88, 9P-10P.

Reid, W. L. 1929. Amer. Jour. Physiol. 90, 168-171.

Rein, H., und Schneider, M. 1930. Z. Biol. 91, 13-25.

Rein, H., und Schneider, M. 1937. Arch. ges. Physiol. 239, 464-475.

Rey, P. 1937. Ann. Physiol. 13, 1081-1144.

Rey, P. 1938. Ann. Physiol. 14, 1-66.

Richter, C. P. 1936. Proc. Asso. Res. Nerv. Ment. Dis. 17, 392-409.

Richter, C. P. 1937. Science 86, 354-355.

Richter, C. P. 1938. Amer. Jour. Physiol. 122, 668-675.

Richter, C. P., and Brailey, M. E. 1929. Proc. Nat. Acad. Sei. 15, 570-578.

Richter, C. P., and Eckert, J. F. 1938a. Endocrinol. 22, 214-224.

Richter, C. P., Holt, L. E. Jr., Barelare, B. Jr., and Hawkes, C. D. 1938. Amer. Jour. Physiol. 124, 596-602.

Riegel, C. 1927. Jour. Biol. Chem. 74, 135-148.

Rioch, D. M. 1927. Arch. Int. Med. 40, 743-756.

Rioch, D. M. 1930. Jour. Physiol. 70, 45-52.

Ritzman, E. G., and Benedict, F. G. 1938. Nutritional physiology of the adult ruminant. Carn. Inst. Washington Publ. No. 494.200 pp.

Robertson, J. D. 1939a. Brit. Jour. Ophthal. 23, 106-124.

Robertson, J. D. 1939b. Jour. Exp. Biol. 16, 387-397.

Robinson, E. A., and Adolph, E. F. New data.

Robinson, S., Edwards, H. T., and Dill, D. B. 1937. Science 85, 409-410.

Rockwell, F. V. 1935. Jour. Clin. Inves. 14, 202-211.

Rominger, E. 1920. Z. Kinderhk. 26, 23-64.

Rose, W. B., Stucky, C. J., Mendel, L. B., and Cowgill, G. R. 1931. Amer. Jour. Physiol. 96, 132-138. 
Ross, L. G. 1930. Biol. Bull. 59, 326-338.

Rost, E. 1903. Arch. Physiol. 1903, 369-377.

Rous, P., and Turner, J. R. 1916. Jour. exp. Med. 23, 219-237.

Rowntree, L. G. 1922. Physiol. Rev. 2, 116-169.

Rowntree, L. G. 1923. Arch. Int. Med. 32, 157-174.

Rowntree, L. G. 1926. Jour. Pharm. 29, 135-159.

Rubner, M. 1881. Z. Biol. 17, 214-238.

Rubner, M. 1894. Z. Biol. 30, 73-142.

Rubner, M. 1902. Die Gesetze des Energieverbrauchs bei der Ernährung. Deuticke, Leipzig und Wien. $426 \mathrm{~S}$.

Rubner, M. 1908. Arch. Hyg. 66, 127-208.

Rubner, M. 1924. Biol. Z. 148, 187-221.

Rydin, H., and Verney, E. B. 1938. Quart. Jour. Exp. Physiol. 27, 343-374.

Sato, H. 1930. Tohoku Jour. Exp. Med. 16, 487-493.

Sayers, R. R., and Yant, W. P. 1923. Publ. Health Rep. 38, 2053-2074.

Schade, H. 1927. Ergeb. inn. Med. 32, 425-463.

Schiff, E., Bayer, W., und Choremis, K. 1925. Jahrb. Kinderhk. 109, 287-299.

Schiödt, E. 1938. Acta med. scand., suppl. 89, 169-172.

Schmidt, P. 1918. Jour. Exp. Zool. 27, 57-72.

Scholles, W. 1933. Z. vergl. Physiol. 19, 522-554.

Schretter, G., und Nevinny, H. 1931. Z. Geburts. Gyn. 98, 258-276.

Schuchardt, B. 1847. Quaedam de effectu, quem privatio singularum partium nutrimentum constituentium exercet in organismum ejusque partes. Inaug. Diss., Marburgi. $53 \mathrm{pp}$.

Seott, E. L. 1927. Jour. Biol. Chem. 73, 81-112.

Scott, E. L., and Ford, T. H. 1923. Amer. Jour. Physiol. 63, 520-534.

Sedlmair, A. C. 1899 . Z. Biol. 37, 25-58.

Shannon, J. A. 1935. Amer. Jour. Physiol. 112, 405-413.

Shannon, J. A. 1936a. Amer. Jour. Physiol. 114, 362-365.

Shannon, J. A. 1936b. Amer. Jour. Physiol. 117, 206-225.

Shannon, J. A. 1938. Amer. Jour. Physiol. 122, 782-787.

Shapiro, H., and Parpart, A. K. 1937. Jour. Cell. Comp. Physiol. 10, 147-153.

Shaw, L. A. 1926. Amer. Jour. Physiol. 79, 91-99.

Shear, M. J. 1935. Amer. Jour. Cancer 23, 771-783.

Shear, M. J., and Fogg, L. C. 1934. Publ. Health Rep. 49, 225-240.

Shelley, W. B., and Hemingway, A. 1940. Amer. Jour. Physiol. 129, 623-630.

Shelling, D. H., Kajdi, L., and Guth, L. 1938. Endocrinol. 22, 225--235.

Skinner, B. F. 1932. Jour. Gen. Psychol. 6, 22-37.

Skinner, B. F. 1936. Jour. Gen. Psychol. 15, 205-210.

Skinner, B. F. 1938. The behavior of organisms: an experimental analysis. AppletonCentury, New York and London. $457 \mathrm{pp}$.

Smirk, F. H. 1933a. Jour. Physiol. 78, 113-126.

Smirk, F. H. 1933b. Jour. Physiol. 78, 127-146.

Smith, H. P. 1920. Amer. Jour. Physiol. 51, 221-231.

Smith, H. P. 1925. Bull. Johns Hopkins Hosp. 36, 325-342.

Smith, H. P., Arnold, H. R., and Whipple, G. H. 1921. Amer. Jour. Physiol. 56, 336360.

Smith, H. P., Belt, A. E., and Whipple, G. H. 1920. Amer. Jour. Physiol. 52, 54-71.

Smith, H. W. 1930. Amer. Jour. Physiol. 93, 480-505.

Smith, H. W. 1937. The physiology of the kidney. Oxford Univ., New York. 310 pp.

Smith, V. D. E., and Jackson, C. M. 1931. Biol. Bull. 60, 80-93. 
Snell, A. M., and Rowntree, L. G. 1928. Amer. Jour. Physiol. 85, 577-590.

Soskin, S., and Levine, R. 1937. Amer. Jour. Physiol. 120, 761-770.

Spencer, H. 1866. The principles of biology. Appleton, New York. 2 vols.

Spiegler, A. 1901. Z. Biol. 41, 239-270.

Stadie, W. C., and Martin, K. A. 1925. Jour. Clin. Inves. 2, 77-91.

Stanbury, J. B., Warweg, E., and Amberson, W. R. 1936. Amer. Jour. Physiol. 117, 230-236.

Starling, E. H. 1896. Jour. Physiol. 19, 312-326.

Stewart, G. N. 1921. Amer. Jour. Physiol. 58, 45-52.

Stier, T. J. B. 1933. Proc. Nat. Acad. Sci. 19, 725-729.

Stöhr, R. 1934. Z. ges. exp. Med. 95, 55-66.

Straub, W. 1899. Z. Biol. 38, 537-566.

Summer, F. B. 1913. Jour. Exp. Zool. 15, 315-377.

Sunderman, F. W., and Austin, J. H. 1930. Amer. Jour. Med. Sci. 179, 167-177.

Sunderman, F. W., and Austin, J. H. 1936. Amer. Jour. Physiol. 117, 474-486.

Sutherland, G. F. 1921. Amer. Jour. Physiol. 55, 258-276.

Sveinsson, S. L. 1940. Skand. Arch. Physiol. 83, 188-194.

Szwejkowska, G. 1938. Ann. physiol. 14, 122-144.

Takahashi, S. 1935a. Tohoku Jour. Exp. Med. 25, 531-549.

Takahashi, S. 1935b. Tohoku Jour. Exp. Med. 26, 60-72.

Takahashi, S. 1935c. Tohoku Jour. Exp. Med. 26, 73-82.

Takeda, K. 1936. Tohoku Jour. Exp. Med. 29, 137-143.

Taylor, H. S. 1924. A treatise on physical chemistry. Van Nostrand, New York. 2 vols.

Terroine, E. F., et Trautmann, S. 1927. Ann. physiol. 3, 422-257.

Theobald, G. W. 1934. Jour. Physiol. 81, 243-254.

Thomas, K. 1911. Arch. Physiol. 1911, 9-38.

Thompson, V., and Bodine, J. H. 1936. Physiol. Zool. 9, 455-470.

Thoreau, H. D. 1863 . Excursions. Ticknor and Fields, Boston. 319 pp.

Tobler, L. 1910. Arch. exp. Path. Pharm. 62, 431-463.

Trimble, H. C., Carey, B. W. Jr., and Maddock, S. J. 1933. Jour. Biol. Chem. 100, 125138.

Trolle, C. 1937. Skand. Arch. Physiol. 76, 220-224.

Turner, C. H. 1924. Biol. Bull. 46, 35-54.

Tuteur, R. 1910. Z. Biol. 53, 361-385.

Ueki, R. 1924. Arch. ges. Physiol. 205, 246-254.

Underhill, F. P. $1919 . \quad$ Arch. Int. Med. 23, 753-770.

Underhill, F. P., and Fisk, M. E. 1930. Amer. Jour. Physiol. 95, 348-363.

Underhill, F. P., and Kapsinow, R. 1922. Amer. Jour. Physiol. 63, 142-145.

Underhill, F. P., and Sallick, M. A. 1925. Jour. Biol. Chem. 63, 61-69.

Utheim, K. 1920. Amer. Jour. Dis. Child. 20, 366-392.

Uyldert, I. E. 1938. Acta brevia neerl. 8, 112-114.

Vassiliev, I. M., and Vassiliew, M. G. 1936. Plant Physiol. 11, 115-125.

Verworn, M. 1898. General physiology. Macmillan, London. 615 pp.

Verzár, F. 1936. Absorption from the intestine. Longmans, London. $294 \mathrm{pp}$.

Voigt, O. 1932. Zool. Jahrb., Abt. allg. Zool. 52, 677-708.

Voit, C. 1866 . Z. Biol. 2, 307-365.

Voit, E. 1929. Z. Biol. 89, 114-138.

Voit, E., und Korkunoff, A. K. 1895. Z. Biol. 32, 58-175. 
Volkmann, A. W. 1874. Ber. Verh. k. sächs. Ges. Wiss., Math.-phys. Cl. 26, 202-247.

Vozarik, A. 1906. Arch. ges. Physiol. 111, 526-536.

Wada, H. 1933. Mitt. med. Ges. Tokio 47, 1873-1913.

Wada, M., Seo, M., and Abe, K. 1935. Tohoku Jour. Exp. Med. 26, 381-411.

Wallace, G. B., and Brodie, B. B. 1937. Jour. Pharm. 61, 397-411.

Warden, C. J. 1931. Animal motivation: experimental studies on the albino rat. Columbia Univ., New York. 502 pp.

Weech, A. A., Goettsch, E., and Reeves, E. B. 1933. Jour. Clin. Inves. 12, 217-227.

Weech, A. A., Reeves, E. B., and Goettsch, E. 1935. Jour. Biol. Chem. 113, 167-174.

Weed, L. H. 1938 . Jour. Anat. 72, 181-215.

Weir, E. G. 1940. Amer. Jour. Physiol. 130, 608-611.

Weir, J. F., Larson, E. E., and Rowntree, L. G. 1922. Arch. Int. Med. 29, 306-330.

Weiss, S., and Baker, J. P. 1933. Medicine 12, 297-354.

Wendt, E. 1876. Arch. Heilk. 17, 529-546.

Wermel, E. M., und Portugalow, W. W. 1935. Z. Zellf. 22, 185-194.

Wettendorff, H. 1901. Institut Solvay, Trav. de lab. 4, 353-484.

Wheeler, W. M. 1928a. Emergent evolution. Norton, New York. 80 pp.

Wheeler, W. M. 1928b. Foibles of insects and men. Knopf, New York. 217 pp.

Whipple, G. H., and Robscheit-Robbins, F. S. 1925. Amer. Jour. Physiol. 72, 395-407.

White, H. L., and Findley, T. Jr. 1937. Amer. Jour. Physiol. 119, 740-748.

Whitehouse, A. G. R., Hancock, W., and Haldane, J. S. 1932. Proc. Roy. Soc. 111 B, $412-429$.

Whiting, M. H. 1915. Biometrkia 11, 1-37.

Widmark, E., und Tandberg, J. 1924. Biochem. Z. 147, 358-369.

Wierzuchowski, M. 1936. Jour. Physiol. 87, 311-335.

Wierzuchowski, M. 1937a. Jour. Physiol. 90, 440-464.

Wierzuchowski, M. 1937b. Jour. Physiol. 91, 140-171.

Wigglesworth, V. B. 1931. Jour. Exp. Biol. 8, 411-427.

Wigglesworth, V. B. 1939. The principles of insect physiology. Methuen, London. $434 \mathrm{pp}$.

Wiley, F. H., and Wiley, L. L. 1933. Jour. Biol. Chem. 101, 83-92.

Wiley, F. H., Wiley, L. L., and Waller, D. S. 1933. Jour. Biol. Chem. 101, 73-82.

Winslow, C. E. A., Herrington, L. P., aud Gagge, A. P. 1937. Amer. Jour. Physiol. 120, $1-22$.

Winsor, A. L. 1930. Amer. Jour. Psychol. 42, 602-607.

Witsch, K. 1926. Arch. ges. Physiol. 211, 185-212.

Wittig, H. 1938. Zentrabl. Agrikulturchem. Abt. B, Tierernährung 10, 481-495.

Wolf, A. V. 1938a. Ecology 19, 233-242.

Wolf, A. V. 1938b. Ecology 19, 346-348.

Wolf, A. V. 1940a. Physiol. Zool. 13, 294-308.

Wolf, A. V. 1940b. Jour. Cell. Comp. Physiol. 15, 355-361.

Wolf, A. V., and Adolph, E. F. New data.

Wolff, L. 1935. Arch. exp. Path. Pharm. 179, 200-203.

Wolvekamp, H. P., und Lodewijks, J. M. 1934. Z. vergl. Physiol. 20, 382-387.

Woodbury, R. A., and Hamilton, W. F. 1937. Amer. Jour. Physiol. 119, 663-674.

Yagloglou, C. P. 1924. Jour. Amer. Soe. Heat. Vent. Engin. 30, 597-609.

Zeleny, C. 1916. Illinois Biol. Monog. 3, 1-170.

Zucker, T. F., and Berg, B. N. 1937. Amer. Jour. Physiol. 119, 539-548.

Zuntz, N., und Hagemann, O. 1898. Untersuchungen über den Stoffwechsel des Pferdes bei Ruhe und Arbeit. Parey, Berlin. $438 \mathrm{~S}$. 



\section{INDEX}

Abstract, of the investigation, ix, 471

Acceleration, of exchanges, $178 \mathrm{f}$., 406

Acclimatization, 376, 412

to heat, 306

to water, 376

Accommodation, 377

Aecumulation rate, defined, 388

Adaptation, 377, 464

Adrenal glands, 412

insufficiency, dog, 60, 174

Age, and heat exchanges, $316 \mathrm{f}$., 320

and water exchanges, 263-269

Agents, in relation to loads, 294, 385, 475

Alcohol, ethyl, elimination of, dog, 380

Alimentary tract, exchanges through, 381

$A m e b a$, water in, 148

Anestheties, and water exchange, dog, 51

Anorexia, 228, 326

Apnea point, 341

Appetites, 331

Applications of regulatory physiology, 475

Arbacia egg, water in, 144-146, 160

Arm, volume of, man, 158 f., 166

Arterio-venous oxygen difference, 406

Asterias egg, water in, 160

Augmentation ratio, defined, 397

heat, man, 311

water, various species, $172 \mathrm{f}$.

Balance, defined, 35, 385, 397

Behavior, 5, $364 \mathrm{ff}$.

in relation to maintenance, water, 72 , 129-131, 195, 243 f.

Bile, flow of, $227 \mathrm{f}$.

duct, ligation of, dog, 69; rabbit, 157

Bipalium (flatworm), water in, 144

Blood, volume of, 153-158, 166, 213-224, 257 concentrations in, 213-224, 239-241, 410 pressure, see Pressure, arterial

Body, mass, 397

size, 177, 180, 263-275

surface, $264 \mathrm{ff}$.

weight, 397, see also Weight

Bovine, water exchanges, 182, 198 young, and water turnover, $265 \mathrm{f}$.

Breathing, frequency, dog, 361

Bromide, elimination of, man, 372
Burns, superficial, 60, 233

Burro, water exchanges in, 182

Calcium, demand for, 331

elimination of, 392

velocity quotient of, dog, 380

Camel, water storage in, 194, 212

Capacities, functional, 456

Carbohydrate, loss, dog, 338

Carbon, total, exchange of, dog, 329

Carbon dioxide, content, eat and dog, $341 \mathrm{f}$. paths of exchange of, mammals, 381 recovery of content, man, $340 \mathrm{f} ., 372$ tolerance eurve, man, 392

velocity quotient of, man, 379

Carbon monoxide, elinination of, dog, 378, 380 ; man, 372,378

Cardiac output, recovery of, man, $353 \mathrm{f}$., 372

Catharsis, of bowel, dog, 60

Causes, 432

Cells, volumes of, 160, 164-166

Ceratocephale (worn1) egg, water in, 160

Characterizations, of regulation, 105, 288, 390

Chicken, heat load in, 305

Chloride, load, 411

depletion, 411

in blood, 219

paths of exchanges, mammals, 381

Chloroform, poisoning, dog, 69

Cholate, elimination of, dog, 380

Cicatrization, of skin wounds, 357

Classification of variables, 397

Clearances, defined, 387

renal, 229 f., 243

Coefficient of variation (C.V.), 74, 175, 361 of difference (C $\triangle$ ) , 74, 175, 362

Combinations, of components, 415

of exchanges, 193, 395

of variables, $12,263,407,408,418,425$

Comparisons, of correlatives of water, 251254

of heat regulations, 318

of species, water, $169 \mathrm{ff}$.

of volume recoveries, 166

of water regulations, $292 \mathrm{f}$. 
Compartments, and maintenances, 393

in body, 236

Compatibility, among exchanges, 375

among properties, $413 \mathrm{f}$.

Compensation, reactions of, $5,390,396$

Complacency, 446

Components, 2, 397

adjustment, in man, 372

relative rates of exchange, $391 \mathrm{ff}$., $400 \mathrm{ff}$.

Composition, biochemical, 241, 459

Conclusions, about constancies, $477 \mathrm{ff}$.

Conditions, environmental, defined, 397 diversity of, 169

Constancies, see Maintenance, Regulations

Content, defined, 397

water, 195-201

Contributions to biology, in this investigation, 476

Coordination, among physiological facts, 436,467

Corixidae (bugs), 375

Correlations, empirical, 409

multiple, 415

types of, 398

Correlatives, search for, 424

Creatinine, elimination of, dog, 378, 380 man, 372,378

Cuvier's rule, 412

Death, see Irreversible change, Reversibility of loads, Survival

Deceleration, of exchanges, $178 \mathrm{f}$., 407

Defense, reactions of, 390

Deficit, absolute and relative, 63 see also Water, deficit

Dehydration, 50, 60, 65, 411 see also Water, deficit

Description, use of, $8,427 \mathrm{ff}$.

Design of experiments, 417 f., 424

Detection, of water, 195 see also Behavior

Deviations, 361

Diagram, see Equilibration, Tolerance

Difference, coefficients of, $74,175,362$

Dilutions, of parts of body, 210-213, 398

Dipodomys (kangaroo rat), 198

Disappearance curves, 358

Disease, $450 \mathrm{f}$.

Displacement, see Load

Disposal, see Exchanges

Distribuend, of volume, 153
Distribution, volume of, see Volume of distribution

Diuresis, see Water exchange

Dog, earbon in, 329

Eck-fistula, 58, 68 f.

esophageal-fistula, 47

glucose in, $332-340$

heat in, 305

nitrogen in, $330 \mathrm{f}$.

various components in, 361, 378, 380, 391

water in, 17-87, 184, 186

young, water relations in, 266-268

Drinking, 27-30, 47, 76, 180-183

Duck, heat load in, 305

Dynamics, 256

see also Forces

Earthworm, see Lumbricus

Echinoderm egg, water in, 144-146 see also Arbacia

Eck-fistula, $68 \mathrm{f}$. reversed, 58

Economy, of structure, 383 quotients, at various heat loads, 305 ; at various water loads, $82,170 \mathrm{f}$; ; defined, 48, 203, 374, 397

Electrolytes, in man, 411

Elephant, 182, 198

Emergents, 416

Energy, in water turnover, 200 laws of, 302 metabolism of, $229,274 \mathrm{f}$. total, in body, dog, 328

Environments, 475

Equilibration, $\mathrm{x}, 397,446,463,474$ diagrams, defined, 32 ; dog, 32, 40, 391; heat, 308; in general, 40, 190-194, 203, 394 ff.; net, 190 f.; total, 190 f.; water, 188-195

Equilibrium, kinetic, 192, 364, 397, $444 \mathrm{ff}$. positions of, 189

Equivalences, physiological, 416

Erythrocytes, count of, 240 volumes of, 155, 164

Evaporation, see Heat, paths of exchanges

Exchange diagram, 40

Exchange-velocity diagram, 40

Exchanges, rates of, $369,373 \mathrm{ff} ., 397$

in general, 4

in relation to content, 205

maximal, man, 372 
net, 203

partitional, $203 \mathrm{f}$., 381

total, 203

see also Recovery

Excitability, in nerve, 357, 374, 377

Excretion, exchanges by, 243, 381 see also Exchanges, Kidneys, Water

Excretory ratio, defined, 387

Exercise, physical, 405 ff., 418 and water exchange, 56, 59

Explanations, 410

Factors, limiting, 457 of safety, 458

Fat, elimination of, 392

Fecal exchanges, 173, 275, 381

Fibroblasts, volumes of, $164 \mathrm{f}$.

Fidelity, of turnover, 82

Fitness, characteristic of regulations, 465

Flow, volume, of blood, $353 \mathrm{f}$.

see also Cardiac output

Fluctuations, in time series, 75

Food, privation of, 323-326

relation to water exchanges, $54,59,60$, $228 \mathrm{f}$.

total intake of, 198, $323 \mathrm{ff}$.

Forces, electrostatic, 258

in equilibrium, 445,461

in water exchanges, 255-258, 297

Frequencies, of occurrence, 362 of water contents, 81

Frog (Rana), larva of, see Tadpole muscles of, volume change in, $162 \mathrm{f}$., 184 water in, 110-121, 184, 186, 246-248

Gain, see Exchanges

Galactose, velocity quotient of, dog, 380

Gap, in time series, 75

Gas, poison, in dog, 60

Generalizations, 9, 434

concerning water regulations, 290-292

defined, 454

of physiology, 454

Gila monster (Heloderma), 316

Glucose, exchanges of, dog, 332-338, 391

in blood, variability of, 336

infusion of, effects of, $\operatorname{dog}, 66,332 \mathrm{ff}$., 410

paths of exchanges, $334 \mathrm{f}$., 381

velocity quotient of, $\operatorname{dog}, 380$

Growth, curves of, 359

in size, 211

rate of, defined, 388
Guinea pig, heat load in, 305

newborn, temperature regulation, 320

Half-life, of recovery, 166, 369, 381

Health, preservation of, 1,3

Heart, frequency of beat, dog, 361, 378; man, $350,372,378,405,412$; tolerance curve, man, 392 ; relocity quotient of, $379 \mathrm{f}$.

see also Cardiac output

Heat, exchanges, 302-322

load, 302-318; in relation to heart frequency, $407 \mathrm{f}$; ; in relation to water load, $400 \mathrm{ff}$.

partition of disposal, man, $310 \mathrm{f}$.; rabbit, $313 \mathrm{f}$.; variabilities of, 313

paths of exchanges, mammals, 310-313, 381

production, man, variability of, $303 \mathrm{f}$.

recovery of content, man, 307 ff., 381; various species, $316-318$

regulation of content, 301-322; man, 302-312 ; mouse, $319 \mathrm{f}$.; rabbit, 312319 ; variabilities, 303

tolerance eurve, man, 392

velocity quotient of, man, 379

Helix (snail), water in, 135-137

Hemoglobin, $220 \mathrm{f}$.

Hemorrhage, dog, 60 ; in relation to other loads, 256

Histamine, dog, 60

Homeostasis, 446

Hydration, see Water, excess

Hydremia, 57

Hypertrophy, rat viscera, 357

Hypotheses, types of, $431 \mathrm{ff}$.

use of, 431

Imbibition, through body surface, 110 ff.

Increment, of component, defined, 203

Individuals, as physiological units, 281

Inductions, 426, 455

Inertia, of processes, 363

Insects, behavior toward humidity, 138

water in, 137-138, 183

Insulin, in dog, 60

Intake, see Exchanges

Integrations, of functions, $466 \mathrm{f}$.

Interdependencies, 420

Internal medium, of body, 298

Interrelations, among components, 383,386 , $400 \mathrm{ff}$.

among variables, 12,284 ff. 
Invasion coefficient, defined, 388

Inversions, in time series, frequency of, 74 , 80,82

Invertebrates, water in, 134-149, 160

Investigation, plan of, $2-13,423$

Iodide, elimination of, man, 372, 392

Irreversible change, 371

Juices, digestive, flow of, 227 f., 243

see also Bile, Pancreatic, Saliva

Kidneys, as path of exchange, 231, 383 excised, 157

see also Clearances, Urine

Kinetics, see Exchanges, rates of

Lactate, paths of exchanges, mammals, 381 recovery of content, dog, 378 ; man, 372 , $378,346 \mathrm{ff}$.

tolerance curve, man, 347,392

velocity quotient of, man, 379

volume of distribution of, man, 346

Lead, elimination of, 392

LeChatelier's theorem, 445

Leucocytes, volumes of, $164 \mathrm{ff}$.

Limax (slug), water in, 134-135

Limiting factors, in recoveries, 382

Load, defined, 18, 397

measurement of, 370

methods of producing, 204

of component, 370

tolerated extremes of, $201 \mathrm{f}$., 373

types of, water, 50-67, 107, 204, 294

Load-velocity diagram, 40

Loading, types of, $385 \mathrm{f}$., 397

Loss, see Disappearance, Exchanges, Water, rates of exchange

Lumbricus (earthworm), water in, 142-144

Maintenance, of constancy, 1-4, 192 of physiological state, 446

Man, arterial pressure in, 352

carbon dioxide in, $332-340$

glucose of blood in, 336

heart beat in, 350-352

heat in, 302-312

lactate in, $346-348$

total substance in, $325 \mathrm{f}$.

various components in, 238-246, 362, 372, 378,392

water in, 88-109, 182, 184, 186
Maxima, 455

Mechanisms, 432

Metabolic rate, defined, 387

Metabolisms, changes in, 283, 389

dog, 227-233

man, 242

Methods, of representing relations, 425

Minima, 455

Minimal work, 456

Modification, of behavior, 366,439 ; by agents, 376

of exchanges, 203, 441

ratio, defined, 34 , 397 ; heat, 311 ; water, 34,172

types of, 395

Monkey, heat load in, 305

water exchanges in, 198

Monotremes, heat regulation in, 319

Mouse, heat regulation in, 316-320 water exchanges in, 198

Muscles, volumes of, 161-164, 166, 184

Narcotics, and water exchange, dog, 51

Nerve, excitability of, 357,377

Neuromuscular activities, and water, 232

Newborn, dog, water exchanges of, $267 \mathrm{f}$. man, temperature of, 304

rabbit, heat exchanges of, 317

Nitrogen, excretory rate, dog, 361

retention of, 225

total body, exchanges of, dog, $329 \mathrm{f}$., 391 ; velocity quotient of, $\operatorname{dog}, 380$

Nomenclature, of variables, 423

Normal, concept of, 364

Novasurol, and water exchanges, dog, 58

Nuclei, volumes of, 160, 164-166

Obstruction, intestinal, 60 pylorie, 60

Oligoposia, 71

Oliguria, 71

Ontogeny, of body temperature, 317-320

of regulations, 458

of water exchanges, 264-269

Organization, physiological, 414, 419

Organs, adjustments independent of, $190 \mathrm{ff}$. see also Paths, Tissues

Origins, hypotheses concerning, 433 of equilibrations, $458 \mathrm{ff}$.

Osmotic flow, coefficient of, 262 pressure, see Pressure 
Output, see Cardiac output, Water output

Overshooting, during recovery, 371

Oxygen, consumption, man, 242, 372, 405; various species, 252

debt or deficit, $343 \mathrm{ff}$.

paths of exchanges, mammals, 381

pulse, 406

reciprocity with earbon dioxide, 345

recovery of content, man, $342 \mathrm{ff}$., 372

tolerance curve, man, 345,392

Pancreatic juice, loss of, dog, 60

Parameters, of exchanges, $139 \mathrm{ff}$., $386 \mathrm{ff}$.

Parathyroid, treatment with, dog, 60

Parts of organisms, water in, 151-167, 281

Paths of exchanges, 189 f., 274, 281, 381

Patterns, of exchanges, ix, $191 \mathrm{f}$.

physiological, 298, 413, 421, 478

Periodicities, 75

Permeability, 258-263, 384

coefficient, defined, 259, 388

inconstancy of, 260

to salts, $164 \mathrm{f}$.

to water, $139,145,261$

Peromyscus (deer mouse), 198, 277

Phascolosoma (worm), water in, 138-142, 184,186

Phosphorus, poisoning, 55 f., 58, 59 retention of, 225

Phylogeny, of regulations, 459

Physiology, comparative, 169 ff., 475 descriptive, $427 \mathrm{ff}$.

general, 205

organ, 475

Physiometry, 436

Pig, heat load in, 305

Pinocytosis, 165

Pituitrin (infundin), and water exchange, $55,58 \mathrm{f}$.

Plants, water in, $148 \mathrm{f}$.

Plasma, concentrations in, $213 \mathrm{ff}$.

volume of, 153-158, 166, 184, 213-224

Polyposia, 71

Polyuria, 71

Precision, of turnover, 82

see also Economy quotient

Preferences, for environment, see Behavior

Pressure, arterial, recovery of, man, 352, 372 ; velocity quotients of, 352 ; turnover, 353

osmotic, 255-257
Procedures, of the investigation, 8, 285, 422, 431

Properties, fixation of, 421

as physiological variables, 282

Propylene glycol, velocity quotient of, dog, 380

Protein, plasma, velocity quotient of, dog, 380

Protozoa, water in, 146-148

Purposes, hypotheses of, 433

Quotient, see Economy quotient, Respiratory quotient, Velocity quotient

Rabbit, glueose of blood in, 336

heat load in, 305, 312-318

water in, 123-126, 182, 248-250

Races, and water exchanges, deer mouse, 276

Random distributions, 74

Rapidity, of turnover, 82

Rat, water in, 126-132, 182, $250 \mathrm{f}$.

young, and water turnover, 265

Rates of exchange, maximal, 176, 178, 311, 314,372

ratio of, water, 18,174

variations of, $78-85,175$

Ratio, see Augmentation ratio, Modification ratio

Reciprocal action, of paths of exchange, 35 , 381

Recovery, defined, 388, 397

exponential, 369,380

net, 397

rates of, see Exchanges

water, see Water, recovery

Refractive index, of plasma, dog, $218 \mathrm{f}$.

Regeneration, skin, see Skin

tadpole tail, $354 \mathrm{ff}$.

Regulations, physiological, $437 \mathrm{ff}$; classes of, $439 \mathrm{ff}$; history of, 437,446 ; meanings of, $1 \mathrm{f}$., $437 \mathrm{ff}$; multiple, $446 \mathrm{f}$.; specifications of, 443

Representation, of relations, methods, 425 ; by mapping, $427 \mathrm{f}$.

Reptiles, water in, 132-134

Resistance, to exchanges, 380, 439, 462

Respiratory exchanges, 381 quotient, dog, 361

Resultant, of correlated components, 416

Returns, of water, 23, 90, 289

Reversibility, of exchanges, 382 of loads, 371, 447, $465 \mathrm{f}$. 
Rhodnius (bug), water in, $137 \mathrm{f}$.

Rhythms, physiological, 420

Run, in time series, 74

Saliva, flow of, 227,243

Schemes, conceptual, 426

Season, and water turnover, 276

Selection, among environments, 5-6 see also Behavior

Sensibility, of turnover, 82

Sensitivity, to load, 5

Sequences, in time, 74, $366 \mathrm{ff} ., 414,420$, $439 \mathrm{ff}$.

Significances, of facts, 410

Signs, of abnormality, 448

of load, $448 \mathrm{f}$.

Size, of body, and water exchanges, 177, $180,263-275$

Skin, healing of, rate, man, 356, 372; tolerance curve, 392 ; velocity quotient, 379

Snake, 362

Boa, 318

garter, 132-134

Sodium, deficit of, 411

Sodium chloride, solutions infused, dog, 57 , $62 \mathrm{f}$.

Solutions, and water exchanges, dog, $56 \mathrm{f}$.

Species, diversities among, $390 \mathrm{ff}$.

physiological characterizations of, 281, 390

Specific gravities, of animals, 195; of urine, 224

Speculations, about regulations, $453 \mathrm{ff}$.

Stability, of function, 103

Stabilization, 440

see also Maintenance, Regulations

Standard deviation $(\sigma), 74$

difference $(\mathrm{S} \Delta), 74$

error (S.E.), 74

States, physiological, 366 ff., 385, 397; balanced, 35,367 ; control, 367 ; loading, 367 , 397 ; normal, 364 ; recovery, 367 369 ; stationary, of exchanges, 41-48, 93-95; of load, 41 f., 367 f., 385,397

Storage, $212 \mathrm{f}$.

Stroke volume, of heart, 354, 406

Substance, body, total, exchanges of, man, $323,326,372,378$; dog, 324 ff., 378, 380 ; rabbit, $323 \mathrm{ff}$.; rat, $325 \mathrm{ff}$.; paths of, mammals, 381
Sucrose, elimination of, man, 372

infusion of, $\operatorname{dog}, 59,61,66$

Surface area, of body, $264 \mathrm{ff}$.

Sweat, chloride in, 376

Survival, 447, 465 f.

Synthetic (chemical) exchanges, 381

System, water-time, 40, 86, 168, 263

water-time-gradient, 263

Tadpole, tail of, regeneration in, $354 \mathrm{ff}$. water turnover in, 269

Temperature, and water exchanges, 275

rectal, dog, 36, 303; man, 303; rabbit, $313,315,317$

regulation, see Heat

Termites, water relations of, 183

Tests, of load, 105, 295, 449

water, $105 \mathrm{f}$.

Theories, defined, 454

of regulations, $467 \mathrm{ff}$.

of water maintenance, 296

Thermostat, in body, 321

Therapeuties, rationale of, 450

Thirst, 60, 232, 245

Thyroxin, and water exchange, dog, 55, 58

Time, as a variable, 283

constant, defined, 388

intervals, 203-205

physiological, 169

relations, in water exchanges, 178-188, 203

series, analysis, 74

Tissues, changes in, 253, 289, $389 \mathrm{f}$; dog, 212 ; frog, 246

dilution of, $\operatorname{dog}, 251$; frog, 247-248, 251; rabbit, 249,251 ; rat, 250 f.

Tolerance curves, 40, 359, 367, 392, 397 diagram, see curves

Tolerated loads, total substance, 325 water, $201 \mathrm{f}$.

Transfusions, rabbit, 153-157

Turnover (rates), measurement of, 363,374 , 397

man, 372

variabilities, $78-84$

water, 78, 195-201

Turtle (Gopherus), 318

Uniformities, see Generalizations

Unit, living, 397 
Urea, retention of, 225

velocity quotient of, dog, 380

washing out of, $230 \mathrm{f}$.

Ureters, ligation, and blood volume, 157

Urine, concentrations in, 224 f., 241

hypotonic to plasma, in frog, 413

production, 110, 215, 223

see also Water, paths of exchanges

Value, physiological, measures of, 417

Vaporization, see Heat, paths of exchanges

Variabilities, 361-364

of blood volume, $156 \mathrm{f}$.

of contents, heat, 6,303 ; water, dog, $73-$ 78 ; frog, 116, various species, 175

of exchanges, water, dog, 78-85; various species, $174 \mathrm{f}$.

of loads, 5, 361

various components, 362

Variables, choice of, for study, $422 \mathrm{ff}$.

combinations of, see Combinations

independent, 416

interrelations among, $284 \mathrm{ff}$.

kinds of, 12, 206 f., 279,396 ff., 423 ff.

physiological, defined, 422

scope of, 280-284

Variation, coefficients of, 74, 175, 361

Velocity diagram, 40

Velocity quotients (k), defined, 37, 203, 388,397

excitability, 358

in dog, glucose, 337, 378, 388; water, 37, 58

in man, $185,372,377 \mathrm{ff}$.

in relation to load, 378

in tissues, 166

net, 37,379

total, 37,379

water, 187, 283

Ventilation, pulmonary, 342, 345, 361

Vole (Microtus), 198

Volume, erythrocyte, 210, 239

extracellular, 209, 248

measurement of changes in, 159; cells and nuclei, 160; isolated cells, $164 \mathrm{f}$.; isolated muscles, 163 ; isolated parts, 161; method, 152, $159 \mathrm{f}$., 207

non-solvent, 260

of blood, and water load of body, 156, 213-224; compensations, 154, 166,
257 ; dog, 213-224; equilibrations, $156 \mathrm{ff}$.; in general, 257; method, 154-155; negative increment by transfusion, 154; rabbit, 153-158, 166 ; rates of exchange, 154, 156; rates of recovery, $156 \mathrm{f}$.; tissues concerned in restoration, 157; total, 156 ; variability of, $156 \mathrm{f}$; see also Blood

of distribution, defined, $151 \mathrm{f}$.; in relation to body water, dog, 208-224; man, $238 \mathrm{f}$.; mean, in dog, man, rabbit, 153

of parts, $207-210$

of plasma, equilibration, $157 \mathrm{f}$; ; load, 157, 208; variability, $156 \mathrm{f}$; see also Plasma

of tissues, in water loads, dog, 207-210; frog, 246 ; man, $238 \mathrm{f}$.

regulation of, in general, 151-167, 397 relations, in general, 151-167

velocity quotients of, 166

Water, absorption, dog, 22, 24, 26, 30 ; man, 101 ; rat, 250

administration, route of, $51-54,58$

balance, 68

behavior toward, dog, 72 ; rat, 129-131; various species, 195

comparisons of exchanges, 102, 172 correlations, similarities and differences in, 168

content, analytical, of various species, 195-197; maintenance of, theories about, 235, 296; measures of, 77 , 207

deficit, Ameba, 148; Arbacia egg, 145; Bipalium, 144; dog, 27-32, $47 \mathrm{f}$.; frog, 110, 114; Helix, 135; insects, 137 f. ; Limax, 134 ; Lumbricus, 143 ; man, 95-99; marine animals, 159; plants, 148; rabbit, 123, 125; rat, $126 \mathrm{f}$. ; reptiles (snake), 132-134

drinking, 150; dog, 27-30,47,61, 66, 267 ; insects, 138 ; man, 96-98; rabbit, 123 ; rat, 126 ; reptiles, 132 ; various mammals, 182

economy quotients, 170 equilibration, 166 ; Ameba, 148; Arbacia egg, 145; dog, 36, 268; frog, 120; Helix, 135; insects, 138; Limax, 
Water, equilibration (continued)

135; Lumbricus, 143; man, 100; marine animals, 149; plants, 149; rabbit, 125; rat, 128; reptiles, 134 ; various species, 188; Zoothamnium, 146

evaporation, dog, 36 ; man, 100 ; see also paths

excess, Ameba, 148; Arbacia egg, 145; dog, 18, 27, 41, 47, 50; frog, 110, 114, 269; Helix, 135 ; insects, 137 ; Limax, 134; Lumbricus, 143 ; man, 88-95, $268 \mathrm{f}$.; marine animals, 149 ; rabbit, 123 ; rat, 126 ; reptiles, $132-$ 134; Zoothamnium, 146-147

exchange, general features, 168 ; in tissues, 210 ; see also rates of exchange gain, see deficit

intake, in relation to body weight, 269275 ; see deficit, drinking

loads, classification, 24 ; in relation to heat loads, $400 \mathrm{ff}$; tolerated, $201 \mathrm{f}$.; types of, 107, 204, 245, 282, 295

loss, see excess, rates of exchange modification ratios, 172

output, in relation to body weight, $270-$ 275 ; see also excess

paths of exchanges, dog, 18, 33, 38, 63; mammals, 172,381 ; man, 89-100; various species, $172 \mathrm{f}$., $189,198-200$ permeability to, see Permeability rates of exchange, 106, 173-176; Ameba, 148; aquatic species, 199; Arbacia egg, 145; Bipalium, 144; bovine, 198, 265 f.; dog, 19-72, 198; frog, 112; Helix, 135 ; insects, 137 ; Limax, 134; Lumbricus, 143; man, 88-107,
198 ; marine animals, 149, 199 ; maximal, 176; plants, 149; rabbit, 123, 198; rat, 126, 198, 265; reptiles, 134 ; various species, 198; Zoothamnium, 146

recovery of content, Ameba, 148; $A r b a$ cia egg, 145; Bipalium, 144; dog, 19, 28, 378; frog, 110, 115-116; Helix, 135; insects, 137; Limax, 134 ; Lumbricus, 144 ; man, 90 ; marine animals, 149; plants, 149; Phascolosoma, 139; rabbit, 123 ; rat, 126-129; reptiles, 132-134; Zoothamnium, 146

relations, in general, 15-298, especially 286-290

requirements, 296

reserves, in tissues, 207

return, dog, 23 ; man, 90

tolerance curves, blood, $154 \mathrm{f}$.; dog, $23 \mathrm{f}$.; frog, 111; leucocyte, 165; man, 88 f., 392; rabbit, 124; rat, 128; snake, 133; various species, $179 \mathrm{f}$.

turnover, 175, 198-200, 269-275

variability, of content, 73-78, 104, 116, 175,195 ; of exchange, $78-85,104$, $123-125, \quad 173-175$; see also exchange

velocity quotient of, Ameba, 148; Bipalium, 144; defined, 37 ; dog, 37, 58, 380; frog, 114; man, 91, 93; marine animals, 149 ; total, 37 ; various species, 187

Weight, body, 70, 361

Worms, see Bipalium, Lumbricus, Phascolosoma

Zoothamnium (ciliate), water in, 146-148 







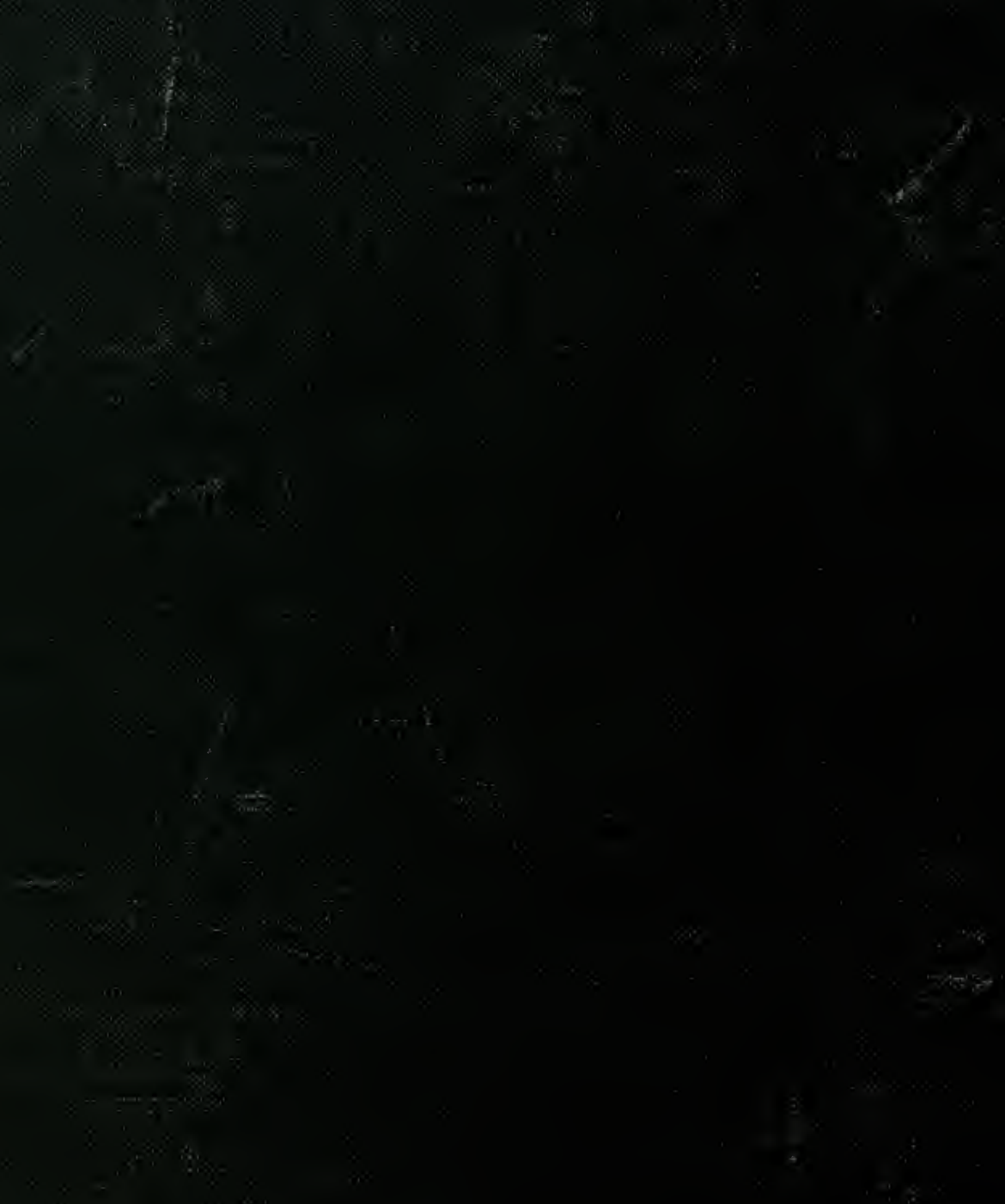

